
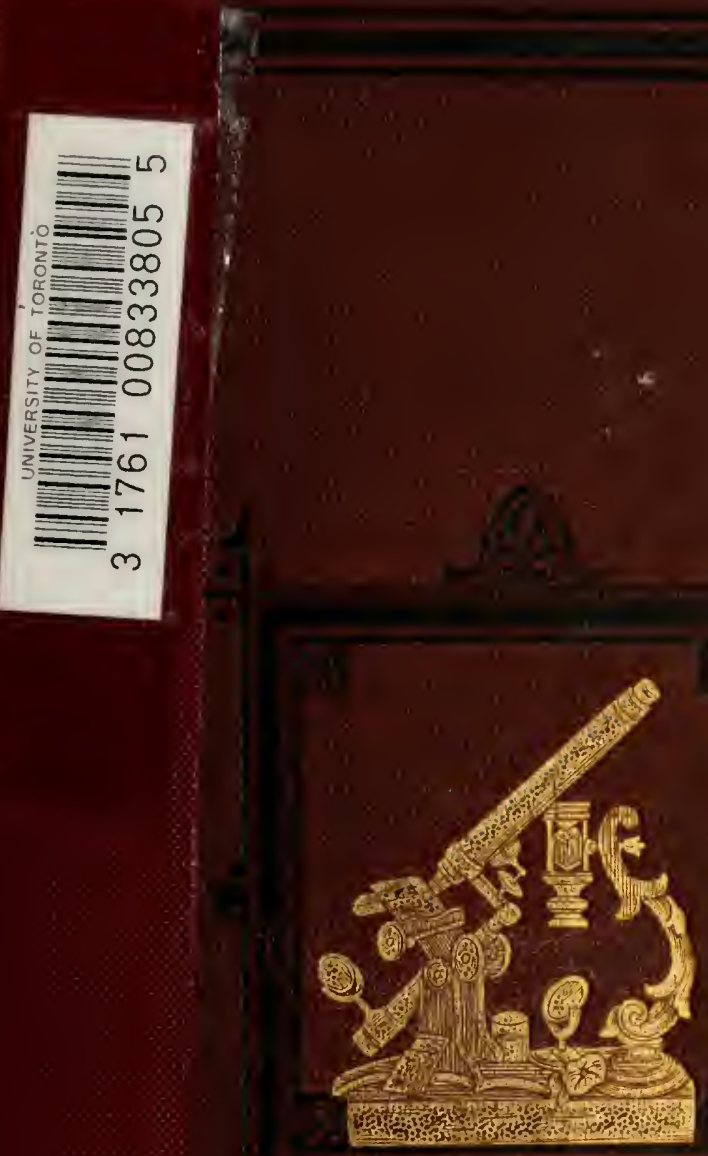

\title{
Handle with EXTREME CARE
}

This volume is BRITTLE

and cannot be repaired

Photocopy only if necessary GERSTEIN SCIENCE INFORMATION CENTRE 


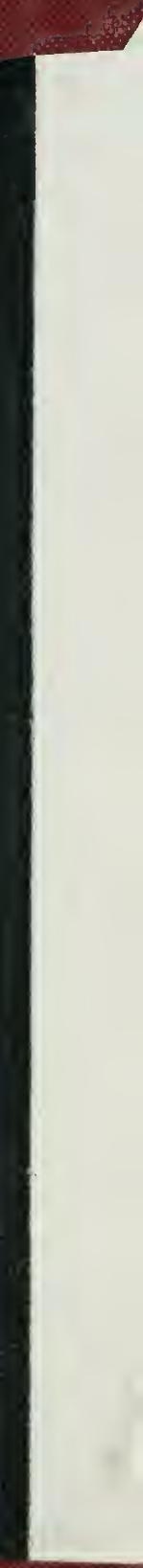

ex: 
Digitized by the Internet Archive in 2008 with funding from Microsoft Corporation 


\section{THE MICROSCOPE.}




\section{THE MICROSCOPE}

ITS

\section{HISTORY, CONSTRUCTION, AND APPLICATION}

BEING

A EAMILIAR INTRODUCTION TO THE USE OF THE INSTRUMENT

AND THE STUDY OF MICROSCOPICAL SCIENCE

BY

\section{JABEZ HOGG}

Senior-Surgeon to the Royal Westminster Ophthalmic Hospital Ophthalmic Surgeon to the Royal Masonic Schools

Consulting Ophthalmic Surgeon to the Hospital for Diseases of Women and Children

Fellow and late Vice-President of the Medical Society of London President of the Medical Microscopical Society; F.R.M.S., $\sigma^{2} c_{*} \xi^{2} c$.

WITH MORE THAN FIVE HUNDRED ENGRAVINGS, AND COLOURED ILLUSTRATIONS BY TUFFEN WEST.

\section{题的 Evition}

LONDON AND NEW YORK

GEORGE ROUTLEDGEAND SONS 


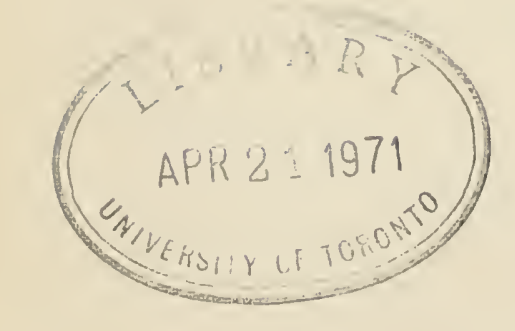




\section{PREFACE TO THE SIXTH EDITION.}

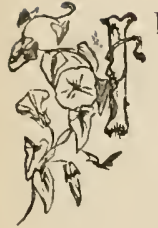

$\mathrm{N}$ issuing the Sixth Edition of this Work on the Mroroscope, we may state that it has been thoroughly revised and for the most part re-written. Eight carefully and beautifully executed Plates are added, which were drawn by Tuffen West from natural objects, engraved and printed by Edmund Evans in the first style of colourprinting.

The Author cannot but express his grateful surprise at the extraordinarily popular reception which his book has met with : a sale of fifty thousand is an unprecedented event for a work of the kind. This circumstance is extremely gratifying to him, because it affords reasonable grounds for believing that his work has been useful, and encourages renewed effort to make the volume still more acceptable. It has been his endeavour to bring the information contained in its pages up to the most recent discoveries; although, in a daily progressing field of science, it is almost impossible to keep pace with the advance of knowledge in all its ramifications.

The passing remarks the Author has seen occasion to make upon the various objects that have fallen under his notice are intended to serve, as would a finger-post or 
guide in a country abounding in treasures. It was impossible to have attempted a more detailed description than has been given in dealing with the vast expanse of natural objects presented to his contemplation. If, however, the notices have been sufficiently ample and precise to assist the study of the reader, the Author will have accomplished the most cherished object he had in view in presenting the work to the public.

\section{Bedrohd Squarf,}

Octaber, :8c7. 


\section{PREFACE TO THE FIRST EDITION.}

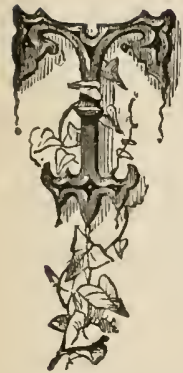

HE Author of this Publication entered upon his task with some hesitation and diffidence; but the reasons which influenced him to undertake it may be briefly told, and they at once explain his motives, and plead his justification, for the work which he now ventures to submit to the indulgent consideration of his readers.

It had been to him for some time a subject of regret, that one of the most useful and fascinating studies-that which belongs to the domain of microscopic observation-should be, if not wholly neglected, at best but coldly and indifferently appreciated by the great mass of the general public; and he formed a strong opinion, that this apathy and inattention were mainly attributable to the want of some concise, yet sufficiently comprehensive, popular account of the Microscope, both as regards the management and manipulation of the instrument, and the varied wonders and hidden realms of beauty that are disclosed and developed by its aid. $\mathrm{He}$ saw around him valuable, erudite, and splendid volumes, which, however, being chiefly designed for circulation amongst a special class of readers, were necessarily pub- 
lished at a price that renders them practically unattainable by the great bulk of the public. They are careful and beautiful contributions to the objects of science, but they cannot adequately bring the value and charm of microscopic studies home, so to speak, to the firesides of the people. Day after day, new and interesting discoveries, and amplifications of truth already discerned, have been made, but they have been either sacrificed in serials, or, more usually, devoted to the pages of class publications; and thus this most important and attractive study has keen, in a great measure, the province of the few only, who have derived from it a rich store of enlightenment and gratification: the many not having, however, participated, to any great extent, in the instruction and entertainment which always follow in the train of microscopical studies. $^{1}$

The manifold uses and advantages of the Microscope crowd upon us in such profusion, that we can only attempt to enumerate them in the briefest and most rapid manner in these prefatory pages.

It is not many years since this invaluable instrument was regarded in the light of a costly toy; it is now the inseparable companion of the man of science. In the medical world, its utility and necessity are fully appreciated, even by those who formerly were slow to perceive its benefits; now, knowledge which could not be obtained even by the minutest dissection is acquired readily by its assistance, which has become as essential to the anatomist and pathologist as are the scalpel and bedside observation. The smallest portion of a diseased structure, placed under a Microscope, will tell more $i_{u}$ one minute to the experienced eye, than could be ascertained by long examina-

(1) At the time this work was written, scarcely a book of the kind had been published at a price within the reach of the working classes. 
tion of the mass of disease in the ordinary method. Microscopic agency, in thus assisting the medical man, contributes much to the alleviation of those multiplied "ills which flesh is heir to." So fully impressed were the Council of the Royal College of Surgeons with the import ance of the facts brought to light in a short space of time, that, in 1841, they determined to establish a Professorship of Histology, and to form a collection of preparations of the elementary tissues of both animals and regetables, healthy and morbid, which should illustrate the value of microscopical investigations in physiology and medical science. From that time, histological anatomy deservedly became an important branch of the education of the medical student.

In the study of Vegetable Physiology, the Microscope is an indispensable instrument; it enables the student to trace the earliest forms of vegetable life, and the functions of the different tissues and vessels in plants. Valuable assistance is derived from its agency in the detection of adulterations. In the examination of flour, an article of so much importance to all, the Microscope enables us to judge of the size and shape of the starch-grains, their markings, their isolation and agglomeration, and thus to distinguish the starch-grains of one meal from those of another. It detects these and other ingredients, invisible to the naked eye, whether precipitated in atoms or aggregated in crystals, which adulterate our food, our drink, and our medicines. It discloses the lurking poison in the minute crystallisations which its solutions precipitate. "It tells the murderer that the blood which stains him is that of his brother, and not of the other life which he pretends to have taken; and as a witness against the criminal, it on one occasion appealed to the very sand on which he trod at nidnight." 
The zoologist finds in the Microscope a necessary cooperator. To the geologist it reveals, among a multiplicity of other facts, "that our large coal-beds are the ruins of a gigantic vegetation; and the vast limestone rocks, which are so abundant on the earth's surface, are the catacombs of myriads of animal tribes, too minute to be perceived by the unaided vision."

By "conducting the eye to the confines of the visible form," the Microscope proves an effective auxiliary in defining the geometric properties of bodies. Its influence as an instrument of research upon the structure of bodies has been compared to that of the galvanic battery, in the hands of Davy, upon Chemistry. It detects the smallest structural difference, heretofore inappreciable, and, as an ally of Chemistry, enables us to discovar the very small changes of form and colour effected by test-fluids upon solids; and dissects for us, so to speak, the most multiplex compounds. It opens out to the mind an extended and vast tract, opulent in wonders, rich in beauties, and boundless in extent.

The Microscope not only assists studies, and develops objects of profound interest, but also opens up innumerable sources of entertainment and amusement, in the ordinary conventional acceptation of these terms;-discrosing to us peculiarities and attractions in abundance;impressing us with the wonderful and beautifully-skilful adaptation of all parts of creation, and filling our minds with additional reverence and admiration for the beneficent and Almighty Creator.

The Author will conclude these prefatory observations with a few words in explanation of his arrangements, and by way of acknowledgment to those to whom he is indebted. He has sought, in the volume that he now lays before the public, to point out and elucidate at once in a 
practical manner and in a popular style, the vast fund of utility and amusement which the Microscope affords, and has endearoured to touch upon most of the interesting subjects for microscopic observation as fully as the restrictions of a limited space, and the nature of the succinct summary, would permit. To have dwelt upon each in complete detail would have necessitated the issue of many expensive volumes - and this would have entirely frustrated the aim which the writer had in view; he has, therefore, contented himself with the humble, but, he trusts, not useless, task of setting up a finger-post, so to say, to direct the inquirer into the wider road. In the section of the work devoted to the minuter portion of creation, he has ventured to dwell somewhat longer, in the belief that that department is more especially the province of the microscopist. $\mathrm{He}$ has arranged his topics under special headings, and in separate chapters, for the sake of perspicuity and precision; and has brought the ever-welcome aid of illustrations to convey his explanatory remarks more vividly to the minds of his readers. $\mathrm{He}$ is peculiarly indebted to Professor Quekett, whose valuable lectures, delivered annually in the Royal College of Surgeons, and whose multifarious and successful researches, have pre-eminentiy distinguished him as the microscopist of the day. From notes made during the lectures spoken of, and from the many admirable papers which this gentleman has published, much sound information has been gleaned; and the Author has to thank him, in the most sincere and cordial manner, for placing at his disposal the mass of contributions with which he has enriched microscopical science. A free use has been made of the researches of scientific investigators generally -Leeuwenhoek, Ehrenberg, Carpenter, Johnston, Ralfs, Busk, Gosse, Huxley, Hassall, Lobb, Davies, and other members of the Microscopical Society of London. His 
acknowledgments are likewise due to Mr. George Pearson, for the great care he has bestowed upon the engravings which illustrate these pages.

Finally, it is the Author's hope that, by the instrumentality of this volume, he may possibly assist in bringing the Microscope, and its most valuable and delightful studies, before the general public in a more familiar, compendious, and economical form than has hitherto been attempted; and that he may thus, in these days of a diffused taste for reading and the spread of cheap publications, supply further exercise for the intellectual faculties,-contribute to the additional amusement and instruction of the family circle,--and aid the student of nature in investigating the wonderful and exquisite works of the Almighty. If it shall be the good fortune of this work, which is now confided with great diffidence to the consideration of the public, to succeed in however slight a degree, in furthering this degign, the Author will feel sincerely happy, and will be fully repaid for the attention, time, and labour that he has expended.

LeNDON, May, 1851. 


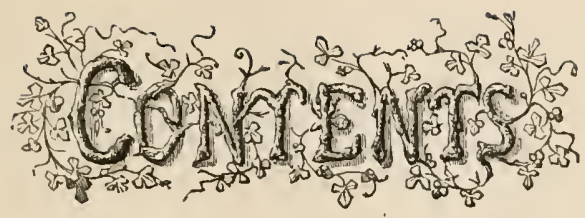

PART I.

HISTYRY OF THE INVENTION AND IMPROVEMENI'S OF THE MICROSCOPE.

\section{CHAPTER I.}

\section{PAGE}

HISTORY OF THE INVENTION AND IMPROVEMENTS OF THE MICROSCOPE •. • . . . . . . . . . 1

\section{CHAPTER II.}

MECHANICAL AND OPTICAL PRINCIPLES INVOLVED IN THE CONSTRUCTION OF THE MICROSCOPE-LENSES-MODE OF ESTIMIATING THEIR POWER - MICROMETERS - POLARISED LIGHT-CAMERA LUCIDA - BINOCULAR INSTRUNENTPHOTOGRAPHIC-DRAWING-MICROSPECTROSCOPY - ACHROMATIC LENSES - MAGNIFYING POWER - WOLLASTON'S DOUBLET-CODDINGTON'S LENG-SIMPLE AND COMPOUND MICROSCOPES - QUEKETT'S, RUSS'S, POWELL AND LEALAND'S, BAKER'S, PILLISCHER'S, LADDS', MURRAY'S, HGHLFX's, COLLINS'S, AND OTHER MICROSCOPES . . . . 
CHAPTER III.

PRELIMINARY DIRECTIONS-ILLUMINATION-ACCESSORY APPARATUS-ACHROMATIO ILLUMINATOR - GILLETT's, ROSS'S, WEBSTER'S, AND OTHER CONDENSERS-OBLIQUE ILLUMINATION-DOUBLE PRISM ILLUMINATION-LIEBERKÜHN -SIDE-REFLECTOR-OBJECT-FINDERS - SECTION CUTTERS -COLLECTING OBJECTS-MODE OF INJECTING-PREPARING AND MOUNTING OBJECTS, ETC. . . . . . . . . 159

\title{
PART II.
}

\author{
CH A P T E R I.
}

VEGETABLE KINGDOM-VITAL AND CHEMICAL CHARACTERISTICS OF CELLS-MICROSCOPIC FORMS OF VEGETABLE CELLSFUNGI-FUNGOID DISEASES-ALGE-CONFERVA-DESMIDIACEA - MOSSES - FERNS - STRUCTURE OF PLANTSADULTERATION OF ARTICLES USED FOR FOOD-PREPAIRATION AND MOUNTLNG-FOSSIL PLANTS . . . . . . $\$ 55$

\section{CHAPTER II.}

PBOTOZOA-GREGARINE-RHIZOPODA - POLICYSTINA - DIATOMACEA-FOSSIL INFUSORIA-SPONGES - VORTICELLAACTINIZOA - ROTIFERA-POLYPIFERA-ACALEPIIA - ECHIMODERMATA 


\section{CHAPTER III.}

POLYZOA-MOLLUSCA-GASTEROPODA-BRACHIOPODA-CONCH1FERA - CEPHALOYODA - PTEROPODA - TUNICATA - CRUSTACEA-ENTOMOSTRACA-CIRRIPEDA - ENTOZOA - ANNU LGSA-ANNELIDA . . . . . . . . . . . . . E12

\section{CHAPTER IV.}

SUB-EINGDOM ARTICULATA-INSECTA-ARACHNIDA . . . . 579

\section{CHAPTEK V.}

VERTEBIDATA - PHYSIOLOGF - HISTOLOGY - BOUNDARY BETWESN THE TWO KINGDOMS-CELL THEORY-GROWTH OF TISSUES-SPECIAL TISSUES-SKIN, NERVES, CARTILAGE, TEETH, BONE, ETC. . . . . . . . . . . . 654

\section{CHAPTER VI.}

INORGANIC OR MINERAL KINGDOM-FORMATION OF CRYSTALS -POLARISATION-SPECTRUM ANALYSIS, ETC. . . . . 728 



\title{
DESCRIPTION OF COLOURED PLATES.
}

\author{
PLATE I.-Page 255.
}

\section{PROTOPHYTA.}

Fig. 1. Peziza bicolor-2. Truffle-3. Sphæria herbarum : $a$. piece of dead plant, with S. herbarum natural size; $b$. section of same, slightly magnified; $d$. $\Lambda$ scus with spores, and paraphyses more magnified-4. Peziza pygmaea-5. Proliferous form of same-6. P. corpulasis; Ascus with spores and paraphyses, merely given as a further illustration of structure in Peziza-7. Yeast, good-8. Teast, exhausted-9. Phyllaetinia guttata-10. Ycast with favis spores and nycelium-11. Favus ferment; with torula and bacteria-like lodies-12. Puccinia-like-form in ditto, growing from saccharine solution13. Aërozoa-14. Sporules, de. from eczema produced by ycast-15. Volvox globator-16. Amœeloid condition of portion of volvox-17. Puccinia buxi18. Ditto, more enlarged-(17 to 20 illustrate Coniomycetes $)-19$. Acidium grossularix, transverse section of leaf of currant aftected by: $a$. spermogones on upper surtace; $b$. perithecia with spores-20. Phragmidium bulbosum, development of-21. Parmelia parietina, trans. section through a spermogone, showing green gonidia and spermatia escaning-22. Aeidium berberidis, from leaf of berberry-23. Vaucheria sessilis-24. Stephanospliæra pluvialis: $a$. Full-grown example, germ-cells spindle-shaped, with protoplasmic elongations. $b$. Resting-cell. c. division into four. $d$. Free swimming ciliated young specimen. e. Amœboid condition-25. $a, b, c, d, e$, $f$ and $g$, Development of Lichen gonidia-26. Parmelia stellaris (Lichen), vertical section tlirough apotheeium, showing asci, spores, and paraphyses, with gonidia and filanentous medulla. a. Spermatophore with spermatia -27. Moss gonidia assuming amœboid form.

The intention of this plate has been, first to show illustrations of various forms of Protophytes, as the lowest types of vegetable structure; (from 7 to 14), supposed modes of development or rudimentary conditions; and Confervoidere illustrated by Vaucheria in the Siphonacer, f. 23, Stephanosphrra 24, and Volvox 15, in the Volvoeiner; and their remarkable amœboid conditions illustrated by $16,24 e$, and 27 . The protean forms assumed in developmeni by 7 to $14 ; 24 a$ to $e ; 25 a$ to $g$, and 27 .

\section{PLATE II.-Page 274.}

\section{PROTOPHYTA. ALG正.}

Fig. 27. Ceramium acanthonotum -28 . Triploceras gracile -29 . Cosmanum radiatum -30 . Micrasterias denticulata-31. Docidium pristid -32 . Call:thannion plumula-33. Diatomacex, living: $\alpha$. Licmophora splendida: $b$. Achnanthes longipes; c. Grammatophora marina. These figures are inteuded to show the general claracter of the eudochrome and growth of frustule-34. Callithammion refractum-35. Jungermannia albicans. b. represents elater and spores-36. Leaf with antheridia, or male elements, which are represented nıore magnified at $a$ to the left of the figure-37. Ceramiumechinotum-38. Pleurosigma angulatum, side view-39. Delesseria hypoglossum-40. Pleurosigma angulatum, front view, endochrome not reproBented-41. Ceramium flabelligerum.

The intention of this plate is chiefly to show various forms of Algre. The figures of Desinids, 28 to 33 , illustrate the appearance 


\title{
xviii DESCRIPTION OF COLOURED PLATES.
}

of the endochrome, and introduce some new forms, described by E. G. Lobb and W. Archer, Quar. Jour. Micro. Sci. vol. v. 1865, p. 255 : and 38,40 to illustrato diatom circulation.

\section{PLATE III.-Page 376.}

\author{
PROTOZOA-RHIZOPODA.
}

rigs, 43, 44, 45, 46, 47, 48, 49, 50, 51, 52. These figures are from diagrams by Major Owen, to illustrate a paler given in Jour. Linn. Soc. vol. viii. p. 202. They illustrate forms of living Polycystina, sketched from life by Major Owen, and show the riclıly coloured appearance of the sarcode; figs. 48 to 52 -53 . Monocystis lumbrieorum, round form-54. Monocystis lumbricorum, the usual elongated sliape-55. Monoeystis serpulæ-56. Gregarina Sieboldii ; illustration of septate form, with retlexed hook-like lirocesses57. Monocystis lumbricorum, eneysted - 58. Monocystis lumbrieorum, more advanced and nseudo-navice'læ forming -59. Monocystis lumbricorum, free pseudo-navicella of $-60,61$. Monocystis lumbricorum, amœboid forms of -62 . Cruciate sponge-spicule-63. Asteromua Humboldtii-64. Eözoon Canadense, rejresents appearance of a portion of the natural size-65. Eözoon Canadense, magnified, showing portions of cell-walls left uncoloured, the animal sar'code inhabiting it coloured dark green as in nature, and converted by fossilization into a siliceous nineral: the narrow bands passing between these are processes (stolons) of the same substance-60. Actinophrys Sol, budding- 67 . Euglena viridis : $a$. contracted, $b$. elongated form -68. Acineta tuberosa - 69. Eeistes longicornis (Davis). - 70. Oxytricha gibba (side view) -71. Oxytricha pellionella - 72. Limnias (? n. sp.) - 73. Cyclidium glancoma-74. Glaucoma scintillans -75 to 79,80 to 85 , illustrate forms of Foraminifera found by Major Owen, living-75. Globigerina (Orbulina) acerosa, n. sp., broken open to show interior-76. Globigerina (Orbulina) continens, n. sp. broken open to show interior-77. Globigerina hirsuta-78. Globigerina (Orbulina) universa-79. Globigerina bulloides80. Conochilus vorticella - 81 . Globigerina bulloides-82. Globigerina inflata, sinistral shell-83. Pulvinulina Micheliniana-84. Pulvinulina Canariensis-85. P. Menardii.

The figures of recent Polycystina illustrate the surface-fauna of mid-oceall; the original drawings were made from living specimens, as were those of the Foraminifera; they well represent in their natural state these elegant and interesting objects. 53 to 61 give some idea of the state of our kuowledge of forms and life-history of the Gregarine. Figures of Infusoria 67, 68, 70, 71, 73, 74: 69,72 , new forms of Rotifera.

\section{PLATE IV.-Page 511.}

\section{POLYZOA.}

Fig. 86. Hartea elegans-87. Side view of Synapta spicula-88. Ophiocoma rosula (very immature specimen), $a$. Claw hooks, b. Palmate spicula. The development of this creature has been deseribed by G. Hodge, in Transactions of Tyneside Naturalists' Field-Club-s9. Spine of a star-fish, particularly interesting as showing the reticular calcareous network obtaining in this as in all other hard parts of the Echinodermata - 90. Very minute Syatangus, obtained from stomach of a bream: many of the spines are gone, but the structure of the shell is intact and forms a beautiful object, interesting in connexion with the source whence obtained-91. Ophiocoma neglecta ; wriggling or brittle Starfisli. The plate does not admit of a figure on a scale sufficient to show the full beauty of this object-92. Tubularia Dumortieri-93. Pedicellaria mandibulata from Uraster glacialis-94. Pedi- 
cellaria forcepiforma, from the same- 95 . Cristatella mucedo, statoblast96. Cristatella mucedo, statoblast, edge-view-97. Early stage of development of same-9S. Lopliopus crystallinus-99. Plumatella repens and ova, on a piece of submerged stem-100. Tienia echinococcus-101. Mydatids in human liver-102. Bilharzia hrematobia-103. Amplistoma conicum-104. Trichina spiralis from Hambro' pork-105. Trichina spiralis extracted106, 107. Fasciola gigantea, after Cobbold.

\section{PLATE V.-Page 538.}

\section{MOLLCSCA.}

Fig. 10S. Velutina lævigata, portion of lingual mem̄brane-109. Velutina liev. gata, part of mandible-110. Hybocystis blennius, portion of palate-111. Sepia officinalis, portion of palate-112. Aplysia liybrida, part of mandble-113. Loligo vulgaris, part of palate-114. Haliotis tuberculatus, part of palate-115. Cistula catenata, part of palate-116. Patella radiata, part of palate-11\%. Acmeoea virginea, part of palate-118. Cymba olla, part of palate - 119. Scapander liguiarius - 120. Oncidoris bilamellata, part of palate-121. Testacella Maugei, part of palate-122. Pleurobrauchus plumula, part of mandible-123. Turbo marnoratus, part of palate.

Figs. 108 to 123, Lingual membranes of Molluses ; the drawings made by Mrs. Maples from specimens in the late S. P. Wood. ward's collection, now the property of F. E. Filwards, Esq.

Chosen without any special order, and simply as showing good examples of the wonderful forms met with in the mouth-armature of Gasteropod and Cephalopod Mollusca; viewed by polarised light and selenite stage.

\section{PLATE VI.-Page 576.}

\section{INSECTA.}

Fig. 124. Egg of Caradina Morphens, Mottled Rustic Moth-125. Egg of Tortoiseshell Butterfiy, Vanessa Urtica-126. Egg of Common Footman, Lithosia complanula - 127. Egg of Shark Jloth, Cucullia Umbratica - 128. Mapleaphis-129. Egg-shell of Acarus, emlty - 130. Egg of House-Fly - 131. Mouth of Tsetse-Fly, Glossina morsitans - 132. Vapourer Moth, Orgyia antiqua: antenna of male-133. Vapourer Moth, antenna of female: $\boldsymbol{a}$. branch more magnified to show rudimentary condition of the parts 134. Tortoise-shell Butterfly; head in profile, showing large compound eye, one of the palpi, and spiral tongue-135. Tortoise-beetle, Cassida viridis; under surface of left fore-foot, to show the bifurcate tenent ap: pendages, one of which is given at $a$, more magnified. This form of appendage is characteristic of the family. See West on Feet of Insects, Linn. Trans. vol. xxiii. tab. $43-136$. Egg of lise Argus Butterfly, Polyonmatus Argus - 137. Egg of Mottled Umber, Eranuis Defolaria138. Egg of Ennomos erosaria, Thorn-Moth-139. Egg of Aspilates Gilvaria, Straw Belle - 140. Blow-lly, Musca Vomitoria; left fore-foot, undersurface, to show teuent hairs; $a b$ more magnified; $a$ from below, $b$ from the side-14l. House-lly larva-142. Amara comniunis, left fore-foot, under-surface, to show form of tenent appendages, of which one is given more magnitied at $a$. These, in the ground beetles, are met with only in the males, and seem to be used for sexual purposes. The way in which they are protected when not in use is shown by T. West-l43. Epliydra riparia: left fore-foot, uuder-surface. This fly is met with sometimes in immense numbers on thic water in salt-marshes; it has no power of elimbing on glass, wlich is explained by the structure of the tenent hairs; che central tactile organ also is very peculiar, the whole acting as a tloat, oue to each foot, to enable the fly to rest on the surface of the water; a. ono 
of the external hairs-144. Egg of Bot-Fly ; the larva just escaping-145. Fgg of parasite of Pheasant-146. Egg of Seatophaga-147. Egg of parasite of magpie-148. Egg of Jodisvernaria (Small Emerald Butterfly).

\title{
PLATE VII.-Page 6.54.
}

\author{
V E R T E B R A. T A.
}

Fig. 149. Toe of monse, integuments, bone of foot, and vessels-150. Tongue of mouse, showing erectile papille, muscular layer, \&c.-151. Brain of rat, showing its vascular supply-152. Tongue of cat, showing fungi-form papillas, with capillary loojs passing into them, vessels, \&c.; perfendicular section -153. Kidney of eat, showing Malpighian turfts and arteries-154. Small tntestine of rat, showing villi and layer of mucous membrane-155. Nose of mouse, showing vaseular supply to roots of whiskers -156 . Vascular supply to internal gill of tadpole, during one phase of its development-15T. Seetion through sclerotic and retina of cat's-cye, slowing vascular supply of choroid and other coats-158. Internal gill of tadpole, fully developed, exhibiting crests and vascular system, after Whitney.

This plate is designed to show the value of injected preparations in the delineation of animal structures. By thus artificially restoring the blood and distending the tissuc, a much better idea is obtaiued of the relative condition of parts during life, while we receive much assistance in the elucidation of complicated and delicate membranes, the appearance of erectile tissues, papillæ, \&c.

\section{PLATE VIII.-To face Title.}

\section{POIAARISCOPE OBJECTS.}

Fig. 158. New Red Sandstone-159. Qriartz-160. Granite-161. Sulph. Copper -162. Saliginine - 163. Sulpk. Iron and Cobalt, crystallized in the way described by Thomas - 164. Borax - iô. Salph. Nickel and Potash-166. Kreatine - 167. Starch granules - 16s. Aspartic Acid - 169. Fibro-Cells, Orchid. -170 . Equisetum euticle $\rightarrow$ 171. Spicula Holothuria, Australia-172. Spicula Holothuria, Port Essington-173. Dentzia Scabra; npper and under surface-174. Cat's tongue, process-175. Prawn shell, exuvia with crystals of lime-176. Grayling scale - 177. Scyllium eaniculum seale -178 . Rhinoceros horn, transverse section - 179. Horse hoof-180. Dytiscus, elytra with erystals of lime.

This plate is especially intended to illustrate the beautiful and gorgeous spectacle produced by polarised light on the various olvjects here grouped together. It will be seen that all structures belonging either to the animal, vegetable, or mineral kingdoms in which the power of unequal or double refraction is suspected to be present, are those which may be submitted to this mode of micro. chemical investigation.

PLATE IX.-Woodcut.-Page 49 S.

ASTEROIDEA-ECHINIDE-CRUSTACEA, \&C. 


\section{, ERRATA.}

Page 134, line 32, for "polarised" read "common."

_ 459, line 13, for "Honey" read "Horney."

— 636, line 25, for "Acarus" read "Sarcoptes." 



\section{THE MICROSCOPE.}

\section{PART I.}

HISTORY OF THE INVENTION AND IMPROVEMENTS OF THE MICROSCOPE.

\section{CHAPTER I.}

HISTORY OF THE MICROSCOPE.

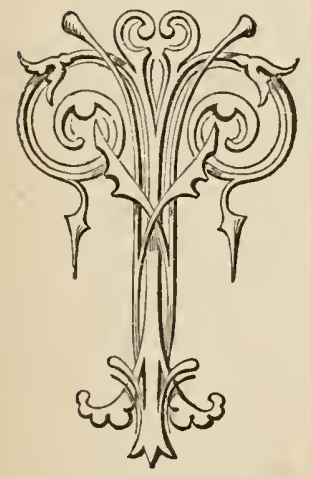

HE instrument known as the Microscope derives its name from

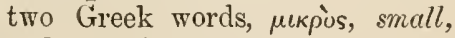
and $\sigma \kappa о \pi \epsilon \omega$, to view; that is, to see or view such minute objects as without its aid would be invisible.

The honour of the invention is claimed by the Italians and the Dutch; the name of the inventor, however, is lost. Probably the discovery did not at first appear sufficiently important to engage the attention of those men who, by their reputation in science, were able to establish ar. opinion of its merit, and to hand down the name of ite inventor to succeeding ages.

If we consider the microscope as an instrument con , sisting of one lens only, it is not at all improbable that i: was known at a very early period, nay even in a degree $t$ : 
the Greeks and Romans; at any rate, it is tolerably certain that spectacles were used als early as the thirteenth century. Now as the crlasses of these were made of different cunvexities. and consequently of different magnifying powers, it is natural to suppose that smaller and more convex lenses were made, and apulied to the examination of minute oljects. Many anong the learned refuse to the aucients a knowledge of mangifying lenses, and à fortiori that of refracting $t$ lescopes, since, according to them, the Greeks and Rumans had only very imperfect notions with respect to the fabrication of glass.

From a passage in Aristophanes it is plain that globules of glass were sold at the shops of the grocers of Athens, in the time of that comic author. He speaks of them as "burning spheres."

Pliny states that the immense theatre (it was capable of containing eighty thousnnd prersons) erected at Rome by Scanrus, son-in-law of Sylla, was three stories in height, and that the second of these stories was entirely inlaid with a mosaic of glass.

Ptolemy, in his "Optics," has inserted a table of the refractions which light experiences under different angles of incidence, in passing from air into glass. The values of these angles, which differ only in a slight degree from those obtained in the present day by means of similar experiments, prove that the glass of the ancients differed very little from that manufictured in our own times.

There is in the French Cibinet of Medals a seal, said to have belonged to Nichael Angelo, the fabrication of which, it is believed, ascends to a very remote epoch, and upon which fifteen tigures have been engraven in a circular space of fourteen millimètres in diameter. These figures are not all visible to the nakid eye.

Cicero makes mention of an Iliad of Homer written upon parchment, whuch was comprised in a nutshell.

Pliny relites that Mymmecides, a Milesian, execnted in ivory a square figure which a fly covered with its wings.

Unless it be maintained that the powers of vision of our ancestors surpassed those of the most skilful modern artists. these ficts establish that the magnifying property of leuses was known to the Greeks and Romans nearly 
two thousand years ago. We may besides advance a step fiurther; and borrow from Seneca a passage whence the same truth will emerge in a mamner still more direct and decisive. In the "Nitural Questions" we read: "However small and obsetive the writing may be, it appears larger and clearer when viewed through a globule of glass filled with water."

Dutens has seen in the Museurn of Portici ancient lenses which had a focal length of only uine mullimètres. He actually possessed one of these lenses, but of a longe: forus, which was extracted from the ruins of Hereulaneum.

At the meeting of the British Association, held at Belfast in the year 1852, Sir David Brewster showed a plate of rock-crystal worked into the form of a lens, which was recently found among the ruins of Nineveh. Sir David Brewster, so competent a judge in a question of this kind, maintained that this lens had been destined for optical purposes, and that it never was an article of dress.

It is not difficult to fix the period when the microscope first beyan to be generally knuwn, and to be used for thie purpose of examining minnte ohjects; for though we are ignorant of the name of the first inventor, we are acquainted with the names of those who introduced it to public view. Zacharias Jansens and his son are sail to have made microseopes before the year 1590 : about that time the ingenious Cornelius Drebell hrought one made by them with him to England, and showed it to William Borrell and others. It is possible this instrument of Drebell's was not strictly what is now ealled a microseope, but was rather a kind of mieroscopic telescope, something similar in principlo to that lately described by M. Aepinus in a letter to the Academy of Sciences at St. Petersburg. It was formed of a copper tube six fect lung and one inch in dinmeter, supported by three brass pilliurs in the shape of dolphins; these were fixed to a bise of ehony, on which the objects to be viewed by the microseope were placed. Fontana, in a work which he published in 1646 , says that he had made microseopes in the year 1618 : this may be perfectly true, without derogating fion the merit of the Jansens; for we bave many instancer in our own times of more than 
one person having made the same invention nearly simu]taneously, without any communication from one to the vther. In 1685 Stelluti published a description of the parts of a bee, which he had examined with a microscope.

The history of the microscope, like that of nations and arts, has had its brilliant periods. in which it shone with uncommon splendour, and was cultivated with extraordinary ardour; and these have been succeeded by intervals marked with no discovery, and in which the science seemed to fade away, or at least to lie dormant, till some favourable circumstance-the discovery of a new object, or some new improvement in the instruments of observationawakened the attention of the curions, and reanimated their researches. Thus, soon after the invention of the microscope, the field it presented to observation was cultivated by men of the first rank in science, who enriched almost every branch of natural history by the discoveries they made by means of this instrument.

The Single, or Simple Microscope. - We shall first speak of the single microscope, that having been invented and used long before the double or compound microscope. When the lenses of the single microscope are very conrex, and consequently the magnifying power great, the field of view is small ; and it is so difficult to adjust with accuracy their focal distance, that it requires some practice to render the use of them familiar. It was with an instrument of this kind that Leeuwenhoek and Swammerdam, Lyonet and Ellis, examined the invisible forms of nature, and by their example stimulated others to the same pursuit.

About the year 1665 , small glass globules began to be occasionally applied to the single microscope, instend of conrex lenses; and by these globules an immense magnifying power was obtained. Their invention has been generally attributed to M. Hartsoeker; though it appears that we are really indebted to the celebrated Dr. Hooke for this discovery, for he described the manner of making them in the preface to his Micrographia Illustrata, published in the year 1656 .

Mr. Stephen Gray ${ }^{1}$ having observed some irregular particles within a glass globule, and finding that they

(1) Phjosophical Transactions, 1696. 
appeared distinct and prodigiously magnified when held close to his eye, concluded, that if he placed a globule of water in which there were any particles more opaque than the water near his eye, he should see those particles distinctly and highly magnified. The result of this idea far exceeded his expectation. His method was, to take on a pin a small portion of water which he knew contained some minute animalcules; this he laid on the end of a small piece of brass wire, till there was formed somewhat more than a hemisphere of water; on applying it then to the eye, he found the animalcules enormously magnificd; for those which were scarcely discernible with his glasis globules, with this appeared as large as ordinary-sized peas.

Dr. Hooke thus describes the method of using this water-microscope: "If you are desirous," he says, "of obtaining a microscope with one single refraction, and consequently capable of procuring the greatest clearness and brightness any one kind of microscope is susceptible of, spread a little of the fluid you intend to examine on a glass plate; bring this under one of your globules, then move it gently upwards till the fluid touches the globule, to which it will soon adhere, and that so firmly as to bear being moved a little backwards or forwards. By looking through the globule, you will then have a perfect view of the animalcules in the drop."

The construction of the single microscope is so simple, that it is susceptible of but little improvement, and has therefore undergone few alterations; and these have been chiefly confined to the mode of mounting it, or to additions to its apparatus. The greatest improvement this instrument has received was made by Lieberkuhn, ${ }^{1}$ about the year 1740 : it consists in placing the small lens in the centre of a highly-polished concave speculum of silver, by which means a strong light is reflected upon the upper surface of an object, which is thus examined with great ease and pleasure. Before this contrivance, it was almost impossible to examine small opaque objects with any degree of exactness; for the dark side of the object being next the eye, and also overshadowed by the proximity of

(1) Dr. Nathaniel Lieberkubn of Berlin. 
the instrument, its appearance was necessarily ohscure and indistinct. Lieberkuhn alapted a separate microscope to

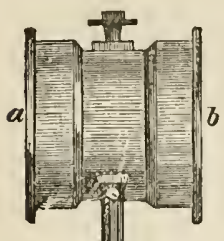
every object: but all this labour was not hestowed on trifling ohjects; his were generally the most curious anatomical preparations, twelve of which, with their microscopes. are deposited in the Museum of the Royal College of Surgeons.

Lieherkuhu's instrument, fig. 1 , is thus described by Professur Quekett : ${ }^{1} a b$ represents a piece of brass tube. about an inch long and an inch in dianeter. which is provided with a cap at each extremity; the one at $a$ carries a small domble-convex lens of half an inch in focal length, whilst the one at $b$ carries a condensing lens threequarters of an inch in diameter.

A vertical section of one of these instruments is seen in fig. 2 : a represents the magnifier, which is lodged in a cavity formed partly by the cap $a$, and by the silver cup or speculum $l$. In front of the lens is the speculum $l$, which is a quarter of an inch thick at its edge, and whose focus is about half an inch; in front of this agiln there is a disk of metal $c$, three-eighths of an inch in diameter, comnected by a wire with the small Fig. 1. knob $d$; upon this disk the injected ohject is fastened, and is covered over with some kind of varnish which has dried of a hemispherical figure.

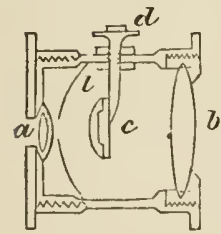

Fig. 2.

Between this knob and the inside and ontside of the tube tbere are two slips of thin brass, which act as springs to keep the wire and disk steady. When the knob is moved, the injected object is curried to or from the lens, so as to be in its focus. and to be scen distinctly, whilst the condensing lens $b$ serves to concen-

Lieberkuhn's Microscope trate the light on the speculum. To the

(1) Practical Treatise on the Microscope, p. 16. 
lower part of the tube a hindile of ebouy, about three inches in length, is attached by a brass ferrule and two screws. The use of this instrument is obvious: it is held in the hand in such a position that the lays of light from a lamp or white cloud may fall on the eondenser $b$, by which they are concentrated on the speculum $l$; this, agnin, further eondenses them on the object and the disk $c$, which object, when so illuminated, can readily be adjusted by the little knob $d$, so as to be in the focus of the small magnifier at $a$.

We must not omit in this place some account of Leeuweuhoek's microscopes, which were rendered famous throughout all Europe, on account of the numerous discoveries he had made with them. At his death he bequeathed a part of them to the Royal Society.

The microscopes he used were all single. and fitted up in a convenient and simple minuer : each consisted of a very small double-eonvex lens, let into a socket between two plates riveted together, and piereed with a small hole; the object was placed on a silver point. or neerile, which, by means of screws adapted for that purpose, might be turned about, raised or depressed at pleasure, and thus be lrought nearer to, or be removed farther from the glass, as the eye of the observer, the nature of the object, and the convenient examination of its parts required.

Leeuwenhoek fixed his objects, if they were solid, to these points with glue; if they were fluid, he fitted them on a little plate of tale, or thin-blown glass, which he afterwards glued to the needle in the same manner as hi other objects. The glasses were all exceedingly clear, and of different magnifying powers, proprortioned to the nature of the object and the parts designed to be examined. He observed, in his letter to the Royal Society, that "from upwards of forty years' experience, he had funnd the most considerable discoveries were to he made with glasses of moderate magnifying power, which exhibited the object with the most perfeet brightuess and distinetness." Each instrument was devoted to one or two objects; hence he had always some hundreds by him.

The three first compound microseopes that attract our notice are those of Dr. Hooke, Eustachio Divini, and Philip 
Bontuani. Dr. Hooke gives us an account of his in the preface to his Micrographia, published in the year 1667: it was about three inches in diameter, seven inches long, and furnished with four draw-out tubes, by which it might be lengthened as occasion required; it had three glassesa small object-glass, a middle glasi, and a keep eye-glass. Dr. Hooke used all the glasses when he wanted to take in a considerable part of an object at once, as by the middle glass a number of radiating pencils were conseyed to the eye which would otherwise have been lost; but when he wanted to exanine with accuracy the small parts of any substance, he took out the middle glass, and only made use of the eye and object lenses; "for," he writes, "the fewer the refiactions are, the clearer and brighter the object appears."

Dr. Hooke also gave us the first and most simple method of fiuding how much any compound microscope magnifies an object. He placed an accurate scale, divided into very minute parts of an inch, on the stage of the microscope; adjusted the microscope till the divisions appeared distinct, and then observed with the other eye how many divisions of a rule similarly divided and laid on the stage were included in one of the magnified divisions; "for if one division, as seen with one eye through the microscope, extends to thirty divisions on the rule, which is seen by the naked eye, it is evident that the diameter of the object is increased or magnified thirty times."

An account of Eustachio Divini's microscope was read at the Royal Society in 1668. It consisted of an objectlens, a middle glass, and two eye-glasses, which were planoconvex lenses, and were placed so that they touched each other in the centre of their convex surfaces. The tube in which the glasses were enclosed was as large as a man's leg, and the eye-glasses as broad as the palm of the hand. It had four several lengths: when shut up was 16 inches long, and magnified the diameter of an object $4 \mathrm{l}$ times, at the second length 90 , at the third length 111, and at the fourth length 143 times. It does not appear that Divini varied the object-glasses.

Philip Bonnaui published an account of his two microscupes in 1698. Both were compound. The first was 
similar to that which Mr. Martin published as new, in his Micrographia Nova, in 1712. His second was like the former, composed of three glasses, one for the eye, a middle glass, and an object lens; they were mounted in a cylindrical tube, which was placed in a horizontal position; behind the stage was a small tube with a convex lens at each end; beyond this was a lamp; the whole capable of various adjustments, and regulated by a pinion and rack. The small tube was used to condense the light on to the object.

A short time before this, Sir Isaac Newton having discovered his celebrated theory of light and colours, was led to improve the telescope, and apply his principles mosi successfully to the construction of a compound reflecting microscope. On the 6 th of February, 1672, he communicated to the Royal Society his "design of a microscope by reflection." It consisted of a concave spherical speculum of metal, and an eye-glass which magnified the reflected image of any object placed between them in the conjugate focus of the speculum. He also pointed ont the proper mode of illuminating objects by artificial light, as he describes it, "of any convenient colour not too much compounded," mono-chromatic. We find other two plans of this kind; the first that of Dr. Robert Barker, and the second that of Dr. Smith. In the latter there were two reflecting mirrors, one concave, and the other convex: the image was viewed by a lens. This microscope, though far from being executed in the best manner, performed, says Dr. Smith, very well, so that he did not doubt it would have excelled others, had it been properly finished.

In 1738, Lieberkuhn's invention of the solar microscope was communicated to the public. The vast magnifying power obtained by this instrument, the colossal granden. with which it exhibited the "minutiæ of nature," the pleasure which arose from being able to display the same object to a number of observers at the same time, by affording a new source of rational amusement, increased the number of microscopic observers, who were further stimulated to the same pursuits by $\mathrm{Mr}$. Trembley's famous discovery of the polype. The discovery of the wonderful properties of this little aninal, together with the works of Mr. Trembley, 
Mr. Baker, and Mr. Adams, combined to spread the reputation of the instrument.

In 1742, Mr. Henry Baker, F.R.S., published an admirable treatise on the microscope. He also read several papers before the Proyal Society on the subject of his microscopic discoveries. In the wood-cut (figr. 3) at the end of this chapter we have represented an elegant scroll "pocket microscope with a speculum," described by him as a new invelution.

In 1770, Dr. Hill published a treatise, in which he endeavours by means of the microscope to explain the construction of timber, and to show the number, the nature, and office of its several parts, their various arrangements and proportions in the different kinds; and he points out a way of judging, from the structure of trees, the uses they will best serve in the affairs of life.

M. L. F. Delabarre published an accuunt of his microscope in 1777 . It does not appear that it mas superior in any respect to those that were then made in England. It was inferior to some; for those made by Mr. Adams, in 1771, possessed all the advantages of Delabarre's in a higher degree, except that of changing the eye-glasses.

In 1774, Mr. George Adams, the son of the above, improved his father's invention, and reudered it useful for viewing opaque as well as transparent objects. This instrument, made and described by him, ${ }^{1}$ continued in nse up to the time of the invention of the achromatic improvement, proposed and made in 1815 for Amici, who subsequently gave so much time to the investigation of polarised light. and the adiptation of a polarising apparatus to the micruscope.

In 1812, Dr. Wullaston proposed a doublet in which the glasses were in contact, under the name of a "Periscopic Microscupe." And he says, "with this doublet I have seen the finest strix and serratures on the scales of the lepisma and podura. and the scales on a gnat's wing."

In the year 1816. Franenhofer, a celebrated optician of Munich, constructed object-glasses for the microscope of a single achromatic lens, in which the two glasses, although in juxtaposition, were not cemented together: these glasses

(1) Microscopical Essays, 1787. 
were very thick, and of long focus. Although such con. siderable improvements had taken place in the making of achromatic object-crlasses since their first discovery by Euler in 1776 , we find, even at so late a period as 1821 , M. Biot writing, "that opticians regarded as impossible the construction of a cood achromatic microscope." Dr. Wollaston also was of the same opinion, "that the compound instrument would never rival the single."

In 18\%3, experiments were commenced in France by M. Selligues, which were followed up by Frauenhofer in Munich, by Amici in Modena, by M1. ( hevalier in Paris, and by the late Dr. Goring and Mrr: Tulley in London. 'To M. Selligues we are indelsted for the first plan of making an object glass composed of four achromatic compound lenses, each consisting of two lenses. The focal length of each olject-class was eighteen lines its diameter six lines, and its thickness in the centre six lines, the aperture only one line. They could be used combiued or separated.

A microscope constructed on this principle, hy M. Chevalier, was presented by M. Sellignes to the Académie des Sciences on the 5 th of April, 18:4. In the same year, and without a knowledge of what had been done on the Contineut, the late Mr. Tulley, at the suggestion of Dr. Goring, constructed an achromatic ohject-glass for a compouud microscope of nine-tenths of an inch focal length, composea of three lenses, and transmitting a prencil of eighteen degrees; this was the first that had been made in England.

Sir David Brewster first pointed out in 1813 , the value of precions stones, the diamond, ruby, garnet, \&c., for the construction of microscopes. "The durability," he says, "of lenses made of precious stones is one of their greatest recommendations. Lenses of gliss undergo decomposition, and lose their polish in course of time. Mr. Baker found the glass lenses of Leeuwenhoek utterly useless after they became the property of the Royal society. The glass articles found in Nimrond were deconmosed, while the rock crystal lens was uninjured." $\mathrm{Mr}$ Pritchard at one time made two plano-convex lenses from a very perfect diamond, one the twentieth of an inch focus, which was 
purchased by the late Duke of Buckingham, and another the thirtieth of an inch focus.

In March 18ะ5, M. Chevalier presented to the Society for the Encouragement of the Sciences. an achromatic leus of four lines focus, two lines in diameter, and one line in thickness in the centre. This lens was greatly superior to the one before noticed, which had been made by him for M. Selligues.

In 1826, Professor Amici, of Modena, who from the year 1815 to 1824 had abandoned his experiments on the achromatic object-glass, was induced, after the report of Fresnel to the Academy of Science, to resume them; and in 1827 he brought to this country and to Paris a horizontal microscope, in which the object-glass was composed of three lenses superposed, each having a focus of six lines and a large aperture. This microscope had also extra eyepieces, by which the magnifying power could be increased. A microscope constructed on Amici's plan by Chevalier, during the stay of that physician in Paris, was exhibited at the Louvre, and a silver medal was awarded to its maker. ${ }^{1}$

"While these practical investigations were in progress," says Mr. Ross, "the subject of achromatism engaged the attention of some of the most profound mathematicians in England. Sir John Herschel, Professors Airy and Barlow, Mr. Coddington, and others, contributed largely to the theoretical examination of the subject; and though the results of their labour's were not immediately applicable to the microscope, they essentially promoted its improvement."

Mr. Jackson Lister, in 1829, succeeded in forming a combination of lenses upou the theory propounded by these gentlemen, and effected one of the greatest improvements in the manufacture of object-glasses, by joining together a plano-concave flint lens and a convex, by means of a transparent cement, Canada balsam. This is desirable

(b) In 1855, when the Jury on Microscopes at the Paris Exposition were comparing the rival instruments, Professor Amici brought a compound achromatic microscope, comparatively of small dimensions, whicb exhibited certain striæ in test objects better than any of the instruments under examination. This superiority was produced by the introduction of a drop of water between the object and the object-glass. 
to be taken as a basis for the microscopic object-glass: it diminishes very nearly half the loss of light from reflection, which is considerable at the numerous surfaces of a combination; the clearness of the ficld and brightness of the picture is evidently increased by doing this; and it prevents any dewiness or vegetation from forming on the inner surfaces. Since this time, Mr. Ross has been constantly employed in bringing the manufacture of objectglasses to their greatest perfection, and at length they have attained to their present improved manufacture. Having applied Mr. Lister's principles with a degree of success never anticipated, so perfect were the corrections given to the achromatic object-glass, so completely were the errors of sphericity and dispersion balanced or destroyed, that the circumstance of covering the object with a plate of the thinnest glass or talc disturbed the corrections, if they had been adapted to an uncovered object, and rendered an object-glass which was perfect under one condition sensibly defective under the other. Here was another and unexpected difficulty to be overcome, but which was finally accomplished; for in a communication made to the Society of Arts in 1837, Mr. Ross stated, that by separating the anterior lens in the combination from the other two, he had been completely successful. The construction of this object-glass will be illustrated and explained in a future chapter.

The rapid improvement in the manufacture of the achromatic compound microscope in this country has been greatly furthered by the spirit of liberality evinced by Sir David Brewster, the late Dr. Goring, Mr. R. H. Solly, and Mr. Bowerbank. To the patronage of Dr. Goring we owe the construction of the first triplet achromatic object-glass, of the diamond lens, and of the improved reflecting instrument of Amici by Cuthbert.

The achromatic microscopes now manufactured by our London makers, Mr. Ross, Messrs. Powell and Lcaland, and Messrs. Smith and Beck, are unequalled in any part of the world. This opinion is confirmed by the reports of the juries on the Exhibition of Works of Industry of all Nations, 1851 ; at that time the instruments exhibited by Mr. Ross and Messrs. Smith and Beck, by fir excelled 
those of all other countries. Messrs. Powell and Lealand's microscope, with object-glasses, was selected by the Ruyal Society as the best agaiust all competitors. See Juries' Reports for much interesting matter ou this subject; article "Microscope," I'enay Cyclopuedia, by Mr. Koss; Practical Treatise on the Microscope, by Professor Quekett; Sir David Brewster's T'reatise on the Microscope; Dujardin's Observateur; Maudl, Traité pratique du Microscope: Dr Robin, Du Microscope, \&c.

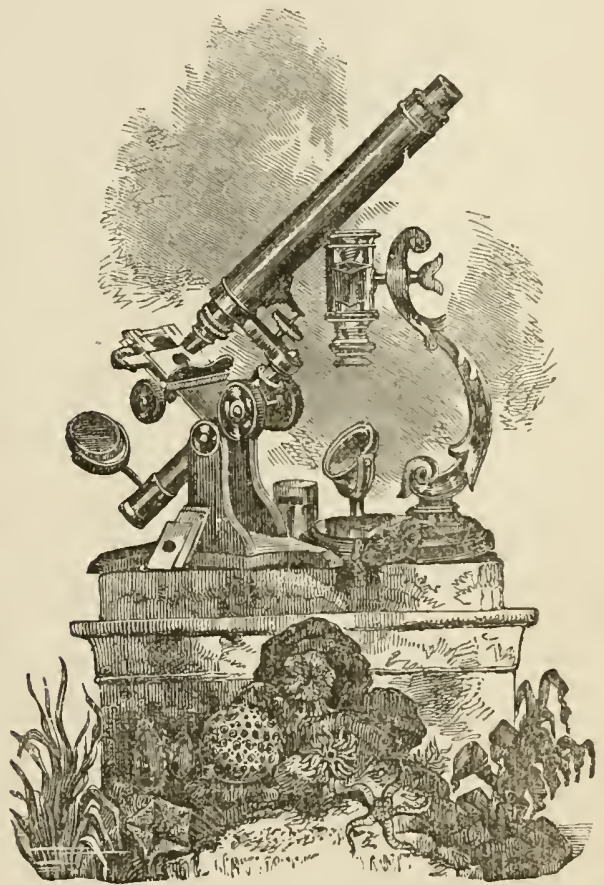

Rig. 5.-Baker's Scroll or Pocket Microscope, and the Modern Compound Mistoscope of $W$. Lard's marufacture. 


\section{CHAPTHR II.}

MFCHANICAL AND OPTICAL PRINCIPLES INYOLVED IN THE CONSTRUCTOM OF THE MICROSCOPE-LENSIS-MODE OF ESTIMATING THEIR FWER, ETC. - ACHROMATIC LENSFS - MAGNIFYING FOWER WULLASTON'S DOUBLET - CODDINGTONS LENS - NOSS'S SIMPLE AND COMPOUND MICROSCOPES - MICROMETERS, ETC.

$\mathrm{N}$ the construction of the modern microscope, optical and mechanical principles of some importance are involved. These principles we shali briefly explain, together with the more recent imiprovements effected in the instrument generally. ${ }^{1}$

The microscope depends for its utility and operation uprols concave and convex lenses, and the course of the lays of light passing through them. Lenses are usially defined as pieces of glass, or other transparent substances, having their two surfaces so formed that the rays of light, in passing through them, have their direction changer, and are made to converge or diverge from their original parallelism, or to become parallel after converging or diverging. When a ray of light passes in an oblique direction from one trimsparent nedium to another of a different density, the direction of the ray is changed both on entering and leaving ; this influence is the resnlt of the well-known law of refraction,--that a ray of light passing from a rare into a dense medium is refiacted towards the perpendicular, and vice versa.

(1) For a full explanation of the laws of optics, and their application to the construction of lenses, the reader is referred to Dr. Bird and Mr. Brooke' "Manual of Natural Philosoplyy," Professor Potter's "Elementary Treatise or optics," Sir David Brewster"s "Optics," \&c. 
Dr. Arnott remarks: "But for this fact, which to many persons might at first appear a subject of regret, as preventing the distinct vision of objects through all transparent media, light could have been of little utility to man. There could have been neither lenses, as now; nor any optical instruments, as telescopes and microscopes, of which lenses form a part; nor even the eye itself." Rays of light falling perpendiculariy upon a surface of glass or other transpareut substance, pass through without being bent from the original line of their direction. Thus, if a

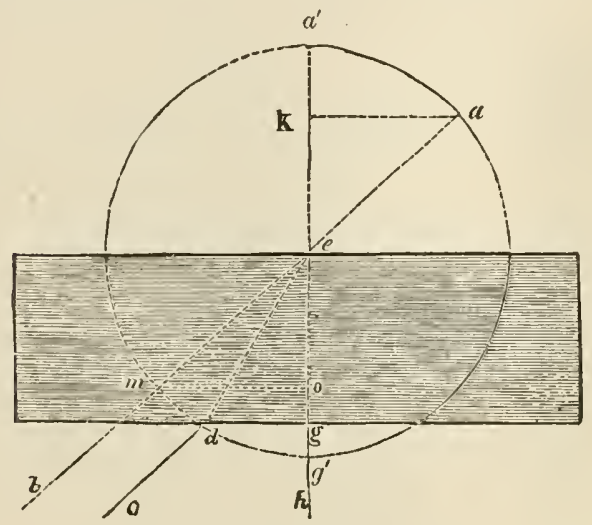

Fig. 4.

ray pass from $k$ perpendicularly to the surface of the piece of glass at $e$ (fig. 4), it will go on to $h$ in the right line $k e 0 g h$. But if the same ray be directed to the surface $\epsilon$ obliquely, as from $a$, instead of passing through in a direct line to $b$ in the airection $a e m b$, it will be refracted to $d$, in a direction approaching nearer to the perpendicular line $k h$. The ray $a e$ is termed the ray of incidence, or the incident ray; and the angle $a c k$ which it makes with the perpendicular $k h$ is called the angle of incidence. That part of the ray from $e$ to $d$ passing through the transparent medium is called the ray of refraction, or the refracted ray: and the angle $d e g$ which it makes with the 
perpendicular is called the angle of refraction. The ray projected from $a$ to $e$ and refracted to $d$, in plassing ont of the transparent medium as at $d$, is as much bent from the line of the refracted ray $e d$ as that was from the line of the original ray $a e b$; the ray then passes from $d$ to $c$, parallel to the line of the original ray $a e b$. It follows, then, that any ray passing through a transparent medium, whose two surfaces, the one at which the ray enters, and the one at which it passes out, are parallel planes, is first refracted from its original course; but in passing out is bent into a line parallel to, and rumning in the same direction as the original line, the only difference being, that its course at this stage is shifted a little to one side of that of the original. If from the centre $e$ a circle be described with any radius, as $d e$, the arc $a \alpha^{\prime}$ measures the angle of incidence $a e k$, and the arc $g^{\prime} d$ the angle of refraction ged. A line $a k$ drawn from the point $a$ perpendicular to $k h$ is called the sive of the angle of incidence; and the line $d g$ drawn from the point $d$ perpendicular to $k h$ is called the sine of the angle of refraction. From the conclusions drawn from the principles of geometry, it has been found, that in any particular transpareut substance the sine of the angle of incidence $a \cdot k$ has always the same ratio to the sine $d g$ of the angle of refraction, no matter what be the degree of obliquity with which the ray of incidence $a e$ is projected to the surface of the transparent medium. If the ray of incidence passes from air obliquely into water, the sine of incidence is to that of refraction as 4 to 3 ; if it passes from air into glass, the proportion is as 3 to 2 ; and if from air into diamond, it is as 5 to 2.

By the help of glasses of certain forms, we unile in the same sensible point a great number of rays proceeding from one point of an olject; and as each ray carries with it the image of the point from whence it proceeded, and all the rays united must form an image of the object from whence they were emitted, this image is brighter in proportion as there are more rays united, and more distinct in proportion as the order in which they proceeded is better preserved in their union. The point at which parallel rays meet after converging through a lens is called the principal focus, and its distance from the middle of 
the lens the focal length. The radiart point and its image after refraction are called conjugate foci. These foci vary according to the distance of the radiant points. In every lens the right line perpendicular to the two surfices is called the axis of the lens, and is seen in the aunexed figure ; the point where the axis cuts the surface is called the vertex of the lens.

Fig. 5 is intended to represent the different forms of lenses in use; $a$ is a plane glass of equal thickness

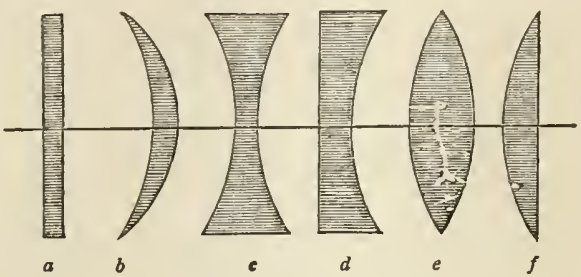

Fig. 5.

throughout; $b$, a meniscus, concave on one siae, convex on the other ; $c$, a double-concave; $d$, a plano-concave; $e, a$ double-convex; $f$, a plano-convex.

The lenses employed in the construction of microscopes are chiefly convex; concave lenses being only used to make certain modifications in the comrse of the rays passing through those of a convex form, whereby their performance is rendered more exact. In accordance with the laws of refraction, when a pencil of parallel rays, passing through the air, inpinges upon a convex surface of glass, the rays are made to converge; for they will be bent towards the centre of the circle, the radius heing perpendicular to each point of curvature. Parallel rays, falling on a plano-convex lens, are brought to a focus at the distance of its diameter; and conversely, rays diverging from that point are rendered parallel. Plano-convex leuses possess properties which render them valuable in the construction of microscopes.

Parallel rays, falling on a double-convex lens are brought to a focus in the centre of its diameter; conversely, rays 
diverging from that point are rendered parallel. Hence the focus of a double-convex lens will be at just half the distance, or half the length, of the focus of a flano-convex lens having the same curvature on one side. The distance of the focus from the lens will depend as much on the degree of curvature as upon the refracting power (called the index of refraction) of the glass of which it may be formed. A lens of crown-glass will have a longer focus than a similar one of flint-glass; since the latter has a greater refracting power than the former. For all ordinary practical purposes, we may consider the principal focusas the focus for parallel rays is terrned-of a double-convex lens to be at the distance of its radius, tlat is, in its centre of curvature; and that of a plano-convex lens to be at the distance of twice its radius, that is, at the other end of the diameter of its sphere of curvature. The converse of all this occurs when divergent rays are made to fall on a convex lens. Rays already converging are brought together at a point nearer than the principil fucus: whereas rays diverging from a point withiu the principal focus are rendered still more diverging, though in a diminished degree. Rays diverging from points more distant than the priucipal focus on either side, are brought to a focus beyond it; if the point of divergence be within the circle of curvature, the focus of convergence will be beyoud it; and vice versâ. The same principles apply equally to a planoconvex lens; allowance being made for the doulle distance of its principal focus. They also apply to a lens whose surfaces have different curvatures; the principal focus of such a lens is found by multiplying the radius of one surface by the radius of the other, and dividing this product by half the sum of the radii.

The refracting influence of concave lenses will be precisely the opposite of that of convex. Rays which fall upon them in a parallel dircction, will be made to diverge as if from the principal focus, which is here called the negative focus. This will be, for a plano-concave lens, at the distance of the diameter of the sphere of curvature; and for a double-concave, in the centre of that sphere. If a lens be convex on one side and concave on the other, 
forming what is termed a meniscus, its effect will depend upon the proportion between the two curvatures.

The rules by which the foci of all lenses may be found, will be more advantageously studied in works on Optics.

As each ray carries with it the image of the object from whence it proceeded, it follows, that if those rays, after intersecting each other, and having formed an image at their intersection, are again united by refraction or reflection, they will form a new image, and that repeatedly, so long as their order is not disturbed. It follows, also, that when the course of the luminons ray throngh several lenses is under consideration, we may look on the image first produced as an object in reference to the second lens, and may consider the second image as produced by this object, and so on successively. This is, indeed, a principle involved in the adaptation of leuses to magnifying objects; and in fig. 6 , it is seen that if the point of light be situated

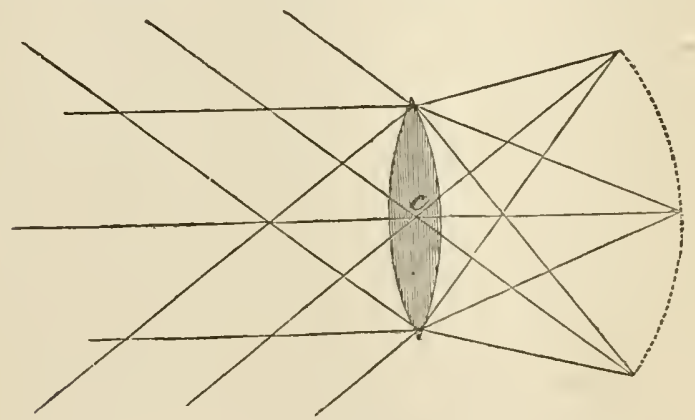

Fig. 6.

above the line of the axis, the focus will then be below it, and vice versa ; but the surface of every luminous body may be regarded as comprehending an infinite number of such points, from all of which a pencil of light-rays proceeds, and is refracted according to the general law; so that a perfect but inverted image or picture of the object is formed upon any surface placed in the focus, and adapted to receive the rays. If any object be placed at twice the 
distance of the principal focus, the image being formed at an equal distance on the other side of the lens, will be of the same dimensions with the object, as in fig. 7 ; but if

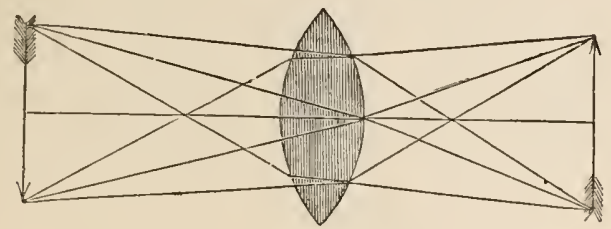

Fig. 7.

the object be placed nearer to the lens, the image will be farther from it, and of larger dimensions, as in fig. $\delta$; and,

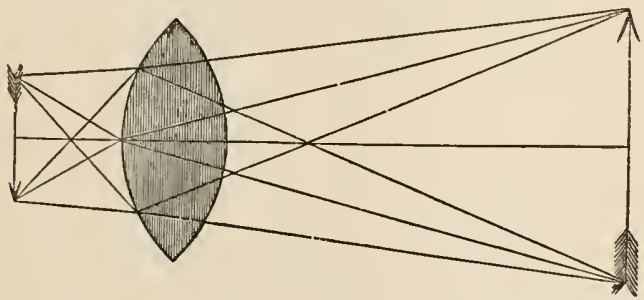

Fig. 8.

$\mathrm{cn}$ the other hand, if the object be farther from the lens, the image will be nearer to it, and smaller than itself. But it is to be observed, that the larger the image is in proportion to the object, the less bright it will be, because the same amount of light has to be spread over a greater surface; whilst a smaller image will be much more brilliant.

Aberration of Lenses. - Although the image of an object produced by the convex lens, fig. 8 , appears at first view to be an exact reproduction of the object, it is found, when submitted to rigorous examination, to be more or less confused and indistinct : which is augmented when viewed in a microscope. This indistinctness and confusion arises from two causes, one depending on form, and the other on the material of the lens. That which depends on the form of the lens we shall now proceed to explain. 
In optical instruments the curvature of the lenses employed is spherical, that being the only form which cau be given by grinding with the requisite degree of truth. But convergent lenses, with spherical curvatures, have the defect of not bringing all the rays of light which pass through them to one and the same focus. Each circle of rays from the axis of the lens to its circumference has a different focus, as shown in fig. 9. The rays $a a$,

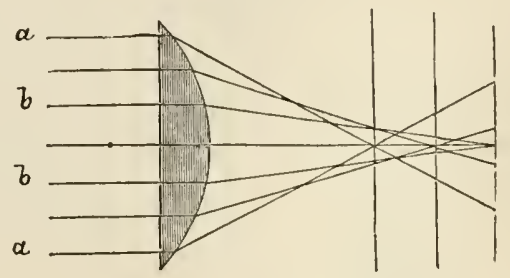

Fig. 9.

which pass through the lens near its circumference, it is seen to be more refracted, or come to a focus at a shorter distance behind it than the rays $b b$, which pass through near its centre or axis, and are less refracted. The consequence of this defect of lenses with spherical curvatures, which is called spherical aberration, is that a well defined image or picture is not formed by them, for when the object is focused, for the cilcumferential rays, the picture projerted to the eye is reudered indistinct by a halo or conris: $n$ produced by the central rays falling in a circle of dissipation, before they lave come to a focus. On the other hand, when pliced in the focus of the central rays, the picture formed by them is rendered indistiuct by the halo produced by the circunferential rays, which have already come to a focus and crossed, now fall in a state of divergence, forming a circle of dissipation. The grosser effects of this spherical aherration are corrected by cutting off the passage of the rays $a \alpha$. throigh the circumferences of the leus, by meins of a stop diaphragm, so that the central rays, $b b$, only are concerned in the formation of the picture. This defect is reduced to a minimum, by 
using the meniscus form of lens, whic'. is the segment of au ellipsoid instead of a sphere.

The ellipse and the hyperbola are curves of this kind, in which the curvature diminishes from the central ray, or axis, to the circumference $b$; and mathematicians have shown how spherical aberration may be entireiy removed by lenses whose sections are ellipses or hyperbolas. For this curious discovery we are indehted to Descartes.

If $a l, a l^{\prime}$, for example, fig. 10, be part of an ellipse

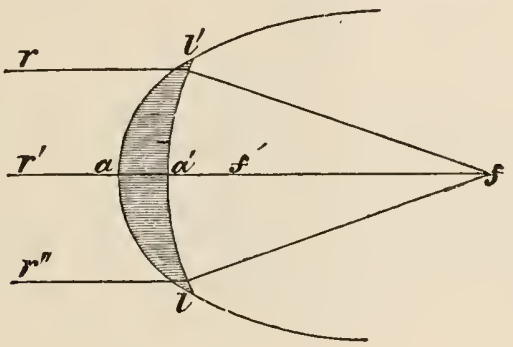

Fig. 10.

whose greater axis is to the distance between its foci $f f$ as the index of refraction is to unity, then parallel rays $r l^{\prime}, r^{\prime \prime} l$ incident upon the elliptical surface $i^{\prime} a l$, will be refracted by the single action of that surface into lines which would meet exactly in the farther focns $f$, if there were no second surface intervening between $l a l^{\prime}$ and $f$. But as every useîui lens must have two surfaces, we have only to describe a circle $l a^{\prime} l^{\prime}$ round $f$ as a centre, for the second surfice of the lens $l^{\prime} l$.

As all the rays refracted at the surface $l a l^{\prime}$ converge accurately to $f$, and as the circular surfice $l a^{\prime} l^{\prime}$ is perpendicular to every one of the refracted rays, all these rays will go on to $f$ without suffering any refraction at the circular surface. Hence it shouid follow, that a meniscus whose convex surface is part of an ellipsoid, and whose convex surface is part of any spherical surface whose centre is in the farther focus, will have no aprreciable spherical aberration, and will refract parallel rays iucident on its convex surface to the farther focus. 
In like manner, a concavo-convex lens, fig. 11, $l l^{\prime}$, whose soncave surface $l a^{\prime} l^{\prime}$ is a circle described round the farther

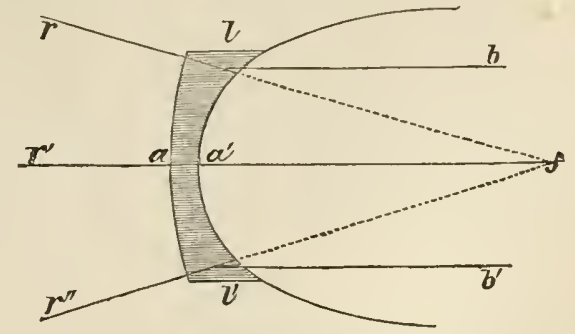

Fig. 11.

tocus of the ellipse, will cause parallel rays $b l, b^{\prime} l$, to diverge in directions $l r, l^{\prime} r^{\prime \prime}$, which, when continued backwards, will meet exactly in the focus $f$, which will be its virtual focus.

If a plano-convex leus, fig. 12, has its convex surface $l a l^{\prime}$ part of a hyperboloid, formed by the revolution of a

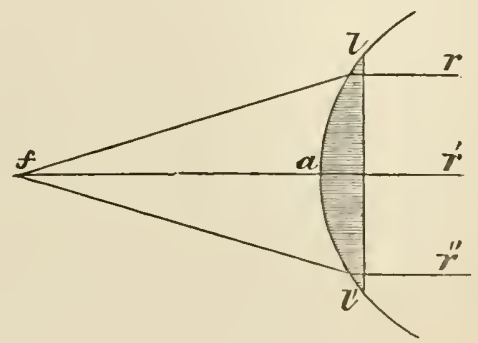

Fig. 12.

hyperbola whose greater axis is to the distance between the foci as unity is to the index of refraction, then parallel rays $r l, r^{\prime \prime} l^{\prime}$ falling perpendicularly on the plane surface, will be refracted without aberration to the further focus of the hyperboloid. The same property belongs to a plano-concave lers having a similar hyperbolic surface, and receiving parallel rays on its plane surface. ${ }^{1}$

(1) It must be borne in mind, that in none of those lenses would the objec. he correctly seen in focus, exce?t at the one point known as :he mathematica or geometrica' axis of the lens. 
When the convex side of a plano-convex lens is exposed to parallel rays, the distance of the focus from the plane side will be equal to twice the radius of its convex surface diminished by two-thirds of the thickness of the lens; but when the plane is exposed to parallel rays, the distance of the focus from the convex side will be equal to twice the radius.

A meniscus with spherical surfaces, fig. 13, has the property of refracting all converging rays to its focus, if

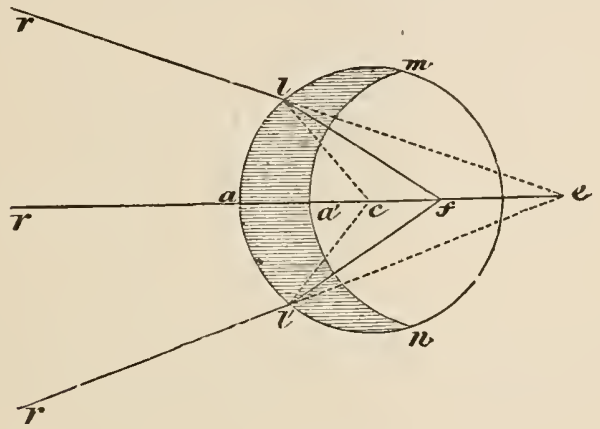

Fig. 13.

its first surface be convex, provided the distance of the point of convergence or divergence from the centre of the first surface is to the radius of the first surface as the index of refraction is to unity. Thus, if $m l l^{\prime} n$ be a meniscus, and $r l, r^{\prime} l^{\prime}$ rays converging to the point $e$, whose distance $e c$ from the centre of the first surface $l a l^{\prime}$ of the meniscus is to the radius $c a$, or $c l$, as the index of refraction is to unity, that is as 1.500 to 1 in glass; then if $f^{\prime}$ is the focus of the first surface, describe, with any radius less than $f a$, a circle $m a^{\prime} n$ for the second surface of the lens. Now it will be found by projection, that the rays $r l, r l^{\prime}$, whether near the axis $a e$ or remote from it, will be refracted accurately to the focus $f$; and as all these rays fall perpendicularly on the second surface $m n$, they will still pass on, without refraction, to the focus $f$. In like manner, it is obvious that rays $f l, f l^{\prime}$, diverging from $f$ will 
be refracted into $r l, r^{\prime} l^{\prime}$, which diverge accurately from the virtual focus. ${ }^{2}$

Spherical aberration is not so much connected with the focil length of the lens as depending on the relative convexity of its surfaces, and is much reduced by observing a certain ratio between the radii of its anterior and pos. terior surfaces; thus the spherical aberration of a lens, the radius of one surface of which is six or seven times greater than that of the other, as in fig. 14, is very much

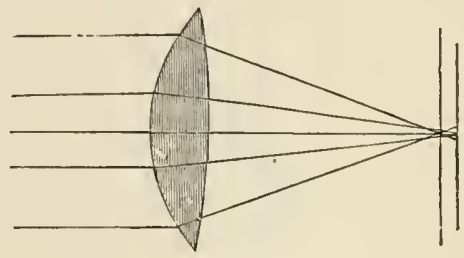

Fig. 14.

less when its more convex surface is turned forward to receive parallel rays, than when its less convex surface is turned for'wards.

This is still better effected, or even got rid of altogether, by using combinations of lenses, so disposed thiat thoir opposite aberritions shall correct each other, whiist magnifying power is gained. For it is seen that, as the aberration of a concave lens is just the opposite of that of a convex lens, the aberration of a convex lens

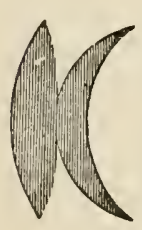
placed in its most filvourable position may be corrected by a concave lens of much less power in its most favourable position. This is the principle of a combination proposed by Sir John F. W. Herschel, fig. 15, an "aplanatic doublet," consisting of a double-convex lens and a meniscus; a doullet of this kind is found extremely useful and available for micro-

Fig. 15. the Coddington lens. scopic purposes: it affcrds a large field, like

Chromatic aberratim. - Another and serious difficulty arises, in the unequal refrangibility of the different 
coloured rays which together make up white light, so that they are not all brought to the same focus, even by a lens free from spherical aberration. It is, indeed, this difference in their refrangibility which canses their complete separation by the prism into a spectrum.

The correction of chromatic with spherical aberration is effected in a most ingenious manner, by combining a convex lens made of crown-glass, and a concave lens of flintglass. If we examine closely the image projected on the table of a camera obscura provided with a common lens, we see that it is bordered with the colours of the rainbow; or if we look through a common magnifying-glass at the letters on the title-page of a book, we see them shightly coloured at their edges in the same manner. The cause of this iridescent border is that the primitive rays-red, yellow, and blue, - of which a colomless ray of light is composed, are not all equally refiangible. Hence they are not all brought to one point or focus, but the blue rays veing the most refrangible, cone to a focus nearer the lens than the yellow ones, which are less refrangible, and the yellow rays than the red, which are the least refrangible. Thus, in fig. 16, chromatic aberration proves still more

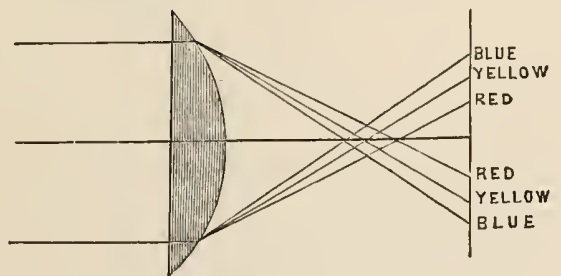

Fig. 16.

detrimental to the distinct definition of images formed by a lens, than spherical aberration. This arises more from the size of the circles of dissiuation, than from the iridescent border, and it may still exist, although the spherical aberration of the lens be altogether corrected. Chromatic aberration is, as hefore stated, corrected by combining, in the construction of lenses, two media of opposite form, and differing from each other in the proportion in which they 
respectively refract and disperse the rays of light; so that the one medium may, by equal and contrary dispersion, neutralize the dispersion caused by the other, without, at the same time, wholly neutralizing its refraction. Remarkable enough, the media found the most valuable for such a purpose should be the combination of pieces of crown and flint glass, of crown-glass whose index of refraction is 1.519 , and dispersive power 0.036 , and of fint-glass whose index of refraction is 1.589 , and dispersive power 0.0393 . The focal length of the convex crown-glass leus must be $4 \frac{1}{3}$ inches, and that of the concave flint-glass lens $7 \frac{2}{3}$ inches, the combined focal length of which is 10 inches. The following tig. 17 will serve us to explain how rays of light are brought to a single focus, free from colour.

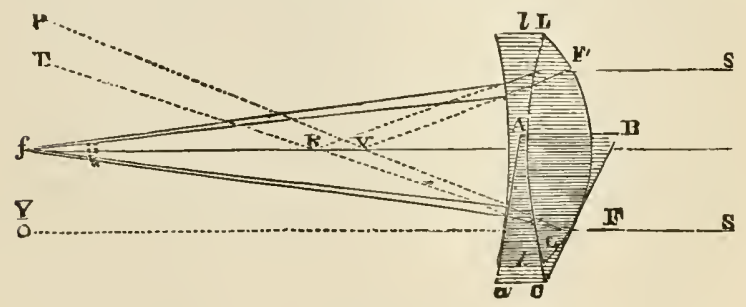

Fig. 17.

In this diagram, $\mathrm{L} \mathrm{L}$ is a convex lens of crown-glass, and $l l$ a concave one of flint-glass. A convex lens will refract a ray of light ( $\mathrm{s}$ ) falling at $\mathrm{F}$ on it exactly in the same manner as the prism A B C, whose faces touch the two surfaces of the lens at the points where the ray enters, and quits. The ray $\mathrm{S} F$, thus refracted by the lens $\mathrm{L} L$, or prism A B C, would have formed a spectrum ( $P$ T) on a screen or wall, had there been no other leus, the violet ray (F v) crossing the axis of the lens at v, and going to the upper end (P) of the spectrum; and the red ray (F R) going to the lower end (T). But, as the flint-glass lens $(l l)$ or the prism $\mathrm{A} a \mathrm{C}$, which receives the rays $\mathrm{FV}, \mathrm{F}$, at the same points, is interposed, these rays will be united at $f$, and form a small circle of white light, the ray (s F) being now refracted without colour from its primitive direction 
( $S F Y$ ) into the new direction ( $F f$ ). In like manner, the corresponding ray $\left(\mathrm{s}^{\prime} \mathrm{F}^{\prime}\right)$ will be refracted to $f$, and a whitc and colourless image there formed by the two lenses.

The Magnifying Power of Lenses.-To assist us in gaining a clearer notion of the mode in which a single lens serves to magnify minute objects, it is necessary to take a passing glance at the ordinary phenomena of vision. The human eye is so constituted, that it can only have distinct vision when the rays falling upon it are parallel or slightly divergent; because the retina, on which the image impinges, requires the intervention of the crystalline lens to bring the rays to an accurate focus upon its surface. The limit of distinct vision is generally estimated at from six to ten inches; objects viewed nearer, to most persons, become indistinct, although they may be larger. The apparent size of an object is, indeed, the angle it subtends to the eye, or the angle formed by two lines drawn from the centre of the eye to the extremity of the object. This will be understood upon reference to fig. 18. The lines drawn from the eye to $\mathrm{A}$ and $\mathrm{R}$ form an angle, which, when the distance is small, is nearly twice as great as the angle

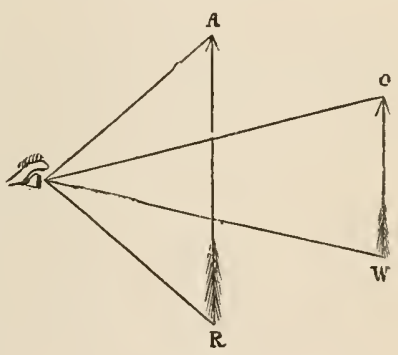

Fig. 18. from the eje to $0 \mathrm{w}$, formed by lines drawn at twice the distance. The arrow at $\& \mathrm{R}$ will therefore appear nearly twice as long as $0 \mathrm{w}$, being seen under twice the angle; and in the same proportion for any greater or less difference in distance. This, then, is called the angle of vision, or the visual angle. Now the utility of a convex lens interposed between a near object and the eye consists in its reaucing the divergence of the rays forming the several pencils issuing from it; so that they enter the eye in a state of moderate divergence, as if they had issued from an object beyond the nearest limit of distinct vision ; and in well-ảefined image is consequently formed upon the retina. In fig. 19, a double-convex lens is placed before 
the eye, near which is a small arrow, to represent the object uuder examination; and the cones drawn from it

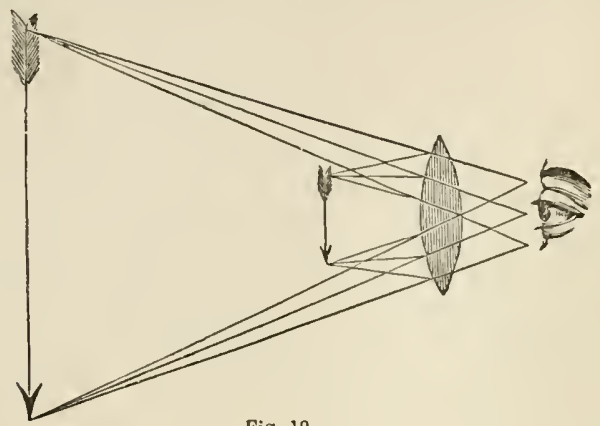

Fig. 19.

are portions of the rays of light diverging from those points and filling upon the lens. These rays, if permitted to fall at unce upon the pupil, would be tro divergent to allow of their being brought to a focus upon the retina by the dioptric media of the eye. But being first passed through the lens, they are bent into nearly parallel lines, or into lines diverging from some points within the limits of distinct vision. Thus altered, the eye receives them precisely as if they had emanated directly from a larger arrow placed at ten inches from the eye. The difference between the real and the imaginary arrow is called the magnifying power of the lens. The object, when thus seen, appear's to be magnified nearly in the proportion which the focal distance of the leus bears to the distance of the object when viewed by the unassisted eye; and is entirely owing to the object being distinctly viewed so much nearer to the eye than it could be without the lens. ${ }^{1}$ With these preliminary remarks as to the medium by which microscopic power is obtained, we shall proceed to apply them to the construction of a perfect iustrument.

I'he Microscope.-A mieroscope, as we have before ixplained, may be either a single, or simple, or a compound

(1) "The Magnifying Power of Short Spaces" has been most ably elucidated in John Gorham, Esq. M.R.C.S. See Journal of Microscopical Society, Octobex. 1854. 
instrument. The simple microscope may consist of one, as seen in ing. 19, or of two or three lenses; but theso latter are so arranged as to have the effeet only of a single? leus. In the compound mieroseope, not less than two leuses must be cmployed: one to form an inverted image of the object, which, being the nearest to the object, is called the oliject-glass; and the other to magnity this image, and from being next the eye of the observer, called the eye-glass. Both these may be formed out of a combiuation of lenses, as will be hereafter seen.

We have hitherto considered a lens only in reference to its enlargement of the object, or the increase of the angle under which the object is seen. A further and equally important consicleration is that of the number of ritys or quantity of light by which every point of the object is rendered visible; and much may be accomplished, as we have before pointed out, by the combination of two or more lenses instead of one, thus reducing the angles of incidence and refraction. The first satisfictory arrangement for this purpose was the invention of the celebrated Dr. Wollaston. His doublet (fig. 20) consisted of two plano-eonvex lenses having their foeal lengths in the proportion of one to three, or nearly so, aud placed at a distance which can be ascertained best by actual experimeut. Their plane sides are placed towards the object, and the lens of shortest foeal length next the object.

It appears that Dr. Wollaston was led to this invention by considering that the achromatic Huyghenian eyepiece, which will be presently described, would, if reversed, possess similar good properties as a simple nicroseope. But it will be evilent, when the eye-piece is understood, that the circumstances which render it achromatic are very imperfectly applicable to the simple mieroscope, and that the doublet, withcut a nice adjustment of the stop, would be valueless. Dr. Wollaston nakes no allusion to a stop, nor is it certain that he contemplated its introduction: although his illness, which terminated fatally soon after the presentation of his paper to the Ruyal Society, may account for the omission.

The nature of the corrections which take place in the doublet is explained in the annexed diagram, where $l o i^{\prime}$ is 
the object, $p$ a portion of the cornea of the eye, and $d d$ the stop, or limiting aperture.

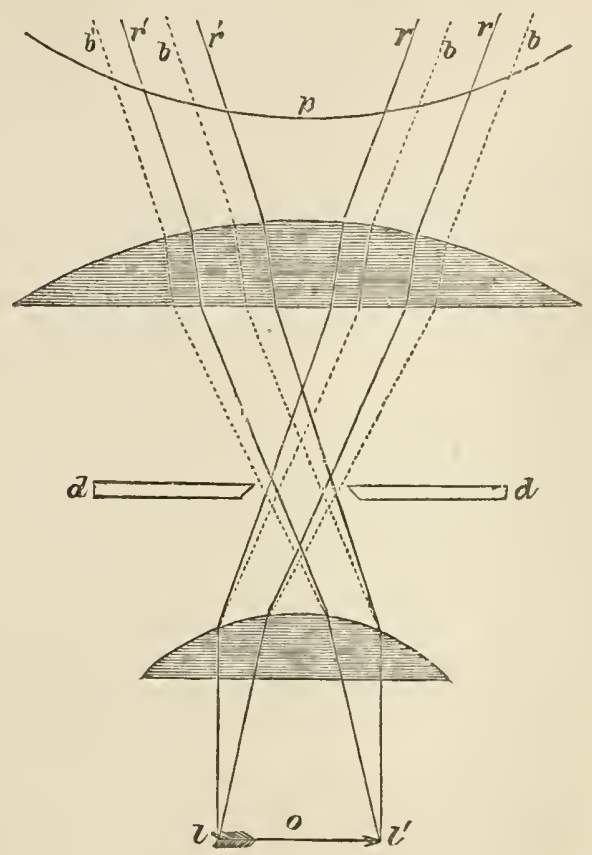

Fig. 20.

Now it will be observed that each of the pencils of light from the extremities $l l^{\prime}$ of the object is rendered excentrical by the stop; consequently, each passes through the two lenses on opposite sides of their common axis $0 p$; thus each becomes affected by opposito errors, which to some extent balance and correct each other. To take the pencil $l$, for instance, which enters the eye at $r b, r b$ : it is bent to the right at the first lens, and to the left at the second; and as each bending alters the direction of the blue rays more than the red, and moreover as the blue rays fail 
nearer the margin of the second lens, where the refraction being more powerful than near the centre, compensates in some degree for the greater focal length of the second lens, the blue rays will emerge very nearly parallel, and of consequence colorrless to the eye. At the same time, the spherical aberration has been diminished by the circumstance that the side of the pencil which passes one lens nearest the axis passes the other nearest the margin.

This explanation applies only to the pencils near the extremities of the object. The central pencils, it is obvious, would pass both lenses symmetrically, the same portious of light occupying nearly the same relative places on both lenses. The blue light would enter the second lens nearer to its axis than the red; and being thus less refracted than the red by the second lens, a small amount of compensation would take place, quite different in principle, and inferior in degree, to that which is produced in the excentrical pencils. In the intermediate spaces the corrections are still more imperfect and uncertain; and this explains the cause of the aberrations which must of necessity exist even in the best-made doublet. It is, however, infinitely superior to a single leus, and will transmit a pencil of an angle of from $35^{\circ}$ to $50^{\circ}$ without any very sensible errors. It exhibits, therefore, many of the usual test-objects in a very beautiful manner.

The next step in the improrement of the simple microscope bears more relation to the eye-piece; this was effected by Mr. Holland: it consists in substituting two lenses for the first in the doublet, and retaining the stop between them and the third. The first bending being thus effected by two lenses instead of one, is accompanied by smaller aberrations, which are, therefore, more completely balanced or corrected at the second bending, in the opposite direction, by the third lens.

Hand Magnifiers.-Before we proceed further, it will be as well to bestow a passing notice on the simple hand magnifier, so often employed by microscopists in the preliminary examinations of objects.

A very good form of lens wis proposed by Dr. Wollaston, and called by him the Periscopic lens: which consisted of 
two hemispherical lenses cemented together by their plane faces, having a stop between them to limit the aperture. A similar proposal was made by Sir David Brewster in 1820 , who, however, executed the project in a better manner, by cutting a groove in a whole sphere, and filling the groove with opaque matter. His lens, which is better known as the Coddington lens, ${ }^{1}$ is shown at fig. 21 : it gives a large field of view, which is equally good in all directions, as it is evident that the pencils $a b$ and $b a$ pass
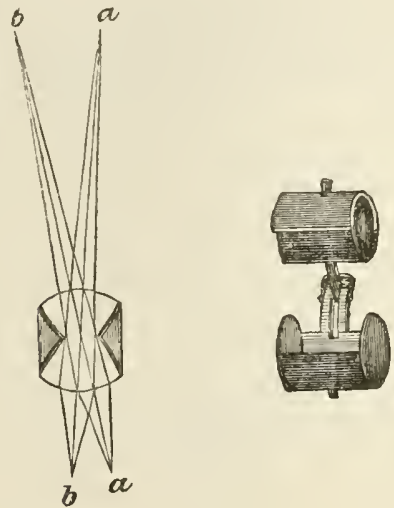

Fig. 21.

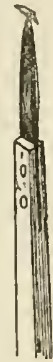

Fig. 22

through under precisely the same circumstances. Its spherical form has the further advantage of rendering the position in which it is beld of comparatively little consequence. It is therefore very convenient as a hand magnifier; but its definition is, of course, not so good as that of a well-made doublet or achromatic lens. It is generally set in a folding case, as represented in the figure, and so contrived that it is admirably adapted for the waistcoatpocket; which, together with the small holder, fig. 22 , for

(1) The late Mr. Coddington, of Cambridge, who had a high opinion of the value of this lens, had one of these grooved spheres executed by Mr. Carey, who gave it the name of the Coddington Lens, supposing that it was invented by the person who employed him, whereas Mr. Coddington never laid claim to it, and the circumstance of his liaving one made was not until nine years after it was described by Sir David Brewster in the "Edinburgh Journal." 
securing small objects and holding them during examination, are all that is required for a field instrument during a day's ramble. 'This useful little holder may be purchased in a case at Mr. Weedon's, 41, Hart-street, Bloomsbury. The Stanhope lens is similarly constructed, although not so good and convenient as the former, and is but soldom to be purchased properly made.

When the magnifying power of a lens is considerable, or when its focal length is short, and its proper distance from the object equally short, it then. becomes necessary to be placed at a proper distance with great precision; it cannot therefore be held with sufficient accuracy and steadiness by the unassisted hand, but must be mounted in a frame, having a rack or screw to move it towards or from another frame or stage which holds the object. It is then called a microscope; and it is furnished, according to circumstances, with lenses and mirrors to collect and reflect the light upon the object, with other conveniences.

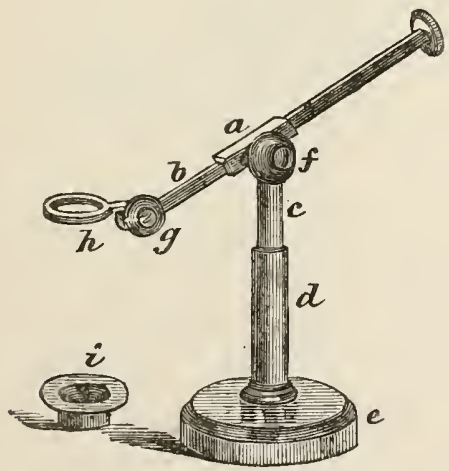

Fig. 23.-Ross's Simple Microscope.

The best of the kind was that contrived by Mr. Rosis, represented in fig. 23 ; and consists of a circular foot $e$, from which rises a short tubular stem $d$, into which 
slides another short tube $c$, carrying at its top a joint $f$; to this joint is fixed a square tube $a$, through which a rod $b$ slides; this rod has at one end another but smaller joint $g$, to which is attached a collar $h$, for receiving the lens $i$. By means of the joint at $f$, the square rod can be moved up or down, so as to bring the lens close to the object; and by the rod sliding through the square tube $a$, the distance between the stand and the lens may be either increased or diminished: the joint $g$, at the end of the rod, is for the purpose of allowing the lens to be brought either horizontally or at an angle to the subject to be investigated. By means of the sliding arm the distance between the table and the jointed arm can be increased or diminished. This microscope is provided with lenses of one-inch and half-inch focal length, and is thereby most useful for the examination and dissection of objects. It is readily unscrewed and taken to pieces, and may be packed in a small case for the pocket.

Another highly-useful and more complete simple microscope was contrived by Mr. W. Valentine, and made for him by Mr. Ross in 1831. It is thus described by the latter gentleman, and is represented in fig. 24 . It is supported on a firm tripod, made of bell-metal, the feet of which, $a a a$, are made to close up for the purpose of packing it in a box. The firm pillar $b$ rises from the tripod, and carries the stage $e$; this is further strengthened by the two supports $r r$. From the pillar a triangular bar $d$, and a triangular tube $c$, is moved up and down by a screw, having fifty threads in the inch, and turned by a large milled head $v$, which is situated at the base of the pillar: this is the fine adjustment. The small triangular bar $d$ is moved up and down within the triangular tube $c$, by turning the milled head $t$, forming the coarse adjustment: this bar carries the lens-holder $m n \circ p$. The stage $e$ consists of three plates; the lowest one is firmly attached to the pillar, and upon this the other two work. The upper one carries a small elevated stage $g$, on which the objects are placed; this stage is mounted on a tube $f$, and has a spring clip $h$, for holding, if necessary, the objects under examination. By means of two screws placed diagonally, one of which is seen at $s$, this elevated stage can be moved 
in two directions, at right angles to one another; and thus different parts of objects can be brought successively into

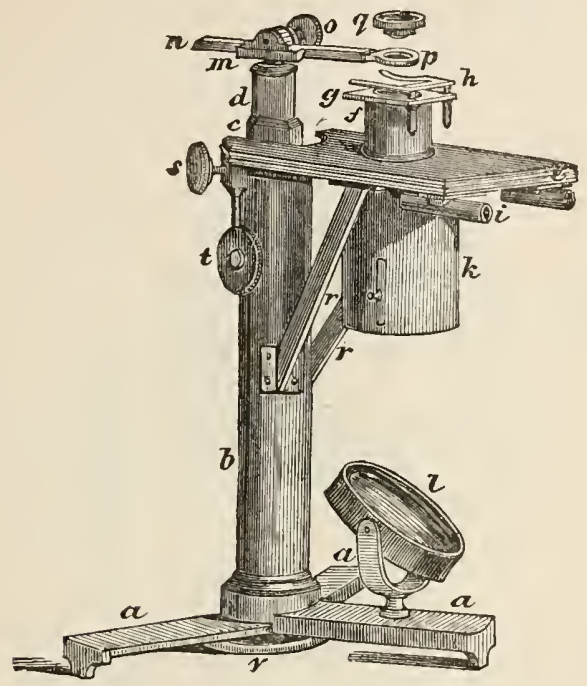

Fig. 24.-Valentine's Microscope.

the field of view. The arm $n p$, for carrying the lenses, is attached to the triangular bar $d$ by a conical pin, on which it is made to turn horizontally, and the arm itself can be lengthened or shortened by means of the rack and pinion $m o$; hence the lens $q$ can be applied to every part of an object without moving the stage.

The mirror $l$ is fitted into the largest of the three legs, and consists of a concave and plane glass reflector. To the under side of the stage is fitted a Wollaston's condenser $k$; and the lens is made to slide up and dowr by means of two small handles projecting from the cell in which the lens is set. 'Two small tubes $i$, with either a condensing lens for opaque objects, or a pair of forceps, may be attached to this side of the stage. The magnifiers are 
either simple lenses or doublets; or it could be ensily converted into a compound microscope by inserting a compornd body, supported on a bent arm, in the place of the one carrying the single lenses.

An arrangement devised by the late MIr. Quekett, for a dissecting microscope, represented in fig. 25 , is one of value

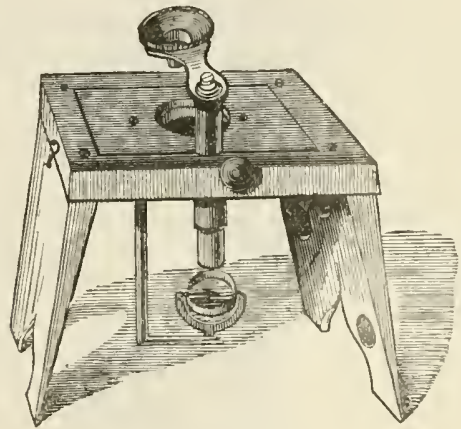

Fig. 25.-Quekett's Simple Hicroscope.

and convenience. The instrument is made by Mr. Ladd of Beck Street, and is furnished by him with three magnifiers, namely, an inch, and half-inch, ordinary lenses, and a quarter-inch Coddington; these will be found to be the powers most useful for the purposes to which this instrument is specially adapted. The lenses, mirror, condenser, vertical stem, \&c., all fit into hollows cut for their reception on the under side of the stage, and are then covored and kept in place by the side flaps: so that, when packed together, and the flaps kept secure by an India rubber band, the instrument is rery conveniently portable. The size and firmuess of the stage afford great facilities for dissection, and other scientific investigations.

The Compouxd Microscope.-The compound microscope may, as before stated, consist of only two lenses, while a simple microscope has been shown to contain sometimes three. In the triplet for the simple microscope, however, it was explained that the object of the first two lenses was 
to do what might have been accomplished, though not $\mathrm{so}$ well, by one; and the third merely effected certain modi. fications in the light before it entered the eye. But in the compound microscope the two lenses have totally different functions: the first receives the rays from the object, and bringing them to new foci, forms an image, which the second lens treats as an original object, and magnifies it just as the single microscope magnified the object itself.

Fig. 26 shows the earliest form of the compound microscope, with the magnified image of a fly, as given by Adams, which he describes as consisting of an object-glass, $l n$, a field glass $d e$, and an eye-glass, $f g$; the object, $b^{\prime} o^{\prime}$, being placed a little further from the lens than its principal focal distance, the pencil of rays from which converge to a focus, and form an inverted image of the object at $p q$, which image is viewed by the eye placed at a through the eye-glass $f g$. The rays remain parallel after passing out until they reach the eye, when they will converge by the refractive powers of this organ, and be collected on the retina. But the image differs from the real object in a very essential particular. The light being emitted from the object in every direction, renders it visible to an eye

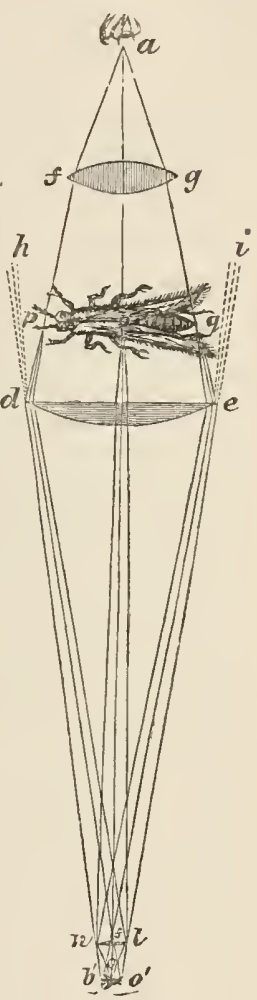

Fig 26. placed in any position; but the points of the image formed by a lens emitting no more than a small conical body of rays, which it receives from the glass, can be visible only to the eye situate within its range. Thus the pencil of rays emanating fiom the object at $o^{\prime}$, unless converged by the ficld-lens to $f$, would cross each other, and diverge 
towards $h$, and therefore would never arrive at the lens $f g$, without the interposition of the plano-convex lens at $d e_{\text {; }}$ placed at a smaller distance from the object; and by this means the pencil $d n$, which would have proceeded to $h$, is refracted or bent towards the lens $f g$, having a radial point at $p q$. The object is magnified upon two accounts: first, because if we view the image with the maked eye, it would appear as much longer than the object as the image is really longer than it, or as the distance $f b$ is greater than the distance from the real object to $f^{\prime}$; and secondly, because this picture is again magnified by the eye-glass. The compound microscope, then, consists of an object-lens, $l n$, by which the image is formed, enlarged, ond inverted; an amplifying lens, $d e$, by which the field of view is enlarged, and is consequently called the field-glass; and an eye-glass or lens $f g$, by which the eye is permitted to approach very near, and consequently enabled to view the image under a large angle of apparent magnitude. The two, when combined, are termed the eye-piece.

Mr. Lister's investigations in the year 1829, made for the purpose of improving and correcting the imperfections of the object-glasses of the compound microscope, led to the most important results. Mr. Ross also presented to the Society of Arts, in 1837, a paper on the subject, this was published in the 5lst volume of their Transactions: he this writes:-

"In the course of a practical investigation, with the view of constructing a combination of lenses for the objectglass of a compound microscope which should be free from the effects of aberration, both for central aud oblique pencils of great angle, I obtained the greatest possible distance between the object and object-glass; for in object-glasses of short focal length, their closeness to the object has been an obstacle in many cases to the use of high magnifying powers, and is a coustant source of inconvenience.

"In the improved combination the diameter is only sufficient to admit the proper pencil; the convex lenses are wrought to an edge, and the concave have only sufficient thickness to support their figure : consequently the combination is the thinnest possible, and it follows that there 
will be the greatest distance between the object and the object-glass. The focal length is $\frac{1}{8}$ of an inch, having an angnlar aperture of $60^{\circ}$, with a distance of $\frac{1}{25}$ of an inch, and a magnifying power of 970 times linear, with perfect definition on the most difficult Podura scales. I have made object-glasses $\frac{1}{1 \sigma}$ of an inch focal length; but as the angular aperture cannot be advantageously increased if the greatest distance between the object and object-glass is preserved, their use will be very limited.

"The quality of the defivition produced by an achromatic compound microscope will depend upon the accuracy with which the aberrations, both chromatic and spherical. are balanced, together with the general perfection of the workmanship. Now in Woliaston's doublets anci Holiand s triplets there are no meass of producing a balance of the aberrations, as they are composed of convex lenses only; therefore the best thing that can be done is to make the aberrations a minimum. The remaining positive aberration in these forms produces its peculiar effect upon objects (particularly the detail of the thin transparent class), which may lead to misapprehension of their true structure; but with the achromatic object-glass, where the aberrations are correctly balanced, the most minute parts of an object are accurately displayed, so that a satisfactory judgment of their character may be formed. When an object has its aberrations balanced for viewing an opaque object, and it is required to examine that object by transmitted light, the correction will remain; but if it is necessary to immerse the object in a fluid, or to cover it with glass, an aberration arises from these circumstances which will disturb the previous correction, and consequently deteriorate the definition; and this defect will be more obvious from the increase of distance between the object and object-glass.

"If an object-glass is constructed as represented in fig. 27, where the posterior combination $p$ and the middle $m$ have together an excess of negative aberration, and if this be corrected by the anterior combination $a$ having an excess of positive aberration, then this latter combination can be made to act more or less powerfully upon $p$ and $m$, by making it approach to or recede from them; for when 
the three act in close contact, the distance of the object from the object-glass is greatest, and consequently the
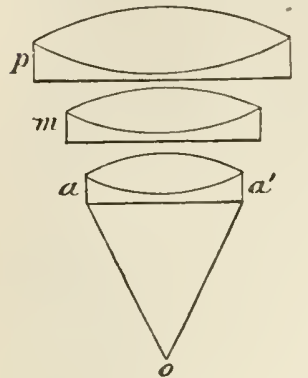

Fig. 27 . lays from the object are diverging from a point at a greater distance than when the combinations are separated; and as a lens bends the rays more, or acts with greater effect, the more distant the object is from which the rays diverge, the effect of the anterior combination $a$ upon the other two, $p$ and $m$, will vary with its distance from thence.

"When, therefore, the correction of the whole is effected for an opaque object, with a certain distance between the anterior and middle combination, if ther are then put in contact, the distance between the object and object-glass will be imcreased ; consequently, the anterior combination will act more powerfully, and the whole will have an excess of positive aberration. Now the effect of the aberration produced by a piece of flat and parallel glass being of the negative character, it is obvious that the above considerations suggest the means of correction, by moving the lenses nearer together, till the positive aberration thereby prodnced balances the negative aberration cansed by the medium.

" The preceding refers only to the spherical aberration; but the effect of the chromatic is also seen when an object is covered with a piece of glass: for in the course of my experiments I observed that it produced a chromatic thickening of the outline of the Podura and other delicate scales; and if diverging rays near the axis and at the margiu are projected through a piece of flat parallel glass, with the various indices of refraction for the different colours, it will be seen that each lay will emerge, separated, into a beam consisting of the component colonrs of the ray, and that each beam is widely different in form. This difference, being magnified by the power of the microscope, readily acconuts for the chromatic thickening of the outline just mentioned. Therefore, to obtain the 
finest definition of extremely delicate and minute objects, they should be viewed without a covering; if it be desirable to immerse them in a fluid, they should be covered with the thinnest possible film of talc, as, from the character of the chromatic aberration, it will be seen that varying the distances of the combinations will not sensibly affect the correction; though object-lenses may be made to include a given fluid, or solid medium, in their correction for colour.

"The mechanism for applying these principles to the correction of an object-glass under the various cireumstances, is represented in fig. 28 , where the anterior lens is set in the end of a tube $a$, which slides on the cylinder $b$, containing the remainder of the combination; the tube $a$, holding the lens nerrest the object, may then be moved upon the cylinder $b$, for the purpose of varying the distauce, according to the thickness of the glass covering the object, by turning the screwed ring $c$, or more simply by sliding the one on the other, and clamping them together when adjusted. An aperture is made in the tube $a$, within which is seen a mark engraved on

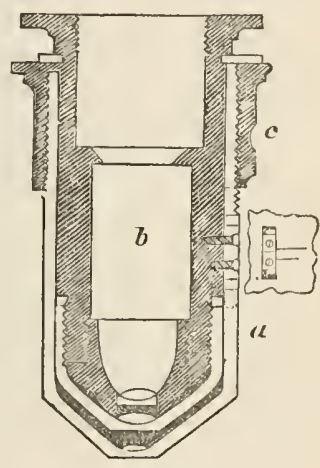

Fig. 28. the cylinder; and on the edge of which are two marks, a longer and a shorter, engraved upon the tube. When the mark on the cylinder coincides with the longer mark on the tube, the adjustment is perfect for an uncovered object; and when the coincidence is with the short mark, the proper distance is obtained to balance the aberrations produced by glass the hundredth of an inch thick, and such glass can be readily supplied. 'This adjustment should be tested experimentally by moving the milled edge, so as to separate or elose together the combinations, and then bringing the object to distinct vision by the screw adjustment of the microscope. In this process the milled edge of the object-glass will be employed to adjust for character 
of definition, and the fine screw movement of the microscope for correct focus.

"It is hardly necessary to observe, that the necessity for this correction is wholly independent of any particular constrnction of the object-glass, as in all cases where the object-glass is corrected for an object uncovered, any covering of glass will create a different value of aberration to the first lens, which previously balanced the aberration resulting from the rest of the lenses; and as this disturbance is effected at the first refraction, it is independent of the other part of the combination. The visibility of the effect depends on the distance of the object from the object-glass, the angle of the pencil transmitted, the focal length of the combination, the thickness of the glass covering the object, and the general perfection of the corrections of chromatism and the oblique pencils.

"With this adjusting object-glass, therefore, we can have the requisites of the greatest possible distance between the object and object-glass, an intense and sharply-defined image throughout the field, from the large pencil transmitted, and the accurate correction of the aberrations; also, by the adjnstment, the means of preserving that correction under all the varied circumstances in which it may be necessary to place an object for the purpose of observation."

Angle of Aperture.-The definition of an object-glass

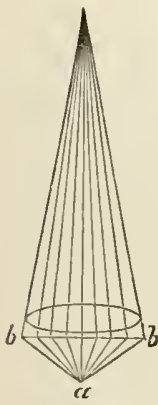

Fig. 29.

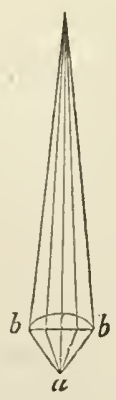

Fig 30. much depends upon the increased "angle of aperture." The angle of aperture is that angle, which the most extreme rays that are capable of being transmitted through the object-glass make at the point of focus : $b \quad a b$, in figs. 29 and 30 , is the angle of aperture; but it will be seen that the angle of aperture is much greater in fig. 29 than in fig. 30 , which represents an uncorrected lens; ensequently, a much larger quantity of light is trans- 
witted by the former than by the latter, when any object is subjected to examination. In order to see an object distinctly with an uncorrected lens, it is necessary to diminish the aperture so much, by the aid of stops, as to interfere with the transmission of the amount of light required to see the object perfectly. The greatest angle of aperture of which a given lens is capable, will be found by determining the greatest obliquity with which it is possible for rays to fall upon the object-glass, so as to be refracted to the eye-glass. Dr. Goring and Mr. Pritchard contrived an instrument for ascertaining this, for any given object-glass; which instrument is fully described in Dr. Lardner's useful little work on the microscope.

A very perfect instrument for measuring the angle of aperture, designed by Mr. Gillett, consists of two microscopes, the optical axes of which may be adjusted to coincidence. One of these is attached horizontally to the traversing arm of a horizontal graduated circle, and is adjusted so that the point of a needle, made to coincide with the axis of motion of the movable arm, may be in focus and in the centre of the field of view. The other microscope, to which the object-glass to be examined is attached, is fixed, and so adjusted that the point of the same needle may be in focus in the centre of its field. The eye-piece of the latter is then removed, and a cap with a very small aperture is substituted, close to which a lamp is placed. It is evident that the rays transmitted by the aperture will pursue the same course in reaching the point of the needle as the visual rays from that point to the eye, but in a contrary direction; and being transmitted through the movable microscope, the eye will perceive an image of the bright spot of light throughout that angular space that represents the true aperture of the object-glass examined. The applications of this instrument in the construction of object-glasses are too numerous to be here detailed: amongst the most obvious of which may be mentioned the ready means it presents of determining the nature, and measuring the amount of aberration in any given optical combination.

There is yet another source of inaccuracy which is more mechanical than optical. All the lenses composing the 
microscope require to be set in their respective tubes, sc that their sereral axes shall be directed in the same straight line with the greatest mathematical precision. This is what is called centering the lenses, and it is a process which, in the case of microscopes, demands great skill on the part of the manufacturer. The slightest

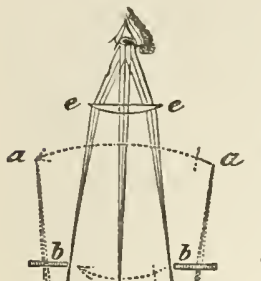

deriation from true centering would cause the images produced by the lenses to be laterally displaced, one heing thrown more or less to the right, and the other to the left, or one upwards and the other downwards; and eren thongh the aberrations should be perfectly effaced, the superposition of such displaced images would effectually destroy the efficiency of the instrument. It should also be so accurate, that the optical axis of the instrument should not be in the least altered by morement in a vertical direction; so that, if an object be brought into the centre of the field with a low power, and a higher power be then substituted, it should be found in the centre of its field, notwithstanding the great alteration in focus.

Fig. 31 represents the body of one of Mr. Ross's compound microscopes with the triple object-glass, where $o$ is an object; and above it is seen the triple achromatic object-glass, in connection with the eye-piece $e e, f f$ the plano-convex lens; $e$ e being the eyeglass, and $f f$ the field-glass, and between them, at $b b$, a dark spot or diaphragm. The course of the light is shown by three rays drawn from the centre, and three from each end of the object $o$; these rays, if not prevented by the lens $f f$, or the diaphragm at $b b$, would form an image at $a a$; but as they meet with the lens $f f$ in their passage, they are 
converged by it and meet at $b \quad b$, where the diaphragm is placed to intercept all the light except that required for the formation of a perfect image; the image at $b b$ is further magnified by the lens $e e$, as if it were an original object. The triple achromatic combination constructed on Mr. Lister's improved plan, although capable of transmitting large angular pencils, and corrected as to its own errors of spherical and chromatic aberration, would, in some instances, be less effective without an eye-piece of peculiar construction.

The eye-priece, which up to this time is considered to be the best to employ with achromatic object-glasses, to the performance of which it is desired to give the greatest possible effect, is described by Mr. Cornelius Varley, in the fifty-first volume of the Transactions of the Society of Arts. The eye-piece in question was invented by Huyghens for telescopes, with no other view than that of diminishing the spherical aberration by producing the refractions at two glasses instead of one, and of increasing the field of view. It consists of two plano-convex lenses, with their plane sides towards the eye, and placed at a distance apart equal to half the sum of their focal lengths, with a stop or diaphragm placed midway between the lenses. Huyghens was not aware of the value of his eye-piece; it was reserved for Boscovich to point ont that he had, by this important arrangement, accidentally corrected a great part of the chromatic aberration. Let fig. 32 represent the Huyghenian eye-piece of a microscope, $f f$ being the fieldglass, and $e e$ the eye-glass, and $l m n$ the two extreme rays of each of the three pencils emanating from the centre and ends of the object, of which, but for the fieldglass, a series of coloured images would be formed from $r r$ to $b \quad b$; those near $r r$ being red, those near $b \quad b$ blue, and the intermediate ones green, yellow, and so on, corresponding with the colours of the prismatic spectrum. 'This order of colours is the reverse of that of the common compound microscope, in which the single object-glass projects the red image beyond the blue.

The effect just described, of projecting the blue image beyond the red, is purposely produced for reasons presently to he given, and is called orer correcting the object-glass 
sis to colour. It is to be observed also, that the images $b b$ and $r r$ are curred in the wrong direction to be distinctly seen by a convex eye-lens, and this is a further defect of the compound microscope of two lenses. But

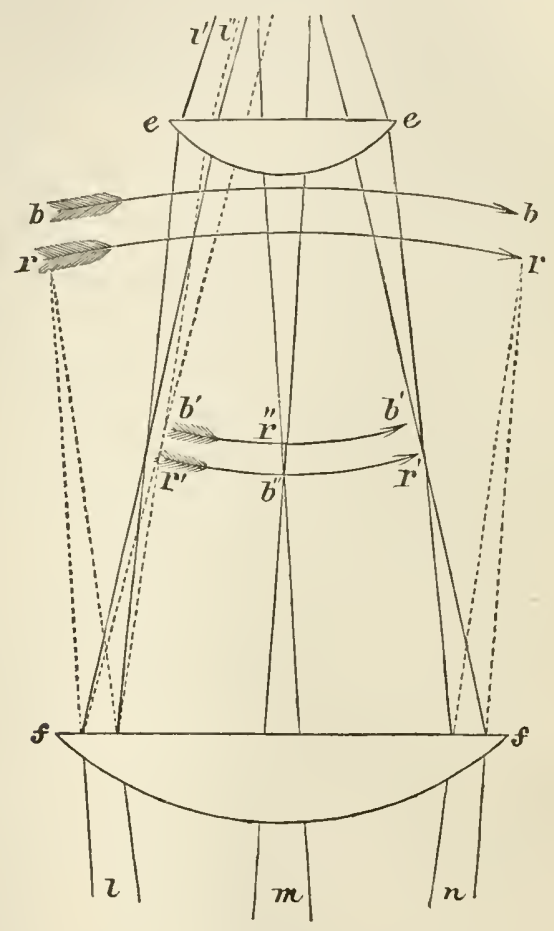

Fig. 32 .

the field-glass, at the same time that it bends the rays and converges them to foci at $b^{\prime} b^{\prime}$ and $r^{\prime} r^{\prime}$, also reverses the curvature of the images as there shown, and gires them the form best adapted for distinct vision by the eye-glass $e e$. The field-glass bas at the same time brought the blue and red images closer together, so that they are adapted to 
pass uncoloured through the eye-glass. To render this important point more intelligible, let it be supposed that the object-glass had not beeu over-corrected, that it had been perfectly achromatic; the rays would then have become coloured as soon as they had passed the field-glass; the blue rays, to take the central pencil, for example, would converge at $b^{\prime \prime}$, and the red rays at $r^{\prime \prime}$, which is just the reverse of what the eye-lens requires; for as its blue focus is also shorter than its red, it would demand rather that the blue image should be at $r^{\prime \prime}$, and the red at $b^{\prime \prime}$. This effect we have shown to be produced by the overcorrection of the object-glass, which protrudes the blue foci $b b$ as much beyond the red foci $r r$ as the sum of the distances between the red and the blue foci of the field-lens and eye-lens; so that the separation $b r$ is exactly taken up in passing through those two lenses, and the whole of the colours coincide as to focal distance as soon as the rays have passed the eye-lens. But while they coincide as to distance, they differ in another respect,- the blue images are rendered smaller than the red by the superior refractive power of the field-glass upon the blue rays. In tracing the pencil $l$, for instance, it will be noticed that, after passing the field-glass, two sets of lines are drawn, one whole and one dotted, the former representing the red, and the latter the blue rays. This is the accidental effect in the Huyghenian eye-piece pointed out by Boscovich. The separation iuto colours of the fieldglass is like the over-correction of the object-glass,-it leads to a subsequent complete correction. For if the differently coloured rays were kept together till they reached the eye-glass, they would then become coloured, and present coloured images to the eye; but fortunately, and most beautifully, the separation effected by the fieldglass causes the blue rays to fall so much nearer the centre of the eye-glass, where, owing to the spherical figure, the refractive power is less than at the margin, that that spherical error of the eye-lens constitutes a nearly perfect balance to the chromatic dispersion of the field-lens, and the blue and red rays $l^{\prime}$ and $l^{\prime \prime}$ emerge sensibly parallel, presenting, iu consequence, the perfect definitiou of a single point to the eye. The same reasoning 
is true of the intermediate colours and of the other pencils. The eye-glass $e e$ not only brings together the images $b^{\prime} b^{\prime}, r^{\prime} r^{\prime}$, but it likewise has the most important effect of renderiug them flat, thus at once correcting both the chromatic and spherical aberration.

The Huyghenian eye-piece, which we have described, is the best for merely optical purposes; but when it is

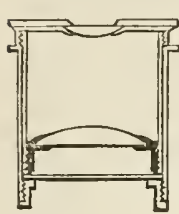

Fig. 33. required to measure the magnified image, we use the eye-piece invented by $\mathrm{Mr}$. Ramsden, and called by him the micrometer eye-piece. The arrangement may be readily understocul upon reference to fig. 33 . The field-glass has now its plane face turned towards the object; the rays from the object are made to converge immediately in front of the field-glass; and here is placed a plane-glass, on which are engraved divisions of 1-100th of an inch or less. The markings of these divisions come into focus, therefore, at the same time as the image of the object, and both are distinctly seen together. The glass with its divisions is shown in fig. 34 , on which, at $\mathrm{A}$, are seen some magnified grains of starch. Thus the measure of the magnified image is given by mere inspection; and the Fig. 34 . value of such measurements, in reference to the real object, when once obtained, is constant for the same object-glass.

It is affirmed by Mr. Ross, that if the achromatic principle were applied to the construction of eye-pieces, the latter is the form with which the greatest perfection would be obtained. That such an adaptation might be productive of valuable results, appears from Mr. Brooke's statement, that he has employed as an eye-piece, a triplet objective of one inch focus, the definition obtained by it being superior to that afforded by the ordinary Huyghenian eye-piece. Some of the lowest French achromatic 
object-glasses answer extremely well for this purpose ; and as the sets are usually made removable, the front pair call be readily separated for the experiment.

Mr. Lister places on the stage of his micruscope a divided scale, the value of which is known ; and viewing the scale as the microscopic object, observes how many or the divisions on the scale attached to the eye-piece correspond with one of those in the magnified image. If, for instance, ten of those in the eye-piece correspond with one of those in the image, and if the divisions are known to be equal, then the image is ten times larger than the object, and the dimensions of the object are ten times less than indicated by the micrometer. If the divisions on the nicrometer and on the magnified scale are not equal, it becomes a mere rule-of-three sum; but in general this tronble is taken by the maker of the instrument, who furiishes a table showing the value of each division of the micrometer for every object-glass with which it may be used.

Mr. Jackson invented the simple and cheap form of micrometer, represented in fig. 35, which he described in the Microscopical Society's Transactions, 1840. It consists of a slip of glass placed in the focus of the eyeglass, with the divisions sufficiently fine to have the value of the ten-thousandth of an inch with the quarter-inch object-glass, and the twenty-thousandth with the eighth; at the same time the half, or even the quarter of a division may be estimated, thus affording the means of attaining all the accuracy that is really available. It may therefore entirely supersede the more complicated and expensive screw-inicremeter, being much handier to use, and not liable to derangement in inexperienced hands.

The positive eye-piece gives the best view of the micrometer, the negative of the object. The former is quite free from distortion, even to the edges of the field; but the object is slightly coloured. The latter is free from colour, but is slightly distorted at the edges. In the centre of the field, however, to the extent of half its diameter, there is no perceptible distortion; and the clearness of the definition gives a precision to the measure- 
mont which is very satisfactory. For this reason $\mathrm{Mr}$ Jackson gives it the preference.

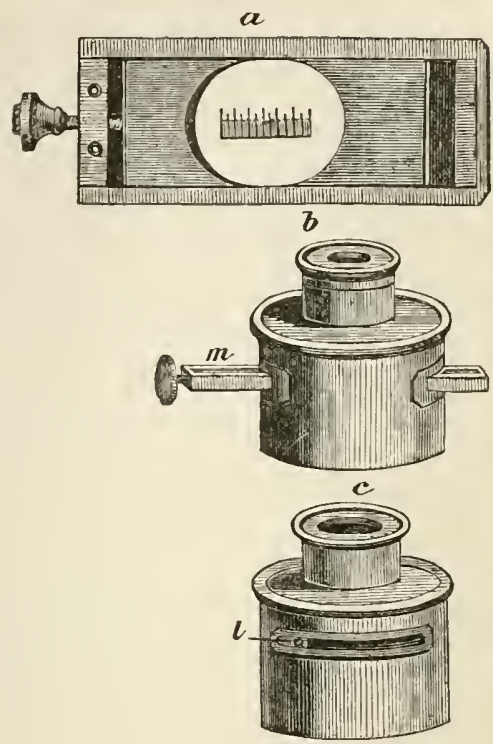

Fig. 35.-Mr. Jackson's Micrometer eye-piece.

Short bold lines are ruled on a piece of glass, $a$, fig. 35 ; and to facilitate counting, the fifth is drawn longer, and the tenth still longer, as in the common rule. Very finely levigated plumbago is rubbed into the lines, to render them visible; and they are covered with a piece of thin glass, cemented by Canada balsam, to secure the plumbago from being wiped out. The slip of glass thus prepared is placed in a thin brass frame, so that it may slide freely; and is acted on at one end by a pushing-screw, and at the other by a slight spring.

Slips are cut in the negative eye-piece on each side, $b$, so that the brass frame may be pressed across the field in the focus of the eye-glass, as at $m$; the cell of which should have a longer screw than usual, to admit of adjust- 
ment for different eyes. The brass frame is retained in its place by a spring within the tube of the cye-piece; and in using it the object is brought to the centre of the field by the stage movements; and the coincidence between one side of it and one of the long lines is made with great accuracy by means of the small pushing-screw that moves the slip of glass. The divisions are then read off as easily as the inches and tenths on a common rule. The operation, indeed, is nothing more than the laying a rule across the body to be measured; and it matters not whether the object be transparent or opaque, mounted or not mounted, if its edges can be distinctly seen, its diameter can be taken.

Previously, however, to using the micrometer, the value of the divisions should be ascertained with each objectglass; the mode of doing which is best performed as follows :-

"Lay a slip of ruled glass on the stage; and having turned the eye-piece so that the lines on the two glasses are parallel, read off the number of divisions in the eyepiece which cover one on the stage. Repeat this process with different portions of the stage-micrometer, and if there be any difference, take the mean. Suppose the hundredth of an inch on the stage requires eighteen divisions in the eye-piece to cover it : it is quite plain that an inch would require eighteen hundred, and an object which occupied nine of these divisions would measure the twohundredth of an inch. This is the common mode of expressing microscopical measurements; but I am of opinion that a decimal notation would be preferable, if universally adopted.

"Take the instance supposed, and let the microscope be furnished with a draw-tube, marked on the side with inches and tenths. By drawing this out a short distance, the image of the stage micrometer may be expanded until one division is covered by trenty in the eye-piece. These will then have the value of two-thousandths of an inch, and the object which before measured nine will then measure ten; which, divided by 2,000 , gives the decimal traction 005 .

"Enter in a table the length to which the tube is drawn 
out. and the number of divisions on the eye-piece micrometer equivalent to an inch on the stage; and any measurements afterwards taken with that micrometer and object-glass may, by a short process of mental arithmetic, be reduced to the decimal parts of an inch, if not actually observed in them.

"In ascertaining the value of the micrometer with a deep object-glass, the hundredth of an inch on the stage will occupy too much of the field; the two-hundredth or five-hundredth should then be nsed and the number of divisions corresponding to that quantity be multiplied by two hundred or five hundred, as the case may be.

"The micrometer shond not be fitted into too deep an eye-piece, for it is essential to preserve clear definition. The middle eye-piece is for most purposes the best, pro vided the object-glass be of the first quality; otherwise, use the eye-piece of lowest power. The lens above the micrometer should not be of shorter focus than threequarters of an inch, even with the best object-glasses; and the slit cut in the tube can be closed at any time by a small sliding bar, as at $l$, fig. 35."

We subjoin the following comparative micrometrical measures given by Dr. Hannover, as a reference-table.

\begin{tabular}{|c|c|c|c|c|}
\hline Millemetre. & Paris lines. & Vienna lines. & Rhenish Iines. & English inch. \\
\hline $\begin{array}{c}1 \\
2 \cdot 25.5829 \\
2 \cdot 195149 \\
2 \cdot 179538 \\
25 \cdot 39954\end{array}$ & $\begin{array}{c}0.143296 \\
1 \\
0.973101 \\
0.466181 \\
11.25952\end{array}$ & $\begin{array}{c}0 \cdot 4555550 \\
1027643 \\
1 \\
0.992888 \\
11.57076\end{array}$ & $\begin{array}{c}0 \cdot 458813 \\
1 \cdot 035003 \\
1 \cdot(071625 \\
1 \\
11 \cdot 65364\end{array}$ & $\begin{array}{c}0.0393708 \\
0.0858138 \\
0.0864248 \\
0.0858101 \\
1\end{array}$ \\
\hline
\end{tabular}

The wonderful tracing on glass executed by M. Nobert, of Barth, in Prussia, deserves attention. The plan adopted by him is, to trace on glass ten separate bands at equal distances from each other, each band being composed of parallel lines of some fraction of a Prussian inch apart; in some they are 1-1000th, and in others ouly 1-4000th of $a$ Prussian inch separated. The distance of these paralle] lines forms fart of a geometric series :- 


$\begin{array}{ll}0.001000 & \text { lines. } \\ 0.000857 & " 1 \\ 0.000735 & \text { "1 } \\ 0.000630 ~ ", \\ 0.000540 \quad "\end{array}$

0.000463 lines.

0.000397

$0.000340, "$

$0.000292 "$,

0.000225,

To see these lines at all, it is requisite to use a microscope with a magnifying power of 100 diameters; the bands containing the fewest, number of lines will then be visible. To distinguish the finer lines, it will be necessary to use a magnifying power of $3 \cup 0$, and then the lines which are only 1-4700th of an inch (Prussian) apart will be seen perfectly traced. Of all the tests yet found for object-glasses of high power, these would seem the most valuable. These tracings have tended to confirm the undulating theory of light, the different colours of the spectrum being exhibited in the ruled spaces according to the separation of the lines; and in those cases where the distances between the lines are smaller than the length of the violet-coloured wares, no colour is perceived; and it is stated, that if inequalities amounting to 000002 line occur in some of the systems, stripes of another colour would appear in them.

Achromatic object-glasses for microscopes are of various foci, differing from 2 inches to 1-50th of an inch.

IIagnifying Power of Mr. Ross's Object-Glasses with liis various Eye-Pieces.

\begin{tabular}{|c|c|c|c|c|c|c|}
\hline \multirow{2}{*}{$\begin{array}{c}\text { EyE Glasses, } \\
\text { or } \\
\text { Eye-PIEces. }\end{array}$} & \multicolumn{6}{|c|}{ ОвJесt GLasses. } \\
\hline & 2-inch. & 1-inch. & $\frac{1}{2}$-inch. & $\frac{1}{4}$-inch. & $x_{s}$-inch. & $\frac{1}{12}-i n c h$ \\
\hline A & 20 & 60 & 100 & 220 & 420 & 600 \\
\hline B & 30 & 80 & 130 & 350 & 670 & $8 \% 0$ \\
\hline C & 40 & 100 & 180 & 500 & 900 & 1200 \\
\hline $\begin{array}{l}\text { Value of each } \\
\text { space in the MI- } \\
\text { crometer eye- } \\
\text { glass, with the } \\
\text { various object- } \\
\text { glasses. }\end{array}$ & $\begin{array}{l}\frac{1}{400} \\
.0025\end{array}$ & $\begin{array}{l}\frac{1}{970} \\
.001031\end{array}$ & $\begin{array}{c}\frac{x}{180 \overline{0}} \\
.0005263\end{array}$ & $\begin{array}{c}\frac{1}{4300} \\
.0002325\end{array}$ & $\begin{array}{c}\frac{2}{8000} \\
.0001111\end{array}$ & $\begin{array}{l}\frac{1}{18500} \\
000074\end{array}$ \\
\hline
\end{tabular}


Magnifying Power of Messrs. Powell and Lealand's Achromatic Object-Glasses.

\begin{tabular}{|c|c|c|c|c|c|c|c|}
\hline $\begin{array}{l}\text { Object } \\
\text { Glasses. }\end{array}$ & $\begin{array}{l}\text { Angular } \\
\text { Aperture. }\end{array}$ & \multicolumn{5}{|c|}{$\begin{array}{l}\text { Magnifying Power with the various } \\
\text { Eye-Pieces. }\end{array}$} & Price. \\
\hline & & No. $J$. & No. 2. & No. 3. & No. 4. & No. 5 . & \\
\hline Inches. & Deg. & & & & & & $£ 8$. \\
\hline 2 & 14 & 25 & 37 & 50 & 100 & 150 & 215 \\
\hline 1 & $\begin{array}{l}28 \\
70\end{array}$ & $\begin{array}{r}50 \\
100\end{array}$ & $\begin{array}{r}144 \\
148\end{array}$ & $\begin{array}{l}100 \\
200\end{array}$ & 400 & 600 & \\
\hline$\frac{2}{4}$ & .95 & 200 & 296 & 400 & 800 & 1200 & 5 \\
\hline${ }_{8}^{4}$ & 125 & 400 & 592 & 800 & 1606 & 2400 & $\begin{array}{ll}8 & 8\end{array}$ \\
\hline$\frac{1}{12}$ & 145 & 600 & 888 & 1200 & 2400 & 3600 & 1010 \\
\hline$\frac{2}{20}$ & 175 & 800 & 1184 & 1600 & 3200 & 4800 & 1616 \\
\hline
\end{tabular}

Schmidt's goniometer positive eye-piece, for measuring the angles of crystals, is so arranged as to be easily rotated within a large and accurately graduated circle. In the focus of the eye-piece a single cobweb is drawn across, and to the upper part is attached a vernier. The crystals being placed in the field of the microscope, and care being taken that they lie perfectly flat, the vernier is brought to zero, and then the whole apparatus turned until the line is parallel with one face of the crystal; the frame-work bearing the cobweb, with the vernier, is now rotated until the cobweb becomes parallel with the next face of the crystal, and the number of degrees which it has trarersed may then be accurately read off.

To the most complete instruments a set of eye-pieces, consisting of not less than three, is usually made. These differ in power; the longest is always the lowest power, and is marked A. Its angular aperture, which determines the size of the field of view, is generally less than that of the others (if constructed on the Huyghenean plan), being limited by the diameter of the body. It is usually about 20 degrees. The next eye-piece, or middle power, marked B, and the deepest, C, have more than 30 degrees of angular aperture.

For viewing thin sections of recent or fossil woods, coal, the fructification of ferns and mosses; fossil-sheils, seeds, small insects, or parts of larger ones; molluscs or 
the sirculation in the frog, \&c., the eyc-piece $\mathrm{A}$ is best adavted.

For examining the details of any of the above objects, : ill be advisable to substitute the eye-piece $B$, which also should be used in the observation of crystals when illuminated by polarised light, the pollen of flowers, minute dissection of insects, the vascular and cellular tissues of plants, the Haversian canals and lacunæ of bone, and the serrated laminæ of the crystalline lens in the eyes of birds and fishes.

The eye-piece $\mathrm{c}$ is of use when it is requisite to investigate the intimate structure of delicate tissues; and also in observations upon fossil infusoria, volvox, scales from moths' wings, raphides, \&c. The employment of this eyepiece, when a ligher power is required, obviates the necessity of using a deeper object-glass, which always occasions a fresh arrangement of the illunination and focus. It must be borne in mind, that the more powerful the eyepiece, the more apparent will the imperfections of the object-glass become; hence less confidence should be placed in the observations made under a powerful eyepiece than when a similar degree of amplification is obtained with a shallow one and a deeper object-glass.

The degree of perfection in the construction of the optical part of a microscope is judged of by the distinctness and cornfort with which it exhibits certain objects, the details of which can only be made visible by combinations of lenses of high magnifying power, and a near approach to correctness. Such are termed by the microscopist test-objects. Mr. C. Brooke, F.R.S., whose labours have been devoted to the correction of errors which have crept into this part of philosophical research, says:- In order to arrive at any satisfactory conclusions regarding the action of any transparent medium on light, it is necessary to form some definite conceptions regarding the external form and interual structure of the medium. This observation appears to apply in full furce to microscopic test-objects; and for the purposes of the present inquiry, it will suffice to limit our observations to the structure of two well-known test-objects, - the scales of Podura plumbea, and the siliceous loricre, or valves of the genus Pleurosigma. 
freed from organic matter: the former of these is commonly adopted as the test of the defining power of an achromatic object-glass, and the several species of the latter as the tests of the penetrating or separating power, as it has been termed. The defining power depends only on the due correction of chromatic and spherical aberrations, so that the image of any point of an object. formed on the retina may not overlap and coufuse the images of adjacent points. This correction is never theoretically perfect, since there will always be residual terms in the general expression for the aberration, whatever practicable number of surfaces we may introducc as arbitrary constants; but it is practically perfect when the residna? error is a quantity less than that which the eye can appreciate. The separation of the markings of the Pleurosigmata and other analogous objects is found to depend on good defining power associated with large angle of aperture.

The Podura scale appears to be a compound structure, consisting of a very delicate trausparent lamina or membrane, covered with an imbricated arrangement of epithelial plates, the length of which is six or eight times their breadth, somewhat resembling the tiles on a roof, or the long pile of some kinds of plush. This structure may be readily shown by putting a live Podura into a small test-tube, and inverting it on a glass-slide; the insect. should then be allowed for some time to leap and run about in the confined space. By this means the scales will be freely deposited on the glass; and being subsequently trodden on by the insect, several will be found from which the epithelial plates have been partially rubbed off, and at the margin of the mndisturbed portion the form and position of the plates may be readily recoguised. This structure appears to be rendered most evident by mounting the scales thus obtained in Canada balsam, and illuminating them by meaus of Wenham's parabolic reflector. The structure may also be very clearly recognised when the scale is seen as an opaque object under a Ross's ${ }_{1}^{2}$ th (specially adjusted for uncovered objects), illuminated by a combination of the parabola and a flat Lieberkuhn. The under-side of the scale thus appears as a smonth 
glistening surface, with very slight markings, corresponding, probably, to the points of insertion of the plates on the contrary side. The minuteness and close proximity of the epithelial plates will readily account for their being a good test of definition, while their prominence renders them independent of the separating power due to large angle of aperture.

The structure of the second class of test-objects above mentioned differs entirely from that above described; it will suffice for the present purpose to notice the valves of three species only of the genus Pleurosigma; which, as arranged in the order of easy visibility, are, $P$. formosum, $P$. hippocampus, $P$. angulatum. These appear to consist of a lamina of homogeneous transparent silex, studded with rounded knobs of protuberances, which, in $P$. formosum and $P$. angulatum, are arranged like a tier of round shot. in a triangular pile, and in hippocampus like a similar tier in a quadrangular pile, as has frequently been described; and the visibility of these projections is probably proprotional to their convexity. The "dots" have by some been supposed to be depressions; this, however, is clearly not the case, as fracture is invariably observed to take place between the. rows of dots, and not through them, as would naturally occur if the dots were depressions, and consequently the substance is thimner there than elsewhere.

This, in fact, is always observed to take place in the siliceous loricæ of some of the border tribes that occupy a sort of neutral, and yet not undisjuted, ground between the confines of the animal and vegetable kingdoms; as, for example, the Isthmia, which possesses a reticulated structure, with depressions between the meshes, somewhat analogous to that which would result from pasting together bobbin-net and tissue-paper. The valves of $P$. angulatum, and similar other objects, have been by some writers supposed to be made up of two substances possessing different degrees of refractive power; but this hypothesis is purely gratuitous, since the observed phenomena will naturally result from a series of rounded or lenticular protuberances of one homogeneous substance. Moreover, if the centres of the markings were centres of greatest density, if, in fact, the structure were at all analogous to that of the crystallino 
lens, it is difficult to conceive why the oblique rays only should be visibly affected. When $P$. hippocampus or $P$. formosum is illuminated by a Gillett's condenser, witl a cenural stop placed under the lenses, and viewed by a quarterinch object-glass of $70^{\circ}$ aperture, both being accurately adjusted, we may observe in succession, as the object-glass approaches the object, first a series of well-defined bright dots; secondly, a series of dark dots replacing these; and thirdly, the latter are again replaced by bright dots, not, however, as well defined, as the first series. A similar succession of bright and dark points may be observed in the centre of the markings of some species of Coscinodiscus from Bermuda.

These appearances would result if a thin plate of glass were studded with minute, equal, and equidistant planoconvex lenses, the foci of which would necessarily lie in the same plane. If the focal surface, or plane of vision, of the object-glass be made to coincide with this plane, a series of bright points would result from the accumulation of the light falling on each lens. If the plane of vision be next made to coincide with the surfaces of the lenses, these points would appear dark, in consequence of the rays being refracted towards points now out of focus. Lastly, if the plane of vision be made to coincide with the plane beneath the lenses that contain their several foci, so that each lens may be, as it were, combined with the object-glass, then a second series of bright points will result from the accumulation of the rays transmitted at those points. Moreover, as all rays capable of entering the object-glass are concerned in the formation of the second series of bright focal points, whereas the first series are formed by the rays of a conical shell of light only, it is evident that the circle of least confusion must be much less, and therefore the bright points better defined in the first than in the last series.

If the supposed lenses were of small convexity, it is evident that the course of the more oblique rays only would be sensibly influenced; hence probably the structure of $P$. angulatum is recognised only by object-glasses of large angular apertures, which are eapable of admitting very oblique rays.

It does not appear to be desirable that objects should he 
illuminated by an entire, or, as it may be termed, a solid cone of light of much larger angle than that of the objectglass. The extinction of an object by excess of illumination may be well illustrated by viewing with a one-inch object-glass the Isthmia, illuminated by Gillett's condenser. When this is in focus, and its full aperture open, the markings above described are wholly invisible; but as the aperture is successively diminished by the revolving diaphragm, the object becomes more and more distinct, and is perfectly defined when the aperture of the illuminating pencil is reduced to about $20^{\circ}$. The same point may be attained, although with much sacrifice of definition, by gradually depressing the condenser, so that the rays may diverge before they reach the object; and it may be remarkea, generally, that the definition of objects is always most perfect when an illuminating pencil of suitable form is accurately adjusted to focus, that is, so that the source of light and the plane of vision may be conjugate foci of the illuminator. If a condenser of $120^{\circ}$ aperture, or upwards, be used as an illuminator, the markings of Diatomaceæ will be scarcely distinguishable with the best object-glass, the glare of the central rays overpowering the structure of those that are more oblique.

There are indeed almost as many methods employed for bringing out the markings on test-objects, as there are skilled and efficient microscopists, each one preferring his own, simply because he has been at great pains in working it out with the utmost nicety. The late Professor Quekett, in his treatise on the microscope, recommends "oblique light with the mirror and lamp, removing all appliances under the stage; then, after much patience and perseverance, Grammatophora subtilissima and the Amician test may be resolved." Another plan is to use the flat mirror, the achromatic condenser, and a paraffin lamp, daylight not being so easily managed, or even so good for the $\frac{1}{8}$ th or higher objectives.

Mr. Lobb's method is seen in Fig. 36:-“The microscope is placed in the horizontal position, and a small camphine lamp so adjusted that its reservoir may be close against the end of the rack-tube; having the A eye-piece, the one-inch objective, and the achromatic condensar of $170^{\circ}$ 
aperture, place No. 1 aperture of the wheel of diaphragms in the field, then, looking through the eye-piece, centre the aperture accurately; then bring No. 11 aperture in the field, and again centre the lamp flame, and be careful that it corresponds with the axis of the pupil of the eye.

"Should we wish to examine hairs, scales, or morbid structures, use No. 3, 4, or 5 apertures of the wheel of diaphragms without a stop, the $\frac{1}{8}$ th, $\frac{\pi}{12}$ th, $\frac{x}{16}$ th, $\frac{\pi}{25}$ th, or soth object-glasses, and the $\mathrm{A}, \mathrm{B}$, or $\mathrm{C}$ eye-piece as

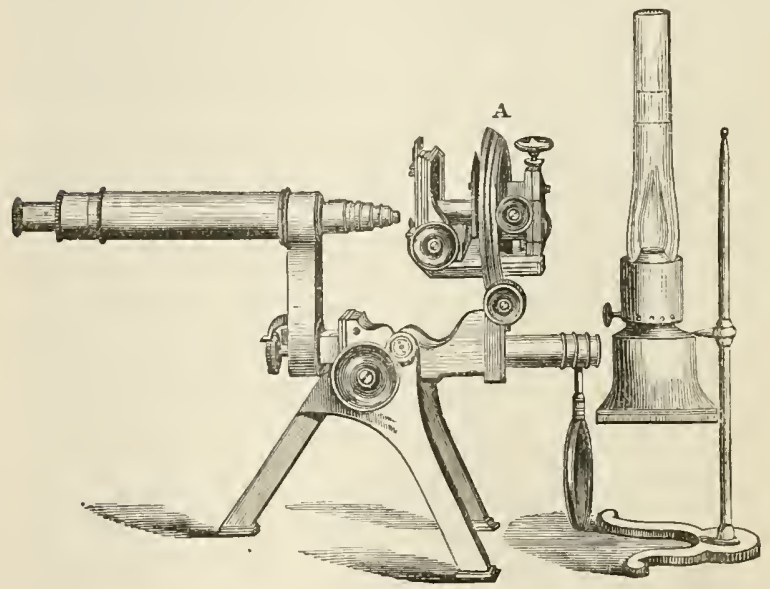

r'ig. 36. -Powell and Lealand's Microscope arranged for Lobb's illumination.

A. Secondary Stage earrying Achromatic Condenser.

thought proper; now bring the object into focus, and by racking up the condenser for the best light, very clear and satisfuctory definition will be obtained. Nothing more is required when examining these objects than the selection of the most suitable aperture, and focussing the condenser for the illumination which defir es the best; too much and too little light being equally bad.

"If we wish to examine objects such as Pleuresigma 
formosum, I'. quadratum, P. angulatum, and their allics, bring No. 11 aperture in the field, rack up the condenser until the field is very bright, then put on No. I stop, and rack up the condenser until the stop disappears: if the desired effect be not obtained, try No. 2 stop; the condenser will then require to be racked up stiil higher, and the dots will come out easily. To examine Navicula cuspidatum, N. rhomboides, Pleurosigma fasciola, Pluerosigma macrum, \&c., still use No. 11 aperture, and stop No. 2, which will require a slight alteration in position only, when the checks will appear distinctly.

"For the Amician test use the slots instead of No. 2 stop; and for very difficult lines, such as those of the Acus, the condenser must be so arranged (when focussed up till the field becomes exceedingly bright) that a shaderv is thrown on the object from the left hand, and No. 1 and No. 2 stop used, so as to darken a little the right hand side of the field. The $\frac{1}{8}$ th and $\frac{1}{12}$ th object-glasses are generally found the best suited for the Acus with the $\mathrm{B}$ eye-piece ; in fact, the $\mathrm{B}$ eye-piece is mostly to be preferred with high powers."

In short, the whole requires perfect centering as well as careful illumination, and the condenser used must have a wide aperture: we have found Collins's answer: exceedingly well, and with it we have brought out the markings on the $P$. fasciola,. . Acus, \&c.

When employing high powers for viewing test, or indeed any objects, only that part which is exactly in focus can be perfectly seen at one time; and even a very slight turn of the fine adjustment, or "slow motion," often causes a totally different set of appearances to present themselves. It is desirable during our examination to keep the finger on the slow motion, and thus bring into view different parts or thicknesses of an object, so that in the most careful manner layers or sets of vessels may be seen and continuously traced. A clearer idea of the nature of a doubtful structure is in this way obtained, while we are in the act, as it were, of changing the focus. When different structural features present themselves on different planes, it is often a most difficult task to determine which of them is the nearer. This may be regarded 
as a result inherent in our mode of viewing objects by transmitted light; nevertheless it is often productive of serious embarrassment when we are dealing with the almost inappreciable markings on the silicuous diatoms, \&c. The following general rule is given by Mr. Wenham (Quart. Joum. Micros. Scien. 1854, p. 138) for securing the most efficient performance of an object-glass with any ordinary object :- " Select any dark speck or opaque portion of the object, and bring the outline into perfect focus; then lay the finger on the milled head of the fine adjustment, and move it briskly backwards and forwards in both directions from the first position. Observe the expansion of the dark ontline of the object, both when within and when without the focus. If the greater expansion, or coma, be when the object is without the focus, or farther from the objective, the lenses must be placed further asunder, or towards the mark 'uncovered.' If the greater coma be when the object is within the focus, or nearer to the objective, the lenses must be brought closer together, or towards the mark 'covered.' When the object-glass is in proper adjustment, the expansion of the outline is exactly the same both within and without the focus. A different indication, however, is afforded by such test-objects as present (like the Podura-scale, the Diatoms, \&c.) a set of distinct dots or other markings. For if the dots have a tendency to run into lines when the object is without the focus, the glasses must be brought closer torether; on the contrary, if the lines appear when the object is within the focal point, the glasses must be further separated." Mr. Wenham remarks upon the dif ficulty of accurately making this adjustment even in the best made objectives.

Mr. R. Beck, in a paper contributed to the proceedings of the Royal Microscopical Society, directs particular attention to avoidance of errors of interpretation. Viewing objects by trausmitted light, he observes, is a method peculiar to the microscopist ; it is, however, one to which our vision, applied in the ordinary way, is so much unaccustomed, t.1at in many instances it not only conveys imperfect notions of an object, but produces appearances which bear little or no resemblance to the true structure 
of the object. An instance of this is furnished in an examination of the scales of Lepisma saccharina.

"Upon the more abundant scales the most prominent markings appear as a series of double lines, which run parallel and at considerable intervals from end to end of the scale, whilst other lines, generally much fainter, radiate from the quill, and take the same direction as the outline of the scale when near the fixed or quill end; but there is, in addition, an interrupted appearance at the sides of the scale which is very different from the mere union, or 'cross-hatchings,' of the two sets of lines." (Fig. 37, Nos. 1 and 2, the upper portions.)

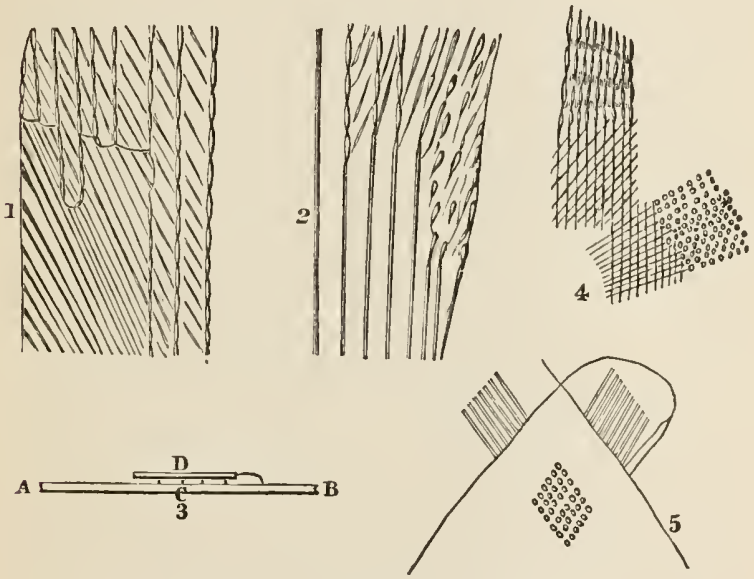

Fig. 37.-Portions of Scales of Lepisma, after Beck.

The scales themselves are formed of some truly transparent substance, for water instantly and almost entirely obliterates their markings, but they reappear unaltered as the moisture leaves them; therefore the fact of their being visible at all, under any circumstances, is due to the refraction of light by superficial irregularities, and the following experiment establishes this fact, whilst it determines at the same time the structure of each side of the scale, a 
matter which it is impossible to do from the appearance of the markings in their unaltered state :-

"Remove some of the scales by pressing a clean and dry slide against the body of the insect, and cover them with a piece of thin glass, which may be prevented from moving by a little paste at each corner. No. 3 may then be taken as an exaggerated section of the various parts. $A B$ is the glass slide, with a scale, $C$, closely adherent to it, and $D$ the thin glass cover. If a very small drop of water be placed at the edge of the thin glass, it will run under by capillary attraction; but when it reaches the scale, $C$, it will run first between it and the glass slide $A B$, because the attraction there will be greater, and consequently the markings on that side of the scale which is in contact with the slide will be obliterated, while those on the other side will, for some time at least, remain unaltered: when such is the case, the strongly marked vertical lines disappear, and the radiating ones become continuous. (See No. 1, the lower left hand portion.) To try the same experiment with the other, or inner surface of the scales, it is only requisite to transfer them, by pressing the first piece of glass, by which they were taken from the insect, upon another piece, and then the same process as before may be repeated with the scales that have adhered to the second slide; the radiating lines will now disappear, and the vertical ones become continuous. (See No. 2, left portion.) These results, therefore, show that the interrupted appearance is produced by two sets of uninterrupted lines on different surfaces, the lines in each instance being caused by corrugations or folds on the external surfaces of the scales. Nos. 1 and 2 are parts of a camera lucida drawing of a scale which happened to have the opposite surfaces obliteraied in different parts. No. 4 shows parts of a small scaie in a dry and natural state; at the upper part the interrupted appearance is not much unlike that seen at the sicles of the larger scales, but lower down, where lines of equal strength cross nearly at right angles, the lines are entirely lost in a seriec of dots, and exactly the sime apfearance is shown in No. 5 to be produced by two scales at a part where they overlie each other, altnough ach one separately shows only parallel vertical lines." 
Another very characteristic fallacy resulting from confguration is furnished in the supjosed tubular structure of human hair. When we view this object by transmitted light, it presents the appearance of a darkish flattened band down the centre; this, however, is entirely due to the convergence of the rays of light produced by the convexity of the surface of the hair. That it is a solid structure is proved by making a transverse section of the hair-shaft, when it is seen filled up with medullary substance, with the centre somewhat darker than the other part. It is, in fact, a spiral outgrowth of epithelial scales, overlarping each other like tiles on a house-top, and this gives a striated appearance to the surface. A cylindrical thread of glass in balsam appears as a flattened, band-like streak, of little brilliancy. Another instance of fallacy arising from diversity in the refractive power of the internal parts of an object, is furnished by the mistakes formerly made with regard to the true character of the lacunce and canaliculi of bone-structure, which were long mistaken for solid corpuscles, with radiating opaque filaments proceeding from a dense centre; we now know them to be minute chambers, with diverging passages, excavated in the solid osseous substance. That such is truly the case, is shown by the effects of Canada balsam : as this infiltrates the osseous substance, and fills up the excavations, it quite obliterates the bone corpuscles.

The molecular movements of finely divided particles, seen in nearly all cases when certain objects are first suspended in water, or other fluids, is often a source of embarrassment to beginners. If a minute portion of indigo or carmine be rubbed up with a little water, and - a drop placed on a glass slide under the microscope, it will at once exhibit this peculiar perpetual motion appearance. This movement was first observed in the granular particles seen among the pollen grains of plants, known as fovilla, which are set free when the pollen is crushed. Important vital endowments were formerly attributed to these particles, but Dr. Robert Brown showed such granules were common enough both in organic and inorganic substances, and therefore they were in no way particularly "indicative of life." We must especially notice another point which 
is a common cause of annoyance, as well as a source of embarrassment, to the microscopist, the disturbance produced by the passage of light through transparent bodies; this greatly impairs the sharpness of outline, and is caused by what is called the inflection or diffraction of light.

If a divergent pencil of light fall upon any opaque object placed between the light and a screen, it is eviclent that a shadow will be cast upon the screen: from our previous observation that light always moves in straight lines, we should expect to find that the image would bo clear, sharp, and well-defined; but we know from experiment that this is not so, and that there will be no exact margin between the strongly illuminated part outside, and that part of the screen which is covered by the object, but there will be more or less of a shading off at the edges of the image.

From this it is inferred that the rays of light which pass the edges of the opaque object do not proceed in the same straight lines in which they would have proceeded if the object had not been present. This effect is called inflection or diffraction, and is a consequence of the general property of undulation. When any system of waves meets with an obstacle, subsidiary systems of undulation will be formed round the extremities of the obstacle, and will be propagated from those points independently of, and simultaneously with, the original system of waves. And for a certain space around the lines in which the rays, grazing the edge of the opaque body, would have proceeded, the two systems of undulation will intersect and produce the phenomena of interference.

If the opaque bolly be very small, and the distance from the luminous point very large in proportion, the two pencils formed by inflection will intersect, and all the phenomena of interference will ensue. Thus, if the light be homogeneous, a bright line of light will be formed under the centre of the opaque object, outside of which will be dark lines, and then bright and dark lines alternately. If the light be compound solar light, a series of coloured fringes will be formed. The following is a very good illustration of how errors of interpretation may easily arise. If a small sphere formed of any opaque sub- 
stance be suspended in a dark room, and a pencil of homogeneous light be allowed to fall upon it, so that its shadow may be received on a screen, it will be found that a bright spot will appear in the middle of the shadow, outside of which will be a dark circle, and beyond this a bright circle, and so on, the circles corresponding successively to the interference of the rays, by which their brilliancy is either doubled or extinguished ; the colour of the bright circles corresponds to that of the light. If common compound light be used, the central spot will be white, and will be surrounded by a series of coloured fringes.

No rule can be laid down for the avoidance of errors such as we have been divelling upor, but the practised microscopist will, however, in time learn, almost as it were instinctively, to overcome the difficulties of diffraction, as well as those due to oblique illumination at certain angles, particularly in that which results in the production of a double image or overlying shadow, which we have seen nearly as strongly marked as the real image. This kind of shadow is sometimes called the "diffraction spectrum," although it must be evident to anyone who will be at the trouble to study the subject, that it is due to a different cause. It cannot be denied that errors of interpretation are not unfrequently due, as Mr. Brooke has shown, to the increasing desire there is to produce object-glasses with large angles of aperture. Mr. Brooke observes:- "The superiority in resolving power possessed by objectives of large angular aperture is obtained at the expense of other advantages." And Messrs. Sullivant and Wormley, in their paper ou "Nobert's Test-Plate and the Stria of Diatoms," American Journal of Science, 1861, are convinced "that when the resolving porver of an objective is near its limit, 'spectral' or spurious lines are to be seen, and these are only to be distinguished from the true by a practised eye. Hence they think "that the mere exhibition of lines is not always conclusive evidence of their ultimate resolution." - For the same reason we have not the amount of confidence in the higher powers that some observers seem to have; we know, indeed, with every increase in this direction, how liable we are to encounter unforeseen errors and exaggerations, and we still prefer the $\frac{1}{6}$ th or 
$\frac{1}{4}$-inch of Ross, because we know its precise working and defining power, and that most of the great achievements and discoveries of the microscope have been made with powers in no way superior to the last-mentioned $\frac{1}{4}$ oljective. The many important contributions to microscopy by Owen, Carpenter, Quekett, Ralfs, \&c. have been made with powers of limited capacity.

\section{MECHANICAL ARRANGEMENTS.}

Having explained the more important optical principles of the achromatic compound microscope, it remains for us to notice the mechanical and accessory arrangements for giving these principles their full effect. In few philosophical instruments is theoretical perfection so nearly reached as in the microscope: in it the highest optical skill is combined with the most consummate mechanical contrivance, and the mechanism of the instrument is of much more importance than might be imagined by those who hare not studied the subject. In the first place, steadiness, or freedom from vibrations not equally communicated to the object under examination, and to the object-glass or lenses by which it is viewed, is a point of the utmost importance. The various improvements which of late years have combined to make the microscope the superior instrument it is, may be classed under eight heads :

1. The circular rack, which is placed immediately under the stage, and which is capable of carrying it round, in some instruments, the entire revolution of a circle, is a very convenient movement for altering the angle at which an object is viewed, without putting it out of field or focus; it may also be used as a goniometer, for measuring crystals.

2. The clamping arc, peculiar to Ross's microscopes, fixes the microscope at any inclination, even after the suspension joint has become supple by long use.

3. The advantage of the sub-stage for holding and adjusting, by universal motions, all the illuminating and polarising apparatus placed beneath the object, can hardly be overrated.

4. The dark-ground illuminator is an excellent contrivance by which a bright light is apparently thrown on semi-opaque objects, though really coming beneath them, 
but so obliquely, that none of it enters the object-glass but that which is interrupted by the object.

5. Mr. Brooke's double nose-piece, or Baker's treble, is a useful piece of mechanism attached to the end of the microscope body, by which two or three object-glasses can be screwed on to it at once, and rapidly changed.

6 . The double arm to the mirror allows it to be extended so as to cast a very oblique light on objects, as well as to be raised near them without any preparatory movement.

7. The separation of the outer and inner lenses of objectglasses is an arrangement by which the greatest possible flatness of field and penetration are secured.

8. The great and manifold advantages of the binocular arrangement are very obvious; objects stand out with stereoscopic effect, and the ease to the eyes of the observer is very great. When high powers are required, the prism can be drawn aside, and the microscope used as a monocular instrument.

As regards recent optical improvements, Mr. Ross (the elder), as we have already explained (page 40), was the first to notice that however perfect the correction of an object-glass might be, "the circumstance of covering the object with a piece of the thinnest glass or talc, disturbed the corrections if they had been adapted to an uncovered object, and rendered an object-glass which was perfect under one condition, seriously defective under the other." To remedy this defect, he devised the well-known contrivance called the adjustment of object-glasses, by which they are rendered equally correct for covered or uncovered objects

The following diagrams (fig. 38) of the different objectglasses will help to explain the complex structure, and consequent costliness, of an achromatic combination. The double-convex and plano-convex lenses are of crown glass, and the plano- and double-concave, and meniscus lenses, of flint glass.

It will be seen, therefore, that each of the object-glasses from the $\frac{1}{2}$ to the $\frac{1}{25}$, is made up of as many as eight distinct lenses, the back combination being a triplet composed of two double-convex lenses of crown, with a double. 
concave lens of flint glass between them, the intermediate combination being a doublet composed of a double-convex. lens of crown glass with a double-concave of flint glass; and the front combination being another triplet composed of two plano-convex lenses of crown, and a plano-concave lens of flint glass between them. 'This will also explain the composition of the shallower object-glasses; the distance between the different combinations throughout all the objectives, and their size, depending on the amount of magnifying power required. It may be briefly stated that the principal qualities to be looked for in an object-glass
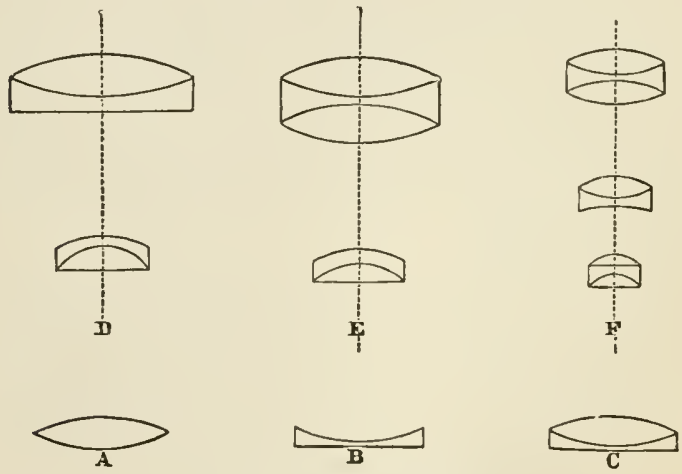

Fig. 38.-Forms of Olject-glasses.

$A$, Double-convex lens; $B$, Plano-concave; $C$, Bi-convex ana plano-contave, united: shown in their various combinations, as at $D$, form the 3 -in. 2 -in, of $1 \frac{1}{2}$-in. ; at $E, 1$-in. and $\frac{3-i n}{3}$; and at $F$, the $\frac{1}{2}$-in. $\frac{4}{20}$-in. $\frac{1}{4}-i n$ and $\frac{1}{2}-$-in. ob jectives.

are, 1st, Resolution, or the power of showing clearly minute details, which is chiefly effected by increase of light admitted through the objective, by what is termed angular aperture; 2d, Penetration, or that power which enables the observer to see deep into the structure of objects without any alteration of focus; $3 \mathrm{~d}$, Definition, or the capabilities of an objective for showing the various details of an object, especially its boundaries, with the most perfect and exquisite sharpness ; 4 th, Flatness of field, or marginal and central definition, which denotes the exact capability 
of the objective to show the peripheral or marginal portions of the field with the same sharpness as the central.'

A considerable accession of available power has been effected by both Foreign and English manufacturers since the appearance of the third edition of our book, 1859. In those by continental makers, this has been achieved by the intervention of a third medium between the exterual surface of the objective, and the covering glass of the object. We may particularize those of M. Hartnack Oberhauser's successor, as being most couspicuous; but the objective, which is said to be superior to all yet manufactured, is the $\frac{1}{50}$ th of an inch of Powell and Lealand, who, some time before, produced good objectives of th th and $\frac{x}{25}$ th of an inch focus. In these object-glasses, exces. sive angular aperture has been judiciously sacrificed to a more comprehensive and practical utility. It should be stated, however, that the priority of construction of an effective $\frac{1}{25}$ th objective is due to $\mathrm{Mr}$. Wenham, to whom the microscope was largely indebted before he made this glass in 1856. It was constructed on a principle differing from that usually adopted in the high powers, in having a single front leus. A single anterior lens transmits more light, gives clearer definition, with any required extent of aperture; from its simplicity, it is comparatively freer from errors of workmanship, and the chromatic and spherical aberrations can be more perfectly corrected. Mr. Wenham says, "that such object-glasses will challenge comparison with the best made and more expensive forms."

Dr. Beale's account of the performance of the $\frac{1}{50}$ th is, that it is even better than the $\frac{1}{25}$ th inch. Plenty of light for illuminating the objects to be examined is obtained by the use of a condenser provided with a thin cap, having an opening of not more than $\frac{1}{30}$ th inch in diameter. The objects should be covered with the thinnest glass, or with mica, and then there is room to focus to the lower surface of very thin specimens, which can alone be examined by such high powers. Particular attention is drawn to these very high powers, because the statements recently made in

(1) See an excellent paper by J. Plumer, Esq. "On the Choice of a Microvcope." Micros. Journ. vol. iii. p. 153. 
favour of the spontaneous generation theory, may probably be studied with some advantage. Not only are the minuter atoms too small to be studied by the $\frac{1}{12}$ th or $\frac{1}{15}$ th, but particles too transparent to be observed by the $\frac{7}{25}$ th are, according to Dr. Beale, distinctly demonstrated by the $\frac{1}{50}$ th ; he observes, "I feel sure that further careful study, by the aid of these high powers, of the development and increase of some of the lowest organisms, and the movements which have been seen to oceur in certain forms of living matter (amcba, white blood-corpuscles, young epithelial cells, \&c.), will lead to most valuable results, bearing upon the much debated question of vital actions.

"The most delicate constituent of the nerve-fibres of the plexus in the summit of the papilla (see Phil. Trans for 1864) can be readily traced by the aid of this power. The finest nerve-fibres thus rendered visible, are so thin, that in a drawing they would be represented by fine single lines. Near the sumnit of the papilla there is a very intricate interlacement of nerve-fibres, which, although scarcely brought out by the $\frac{x}{25}$ th, is very clearly demonstrated by this power. In this object, the separation of the fibres, as they ramify in various places, one behind anothe $_{1}$, is remarkable, and the flat appearance of the specimen as seen by the $\frac{1}{25}$ th gives place to that of considerable depth of tissue and perspective. The finest nerve-fibres, ramifying in the cornea of the eye, and in certain forms of connective tissue, are beautifully brought out; and their relation to the delicate processes frum the connective-tissue corpuscles can be more satisfactorily demonstrated than by the $\frac{1}{25}$ th. The advantage of the $\frac{1}{50}$ th in such investigations seems mainly due to its remarkable power of penetration."

The one great drawback to the use of this objectire, and a very serious one to most microscopists, is its costliness. The price of this power alone is almost more than many can afford to give for a complete instrument.

In flatness of field, and in perfection of definition, both at the centre and margin of the field of view, few objectives have equalled the recent $\frac{1}{12}$ th of $M \Gamma_{1}$. Ross, who appears to have inherited his late father's well-known skill 
in all that appertains to the microscope. Mr. Baker has also made considerable advances in the same direction: his objectives have been made with a greater angle of aperture than formerly, and with more penetration. Much nisconception, however, exists with regard to the available angle of aperture for optical requirements; it will be as well to remark that an admirable method of determining this was proposed by Professor Govin, of Turin, which consists in placing the micruscope perpendicularly to any plane dark non-reflecting surface (as a table covered with a green cloth), and having converted the instrument into a telescope, by placing above the eye-piece a suitable combination of two lenses (such as the Examining-Glass of Mr. Ross), and then examining and marking the greatest lateral distance on either side at which a clear image of some distinct object, such as a narrow strip of white cardboard or paper, laid on the table can be perceived. "Half the distance between these two points, divided by the vertical distance of the focal point of the objective from the surface of the table, will, by reference to a table of natural tangents, give half the required angle of aperture. This will, in many cases, be found to be considerably less than what may be termed the angle of admission of diffused light. To illustrate this, supposing the focal point to be at a distance of 0.01 inch from the surface of the objective, a reference to a table of natural tangents will show that an angular aperture of $17^{\circ}$ wili necessitate a linear aperture of 0.22 inch : an aperture of $170^{\circ}$ will require 0.28 inch, and one of $174^{\circ}$, of $0.38 \mathrm{inch}$, in order to admit the extreme rays, which, for objectives of $\frac{1}{8}$ th inch focus, is manifestly impissible."

In regard to angle of aperture, it may be stated once and for all, that large angle of aperture is necessarily incompatible with that far more generally useful quality of penetration. Penetrating power ${ }^{1}$ is synonymous with

(1) Penetrating Power. "The origin of this teras will be found in the Phil. rrans, for 1500 , in an article by Sir Wm. Herschel, entitled 'On the Power of Penetration into space possessed by Telescopes.' in that article, we are told that when, owing to the darkness, a distant church steeple was invisible, a certain telescope described, clearly slowed the time upon the clock. This, adds Sir William, was not owing to magnifying power alone, for the steeple could not be liscerned by the naked eye. And he has shown in the same article, that the 
depth of focus, that is, extreme distance of two planes, the points of which are at the same time sufficiently in focus for the purpose of distinct vision. 'This distance will manifestly increase as the angle of aperture diminishes, just as in a landscape camera the fore and back grounds can be brought into sensible focus simultaneously only by the use of a small diaphragm, which greatly diminishes the angular aperture of the incident pencils. But, at the same time, it must be borne in mind, that illumination, cateris paribus, increases or dininishes with angle of aperture, and the best working glass will be that in which a compromise is effected between these two conflicting requisites.

We entirely agree with $M r$. Brooke, that "for all practical purposes, except developing the markings of diatoms, an objective of moderate aperture will be found most available. It may reasonably be doubted whether the development of the dottings of difficult diatoms is not an object rather of curiosity than of utility, and whether it is worth the labour that has been bestowed upon the production of glasses for that especial purpose; the labour of construction being immensely augmented by the difficulty of duly balancing the aberrations of the more oblique pencils. So much is this the case, that in the best constructed objectives of the

words 'penetrating power' bave a deflnite meaning, and that the amount of this power possessed by a telescope, can be obtained by calculation. And of course, this must also be true of a microscope. This power, says Mr. Ryland, in a very interesting paper, must not be confused with angular aperture, which has reference to the objective alone; neither has it any ennnexion with either definition or thickness of field. In a word, as magnifying power expresses the angle subtended by an object or image at the eye of the observer, so penetrative power is the measure of the angle subtended by the eye at the object, or the equivalent of that angle in the case of telescopic or microscopic vision. The one is the measure of size, the other of brightness. The latter, however, must not be confused with 'illuminatiou.' The one power is neither less important nor less essential to distinct vision than the other. There required little mag. nifying power, and there was no illumination, in the case of the church steeple, still the hour could be read on the dial."

The third power, - the visual power of microscopes, - is one which has rarely been recognised as distinct.

For an object to be magnified 100 times, that is s.en at 100 th part of the distance, it is necessary not only that the angle subtended by it at the eye-the magnifying power-but also the angle subtended by the eye at the object-the penetrating power-shall be increased one hundred-fold. When this is the case, the visual jower will be 100 also.

If we approach an object bodily, these angles naturally increase in the same proportion, but it is not so where optical instruments are exployed.- $R$. $G$. Ryland "On the Optical Powers of the Microscope."-Micros. Jour. Science vol. vii. p. 27. 
iargest angles, the visual effect is sensibly impaired wheu the rays are transmitted through any other thickness of covering-glass than that for which they have been specially corrected."

The value of penetrating power is especially felt when the binocular arrangement is employed, since the assistance which it is able to give in the estimation of the solid forms of objects, is altogether neutralized by the employ ment cf objectives of such wide angular aperture as not to show any part of the object, save what is precisely in focus; and, therefore, there cannot be a doubt of the superior value of objectives of a moderate angle of aperture for ordinary working purposes.

"Successful observation, with very high power, is mainly dependent upon illumination. Indeed, by ordinary means, it is not possible to obtain a light sufficiently intense to illustrate an object magnified 3,000 diameters. I have tried various plans, and have come to the conclusion, that the most satisfactory results are obtained by the ase of Kelner's eye-piece as a condenser. By this means $I$ zan obtain a light sufficient for a magnifying power of 10,000 linear.

"My method of increasing the size of the image, without altering the object-glass, is as follows:-

"Supposing the limits of magnifying power of the object-glass to have been reached, I then increase the distance between it and the eye-piece, by lengthening the body by the aid of a draw-tube. The $\frac{x}{25}$ th objective being applied when the tube is increased in length, so that from the lowest glass of the object-glass to the eyoglass of eye-piece, the distance measures 24 inches, tho magnifying power correspouds to upwards of 10,000 diameters, 20 inches about $6,000,15$ inches about 2,600, 11 inches about 1,800 .

"If there is any reflection from the interior of the tube which renders the image indistinct, let the tube be lined with black velvet; of course the practical utility of increasing the magnifying power entirely depends upon the character of the specimen, and the preparations best suited for examination by very high powers must be innmersed in the strongest giycerine that can be procured. 
In this way, I have been able to see points which I have failed to bring out in any other way." 1

Magnifying power, as we have already explained, has to do with size only, and only expresses the magnitude of the angle subtended by the enlarged image, at the eye, as compared with that subtended by the object itself under the circumstances of ordinary standard vision; namely, at a distance of ten inches from the eye.

The Kelner eye-piece, while it increases the magnification, detracts from the definition; on this account, it is only used when desirous to show the whole of an object at once, as an insect, or in viewing the series of rings formed by different crystals with polarized light. This differs from the ordinary Huyghenian eye-piece in having a double-convex field-glass, and an achromatic meniscus eye-glass.

\section{COMPOUND MICROSCOPES.}

It should be understood that the descriptions we are about to give of Compound Microscopes cannot embrace that of every manufacturer. Want of space forbids this, and therefore we particularly wish to disclaim anj intention of instituting invidious comparisons; our desire is solely that of giving, as concisely as possible, a description of those instruments that have more immediately fallen under our notice, or have been the companions of our microscopical pursuits. At the head of our list stands-

Ross's microscope, an instrument of which the aim is not simplicity, but perfection-not the production of the best effect compatible with limited means, but the attainment of everything that the microscope can accomplish, without regard to cost or complexity. Without any undue preference, the first place may fairly be assigned to this large Compound Microscope of Mr. Ross, as the one which was earliest brought (in all essential features, at least) to its present form. The general plan of Mr. Ross's microscope will be seen to be essentially that which has been adopted in a simpler form by many other makers; but it is carried out with the greatest attention to solidity

(1) Beale, "How to Work with the Microscope." 
of construction, in those parts especially which are most liable to tremor, and to the due balancing of the weight of the different parts upon the horizontal axis. The "coarse" adjustment is made by the large milled-head

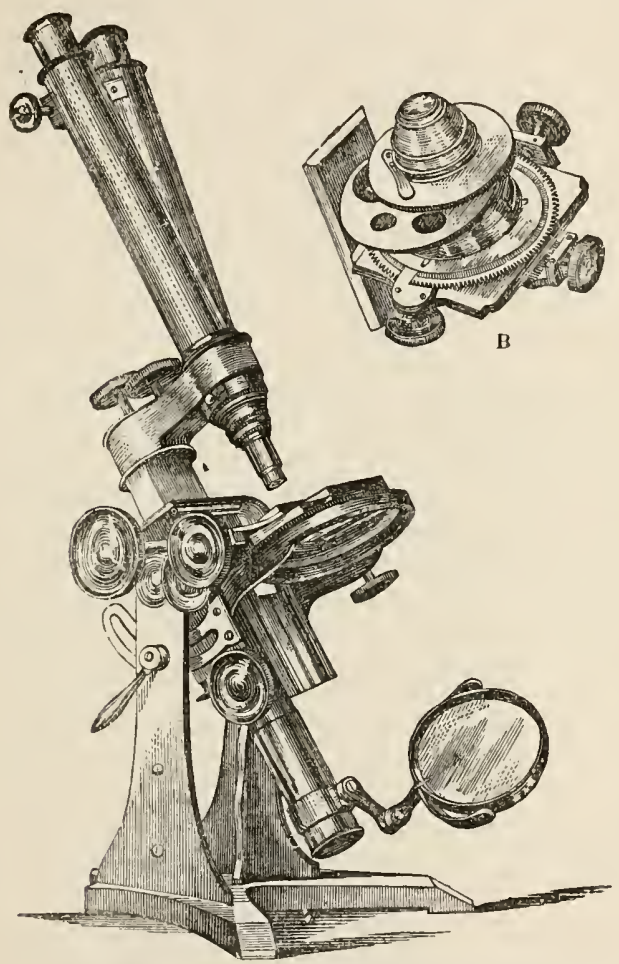

Fig. 39. - Ross's No. 1a Large Microscope, with Binocular arrangement and Object-glass. Scale $\frac{1}{6}$.

B. Sub-stage of ditto, with new Achromatic Condenser. Scale $\frac{1}{3}$.

situated just behind the summit of the uprights, which turns a pinion working into a rack cut on the back of a very strong flattened stem, that carries the transverse arm at its enmmit. A second milled-head (whicb is here con- 
cealed by the stage-fittings) is attached to the other end of the axis of the pinion (as in fig. 39), so as to be worked with the left hand. The "fine" adjustment is effected by the milled-head on the transverse arm, just behind the base of the "body." This acts upon the "nose" ur tube projecting below the arm, wherein the objectives are screwed. The other milled-head seen at the summit of the stem serves to secure the transverse arm to this, and may bo tightened or slackened at pleasure, so as to regulate the traversing movement of the arm. This movement is only allowed to take place in one direction; namely, towards the right side, being checked in the opposite by a "stop," which secures the coincidence of the axis of the body with the centre of the stage, and with the axis of the illuminating apparatus beneath it. It is in the movements of the stage that the greatest contrivance is shown. These are three; namely, a traversing movement from side to side, a traversing movement from before backwards, and a rotatory movement. The traversing movements, which allow the platform carrying the object to be shifted about an inch in each direction, are effected by the two milledheads situated at the right of the stage; and these are placed side by side, in such a position that one may be conveniently acted on by the fore-finger, and the other by the middle-finger, the thumb being readily passed from one to the other. The traversing portion of the stage carries the platform whereon the object is laid, which has a ledgo at the back for it to rest against ; and this platform has a sliding movement of its own from before backwards, by which the object is first brought near to the axis of the microscope, its perfect adjustment being then obtained by traversing movements. To this platform, and to the traversing slides which carry it, a rotatory movement is imparted by a milled-head placed underneath the stage on the left-hand side; for this milled-head turns a pinion which works against the circular rack (seen in the figure), whereby the whole apparatus above is carried round about two-thirds of a revolution, without in the least disturbing the place of the object, or removing it from the field of the microscope. This rotatory movement is useful for twe purposes-first, in the examination of very delicate objects 
by oblique light, in order that, without disturbing the illuminating apparatus, the effect of the light and shadow may be seen in every direction, whereby important additional information is often gained; and, secondly, in the examination of objects under polarized light-a class of appearances being produced by the rotation of the object between the prisnis, which is not developed by the rotation of either of the prisms themselves. The graduation of the circular rack, moreover, enables it to be used as a goniometer. In the improved form of this instrument hore represented, the whole stage-apparatus is made so thin, and the opening beneath so large, as to permit the employment of light of extreme obliquity ; and to enable the mirror to afford this, it is mounted upon an extending arm, the socket of which slides upon a cylindrical stem. Below the stage, and in front of the stem that carries the mirror, is a dovetail sliding-bar, which is moved up and down by the milled-head shown at its side. This slidingbar carries what is termed by Mr. Ross the "secondary stage" (shown separately at B), which consists of a cylin. drical tube for the reception of the achromatic condenser, the polarizing prisms and other fittings as in Messrs. Powell and Lealand's arrangement; it is here shown as fitted with a condenser specially devised by Mr. T. Ross for the illamination of a large field under low magnifying powers. To this secondary stage, also, a rotatory motion is communicated by the turning of a milled-head; and a traversing movement of limited extent is likewise given to it by means of two screws, one on the front and the other on the left-hand side of the frame which carries it, in order that its axis may be brought into perfect coincidence with the axis of the "body."

The mechanical movements and general finish of the instrument is all that can be desired by the practised observer, and tends towards that saviug of time and labour as essential in microscopical pursuits as in other branches of science. Although our description is confined to Mr. Ross's first-class microscope, other instruments without the accessory arrangements described may be had equally well made and suited to the means of the student; ranging in prices from $12 l$. 12s. upwards. 
The general plan of Powell and Lealand's Compound Microscope is represented in fig. 40 . The tripod-stand gives a firm support to the trunnions that carry the tube to which the stage is attached, and from which a triangular stem is raised by the rack-and-pinion movement set in

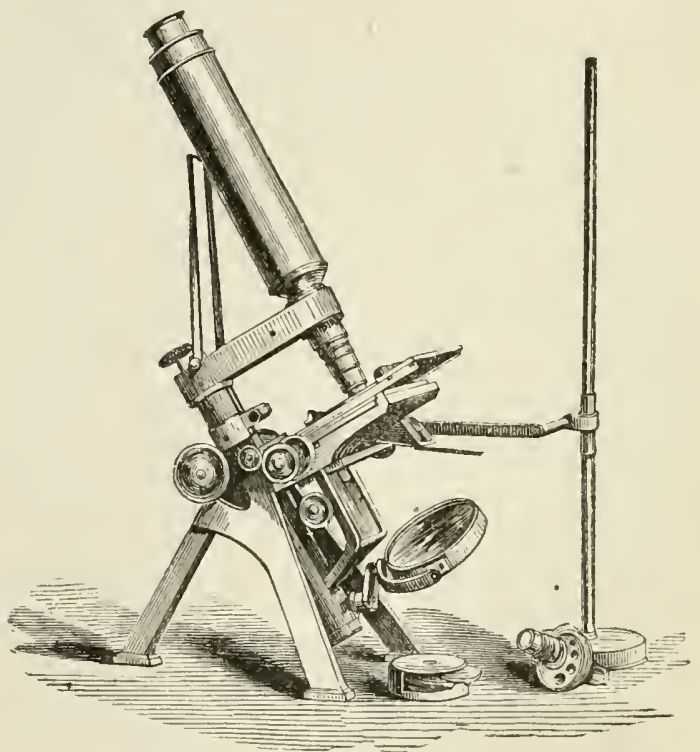

Fig. 40.-Powell and Lealand's Microscope, with Amici prism arranged for oblique illumination.

action by the double-milled head, whereby the coarse adjustment of the focus is obtained. To the upper part of the triangular bar a broad arm is fixed, bearing the com- pound body; this arm is hollow, and contams the mechanism for the fine adjustment, which is effected by turning the small milled-head. The arm is connected with the triangular bar by a strong conical pin, on which it turns, so that the compound body may be moved aside from the stage when necessary; by a mechanical arrangement it 
stops when central. The stage is of an entirely new construction, having vertical, horizontal, and circular movements, and graduated for the purpose of registering objects so as to be found at pleasure; and in order to do this effectually a clamping piece is provided against which the object slide rests, and the circular motios of the stage is stopped. It is an exceedingly effectual method of finding a favourite object. The stage is remarkably strong, and at the same time so thin, that the utmost obliquity of illumination is attainable, the under portion being entirely turned out: it has a dove-tailed sliding bar moveable by rack and pinion; into this bar slides the under stage, having vertical and horizontal motions for centering, and also a circular motion; into the stage are affixed the various appliances for underneath illumination, removed in our woodcut. Their achromatic condenser is of $170^{\circ}$ aperture, with nine openings and five central stops. The openings and stops having independent movements, the manipulator can regulate these at will, which is admitted to be an improvement. There is an especial appliance provided for the use of a set of dark-wells, with or without the condenser. The mirror is attached to a quadrant of brass and two arms, in order to obtain greater obliquity of illumination; the whole fits into a short piece of tube made to slide either up or down the long tube attached to the bottom of the stage by which the mirror is connected with the other part of the stand; the reflectors are both plane and concave, as in other instruments.

Powell and Lealaud have another pattern, somewhat resembling the instrument just describel, but larger and more massive in its general arrangements. The construction of the stage and sub-stage differ entirely : hoth of these rest on a large solid brass ring, firmly attached to the stem of the instrument. The upper side of this ring bears a sort of carriage that supports the stage, and to this carriage a rotatory motion is given by a milled-head, the arnount of the movement which may be carried through an entire revolution being exactly measured by the graduation of a circle of gun-metal, which is borne on tte upper surface of the ring. The rotatory action of the stago buing thus effected beneath the traversing movement, the cence- 
ing of an object brought into the axis of the microscope is not disturbed by it; and the workmanship is so accurate that the stage may be driven through its whole revolution without throwing out of the field an object viewed with the $\frac{1}{10}$ th objective. The stage withal is made thin enough to admit of the most oblique light being thrown on the object, such as that obtained by the use of the Amici prism, as shown arranged in the preceding figure. The sub-stage is also provided with rotatory, rectangular, and vertical movements, and is made in such a manner as to admit of the simultaneous use of the polarising prism and of the achromatic condenser. This instrument combines remarkable steadiness with great solidity, and is so well balanced on its horizontal axis that it requires no clamping in whatever position it may be placed.

Cheaper instruments are furnished by Powell and Lealand, such as a student's microscope, with $\frac{3}{4}$-inch stage movement, coarse and fine adjustments to body, plane and concave mirrors, revolving diaphragm, Lister's dark-wells, and two eye-pieces, for the price of $8 l$.

An improved form of microscope (fig. 41) is manufactured by Mr. Ladd, of Beak Street, Regent Street; having a stand so simple and light in its construction as to render it very portable and useful. It is fitted with a magnetic stage, which facilitates the moving of the objects when placed on it by the unaided fingers; a point of some importance to such microscopists as desire to retain and cultivate delicacy of touch in preference to that growing dependence upon mechanical movements. The main features of this form of microscope are, that the bearings for the compound body, stage, and sub-stage are all fitted, while connected together into the dovetailed brass bar running from top to bottom of the instrument. The magnet is attached to the under part of the stage, and a gilt iron bar, ledge, or keeper, serves for an object-rest. The sub-stage is constructed of three thin plates having rectangular movements, the top one having a tube attached, into which is fitted the Polariscope, spotted lens, \&c., the focussing of which is by a rack placed below. The mirror, being provided with a double-jointed arm, can be used with any amount of obliquity. The stand forms a tripod 
atrengthened by cross bars; the beanty of the chain movement (with which all Mr. Ladd's microscopes are furnished is made apparent by a simple and effective fine adjustmei.

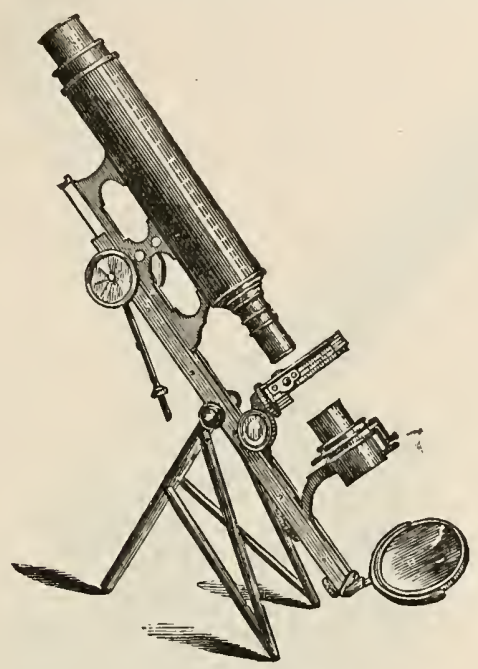

Fig. 41.-Ladd's Student's Microscope.

attached to the miiled head, thus making the one adjustment subsidiary to both purposes. The general appearance of the instrument is one of elegance, stability, lightness, and compactness.

Mr. Baker (Holborn) has kept pace with our leading manufacturers, and his first-class microscopes fairly entitlo him to take his place among the makers of superior instruments. One of his best forms, shown in fig. 42, combines good workmanship with remarkable solidity and completeness in all its details.

In this instrument, two uprights are strengthened by two internal buttresses mounted on a solid tripod. At the upper part, and between the uprights, is an axis upon which the whole of the upper part of the instrument turns, so as to enable it to take a horizontal, vertical, or 
any intermediate position-such, for instance, as that shown in the drawing. This moveable part is fixed to the axis near the centre of gravity, and consists of the stage and the arm screwed into the square bar which

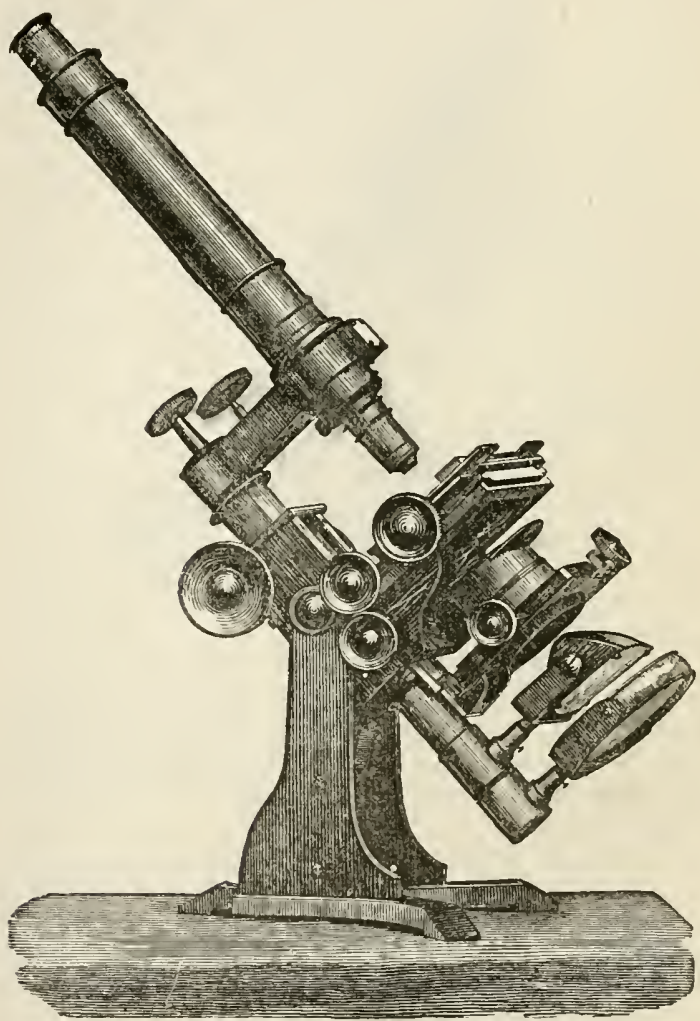

Fig. 42.-Baker's No. I Lumpounu an ceroscope.

carries the tube or body, with object-glass screwed into its place. The upper or table-stage has rectangular rackand-pinion movements, working one inch in each direction. A circular and sliding motion is also given to the top plate. 
The square bar, together with the arm and nicroscopetube, is moved by the larger milled-heads, and a more

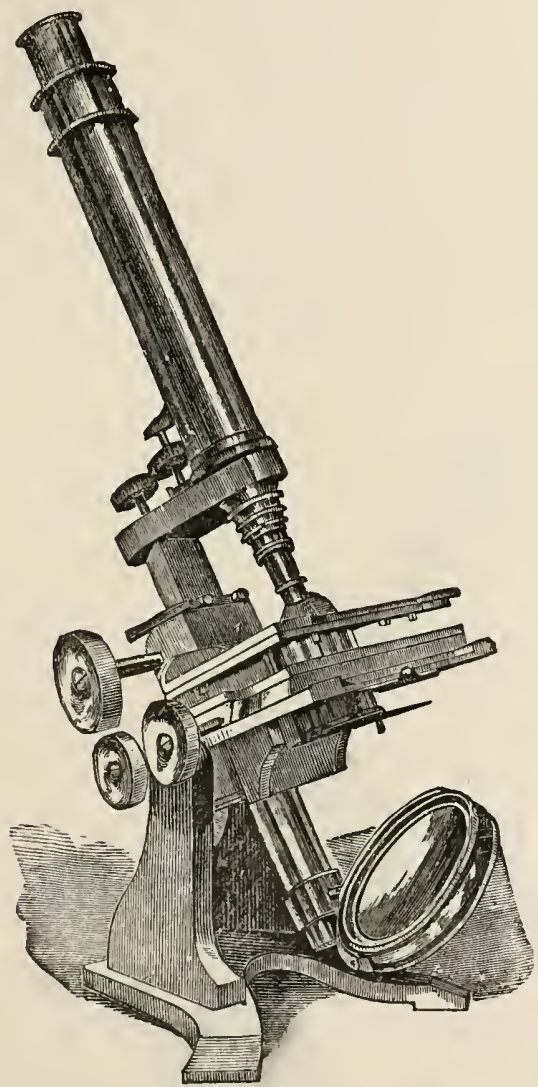

Fig. 43.-Balcer's No. 2 Compound Microssope.

delicate adjustment of this optical part is effected by the smaller milled-head seen behind the body. This milledhead is graduated, affording a means of measuring the 
thicknese of an object or the thin covering-glass. The other milled-head fixes the arm to the square bar. Below the table-stage is the secondary or sub-stage, into which is fitted the diaphragm, polariscope, achromatic condenser, and other illuminating apparatus. It is supplied with centering screws, circular and focussing rack-work, giving perfect and accurate adjustments. Sliding upon the lower end of the instrument is a large double mirror, with double-jointed arm, and above this a full-size Amici prism for oblique illumination. The microscope is furnished with a draw-tube, divided into tenths of an inch, and is thus rendered as perfect as is necessary for all purposes.

Fig. 43 represents Mr. Baker's smaller Compound Microscope, differing in some respects from that just

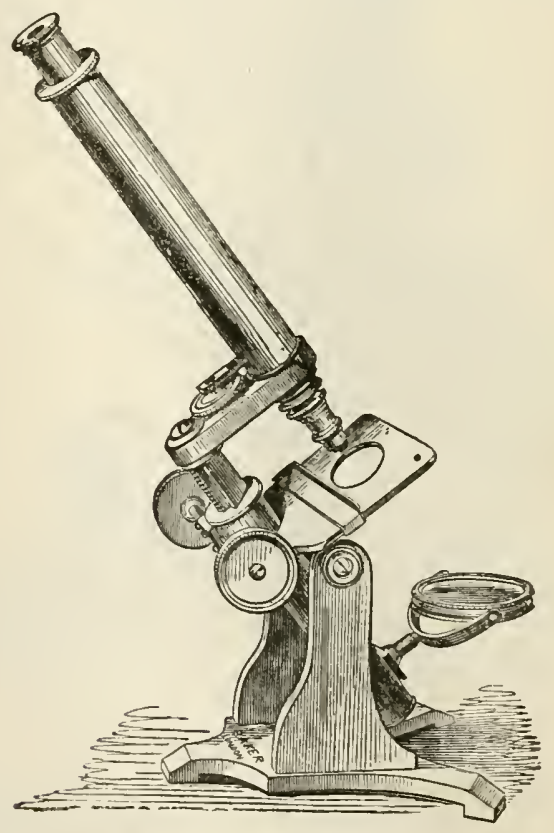

Fig. 41.-Baker's Educational Microscope. 
described. It is not fitted with sub-stage, but such an appliance may be readily attached, dovetailed grooves being left for the purpose. The motions to stage and bedy, and general finish, are similar to those of the best instruments, and it is altogether such a microscope as can be well recommended. for medical or other purposes where a good stand is required, and to which it is intended that further additions shall be hereafter made.

A smaller compound achromatic microscope, called the "Educational," fig. 44, is made by Mr. Baker. This is peculiarly adapted for students, and is supplied in a neat mahogany case, with the necessary apparatus and excellent object-glasses, for the small price of $4 l$. $4 s$. If with iron stand and in portable case, $3 l$. $3 s$.

Mr. Baker, in his binocular instrument, fig. 46, has succeeded in reducing some of the difficulties to a minimum. The setting of the prism, with its necessary stops, is so contrived that it is contained in one piece or fitting, so that when the monocular body is required to be used, this piece can be removed, and an uninterrupted field obtained, the light entering the tube at the utmost obliquity for high powers. At fig. 45, the body and nose-piece is seen detached. It has this advantage, that the prism remains in permanent adjustment when the brass nose-piece $B$ is screwed home.

Another feature in connexion with the use of the binocular, and not the least important, is that of illumination; we have been much pleased with the simple arrangement of a cheap condenser by the same optician. It

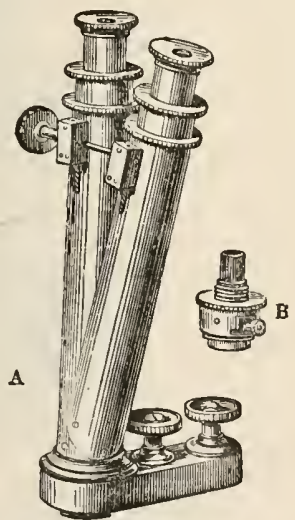

Fig. 45.-Baker's arrangement of Wenham's Binocular.

A. Bodies detached from Stand. B. Nose-piece containing Prism. consists of two plano-convex lenses, the lower one being hemispherical; the upper lens (of a somewhat smaller diameter) is placed just within its focus; the two are fitted into a sliding-tube which admits of easy adjustment 
This condenser has the property of throwing a sofi white-cloud with low powers, adding greatly to the definition and stereoscopic effect of the binocular; it is moreover useful in giving a full, clear field with the higher powers.

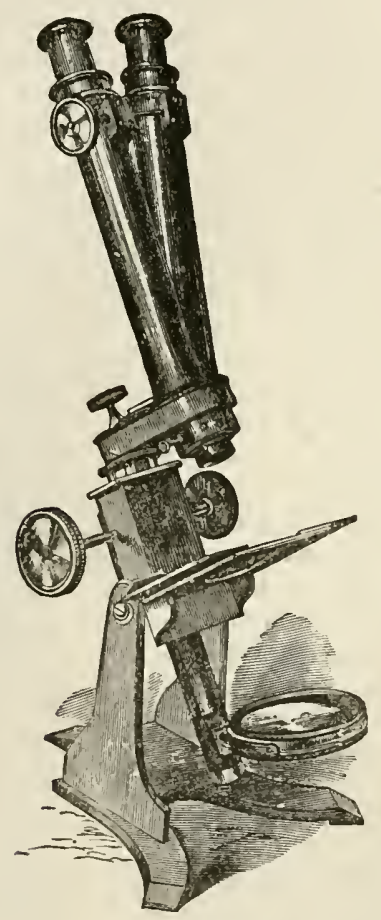

Fig. 46.-Baker's Student's Binocular Microscope.

We can also refer with some confidence to the Student's Binocular Microscope produced by Mr. Baker; it possesses remarkable excellence for the small sum charged for it. This instrument is rather larger than the Educa tional by the same maker, and is fitted with sliding-stage. It has the usual coarse and fine movements, and is supplied 
with a double mirror and one pair of eye-picces, the latter having rack and pinion adjustment. It is seen in fig. 46 , and sold as there represented for 6l. - a less sum than is charged for altering the larger microscopes.

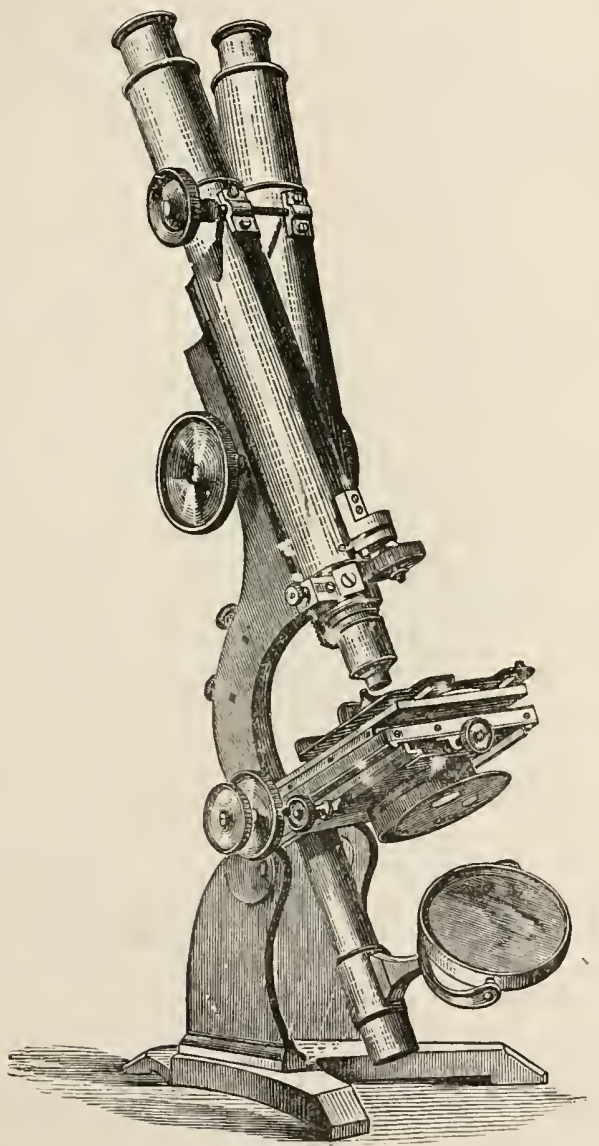

Fig. 47. - Fillisther's No. I Binomilar Microsape. 
Mr. Pillischer (New Bond Street) is favourably known for the excellency of his instruments. His No. 1 Microscope (fig. 47) is of good workmanship, and somewhat novel in design. It is constructed on a plan which may be described as intermediate between that of Smith and Beck and Ross's well-known pattern, and in point of finish is quite equal to the microscopes of the firstmentioned manufacturers. The bent form given to the arm carrying the body gives increased strength and solidity to the instrument, although it is doubtful whether it adds to its steadiness whon placed in the horizontal position. The straight body rests for a great part of its length upon a straight bar of solid brass, ploughed into a groove for the reception of the rack which is attached to the body, the groove being of such a form that the rack is held firmly while it glides smoothly through it. This is so firm, and gires such a steady uniform motion, as almost to render the fine adjustment unnecessary. The fine adjustment screw is removed from the usual position and placed in front of the body, just above and in front of the Wenham prism. The binocular bodies are inclined at a smaller angle to one another than in most makers, which, with the range of motion given to the eye-pieces by the rack and pinion, enables observers whose eyes differ greatly in separation to use the instrument with equal facility. The prism is so well set that it illuminates both fields with equal intensity. The stage is provided with rectangular traversing movements to the extent of an inch and a quarter in each direction. The milled-heads which effect these are placed on the same axis, instead of side by side, one of them-the vertical one-being repeated on the left of the stage, so that the movements may be communicated either by the right hand alone or by both hands acting in concert. The stage-plate has the ordinary vertical and rotatory motions, but to a much greater extent than usual ; and the platform which carries the object is provided with a spring clip to secure the object when the stage is placed in the vertical position. A regularly fitted sub-stage with centering screws is made in the usual way to carry an achromatic condenser, diaphragm, polarising, and other apparatus-in short, no 
instrument can be better adapted than this to all the ordinary wants of the pathologist or skilled microscopist. The object-glasses furnished with this instrument are thoroughly good working powers.

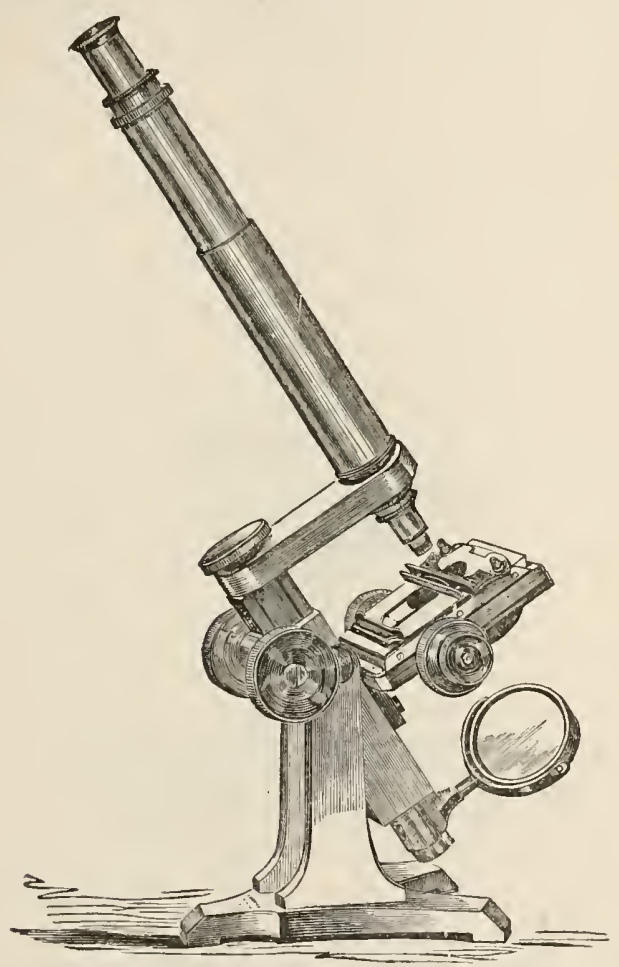

Fig. 4S.-Pillischer's larger Student's Microscope.

Fig. 48 is a compound microscope, made by Mr. Pillischer for the use of students. In every respect it is a compact, handy instrument, and well finished in its mechanical details. The body is furnished with a drawtube, by which its length can be increased about six 
inches; coarse and fine adjustments; moveable mecnanical stage as in the larger instrument; two eye-pieces; superior objectives of 1 inch and $\frac{1}{4}$-inch focus, of $15^{\circ}$ and $80^{\circ}$ angular aperture; condenser, polarising apparatus anả selenite, parabolic reflector, diaphragm, \&c., packed in a mahogany case, for the price of $15 \%$. 15s. This instrument takes its place among the best of its class.

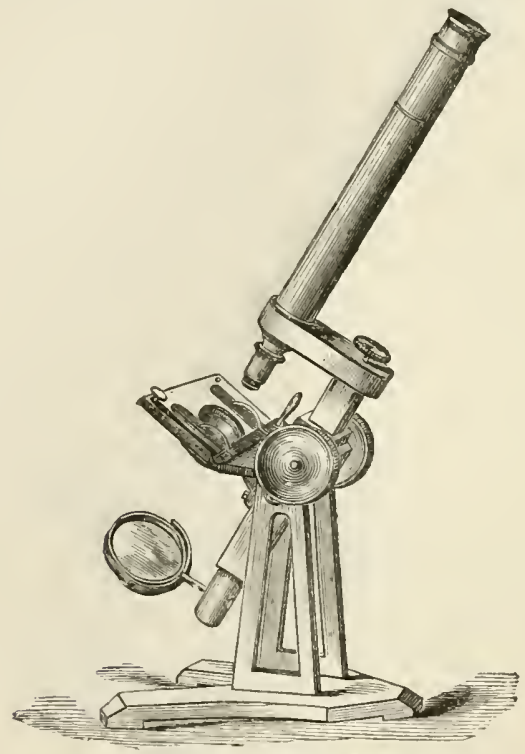

Fig. 49.-Pillischer's 5l. Prize Medal Microscope.

The instrument fig. 49 Mr. Pillischer designates his 5l. Prize Medal Microscope, is an excellent student's instrument, simple and novel in its construction, and well adapted to almost any description of work. The body is furnished with a draw-tube and fine adjustment; the arm which supports this derives additional steadiness from the square solid form given to the box in which the rack and pinion move. A convenient and simple lever-stage, with a double joint, enables the right hand to move the object 
in any direction with great facility. This can be readily detached when it is desired to clear the stage for a frogrplate, \&c. The instrument is furnished with a dividing set of powers, working with a tolerably flat-field; diaphragm, condenser for opaque objects, live box, \&c. The whole packs in a neat case, measuring only $8 \times 6$ inches; forming one of the most portable microscopes for the use of the student we have seen.

The Binocular manufactured by Mr. Collins (Great Titchfield Street, Portland Place), is constructed on the model suggested by Dr. Harley, and contains all the recent improvements for combining rapidity of application with simplicity in manipulation. Indeed, so far as the saving of time is concerned, we scarcely know how a change for the better could be devised. The whole of the appliances of the instrument, prism, polariscope, stage condenser, objectives of both high and low powers, \&c. are attached to the microscope itself, and that, too, in such a manuer as to enable the observer to place them in exact position without the turn of a single screw or a moment's delay.

Collins's Binocular, represented in fig. 50 , is fitted into the bottom of a mahogany box, which forms at the same time the stand; round it a groove is run to receive the lip of a glass shade. The instrument itself is made of polished brass, and is eighteen inches high. The eyepieces are supplied with shades $(a a)$ to protect the eyes.

At the and of the transverse arm $(f)$ is the box which contains both Wenham's binocular prism and the analyser of the polariscope ; and by merely drawing it a little out, or pushing it further in, the instrument can be instantly changed from a binocular to a monocular, and still further to a polarising microscope.

Immediately beneath $(f)$ are the two objectives, a quarter and an incli; so that, in order to change the power, all that is necessary is to slide them backwards or forwards. Mloreover, these are fitted with the universal screw, so that either of them may be detached, as in an ordinary instrument, and a quarter, or one-eighth, or other power put in its place, at the option of the observer. The instrument is fitted with a coarse and fine adjustment, and has the additional advantage of a magnctic stage, in tho 
cross-bar $(h)$ of which is a groove, in order that the observer may enjoy the luxury of applying a Maltwood's fnder, as in larger instruments possessing moveable stages.

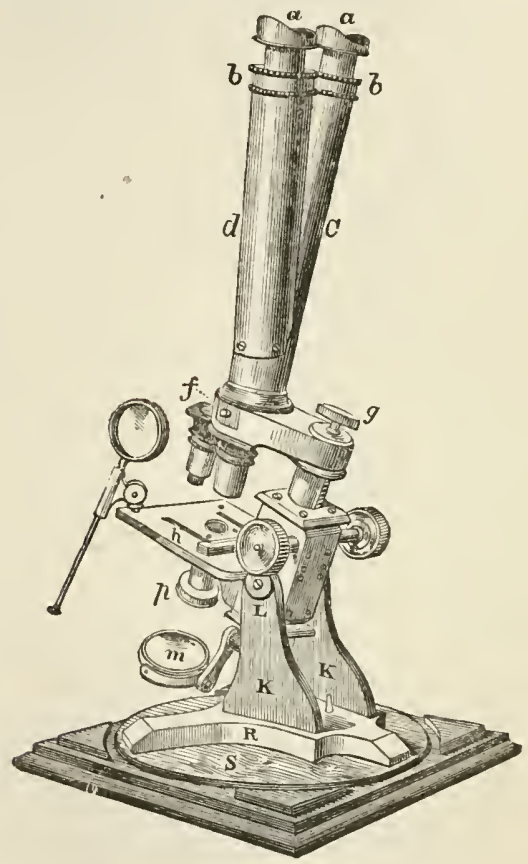

Fig. 50.-Collins's Binocular Microscone.

Beneath the stage is seen the polariser $(p)$, fitted into the circular diaphragm. The donble mirror $(m)$ possesses a triple joint, so that it can be applied obliquely in ail directions.

Collins's Student five-guinea Binocular Microscope consists of two eye-pieces, rack adjustment, top-sliding stage, wheel of diaphragms, concave mirrors with adjustment? axes for inclining to any angle, tweezers and glass plate, 1 in. and $\frac{1}{2}$ in. achromatic objectives, $C$ series. Packed 
in polished mahogany cabinet complete (fig. 51) : an AXceedingly cheap instrument.

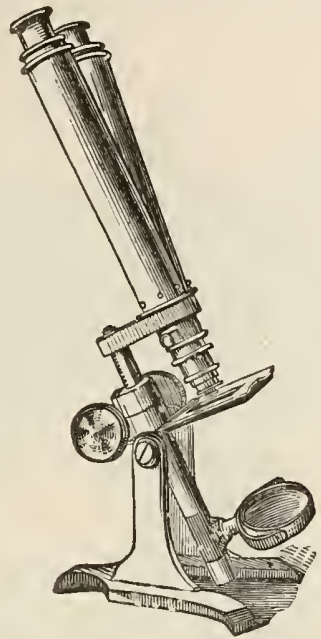

Fig. 51.-Collins's Student's Binocuter Microscope.

Collins's Lawson Binocular Dissecting Microscope.This instrument is intended to supply a want, often felt in anatomical and botanical investigations, when only a moderate magnufying power is required.

In consequence of using both eyes it can be worked with for a length of time with great comfort. A large range of field is obtained, and plenty of room for working. It consists of a neat oblong French-polished mahogany box, measuring, when closed, $6 \frac{1}{2}$ in. by 4 in., fig. 52. The top and front let down by hinges, and on the inside of them are fitted the scissors, needles, and knives necessary for dissecting. The two sides draw out about six inches, and are hollowed out so as to serve as rests for the hands. The magnification is obtained by two lenses mounted in the eye-pieces, as represented in the diagram, and may ho adjusted to the focus by a sliding bar. These show the object beautifully in relief. Beneath is a gutta-percha trough or stage, to pin the object down to, which can be 
filled with water if required. Under this is the mirror for transparent illumination, and the light from it is passed through a circle of glass in the centre cf the trough. The

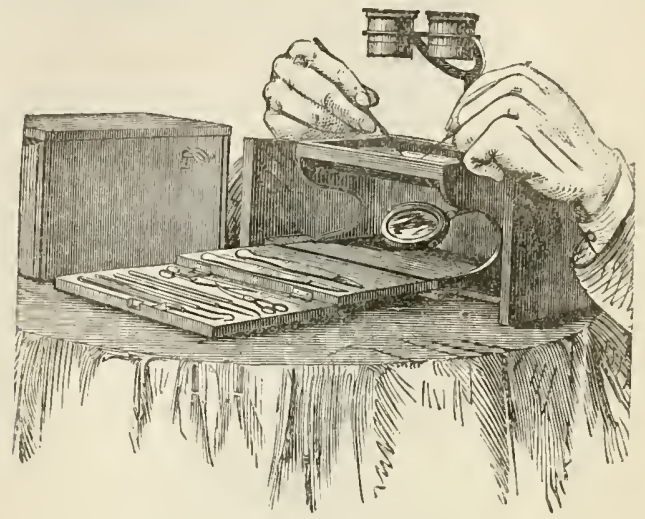

Fig. 52.-Lawson's Dissecting Arivroscopue.

instrument is admirably adapted for the wants of students in the preparation and dissection of microscopic objects, and also answers well for botanical investigations.

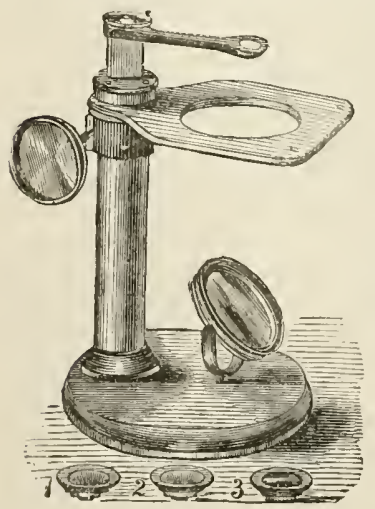

Hin. 33.- Baker's Disszcing diëcroscope

A cheap form of Dissecting Microscope, represented in the annexed woodcut (fig. 53), has been constructed by Mr. Baker. The instrument consists of a solid circular foot of brass, from the border of which arises a firm pillar supporting the stage-which is of ample dimensions-and a firm horizontal bar, into which the lenses are screwed. The latter is elevated and depressed by a rack and pinion movement, the milled-head being situate 
at a level a little below that of the stage. In the centre of the foot is placed the mirror, which moves in an arc of brass; that, in its turn, works upon a pivot in the foot, of the instrument. This handy microscope, with three powers and mahogany case, is sold at thirty-five shillings.

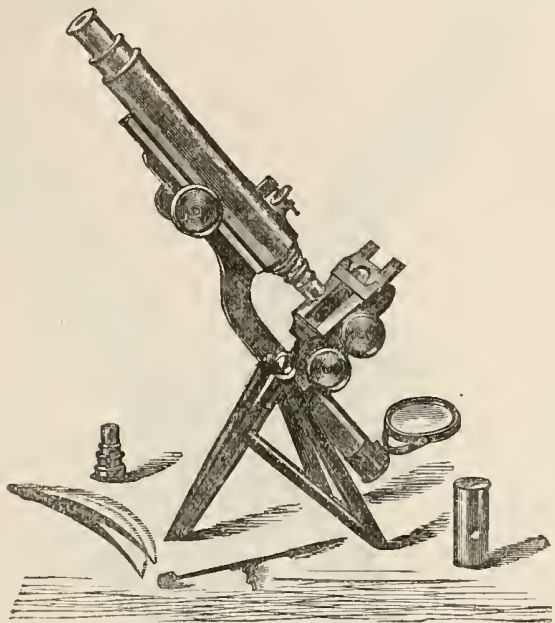

Fig. 54.-Highley's Professional Microseope.

Highley's Professional Microscope, shown in fig. 54, is a useful and well-made instrument, mounted on a tripod, with coarse and fine adjustment, mechanical stage, \&c. The mode in which the body is supported is very good in principle, and the milled-heads for the coarse adjustment are in a position which is easily reached by the lefthand when the elbow is resting on the table, whilst the right-hand finds the milled-heads of the traversing stage, \&c. It is arranged for a secondary stage, and all the necessary apparatus to make a complete instrument. Although the tripod is a very good form for a microscope, it requires all the solidity and metal of a Powell and Lealand, or it will not remain (without clamping) perfectly steady and well balanced when placed in the horizontal 
position. The new form of tripod by this manufacturer is, however, well constructed, and, in our opinion, possesspa advantages both in weight and steadiness.

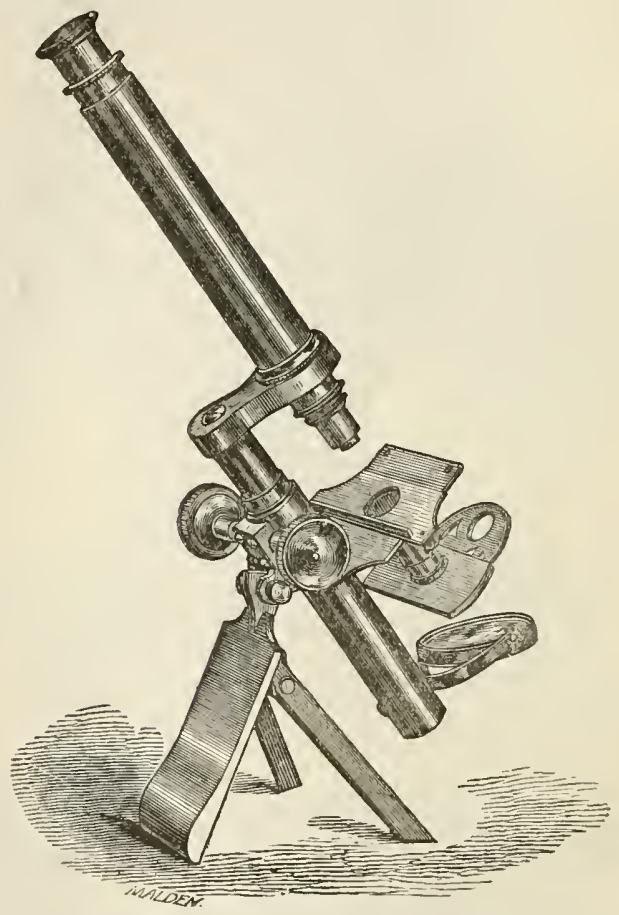

Fig. 55.-Highley's Complete Student's Microscope.

Highley's Complete Microscope, of which the general plan is shown in the accompanying fig. 55, may be classed among the cheapest form of instruments manufacturer for the use of the student. The stand is made in the tripod form, and the coarse adjustment given by the usual rack and pinion motion, whilst the fine adjustment, or slow motion, is given by a milled-head acting on the objective. The roominess. fistness, and thinness of the stage 
especially adapt it for the examination of anatomical and pathological specimens. The usual diaphragm-plate admits the application of illuminating apparatus, such as the Webster condenser, polarising prism, \&c. The cost of the instrument, fitted with two good ordinary objectglasses of 1 inch and $\frac{1}{4}$ inch focus, is $6 l .16 s .6 d$.

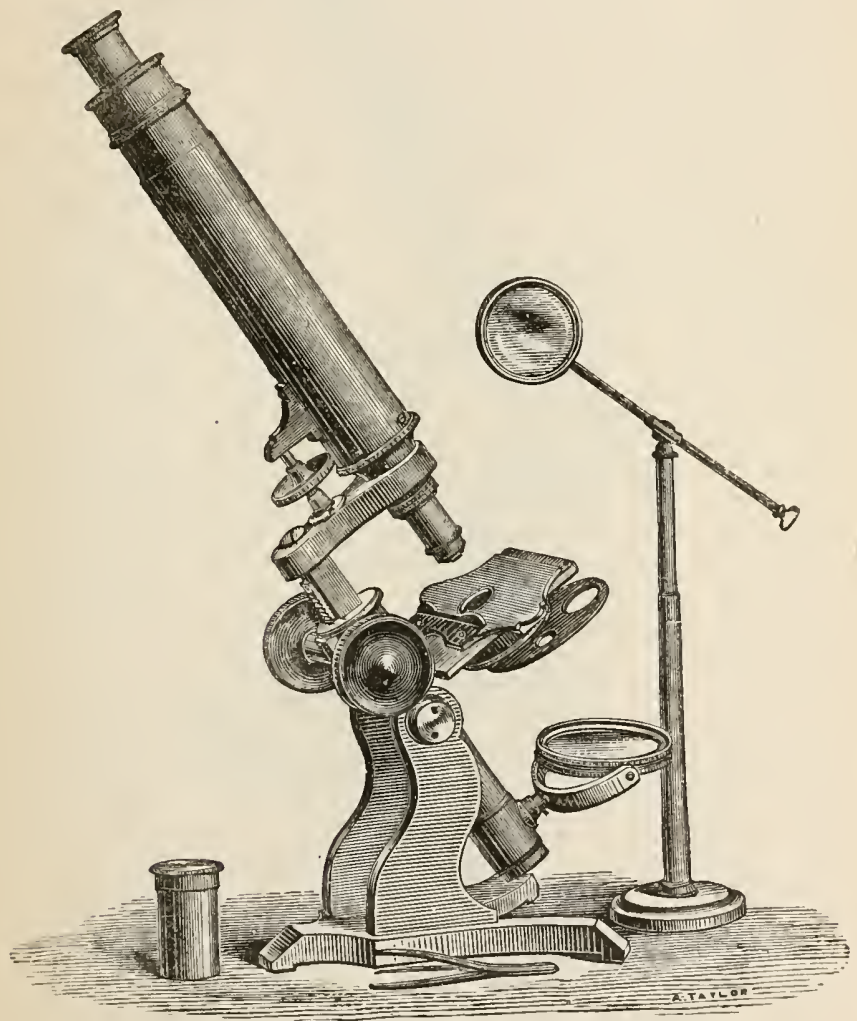

Fig. 56.-How's Student's Microscope.

Mr. How (Foster Lane) is the manufacturer of the imposing-looking instrument fig. 56. It is of fair workman 
ship, suitable for all ordinary investigations, well deserving a place among cheap microscopes for the student. The stand is of brass, firm and well finished; the body is fitted with coarse and fine adjustments for focussing; and a draw-tube for increasing the magnifying power of the eye-piece. The stage has an arrangement, simple but novel in construction, by which a near approach to a universal movement is obtained. The moveable, or upper plate, is held to the fixed lower plate with springs, and, although offering a convenient resistance, allows of a smoothness of motion quite remarkable. It resembles the magnetic stage, but is far more reliable, and can be moved upwards, downwards, laterally, or in a slanting direction, thus enabling the microscopist to follow living objects with great facility, superseding to some extent the more expensive mechanical stage. A dividing set of objectglasses is supplied with the $\mathrm{B}$ eye-piece, thus giving a range of power varying from 40 to about 200 diameters. His powers are of English workmanship, but differ from the higher-priced objectives in having smaller angular apertures, which is, perhaps, a legitimate mode of lessening the cost. The instrument being made with the universal screw, other objectives of a better class can be added at any time. There is also a condenser mounted on a brass stand for the illumination of opaque objects. The whole is fitted in a mahogany case, with drawer for objects, and sold at $5 l .5 s$.

Murray and Heath's (Jermyn Street) Student's Microscope (fig. 57) is a good solid form of instrument with a bent tripod-stand. The great object of furnishing a stand at a low price which shall be capable, if desired, of being adapted to the use of the higher objectives, and fitted for the addition of all accessory apparatus, has been very satisfactorily carried out and obtained in this microscope. The stand is remarkably firm, and, being bronzed over, is well adapted for daily use in the class-room or laboratory. The adjustment is effected by a chain-movement, which gives sufficient delicacy for powers up to the $\frac{1}{8}$ inch. The stage is perfectly flat, and the slide-rest moves smoothly and freely over it. If the instrument is intended for uso in the laboratory, a glass stage is made to replace the brass 
one. The objectives furnished with this microscopo are a $\frac{1}{4}$ inch of $75^{\circ}$ angular aperture, and a 1 inch of $15^{\circ}$, both of excellent quality. A binocular body, with fine adjust-

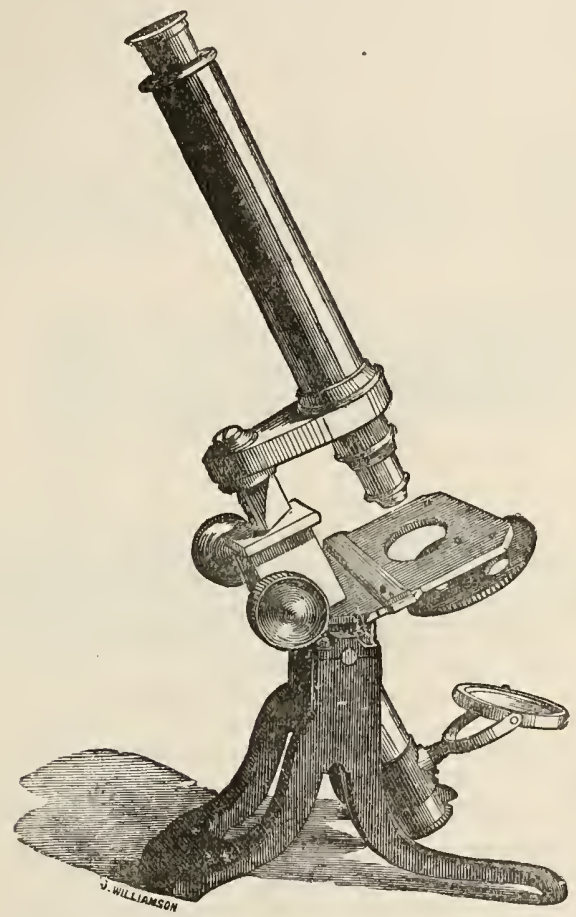

Fig. 57.-Murray and Ileath's 5l. 5s. Student's Fizicroscope.

ment, is added for a small additional sum, and the instrument then becomes all the student can desire.

Mnrray and Heath's Class Microscope, represented in fig. 58, is especially intended for the use of teachers in the demonstration of objects to a class of students. It is but too well known to those who are engaged in teaching how liable the objects exhibited, and sometimes even the object-glass itself, are to be injured in the hands of those 
unaccustomed to use the microscope. In order to avoid this risk, Messrs. Murray and Heath have constructed an instrument intended to combine an ordinary with a demonstrating or class microscope. It consists of the usual microscope body (A), which ean be inclined at any angle, with a mirror (c) on a ball-and-socket joint; and a stageplate with universal movement. When about to be used as a class microscope, the slide is placed in a shallow box into which it is locked by means of a key. The same key locks this box firmly on the stage-plate. When the object has been found, this latter ean be secured firmly on the stage in the same manner. After focussing, the

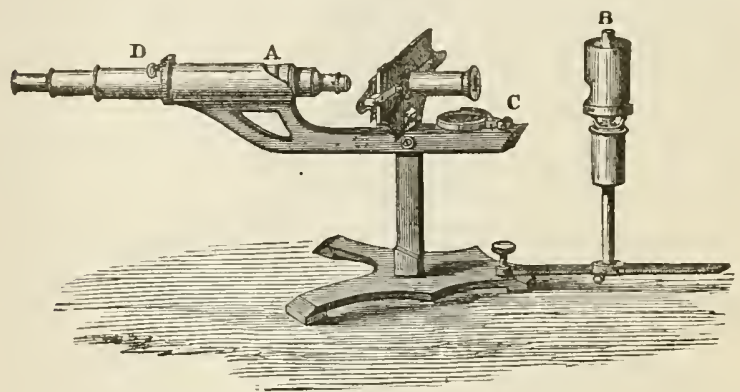

Fig. 58.-Murray and Heath's Class Microscope.

body is also locked in its place with the same key, which is seen at $\mathrm{D}$, the final adjustment being made with the eye-piece. The body is then placed in the horizontal position, and fastened with a screw. The instrument ean now be passed round a class-room without possibility of injury either to object or object-glass. The illumination is obtained either by directing the instrument towards the window, or by means of a small lamp (B), similar to that employed by Dr. Beale, and which ean be so adjusted as to be used either for opaque or transparent objects.

This instrument appears to be particularly well adapted to the purposes for which it is intended, and, at the same time, if without the contrivance for locking, to be a useful portable form for general, professional, or sea-side purposes. 
It should be added, that $\approx$ novel and efficient form of achromatic condenser is supplied with the instrument; a series of small stops of various sizes are made to drop into a minnte hole drilled in the centre of the anterior plano-convex lens, which convert it into a spot-glass, or dark-ground illuminator. The whole is packed in a mahogany case, and sold for $5 l .5$ s.

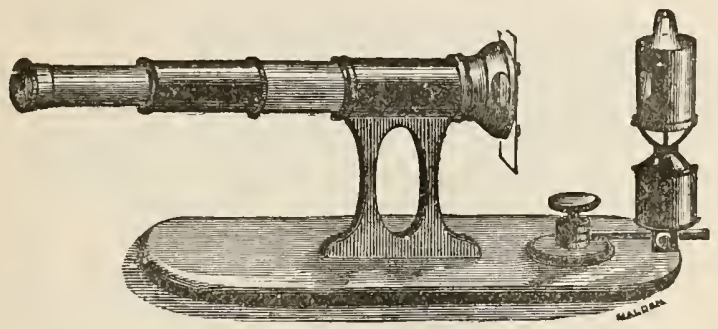

Fig. 59.-Beale's Clinical Microscope.

Dr. Beale devised an exceedingly simple and convenient form of microscope, for the purposes of clinical instruction and of class demonstration (fig. 59). Over the body of the microscope, which is of small dimensions, a tube is fitted with a bell-shaped mouth at the end. This tube slides freely over the body, but is capable of being fixed at will by means of a clamping-screw. The slide containing the object is placed across the bell-mouth, and held there by a spring pressing against the back of it, and is thus maintained perpendicularly to the axis of the instrument. When the focus is adjusted the climping-screw is fixed, and the fine adjustment necessary for the differences of vision in different individuals is effected by drawing out or pressing in the eye-piece. The object and object-glass are thus protected from mutual injury, an accident of by no means unfrequent occurrence in careless or unpractised hands. In this form the instrument is adapted to the clinical examination of secretions, \&c. and must be directed by the hand towards day or artificial light. For demonstration to a class, this instrument is attached horizontally to a small wooden stind by means of a clamp, supported by two legs. To the stand a small 
oil-lamp is likewise attached; and a stem proceeding from the lower edge of the bell-mouth carries any desired form of condensing or illuminating apparatus. This stand is capable of being freely handed round a large class without the focus becoming at all deranged, even when a very deep objective is employed. This instrument is manufactured by Mr. S. Highley. ${ }^{1}$

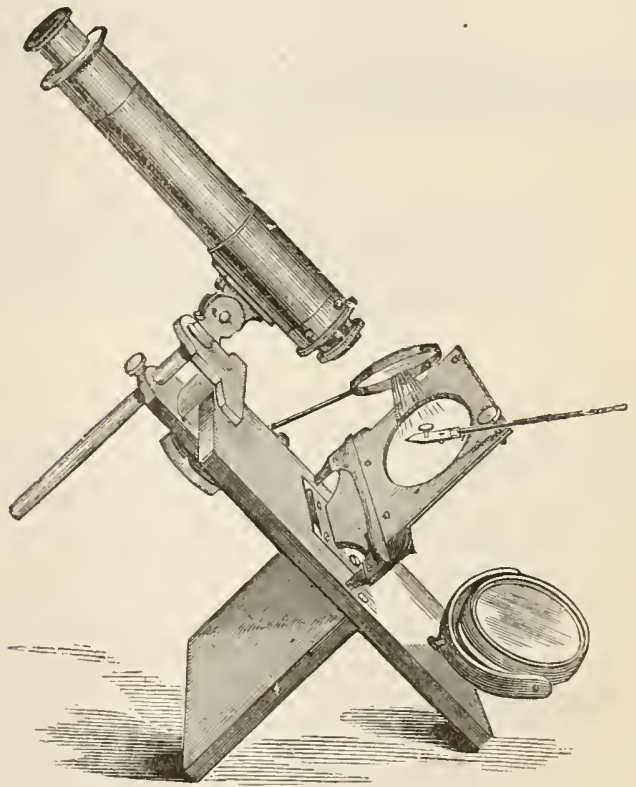

Fig. 60.- Warington's Unitersal Microscope.

While alluding to cheap microscopes, we would mention Warington's Travelling Microscope, made by Salmon, 100, Fenchurch Street. It has a simple, firm, wooden stand, whereby the cost is greatly diminished; and an arrangement of its parts which enables it to be used for

(1) A very simple instrument, contrived by Mr. T. C. Archer for the purpose of being nsed either as a lecture-room or as an ordinary table microscope, is manufactured by Messrs. Parkes of Birmingham, and sold, with a set of achromatic powers, for $2 l .5 s$. in case complete. 
viewing objects in aquaria, and under other circumstances where any ordinary form of instrument could not be made available. It is altogether a useful student's microscope, having the recommendation of folding up into a small compass, and not liable to much injury either from chemical or marine investigations. For $3 l$. this microscope is furnished

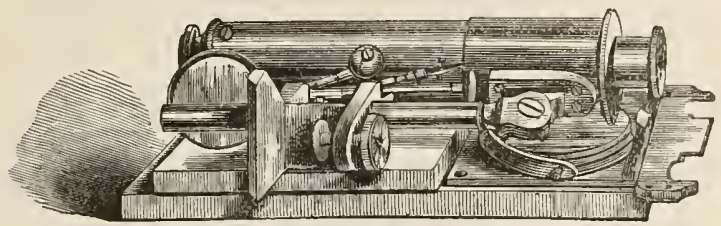

Fig. 61.-Warington's Microscope packed.

complete, with one eye-piece, quite sufficient for all ordinary investigations.

Fig. 60 is a representation of Warington's microscope, as it anpears when put together, and ready for use ; and fig. 61 for packing in a small wood case. The draw-tube itself is the coarse adjustment; whilst a finer is secured by a well-made union-joint, into which the object-glass is made to screw. With an additional arm for the reception of a single lens, it can be converted into a dissecting microscope.

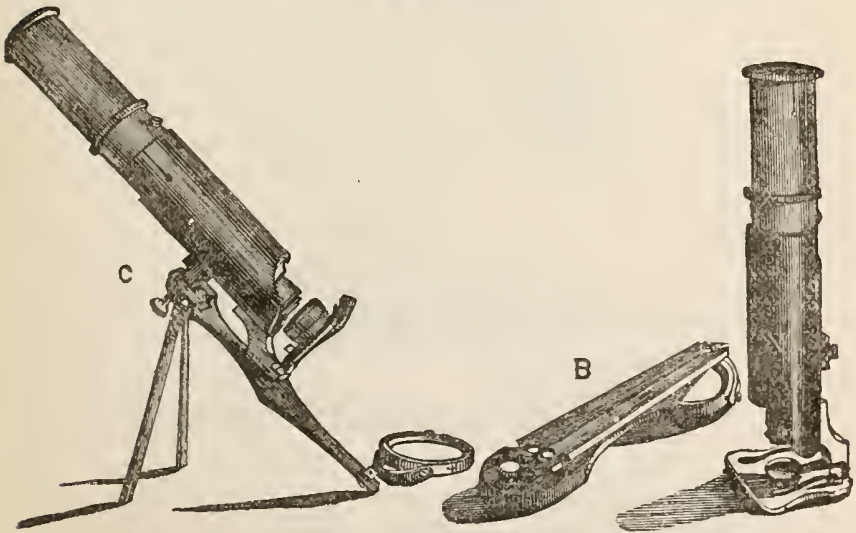

Fig. 62.-Murray and Heath's Sect-side Porket Mierossope. 
Fig. 62 represents a small portable instrument by Murray and Heath, designated "The Sea-side Pocket Microscope," the chief recommendation of which is its simplicity of constructiou and its small compact form ; the whole packs into a case six inches long, and may be carried without incumbrance in the pocket of the field-naturalist.

The body of the instrument is seen at c, supported ou a tripod, which is removed and folded up at B. If desired, it can be used in the upright position, as at A, when a pair of short legs placed near the mirror must be turned down, and forms a tolerably steady support to the body. The adjustment is made by sliding the body through the outer tube, which carries a triple combination of achromatic objectives. A live-box, \&c. is added; and, when packed in a morocco case, is sold for $2 l .5 \delta$.

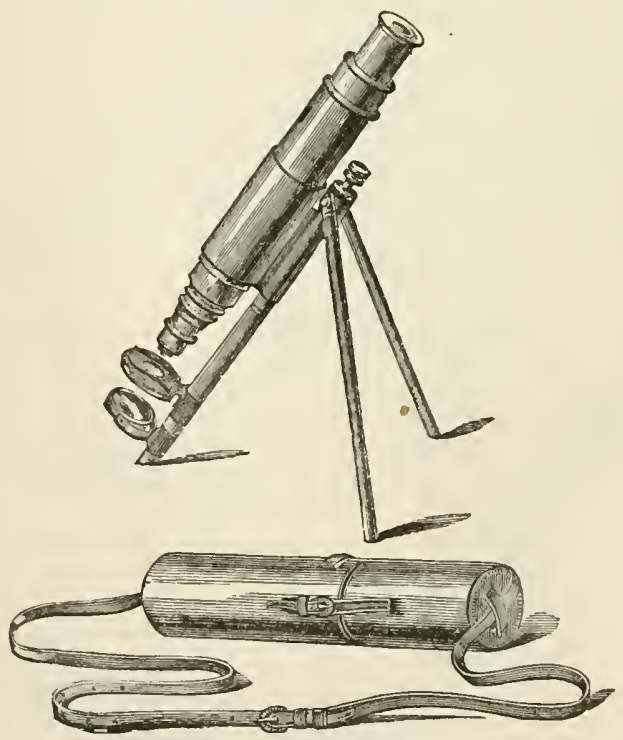

Fig. 63.-Baker's Traveller's Microscope.

An instrument somewhat similar in appearance to the foregoing, but differing so materially in detail as almost to 
claim to be a new invention (fig. 63), has been introduced by Baker, and not inappropriately called "The Traveller's Microscope," from its obvious capabilities. The aim has been to combine steadiness with extremo portability. The compound body is permanently affixed to the fore-leg of the tripod-stand; the two other legs are supported on capstan-bar joints, which can be tightened at pleasure, or folded up parallel with the former when not in use. The difficulty of using high powers with an instrument the body of which slides in eloth is well known; the tube becomes tarnished by continued use, and a firm adjustment, which shall be easy of access, is almost indispensable. To obtain an approximate focus, the inner tube is drawn out until the combined length of the tubes is eight inches; the body is then returned to its "jacket," and placed at a proper distance from the stage to suit the object-glass employed. The fine adjustment is effected by means of a tangent-screw (fifty threads to the inch) placed conveniently behind the body, and worked by a milledhead acting on a spring contained in the upright which supports the body.

This part of the instrument is rery satisfactory; it is steady and works efficiently. A mechanical stage is not generally applied, but can be if required. Sufficient movement is obtained by a plain stage, with two springs to hold the live-box or glass slip.

This microscope is carried in a leathern case 10 inches by 3 , seen in the woodcut (similar to that made for deerstalking telescopes), and fitted with object-glass, eye-piece, live-box, \&c. ; the weight of the whole not exceeding two pounds in weight, and it therefore especially recommends itself to the field-naturalist.

The whole merit of this invention is due to Mr. Moginie, of Mr. Baker's establishment, Holborn, who has devoted much time and thoughtfulness towards bringing it to its present state of perfection.

Highley's Pocket Microscope (fig. 64), for botanical or field uses, consists of a short tube furnished with a sliding eye-tube, fitting into an outer tube. The coarse adjustment is made by sliding the body through the outer tube, which carries the object; the fine adjustment by sliding the eyo- 
tube in or out. The object, if transparent, is illuminated either by holding up the microscope towards a white cloud, or other source of light, or by directing it towards a mirror laid upon the table at such an angle as to reflect the light.

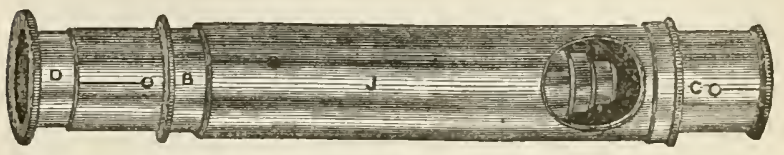

Fig. 64.-Highley's pocket Microscope.

If opaque, it is allowed to receive direst light through an aperture in the outer tube. The extreme simplicity and portability of the instrument-which is only six inches long-constitutes its chief recommendation.

Norman's (178, City Road) Universal Educational Microscope consists of a well-finished stand with tripod foot and two uprights, with axis for giving inclination to the optical part. The body has quick and slow motions, one Huyghenian eye-piece, three achromatic object-glasses, viz. a $\frac{1}{4}$-inch dividing into $\frac{1}{2}$ and 1 inch, all of fair defining power and English made. The stage has a large sliding-piece, and a revolving wheel of diaphragms; the mirror has sliding and oblique motions for the better illumination of the object under examination. The following apparatus is also supplied with the instrument:-a stand condenser with adjustment, stage and hand forceps, livebox or animalculæ cage, a frog-plate for viewing the circulation of the blood in the web of a frog's foot; also three good objects to test the different object-glasses, one hollowed and two plain slips, some thin glass. The whole is packed in a mahogany or walnut cabinet, with a drawer for objects, lock and key, and sold for the small price of $3 l .58$.

24 first-class objects, suited for the object-glasses, are supplied with this instrument for $1 l$. $1 s$.

Mr. E. Wheeler's (Holloway) well-made instruments deserve commendation and notice; they are carefully finished and quite up to the modern standard. The full assorted sets of objects which Mr. Wheeler supplies in a very neat 
book-folding case, the names on which can be real off at a glance, are particularly well selected. Many of the cases are so arranged as to form complete sets of certain classes of specimens admirably suited for educational purposes, and also for the elucidation of general and particular principles ; as, for instance, the various parts of an insect, to show peculiarities of structure for the illustration of entomological lectures, the same for botanical, anatomical, \&c. Many of our drawings have been made from Mr. W'heeler's excellent specimens.

Mr. Piper, of the Old Change Microscopical Society, devised a convenient, cheap, and portable object-case. It is a compact oblong paste-board box, macle to contain six, twelve, or twenty-four shallow trays, with six or twelve divisions just the size of the ordinary glass slides. The objects lie flat in these trays, which pack ore above the other. For handiness, neat packing, facility of finding and reading off names of objects, this case cannot be surpassed. It is but right to add, that it is adapted to the use of those whose aim is the economically useful cabinet for storing and classifying objects. This "universal" object-case is sold by Baker, Holborn.

Several forms of simple microscopes have been devised for field use under various designations, such as "Diatom Finders," \&c. One of the most useful little instruments of the class is that described by Mr. J. N. Tomkins, in the Trans. Micros. Soc. vol. vii. p. 57, 1859. Another was invented by Dr. W. Gairdner of Edinburgh, and made by MIr. Bryson of that city, neatly packed in a case for the waistcoat-pocket. Want of space will not permit us to enter further into this department, nor ean we go into a critical examination of the productions of numerous well-known makers of microscopes; as, for instance, the Educational Microscopes of Messrs. Smith and Beck, of Cheapside; Mr. Browning, 111, Minories; Mr. Matthews of Portugal Street; Mr. Dancer of MIanchester; Messrs. Abraham of Liverpool ; Mr. King of Bristol, \&c.-all of whom have obtained a deservedly high reputation for their convenient forms of educational and other wellmanufactured instruments. 
APPLICATION OF BINOCULARITY TO THE MICROSCOPE.

The application of this principle to microscopic purposes seems to have been tried as early as 1677 , by a French philosopher, le Père Cherubin, of Orleans, a Capuchin friar. The following is an extract from the description given by him of his instrument: "Some years ago I resolved to effect what I had long before premeditated, to make a microscope to see the smallest objects with the t.wo eyes conjointly; and this project has succeeded even beyond my expectation, with advantages above the single instrument so extraordinary and so surprising, that every intelligent person to whom I have shown the effect, has assured me that inquiring philosophers will be highly pleased with the communication."

This communication long slumbered and was forgotten, and nothing more was heard of the subject until Professor Wheatstone's very surprising invention of the stereoscope, which he evidently expected to apply to the microscope, for he applied to both Ross and Powell to make him a binocular microscope. But this was not clone; and during the year 1853 a notice appeared in Silliman's American Joumal of a binocular instrument constructed by Professor Riddel of America, who contrived a binocular microscope in 1851, with the view " of rendering both eyes serviceable in microscopic observations." "Behind the objective," he says, "and as near thereto as possible, the light is equally divided and bent at right angles, and made to travel in opposite directions, by means of two rectangular prisms, which are in contact by their edges somewhat ground away, the reflected rays are received, at a proper distance for binocular vision, upon two other rectangular prisms, and again bent at right angles, being thus either completely inverted for an inverted microscope, or restored to their first direction for the direct microscope." M. Nachet also constructed a binocular microscope, upon the same principle as his double microscope, with the tubes placed vertically and $2 \frac{1}{2}$ inches distant. This had many disadvantages and inconveniences, which Mr. F. H. Wenham ingeniously succeeded in modifying and improving. 
Tu describing his improvements, he observes : "That in obtaining binocularity with the compound achromatic microscope, in its complete acting state, there are far greater practical difficulties to contend against, and which it is highly important to overcome, in order to correct some of the false appearances arising from what is considered the very perfection of the instrument.

"All the object-glasses, from the one-inch upwards, are possessed of considerable angular aperture ; consequently, images of the object are nbtained from a different point of riew, with the two opposite extremes of the margin of the cone of rays; and the resulting effect is, that there are a number of dissimilar perspectives of the object all blended together upon the single retina at once. For this reason. if the object has any considerable bulk, we shall have a more accurate notion of its form by redncing the aperture of the object-glass.

"Select any object lying in an inclined position, and place it in the centre of the field of view of the microscope; then, with a card held close to the object-glass, stop off alternately the right or left hand portion of the front lens: it will be seen that during each alternate change certain parts of the object will alter in their relative position.

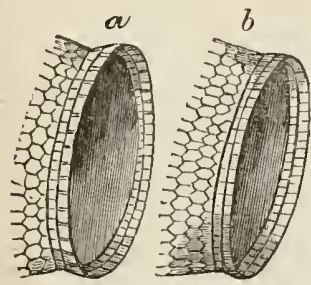

Fig. 65 .

"To illustrate this, fig. $65 a, b$ are enlarged drawings of a portion of the egg of the common bed-bug (Cimex lecticularis), the operculum which covers the orifice having been forced off at the time the young was hatched. The tigures exactly represent the two positions that the inclined orifice will occupy when the right and left hand portions of the object-glass are stopped off. It was illurs:nated as an opaque object, and drawn mnder a two-thiras object-glass of about $28^{\circ}$ of aperture. If this experiment is repeated, by holding the card over the eye-piece, and stopping off alternately the right and left half of the ultimate emergent pencil, exactly the same changes and appearances will be observed in the object under view. 
The two different images just produced are such as are required for obtaining stereoscopic vision. It is therefore evident that if, instead of bringing them confusedly together into one eye, we can separate them so as to bring fig. $96 a b$ into the left and right eye, in the combined effect of the two projections, we shall obtain all that is necessary to enable us to form a correct judgment of the solidity and distances of the various parts of the object.

"Diagram 3, fig. 66, represents the methods that I have contrived for obtaining the effect of bringing the two eyes
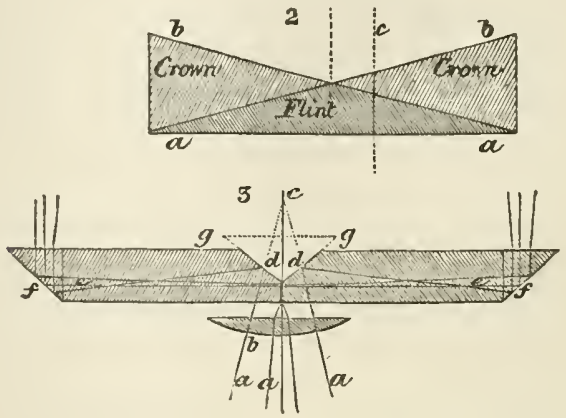

Fig. 66 .

sufficiently close to each other to enable them both to see through the same eye-piece together. a $a$ a are rays converging from the field lens of the eye-piece; after passing the eye-lens $b$, if not intercepted, they would come to a focus at $c$; but they are arrested by the inclined surfaces, $d d$, of two solid glass prisms. From the refraction of the under incident surface of the prisms, the focus of the eyepiece becomes elongated, and falls within the substance of the glass at $e$. The rays then diverge, and after being reflected by the second inclined surface $f$, emerge from the upper side of the prism, when their course is rendered still more divergent, as shown by the figure. The reflecting angle that $I$ have given to the prisms is $47 \frac{1}{2}^{\circ}$. I also find it is requisite to grind away the contact edges of the prisms, as represented, as it prevents the extreme margins 
of the reflecting surfaces from coming into operation, which can seldom be made very perfect.

"The definition with these prisms is good; but they are liable to objection on account of the extremely small portion of the field of view that they take in, and which arises from the distance that the eyes are of necessity placed beyond the focus of the eye-piece, where, the rays being divergent, the pupil of the eye is incapable of taking them all in; also there is great nicety required in the length of the prisms, which must differ for nearly every different observer."

The great disadvantages of the first arrangement of the binocular microscope were the expensive alterations required in their adaptation: to most persons, the view that it gave of the object was pseudoscopic, and not that of solidity and roundness; and the two bodies being united at a fixed angle of convergence, the distance between their axes could not be adapted to the varying distances between the eyes of different individuals. At length, these, as well as other defects, have been completely overcome by the improvements the instrument has recently received at the hands of the inventor; and we have no hesitation in saying that Wenham's prism is the most valuable addition the microscope has received since the perfection of the object-glasses. The adaptation does not at all interfere with the use of the instrument as a monocular microsçope, and such additions as the microspectroscope can be as easily used with it as in the old form; it also affords a ready comparison between the object as seen singly, and by natural double vision, and thus may be obtained an ever ready test, or sight analysis of the structure under examination. Besicles, the relief afforded to the eyes is, to our sensation, something quite marvellous, and we believe, therefore, much less risk is run of doing injury to vision. Objects become easily distinguishable, more especially all opaque or semi-opaque ones; and for the more transparent ones all that is required is to use care in their illuminationdiffuse the light by placing a piece of ordinary tracing or tissue paper, or ground glass, between the iight and the object; or even polarised light for peculiar substances will 
enable us to bring them out in greatcr perfection and beauty. Another advantage gained by the last improvement is, that the ordinary single-bodied microscope can be converted into a binocular instrument by simply fixing another tube at the proper angle, and adding a small prism mounted in a brass box. The latter is made to slide into the lower part of the body immediately over the objective. By its aid the rays of light proceeding from the object are reflected in two directions, which, by means of the double body, are conveyed to both eyes, and thereby a stereoscopic view of the object nnder observation is obtained. The most important point to be observed, when using the binocular, is that each eye has a clear view of the object. This is readily ascertained by closing the eyes alternately without moving the head away from the instrument, when, if it be found that the two images do not quite coincide, it must be corrected by racking out or in the draw-tubes, which should form a part of the bodies of all binoculars. If both fields be not equally illuminated, the object is not rendered stereoscopic.

Mr. Wenham's most important improvement consists in splitting up and dividing the pencil of rays proceeding from the objective by the interposition of a prism of the form shown in fig. 67. This is so placed in the body or tube of the microscope (fig. 68, a) as only to interrupt one-half $(a c)$ of the pencil, the other half $(a b)$ going on continuously to the field-glass, eye-piece, of the principal body. The interrupted half of the pencil, on its entrance into the prism, is subjected to very slight refraction, since its axial ray is perpendicular to the surface it meets. Within the prism it is subjected to two reflections at $b$ and $c$, which sencl it forth again obliquely on the line $b$ towards the eye-piece of the secondary body, to the lefthand side of the figure; and since at its emergence its axial ray is again perpendicular to the surface of the glass, it scffers no more refraction on passing out of the prism than on entering it. By this arrangement, the image received by the right eye is formed by the rays which have passed through the loft half of the objective; whilst the image received by the left eye is formed by the rays which have passed through the right half, and which bave bcen 
subjective to two reflections within the prism, passing through only two surfaces of glass. The prism is held by the ends only on the sides of a small brass drawer, so that all the four polished surfaces are accessible, and should slide in so far that its edge may just reach tho

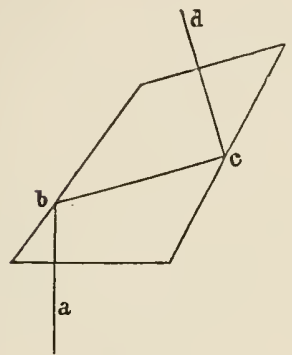

Fig. 67.

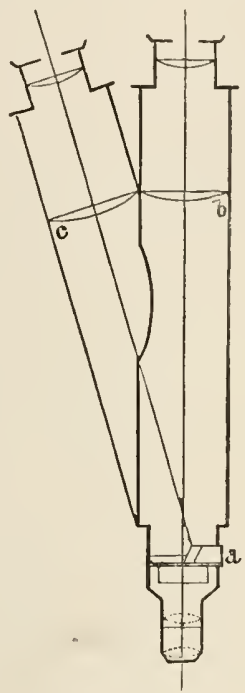

Fig. 68 .

central line of the objective, and oe drawn back against a stop, so as to clear the aperture of the same. In this case the straight tube acts as a single microscope.

"Both the transmitting and reflecting surfaces of the prism should be accessible, for the purpose of wiping off particles of dust or mildew-particles of any kind adhering to the prism will prevent total reflection at the point of contact. If the prism is well made and polisher, and of the smallest size possible for admitting the pencil, the difference between the direct and reflected image is scarcely appreciable.

"The binocular constructed as we have described performs satisfactorily up to the $\frac{1}{4}$ th inch; but for powers above 
this a special arrangement is needed for the prism, which must be set close behind the lens of the $\frac{1}{8}$ th or $\frac{1}{12}$ th inch, in order to obtain an entire field of view in each eye. This it is found to accomplish perfectly when placed in that position; but still, for very delicate testobjects, requiring the utmost extent of aperture for their definition, it will not resolve them as clearly as with a single body, from the fact that the aperture is divided and half only effective in each eye. This difficulty has at length been nearly overeome by Messrs. Powell and Lealand, by means of an inclined disc of glass with parallel sides, the partial reflection from the under surface of which is again reflected into the second eye, by means of a rectangular prism. As the disc is made thinner, so do the images approximate and the distance between them diminish. Therefore, if the glass is made as thin as possible, and a very slight angle given to the two sides, it may be so arranged that both images are ultimately combined at the eye-piece. There would be no difficulty in working the glass to a mean thickness of $\frac{3}{50}$ th of an inch. In this form the angle between the sides would be so exceedingly small that-the chromatic effect, considered as a prism, would be inappreciable in the direct eye-tube. ${ }^{1}$

" A strong light should be avoided for the illumination of objects observed with the binocular microscope, as direct rays tend to destroy the stereoscopic effect. The illuminator that has been found to give an excellent effect consists of three plano-convex lenses, so combined as to give a very large area of light, as well as great intensity. The final emergent pencil should have, if possible, an angular aperture of $170^{\circ}$. Just above the top lens should be placed a sliding-cap, the crown of which is covered with a diffusing film. For this, the best material is the beautiful snow-white powder obtained from turning glass with a diamond turning-tool. This may be obtained from the opticians, and should be well washed to free it from the larger particles. $A$ thin film of this impalpable powder should be compressed between two dises of thin glass, and fixed in the top of the sliding-cap, which is to be raised or lowered till the most intense light is obtained

(1) Quarterly Journal of Microscopical Science, vol. i. 1S61, and vol. vi. 1866. 
on the film. This illuminator is employed in the position of the achromatic condenser, and a disc of slightly coloured neutral tint-glass placed below the bottom lens increases the purity of the light, aud gives greater distinctness to objects. The effect of this diffusing film is sometimes enhanced by coudensing light down on the object from above as well as from below."

An important improvement has been effected in Nachet's binocular by Professor Snith, of Kienyon College, U.S. It consists in the adaptation of Nachet's set of prisms for use as eye-pieces, with any monocular iustrument. 'The prisms being mounted in a light material, vulcanite, are made to fit into the microscope body and take the place of the ordinary eye-piece. The image transmitted by the objective is brougnt to a focus on the face of the first equilateral triangular prism by the intervention of an erector eye-piece placed beneath it. The second set of prisms are by a rack-and-pinion movement adjusted to suit any visual angle; while the illumination of both fields is quite perfect, even with high powers.

\section{SPECTRO-MICROSCOPY.}

The application of the spectroscope to the microscope is one of the most beautiful additions the instrument has lately received. The honour of the invention appears to belong to H. C. Sorby, F.R.S., whose first experiments were made with a simple triangular prism, arranged and fixed below the stage, so that a minute spectrum of any transparent object might be readily examined, when placed in position immediately before the slit. Shortly after the publication of Mr. Sorby's paper, Mr. Huggins proposed to adapt a direct vision spectroscope to the eye-piece, and so enable us to view the spectra of opaque as well as transparent objects. The exact form of prism finally adopted, and now in general use, is that known as the Sorby-Browning Spectroscope.

The first spectroscope made by Mr. Browning (Minories) is represented in tig. 69. A prism is placed at $\mathrm{P}$, which is enclosed in a box, so as to give a black field, by excluding 
extraneous light. The rays of light, after passing between the knife-edges at $\mathrm{k}$, are rendered parallel by means of the lens at $\mathrm{L}$. Then passing through the prism and condenser (c), they reach the object at 0 . The light is placed at $w$, and if it be proposed to examine a liquid, it can be placed in a small tube (T), closed at one end; or a transparent object may be placed on the stage in the usual

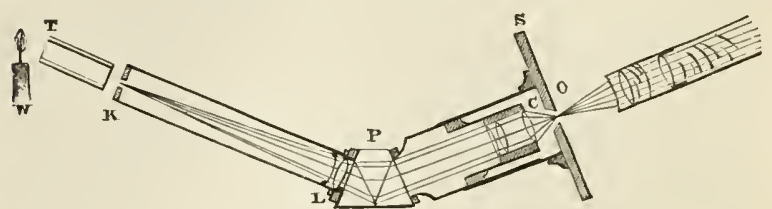

Fig. 69.-Sectional view of the Browning Spectroscoye.

manner. By the addition of a small telescope, instead of a condenser, this contrivance can be applied to a microscope in place of the eye-piece, and it can then be used for the examination of opaque objects.

The great objection to this form is its limited range, and the constant shifting of parts it requires for finding and focussing the object, and the awkward position of the microscope, whether it be used under the stage or as an eye-piece.

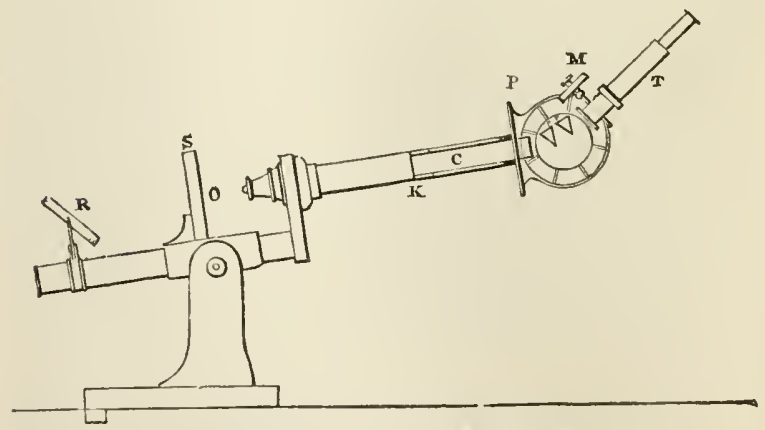

Fig. 70. -The Browning Huggins Micro-spectroscope.

The apparatus used by Mr. Huggins (fig. 70) was a star 
spectroscope, of which the collimativc-tube was inserted in the body of the microscope, instcad of an eye-piece. With this apparatus he has succeeded in obtaining a spectrum showing the absorption-bands from a mere fragment of single blood-disc, when mounted as a transparent object. In fig. $70, \mathrm{~K}$ represents the knife-edges, $\mathrm{c}$ the tube containing the collimating-lens, $\mathrm{P}$ the prisms, $\mathrm{T}$ the telescope, and is the micrometer; the object is placed on the stages at 0 , and must be illuminated from below if transparent, or, if opaque, from above by any kind of condenser.

Mr. Sorby suggested that a prism might be marte of dense flint-glass, of such a form that it could be used in two different positions, and that in one it should give twice the dispersion that it would in the other, but that the angle made by the incident and emergent rays should be the same in both positions.

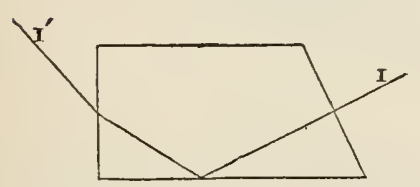

Fig. 71.

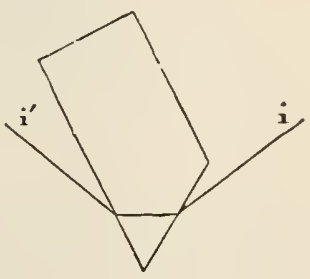

Fig. 72 .

Figs. 71 and 72 represent prisms of the kind made by Mr. Browning, used in two different positions, $i$ and $i^{\prime}$ being the same angle as I and I'.

For most absorption-bands, particularly if faint, the prism would be used in the first position, in which it gives the least dispersion; but when greater dispersion is required, so as to separate some particular lines more widely, or to show the spectra of the metals, or Fräunhofer's lines in the solar spectrum, then the prism must be used as in fig. 72. This answers well for liquids or transparent objects, but it is, of course, not applicable to opaque objects. 
To combine both purposes, some form of direct vision. prisms which can be applied to the body of the microscope is required. Fig. 73 represents the arrangement of direct vision-prisms, invented by $A$. Herschel. The line $\mathbf{R} \mathbf{R}^{\prime}$ shows the path of a ray of light through the prisms, where it would be seen that the emergent ray $\mathrm{R}^{\prime}$ is parallel and coincident with the incident ray $\mathrm{R}$.

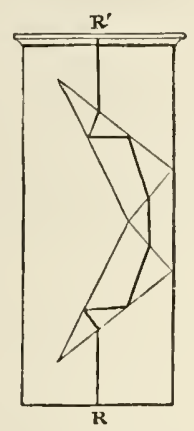

Fig. 73.

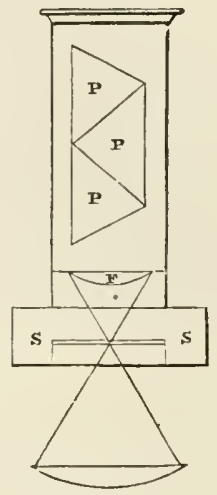

Fig. 74.

A nother very compact combination is shown in fig. 74 . Any number of these prisms ( P P P) may be used, according to the amount of dispersion required. They are mounted in a similar way to a Nicols' prism, and are applied directly over the eye-piece of the microscope. The slit $\mathrm{s} \mathrm{s}$ is placed in the focus of the first glass (F) if a negative, or below the second glass if a positive eyepiece be employed. One edge of the slit is moveable, and, in using the instrument, the slit is first opened wide, so that a clear view of the object is obtained. The part of the object of which the spectrum is to be examined is then made to coincide with the fixed edge of the slit, and the moveable edge is screwed up, until a brilliant coloured spectrum is produced. The absorption-bands will then be readily found by slightly altering the focus. This contrivance answers perfertly for opaque objects, 
without any preparation; and, when desirable, the same prism can be placed below the stage, and a micrometer used in the eye-piece of the microscope, thus avoiding a multiplication of apparatus.

The latest improvement is that shown in fig. 75 , also effected by Mr. Browning, who deserves great credit for the skill displayed in the invention and construction of this new and elegant micro-spectroscope.

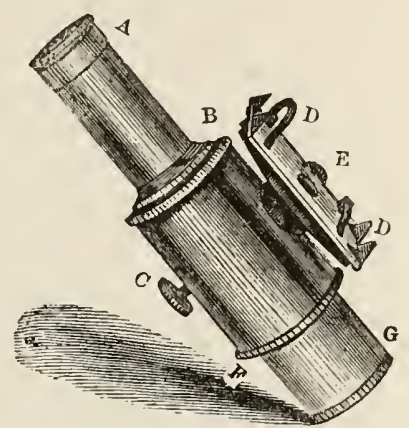

Fig. 75. - The Sorby-Browning Micro-spectroscope.

The prism is contained in a small tube, which can be removed at pleasure. Below the prism is an achromatic eye-piece, having an adjustable slit between the two lenses, the upper lens being furnished with a screw motion to focus the slit. A side slit, capable of adjustment, admits, when required, a second beam of light from any object whose spectrum it is desired to compare with that of the object placed on the stage of the microscope. This second beam of light strikes against a very small prism, suitably placed inside the apparatus, and is reflected up through the compound prism, forming a spectrum in the same fie!d with that obtained from the object on the stage.

$\Delta$ is a brass tube, carrying the compound direct vision prism. $\mathrm{B}$, a milled head, with screw motion to adjust the focus of the achromatic eye lens. c, milled head, witl screw motion to open or shut the slit vertically. Anothar 
screw at right angles to $\mathrm{c}$, but which from. its position could not be shown in the eut, regulates the slit horizoutally. This serew has a larger head, and when once recognised cannot be mistaken for the other. D D is an apparatus for holding a small tube, that the spectrum given by its contents may be compared with that from an object on the stage. $\mathrm{E}$ is a square-headed screw, opening and shutting a slit to admit the quantity of light required to form the second spectrum. A light entering the round hole near $\mathrm{E}$, strilies against the right-angled prism, which wo have mentioned as being placed inside the apparatus, and is reflected up through the slit belonging to the compound prism. If any incandescent object be placed in a suitable position with reference to the round hole, its spectrum will be obtained. F shows the position of the field lens of the eyc-piece. $G$ is a tuibe made to fit the microscope to which the instrument is applied. To use this instrument insert $\mathrm{G}$, like an eye-piece in the microscope tube, taking care that the slit at the top of the eye-piece is in the same direction as the slit below the prism. Screw on to the microscope the object-glass required, and place the object whose spectrum is to be viewed on the stage. Illuminate with the stage mirror if it be transparent; with mirror, Lieberkiihn, and dark well, by side reflector, or bull's-eye condenser if opaque. Remove $\mathbf{A}$, and open the slit by means of the milled-head, not shown in cut, but which is at right angles to D D. When the slit is sufficiently open the rest of the apparatus acts like an ordinary eye-piece, and any object can be focussed in the usual way. Having focussed the object, replace $\boldsymbol{A}$, and gradually close the slit till a good spectrum is obtained. The spectrum will be much insproved by throwing the object a little out of focus.

Every part of the spectrum differs a little from adjacent parts in refrangibility, and delicate bands or lines can only be brought out by accurately focussing that particular part of the spectrum. This can be done by the milled head $\mathbf{B}$. Disappointment will occur in any attempt at delicate investigation if this direction be not carefully attended to.

At $\mathrm{x}$ a small mirror is attached, which is omitted in the diagram to prevent confusion. It is like the mirror below 
the stage of a microscope, and is mounterl in a similar manner. By means of this mirror light may be reflecter into the eye-piece, and in this way two spectra may be procured from one lamp.

For observing the spectra of liquids in cells or tubes of considerable diameter, say not less than $\frac{1}{10}$ th of an inch, powers from 2 inch to 1 inch will be the most suitable, and of course low powers only can be used to investigate the spectra of opaque objects; but when the spectra of very minute objects are to be viewed, powers of from half an inch to one-twentieth, or even higher, may be employed.

Blood, madder, aniline red, permanganate of potash, in crystals or solution, are convenient substances to begin experiments with. Solutions when made too strong produce dark clouds instead of absorption bands. Professor Church has recently pointed out that zircon, an almost colourless stone, gives well-defined absorption-bands.

Mr. Sorby says of the correct performance of a spectrum adaptation, "The best tests are, first, that the absorptionbands in blood can be seen when they are very faint; second, to well divide the bands in permanganate of potash; and, third, to see distinctly the very fine line given in the red by a solution of chloride of cobalt dissolved in a concentrated cold solution of chloride of calcium: there is a line so fine that it looks like a Fräunhofer's line. An instrument that shows all these well is all that can be desired.

"The objects most easily obtained, and which furnish us with the greatest variety of spectra, are coloured crystals, coloured solutions, and coloured glasses. The spectrum microscope enables us to examine the spectra of very minute crystals, of very small quantities of material in solution, and of small blow-pipe beads. As previously named, the thickness of the object makes a very great difference in the spectrum. For example, an extremely thin crystal of ferricyanide of potassium cuts off all the blue rays, and leaves merely red, orange, yellow, and more or less green; but on increasing the thickness, tho green and yellow disappear; and when very much thicker, little else but a bright red light is transmitted. In all sucb 
cases, the apparent magnitude of the effect of an increase in thickness is far greater when the object is thin than when thick, and past a certain thickness the change is comparatively very slight. If only small crystals can be obtained, it is well to mount a number of different thicknesses; but when it is possible to obtain erystals of sufficient size, it is far better to make them into wedgeshaped objects, since then the effect of gradual change in thickness can easily be observed. Different kinds of crystals require different treatment, but, as a general rule, I find that it is best to grind them on moderately soft Water-of-Ayr stone with a small quantity of water, which soon becomes a saturated solution, and then to polish them with a little rouge spread on paper laid over a flat surface; or else, in some cases, to dissolve off a thin layer by carefully rubbing the crystal on moist blotting-paper until the scratches are removed. Then, whenever it is admissible, I mount the crystal on a glass, and also cover it with a piece of thin glass with Canada balsam. Strongly coloured solutions may be examined in test-tubes, or may be kept sealed up in small bottles made out of glass tubes, the light then examined being that which passes through the centre of the tube from side to side. (Most of these solutions require the addition of a little gum Arabic to make them keep.) Such tubes may be laid on the ordinary stage, or laid on the stage attached to the eye-piece. Smaller quantities may be examined in cells cut out of thick glass tubes, one side being fixed on the ordinary glass with Canadir balsam, like a microscopic object, and the other covered with thin glass, which readily holds on by capillary attraction, or may be cemented fast with gold size or Canada balsam, if it be desirable to keep it as a permanent object. Such tubes may be made of any length that may be required for very slightly-coloured solutions. Cells made out of spirit thermometer tubes, so as to be about $\frac{1}{10}$ th of an inch in diameter, and $\frac{1}{2}$ an inch long, are very suitable for the examination of very small quantities; but where plenty of material can be obtained, it is far better to use cells cut out of strong tubes, having an interior diameter of about 3 ths of an inch, cut wedgeshape, so that the thickness of the solution may be $\frac{1}{4}$ th 
of an inch, or more, on one side, and not above ${ }_{40}^{\frac{1}{0}}$ th on the other; and then the effect of different thicknesses can easily be ascertained.

"Fortunately, the various modifications of the colouring matter of blood yield such well-marked and characteristic spectra, that there are few subjects to which the spectrummicroscope can be applied with greater advantage than the detection of blood-stains, even when perfectly dry. For this purpose condensed light may be used, provided a sufficiently bright light be thrown on the object by means of a parabolic reflector or bull's-eye condenser. A speck of blood on white paper shows the spectrum very well, provided it be fresh, and the colour be neither too dark nor too light, and the thickness of the colouring matter neither too great nor too little. A mere atom, invisible to the naked eye, which would not weigh above the 1000000 th of a grain, is then sufficient to show the characteristic absorption-bands. They are, however, far better seen in solution. About $\frac{1}{100}$ th of a grain of liquid blood, in a cell of $\frac{1}{10}$ th of an inch in diameter, and $\frac{1}{2}$ an inch long, gives a spectrum as well marked as could be desired. In exhibiting the instrument to a number of persons at a meeting, I have found that no object is more convenient, or excites more attention, than one in which a number of cells are fixed in a line, side by side, containing a solution of various red-colouring matters. In one I mount blood, which gives two well-marked absorption-bands in the green; in another magenta, which gives only one distinet band in the green; and in another I place the juice of some red-eoloured fruit, which shows no well-defined absorption-band. Keeping a larger cell containing blood on the stage attached to the eye-piece, these three objects can be passed one after another in front of the object-glass, and the total difference between the spectrum of blood and that of either fruit-juice or magenta, and the perfect identity of the spectra when both are blood, can be seen at a glanee. By holding coloured glasses, which cut off the red, but allow the green rays to pass, we can readily.show how the presence of any foreign colouring-matter, which entirely alters the general colour, might not in any degreo disguise the eharacteristic part of the spectrum; and by 
changing the cell held on the eye-piece for a tube containing an ammoniacal solution of cochineal, it is easy to show that, though it yields at spectrum with two absorption-bands, more like those due to blood than I have seen in any other substance, they differ so much in relation, size, and position, that therr is no chance of their being confounded when compared together side by side." I

We have been usually taught that the red-blood corprascles consisted of two substances, hematin and glohulin; but later researches lead to the belief that they consist of one erystalline substance, termed globulin or homato-globulin. A solution of this substance, as well as of certain products of its decomposition, produces the absorption-bands referred to. Hoppe was the first to demonstrate this fact: he found that a very dilute solution of blond was sufficient for the purpose. Professor Stokes proved that this colouring-matter is
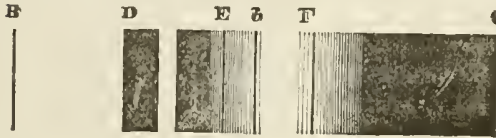

No. 1. Arterial Blood, Scarlct Cruorine.
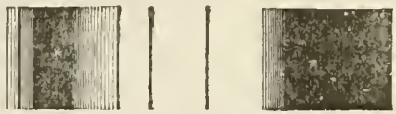

No. 2. Venous Blood, P'urple Cruorine.
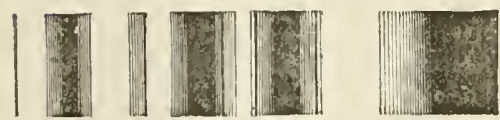

No. 3. Blood treated with Alctic scid.
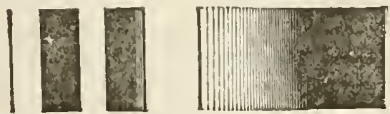

No. 4. Solution of IIrmatin.

ABSORPTION-DANDS, AFTER, STOKES. capable of existing in two states of oxidation, and that

G a very different spectrum is produced according as the substance, which $h$. has termed cruorine, is in a zuore or less oxidised condition." Proto-sulphate of iron, or proto-chloride of tin, causes the resluction of the colouring-matter, and, by exposure to air. oxygen is absorbed, and the solution again exhibits the spectrum character. istic of the more oxidised state. The different substances obtained from blood colouring - matter produce different bands. Thus, homatin gives rise to a band in the red spcctrum ; hamato - globulin produces two bands, the second twice the brearth of the first in the yellow portion of the spectrum between the lines $\mathrm{D}$ and $\mathrm{E}$, No. 1. The absorptionbands difter according to the strength of the solntion employed. and the medium in which the bloodsalt is dissolved; hut an exceedingly minute proportion dissolved in water is sufficient to bring out very distinet bands.

(1) Popular Science Review, January, 1866.

(2) Professor stokes, "Un the Reduction and Oxidation of the Colonringmatter of the Blood" (Procecd. Royal Soc. vol. xiii. p. 355). The oxidising solntion is made as follows:-To a solution of proto-sulphate of iron, enough tartaric acid is added to prevent precipitation by alkalies. A small quantity of this solution, made slightly alkaline by ammonia or carbonate of soda, is to be alded to the weak solution of blood in water. 
THE CAMERA LUCIDA.

The Camera Lucida, fig. 79, invented by Dr. Wollaston, in 1807, is a valuable addition to the microscope, for making drawings of structures, or for obtaining, with a micrometer, measurements of objects. It consists

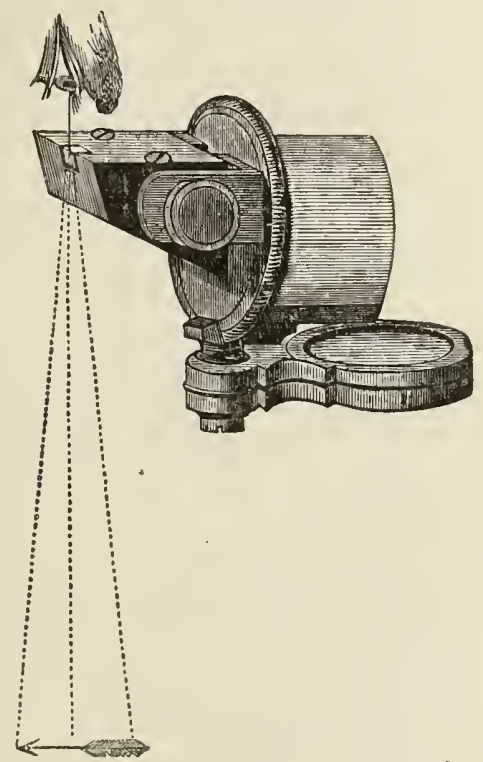

Fig. 79.

of a four-sided prism of glass, set in a brass frame or case, as represented in the figure annexed; and by means of a short tube it is slipped over the front part of either of the eye-pieces, its cap having been previously removed. Mr. Ross attaches the prism, by two short supports, to a circular piece of brass at the end of the tube ; on this it can be slightly rotated, whilst the prism itself can also be curued up or down, by means of two screws with millccl 
hearis. So arranged, the camera may be adapted to the eye-piece, the microscope having been previously placed in a horizontal position; if the light be then reflected up through the compound body, an eye placed over the square loole in the frame of the prism will see the image of any object on the stage upon a sheet of white paper placed on the table immediately beiow it. But should it happen that the whole of the field of view is not well illuminated, then, either by revolving the circular plate or turning the prism upon the screws, the desired object will be effected. The chief difficulty in the use of this instrument is for the artist to be able to see, at one and the same time, the pencil and the image. To facilitate this in some measure, the one or two lenses below the prism will cause the rays from the paper and pencil to diverge at the same angle as those received from the prism, whereby both object and pencil may be seen with the same degree of distinctness.

The following is the method for employing the Camera Lucida with the microscope. The first step to be taken, after the object about to be drawn has been properly illuminated, adjuster, and brought into the centre of the neld of view, is to place the compound body of the microscope in a horizontal position, and to fix it there. The cap of the eye-piece having been removed, the camera is to be slid on in its stead: if the prism be properly adjusted, a circle of white light, with the object within it, will be seen on a piece of white paper placed on the table immediately under the camera, when the eye of the observer is placed over the uncovered edge of the prism, and its axis directed towards the paper on the table. Should, however, the field of view be only in part illuminated, the prism must either be turned round on the eyepiece, or revolved on its axis, by the screws affixed to its frame-work, until the entire ficld is illuminated. The next step is to procure a hard, sharp-pointed pencil, which, in order to be well seen, may be blackened with ink round the point; the observer is then to bring his eye so near the edge of the prism that he may be able to see on the paper, at one and the same time, the pencil-point and the image of the object. When he has accomplished this, the 
pencil may be moved along the outline of the image, so as to trace it on the paper. However easy this may appear in description, it will be found very difficult in practice; and the observer must not be foiled in his first attempts, but must persevere until he accomplishes his purpose. Sometimes he will find that he can see the pencil-point, and all at once it disappear's : this happens from the movement of the axis of the eye. The plan then is to keep the pencil upon the paper, and to move alout the eye until the pencil is again seen, when the eye is to be kept steadfastly fixed on the same position until the entire outline is traced. It will be found the best plan for the beginner to employ at first an inch object glass, and some object, such as a piece of moss, that has a welldefined outline, and to make many traciugs, and examine how nearly they agree with each other; and when he has succeeded to his liking, be may then take a more complicated subject. If the operation is conducted by lamp. light, it will be found very advantageous not to illuminate the object too much, but rather to illuminate the paper on which the sketch is to be made, either by mons of the lamp with the condensing lens, or a small taper placed near it. When the object is so complicated that too much time would be required for it to be completed at one sitting, the paper should be fixed to the table by a weight, or on a board by drawing-pins. An excellent plan to 2dopt is to fix the microscope on a piece of deal about two feet in length and one foot in breadth, and to pin the paper to the same; there will then be no risk of the shifting of the paper, as, when the wood is moved, both microscope and paper will move with it. In all skctches made by the camera, certain things must be borne in mind; the eye, when once applied to it, should be kept stcadily fixcd in one position; and if the sketches are to be reserved for comparison with others, the distance between the paper and the camera should be always the same. A short rule or a piece of wood may be placed between the paper and the under-surface cither of the compound body or the arm supporting it, in order to regulate the distance, as the sizo of the drawing made by the camera will depend upon the distance between it and the paper. It is also very desirable,

$$
\text { K } 2
$$


before the camera is removed, to make a tracing in some part of the paper of two or more of the divisions of the stage micrometer, in order that they may form a guide to the measurement of all parts of the object. Some persons cover the whole of the drawing over with squares, to facilitate, not only the measurement, but in order that a larger or smaller drawing may be made from it than that given by the camera. It must be recollected, that an accurate outline is the only thing the camera will give: the finishing of the picture must depend entirely upon the skill of the artist himself.

\section{ON THE POLARISATION OF LIGHT AS APPLIED TO THE MICROSCOPE.}

Common light moves in two planes at right angles to each other, polarised light moves ouly in one plane. Common light may be turned into polarised light either by transmission or reflection; in the first instauce, one of the planes of common light is got rid of by reflection, in the other, by absorption. Huyghens was among the first to notice that a ray of light has not the same properties in every part of its circumference, and he compared it to a magnet or a collection of magnets; and supposed that the minute particles of which it was said to be composed had different poles, which, wheu acted on in certain ways, arranged themselves in particular positions; and thence the term polarisation, a term having neither reference to cause nor effect. It is to Malus, however, who, in 1808, discovered polarisation by reflection, that we are indebted for the series of splendid phenomena which have since that period been developed; phenomena of such surpassing beauty as far to exceed all ordinary objects presented to our eyes under the microscope. It has been truly observed by Sir David Brewster, that "the application of the principles of double refraction to the examiuation of structures is of the highest value. The chemist may perform the most dexterous analysis; the crystallographer may examine crystals by the nicest determination of their forms and cleavage; the anatomist or botanist may use the dissecting knife and microscope with the most exquisite skill; but there are still structures in the mineral, 
vegetable, and animal kingdoms, which defy all such modes of examination, and which will yield only to the magical analysis of polarised light. A body which is quite transpareut to the eye, and which might be judged as monotonous in structure as it is in aspect, will yet exhibit, under polarised light, the most exquisite organisation, and will display the result of new laws of combination which the imagination even could scarcely have conceived. In evidence of the utility of this agent in exploring mineral, vegetable, and animal structures, the extraordinary organisation of Apophyllite and Analcime may be referred to; also the symmetrical and figurate depositions of siliceous crystals in the epidermis of equisetaceous plants, and the wonderful variations of density in the crystailine lenses of the eyes of animals.
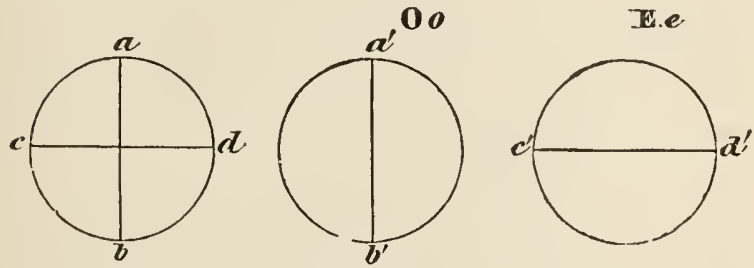

Fig. 80.

If we transmit a beam of the sun's light through a circular aperture into a darkened room, and if we reflect it from any crystallised or uncrystallised body, or transmit it through a thin plate of either of them, it will be reflcted and transmitted in the very same manner, and with the same intensity, whether the surface of the body is held above or below the beam, or on the right side or left, provided that in all cases it falls upon the surface in the same manner; or, what amounts to the same thing, the beam of solar light has the same properties on all its sides; and this is true, whether it is white light as directly emitted from the sun, or from a candle or any burning or selfluminous body; and all such light is called common light. A section of such a beam of light will be a circle, like $a b$ $c d$, fig. 80 ; and we shall distinguish the section of a beam 
of common light by a circle with two diameters $a b, c d$, at right angles to each other.

If we now allow the same beam of light to fall upon a rhomb of Iceland spar, and examine the two circular beams, $O \circ E$ e, formed by double refraction, we shall find, 1st, that the beams $O \circ E$ e have different properties on different sides, so that each of them differs in this respect from the beam of common light.

2 d. That the beam $O$ o differs from $E e$ in nothing excepting that the former has the same properties at the sides $a^{\prime} b^{\prime}$ that the latter has at the sides $c^{\prime}$ and $d^{\prime}$; or in general that the diameters of the beam, at the extremities of which the beam has similar properties, are at right angles to each other, as $a^{\prime} b^{\prime}$ and $c^{\prime} d^{\prime}$ for example.

Thes a two beams, $O o, E e$, are therefore said to be polarised, or to be beams of polarised light, because they have sides or poles of different properties and planes passing through the lines $a b, c d$; or $a^{\prime} b^{\prime}, c^{\prime} d^{\prime}$, are said to be the rlanes of polarisation of each beam, because they have the same property, and one which no other plane passing through the beam possesses.

Now it is a curious fact, that if we cause the two polarised beams $O n, E e$ to be united into one, or if we produce them by a thin plate of Iceland spar, which is not capable of separating them, we obtain a beam which has exactly the same properties as the beam $a b c d$ of common light. Hence we infer that a beam of common light, $a b$ $c d$, consists of two beams of polarised light, whose plane of polarisation, or whose diameters of similar properties, are at right angles to one another. If $O \circ$ be laid above $E$ e, it will produce a figure like $a b c d$; and we shall therefore represent polarised light by such figures. If we were to place $O$ o above $E e$, so that the planes of polarisation $a^{\prime} b^{\prime}$ and $c^{\prime} d^{\prime}$ coincide, then we should have a beam of polarised light twice as luminous as either $O o$ or $E e$, and possessing exactly the same properties; for the lines of similar property in the one beam coincide with the lines of sinilar property in the other. Hence it follows that there nre three ways of converting a beam of common light, $a b$ $c d$, intc a beam or beams of polarised light.

1st. We may separate the beam of common light, $a b c d$, 
component parts $O o$ and $E$ e. 2d. We may turn round the planes of polarisation, $a b c d$, till they coincide or are parallel to cach other. $3 \mathrm{~d}$. We may absorb or stop one of the beams, and leave the other, which will consequently be in a state of polarisation." I

The first of these methods of producing polarised light is that in which we employ a doubly refracting crystal. and was first discovered to exist in a transparent mineral substance called Iceland spar, calcareous spar, or carbonate of lime. 'This substance is admirably adapted for exhibiting this phenomenon, and is the one generally used by microscopists. Iceland spar is composed of fifty-six parts of lime and forty-four parts of carbonic acid; it is found in various shapes in almost all countries; but whether found in crystals or in masses, we can always cleave it or split it into shapes represented by fig. 81 , which is called a rhomb of Iceland spar, a solid bounded by six equal and similar rhomboidal surfaces, whose sides are parallel, and whose angles $b a c$, a $c d$, are $101^{\circ} 55^{\prime}$ and $78^{\circ} i^{\prime}$. 'The

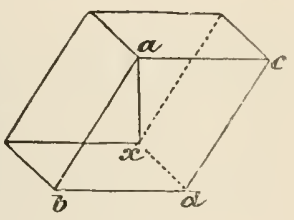

Fig. 81. line $a x$, called the axis of the rhomb, or of the crystal, is equally inclined to each of the six faces at an angle of $45^{\circ} 23$.' It is very transparent, and generally colourless. Its natural faces when it is split are commonly even and perfectly polished; but when they are not so, we may, by a new clevage, replace the imperfect face by a better one, or we may grind and polish an imperfect face.

It is found that in all bodies where there seems to be an irregularity of structure, as salts, crystallised minerals, \&c., on light passing through them, it is divided into two distinct pencils. If we take a crystal of Iceland spar, and look at a black line or dot on a sheet of paper, there will appear to be two lines or dots; and on turning the spar round, these objects will seem to turn round also; and twice in the revolution they will fall upon each other, which occurs when the two positions of the spar are exactly opposite, that is, when turned one-half from the position 
where it is first observed. In the accompanying diagram, fig. 82, the line appears double, as $a b$ and $c d$, or the dot,

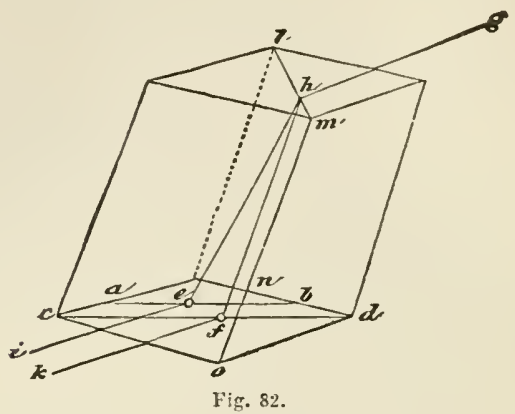

as $e$ and $f$. Or allow a ray of light, $g h$, to fall thus on the crystal, it will in its passage through be separated into two rays, $h f, h e$; and on coming to the opposite surface of the crystal, they will pass out at $e f$ in the direction of $i k$, parallel to $g h$. The plane $l m n o$ is designated the principal section of the crystal, and the line drawn from the solid angle $l$ to the angle $o$ is where the axis of the crystal is contained; it is also the optic axis of the mineral. Now when a ray of light passes along this axis, it is undivided, and there is only one image; but in all other directions there are two.

If two crystals of Iceland spar be used, the only difference will be, that the objects seem farther apart, from the increased thickness. But if two crystals be placed with their principal sections at right angles to each other, the ordinary ray refracted in the first will be the extraordinary in the second, and so on vice vers $\hat{a}$. At the intermediate position of the two crystals there is a subdivision of each ray, and therefore four images are seen; when the crystals are at an angle of $45^{\circ}$ to each other, then the images are all seen of equal intensity.

Mr. Nicol first succeeded in making rhombs of Iceland spar into single-image prisms, by dividing one into two equal portions. His mode of proceeding is thus described in the Edinburgh Philosophical Jomnal (vol. vi. p. 83): 
"A rhomb of Iceland spar of one-fourth of an inch in length, and about four-eighths of an inch in breadth and thickness, is divided into two equal portions in a plane, passing through the acute lateral angle, and nearly touching the obtuse solid angle. The sectional plane of each of these halves must be carefully polished, and the portions cemented firmly with Canada balsam, so as to form a rhomb similar to what it was before its division; by this management the ordinary and extraordinary rays are so separated that only one of them is transmitted: the cause of this great divergence of the rays is considered to be owing to the action of the Canada balsam, the refractive index of which $(1.549)$ is that hetween the ordinary $(1.6543)$ and the extraordinary (1.4833) refraction of calcareous spar, and which will change the direction of both rays in an opposite manner before they enter the posterior half of the combination." The direction of rays

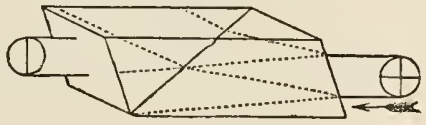

Pig. 83.

passing through such a prism is indicated by the arrow, fig. 83 , and the combination is shown mounted, one for

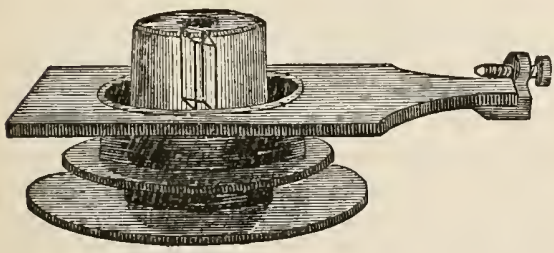

Fig. 84.

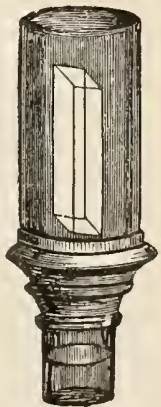

Fig. 85 .

use under the stage of the microscope, fig. 84, termed the polariser; another, fig. 85 , screwed on to and above the 
object-glasses, is called the anayser. The definition is letter if the analyser be placed at top of the $A$ eye-piece, and it is more easily rotated than the polariser.

Meihod of using the polarising Prism, fig. 84.-After having adapted it to slide into a groove on the under-surface of the stage, it is held in its place by turning the small milled-head screw at one end: the other prism, fig. 85, is ferewed on above the object-glasses, and made to pass into the body of the microscope itself. The light having been reflected through them by the mirror, it becomes necessary to make the axes of the two prisms coincide; this is done by regulating the milled-head screw, until by revolving the polarising prism, the field of view is entirely darkened twice during one revolution. This should be

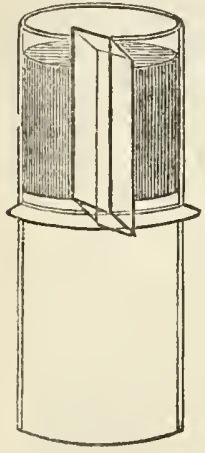

Fig. 86. . ascertained, and carefully corrected by the maker and adapter of the apparatus. If very miuute salts or crystals are to be viewed, it is preferable to place the analyser above the eye-piece; it will then require to be mounted as in fig. 86 . 'Thus the polariscope consists of two parts; one for polarising, the other for analysing or testing the light. There is no essential difference between the two parts, except what convenience or economy may lead us to adopt; and either part, therefore, may be used as polariser or analyser ; but whichever we use as the polariser, the other becomes the analyser.

The tourmaline, a precious stone of a neutral or bluish tint, forms an excellent analyser; it should be cut about $\frac{1}{20}$ th of an inch thick, and parallel to its axis. The great objection to it is, that the transmitted polarised beam is more or less coloured. The hest tourmaline to choose is the one that stops the most light when its axis is at right angles to that of the polariser, and yet admits the most when in the same. plane. It is necessary to choose the stone as perfect as possible, the size is of no importance when used with the microseope.

In the illumination of objects by polarised light, when under view with high powers, for the purpose of obtaining 
the maximum effect, it is also requisite that the angle of aperture of the polariser should be the same as the objectglass, each ray of which should be directly opposed by a ray of polarised light. The Polarising Condenser is merely an ordinary achromatic condenser of large aperture, close under the bottom leris of which is placed a plate of tourmaline, used in combination with a superposed film of selenite or not, as required. The effect of this arrangement on some objects is very remarkable, bringing out strongly colours which are almost invisible by the usual mode.

The production of colour by polarised light has been thus most clearly and comprehensively explained by $\mathrm{Mr}$. Woodward, in his "Introduction to the Study of Polarised Light." 1

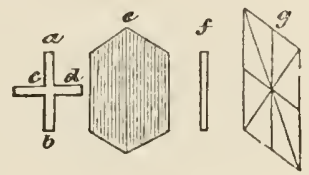

Fig. 87 A.
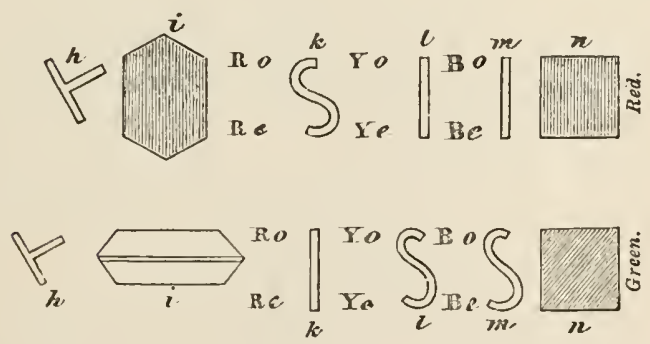

Fig. 87 B.

$a b c d$ represent the rectangular vibrations by which a ray of common light is supposed to be propagated.

$e$, a plate of tourmaline, called in this situation the polariser, and so turned that $a b$ may vibrate in the plane of its crystallographical axis.

(1) Mr. Wordward constructed a very available form of polariscope for mont purposes; the instrument is described in Elements of Natural Philosophy, by Jabez Hogg. 
$f$, light polarised by $e$, by stopping the vibrations $c d$, and transmitting those of $a b$.

$g$, a piece of selenite of such a thickness as to produce red light, and its complementary colour green.

$h$, the polarised light $f$ bifurcated, or divided into ordinary and extraordinary rays, and thus said to be depolarised by the double refractor $g$, and forming two planes of polarised light, $o$ and $e$, vibrating at right angles to each other.

$i$, a second plate of tourmaline, here called the analyser, with its axis in the same direction as that of $e$, through which the several systems of waves of the ordinary and extraordinary rays $h$, not being inclined at a greater angle to the axis of the analyser than that of 45 degrees, are transmitted and brought together under conditions that may produce interferences.

$k$, the waves $\mathrm{R} o$ and $\mathrm{R} e$, for red light of the ordinary and extraordinary systems meeting in the same state of vibration, occasioned by a difference of an even number of half undulations, and thus forming a wave of doubled intensity for red light.

$l m$, the waves $\mathrm{Y} o$ and $\mathrm{Y} e$ and $\mathrm{B} o$ and $\mathrm{B} e$ for yellow and blue of the ordinary and extraordinary systems respectively meeting together, with a difference of an odd number of half undulations, and thus neutralising each other by interferences.

$n$, red light, the result of the coincidence of the waves for red light, and the neutralisation by interferences of those for yellow and blue respectively.

$h$, fig. $87 \mathrm{~B}$, depolarised light, as fig. $87 \mathrm{~A}$.

$i$, the analyser turned one quarter of a circle, its axis being at right angles to that of $i$ in fig. $87 \mathrm{~A}$.

$k$, the waves $\mathrm{R} o \mathrm{R} e$, for red light of the ordinary and extraordinary systems meeting together with a difference of an odd number of half undulations, and thus neutralising each other by interference.

$l m$, the waves $\mathrm{Y} o$ ч $e$ and в $о$ В $e$, for yellow and blue of the two systems severally meeting together in the same state of vibration, occasioned by the difference of an even number of half undulations, and forming by their coincidences wares of doubled intensity for yellow and blue light. 
$n$, green light, the result of the coincidences of the waves for yellow and blue light respectively, and the neutralisation by interference of those for red light.

By substituting Nicol's prisms for the two plates of tourmaline, and by the addition of the object-glass and eye-piece, the diagrams would then represent the passage of polarised light through a microscope.

For showing objects by polarised light under the microscope that are not in themselves doubly refractive, put upon the stage a film of selenite, which exhibits, under ordinary circumstances, the red ray in one position of the polarising prism, and the green ray in another, using a double-image prism over the eye-piece; each arc will assume one of these complementary colours, whilst the centre of the field will remain colourless. Into this field introduce any microscopic object which in the usual arrangement of the polariscope undergoes no change in colour, when it will immediately display the most brilliant effects. Sections of wood, feathers, algæe, and scales, are among the objects best suited for this kind of exhibition. The power suited for the purpose is a two-inch objectglass, the inteusity of colour, as well as the separating power of the prism, being impaired under much higher amplification; although in some few instances, such as in viewing animalcules, the one-inch object-glass is perhaps to be preferred.

Selenite is the native crystallised hydrated sulphate of lime. A beautiful fibrous variety called satin gypsum is found in Derbyshire. It is found also at Shotover Hill, near Oxford, where the labourers call it quarry-glass. Very large crystals of it are found at Montmartre, near Paris. The form of the crystal most frequently met with is that of an oblique rectangular prism, with ten rhomboidal faces, two of which are much larger than the rest. It is usually slit into thin laminæ parallel to these large lateral faces; the film having a thickness of from onetwentieth to the one-sixtieth of an inch. In the two rectangular directions they allow perpendicular rays of polarised light to traverse them unchanged; these directions are called the neutral axes. In two other directions, however, which form respectively angles of $45^{\circ}$ with the 
neutral axes, these films have the property of double refraction. These directions are known as the depolarising axes.

The thickness of the film of selenite determines the particular tint. If, therefore, we use a film of irregular thickness, different colours are presented by the different thicknesses. These facts admit of very curious and beautiful illustration, when used under the object placed on the stage of the microscope. The films employed should be mounted between two glasses for protection. Some persons employ a large film mounted in this way between plates of glass, with a raised edge, to act as a stage for supporting the object, it is then called the "selenite stage." 'The best film for the microscope is that which gives blue, and its complementary colour yellow. Mr. Darker has constructed a very neat stage of brass for this purpose, producing a mixture of all the colours by superimposing three films, one on the other; by a slight variation in their positions, produced by means of an endless-screw motion, all the colours of the spectrum are shown. When objects are thus exhibited, we must bear in mind that all the negative tiuts, as we term them, are diminished, and all the positive ones increased; the effect of this plate is to mask the true character of the phenomena. Polarised structures should therefore never be drawn and coloured under such conditions.

Dr. Heripath, of Bristol, described a salt of quinine, which is remarkable for its polarising properties. The salt was first accidentally observed by $\mathbf{N}[\boldsymbol{r}$. Phelps, a pupil of Dr. Herapath's, in a bottle which contained a solution of disulphate of quinine: the salt is formed by dissolving disulphate of quinine in concentrated acetic acid, then warming the solution, and dropping into it carefully, and by small quantities at a time, a spirituous solution of iodine. On placing this mixture aside for some hours, brilliant plates of the new salt will be formed. The crystals of this salt, when examined by reflected light, have a brilliant emerald-green colour, with almost a metallic lustre; they appear like portions of the elytrx of cantharides, and are also very similar to murexide in appearance. When examined by transmitted light, they scarcely possess 
\&ny colour, there is only a slightly olive-green tinge; but if two crystals, crossing at right angles, be examined, the spot where they iutersect appears perfectly black, even if the crystals are not one five-hundredth of an inch in thickness. If the light be in the slightest degree polarised-as by reflection from a cloud, or by the blue sky, or from the glass surface of the mirror of the microseope placed at the polarising angle $56^{\circ} 45^{\prime}$-these little prisns immediately assume complementary colours: one appears green, and the other pink, and the part at which they cross is a chocolate or deep chestnut-brown, instead of black. As the result of a series of very elaborate experiments, Dr. Herapath finds that this salt possesses the propertics of tourmaline in a very exalted degree, as well as of a plate of selenite; so that it combines the properties of polarising a ray and of depolarising it. Dr. Herapath has succeeded in making artificial tcurmalines large enough to surmount the eye-piece of the microscope; so that all experiments with those crystals upon polarised light may be madee without the tourmaline or Nicol's prism, The brilliancy of the colours is much more intense with the artificial crystal than when employing the natural tourmaline. As an analyser above the eye-piece, it offers some advantages over the Nicol's prism in the same position, as it gives a perfectly uniform tint of colour over a much more extensive field than can be had with the prism. ${ }^{1} A$ s these crystals are liable to be injured by damp, and thus lose their polarising property, when out of use they should be kept in a dark dry place.

"The following experiments, if carefully performed, will illustrate the most striking phenomena of double refraction, and form a useful introduction to the practical application of this principle.

(1) Dr. Herapath lias given a later and better proeess for the manufacture of tliese artificial tourmalines in the Quarterly Joumal of Microscopical Science for January, 1854. "These beautiful rosette crystals are best made as follows :Take a moderately strong solution of Cinchonidine in Heraprath's test-fluid (as already described). A little of this is dropled on the centre of a slide and laid down for a time, until the first erystals are observed to be formed near the anargin. The slide slionld now be placed mpon the stage of the microseone, and the progress of formation of the erystals closely watelied. When these are sten to be large enough, and it, is deemed necessary to stop. their further development. the slide must be quickly transterred to the palin of the liand, the warmth of winch will be found suflicient to stop further erystallization." 
"A plate of brass, fig. $\$ 8$, three inches by one, perforated with 2 series of holes from about one-sixteenth to one-

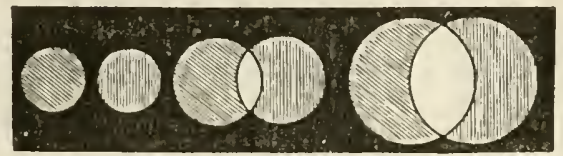

Fig. 88.-Red is represented by perpendicular lines; Green by oblique.

fourth of an inch in diameter; the size of the smallest should be in accordance with the power of the object-glass, and the separating power of the double refraction.

"Experiment 1.-Place the brass plate so that the smallest hole shall be in the centre of the stage of the instrument; employ a low power ( $1 \frac{1}{2}$ or 2 inch) object-glass, and adjust the focus as for an ordinary microscopic object; place the double image prism over the eye-piece, and there will appear two distinct images; then, by revolving the prism, these will describe a circle, the circumference of which cuts the centre of the field of view; the one is called the ordinary, the other the extraordinary ray. By passing the slide along, that the larger orifices may appear in the field, the images will not be completely separated, but will overlap, as represented in the figure.

"Experiment 2.-Screw the Nicol's prism into its place under the stage, still retaining the double image prism over the eye-piece; then, by examining the object, there will appear in some positions two, but in others only one image; and it will be observed, that at $90^{\circ}$ from the latter position this ray will be cut off, and that which was first observed will become visible; at $180^{\circ}$, or one-hilf the circle, an alternate change will take place; at $270^{\circ}$, another change; and at $360^{\circ}$, or the completion of the circle, the original appearance.

"Before proceeding to the next experiment, it will be as well to observe the position of the Nicoi's prism, which should be adjusted with its angles parallel to the square parts of the stage. In order to secure the greatest brilliancy in the experiment, the proper relative position of the selenite may be determined by noticing the natural 
flaws in the film, which will be observed to run parallel with each other; these flaws should be adjusted at about $46^{\circ}$ from the square parts of the stage, to obtain the greatest amount of depolarisation.

"Experiment 3.-If we now take the plate of selenite thus prepared, and place it under the piece of brass on the stage, we shall see, instead of the alternate black and white images, two coloured images composed of the constituents of white light, which will alternately change by revolving the eye-piece at every quarter of the circle ; then, by passing along the brass, the images will overlap; and at the point at which they do so, white light will be produced. If, by accident, the prism be placed at an angle of $45^{\circ}$ from the square part of the stage, no particular colour will be perceived; and it will then illustrate the phenomena of the neutral axis of the selenite, because when placed in that relative position no depolarisation takes place. The phenomena of polarised light may be further illustrated by the addition of a second double image prism, and a film of selenite adapted between the two. The systems of coloured rings in crystals cut perpendicularly to tho principal axis of the crystal are best seen by employing the lowest object-glass."

To show the phenomena of the rings round the optic axes of the crystals, Mr. Lobb adopts the following plan, which is by far the best, and the rings are exhibited in the greatest perfection :-

1. The в eye-piece without a diaphragm, and the lenses so adjusted that the field-lens may be brought nearer to, or farther from the eye-lens as occasion may require; thus giving different powers, and different fields, and when adjusted for the largest field it will be full 15 inches, and take in the widest separation of the axis of the aragonite.

2. A crystal stage to receive the crystals, and to be placed over the eye-piece, so constructed as to receive a tourmaline, and that to turn round.

3. A tourmaline of a blue tint.

4. A large Nicol's prism as a polariser.

5. A common two-inch lens, not achromatic; which must be set in a brass tube long enough when screwed into 
the microscope to reach the polariser, that all extraneous light may be excluded.

The concave mirror should be rised with a bull's-eye condenser by lamplight. The condenser may be dispensed with by daylight. The above apparatus is furnished by Messrs. Powell and Lealand.

The crystals best adapted to show the phenomena of rings round the optic ases, are:-

Quartz.-A uniaxial crystal, one system of rings, no entire cross of black, only the ends of it, the centre being coloured, and as the tourmaline is revolved, the colour gradually chinging into all the colours of the spectrum, one colour only displayed at once.

Quartz.-Cut so as to exhibit right-handed polarisation.

Quartz. - Cut so as to exhibit left-handed polarisation; that is, the one shows the same phenomena when the tourmaline is turned to the right, as the other does when turned to the left.

Quartz.-Cut so as to exhibit straight lines.

Calc Spar.-A uniaxial crystal, one system of rings, and a black cross, which changes into a white cross on revolving the tourmaline, and the colours of the rings into their complementary colours.

Topaz.-A biaxial crystal, al though it has two axes, only exhibits one system of rings with one fringe, owing to the wide separation of the axes. The fringe and colours change on revolving the tourmaline; this is the ease in all the crrstals.

Borax.-A biaxial crystal; the colours more intense than in topaz, but the rings not so complete,-only oue set of rings taken in, from the same cause as topaz.

Rochelle Salt.-A biaxial crystal; the colours more widely spread. Very beautiful. Only one set of rings taken in.

Carbonate of Lead.-A biaxial crystal, axes not much separated, bath systems of rings exhibited, far more widely spread than those of nitre.

Aragonite.-A biaxial crystal, axes widely separated; but both systems of rings exhibited, and decidedly the best crystal for displaying the phenomena of biaxial crystals.

The field-lens of the eye-piece requires to be brought as 
close as possible to the eye-lens, to see properly the phenomena in quartz and aragonite; it must be placed at an intermediate distance for viewing topaz, borax, Rochelle salt, and carbonate of lead; it must be drawn out to its full extent to view nitre and calc spar.

The powers of the micro-polariscope cannot be better displayed than in the exhibition of the foregoing phenomena; there is nothing more beautiful, and fow studies more interesting and enlarging to the mind than that of light, whether common or polarised, which must be entered upon if the phenomena are to be understood.

The crystal eye-piece, with an artificial tourmaline as an aualyser, will be found very useful for polariscope oljects - generally; there is some spherical aberration, but the largeness of the field far more than compensates for the same; it does best for those ohjects that require the twoinch object glass.

Mr. Darker of Lambeth, Messrs. Elliott of the Strand, Messrs. Horne and Co., Newgate St., and M. Soliel of Paris, supply properly cut crystals.

It was long believed that all crystals had only one axis of double refinction; but Brewster found that the great body of crystals, which are either formed by art, or which occur in the mineral kingdom, have two axes of double refraction, or rather axes around which the double refraction takes place; in the axes themselves there is no double refraction.

Nitre crystallises in six-sided prisms with angles of about 120. It has two axes of double refraction, along which a ray of light is not divided into two. These axes are each inclined about $2 \frac{1}{2}^{\circ}$ to the axes of the prism, and $5^{\circ}$ to eacl other. If, therefore, we cut off a piece from a prism of nitre with a knife driven by a smart blow of a hammer, and polish the two surfaces perpendicular to the axes of the prism, so as to leave the thickness of the sixth or eighth of an inch, and then transmit a ray of polarised light along the axes of the prism, we shall see the double system of rings shown in figs. 89 and 90.

When the line comnecting the two axes of the crystal is inclined $45^{\circ}$ to the plane of primitive polarisation, a cross is seen as at fig. 89 , on revolving the nitre, it gradually 
assumes the form of the two hyperbolic curves, fig. 90. But if the tourmaline be revolved, the black crossed lines will

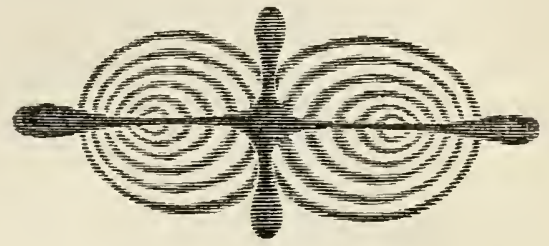

Fig. 89 .

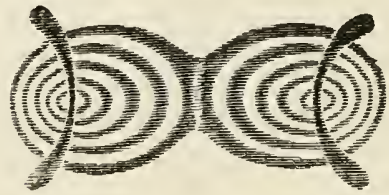

Fig. 90.

be replaced by white spaces, and the red rings by green, the yellow by indigo, and so on. These systems of rings have, generally speaking, the same colours as those of thin plates, or as those of a system of rings round one axis. The orders of the colours commence at the centres of each system; but at a certain distance, which corresponds to the sixth ring, the rings, instead of returning and encircling each pole, encircle the two poles as an ellipse does its two foci. When we diminish or increase the thickness of the plate of nitre, the rings are diminished or increased accordingly.

Small specimens of salts may also be crystallised and mounted in Canada balsam for viewing under the stage of the microscope; by arresting the crystallisation at certain stages, a greater variety of forms and colours will be obtained: we may enumerate salicine, asparagine, acetate of copper, phospho-borate of soda, sugar, carbonate of lime, chlorate of potassa, oxalic acid, and all the oxalates found in urine, with the other salts from the same fluid, a few of which are shown at fig. 91 .

Dr. W. B. Herapath contributed an interesting addition to the uses of polarised light, by applying it to discover the salts of alkaloids, quinine, \&c. in the urine of patients. 
Ilo says: "It has long been a favourite subject of inquiry with the professional man to trace the course of remedies
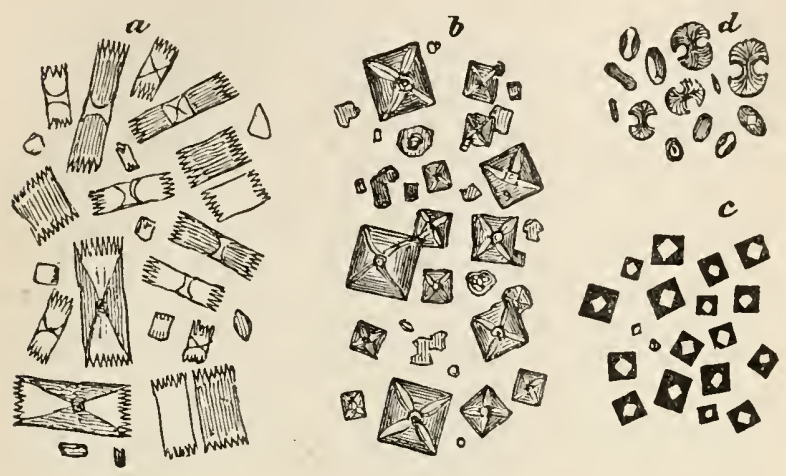

Fig. 91.-Urinary Salts.

$a$, Uric acid; $b$, Oxalate of lime, octahedral crystals of; $c$, Oxalate of lime allowed to dry, forming a black cube; $d$, Uxalate of lime, as it occasionaily appears, termed the dumb-bell crystal.

in the system of the patient under his care, and to know what has become of the various substances which he might have administered during the treatment of the disease.

"Having been struck with the facility of application, and the extreme delicacy of the reaction of polarised light, when going through the series of experiments upon the sulphate of iodo-quinine, I determined upon attempting to bring this method practically into use for the detection of milute quantities of quinine in organic fluids; and after more or less success by different methods of experimenting, I have at length discovered a process by which it is possible to obtain demonstrative evidence of the presence of quinine, even if in quantities not exceeding the one-millionth part of a grain; in fact, in quantities so exceedingly minute, that all other methods would fail in recognising its existence. Take for test-fluid a mixture of three drachms of pure acetic acid, with one fluid-drachm of rectified spirits-ofwine, to which add six drops of diluted sulphuric acid.

"One drop of this test-fluid placed on a glass-slide, and the merest atom of the alkaloid added, in a short time 
solution will take place; then, upon the tip of a very fine glass-rod let an extremely minute drop of the alcoholic solntion of iodine be added. The first effect is the production of the yellow or cinuamon-coloured eompound of iodine and quinine, which forms as a small circular spot; the aleohol separates in little drops, which by a sort of repulsive movement, drive the lluid away ; after a time, the acid liquid again flows over the spot, and the polarising crystals of sulphate of iodo-quinine are slowly produced in beautiful rosettes. This succeeds best without the aid of heat.

"To render these crystals evident, it merely remains to bring the glass-slide upon the field of the microscope, with the selenite stage and single tourmaline, or Nicol's prism, heneath it; instantly the crystals assume the two complementary colours of the stage; red and green, supposing that the pink stage is employed, or blue and yellow, provided the blue selenite is made use of. All those crystals at right angles to the plane of the tourmaline, producing

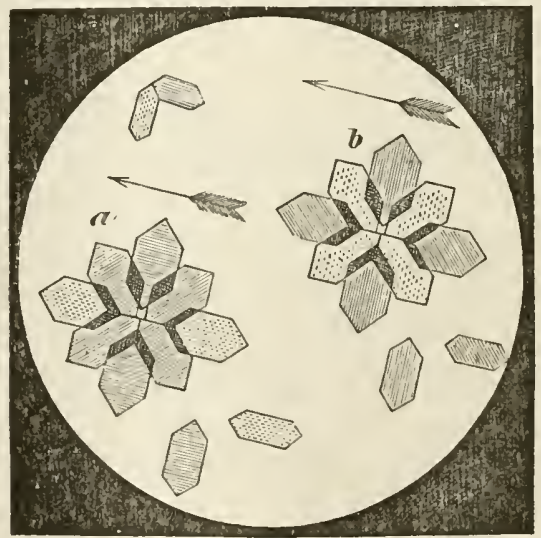

Fig. 92.-In this figure heraldic lines are adopted to denote colour. The dotted parts indicate yellow, the straight lines red, the horizontal lines blue, and the diagonal,-or oblique lines, yreen. The arrows show the plane of the tourmaline, $a$, blue stage; $b$, red stage of selenite employed.

that tint which an analysing-plate of tourmaline would produce when at right angles to the polarising-plate; 
whilst those at $90^{\circ}$ to these educe the complementary tint, as the analysing-plate would also have done if revolved through an arc of $90^{\circ}$.

"This test is so ready of application, and so delicate, that it must become the test, par excellence, for quinine: fig. $92, a$ and $b$. Not only do these peculiar crystals act in the way just related, but they may be easily proved to possess the whole of the optical properties of that remarkable salt of quinine, the sulphate of iodo-quinine.

"To test for quinidine, it is merely necessary to allow the drop of acid solution to evaporate to dryness upon the slide, and to examine the crystalline mass by two tomrmalines, crossed at right angles, and without the stage. Immediately little circular dises of white, with a welldefined black cross very vividly shown, start into existence, should quinidine be present even in very minute traces. These crystals are represented in fig. 93.

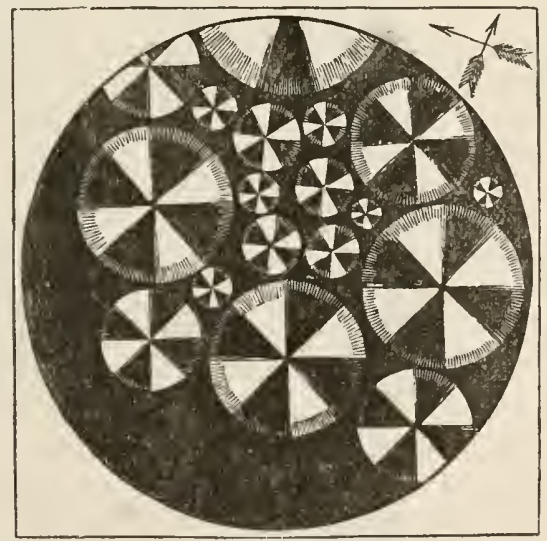

Fig. 93.

"If we" employ the selenite stage in the examination of this object, we obtain one of the most gorgeous appearances in the whole domain of the polarising microscope: the black cross at once disappears, and is replaced by one which consists of two colours, being divided into a cross 

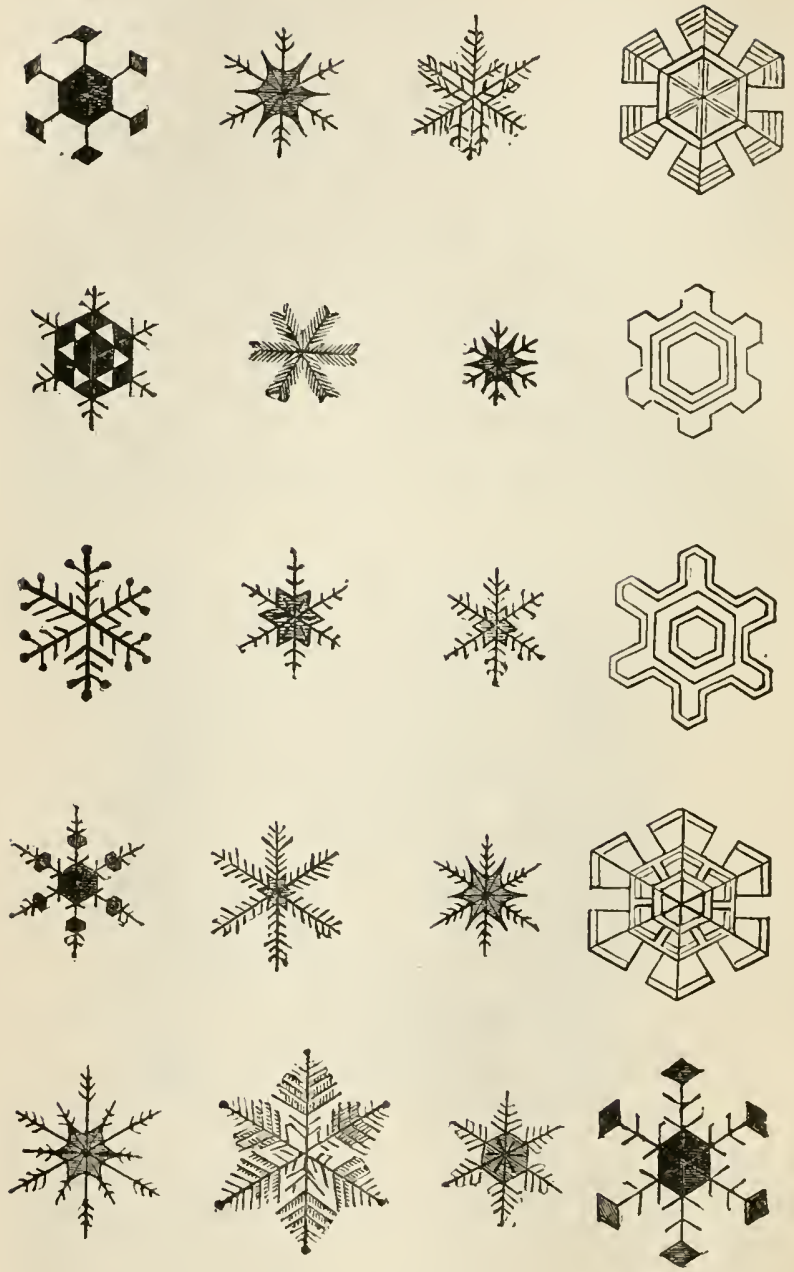

Fib. 94.-Snow Crystale. 
having a red and green fringe, whilst the four intermediate sectors are of a gorgeous orange-yellow. These appearances alter upon the revolution of the analysing-plate of tourmaline; when the blue stage is employed, the cross will assume a blue or yellow tint, according to the position of the analysing-plate. These phenomena are analogous to those exhibited by certain circular crystals of boracic acid, and to those circular discs of salicine (prepared by fusion); the difference being, that the salts of quinidine have more intense depolarising powers than either of the other substances; besides which, the mode of preparation effectually excludes these from consideration. Quinine prepared in the same manner as quinidine has a very different mode of crystallisation; but it occasionally presents circular corneous plates, also exhibiting the black cross and white sectors, but not with one-tenth part of the brilliancy, which of course enables us readily to discriminate the two."

Ice doubly refracts, while water singly refracts. Ice takes the rhomboidic form; and snow in its crystalline form may be regarded as the skeleton crystals of this system. A sheet of clear ice, of about one inch thick, and slowly formed in still weather, will show the circular rings and cross if viewed by polarised light.

It is probable that the conditions of snow formation are more complex than might be imagined, familiar as we are with the conditions relating to the crystallisation of water on the earth's surface. Dr. Smallwood, of Isle Jesus, Canada East, has traced an apparent connection between the form of the componud varieties of snow crystals and the electrical condition of the atmosphere, whether negative or positive; and is instituting experiments for his better iuformation on the subject.

A great variety of animal, vegetable, and other substances possess a doubly refracting or depolarising structure, as : a quill cut and laid out flat on glass; the cornea of a sheep's eye ; skin, hair, a thin section of a finger-nail ; sections of bone, teeth, horn, silk, cotton, whalebone; stems of plants containing silica or flint; barley, wheat, \&c. The larger-grained starches form splendid objects; tousles-mois, being the largest, may be taken as a type of all 
the others. It presents a black cross, the arms of which meet at the hilum. On rotating the analyser, the

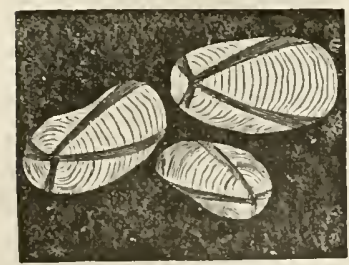

Fig. 95.

Potaln Starch, seen under polarised light. black cross disappears, and at $90^{\circ}$ is replaced by a white cross; another, but much fainter black cross being perceived between the arms of the white cross. Hitherto, however, no colcur is perceptible. But if a thin plate of selenite be interposed between the starch-grains and the polariser, most splendid and delicate colours appear. All

the colours change by revolving the analyser, and become complementary at every quadrant of the circle. TVest and East India arrow-root, sago, tapioca, and many other starch-grains, present a similar appearance; but in proportion as the grains are smaller, so are their markings and colourings less distinct.

"The application of this modification of light to the jlurnination of very minute structures has not yet been fully carried out; lut still there is no test of differences in density hetween any two or more parts of the same substance that can at all approach it in delicacy. All structures, therefore, belonging either to the animal, vegetable, or mineral kingdom, in which the power of unequal or double refraction is suspected to be present, are those that should especially be investigated by polarized light. Some of the most delicate of the elementary tissues of animal, such as the tubes of nerves, the ultimate fibrillæ of muscles, \&c., are amongst the most striking subjects that may be studied with advantage under this method of illumination. Every structure that the microscopist is investigating should be examined by this light, as mell as by that either transmitted or reflected. Objects momited in Canada balsam, that are far too delicate to exhibit any structure under transmitted, will often be well seen under polarised light; its uses, therefore, are manifold."1

(1) Quekett's Practical Treatise on tie Use of the Mitroscope. 
A fine effect may be obtained by using Furze's s]otted lens, with a Herapathite polariser; see Mic. Soc. Trans. $2 d$ series, vol. iii. p. 63 .

\section{APPLICATION OF PHOTOGRAPHY TO THE MICROSCOPE.}

At the time this book was projected, it was thought that if the objects so beautifully exhibited under the microscope corid be drawn by light on the page of the book, or on the wood-biocks, so that the engraver might work directly from the drawings thus made, truthfulness would be insured, and we should present to the reader a valuable record of microscopic research never before seen or attempted. But in this we were doomed to disappointment by the existence of a patent, which presented obstacles too great to be surmounted; and the idea was abandoned, with the exception of a few drawings then prepared, and ready to hand: the patent restrictions having been since removed, we have embodied them in our pages. The eye and feet of fly, antenna of moth, paddles of whirligig, with a few others, were first taken on a film of collodion, then floated off the glass on to the surface of a block of wood, the wood having been previously and lightly inked with printer's ink or amber-varnish, and the film gently rubbed or smoothed down to an even surface, at the same time carefully pressing out all bubbles of air or fluid.

For the purposes of photograplyy the only necessary addition to the ordinary microseope is that of a dark chamber; it should indeed form a camera ohscura, having at one end an aperture for the insertion of the eye-piece end of the microscopic tube, and at the other a groore for carrying the crown-glass for focussing. This dark chamber must not exceed eighteen inches in length; for if longer, the peneil of light transmitted by the object-glass is diffused over too large a surface, and a faint and unsatisfactory pieture results therefrom. Another advantage is, that pietures at this distance are in size very nearly equal to the object seen in the microscope. In some instances, better pictures are produced by taking away the eye-p:ece 
of the microscope altogether. The time of producing the picture varies from five to twenty seconds, with the strength of the daylight. A camphine lamp, light Cannel coal-gas, or the lime-light, will enable a good manipulator to produce pictures nearly equal to those produced by sun-light. Collodion offers the best medium, as a strong negative can be made to produce any number of printed positives.

The light is transmitted from the mirror through the object and lenses, and brought to a focus on the groundglass, or prepared surface of collodion, in the usual manner. Care must be taken not to use the burning focus of the lenses. The gas microscope may be used to make an enlarged copy of an object, it is only necessary to pin up against the screen a piece of prepared calotype paper to receive the reflected image. Mr. Wenham gives directions for improving "microscopic photography" in the Quarterly Journal of Microscopical Science for January, 1855. In this paper he has shown how to insure quick and accurate focussing; or, in other words, the making of the actinic and visual foci of the objective coincident. The simplest and cheapest way of producing coincidence is to screw a biconvex lens into the place of the back-stop of the object-glass, which thus acts as part of its optical combination. An ordinary spectacle lens, carefully centred and turned down to the required size, answers the purpose exceedingly well.

An excellent method has been proposed and adopted by Mr. Wenham, for exhibiting the form of certain very minute markings upon objects. A negative photographic impression of the object is first taken on collodion, in the ordinary way, with the highest power of the microscope that can be used. After this has been properly fixed, it is placed in the sliding frame of an ordinary camera, and the frame end of the latter adjusted into an opening cut in the shutter of a perfectly dark room. Parallel rays of sunlight are then thrown through the picture by means of a flat piece of looking-glass fixed outside the shutter at such an angle as to catch and reflect the rays through the camera. $\Lambda$ screen standing in the room, opposite the lens of the camera, will now receive an image, exactly as from a magic lantern, and the size of the image will be propor- 
tionate to the distance. On this screen is placed a sheet of photogenic paper intended to receive the magnified picture. We ought to add, however, that it requires considerable practice to avoid the distortion and error of definition occasioned by a want of coincidence in the chemical and visual foci. Imperfections are much increased when the highest powers of the microscope are employed; false notions of structure are also given, which is the case in Mr. Wenham's photograph of $P . A n-$ gulatum.

Mr. S. Highley has a mode of adapting an object-glass to the ordinary camera, for the purpose of taking microscopic objects on collodion and other surfaces, fig. 96 ; a sectional view of his arrangement is here given, which is

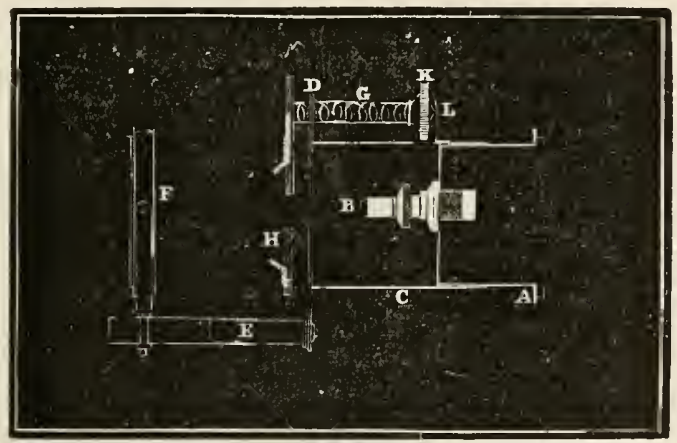

Fig. 96.-Highley's Camera.

very compact, steady, and ever ready for immediate use. The tube a screws into the flange of a camera which has a range of twenty-four inches; the front of this tube is closed, and into it screws the object-glass B. Over a slides another tube $\mathrm{C}$; this is closed by a plate, $\mathrm{D}$, which extends beyond the upper and lower circumference of $\mathrm{c}$, and carries a small tube, $\mathrm{E}$, on which the mirror $\mathrm{F}$ is adjusted. To the upper part of $D$ the fine adjustment $G$ is attached; this consists of a spring-wire coil acting on an inner tube, to which the stage-plate $\mathrm{H}$ is fixed, and is regulated by a graduated head, $\mathbf{x}$, acting on a fine screw, likewise attached to 
the stage-plate, after the manner of Oberhauser's microscopes. An index $\mathrm{L}$ is affixed opposite the graduated head k. The stage and clamp slides vertically on II ; and by sliding this up or down, and the glass object-slide horizontally, the requisite amount of movement is obtained to bring the object into the field. The object being brought into view, the image is roughly adjusted on the focussingglass by sliding $\mathrm{C}$ ou $\mathbf{A}$; the focussing is completed by aid of the fine adjustments $\mathrm{G} K$, and allowance then made for the amount of non-coincidence between the chemical and visual fuci of the object-glass. The difference in each glass employed should be ascertained by experiment in the first instance, and then noted. By employing a finely-ground focussing-glass greased with oil, this arrangement forms an agreeable method of viewing microscopical objects with both eyes, and is less fatiguing. As a very large field is presented to the observer, this arrangement might be advantageously employed for class demonstration.

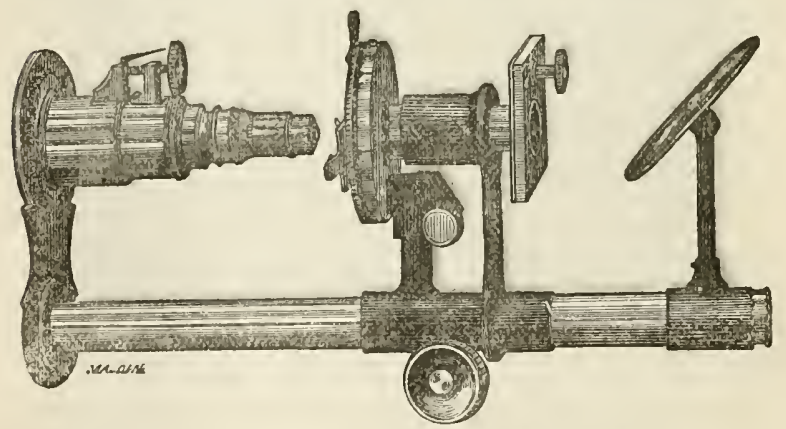

Fig. 97.-Highley's Photo-micrographic Arrangement.

This arrangement combines the most recent improvements of Dr. MIaddox, and consists of a lens-carrier with ordinary adjustments; stage with gymbal motions so as to bring any object parallel to the surface of the objectglass; bright ground illuminator, graduating diaphragm ; and a speculum reflector for giving the light from a single surface. 


\title{
CHAPTER III.
}

\begin{abstract}
PI ELININARY DIRECTIONS - ILLUMINATION - ACCESSORY APPARATUg GILLETT'S, ROSS'S, WEBSTER'S, AND OTHER CONDENSERS - OBLIQUB ELUMINATION-DOUBLE PRISM IILUMINATION-THE LIEDERKUHN-SIDE REFLECTOR-GAS-LAMPS-OBJECT FINDERS-COLLECTING ETICKS-ANIMALCULE CAGES-SECTION CUTTERS-PREPARING AND MOUNIING OB. JECTS, ETC.
\end{abstract}

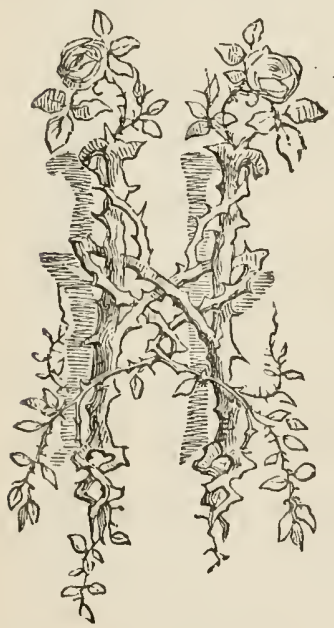

AVING selected an apartment with a northern aspect, and, if possible, with only one window, and that not overshadowed by trees or buildings: in such a room, on a firm, steady table, keep your instruments and apparatus open, and at all times ready for observation. A large bell-glass will be found most convenient for keeping dust from the microscope when set up for use. In winter it will be proper to slightly warm the instrument before it is used, otherwise the perspiration from the eye will condense on the eye-glass, and greatly impede vision. When you clean the eye-glasses, do not remove more than one at a time, and replace it before you touch another; by so doing you will preserve the component glasses in their proper places: recollect that if intermingled they are useless. Keep a piece of well-dusted and very dry chamois leather, slightly impregnated with the finest tripoli or rotten-stone powder, in a small box, to wipe the glasses. 
When you look through the instrument, be sure to place your eye quite close to the eye-piece, otherwise the whole field of view will not be visible; and observe, moreover, if you see a round disc of light, at least when the object is not on the slider-holder : if you do not, it is a sign that something is wrong; perhaps the body is not placed directly before the stage aperture, or may not be properly directed towards the light. Use the smallest amount of light possible, if you work for any length of time. Choose a steady light, with a shade to protect the eyes, one of the old-fashioned fan-shades will be found useful for this purpose : use the eyes alternately. Sit in a comfortable position, and bring the instrument to the proper angle, which will prevent congestion of the ejes; this is indicated if the microscopist is annoyed with little moving objects apparently floating before them: if the eye-lashes are reflected from the eye-glass, you are looking upon the eye-glass instead of through it. Take care that the mirror is properly arranged.

The following are Sir David Brewster's excellent directions for viewing objects:-

"First. Protect the eye from all surrounding light, letting only the rays which proceed from the illuminated centre of the object fall upon it.

"Secondly. Delicate observations should not be made when the fluid which lubricates the cornea is in a viscid state, or there is any irritation or inflammation about any part of the eye.

"Thirdly. The best position for microscopic observations is with the microscope bent to such an angle with the body, that the head may always remain in a natural and easy attitude; consequently, the worst position would be that which compels us to look downwards vertically.

"Fourthly. If we lie horizontally on the back, parallel markings and lines on objects will be seen more perfectly when their direction is vertical, or in a contrary direction to that in which the lubricating fluid desccuds over the cornea of the eye.

"Fifthly. Only a portion of the object should be viewed at one time, and every other part excluded. The light which illuminates that part should be admitted through a 
small diaphragm: at night, from the concentrated light of a sperm-oil 01' gas lamp, having a faint blue-tinted chimney-glass, to correct the yellow colour which predominates in all our artificial illumination. If in the day-time, close a portion of the window-shutters.

"Sixthly. In all cases when high powers are used, the intensity of the illumination should be increased by optical contrivances below the object and stage : this is generally effected by nsing achromatic condenser's beneath the stage. 'The apparatus for illumination should be as perfect as the maguifying power."

If these directions are strictly followed, no injury to the eyes from using a microscope need be feared.

Mr. Ross very properly l'emarks, that the manner in. which an object is lighted is second in importance only to the excellence of the glass through which it is seen. When investigating any new or muknown specimen, it should be viewed in turns by every description of light, direct and oblique, as a transparent object and as an opaque object, with strong and with faint light, with large angular pencils thrown in all possible directions. Every change will probably develope some new fact in reference to the structure of the olject, which should itself be varied in the mode of mounting in every possible way.

It should be seen both wet and dry, and immersed in fluids of various qualities and densities; such as water, alcohol, oil, and Canarla balsam; the last having a refractive power nearly equal to that of glass.

If the object be a delicate vegetable tissue, it will be, in some respects, rendered more visible by gently heating or seorehing before a clear fire, between two plates of glass. In this way the spiral vessels of asparagus and other similar vegetables will be beautifully displayed. Dyeing the objects in tincture of iodine, or some one of the dyewoods, will, in some cases, answer the purpose better.

But the principal question in regard to illumination is the magnitude of the illuminating peneil, particularly in reference to transparent objects. Generally speaking, the illuminating pencil should be not quite so large as can be received by the lens: any light beyond this produces indistiuctnoss and glare. T'he superfluous light from the 
mirror may be cut off by a screen, having váricus-sizeả apertures placed below the stage.

The Diaphragm, fig. 98, is the instrument used for effecting this purpose. It consists of two plates of brass, one of which is perforated with four or five holes of dif-

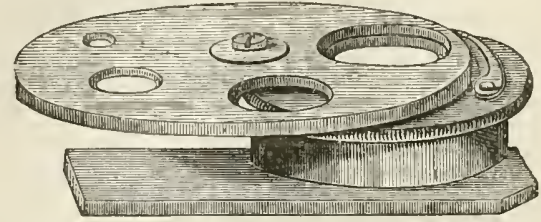

Fig. 98,-The Diaphragm.

ferent sizes; this plate is of a circular figure, and is made to revolve upon another plate by a central pin or axis; this last plate is also provided with a hole as lirge as the largest in the diaphragm-plate, and corresponds in situation to the axis of the compound body. To ascertain when either of the holes in the diaphragm-plate is in the centre, a bent spring is fitted into the second plate, and rubs against the edge of the diaphragm-plate, which is provided with notches. The space between the smallest and largest is great enough to use for the purpose of shutting off all the light from the mirror.

Gilletr's Illuminator, on Condenser.-The advantages of employing an achromatic condenser were first pointed out by Dujardin, since which time an object.-glass has been frequently but inconveniently employed; and more recently achromatic illuminators have been constructed by most of our instrument makers. Some years since, Mr. Gillett was led by observation to appreciate the importance of controlling not merely the quantity of light which may be effected by a diaphragm placed anywhere between the source of light and the object, but the angle of aperture of the illuminating pencil, which can be effected only by a diaphragm placed immediately behind the achromatic illuminating combination. Such a diaphragm is represented in fig. 99, manufactured by Mr. Ross : it consists of an achromatic illuminating lens $c$, which is 
about equal to an object-glass of one-quarter of an inch focal length, having an angular aperture of $80^{\circ}$. This lens is placed on the top of a brass tube, intersecting

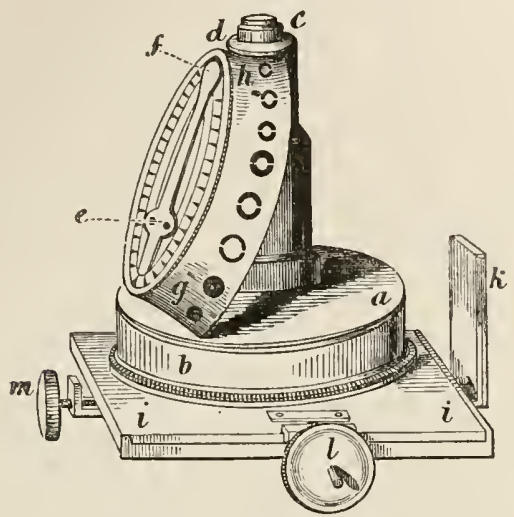

Fig. 99.-Gillell's Condenser.

which, at an angle of about $25^{\circ}$, is a circular rotating brass plate $a b$, provided with a conical diaphragm, having a series of circular apertures of different sizes $h g$, each of which in succession, as the diaphragm is rotated, proportionally limits the light transmitted through the illumiuating lens. The circular plate in which the conical diaphragm is fixed is provided with a spring and catch $e f$, the latter indicating when an aperture is central with the illuminating lens, also the number of the aperture as marked on the graduated circular plate. Three of these apertures have central discs, for circularly oblique illumination, allowing only the passage of a hollow cone of light to illuminate the object. The illuminator above described is placed in the secondary stage $i i$, which is situated below the general stage of the microscope, and consists of a cylindrical tube having a rotatory motion, also a rectangular adjustment, which is effected by means of two screws $l m$, one in front, and the other on the left side of its frame. This tube receives and supports all the var ous 
illuminating and polarising apparatus, or nther auxiliaries which are placed underneath the object. The tube and its frame are affixed to a dovetailed sliding bar $k$, which can be easily moved up or down, or taken off for conveniently attaching the various apparatus. This sliding bar fits into a second sliding bar, which, by means of a milled-head screw, moving a rack and pinion, regulates the distance of the apparatus from the stage.

Directions for. Use by Day or Lamplight.-In the adjustment of the compound body of the microscope with the illuminator above described, two important results are to be sought-first, their centricity, and secondly, the fittest condensation of the light to be employed. IVith regard to the first, place the ilhminator in the cylindrical tube, and press upwards the sliding bar in its place, until checked by the stop; move the microscope body either vertically or inclined for convenient use; and with the rack and pinion which regulates the sliding bar, bring the illuininating lens to a level with the upper surface of the object-stage; then move the arm which holds the microscope body to the right, until it meets the stop, whereby its ceutral position is attained ; adjust the reflecting mirror so as to throw light up the illuminator, and place upon the mirror a piece of clem white paper to obtain a uniform dise of light. Then put on the low eye-piece, and a low power (the half-inch), as more convenient for the mele adjustment of the instrument ; place a transparent object on the stage, adjust the inicroscope-tube, until vision is obtained of the object; then remove the object, and take off the cap of the eye-piece, and in its place fix on the eyeglass called the "centering eye-glass," described below, which will be found greatly to facilitate the adjustment now under consideration, namely, the centering of the compound body of the nicruscope with the illuminating apparatus of whatever description. ${ }^{1}$ The centering-glass, being thus affixed to the top of the eye-piece, is then to

(1) I his centering-glass consists of a tubular cap containing two plano convex lenses, whi h are app.ied and idjusted so that the image of the aperture in the object-glass, and the images of the apertures at the lenses and in the diaphragms contained in the tube which holds the illuminating combination, may all be in focus at the same time, as wh the same aljustment they may be brought sufbcuratly near in focus to recognise their centricity. 
be adjusted by its sliding-tube (withont disturbing the microscope-tube) until the images of the diaphragms in the object-glass and centering lens are distinctly secn. 'llie illuminator should now be noved by means of the left-hand screw on the secondary stage, while looking through the microscope, to enable the oliserver to recognise the diaphragm belonging to the illuminator, and by means of the two adjusting screws, to place this diaphragm central with the others; thus, the first condition, that of centricity, will be accomplished. Remove the white paper from the mirror, and also the centering-glass, and replace the cap on the eye-piece. also the object on the stage, of which distinct vision should then be obtained by the rack and pinion, or fine serew adjustment, should it have become deranged.

The second process is to ascertain that the fittest concentration of light is obtained. For this purpose the mirror should now be so inclined that the image of some intercepting distant object, such as a house-top, or chimney, tree, window-frame, or (if lamp-light be employed) the lamp's flame may be bronght into the field of view; these, though not distinctly seen, may be recognised by partially darkening or otherwise occupying the field; then distinct vision of such object must be obtained by means of the rack and pinion moving the secondary stage to and from the object. Exeepting the case of the lamp's flame, the above objeets are considered as the representatives of the source of light; for when daylight is employed-as, for example, a white clond-its motion prevents the image being eisily produced: then it is convenient to employ a distant object, such as the above,-the difference of the focal length of the illuminating lens for such an object, and for the white cloud, being almost insensible. This last adjustment being effected by the movement of the secondary stage alone, the microscope tube remaining undisturbed, also the objeet on the ohject-stage uninterrupted in focus, the souree of the illuminating light and the object to be examined will both be distinctly seen at the same time. These adjustments, whether for daylight or lamplight, being completed, the mirror may be turned so as wholly to reflect the light either of the sky or of the 
lamp; and the eye-piece and object-glass suitable for exainining the object may be employed, and the focus adjusted accordingly. The couical diaphragm with its various apertures may now be rotated, until that quality of illumination is obtained which gives a cool, distinct, and definite view of the object. Upon changing the object-glass, the centering eye-glass should always be enployed to ascertain that the centricity of the illuminating condenser and microscope body has not been deranged.

It has been stated that the image of a white cloud opposite the sum is the best for illuminating transparent objects when viewed by trausmitted light. $\mathrm{Mr}$. Gillett has successfully imitated this natural surface by an apparatus consisting of a large parabolic reflector, with a small camphine lamp on an adjustable stand, having its flame nearly in the focus; also of two other reflectors of hyperbolic figure, which are employed according to the object-glasses used on the microscope. The parabolic mirror and one of these are attached opposite to each other on the bent arm by which they are supported, having their axes coincident, and the enamel dise placed between them. The small hyperbolic reflector receives the light reflected from the large parobolic reflector, and concentrates the rays on the small enamel disc. The surface of this disc is roughened, so that the forms of all the incident pencils are broken up, and the effect of a white cloud produced.

Several important modifications and valuable improvements in condensers have been introduced by maliers, and, therefore, deserve especial notice. Two things should be required of achromatic condensers intended for general use and for research: First, that the optical combination employed should be adapted to a considerable range of power -say, from an inch, or $\frac{2}{3}$ ds upwards to the highest; and, secomdly, that they should be capable of working with a large aperture through the ordinary glass slides. When low powers are employed, a pleasantly lit field can be obtained by using a condenser a little out of focus, so that the rays cross before reaching the object; but a condenser for general use should send a sufticient quantity, and no more, of oblique rays, when in focus, through an object 
seen with very moderate magnification, and should be capable of giving a dark-ground illumination with a $\frac{1}{2}$ inch or $\frac{2}{3} \mathrm{ds}$ object glass.

In Mr. Ross's $\frac{4}{10}$ ths condenser, represented at $\mathbf{A}$, fig. 39, these desiderata are well provided for. The optical combination is exactly the same as in his large angled $\frac{4}{10}$ ths objectives, and the front lens has a diameter of $\frac{1}{5}$ th of an inch. In all good condensers the diaphragm slould be brought close to the lower lens; that is the case with this instrument, which is provided with two revolving wheels of diaphragms. The upper one is pierced with eight circular apertures; one of which is filled in with a polarizing slice of tourmaline; another is made with a little rim, so as to receive any experimental stops the mieroscopist may wish to try. Omitting this lastmentioned stop, we have seven others, marked respectively $109^{\circ}, 95^{\circ}, 82^{\circ}, 70^{\circ}, 59^{\circ}, 49^{\circ}$, and $40^{\circ}$. These stops allow the lenses to work with the angles of aperture named. "In the wheel of diaphragms, below them, is arranged another set of stops, for combination with the preceding.

A single slot stop keeps ont the central rays, and allows a radial beam, including its proportion of marginal rays, to illuminate the object. This can be used with the larger of the open stops. The two-slot stop gives passages to two such pencils of light, one at right angles to the other, a plan very effective with certain diatoms and other objects. The three-slot stop allows the transmission of three pencils, equidistant from each other. This gives the three readings of the $P$. angulatum. The arrangement for working the diaphragms in the condenser is very convenient, and the whole apparatus rotates with the sub-stage. The lenses are also capable of adjustment to suit different thicknesses of glass.

The proportion which, from the size of the front lens, the marginal and central rays bear to each other, is such that, when the stop allowing $40^{\circ}$ of aperture is employed, the two sets of lines, on Pleurosigma hippocumpus and $P$. angulatum, are distinctly shown with a one-fifth objective and an $A$ eye-piere. $\Lambda$ slight change in the position of the flat mirror makes this stop work excellently with the 
Podura scale. Thus, this arrangement enables an experimenter viewing a new object to see surface markings, and to obtain penetration with one and the same stop; an important gain in original investigation. The slot stops have been found very useful in investigating unknown objects, as well as in displaying those that are known; and with the whole aperture and the two-slot stop, many diatoms with double sets of lines are well brought out. The microscopist will find that from $40^{\circ}$ to $59^{\circ}$ angle of aperture will, in many cases, give the best results, when a $\frac{1}{1}$ or $\frac{1}{5}$ objective is employed.

An excellent condenser of very large angular aperture is made by Messrs. Powell and Lealand, in which every requisite modification of the illuminating pencil may be produced by two revolving dises, one containing apertures of various sizes, and the other various diapliragms for excluding the central portion, or for almitting only angular portions, of the pencil of light. These discs are placed inmediately below the posterior lens of the illuminator. This method of modifying the illuminating pencil was first applied in Gillett's condenser, as constructed by Mr. Ross. The improved hemispherical condenser lately introduced by the Rev. J. B. Reade, answers its purpose remarkably well. The plane surface of the hemisphere is placed upwards, and is covered by a diaphragm in which are marginal apertures, capable of adjustment either to an interval of $90^{\circ}$ with each other, when the arrangement of the dots to be developed is quadrangular, as in $P$. rhomboides, or $P$. hippocampus, or to one of $60^{\circ}$ and $120^{\circ}$, when they are arranged in equilateral triangles, as in $P$. augulatum, \&c. An ingenious plan of illuminating minute objects, mounted in Canada balsam, by reflected light, has been devised by Mr. Wenham: this consists of a small truncated glass paraboloill, which is temporarily attached to the under side of the slide containing the object, by a little gum, oil, or fluid Canada balsam. The rays internally reflected from the convex surface of the paraboloid, impinge very obliquely on the under-surface of the slide, are transmitted in consequence of the fluid-uniting medium, and then internally reflected from the upper surface of the covering glass on to tho 
object. Very minute variations of surface contour may by these means he rendered evident.

Collins's Webeter's Universal Aehromatic Condenser (fig. 100), a superior mechanical contrivance, is provided with a diaphragm which so gradually shuts off all excess of light, that any amount of illumination can be admitted
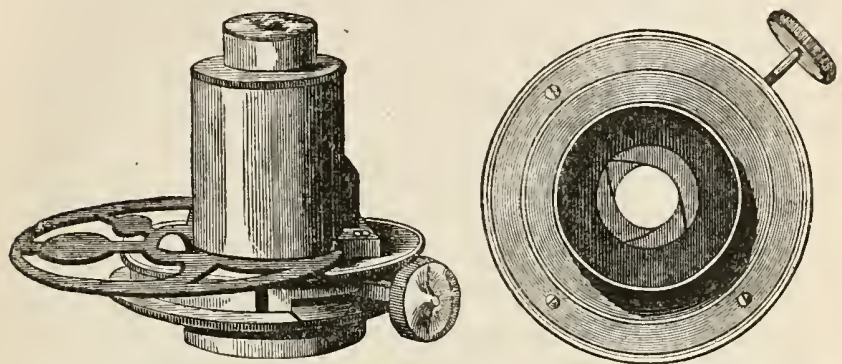

’’in. 100.-Collins's Webster's Universal Condenser. Shutter Diaphragm secn separctely

with the greatest nicety. This valuable addition to the microscope can be fitted to any instrument with or without a sub-stage arrangement. Its chief advantages are that, while its perforning qualities take rank with much more expensive forms, it is moderately cheap, and is at once an achromatic condenser, parabolic illuminator, and graduating diaphragm, with polariser added. By means of a lever, the central aperture can be grudually closed, without losing sight of the object, and at the same time the alteration is effected with a great amount of delicacy. Providerl an object-glass has sufficient "resolving power," its capabilities are greatly increased by this condenser, in bringing out the markings on the difficult test-objects; when in connexion with the Polariscope, a great increase of light is obtained-so much so that, by using a polarising prism with the "spot lens stop," the object appears brilliantly illumined on a dark ground, with fine coloured effects. When high powers are used in connexion with the polariscope, the advantage derived by such an addition to the ordinary mode of illumination will be at once apparent to every microscopist. 
Professor Smith, of Kenyon College, America, invented a condenser for the illumination of opaque objects under high powers. It has been felt that it would be of immense advantage to the pliysiologist if lie could illuminate small bodies, such as blood-globules, and view them as opaque objects with the $\frac{1}{8}$ th or $\frac{1}{12}$ th ; hitherto there have been great difficulties in the way of accomplishing this, but Professor Snith has hit upon a contrivance which promises to be useful.

In this instrument a peneil of light is admitted above the objective and thrown down through it on to the object, by means of a small silver mirror placed on one side, and cutting off a portion of the aperture. Powell and Lealand tevised what they considered to be an improvement, and substituted for the small silver mirror, to which Prufessor Smith gave a preferenee, a flat piece of glass placed at an angle of $45^{\circ}$ across the tube, interposed like an adapter between the objective and the microscope body. A pencil of light entering by a side aperture striking against this flat glass is partly reflected down through the objective and on to the object, the magnified image of which is viewed through the glass. If the flat glass be ground so as to have parallel surfaces, no great amount of error can be detected.

Smith and Beck place a dise of thin covering glass, at an angle of about $45^{\circ}$, in the optic axis of the microscope body, close behind the setting of the object-glass in a special adapter, leaving a suitable aperture for admitting light from a lamp, rays from which are reflected downwards upon the object. The object-glass is thus made its own achromatic condenser.

But the idea of employing the object-glass as its own condenser was suggested by Mr. Hewitt five years ago, and then Mr. Wenham was indueed to give the plan a trial. A concave speculum was fitted at an angle into the body of the microscope, having a central hole sufficiently large to admit the full pericil from the objective, through the back of which the rays from a lamp (the light from which was admitted through a hole in the side of the body) were reflected downwards. The object was strongly illuminated, but there was so much glare from the internal 
fittings, and a reflection from the back of the objectglass-lenses, that an unfavourable opinion was formed of its practicability. It has since been demonstrated that the light was too intense; and the most useful, the central portion of rays, were wanting. A simple dise of thin glass and its partial reflection meets these objections. If such a dise be used with a little care, it is found to be quite as accurate as the other plan, and the natural surface of the glass is a better reflector than any artificial one. It has, however, the disadvantage of extreme fragility. By making the object-glass its own condenser, and examining diatoms as opaque objects under high powers, we may now hope to solve the much vexed question as to the true nature of their markings. Mr. Browning has employed the apparatus in a form much more nearly resembling that of the original inventor, only substituting a small glass reflecting-prism for the metallic reflector. Some advantages are gained by the adoption of this latter arrangement. Such modes of illumination bid fair to correct many errors of interpretation resulting from an exclusive use of trans parent illumination.

Oblique Illumination is of the greatest importance in the demonstration of structure, especially that of testobjects; and much greater obliquity is often required than can be obtained by throwing the mirror out of the axis of the microscope. Various methods are described in the "Transactions" of the Microscopical Society of London, for almost every microscopist has devoted some attention to the subject in his attempts to resolve the more difficult lined or dotted objects, as diatoms; therefore, to give a full description of all is quite uncalled for, if not impossible, in our parges; it is nevertheless most advisable, in doubtful cases, to have recourse to every method which shall present the object uncler a different aspect. One of the earliest methods devised for obtaining oblique light, was the eccentric prism of Nachet, which occupied the place of the present achromatic condenser, and, like it, received its light from the mirror; by simply turuing it in its socket, oblique rays were thrown upon the object from every side. This had, however, defects which Mr. Sollitt proposed to remedy, by employing an achro- 
matic condenser of a very long focus and large aperture, mounted in such a way as to enable its axis to be inclined to that of the microseope through a wide angular range; a condenser of this construction, he says, is also suitable for all ordinary purposes. ${ }^{1}$ A combination of the reflecting and refracting powers of a prism is much preferred by some; such, for instance, as that known as Amici's prism, which causes the rays to be reflected by a plane surface, and made to converge by a lenticular one, so that the prism answers the double purpose of mirror and condenser at the same time. "Abraham's Achromatic Lenticular Prism" is an excellent substitute for the achromatic con. denser, and answers the double purpose of mirror and con denser. This prism, represented half-size in fig. 101, is made

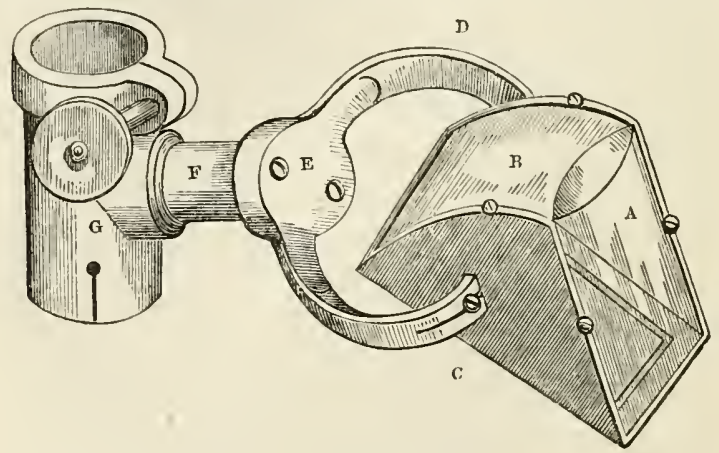

Fig. 101.-Abraham's Achromatic Lenticulur Prism. Half-size.

" p of two kinds of glass, set in a frame of brass; the part employed as the reflector (A) is of flint glass, hollowed ont at its upper surface, and into this is accurately fitted a double convex lens of crown glass (B), so contrived as to have a focus of about four inches. By the tube (G), the prism can be applied to the ordinary support of the mirror; and by means of the flat semicirele (D), and a joint in the connecting piece $(F)$, it can be turned in every possible direction, the semicircle sliding through a spring clip at $\mathrm{E}$. By this

(I) Quart. Journal Micros. Science, vol. iii. p. 87. 
instrument, achromatic condensed light may bo thrown upon any object on the stage. The prism has the usual swinging motion, accomplished by the frame turning on two screws, one of which is seen at C, at the end of the semicircle.

A thin stage is of importance when a very oblique pencil is used, and, to a certain extent, the larger its lower aperture, the more oblique will be the rays we are enabled to transmit to the object. For this reason the thin stage introduced by such makers as Powell and Lealand, is in every way an improvement on the thicker stage of the older instruments. A revolving stage also possesses advantages: it enables the observer to keep the object in the centre of the field of view, and while its various parts are thus presented in succession to rays of greater or less obliquity, an insight into the structure is often afforded which could not otherwise have been obtained. "Thus, suppose that an object be marked by longitudinal striæ, too faint to be seen by ordinary direct light, the oblique light most useful for bringing them into view will be that proceeding in either of the directions $\mathbf{C}$ and $\mathbf{D}$; that which falls upon it in the directions $A$ and $B$ tending to obscure the striæ rather than to disclose them. But if the striæ should be due to furrows or prominences which have one side inclined and the other side abrupt, they will not be brought into view indifferently by light from $\mathrm{c}$, or from $\mathrm{D}$, but will be shown best by that which makes the strongest shadow; hence, if there be a projecting ridge,

$\mathbf{A}$

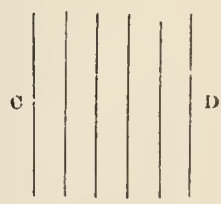

B with an abrupt side looking towards $\mathrm{c}$, it will be best seen by light from $\mathrm{D}$; whilst, if there be a furrow with a steep bank on the side of $\mathrm{c}$, it will be by light from that side that it will be best displayed. But it is not at all unfrequent for the longitudinal striæ to be crossed by others; and these transverse striæ will usually be best seen by the light that is least favourable for the longitudinal, so that, in order to bring them into distinct view, either the illuminating pencil or the object must be moved a quarter round." 
For those who desire to obtain a very oblique illuminating pencil, with objectives of lower powers, but large ang:lar aperture, and with an inexpensive arrangement, the Hemispherieal Condenser (Kettledrum) of the Rev. J. B. Reade will be found serviceable. ${ }^{1}$ This gentleman now, however, uses a double hemispherical condenser for the examination of the most difficult tests; and the great obliquity of the illuminating pencils gives every facility for dealing with the close fine lines of the Amieian test and the Macrum. Diaphragms of tinfoil are placed between the two hemispheres. By looking down the body of the microscope when the eye-piece is removed, and examining the dimensions of the little dises of light, it is seen at a glance whether one or other of the apertures require to be more or less deeply cut. In the one case, the little lappet of tinfoil can be so doubled as to shorten the aperture, and, in the other, it may be cut deeper and thrown further back. To obtain these very slight but not unimportant variations, and in a moment, is an advantage which observers will readily recognise. Mr. Reade also uses more durable diaphragms of thin brass, and, by a new arrangement, the precise amount of light is obtained at once. A fixed diaphragm, with apertures at right angles to each other, and cut nearly to the centre, is placed on the lower hemisphere; and another diaphragm, of which the outline is a right angle upon a semicircle, is placed in a slit of the tube between the condensers, so that the vertex of the right angle divides the space between the long $V$ apertures of the lower diaphragm. When pushed home, it nearly shuts up these apertures of the fixed diaphragm ; but by gently drawing it out, and moving it a little sideways, if necessary, we can obtain, with the utmost nicety, just that length of either aperture which the test-lines under examination require.

The very perfection of oblique illumination for resolving the markings on test-objects, is the double prism made use of by Mr. J. Newton Tomkins. The method of employing the two prisms we give in our friend's words :-

(1) Rev. J. B. Reale, F.R.S. on a New IIemispherical Condenser. Trans Miicros. Soc. 1861, p. 59, and vol. vii. Daga 31867. 
"My first attempts at oblique prism-illumination were made, many years ago, with Abraham's (of Liverpool) Aclromatic Lenticular Prism; the high character given of the performance of this instrument by the late Professor Quekett and by Mr. Sollitt, of Hull, led me to believe that it would entirely supersede the necessity of using a condenser during the examination of the highest class test-objects. In my hands, however, it failed to do this; the pencil of light transmitted proved to be too diffused, and the shadows too faint to enable me to bring out the markings of single-lined tests satisfactorily. I subjoin $\mathrm{Mr}$. Sollitt's directions for using this prism, as communicated

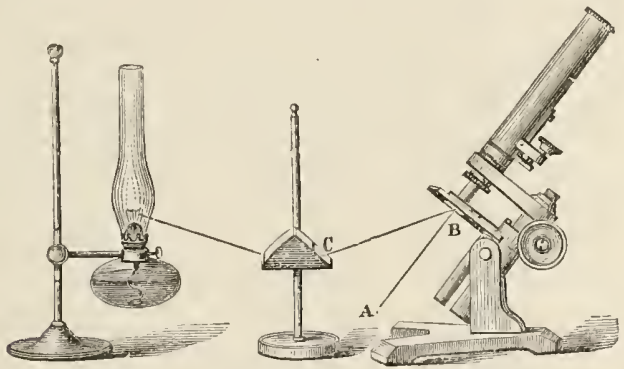

Fig. 102 -Sollitt's Single-Prism Illumination.

to Mr. Abraham :- 'When I require the prism for definition and direct light, I use it in place of the mirror under the stage; but when required for oblique light, that is, for illuminating lined oljects, or for black-ground illumination, I use it on a separate stand, as shown in fig. 102, the object being illuminated by the very oblique ray, $\mathrm{c}$ B. Let A B be a supposed ray of direct light, coincident with the axis of the microscope, then so long as the angle $\mathrm{OBA}$ is less than half the angle of aperture of the object lenses, the field will remain illuminated by oblique light, and the lined objects (if placed in their proper position) will be brought out in the most beautiful manner. I place the prism about five inches in front of the object, and by this method, with my widest apertures, I bring 
out the lines on Navicula Angulata, Ceratoneis Fusciolı, Amician Test, Grammatophora Subtilissima, and even on the Navicula Acus, with the greatest case. In viewing these delicate objects, the lines must be at right augles with the direction of the light. If the angle $\mathrm{C} B \mathrm{~A}$ be greater than half the angle of aperture of the object lenses, then you have the object beantifully illmminated on a black ground; and when the lisht is properly managed, the result is very superior to that ubtained by the parabolic reflector-the objects on the black-ground appear as though they were in full sunlight, bringing out the fine markings on delicate tissues most satisfactorily.'

"My next trial was made with a small rectangular achromatic prism with a foeus of two inches and a halfnot of four or tive inches, as in Mr. Sullitt's case-furnished by Messrs. Powell and Lealand; and I then found it easy to resolve the markiugs of all test objects which were disposed at right angles with the somee of illumination. With a view of resolving the crossed-lines of $P$. Formosum, N. Angulata, and the like, I employed the usual misroscope mirror, so disposed by means of a doublejointed arm that the light from the lamp might be reflected as nearly as possible at right angles with the second set of lines, but met with but moderate success, since the double reflection, due to the primary reflection from the silvered surface of the mirror, and the secondary reflection from the glass of the mirror itself, caused a haze that militated materially against true definition of the markings which characterise such delicate tests as $N$. Rhomboides, $P$. Macrum, \&c.

"The next step was to substitute for the mirror a second lamp and a second prism, also mounted on a separate stand, with rack-work adjustment, and provided with a universaljoint arm. The success of this plan was undoubted; I was now enabled to resolve the crossed lines of $N$. Rhomboides (the Auician Test of the L.olidon, although, perhaps, not of the American microscopists) with a sharpness which was hitherto unattainable; and even the difficult object $P$. Macrum came out in well-defined squares. The advantase of the universal-joint fitted to the second prisu consists in the fact that the diagonal lines of such tests as 
$P$. Rigidum may be brought out with ease by simply inclining the prism to the angle necessary to cause the shadows of this series of lines to fall directly opposite to the source of light.

"The method of using this combination will be readily understood by referring to fig. 103 .

"The microscope (A) is placed at the usual angle of inclination; a valve-say, of $N$. Rhomboides-is selected which lies vertically on the stage; the front lamp (c) is adjusted so that the centre of the flame shall be eleven inches above the table, and ten inches from the object; an object glass of moderate angle, such as a Ross's old. $\frac{1}{2}$ inch, with $60^{\circ}$ of aperture, will be found convenient for this first step in the process; the front prism (D), with its convex surface turned towards the lamp (c), is placed $1 \frac{1}{2}-2$ inches from the stage, and so adjusted that the rencil of transmitted light shall fall with the utmost obliquity on the object; now (referring to the former fig. 102), the angle с в A being about $40^{\circ}$, or greater than

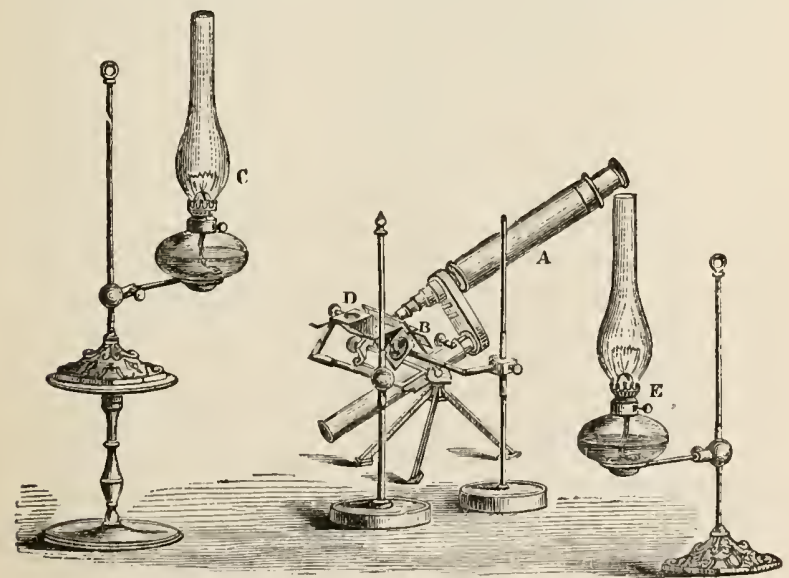

Fig. 103. -Mr. Newton Tomkins' Double Prism Illumination.

half the angle of aperture of the lens, the valve under examination will be brightly illuminated on a black- 
ground. An $\frac{1}{8}$ th, $\frac{1}{12}$ th, or a $\frac{1}{1} 6$ th, is then substituted for the $\frac{1}{2}$ inch lens; and since the angle of aperture of either of these lenses exceeds $140^{\circ}$, the whole field will be illuminated with a very oblique pencil of light. By careful manipulation of the prism, and the closest attention to the due adjustment of the object-glase itself, the transverse markings on the valve (which are by far the most difficult to resolve) should now come out with great clearness.

"The reason for using the $\frac{1}{2}$ inch lens in the first instance, is to find, by 'Maltwood,' or otherwise, the particular object desired, and to determine the exact position of lamp and prism; by commencing to work with the $\frac{x}{12}$ th or $\frac{x}{16}$ th these would be impracticable. The light of the lamp is now to be shut off, and a side prism (B) inclined parallel to the plane of the stage-plate, and brought as near to the under-side of the object as the form of the fixed stage will admit, placed in position. The light of a second lamp (E) is then caused, as before, to fall very obliquely on the object, and the longitudinal markings will be readily shown. On restoring the light of the front lamp (c), and paying particular attention that neither light shall be in excess, the valve will be brought out in squares, with a sharpness and a delicacy of tracing which I venture to assert will bear comparison with the best results obtained by use of the expensive modern condenser. I would by no means undervalue the advantages to be derived from the employment of the condensers now furnished by our eminent makers; but, seeing that these are manifestly articles of luxury, I incline to the opinion, that to those microscopists who are economically disposed, and already possess one rectangular prism, the trifling addition of a second prism and a second lamp will enable them to do good service to the cause of resolving many difficult test-objects hitherto insurmountable, amongst which I would place in the foremost rank Amphipleura pellucida (N. Acus), the markings on which I do not yet despair of seeing 'crossed' by some enthusiastic observer."

We have witnessed with surprise the ease with wlich difficult markings are brought out by this mode of illumiration. 
The Parabolic Reflector.-Mr. F. H. Wenham (Micros. Trans. 1851) proposed a parabolic illuminator, for the purpose of obtaining perfect definition under high powers. Those who have experimented on the subject, may liave observed that there is something in the nature of oblique light reflected from a metallic surface particularly favourable for the purpose of bringing out minute markings, which may, in some measure, be attributed to the circumstance of light so reflected being purely achromatic. In order to render this property available, Mr. Wenham contrived a very ingenious metallic reflector, by which the condensation of lateral light may be effected. ${ }^{1}$

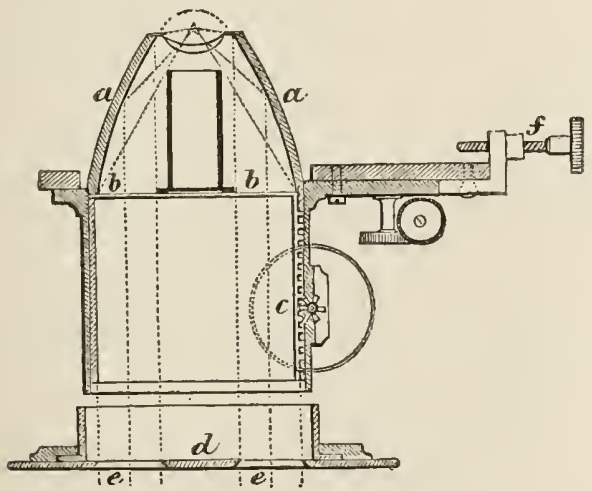

Fig. 104. - Wenham's Parabolic Reflector.

"The apparatus is shown in section in fig. 104; $a$ a is a parabolic reflector, of a tenth of an inch foeus, with a

(1) Mr. Shadbult contrived a modification of Wenham's Reflector, and called it a "Sphero-Annular Condenser:" which consists of a ring of glass whose surface was so shaped as to present a prismatic section, the inclination of the outer side being such as to produce a total reflection of the rays impinging on it, and to direct these through the inner side of the ring, so as to fall at a very oblique angle upon the object from every azimuth of the eircle. A combination of both methods is adopted in the parabolic illuminator now very generally used. This consists of a paraboloid of glass resembling a cast of the interior of Wenham's Parabolic Speculum, but reflecting the rays which fall upon tho eurface of the glass like Shadbolt's Annular Condenser. 
polished silver surface, having the apex so far cut away as to bring the focal point at such a distance above the top of the apparatus (which is closed with a screw-cap) when not in use) as may allow the rays to pass through the thickest glass commonly used for mounting objects upon before coming to a focus.

" $\Lambda$ t the base of the parabola is placed a disc of thin glass $b b$, in the centre of which is cemented a dark well, with a flange equal in diameter to the aperture at the top of the reflector, for the purpose of preventing the direct rays from the source of light passing through the apparatus.

"The reflector is moved to and from the object by means of the rack and pinion $c$, and has similar adjustments for centering, and is fixed mnder the stage of the microscope in the same way as the ordinary achromatic condenser: in addition, there is a revolving diaphragm $d$, made to slide on the bottom tube of the apparatus; it has two apertures $e e$, placed diametrically, for the purpose of obtaining two pencils of oblique light in opposite directions. The effects of the chromatic and sphericil aberrations, in the shape of fog and colour about the objects, caused by the glass slides upon which they are mounted, frequently require compensation; for as the parabola has the property of throwing parallel rays uncoloured to a point, when used alone, it is most suitable for objects without glass underneath.

"By the addition of a meniscus, this compensation is obtained, and also greater purity and intensity of illumination is procured; and as the silver reflector is now closed with glass, it is hermetically sealed, and permanently protected from dust and damp, and will therefore retain its polish. The light most suitable for this method of illumination is lamp or caudle light, the rays of which must in all cases be rendered parallel by means of a large planoconvex lens, or condenser; the light may then be used direct, or reflecied from the plane mirror. The object having been adjusted, the illuminator is moved to and fro till the best effect is produced. For the purpose of viewing some objects, such as naviculæ, the circular diaphragm should be slid on the extremity of the apparatus, and 
revolved till the two pencils of light are thrown most suitably across the object."

The Lieberkïhn.-The concave speculum termed a "Lieberkühn," from its celebrated inventor, was formerly much in use as a reflector, but is now almost abandoned, or rather replaced by other and better contrivances. The Lieberkuhn is generally attached to the object-glass, in the manner represented at fig. 105 , where $a$ exhibits th lower part of the compound body, $l$, the object-glass, Jve which is slid a tube and the Lieberkülı1, $c$, attached to it; the rays of light reflected from the mirror are brought to a focus upon an object $d$, placed between it and the mirror. The object may either be mounted on a slip of glass, or else held in the forceps, $f$; and when too small to fill up the entire field of view, or when transparent, it is necessary to place behind it the dark well, $e$.

Each Lieberkühn being mounted on a short piece of

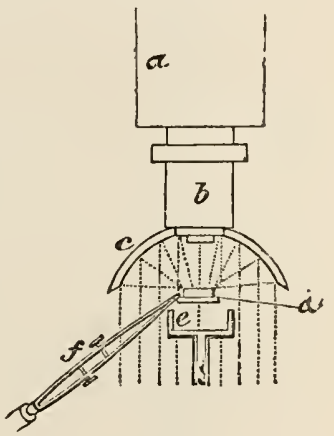

Fig. 105. tube, can be slid up and duwn on the outside of the object-glass, so that the maximum of illumination may be readily oltained. In the higher powers the end of the object-glass is turned swall enough to pass through the aperture in the centre of the Lieberkihn; but in the lower powers, where a great amount of reflecting surface would be lost on account of the large size of the glasses employed if this plan were adopted, the aperture in the centre of the Lieberkuihn is made to admit as many rays as will fill the field of view, and no more.

As the flood of light tlirown back by the Lieberkiuhn is sometimes so great as to obliterate much of the beauty and delicacy of structure of certain objects, it was proposed by Mr. Bridgeman, of Norwich, to cover up a portion of its refiecting surface by gumming a piece of dull-black paper uver oue half of the reflector; then, by rotating the Lieberkühn upon the object-glass, any proportion of oblique 
light may be thrown upon the object, and in any particular direction. In this way points of structure have been brought out which were lost under the full illumination of the Lieberkiihn. Mr. Beck sought to effect an improvement upon the proposal of MIr. Bridgeman, and, by employing the segment of a Lieberkiihn, produced what he terms a "Parabolic reflector." This is made to slide in the ordinary way over the object-glass, and admits of being turned in any direction towards the source of light. The parabolic reflector can also be employed with high powers. Mr. Sorby discovered, while experimenting with a reflector of the kind, the value of studying the peculiarities of objects under every kind of illumination ; for, on viewing specimens of iron and steel with this reflector, he found that, owing to the obliquity of illumination, the more brilliantly polished parts reflected the light beyond the aperture of the objective, and he could not therefore distinguish them from those parts which merely absorbed the light. To throw the illumination more perpendicularly upon the specimen, he was obliged to place a small flat mirror immediately in front of the objective, and cover half its aperture, and at the same time stop-off, by means of a semi-cylindrical tube, the light from the parabolic reflector. By such an arrangement, Mr. Sorby found it produce the reverse appearances of the former mode of illumination, and it proved to be a valuable aid in ascertaining the true condition of the object. Mr. Baker has produced a cheaper and simpler form of this parabolic reflector, which answers its purpose remarkably well, giving increased facilities for the illumination of opaque objects with the microscope set in that direstion most eonvenient for working. The brilliancy of the illumination is very much increased by placing a condensing-lens before the light, or by employing the Bockett lamp. It is, however, only just to Mr. Ross that we should add, that this "parabolie reflector" is but a slight modification of his Side-reflector, contrived several years ago, for the purpose of superseding tibe Lieberkühn.

The Bull's-eye Condenser.-All opaque objects require to be illuminated by rays which, being thrown upon their surface, shall be reflected back into the microscope. The same 
mode of viewing objects is often applied to semi-opaque, or even some transparent ones, to demonstrate peculiaritiss of structure. A condensing lens is used for converging rays from a lamp upon the mirror; or for reducing the diverging rays of the lamp to parallelism, for use either with the parabolic illuminator, or Ross's side-reflector. A plano-convex lens (fig. 106), of about three inches focal length, is the form generally adopted; it is borne upon a swivel-joint, which allows of its being turned in any direction, and placed at any angle; the tube is double, and thus admits of being lengthened or shortened. When used by daylight, its plane side should be turned towards the object, and the same position should be given when used fur converging the rays from a lamp; but when used with the parabolic or sidereflector, the plane side must be turued towards the

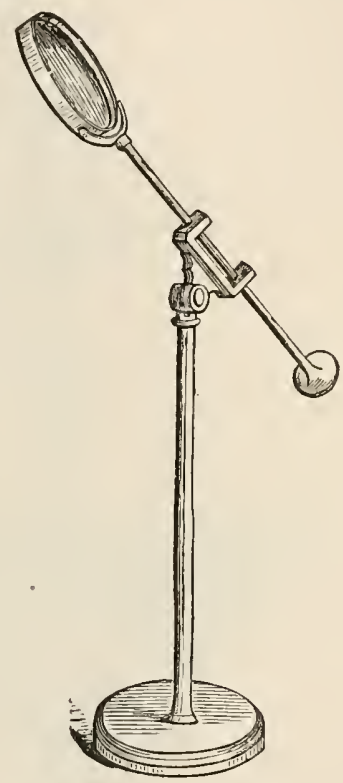

Fig. 166.-The Condensing Lens. lamp. Sometimes a smaller, double convex lens is made use of in addition; this is then often fixed into the stage of the microscope.

In fig. 107 the bull's-eye lens, $c$, slides up and down a brass rod, screwed into a solid foot; and made to concentrate the light upon the object from the table gaslamp, d. Mr. Brooke's method of viewing opaque objects under the lighest powers of the microscope (the $\frac{1}{8}$ and $\frac{1}{2}$ inch object-glass) is effected by two reflections. The rays from a lamp rendered parallel by a condensing lens are received on an elliptic reflector, the end of which is cut off a littie beyond the focus; the rays of light converging 
from this surface are reflected down on the object by a plane mirror attached to the object-glass, and on a level

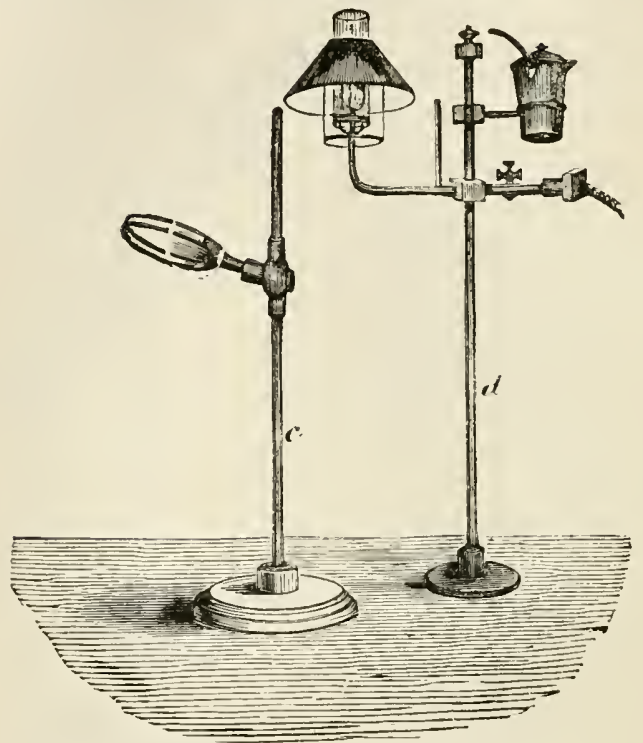

Fig. 107.-Condenser and Table Gas-lamp.

with the outer surface. By such means the structure of the scale of the Podura, and the different characters of its inner and outer surfaces, are rendered distinctly visible.

Lamps. - It is of the utmost importance, both on account of the injury often done to the eyes, as well as for the perfection of illumination when artificial light is employed, that its quality should be of the whitest and purest kind. The most useful and economical lamp is Collins' Bockett lamp; it consists of a good paraffin lamp, reflector, and a $2 \frac{1}{4}$ condenser, mounted together on a brass-rod stand, which admits of being raised from 9 to 16 inches. The pale blue chimney supplied with it gives whiteness to the flame, and for all the requirements of the microscopist it is de- 


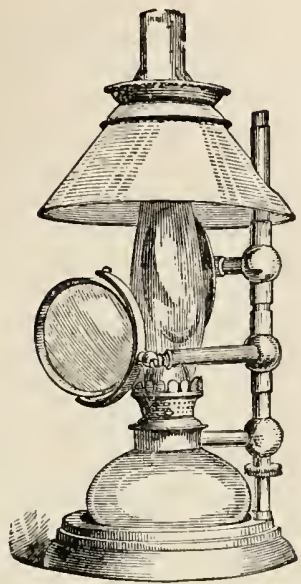

Fig. 108. - Collins' Bockett Lame.

ciledly the most convenient lamp we have seen. Those

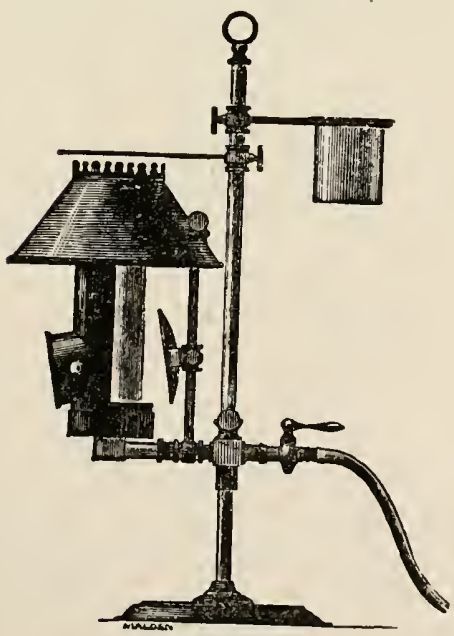

F'ig. 109.-Highley's Gas-lamp, with Steum-bath attoninad. 
among us who have gas at command will, no dout, give it the preference on account of its convenience, and freedom from all kind of trouble. An excellent form of gas-lamp is Highley's (fig. 109).

The mono-chromatic gas-lamp, fig. 110, is very useful and most economical. Gas, as a source of light, presents great advantages over oil and spirit, on account of cleanliness, being ever ready for use, and affording a perfect control over the flame; but when the ordinary gas-lamps are used

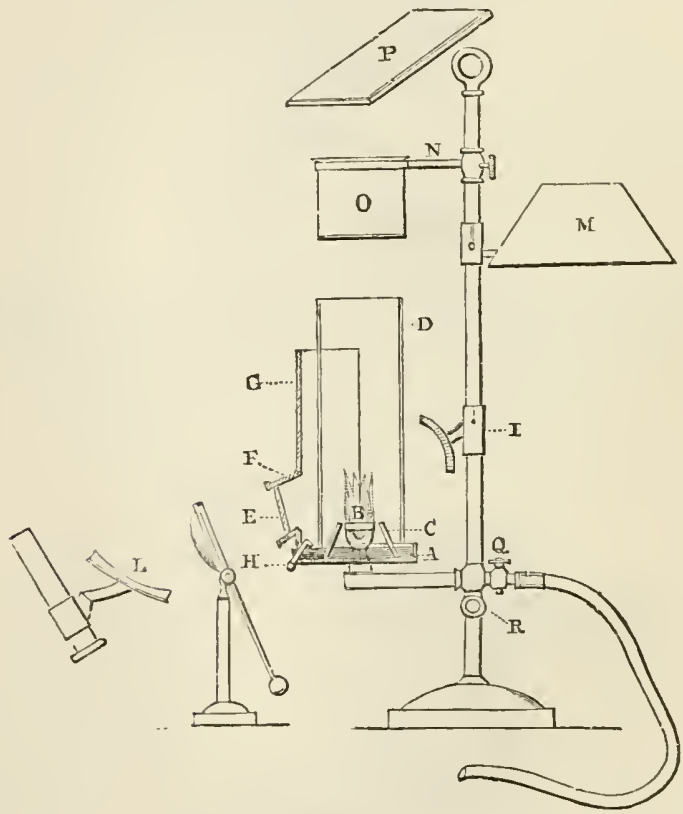

Fig. 110.-Gas-lamp arranged for use.

for the purpose of illuminating the field of the micro scope, a yellow glaring light is given, alike injurious to the eye and the definition of the object under examina. tion. To correct these evils, this lamp was arranged, which 
is also otherwise useful to the microscopist. It consists of a stage $A$, supported by a tube and socket, sliding on an upright rod rising from the stand. This earies an argand burner $\mathrm{B}$; a metal cone $\mathrm{C}$ rises to the level of the burner, and is about one-eighth of an inch from its outer margin.

This arrangement gives a bright cylindrical flame. The brttom of the stage $\mathrm{A}$ is covered with wire-gauze, to cut off irregular currents of air, and thus seemes a steady fame. Over the burner is placed a Leblond's blue glass chimney D. This corrects the colour of the flame to a certain extent; but it is still further rectified by a disc of bluish-black neutral-tint glass $\mathrm{E}$, fitted in a tube $\mathrm{F}$, attached obliquely to the shield G. G is a half-cylinder of metal, which serves to shield the eyes from all extraneous light, but may be rotated on the stage $\mathrm{A}$ by aid of the ivory knot $\mathrm{H}$, when the full light from the flame is desired. $\mathrm{A}$ metallic reflector 1, fixed on its supports, so as to be parallel to $\mathrm{E}$, concentrates the light. By the combination of the two glasses D and E, the yellow rays of the flame are absorbed, and the arrangement affords a soft white light, which may be still further improved by receiving the rays on a concave mirror, backed with plaster-of-Paris $\mathrm{L}$; and where a very strong light is required, a condensing lens should be interposed, as shown in the cut, between the lamp and the mirror of the mieroseope. By removing the shield G, and bringing the shade mover the burner, it may be used as a reading-lamp. A retort ring $N$ supports a water-bath 0 , or a wrought-iron plate $P, 6$ inches by $2 \frac{1}{-2}$ inches, both used in mounting objects. The stop-cock $\bar{Q}$ gives the means of regulating the flame. The screw 1 elamps the lamp-head at any height desired. The lamp may be attached to any gas-supply by vulcauised Indiarubber tubing.

Finders and Indicators.--A finder, as applied to the microseope, is the means of registering the position of any particular object in a slide: as, for instanee, some particularly good speeimen of a diatom, so that it may be referred to at a future time. The subject has been fully diseussed in the pages of the Quarterly Journal of Microscopical Science. The traversing stage admits of such finders as 
those of Mr. Okeden, Mr. Tyrrell, Mr. Amyot, \&c., being used. The tirst named (Mr. Okeden's) finder consists of two graduated scales, one of them vertical, attached to the fixed stage-plate, and the other horizontal, attached to an arm carried by the intermediate plate; the first of these scales enables the observer to "set" the vertically-sliding plate to any determinate position in

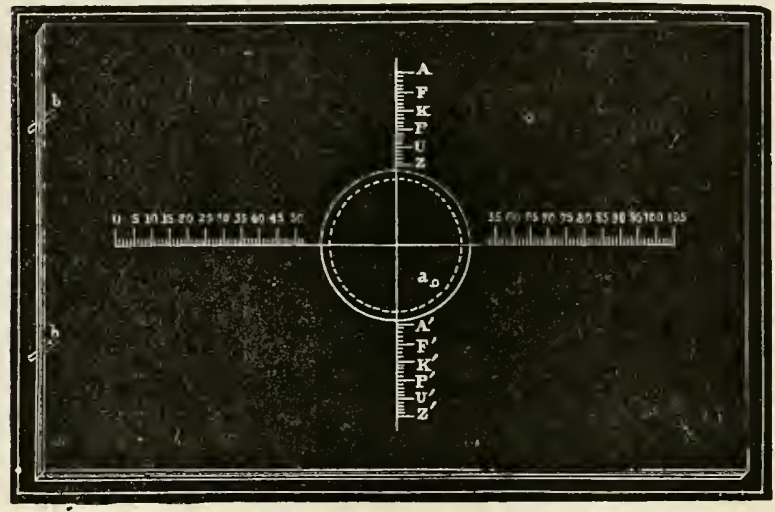

Fig. 1:1.-A myot's Oöject Finder.

relation to the fixed plate, while the second gives him the like power of setting the horizontally-sliding plate by the intermediate. The finder the author has been in the habit of using for some years is a very ingenious and effective instrument described by Mr. Amyot. It consists of a box-wood plate, with a eircular hole $\frac{8}{20}$ ths of an inch in diameter cut out of its centre, into which a disc of ivory, pierced in the exact centre with a very fine needle, fits; this disc can be readily removed by seizing a little brass peg, attached to it, with the forceps. The box-wood is ruled with two axes, vertical and horizontal, which are graduated to 50 ths or 100ths of an inch. The diagram (fig. 111) represents the finder with bone centre-piece in situ. 'The dotted ring shows the rabbet on which the centre-piece rests ; $a$, brass pin attached to centre-piece; $h, b$, two brass pins for steadying the instrument against the 
left side of the microscope stage. The scales niay be rulerl in brass or bronze and inlaid, and horizontal lines might be ruled on the surface of the stage as a guide for placing the slide. This form of instrument possesses the advartage of not requiring the common object slides to be ruled, or in any way prepared.

It is thus used :-1st. Put the finder on the stage and find the central needle hole with the microscope. 2d. Remove the centre-piece with a small pair of forceps. $3 d$. Place the slide on the wood, taking care that the stage screws be not used to move the object after the finder has once been centred.

The position of any object then occupying the centre of the field will be shown by the sides and ends of the slide on the scales of the finder, and can be registered at sight. To find the object again:-Find the centre hole, remove the disk as before, then place the slide according to the letter and number marked upon it, when the object sought will occupy the centre of the field.

For those microscopists whose instruments are without a traversing stage "Naltwood's finder" will be found an efficient substitute for the one just described. This consists of a glass slide, $3 \times 1 \frac{1}{4}$ inches, on which is plotographed a scale occupying a square inch ; this is divided by horizontal and vertical lines into 2,500 squares, each of which contains two numbers marking its "latitude," or place in the vertical series, and its "longitucle," or place in the horizontal series. The scale is in each instance an exact distance from the bottom and left-hand end of the glass slide; and the slide when in use should rest upon the ledge of the stage of the microscope, and be made to abut against a stop, a simple pin, about an inch and a half from the centre of the stage. To use this finder, the objectslide must be placed under the microscope with the same care as the finder; and when some especial object, whose place it is desired to record, has been brought into the field of view, the olject-slide being removed, the finder laid down in its place, the numbers of the square then in the field of view are to be read off and recorded. This should also be recorded upon the object-slide; and at any future time, when it is wished to refer to the same object, 
the process will have to be reversed, by first finding this particular square on the finder, and then replacing it by the object-slide.

A simple form of finder, suggested by $\mathrm{Mr}$. W. K. Bridgeman, of Norwich, and sold by Baker, Holborn, is, from its ingenuity and comparative accuracy, worthy of notice.

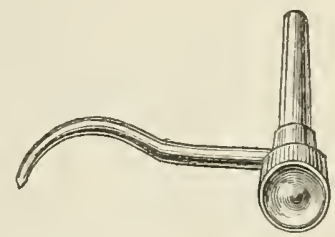

Fig. 112.-Bridgeman's Finder.

This finder (fig. 112) consists of a curved pin of steel, which is attached to the stand by means of a circular fitting of brass. It is so contrived that when the mechanical stage is brought into the rectangular position, and an object laid thereon, it can be pressed down upon the slide. If the latter be covered with paper, the point of the pin leaves a slight puncture: all that is necessary, when we wish to examine the object in future, is to move the stage till the puncture on the slide comes under the point of the finder.

Forceps.-For holding minute oujects, such as parts of plants or insects, to be examined either as transparent or opaque objects, the most useful is represented by fig.113,

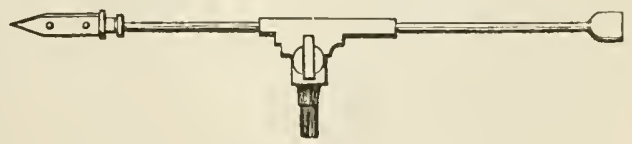

Fig. 113.

and consists of a piece of steel wire, about three inches long, which slides through a small tube, comnected to a stout pin by means of a cradle-joint; to one end of the wire is attached a pair of blades, fitting closely together by their own elasticity, but which, fur the reception of any 
object, may be separated by pressing the two projecting studs; to the opposite end of the wire is adapterl a small brass cup, filled with cork, into which pins, passed through dises of cork, card-board, or other material, having objects mounted on them, may be stuck.

Dipping-tubes are tubes of glass, fig. 114, about nine inches in length, open at both ends, and from one-eighth to one-

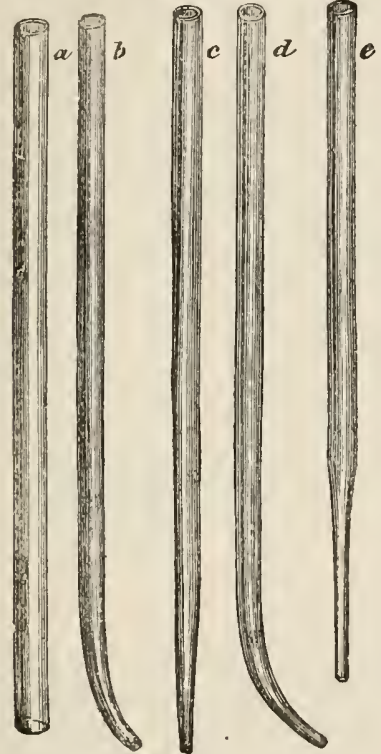

Fig. 114.-Dipping Tubes.

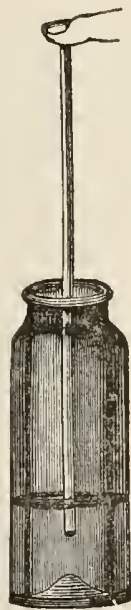

Fig. 115.

fourth of an inch in diameter. The ends must be nicely rounded off in the flame of a blow-pipe; some of them may be made perfectly straight, while others should be drawn out to a fine point, and made of either of the shapes $a, b, c, d, e$. The method of using them is as follows:-Supposing the animalcules are contained in a phial or glass jar (fig. 115), and having observed where they are most numerous,either vith the naked eye, or with a pocket-magnifier,- 
either of the glass-tubes, having one end previously closed by the thumb or fore-finger, wetted for the purpose, is introducel into the phial in the manner represented by the figure: this prevents the water from entering the tube; and when the end is near to the object which it is wished to obtain, the finger is to be quickly removed and as quickly replaced. The moment the finger is taken off, the atmospheric pressure will force the water, and with it, in all probability, the desired objects, up the tube. When the finger has been replaced, the tube containing the fluid should be withdrawn from the phial; and as the tube is almost certain to contain much more fluid than is requisite, the entire quantity must be dropped into a watch-glass, and the particular insect can be caught by putting the tube over it, when a small quantity of fluid is sure to run up by capillary attraction. This small quantity is to be placed upon a glass-slide for examination. It is necessary to add a small quantity of vegetable matter to animalcules, if we wish to keep them alive for many days; and as many species are found on confervæ and duck-weed, some instrument is required to take small portions of such plants

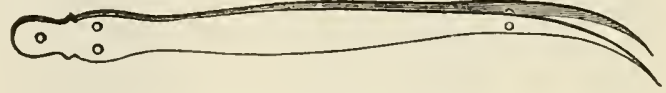

Fig. 116.

out of the jar in which they are growing. For this purpose it will be necessary to make use of the forceps, fig. 116, made of brass; the points are a little curved, to keep them accurately together, and the blades are provided with a hole and a steadying-pin. 'This instrument is also useful for picking up any miuute object.

Fig. 117 , at $\mathrm{B}$, is represented a convenient, cheap, and portable "Collec`ing-bottle and Stick;" simply an ordinary walking-cane divided by a screw, or socket-joint, into two parts for the convenience of packing, and terminated by a brass ring, which is either a fixture or made to screw on and off. This ring is adapted to receive the male-screw of the well-known wide-mouthed bottle, used by perfumers 
and chemists for pomade; this is exactly adapted to fit into the screw of the brass ring. The facilities

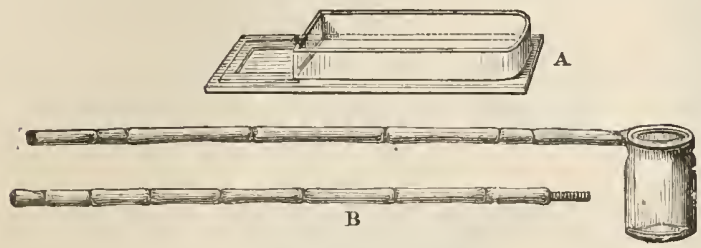

Fig. 117.

A. Trough for showing circulation in fish-tail.

B. Collecting bottle and stick.

afforded by such an arrangement are obvious enough; broken bottles can be readily replaced at a small cost, and each bottle when filled corked up and replaced by another. A small fine-gauze net and a hook is made to scrow into the same stock; the whole packs into a very small compass.

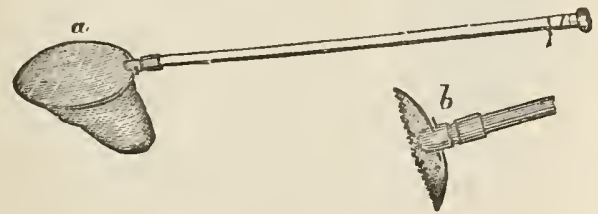

Fig. 11S.-Collecting Net.

Collecting Animalcules.-For collecting fresh water or marine animals, the small net, made similar to a landingnet, fig. 118, will be found useful : this should be securely fastened to the brass ring $a$, and fitted firmly into the socket $b$; when not in use, the socket may be carried in the pocket; and the net, by contracting the diameter of the ring (which the construction admits of) may be carried inside the hat.

For collecting desmids, diatoms, and other small creatures, Mr. Williamson has a cheap and simple contrivance for converting the end of a walking-stick or umbrella into what he terms a "collecting-stick." In fig. 119, a repre- 
sents a piece of whalebone, about 18 inches long, bent sound the end of the stick or umbrella, $b$, and made fast

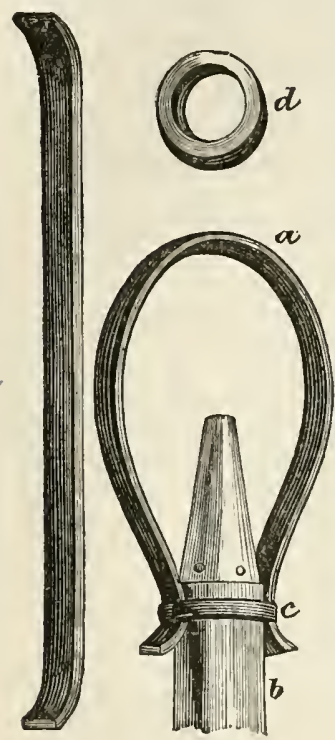

Fig. 119. in that position by one or two rings, $c$, of gutta-percha, india-rubber, or of brass, $d$. A small wide-mouthed bottle, having a rim which will prevent its falling through, is now inserted in the loop thus formed, and is held tightly there by the ends of the whalebone being drawn further through the ring, and thus diminishing the size of the loop. The bottle thus fixed may be used for diping out the animalcules. Whalebone can be moulded to any form by placing it for a short time near the fire.

Fig. 120 is a box containing six bottles for holding the specimens when caught. These bottles should be filled up to the cork with water when and where collected, and care must be taken not to mix the specimens from one brook with those from another, otherwise serious damage may take place, and on reaching home we may find the greater part of our stock either dead or dying. Always separate the various species as speedily as possibie. This can be done easily, by emptying each bottle in its turn into a soup-plate; then with the feather of a pen first lift out the smaller ones, and with the quill-end cut like a scoop lift out the larger, classifying and allotting each to its separate bottle.

Live-Box.-Mr. Varley im-

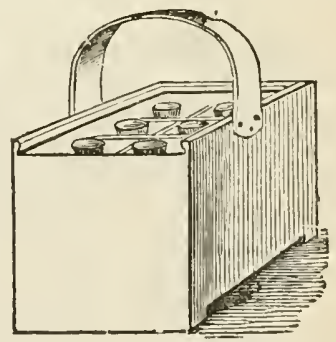

Fig. 120. proved the form of this instrument by making a chamnel 
all round the object-plate, so that the fluid and animalcules in it were retained at the top of the object-plate by capillary attraction. The cover is made in the usual way to slide up and down over the object-plate. The plate of brass to which the tube supporting the tablet and cover is attached, is of a circular form, slightly flattened on its two opposite sides for convenience of package. The in-

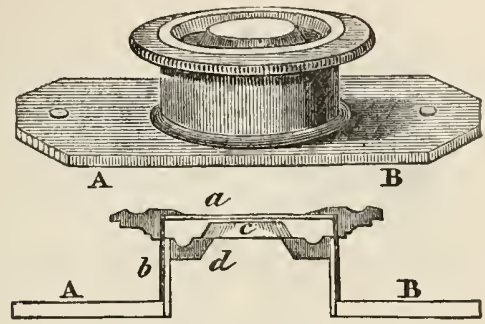

Fig. 121.-Varley's Animalcule-cage.

strument is seen in elevation and in section in fig. 121, $\mathbf{A}, \mathbf{B}$, in both figures, is a flat plate of brass to which the short tube carrying the object-plate or tablet is fixed; $d$, the piece of brass into which the tablet $c$ is fastened; $b$, the tubular part of the cover, into the rim of which the thin plate of glass $a$ is cemented.

Compressorium.-The purpose of this instrument is to apply a gradual pressure to objects whose structure can only be made out when they are pressed or thinned out by extension. The general plan of the compressorium is shown in fig 122

Ross's Compressorium consists of a stout plate of brass $A$, about three inches long, having in its centre a piece of glass like the bottom of a live-box. This piece of glass is set in a framie $B$, which slides in and out so that it can be removed for the convenience of preparing any object upon it, under water if desirable. The upper moveable part $D$ is attached to a screw-motion at $C$; and at one end of the brass-plate $A$, which forms the bed of the instrument, is an upright piece of brass $C$, grooved so as to 
receive a vertical plate, to which a downward motion is given by a single fine screw, surrounded by a spiral spring, which elevates the plate as soon as the screw-pressure is removed. The vertical plate carries an arm at right angles to its own plane, terminating in a square frame $D$, capable of receiving very thin, or somewhat thicker glass. The arm has likewise a horizontal motion, so that the upper
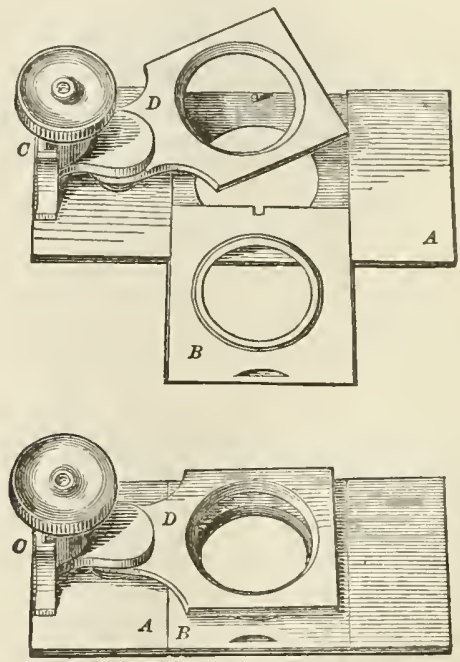

Fig. 122.-Ross's Compressorium.

plate $D$ can be turned completely off the lower one $B$. Should the thin upper glass be broken, it can be instantly replaced, as no cement is required; it is nerely needful to remove the fragments and slip a fresh glass in. It often happens that on account of the trouble attendant upon the use of all ordinary Compressoriums, the microscopist simply uses a slide and a piece of covering-glass; but if he wishes an exact means of regulating the pressure, some such compressorium as Ross's should always be employed. 
Smith and Reck's trough for chara and polypes, a sectional view of which is shown at fig. 123, is made of three pieces of glass, the bottom being a thick strip, and the front $a$ of thinner glass than the back $b$; the whole is cemented together with Jeffery's marine-glue. The method adopted for confining objects near to the front glass varies according $b$ to circumstances. One of the most convenient plans is to place in the trough a piece of glass that will stand across it diagonally, as at $c$; then if the object be heavier than water, it will sink, until stopped by this plate of glass. At other times, when used to view chara, the diagonal plate may be made to press it close to the front by means of thin strips of glass, a wedge of glass or cork, or even a folded spring. When using the trough, it is necessary that the microscope

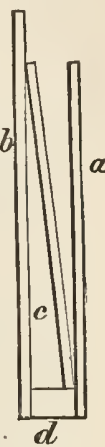

Fig. 128. should be in a position nearly horizontal.

Mr. Walker's trough for exhibiting the circulation of the blood in a fish's tail, \&c. (fig. $117 \mathrm{~A}$ ) consists of a piece of plate-glass about 6 inches long, and 2 inches wide; upon this, three other pieces of plate-glass, about $\frac{1}{2}$ inch wide, are cemented with marine-glue. Three pieces of strong covering glass, about a $\frac{1}{4}$ inch wide, are also cemented on to the plate, and a piece of moderately strong covering glass on to the top of these thin slips; a piece of plate-glass is then cemented to the top of the thin glass, abutting on the ends of the slips; the whole forming an open trough, terminating in a thin cell, which is closed at all parts, except where it communicates with the bottom of the trough.

The fish, wrapped in a little wet linen, is placed in the trough, and the tail is thrust flat into the thin cell; a small quantity of water is placed in the large trough, and the fish kept in its place by one or two elastic bands or strips of thin sheet-lead passed round the glass and over the body of the fish.

The advantages of this arrangement are:-1st. The fish cannot throw up its tail and splash the object-glass with water. $2 \mathrm{~d}$. In consequence of the tail being kept flat in the cell, the view is perfect, and there is little risk 
of the object getting out of focus. $3 d$. If the tail be not pushed too far into the cell, the vessels at its root are not compressed, and the circulation goes on very freely. 4th. The fish may be kept on the stage of the microscope for two or three hours without injury. Mr. Macaulay has suggested, as an improvement, that the three sides of the cell should be made of a single strip of plate-glass, bent to the shape represented in our wood-cut.

Growing-cells.-Considerable attention has been given to various forms of growing-cells for maintaining a continuous supply of fresh water to objects under constant observation, fur the purpose of sustaining vital growth for a long period. The employment of such cells is strongly commended to microscopists, as there is yet much to be discovered concerning the metamorphoses which some of the lower microscopic forms of plant and animal life pass through; a patient investigation will probably show that many which are now classed as distinct species are merely different phases of the same type, which alternate in a higher or lower scale of development according to the varied conditions of temperature and nutrition under which they are grown.

Professor Smith, of Kenyon College, furnished us with what he has called a growing slide, or trough. It consists of two pieces of thinnish glass cemented together; in one comer of the upper cover a small hole is bored, and through this a fresh supply of water is introduced without in any way disturbing the desmid, or living object under inspection. Mr. Beck has contrived an improved form of cell ; but, whilst the first-mentioned may be constructed for a few pence, the latter cannot be had for a less price than ten shillings.

Dissecting Knives, \&c.-Knives and needles of various kinds and sizes are required for microscopic dissection; the best for the purpose are represented in figs. 124, 125, and 126, being, in fact, the very delicately made knives used by surgeons in operations upon the eye. Dissecting needles may be either straight or curved. They may be fixed, or made to take in and out of their handles. The most convenient are shown in fig. 125; those made of Palladium by Mr. Weedon, Hart Street, are very much the best. 

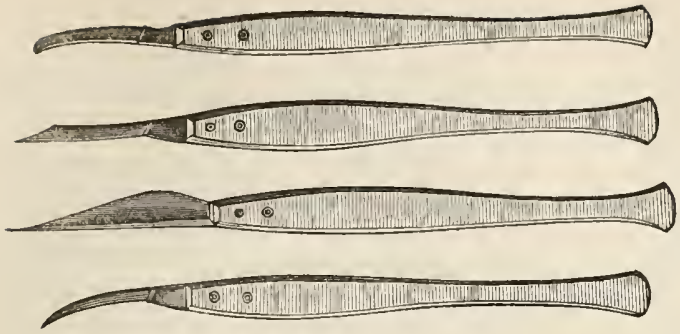

Fig. 124.

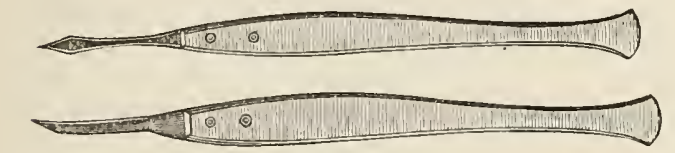

Fir. 125

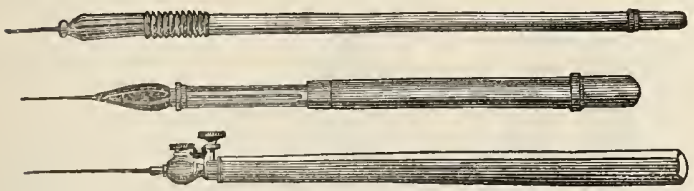

Fig.126.

The mode of using a pair of needles in breaking up tissues into very small pieces is represented in fig. 127.

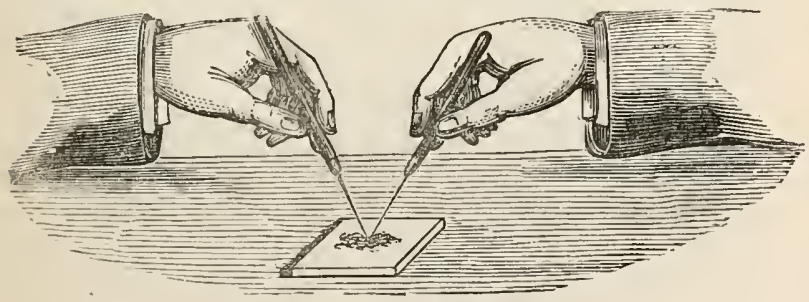

Fig. 127.-Teastng-out Membrane.

With a pair of the small needles held firmly between the fore-finger and thumb, the structure must be teased out; 
an operation which requires care and perseverance, as most of the animal tissues are very difficult of separation. All substances should be carefully separated from dust and other impurities which renders their structure indistinct or confusing. With very delicate membranes, and with those of the nervous system of the smaller animals, insects, \&c., it becomes necessary that the investigation should be carried on under water, or in fluid of some sort, in a glass cell, and having a strong light thrown down upon it by the aid of the condensing lens, as represented in fig. 12S. A certain amount of change of structure must be expected and allowed for; as nearly all membranes imbibe some portion of the fluid. Delicate structures are often advantageously wetted with dilute solutions of sugar or common salt, to prevent the changes from endosmosis, which result from the use of pure water. The contents of bodies are frequently rendered more distinct by the addi-

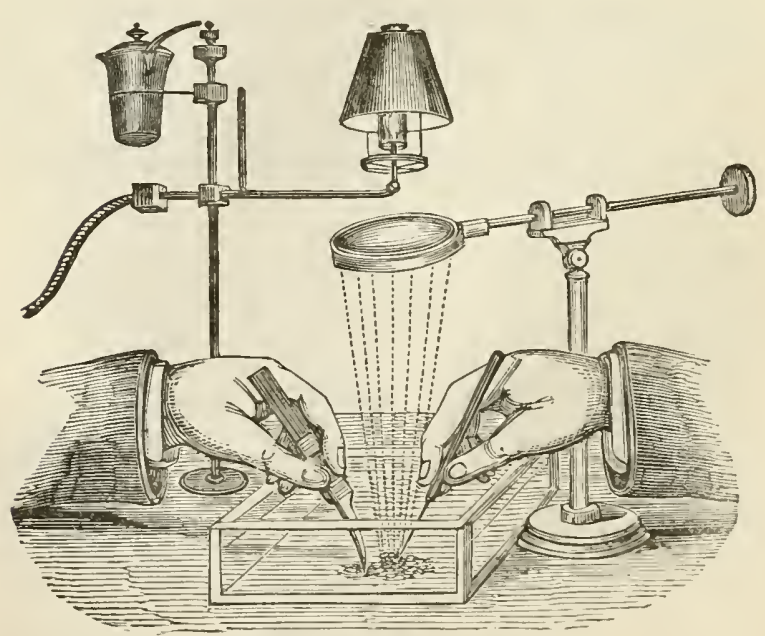

Fig. 128.-Dissecting under water.

tion of re-agents. If the object be a portion of an injected animal, it is better to pin it out on a leaded-cork, 
covered with white wax, and then immerse it in the watertrough; the more delicate the structure, the sooner after death should it be examined, especially animal tissues. With some vegetable structures, the dissection should be carried on under water. The separation of the woorly and vascular tissues, and the spiral vessels, is best effected by maceration and tearing with fine needles.

Valentin's Knife.-For making fine sections of large substances, or those soft in structure, such as the liver, spleen, kidney, \&c., the dlouble-bladed knife, the invention of Professor Valentin, may be used with advantage. An improved construction of this knife, by the late $\mathrm{Mr}$. John Queketi, is represented in fig. 129.1 It consists of two blades, one of which is prolonged by a flat piece of steel to form a handle, and having two pieces of wood riveted to it, for the purpose of its being held more steadily; to this blade another one is attached by a screw; this last is also lengthened by a shorter piece of steel, and both it and the preceding have slots cut out in them exactly opposite to each other, up and down which slot a rivet with two heads is made to slide, for the purpose either of allowing the blades to be widely separated or brought so closely together as to touch. One head of this rivet, being smaller than the hole in the end of the slot, can be drawn
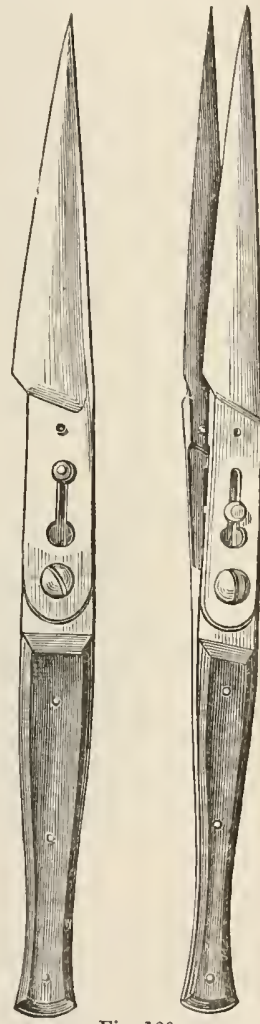

Fig. 129. through it; so that the blade seen in the front of the figure may be turned away from the other in order to be sharpened,

(l) Another form of this instrument is constructed by Mr. Matthews, the blades being made with a convex instead of a straight edge, their distances fru.n each other being regulated by a nilled-head screw, and their separation for cleaning being more readily accomplished. 
or allow of the section made by it being taken away from between the blades. The blades are so constructed that their opposed surfaces are either flat or very slightly concave, that they may fit accurately to each other, which is effected more completely by a steadying pin, seen at the base of the front blade. When the instrument is required to be used, the thickness of the section about to be made will depend upon the distance the blades are apart; and this is regnlated by sliding up and down the rivet, as the blades, by their own elasticity, will always spring open and keep the rivet in place; a cut is then to be made by it, as with an ordinary knife, and the part cut will be found between the blades, from which it may be separated either by opening them as wide as possible by the rivet, or by turning them apart in the manner before described, and floating the section out in water.

Dissecting Scissors. - In addition to the forceps and knives, scissors will be necessary for the purposes of dissection: of these the most useful are shown in fig. 130. They

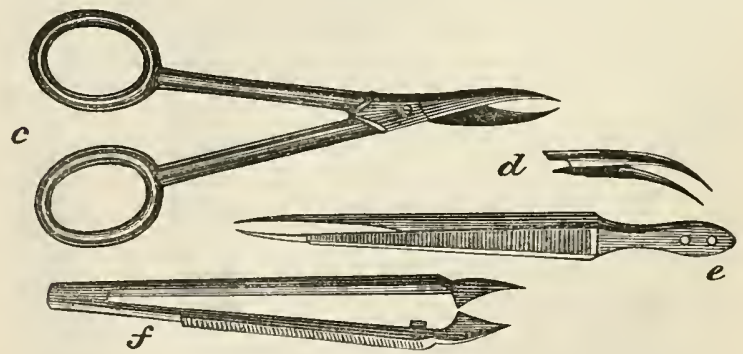

Fig. 130.-Dissecting Scissors.

are made both straight and curved; of the first kind, two pairs will be required, one having the extremities broad, and the other sharp-pointed; if large dissections be undertaken, a still stronger pair, with the extremities broad, and made rough like a file, will be necessary. In dissecting under the microscope, the curved-pointed pair shown at $f$ are the most convenient. In all of these instruments the points should fit accurately together: sometimes those that are very sharp are apt to cross; this 
may in a great measure be prevented by having the branches wide at the base where they are riveted. The points can be sharpened on a hone, and a magnifier employed to examine if they fit closely together.

Section-cutting Instruments.-There are a numerous class of substances much too hard to admit of being cut either by scissors or a Valentin's knife, more especially if we require very thin and perfect sections. As most important information is to be gained respecting the structure of various substances, such as stems and roots of plants, horns, hoofs, cartilages, and other firm parts of animals, by cutting very thin sections for mounting as transparent objects, it would be quite impossible for the microscopist to get on without a section-cutting instrument. Among the many mechanical contrivances which have been devised for the purpose, the ingenious little machine invented by Mr. James Smith ${ }^{1}$ will be found to do its work with neatness and precision.

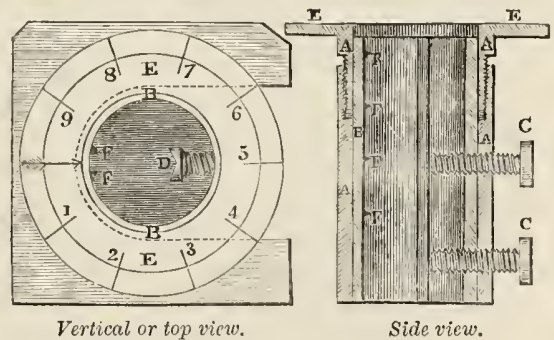

Fig. 131.-Smith's Section Instrument.

Smith's Section Instrument (fig. 131) consists of an cuter tube $\mathrm{A}, \mathrm{A}$, the upper part of which screws into the lower, and has at the top a flat circular plate E, E, which forms the cutting-table. Firmly fixed to the lower part of the tube A, and extending throughout its whole length, is the inner tube $\mathrm{B}, \mathrm{B}$, which forms, with the moveable bar $\mathrm{D}$, a holding for the specimen to be cut, while, at the same time, it supports the upper part of the tube A, A, and gives it greater firmness in screwing up and down. The

(1) "On a Section Instrument, by James Smith." (Micros. Soc. Trane. vol. viii. page 1,1860 .) 
bar $\mathrm{D}$ moving backwards and forwards in the tube $\mathrm{hy}$ weans of the screws $c, c$, serves, in conjunction with tha points F, F, to fix the specimen to be cut, which is effected as follows:-

The cutting surface $\mathrm{E}, \mathrm{E}$, being slightly screwed up, as shown in the drawing, and the bar $\mathrm{D}$ being drawn back a sufficient distance, the specimen to be cut is placed in the instrument, and firmly fixed by turning the screws $\mathrm{c}, \mathrm{c}$; it is then cut level with the surface by a proper knife or chisel, and the table being screwed down one or more divisions (as shown in the left-hand diagram), a section is cut, and if found of sufficient thinness, a number may be cut by continuing to turn the table down a similar number of divisions, until it will screw no further, when the table must be again screwed up, and the specimen loosened and raised if more sections be required. The principal points of the instrument are :-

1st. Its portability - the tube being about two and a half inches long by one inch in diameter. 2d. The specimen to be cut is fixed once for all, and the cuttingsurface screwed down to it, a feature that will render it peculiarly applicable to the cutting of soft substances. $3 d$. The ease with which a number of sections may be cut without disturbing the specimen when once properly fixed, while the size of the tube, and the facility with which it can be adapted to objects of various diameters, enable the operator to get sections of stems of plants, \&c. whole. In cutting sections of hard woods, which require considerable purchase, the instrument may be placed in a semicircular opening in tise edge of the working-table, so that the flat plate or cutting-surface may rest upon it, and the strain thrown on the table. When used for cutting soft substances, it can be held in the hand.

Fig. 130 represents Mr. Gibhon's "Section-cutting Machine." It consists of a stout brass frame, $A$, having an opening in the top plate, for a tube $B$, half an inch in diameter, and in depth one and a half inches. In this tube a loose piston, $c$, works freely, and is steadied by the slot seen in it. T'o a female screw $D$, motion is given by the toothed wheel; and the teeth of which, $E$, answer the triple purposes of thumb-milling, ratchet-stop, and 
graduation. This is screwed to a block of wood, $F$, having a rabbet cut in for the purpose of securing it to the table.

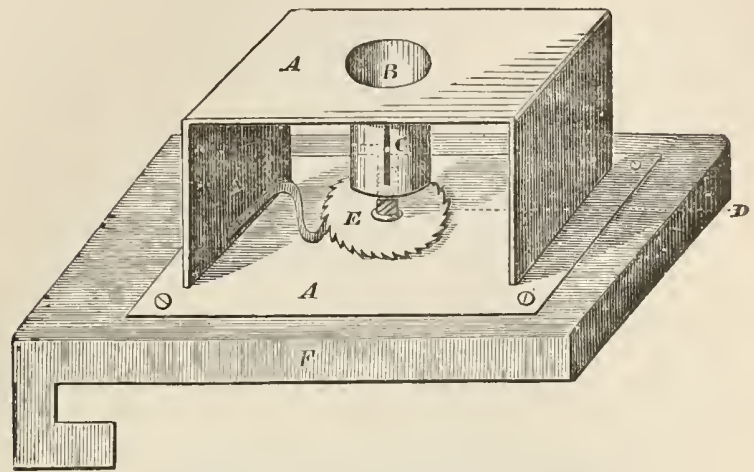

Fig. 132. - Gibbon's Section Cutting Machire.

The machine is self-regulating, and is capable of being worked as rapidly as the skill of the operator may dictate.

Sections of woods, when cut from hard woods containing gum, resin, \&c., should be soaked in essential oil, alcohol, or ether, before they are mounted as transparent objects. A razor may be fixed to the bench for the purpose of cutting these fine sections, or a fine plane will answer very well. The instrument used by Mr. 'Topping, fig. 133, consists of $a b$, a flat piece of mahogany, seven inches long and four wide, to the under surface of which is attached, at right angles, a piece $g$ of same size as $a b$. $d$ is a flat plate of brass, four inches long and three wide, screwed to the upper surface of $a b$; to the middle of this plate is attached a tube of the same metal $e i$, three inches long and half an inch in diameter, and provided at its lower end with a screw $f$, working in a nut, and having a disk $k$ exactly adapted to the bore of the tube ; this disk is connected with the upper end of the screw, and is moved up or down by it. $c$ is another screw connected with a curved piece of brass $h$, which is capable of being carried to the opposite side of the tube by it. The piece of wood abcut 
to be cut is put into the tube $e$, and is raised or depressud by the screw $f$; whilst, before cutting, the curved piece of

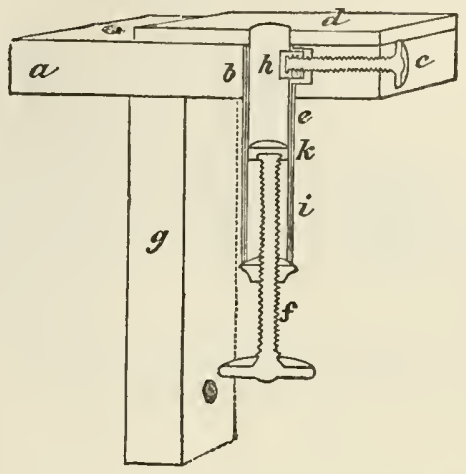

Fig. 133.-Topping's Section Cutting Machine.

metal $h$ should be firmly pressed against it by the screw $c$. This instrument, if fastened to the edge of a bench or table, is always ready for use. The knife employed may be one constructed for the purpose; or a razor ground flat on one side.

Method of making Sections. - If the wood be green, it should be cut to the required length, and be immersed for a few days in strong alcohol, to get rid of all resinous matters. When this is accomplished, it may be soaked in water for a week or ten days; it will then be ready for cutting. If the wood be dry, it should be first soaked in water and afterwards immersed in spirit, and before cutting placed in water again, as in the case of the green wood. The wood, if too large, should be cut so as to fit tightly into the square hole, and be driven into it by a wooden mallet; if, on the contrary, it be round, and at the same time too small for the hole, wedges of deal or other soft wood may be employed to fix it firmly: these will have the advantage of affording support, and if necessary, may be cut with the specimen, from which they may afterwards be easily separated. The process of cutting consists in raising the wood by the micrometer screw, so that the 
thinn sst possible slice may be taken off by the knife; after a few thick slices have been removed to make the surface level, a small quantity of water or spirit may be placed upon it; the screw is then to be turned one or more divisions, and the knife passed over the wood until a slice is removed; this, if well wetted, will not curl up, but will adhere to the knife, from which it may be removed by pressing blotting-paper upon it, or by sliding it off upon a piece of glass by means of a wetted finger. The plan generally adopted is to have a vessel of water by the side of the machine, and to place every section in it: those that are thin can then be easily separated from the thick by their floating more readily in the water; and all that are good, and not immediately wanted, may be put away in bottles with spirit and water, and preserved for future examination. If the entire structure of any exogenous wood is required to be examined, the sections must be made in at least three different ways; these may be termed the transverse, the longitudinal, and the oblique, or, as they are sometimes called, the horizontal, vertical, and tangental: each of these will exhibit different

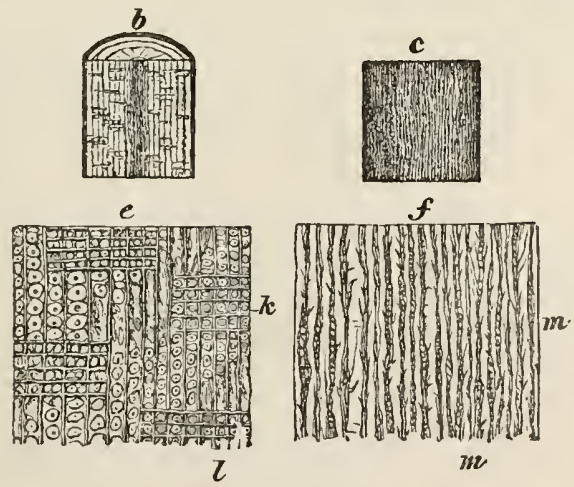

Fig. 234.-Sections of Wood.

appearancas, as may be seen upon reference to fig. 134: $b$ is a vertical section through the pith of a coniferous plant: this exhibits the medullary rays, which are known 
to the cabinet-maker as the silver grain; and at $e$ is a magnified riew of a part of the same: the woody fibres are seen with their dots $l$, and the horizontal lines $k$ indicating the medullary rays cut lengthwise; whilst at $c$ is a tangental section, and $f$ a portion of the same magnified: the openings of the medullary rays $m m$, and the woody fibres with rertical slices of the dots, are seen. Very instructive preparations may be made by cutting oblique sections of the stem, especially when large vessels are present, as then the internal structure of the walls of some of them may oftentimes be examined. The diagram above given refers only to sections of a pine; all exogenous stems, howerer, will exhibit three different appearances, according to the direction in which the cut is made; but in order to arrive at a true understanding of the arrangement of the woody and vascular bundles in endogens, horizontal and vertical sections only will be required. Many specimens of wood that are very hard and brittle may be mach softened by boiling in water; and as the cutting-machine will answer for other structures besides wood, it should be stated, that all horny tissues will also be softened by boiling, and can then be cut very readily.

Preparation of Hard Tissues.-All sections of recent and greasy bones should be soaked in ether for some time, and afterwards dried in the air, before they are fit for the saw, file, and hone; by dissolving out the grease, the lacunr and canaliculi show up very much better. When it is wished to examine the bone-cells of fossil bone, chippings only are required; these may be procured by striking the bone with the sharp edge of a small mineralogical-hammer: carefully select the thinnest of the chips, and mount them at once, without grinding, in Canada balsam. If desirable to compare bone structures, it must be borue in mind that the specimens for comfarison should be cut in one and the same direction; as the bone-cells, on which we rely for our determination, are always longest in the direction of the shaft of the bone, it follows that if one section were transverse, and the other longitudinal, there must be a vast difference in the measurement of the bone-cells, in consequence of their long diameter being seen in the one case, 
and their short diameter in the other. In all doubtful cases, the better plan is to examine a number of fragments, both transverse and lougitudinal, taken from the same bone, and to form an opinion from the shape of bone-cell which most commonly prevails.

The Teeth. - The best mode of examining teeth is by making fine sections. Specimens should be taken, both from young and old teeth, to note the changes. A longitudinal or transverse slice should be first taken off; a circular saw, fitted to the lathe, fig. 135 , cuts sections very quickly - then rub down, first by the aid of the corundum-wheel, - which should also be fitted to the head-stock of the lathe, - then finish them off between two pieces of water-of-Ayr stone, and finally clean and polish between plates of glass, or on a polishing strap with putty powder. The section requires to be washed in ether, to remove all dirt and impurities; when well polished and dried, it may be preserved mnder thin glass, and cemented down

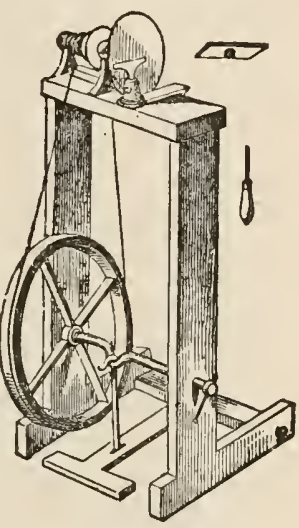

Fig. 135-Small Lathe for polishing. with gold-size or varnish.

Such polished sections are preferable to many others which, on account of their irregular surface, require to be covered with fluids, as Canada-balsam, turpentine, \&c., in order to fit them for examination with high powers. It almcst always happens, that some portion of these fluids enters the dentine, which then becomes indistinct, and almost invisible in its ramifications.

Two sections made perpendicularly to one another through the middle of the crown and fang of a tooth, from before backwards, and from right to left, are sufficient to exhibit the more important features of the teeth; but sections ought also to be prepared, showing the surface of the pulp cavity and that of the enamel; and likewise various ohlique and transverse sections through the dentine 
in the fangs, to exhibit the anastomoses of their branchea. The dental cartilage is easily shown by maceration in hydrochloric-acid, a process which requires a longer or shorter time, according to the concentration of the acid. It is very instructive also to macerate thin sections in acid, and to examine them upon a slip of glass, at intervals, until they entirely break up. The enamel prisms are readily isolated in developing enamel in this way, and the transverse lines readily seen when the section is moistened with hydrochloric acid. The early development may be studied in embryos of two, three, or four months with the simple microscope; and in transverse sections of parts hardened in spirits of wine. 'The pulp of mature teeth is obtained by breaking them in a vice, and the nerves can be made out without difficulty on the addition of a dilute solution of caustic soda.

To cut through the enamel of the tooth, it will be necessary to lessen the friction, by dropping water upon the saw as it is made to revolve. The section is afterwards very quickly ground down by holding it against the flat side of the coruudum-wheel. ${ }^{1}$ A small handle, mounted with shell-lac, to fix the section in, forms a ready holder: polish, as before directed, between two pieces of the water-of-Ayr stone, or on a hone of Turkey-stone kept wet with water. As the flatwess of the polished surface is a matter of the first importance, that of the stones themselves should be tested from time to time; and whenever they are found to have been rubbed down on one part more than another, they should be flattened on a paving stone with fine sand, or on a lead plate with emery. Wheis this has been sufficiently accomplished, the section is to be secured, with Canada balsam, to a slip of thick wellannealed glass, in the following manner:-Some Canad: balsam, previcusly rendered somewhat stiff by evaporation of part of its turpentine, is to be melted on the glinss-slip). so as to form a thick drop, covering a space somewhat larger than the size of the section, and it should then lot set aside to cool ; during which process, the bubbles that may have formed in it will usually burst. When cold, its

(1) Corundum is a species of emery composition; alumina, red oxide of iron, and lime; it is mucb used by dentists as a polishing material. 
hardness should be tested with the edge of the thumb nail, for it should be with difficulty indented by pressure, and yet should not be so resinous as to be brittle. If it be too soft, as indicated by its too ready yielding to the thumb-nail, it should be boiled a little more; if too hard, which will be shown by its chipping, it should be re-melted and diluted with more fluid balsam, and then set aside to cool as before. When of the right consistence, the section should be laid upon its surface, with the polished side downwards; the slip of glass is next to be gradually warmed until the balsam is softened, care being taken to avoid the formation of bubbles, and the section is then to be gently pressed down upon the liquefied balsam in a sort of wave towards the side, and an equable pressure being finaily made over the whole. When the section has been thus secured to the glass, it may be readily reduced in thickness by grinding. When the thinness of the section is such as to cause the water to spread around it between the glass and the stone, an excess of thickness on either side may often be detected by noticing the smaller distance to which the liquid extends. In proportion as the section attached to the glass is ground away, the superfluous balsam which may have exuded around it will be brought into contact with the stone; and this should be removed with a knife, care being taken that a margin be still left round the edge of the section. As the section approaches the degree of thinness which is most suitable for the display of its organization, great care must be taken that the grinding process is not carried too far ; and frequent recourse should be had to the microscope to examine it. The final polish must be given upon a leathern strap, or upon the surface of a board covered with buff-leather, sprinkled with putty-powder and water, until all marks and scratches have been rubbed out of the section.

In mounting sections of bone, or teeth, it is important to avoid the penetration of the Cauada balsam into the interior of the lacunce and canaliculi; since, when these are filled by it, they become almost invisible. The benefit which is derived from covering the surfaces of the specimen with Canada balsam, may be obtained, without the injury resulting from the penetration of the balsam 
into its interior, by adopting the following method:A small quantity of balsam, proportioned to the size of the specimen, is to be spread upon a slip of glass, and to be rendered stiffer by boiling, until it becomes nearly solid when cold; the same is to be done to the thin glass cover; Hext, the specimen being placed on the balsamed surface, and being overlaid by the balsamed cover, such a degree of warmth is to be applied as will suffice to liquefy the balsam, without causing it to flow freely; and the glass cover is then to be quickly pressed down, and the slide to be rapidly cooled, so as to give as little time as possible for the penetration of the liquefied balsam.

Circular Disc.-For the purpose of cutting glass covers or making shallow cells with japanners' gold-size for mounting objects, ${ }^{1}$ fig. 136 will be found useful; it is made

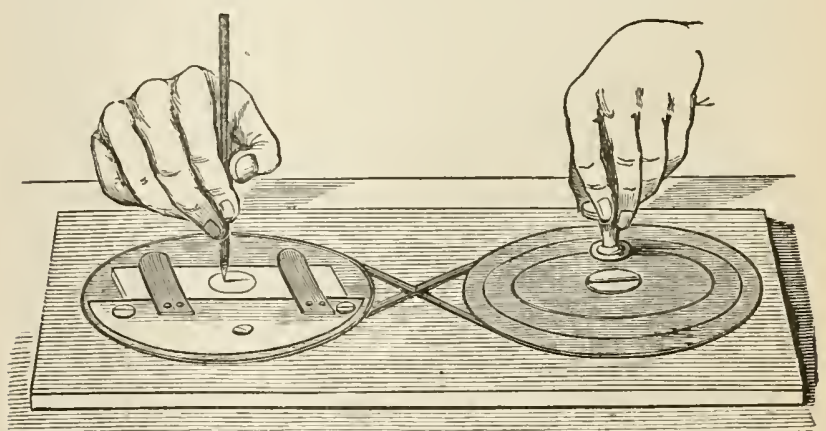

Fig. 130.-Circular Disc Machine.

of two circular wheels of wood, these being let into a solid block of wood, and secured there by central screws. A handle of wood is fixed into the upper part of one, for the purpose of turning it round, the motion being com.municated to the other by an endless band of catgut running in the grooved edge of each. On the upper surface of the wheel, under the right hand, are fixed, by means of screws, two strips of brass, which scrve as springs

(1) Mr. Shadbolt's little turn-table, invented for the same purpose, is in every way superior to this, and is now in very general nse. 
for securing the glass slip; a camel's-hair pencil, previously dipped in japanners' gold-size, is then taken between the finger and thumb, and held as represented in the woodcut, when the wheel is put in motion, and a perfect circle is rapidly formed; the cell is then removed and put aside to dry. In the same way, by securing a sheet of thin glass under the brass springs, and substituting for the pencil a cutting diamond, a circular cover may be readily cut out. A cutting diamond is not only useful to the microscopist for the above purpose, but also for writing the names of mounted objects on the ends of the glass slides. A glazier's diamond for cutting glass slides is both convenient and economical: the mode of using it may be acquired in any glazier's shop.

Cutting glass cells.-Mr. H. H. Brown has also contrived a brass plate for cutting out the centres of pieces of thin glass, to form very shallow cells. It consists of a thin plate of brass, perforated with holes corresponding to the diameter of the cell required, and counter-sunk to a suitable thickness. To use it, it must be heated, and a little sealing-wax or shellac melted into the counter-sunk parts; on this, while hot, the disc of thin glass is to be firmly pressed. When cold, the centres may be easily removed with a common rat's-tail file, the edges being afterwards smoothed over by a fine watch-maker's file. The plate must once more be heated and the glass rings removed, and finally be cleansed from adhesive wax by a little ether. Mr. Brooke has long made use of a small brass press for the purpose of cutting out thin circles of glass and converting the same into shallow cells.

Cells for mounting objects may be made of vulcanite by slicing-tubing made of this material. Glycerine does not act upon it. Mr. Henry Lee uses cells cut from tubes of cardboard; these he finds very useful for mounting dry and opaque objects. Both have the great advantage of cheapness-the former are sold for about $3 d$. a dozen and the latter 1s. a gross. Mr. Suffolk devised a cheap tin-cell, which must partially supersede the more expensive glasscell; it appears to answer equally well for fluid or dry mounting. The metallic rings for making these cells are manufactured in various sizes and thicknesses by 
Collins, of Great Titchfield Street, and sold at 3d. pes dozen.

Dr. Guy brought under the notice of microscopists, a plan for preserving metallic sublimations, crystals, \&c. in small round, or oval tubes, hermetically sealed at both ends. This plan may be made available for the display of insects, parts of flowers, as stamens, pollen, \&zc. Sce his paper in the Micros. Journal, New Series, vol. ii. p. 77.

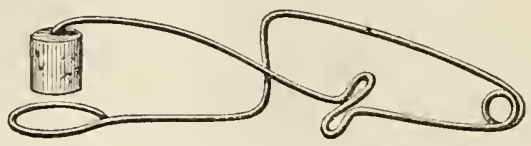

Fig. 137, full size.-Spring-clip for montnting.

Lr. Maddox describes a Wire Spring-clip for mounting purposes, Micros. Soc. Trans. 1865, p. 84 . To make the clip, take a straight piece of brass wire four and threequarter inches in length, turn one end at six-eighths of an inch with a pair of wire-pliers at right angles ; this second portion, at half an inch, again bend at right angles in the same plane; now, at three-quarters of an inch, turn the wire over on itself, leaving at the bend space sufficient to admit a thick slide. At one inch and five-eighths twist the wire completely on itself, and bring the now short ends at right angles to the longest part; file this end quits flat. Give the first portion of the wire a slight curvature, so that the point and bend may act as a stiff spring against the under surface of the slide when applied. This, although a very simple and inexpensive clip to make, has been very much improved upon, as will be admitted by those who have used the little handy spring-clip shown full size in fig. 137. The Universal Clip, as it deserves to be called, may be purchased for $1 s .6 d$. per doz. of Baker, Holborn, so that objects of great delicacy can be mounted and left to dry and harden for any necessary length of time. The little nipple which is seen to press upon the glass cover in fig. 138, is made of a piece of cork or thick leather. The clip simply requires pinching together between the thumb and finger; as soon as it is released the 
little cork nipple comes down with considerable force upon the glass cover. In mounting algæ, \&c. with a gelatine medium, the specimen should be placed on the glass slide with a small quantity of water, and when properly arranged, the thin glass cover must be carefully put on, and a sufficient quantity of gelatine medium placed by its side; gentle heat should be applied to drive ${ }^{\text {Fig. } 138, \text { half size. }}$ ing its modering-clip, show. out the water, when the gela-

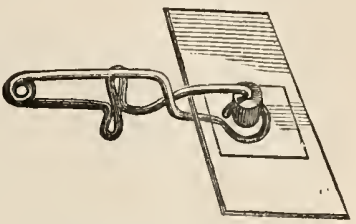
tine flows under to take its place; then put the object aside to dry and harden.

General Directions for the Preparation and Mounting of Objects.-The objects exhibited by the microscope are either opaque or transparent. The former, in the majority of instances, require little or no preparation beyond placing them in such a position as to show their external surface by reflected or condensed light, and covering with thin glass to exclude dust. Those objects, however, which it is intended to examine by transmitted light require, in most cases, to be prepared previously to mounting them, in whateves vehicle may be found most suitalle for exhibiting their structure. The medium most used for mounting trans parent objects is Canada balsam. The pure balsam is, however, too thick for use, anch it requires to be diluted with spirit of turpentine to render it sufficiently fluid to permeate the structure to be exhibited. As a general rulc, it should be just fluid enough to drop readily from the point of a needle. Those who desire to avoid the trouble of mixing their own mounting medium, can procure it ready for use from any of the microscope makers. There are some few objects whose structure is so transparent that they must be mounted dry. Scales from the wings of butterflies and moths, of the podura and lepisma saccharina, and some of the diatomacese are of this class. All that is necessary in preparing objects for dry mounting, is to take care that they are free from extraneous matter, and to fix them permanently in that position in which their structure will show to the best advantage. Care 
should be taken to have no draught of air through the room while handling very delicate objects; many a beautiful object has been wafted from under the hand of the microscopist in this way, sometimes, even by his own breath.

The preparation of very minute objects which require particular chemical treatment before mounting, will be more fully described hereafter. 'To this class belong the diatomacer, whose delicate structure forms one of the most beautiful objects which can be exhibited. In mounting entomological specimens, the first thing, of course, is the dissection of the insect. This is best accomplished by the aid of Collins's Dissecting Microscope, a pair of small brass forceps, and very finely-pointed scissors; the parts to be prepared and mounted should first be carefully detached from the insect with the scissors, then immersed in a solution of caustic alkali (Liquor Potassæ) for a few days, to soften and dissolve out the fat and soft parts : the length of time it is necessary to immerse them can only be ascertained by experience, but, as a general rule, the objects assume a certain amount of transparency when they have been long enough in the alkali ; when this is ascertained to be the case, the object is to be placed in a flat receptacle (a shallow pomatum pot is as good a thing as can be used), and put to soak for two or three hours in soft or distilled water. It is then to be placed between two slips of glass, and gently pressed till the softer parts, \&c. are removed. These will frequently adhere to the edge of the object; it will, therefore, be necessary to wash the latter carefully in water to get rid of the superfluous matter, a process which will be much aided by delicate touches of a camel'shair brush. Place the object now and then under the microscope to see that all extraneous matter is removed, and when this is accomplished take the specimen up carefully with the camel's-hair brush, and lay it on a piece of very smooth paper (thick ivory note is very good for the purpose), arrange it, if necessary, to its natural appearance with the brush and a finely-pointed needle, place a second piece of paper over it, and press it flat between two slips of glass, and compress it by one of the American clips which may be bought for a few pence per dozen. 
When thoroughiy dry (which it will probably be in about twenty-four hours, if in a warm room), separate the glasses, and gently unfold the paper; then, with a little careful manipulation, the object may be readily detached, and should be at once placed in a little spirit of turpentine, where it should remain for a few days till it is rendered transparent and fit for mounting. The time during which it should remain in this liquid will depend on the structure; some objects, such as wings of flies, will be quickly permeated, while horny and dense objects require an immersion of a fortnight or even longer. A pomatum pot with a concrve bottom and well-fitting lid will be found to answer admirably for the soaking process, and it is well, in preparing several specimens at a time, to have two pots, one for large and medium, the other for very small objects, or the smaller ones will be found often to adhere to the larger.

The glasses on which objects are mounted are usually slips of flatted crown or sheet glass cut to a size of three inches by one, and ground at the edges. The mode ot mounting the object is as follows:-Having chosen a glass slide, clean and polish it with a piece of chamois leather, ascertain the centre of the slide by means of a piece of paper or card of exactly the same size as itself, and in which a hole has been eut exactly in the contre, place the piece of paper under the slide, and, having removed the object to be mounted from the turpentine in

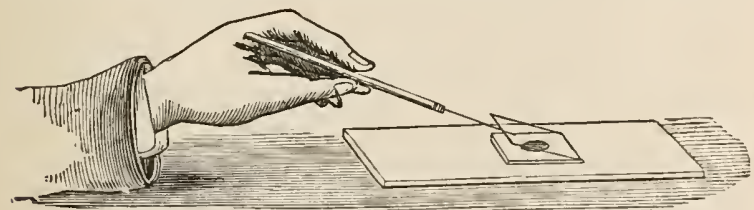

Fig. 139.-Showing the mode of placing Glass Cover on the Object.

which it has been soaked, lay it on the slide on the spot corresponding to the hole in the card underneath; then tak" up a small quantity of the prepared Canada balsam on the point of a large needle or pointed pen-knifc, and 
drop it immediately over the object, slightly warm the under part of the slide over a spirit-lamp to diffuse the balsam and cause it thoroughly to penetrate the object, and immediately cover the latter with one of the small circles of thin glass, sold by opticians for the purpose. In laying the glass cover on the object, eare should be taken to bring the edge of the circle duwn first, and let the other fall slowly on the object (see fig. 139), to prevent the formation of bubbles from the sudden displacement of the air. It requires some little practice to keep the object in the centre of the circle.

Sometimes, notwithstanding all the care of the operator, bubbles will appear. These may generally be got rid of by gently warming the under part of the slide over the spirit-lamp, when the bubbles will usually leave the object, and travel towards the edge of the circle. In most cases they will entirely disappear as the balsam becomes firmer and drier. If it be desired to dry the balsam quickly, the slide may be placed in some warm situation where the heat does not much exceed $100^{\circ}$, and it must be maintained in a perfeetly horizontal position, to prevent displacement, until the balsam has become dry. When this has been aseertained to be the case, the superfluous balsam which surrounds the edge of the circle may be scraped off by the point of a penkuife; and when the major part has been removed in this way, the remainder rnay be got rid of, and the edges of balsam rendered smooth by rubbing gently with an old silk handkerchief moistened with spirit of turpentine. The edge of the circle of balsam will probably appear white and dull, but it may be rendered transparent by gently warming the under part of the slide over a spirit-lamp, and again plasing the object in a warm room till the balsam has a second time become hard and dry ; after which the name of the object should be written with a small writing diamond at one end of the slide. Some microscopists prefer to cover the slide with ornamental paper, which may be procured very cheaply.

In eovering the slides with paper, their edges need not be ground, but may be rubbed with a fine file, which will prevent the sharp glass from damaging the paper cover, and cutting the fingers of the operator. The foregoing is 
the method by which objects are mounted in balsam; there are, however, some specimens, the mounting of which, in balsam, would render them almost invisible, in which case-if not suitable for dry-mounting-they should be placed in fluid in cells, the size and depth of which must be regulated by the proportions of the object. If it be the scale of a fish, or the pollen of a flower, a very shallow cell will suffice, and it may be formed of "Brunswick black" in the manner already described. When the cell is quite dry, take the object (which should have been some time previously snaked in the fluid in which it is to be mounted to dispel the air from its substance), place it in the middle of the circle, fill the space quite full of the mounting fluid, and cover it with a glass circle; place the edge down first, and bring the whole surface of the circle very gradually upon the cell as pointed out in the former case. Some of the fluid will immediately escape under the edge; this may be absorbed by a piece of filtering paper. Should too much escape, a bubble will make its appearance in the cell; in this case the process must be repeated. When this has been performed successfully, secure the glass circle in its place with a small spring-clip; then take a camel's-hair brush, charged with varrish, and carry it round, and slightly over the edge of the cover. Allow the first layer to dry before another is added, and continue to add more gradually until the cell is made perfectly air-tight. Glass or metal cells must be employed for those objects whose bulk renders the method just described inadmissible. Glass-cells may be fastened to the glass-slide either by Canada balsam, by Jefferey's marine glue, or Brunswick black; the latter will be rendered very durable by mixing it with a small quantity of India-rubber varnish (made by dissolving small strips of caoutchouc in gas-tar). The process of mounting in glass-cells is similar to that employed in making varnish-cells, except that a somewhat larger quantity of cementing medium is required on account of the greater weight of the cell. Objects mounted in this way should always be kept in the horizontal position, and a little fresh varnish applied now and then, if the cement show any tendency to crack.

In mounting objects in balsam, great care should bo 
taken to have the speeimens quite dry before soaking them in turpentine. Objeets mounted in eells, on the contrary should have become perfectly saturated with the mounting fluid before being finally secured.

It is preferable to mount and preserve specimens of animal tissues in shallow cells, to avoid undue pressure on the preparation. Cells intended to eontain preparations immersed in fluid must be made of a substance impervious to the fluid used; on the whole, the

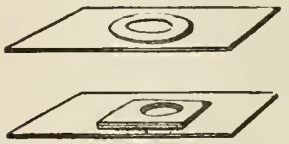

Fig. 140.-Glass-cells for Mounting. most useful are those made with circles of thin glass, cemented to the glass-slide with marine glue, such as we have here represented (fig. 140). The surface of the glass should be slightly roughened before applying the eement.

Different modes of mounting may be employed with advantage, to show different structures; entomologieal specimens, such as legs, wings, spiracles, tracheæ, ovipositors, stings, tongues, palates, cornex, \&c. show best in balsam : the trachea of the house-cricket, however should be monnted dry. Sections of bone show best, when mounted dry, or in a cell with fluid. Seales of butterflies, moths, \&c. should be mounted dry. Other objects, as sections of wood and stones of fruit, exhibit their structure best in a cell with fluid.

There are some objects much more difficult to prepare than others, and which tax the paticnee of the beginner in a manner which ean hardly be imagined by any one who has never made the attempt. The structure of many creatures is so delicate, as to require the very greatest care to prevent mutilation, and eonsequent spoliation of the specimen. The beginner, thereforc, must not be discouraged by a few failures in eommencing, but should persevere in his attempts, and constant practice will soon teach him the best way of managing intricate and diffieult objeets. The room in which he operates should be free from dust, smoke, and intrusion, and everything used should be kept scrupulously clean, since a very small speck of dirt, which may be almost invisible by the naked eye, will assume unpleasant proportions under the microscope, and not only 
mar the beauty, but possibly interrupt a clear view of a very splendid and delicate object. Then, again, if the microscopist prefers to cut and grind his own glass slips, he should be very careful that there are no sand-specks or air-bubbles in the centre of the slide, or of the glasscover: many a good object has been spoiled from neglect of this precaution. A good light by which to work is also highly important. In using the ordinary microscope, the microscopist should keep both eyes open, the practice of closing the eye not in use being injurious to the sight of both. The beginner who is about to purchase a micrnscope, will do well to procure a binocular, the price of which has been reduced so much as to bring it within the reach of those of even moderate means.

The preservation of objects is a matter of much importance. They ought to be kept in a cool and dry place. Sections of bone, the pollen of plants, and the scales of the podura and butterflies, become spoiled if kept in a damp atmosphere. Objects mounted dry should be carefully protected from dust, which will find its way into crevices and corners where one might hardly suppose it could possibly enter.

Some opaque objects require dry mounting; others show best in fluid; the elytra of some beetles are magnificent objects when mounted in balsam. Cells punched out of thick mill-board, or turned in some hard wood, or Mr. Suffolk's metal-rings, are much lighter and more convenient than glass for such objects, and also for those mounted dry. In mounting objects in fluid, the glass-cover should come nearly, but not quite, to the edge of the cell; a slight margin must be left for the cement, which ought to project slightly over the edge of the cover, in order to unite it securely to the cell. In cells, however, that contain balsam, there is no necessity for cement, and the appearance of the slide will be improved by choosing a cover of exactly the same size as the cell.

There are some objects which require considerable dexterity and patience to prepare them for mounting. The petal of the pelargonium is one of these. It is necessary to split. it in order to show its structure to perfection. This is effected by dividing the base of the petal with a 
sharp penknife, and then, with two small pairs of forceps, tearing asunder the two parts; the upper surface, which is the part to be mounted, must then be dried and placed. for a short time in spirit of turpentine, previously to being mounted in balsam. If well prepared, the colour of the petal will be almost as fresh as in its natural state.

In mounting diatoniacex, to show them as nearly as possible in their natural condition, they should be first well washed in distilled water and mounted in a medium composed of one part of spirits of wine to seven parts of distilled water. The siliceous coverings of the diatoms, however, which show such beautiful forms under the higher powers of the microscope, require considerable preparation. The guano, or infusorial earth containing them, should first be washed several times in water till the water is colourless, allowing sufficient time for precipitation between each washing. The deposit must then be put into nitro-hydrochloric acid (equal parts of nitric and hydrochloric acids). A violent effervescence takes place, and when this has subsided, the whole should be subjected to heat, nearly but not quite up to the boilingpoint, for six or eight hours. The acid must now be carefully poured off, and the precipitate washed in a large quantity of water, allowing some three or four hours between each washing, for the subsidence of some of the lighter forms; the sediment must be examined under the microscope with an inch object-glass, and the siliceous valves of the diatoms picked out with a coarse hair or bristle.

When it is desired to exhibit one of the larger forms, it can be placed separately in the middle of a glass-slide, and mounted in Canada balsam; or when it is wished to mount several forms at once, the fluid containing the skeletons should be gently shaken, and a drop spread over the centre of a slide, the glass being held over a spiritlamp till the fluid has all evaporated, when the object may be either dry-covered or mounted in balsam in the usual way, according as its structure may require the one or the other treatment. Diatoms may be kept in distilled water in a small corked phial for any length of time.

For vegetable preparations, distilled water in which 
camphor has been kept is a good mounting medium; some preparers, however, add spirits of wine in the proportion of one draehm to eleven drachms of water; others add to these saltpetre, two grains to the ounce of fluid. The mounting-fluid should be prepared and allowed to settle, when the major part can be poured off clear-a mucl better mode than filtering, as particles of the paper are apt to get mixed with the liquid.

F. M. Rimmington's Glycerine Jelly has become an established preparation, and one found to possess many advantages over Canada balsam, and some other media used for the same purpose, in the facility with which objects can be mounted, and efficiently preserved, and the transparency it gives to many animal and vegetable structures. Its antiseptic powers are sufficient to prevent changes in any object immersed in it, if not too bulky. It is especially alapted for mounting algæ, fungi, vegetable and animal tissues, urinary deposits (as casts, epithelium, crystals), also anatomical specimens, starch granules, des. midiaceæ, diatomaceæ, \&c. Objects to be mounted in this medium only require to be soaked in weak alcohol for a time, greater or less, as may be necessary, for the purpose of dissolving out any soluble matter, or for driving out air entangled in the cellular meshes. When it is thought that this has been accomplished, the object is laid on a slide and freed from superfluous moisture, the slide gently warmed, and a quantity of the jelly, deemed sufficient for the purpose, laid by the side of it, and made to touch it. It will thus be immersed; the cover, previously warmed, is to be laid upon it, and gently pressed. The slicle may now be laid aside and finished by running it round with varnish.

The jelly should be liquefied by holding the bottle at some distance above a lamp, or by dipping it into hot water. No more than what is necessary need be liquefied, and it should never be made hot.

For certain delicate organisms, as the desmidiaceæ and diatomaceæ, whose plasma may be affected by too dense a medium, the jelly may be diluted one-quarter or onethird with camphor-water.

For mounting pathological or injected preparations, the following medium will be found to answer very well :- 
Bichloride of mercury, one grain; chloride of sodium, ninety grains; distilled water, one pint: set aside, and, when perfectly clear, decant and preserve in a stoppered bottle. All preparations used in mounting, and objects themselves, should be carefully preserved from dust. Balsam should be kept in a wide-mouthed bottle, over which a small tumbler or cupping-glass must be inverted. It is advisable to avoid the use of a cork, as it will be broken in attempting to withdraw it, and portions will not only adhere to the neck of the bottle, but are apt to get into the balsam. Objects, and parts of objects, already prepared and dry, may be kept neatly folded up in ivory-paper, as it is not well to have too many objects in the turpentino at the same time, in order to avoid confusion. Glassslides which are soiled with balsam can be cleaned with turpentine and a piece of rag-the glass circles will require to be soaked in turpentine to get rid of any balsam they may bappen to contract. When a greasy appearance is present, it may be removed by a picce of rag moistened with rectitied spirit. Some remove it by rubbing the glass with caustic alkali ; but as this liquid acts on flintglass, and sometimes makes it partially opaque or milky, its use should be entirely discarded-a weak solution of ammonia, or cyanide of potassium, answers the purpose best.

In order to demonstrate the internal structure of animal tissues under very high magnifying powcrs, Dr. Beale ${ }^{1}$ says the specimen must be placed in a viscid medium, and subjected to considerable pressure to make it sufficiently thin. Price's glycerine, having a specific gravity of 1,210 , and a strong syrup, are the best adapted for preserving all soft tissucs. In practice, the specimen is first immersed in a solution of weak glycerine or syrup, and the density of the fluid is gradually increased. In this way, and in the course of two or three days, the softest and most delicate tissues swell out, and become more transparent; but no chemical change is produced in the specimens. The one .thing to be observed is, that the strength of these fluids should be increased very gradnally until the whole of the tissues are thoroughly penetrated and saturated by the strongest that can be obtained. Cerebral tissues, delicate

(1) Beale, "How to Work with the Microscope" 
n Ivous textures, like the retina, may be permented by the strongest glycerine, and, when fully saturated with it, dissected with a,degree of minuteness unattainable in any other way. "The smallest animalcules, entozoa, polyps, molluses, insects, crustacea, fungi, algx, and the delicate parts of vegetahle tissues, are equally well preserved by it." All the various colouring-solutions, as carmine, aniline, \&c., should be always prepared for use with glycerine or syrup as a basis.

The staining or colouring fluid employed by Dr. Beale is made as follows:-Carmine, 10 grains; strong liquil ammonia, $\frac{1}{2}$ drachm ; Price's glycerine, 2 ounces; distilled water, 2 ounces; alcohol, $\frac{1}{2}$ ounce. The carmine is ato be placed in a test-tubc, and the ammonia adder to it. Upon applying the gentle heat of a spirit-lamp, it is dissolved. Boil it up for a few seconds, and allow it to cool before adding the glycerine and rest of the ingredients. Lastly, pass it through a filter, or allow it to stand by and decant off the clear solution. The solution should neither be too alkaline, nor perfectly neutral; if the former, the colouring becomes too intense, and thus much of the soft or imperfectly formed tissue is destroyed; and, if the latter, the miform staining of tissue and germinal matter equally mars the result. The permeating power of the solution may be increased by the addition of a little more water and alcohol.

After the specimen has been properly stained, it should be washed in a solution, consisting of strong glycerine two parts, water one part; and then transferred to the following acid fluil :-Strong glycerine, one ounce; strong acetic acid, five drops; where it must remain three or four days to regain the volume it occupied when fresh. To mount specimens so prepared, take a small portion and spread it out on the glass-slide; add a drop of tresh glycerine, and cover with thin glass; warm it gently, and press it down with a needle-point.

Examine the specimen with a quarter power, and, if a good deal of granular matter appears to be mixed in with it, remove the glass cover, and wash it by adding drop after drop of the glycerine and acid solution. The slide sliould be inclined, and, at the same time, gently warmed 
over the lamp. When a few drops of pure glycerine have been allowed to flow over it, the thin glass cover must be re-applied and pressed tightly down. If the preparation looks clear under an eighth or twelfth, the glass cover may be cemented on, and the specimien left to dry. The success of Dr. Beale's process, it appears, much depends upon the care and patience with which each stage of the soaking, washing, warming, and pressure is carried out. Each point of structural difference is graduaily brought out by subjecting the specimen to a little firmer pressure, or by soaking it in a little fresh glycerine in a watch-glass, and then applying gentle heat. By the aid of needles, and a little careful manipulation, tissues may be laid out perfectly smooth and flat.

A word about making and preparing Transparent Injections.-The great desideratum of a transparent iujecting fluid is, that it shall not, by the action of osmosis, dye the tissue meant to be injected. This at once bars the use of soluble colours, and necessitates the use of insoluble colouring matter in an exceedingly fine state of subdivision. The following composition is stated to succeed admirably, showing vessels of $\frac{1}{2000}$ th of an inch diameter, with a clear outline even under a sth objectire, without a grain of extravasation of the colouring matter:-Take 180 grains best carmine; $\frac{1}{2}$ fluid ounce of ammonia, common strengtli, sp. gr. $0.92 ; 3$ to 4 ounces distilled water. Put these ingredients into a small flask, and allow them to digest without heat for 21 or 36 hours, or until the carmine is dissolved. Then take a Winchester quart-bottle, and mark upon it the line to which 16 ounces of water extend. The coloured solution must then be filtered into the bottle, and pure water must be added until the whole is equal to 16 ounces. Next dissolve 600 grains of potasli-alum in about 10 fluid ounces of water, and add to this, under constant boiling, a solution of carbonate of soda, until a slight permanent precipitate is produced. Filter and add water up to 16 fluid ounces. Boil, and add this solutinn, while boiling, to the cold ammoniacal solution of caruine in the Winchester quart, and shake vigorously for a few minutes. A drop now placed upon white tilter-paper should show no coloured 
ring; if it do, the whole must be rejected. Supposing the precipitation to be complete, cs nearly so, shake vigorously for half an hour, and allow to stand till quite cold; the shaking must then be renewed, and the bottle filled up with cold water. After allowing the precipitate to settle for a day, draw off the clear supernatant fluid with a syphon. Repeat the washing until the clear fluid gives no precipitate with chloride of barium. So much water must be left with the fluid that at last it may measure 40 fluid ounces. For the injection-fluid, take 24 ounces of the above coloured fluid, and 3 ounces of good gelatine; allow these to remain together all night, then dissolve by the aid of a water-bath, and strain through fine muslin. On injecting, the ordinary precautions for a gelatine injection are alone necessary.

Mounting Polyzoa.-Mr. Morris, of Bath, has succeeded in obtaining beautiful specimens of polyzoa and hydroid zoophytes, with expanded tentasles, by adding spirit of wine, drop by drop, to the sali- water cell in which they have been confined. 'The polypidoms should be thus treated as soon as possible after capture.

A plan of mounting objects in a mixture of balsam and chloroform is described by Mr. Wm. Henry Heys in the Microscopical Journal thus :-Take a quantity of the oldest balsam procurable, and place it in an open glass cup, and mix with it as much chloroform as will make the whole quite fluid, so that a very small quantity will drop from the lip of the containing vessel. Then put this prepared balsam into long thin half-ounce vials, and cork and set them aside for at least a month. The advantage of having. it ready-made is, that there is no waste, and none of the usual and troublesone preparation required for putting up objects in Canada balsam; and if it has stood for some time, it loses the yellow tinge which is observable in most samples when first mixed, and, moreover, air-bubbles escape more readily.

Mr. Goadby's fluids are cheap and most effectual for preserving and mounting animal structures in. The following are his formulie:-

Take for No. 1 solution, Lxy sait, 4 oz.; alum, 2 oz.; corrosive sublimate, 2 grains; moling water, 1 quart: mix. 
For No. 2 solution, bay salt, 4 oz.; alum, 2 oz. ; corrosive sublimate, 4 grs.; boiling-water, 2 quarts : mix.

The No. 1 is too strong for most purposes, and should only be employed where great astringency is needed to give form and support to very delicate structures. No. 2 is best adapted for permanent preparation; but neither should be used in the preservation of animals eontaining carbonate of lime (all the mollnsea), as the alum becomes decomposed, sulphate of lime is precipitated, and the preparation spoiled. For such use the following:-

Bay salt, 8 oz.; corrosive sublimate, 2 grs.; water, 1 quart: mix.

The corrosive sublimate is used to prevent the growth of vegetation in the fluid; but as this salt posse-ses the property of eoagulating albumen, these solutions cannot be used in the preservation of ova, or when it is desired to maintain the transparency of certain tissues, such as the cellular tissue, the white corpinscles of the blood, \&c.

Mr. Goadby's method of making marine glue for cementing cells is as follows: dissolve separately equal parts of shell-lae and India-rubber in coal or mineral nitphtha, and afterwards mix the solutions carefully by the application of heat. It may be rendered thinner by the addition of more naphtha, and is always readily dissolved by naphtha, ether, or solution of potash, when it becomes hard or dry in our stock-pots.

Preparation and Preservation of Alga, \&c.-Mr. Ralfs gives excellent directions for making preparations of algre for microseopic iuvestigations:- "The fluid found to answer best is man's in the following way: to sixteen parts of distilled water add onc part of rectified spirits of wine, and a few drops of creosote, sufficient to saturate it ; stir in a small quantity of prepared chalk, and then filter; with this fluid mix an equal measure of camphor. water (water saturated with eamphor); and before using, strain off through a piece of fine linen.

"This fluid I do not find to alter the appearance of the endochrome of algæ more than distilled water alone does after some time; there is certainly less probability of corfervoid filaments making their appearance in the preparations; and there would seem to be nothing tc prevent 
such a growth from taking place, when the object is mounted in water only, provided a germ of one of these minute plants happen to be present, as well as a smali quantity of free carbonic acid.

"My method of making cells in which to mount preparatiuns of algæe is as follows : some objects require very shallow, and other's somewhat deeper cells. The former may be made with a mixture of japanners' gold-size and litharge, to which (if a dark colour is preferred) a small quantity of lamp-black can be added. These materials should be rubbed up together with a painter's muller, and the mixture laid on the slips of glass with a camel-hail pencil as expeditiously as possible, since it quickly becomes hard; so that it is expedient to make but a small quautity at a time. For the deeper cells mariwe-glue auswers extremely well, provided it is not too soft. It must be melted and dropped upon the slip of glass; then flattened, whilst warm, with a piece of wet glass, and what is superfluous cut away with a knife, so as to leave only the walls of the cell; these, if they have become loosened, may be made firm again by warming the under surface of the slip of glass. The surface of the cells must be made quite flat; which can be easily doue by rubbing them upon a wet piece of smooth marble, covered with the finest emerypowder.

"When about to mount a preparation, a very thin layer of gold-size must be put upou the wall of the cell, as well as on the edge of the piece of thin glass which is to cover it; lefore this is quite dry, the fluid with the cbject is to be put into the cell, and the cover of thin gilass slowly laid upon it, beginning at one end; gentle pressure must then be used to squeeze out the superfluolis fluid; and, after carefully wiping the slide dry, a thin coat of gold-size should be applied round the edge of the cell, and a second coat so soon as the first is dry; a thin coat or two of black sealing-wax varnish may then be put ou with advantage, in order to prevent eflectually the admission of air into the cell, or the escape of fluid out of it.

"I would remark, that the gold-size employed shonld be of the consistance of treacle; when purchased, it is usually too fluid, and should be exposed for some time in 
an open vessel ; a process which renders it fit for use. In mounting the Desmidacea, great attention is necessary to exclude air-bubbles, which cannot be avoided unless the fluid completely fills the cells; and also not to use too much fluid, as in this case the smaller species will often be washed away on the escape of the superfluous portion. As the cells cannot be sealed whilst any moisture remains on their edge, it should be removed by blotting-paper, in preference to any other mode. A thin description of glass is manufactured expressly for the purpose of covering specimens when mounted.

"The rare species of Desmidacece are frequently scattered amongst decayed vegetable matter, so that it is difficult to procure good specimens for mounting. In such cases, a small portion of the mass should be mixed with a little of the creosote fluid, and stirred briskly with a needle. After this has been done, the Desmidacea will sink to the bottom, when the refuse should be carefully remored. Successive portions having been thus treated, specimens will at length be procured sufficiently free from foreign matter. Even in ordinary circumstances, if a small extra quantity of fluid be placed in the cell, and the slide gently inclined, most of the dirt may be removed by a needle before the cell is closed; which process will materially increase the beauty of the preparation.

"If the cells are insufficiently baked, the japan occasionally peels off the glass after the specimen has been mounted for some time. To obviate this inconvenience, Mr. Jenner previonsly heats the cell, with much caution, over a rushlight, until the japan becomes of a dark colour, and vapour ceases to arise from it. When gold-size is used for closing the cell, the intrusion of some of it frequently destroys valuable specimens, whatever care may be taken. Mr. Jenner has therefore relinquished it, and now employs a varuish made of coarsely comminuted purified shell-lac or translucent sealing-wax, to which is adced rectified spirits of wine, in sufficient quantity to cover it. This varnish will be ready for use in about twelve hours : when it is too thick, a little more spirit should be added. Mr. Jenner applies three coats of this varnish, and about a week afterwards a fourth, composed of japan varnish or gold- 
size. ${ }^{\text {T }}$ To preserve the brush $\vdots$ a fit state, it shonld always be cleaned with spirits of wine whonever it has been used."

Mr. Topping's fluid for mounting consists of one ounce of rectified spirits to five ounces of distilled water; this he thinks superior to any other combination. $\mathrm{T}=$ preserve delicate colours, however, he prefers to use a srlution of acetate of alumina, one ounce of the acetate to four ounces of distilled water: of other solutions he says, that they teud to destroy the colouring matter of delicate objects, and ultimately spoil them by rendering them opaque.

Injecting Animal Bodies.-For minute injections, the most essential instrument is a proper syringe. This is

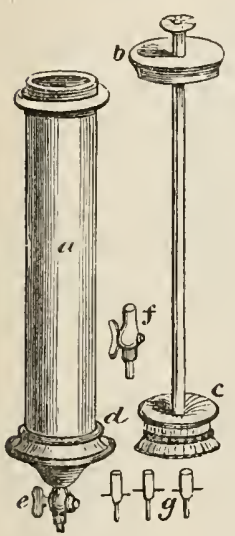

Fig. 141. usually made of brass, of such a size that the top of the thumb may press on the button at the top of the piston-rod when drawn out, while the body is supported between the two fingers. Fig. 141 represents the syringe: $a$ is a cylindrical brass body, with a screw at the top for the purpose of firmly screwing down the cover $b$, after the piston $c$ is introduced into it; this is renderer? air-tight with leathor; the bottom of the syringe $d$ also unscrews for the convenience of cleaning; $e$ is a stop-cock, on the end of which another stop-cock $f$ fits accurately; and on the end of this either of the small pipes $g$, which are of different sizes, may be fixed. The transverse wires across the pipes are intended to secure them more tightly to the ressels, into which they may be inserted with thread, so that they may not slip out. In addition to the syringe, a large tin vessel, to contain hot water, with two or three snaller ones fixed in it, for the injections, will be found useful.

To prepare the material for injecting:-Take of the finest and most transparent glue one pound, break it into small pieces, put it into an earthen pot, and pour on it

(1) "Coachmaker's Black" is an excellent varnish. 
three pints of cold water; let it stand twenty-four hours. stirriug it now and then with a stick; then set it over a slow fire for half an hour, or until all the pieces are perfectly dissolved; skim off the froth from the surface, and strain through a flamnel for use. Isinglass and cuttings of parchment make an excellent size, and are preferable for very particular injections. If gelatine be employed it must be soaked for some hours in cold water before it is warmed. About an ounce of gelatine to a pint of water will be sufficieutly strong, but in very hot weather it is necessary to add a little more gelatine. It must be soaked in part of the cold water until it swells up and becomes soft, when the rest of the water, made hot, is to be added. Good gelatine for injecting purposes may be obtained for two shillings a pound.

The size thus prepared may be coloured with any of the following:-

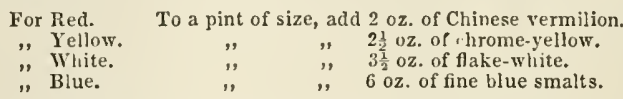

It is necessary to remember, that whatever colouring matter is employed must be very finely levigated before it is mixed with the injection. This is a matter of great importance: for a small lump or mass of colour, dirt, \&c. will clog the minute vessels, so that the injection will not pass into them, and the object will be defeated. The mixture of size and colour should be frequently stirred, or the colouring matter will sink to the bottom. Respecting the shoice of a proper subject for injecting, it may be remarked, that the injection will usually go furthest in young subjects; and the more the fluids have been exhausted during life, the greater will be the success of the injection.

To prepare the subject, the principal points to be aimed at are, to dissolve the fluids, empty the vessels of them, relax the solids, and prevent the injection from coagulating too soon. For this purpose it is necessary to place the animal, or part to be injected, iu warm water, as hot as the operator's haud will bear. This should be kept at nearly the same temperature for some time by occasionally adding hot water. The leugth of time required is in proportion to the size of the part and the amount of its 
rigidity. Ruysch (from whom the art of injecting bas been called the Ruyschian art) rocommends a prevous maceration for a day or two in cold water.

The size must always be kept hot with the aid of warm water; for if a naked fire be used, there is danger of burning it. The size may be placed in a vessel which can be heated by standing it in a common tin sancepan of hot water. A convenient form of apparatus for melting the size, and afterwards keeping it at a proper temperature, is

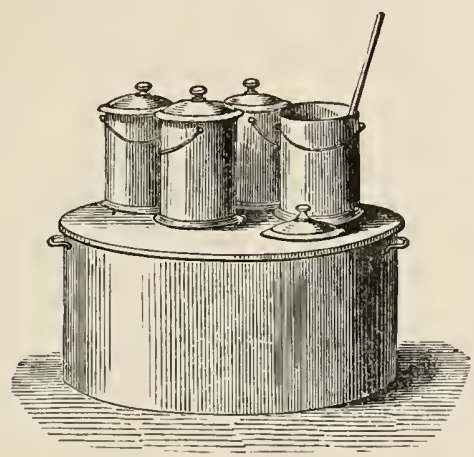

Fig. 142. -Melting Vessel.

shown in fig. 142. It consists merely of a water-bath, in which the cans containing the injecting fluid can be kept hot, and their contents protected from dust by means of their covers. A small apparatus of this kind could be made by any tin-worker, and fitted over a gas jet to stand on the table.

The operator should be provided with several pairs of strong forceps, for scizing the vessel or stopping the escape

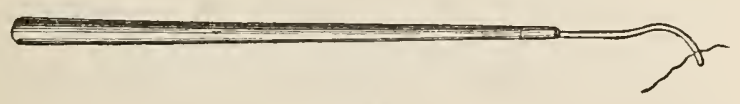

Fig. 143.

of injection. A small needle, fig. 143, will be found useful for passing the thread round the vessel into which the 
injecting pipe is to be inserted. Where the ressels are large, a needle commonly known as an aneurism needle answers the purpose very well. The thickness of thread must vary according to the size of the ressel. The silk used by surgeons will be found the best adapted for the purpose, and not too th:n, or it may cut through the vessel.

When the size and the suljject have both been properly prepared, have the injection as hot as the fingers can well bear. One of the pipes $g$, fig. 141, must then be placed in the largest artery of the part, and made secure by tying. Put the stop-cock $f$ into the open end of the pipe $e$, and it is then ready to receive the injection from successive applications to the syringe $a$, leaving sufficient space only for the piston $c$. The injection should be thrown in by a very steady and gentle pressure on the end of the pistonrod. The resistance of the vessels, when nearly full, is often considerable; but it must not be overcome by violent pressure with the syringe. When as much injection is passed as may be thought advisable, the preparation may be left (with the stop-cock closed in the pipe) for twentyfour hours, when more material may be thrown in.

As the method of injecting the minute capillaries with coloured size is often attended with doubtful success, various other plans have been proposed., Ruysch's method, according to Rigerius, was to employ melted tallow coloured with vermilion, to which in the summer a little white wax was added. Monro recommended coloured oil of turpentine for the small vessels; after the use of which, he threw in the common coarse injection. This is made of tallow and red lead, well mixed and heated before it is used. The cold paint injection succeeds well if thrown carefully into the minute arteries; but its tendency to become brown by age is an objection to its use.

Professor Breschet frequently employed with success nilk, isinglass, the alcoholic solution of gum-lac, spirit rarnish, and spirit of turpentine; but he highly recommends the colouring matter extracted from Campeachy, Feruambone, or Sandal woods. He says: "The colouring matter of Campeachy wood easily dissolves in water and in alcohol : it is so penetrating that it becomes rapidly spread through 
the vascular net-works. The sole inconvenience of this kind of injection is, that it cannot be made to distend any except most delicate vessels, and that its ready penetration does not admit of distingnishing between arteries, reins, and lymphatics." He also recommends a solution of caoutchouc.

Another process, which may be termed the chemical process, was published in the Comptes Rendus, 1841, as the invention of M. Doyers. According to this, an aqueous solution of bichromate of potass, 1,048 grains to two pints of water, is thrown into the vessels; and after a short time, in the same manner and in the same vessels, an aqueous solution of acetate of lead, 2,000 grains to a pint of water, is injected. This is an excellent method, as the material is quite fluid, and the precipitation of the chromate of lead which takes place in the vessels themselves gives a fine sulphur-yellow colour. Mr. Topping prepares many fine injections in this way.

Mr. Goadby has improved upon the process last named by uniting to the chemical solutions a portion of gelatine, as follows:

Saturated solution of bichromate of potash, 8 fluid ounces; water, 8 ounces; gelatine, 2 ounces.

Saturated solution of acetate of lead, 8 fluid ounces; water, 8 ounces; gelatine, 2 ounces.

The majority of preparations thus injected require to be dried and mounted in Canada balsam. Each preparation, when placed on a slip of glass, will necessarily possess more or less of the coloured infiltrated gelatine, (by this is meant the gelatine coloured by the blood, which, together with the acetate of potash resulting from the chemical decomposition, may have transuded throngh the coats of the vessel,) which, when dry, forms, together with the different shades of the chromate of lead, beautiful objects, possessing depth an't richness of colour. The gelatine also separates and defines the different layers of vessels: the arteries are always readily distinguishable by the purity and brightuess of the chromate of lead within them, while the veins are detected by the altered colour imparted by the blood.

Those preparations which require to be kept wet can ke 
preserved perfectly in Mr. Goadby's No. 2 fluid, specific gravity $1 \cdot 100$; the No. 1 fluid destroys then.

"I would recommend that the slips of glass employed for the dry preparation be instantly inscribed with the name of the preparation, written with a diamoud; for, when dry, it is difficult to recognise one preparation from the other, until the operator's eyc be educated to the effects of this chemico-gelatinous injection. Where so much wet abounds, gummed paper is apt to come off. When dry, it is sufficient, for the purpose of brief examination by the microscope, to wet the surface of a preparation with clean oil of turpentine; immediately after examiuation, it should be put away carefully in a box, to keep it from the dust, until it can be mounted in Cauada balsam.

"The bichromate of potash is greatly superior in colour to the chromate, which yields too pale a yellow; and subsequent experience has proved that the acetate of potash frequently effects its liberation by destruction of the capillaries, and this even long after the preparations have been mounted in Canada balsam; perhaps this may be owing to sume chemical action of the acetate of putash upon them. I would suggest the substitution of the nitrate for the acetate of lead, as we should then have, in the liberated nitrate of potash, a valuable auxiliary in the process of preservation. Although lighly desirable, as the demonstrator of the capillaries of normal tissues, I do not think this kind of injection fitted for morbid preparations; the infiltrated gelatine producing appearances of a puzzling kind, and calculated to mislead the pathologist. In preparing portions of dried well-injected skin for examination by the microscope, I have tried the effect of dilute nitric acid as a corroder with very good results. But, probably, liquor potassa would have answered this purpose better.

"When size-injection is to be employed, coloured either with vermilion or the chromate of lead, the animal should be previously prepared by bleeding, to empty the vessels; for if they be filled with coagulated blood, it is quite impossible to trausmit even size, to say nothing of the colouring matter. Hence the difficulty of procuring gcod 
injections of the human subject. But with the chemicogelatinous injections no such preparation is necessary ; and success should always be certain, for the potash liquefies the blood, while constant and long-continued pressure by the syringe drives it through the parietes of the vessel into the cellular tissue."

Transparent Injections.- "Much more strongly," writes Dr. Beale, "can I recommend to you the use of transparent fluids for making injections. It is true, that these are not likely to be so much admired by general observers as opaque injections. Indeed, it is not easy to find any object which will rival in beanty many tissues that have been freely injecterl with vermilion or chromate of lead; althongh it must be confessed that from such preparations we learn but little save the general arrangement of the capillary vessels of the part, their capacity, and the mag. nitude of the meshes of the network. Of the relations which these ressels bear to the elementary structures which give to the texture under examination its peculiar properties, such preparations tell us nothing. Opaque injections are for the most part only adapted for exannination with low powers, while the tissues to which the vessels are distributed can only be seen with the help of very high magnifying powers. Transparent injections, on the other hand, though they fail to excite the wouder of the uninitiated, show us not only the general arrangement of the capillary network, but the precise relation which each little tube bears to the tissue with which it is in contact.

"In order to make injected preparations for examination by transmitted light, several different substances may be used as injecting fluids.

"Injection with Plain Size.-A tissue which has been in. jected with plain size, when cold is of a good consistence for obtaining thin sectious, and many important points may be learned from a specimen prepared in this manner which would not be detected by other modes of preparation. A mixture of equal parts of gelatine and glycerine is, however, much to be preferred for this purpose, and the specimen thus prepared is sure to keep well.

"Colouring Matters for Transparent Injections. - The chief 
colouring matters used for making transparent injections are carmine and Prussian blue. The former is prepared by adding a little solution of ammonia (liqun ammoniæ) to the carmine, and diluting the mixture until the proper colour is obtained, or it may be diluted with size.

"The Prussian Blue consists of an insoluble precipitate, so minutely divided, that it appears like a solution to the eye. The particles of freshly prepared Prussian blue are very much smaller than those of any of the colouring matters employed for making opaque injections.

"Advantages of Employing Prussian Blue.-I have lately" been employing Prussian blue very much, and according to my experience it possesses advantages over every other colouring matter. It is inexpensive,-may be injected cold, - the preparation does not require to be warmed,no size is required-it penetrates the capillaries without the necessity of applying minch force,-it does not run out when a section is made for examination,-neither do any particles which may escape from the larger vessels divided in making the section, adhere to it and thus render the section obscure, - a structure may be well injected with it in the course of a few minutes. Specimens prepared in this manner may be preserved in any of the ordinary preservative solutions, or may be dried and mounted in Canada balsam, (but I give the preference to glycerine, or glycerine jelly,) and they may be examined with the highest magnifying powers. After liaving tried very many methods of making this preparation I have found the following one to succeed best.

"Composition of the Prussian Blue Fluid for MLaking Transparent Injections :-

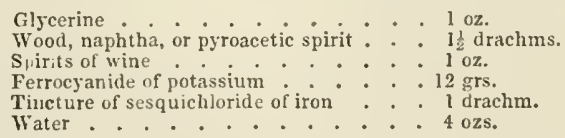

"The ferrocyanide of potassium is to be dissolved in one ounce of the water, and the tincture of sesquichloride of iron added to mother ounce. These schutions should be mixed together very gradually, and well shaken in a bottle 
The iron being added to the solution of the ferrocyanide of potassium. When thoroughly mixed, these solutions should produce a dark blue mixtmre, iu which no precipitate or flocculi are observable. Next, the naphtha is to be mixed with the spirit, and the glycerine and the remaining two ounces of the water added. This colourless fluid is, lastly, to be slowly mixed with the Prussian blne, the whole being well shaken in a large bottle during the admixture. The tincture of sesquichloride of iron is recommended because it can always be obtained of uniform strength. It is generally called the muriated tincture of iron, and may always be purchased of druggists.

"Permit me, then, most earnestly to recommend all who are fond of injecting, to employ transparent injections, and to endeavour, by trying various transparent colouring matters, to discover several which may be employed for the purpose; for I feel sure that by the use of carefully prepared transparent injections, many new points in the anatomy of tissues will be made out.

"Of Injecting Different Systems of Vessels with Different Colours.-It is often clesirable to inject different systems of vessels distributed to a part with different colours, in order to ascertain the arrangement of each set of vessels and their relation to each other. A portion of the gallbladder in which the veins have been injected with white lead, and the arteries with vermilion forms a beantifui preparation. Each artery, even to its smallest branches, is seen to be accompanied by two small veins, one lying on either side of it.

"In this injection of the liver, four sets of tubes have been injected as follows:-The artery with vermilion, the portal vein with white lead, the duct with Prussian blue, and the hepatic vein with lake. There are many opaque colouring matters which may be employed for double injections, but I am acquainted with very few transparent ones, the employment of which affords very satisfactory results.

"Mercurial Injections are not much used for microscopical purposes, although mercury was much employed formerly for injecting lymphatic vessels and the ducts of glandular organs. The pressure of the column of mercury 
supersedes the necessity of any other kind of force for driving it into the vessels. The mercurial injecting apparatus consists of a glass tube, about half an inch in diameter and twelve inches in length, to one end of which has been fitted a steel screw to which a steel injecting pipe may be attached. The pipes and stopcocks must be made of steel, for otherwise they would be destroyed by the action of the mercury.

"Injecting the Lower A nimals. - The ressels of fishes are exceedingly tender; and require great caution in filling them. It is often difficult or quite impossible to tie the pipe in the vessel of a fish, and it will gemerally be found a much easier process to cut off the tail of the fish, and put the pipe into the divided vessel which lies immediately beneath the spinal column. In this sirnple manner beautiful injections of fish may be made.

"Mollusca.-(Shrg, snail, oyster, \&c.) The tenuity of the ressels of the mollusca often renders it impossible to tie the pipe in the usual manner. The capillaries are, however, usually very large, so that the injection runs very readily. In different parts of the bodies of these animals are numerous lacunæ or spaces, which communicate directly with the vessels. Now, if an opening be made through the integument of the muscular foot of the animal, a pipe may be inserted, and thus the vessels may be injected from these lacunæ with comparative facility.

"Insects.-Injections of insects may be made by forcing the injection into the general abdominal cavity, when it passes into the dorsal vessel and is afterwards distributed to the system. The superfluous injection is ther washed away, and such parts of the body as may be required, removed for examination.

"Of the Practical Operation of Injecting.-I propose now to inject a frog and the eye of an ox, in order that you may see the several steps of the process. We must bear in mind that a successful injection cannot be made until the muscular rigidity which comes on shortly after death, and which affects the muscular fibres of the arteries as well as those of the muscles themselves, has passed off. In some few instances in which the fluid does not necessarily pass through arterial trunks before it reaches the 
capillaries (as in the liver), the injection may be effected satisfactorily immediately after the death of the animal.

"The steps of the process are very similar in making the opaque injections, except that when size is employed, the specimen must be placed in warm water until warm throngh, otherwise the size will solidify in the smaller vessels, and the further flow of the injecting fluid will be prevented. Soaking for many hours is sometimes necessary for warming a large preparation thoronghly, and is is desilable to change the water frequently. The size must also be kept warm, strained immediately before use, and well stirred up each time the syringe is filled.

"In the first place, the following instruments must be conveniently arranged :-

"The syringe thoroughly clean and in working order, with pipes, stopcock, and corks.

"One or two scalpels.

"Two or three pair of sharp scissors.

"Dissecting forceps.

"Bull's-nose forceps, for stopping up any vessel through which the injection may escape accidentally.

"Curved needle, threaded with silk or thread, the thickness of the latter depending upon the size of the vessel to be tied.

"Wash-bottle. Injecting fluid in a small vessel.

"I will commence with the frog. An incision is made through the skin, and the sternum divided in the middle line with a pair of strong scissors; the two sides may ensily be separited, and the heart is exposed. Next the sac in which the heart is contained (pericardium) is opened with scissors and the fleshy part of the heart seized with the forceps; a small opeuing is made near its lower part, and a considerable quantity of blood escapes from the wound-this is washed away carefully by the wash-bottle. Into the opening - the tip of the heart being still held firmly by the forceps, a pipe is inserted and directed upwards towards the base of the heart to the point where the artery is seen to be connected with the muscular substance. Before I insert the pipe, however, I draw up a little of the injecting fluid so as to fill it, for if this were not done, when I began to inject, the air contained in the 
pipe would necessarily be forced into the vessels, and the injection would fail.

"The point of the pipe can with very little trouble be made to enter the artery. The needle with the thread is next carried round the vessel and the thread seized with forceps, the needle unthreaded and withdrawn, or one end of the thread may be held firmly, while the needle is withdrawn over it in the opposite direction. The thread is now tied over the vessel, so as to include the tip of the pipe only, for if the pipe be tied too far up, there is greater danger of its point passing through the delicate coats of the vessel.

"The nozzle of the syringe, which has been well washed in warm water, is now plunged beneath the surfice of the fluid, the piston moved up and down two or three times, so as to force out the air completely, and the syringe filled with fluid. It is then connected with the pipe, which is firmly held by the finger and thumb of the left liand, with a screwing movement, a little of the injection being first forced into the wide part of the pipe so as to prevent the prossibility of any air being included.

"The pipe and syringe being still held with the left hand, the piston is slowly and gently forced down with a slightly screwing movement with the right, care being taken not to distend the vessel so as to endanger mupture of its coats. The handle of the syringe is to be kept uppermost, and the syringe should never be completely emptied, in case of a little air remaining, which would thus be forced into the vessels. The injection is now observed rumning into the smaller vessels in differeut parts of the organism.

"I will now proceed to inject the ox-eye in the same manner. The pipe is inserted into this branch of artery close to the nerve. Two minutes will probably be sufficient to ensure a complete injection. In making an injection of the eye, if the globe becomes rery much distended by the entrance of the injecting fluid, an opening may be made in the cornea to allow the escape of the aqueous fluid which will leave room for the entrance of the injection, and permit the complete distension of the ressels. 
"We will now examine these injections. A portion of the intestine of the firog may be removed with scissors, opened, and the mucous surface washed with the aid of the wash-bottle. It may be allowed to soak in glycerine for a short time, and then examined.

"This portion of the delicate choroidal membrane which has been carefully removed in the same manner shows the vessels perfectly injected, and in this preparation of the ciliary processes you will not fail to observe that all the capillary vessels are fully distended with fluid, although the injection was made so quickly.

"Of' Injecting the Ducts of G'lands.-The modes of injecting which we have just considered, although applicable to the injection of vessels, are not adapted for injecting the ducts and glandular structure of glands; for as these ducts usually contain a certain quantity of the secretion, and are always lined with epithelium, it follows that when we attempt to force fluid into the duct, the epithelium and secretion must be driven towards the secreting structure of the gland, which is thus effectually plingged up with a colourless material, and there is no possibility of making ont the arrangement of the parts. In such a case it is obvionsly useless to introduce an injecting fluid, for the greatest force which could be employed would be insufficient to drive the contents through the basement membrane, and the only possible result of the attempt would be rupture of the thin walls of the secretiug structure and extravasation of the contents. As I have before mentioned, partial success has been obtained by employing mercury, but the preparations thus made are not adapted for microscopical observation.

"After death the minute ducts of the liver always contain a little bile. No force which can be employed is sufficient to force this bile throngh the basement membrane, for it will not permeate it in this direction. When any attempt is made to inject the ducts, the epithelinm and mucas, in their interior, and the bile, form an insurmountable barrier to the onward course of the injection. Hence it was obviously necessary to remove the bile from the ducts before on culld hope to make a successful injection. It occurred to me, that any accumulation of fluid in the smallest 
branches of the portal vein or in the capillaries, inust necessarily compress the ducts and the secreting structure of the liver which fill up the intervals between them. The result of such a pressure would be to drive the bile towards the large ducts and to promote its escape. Tepid water was, therefore, injected into the portal vein. The liver' became greatly distended, and bile with much ductal epithelium flowed by drops from the divided extremity of the duct. The bile soon became thinner, owing to its dilution with water, which permeated the intervening membrane, and entered the ducts. These long, narrow, highlytortuous channels were thus effectually washed out from the point where they commenced as tubes not more than 1-300th of an inch in diameter, to their termination in the common duct, and much of the thick layer of epithelium lining their interior was washed out at the same time. The water was removed by placing the liver in cloths with sponges under pressure for twenty-four hours or longer. All the vessels and the duct were then perfectly empty and in a very favourable state for receiving injection. The duct was first injected with a coloured material. Freshly precipitated chromate of lead, white lead, vermilion, or other colouring matter may be used, but for mauy reasous to which 1 have alluded, the Prussian blue injection is the one best adapted for this purpose. It is the only material which furnishes good results when the injected preparations are required to be submitted to high magnifying powers. Preparations injected in this manner should be examined as transparent objects." I

Of Preparing Portions of Injected Preparations for Microscopical Examination. - When thin tissues, such as the mucous membrane of the intestines or other parts, have been injected, it is necessary to lay them perfectly flat, and wash the mucus and epithelium from the free surface, either by forcing a current of water from the washbottle, or by placing them in water and brushing the surface gently with a camel's hair brush. Pieces of a convenient size may then be removed and momnted in a solution of naphtha and creosote, in dilute alcohol, in

(1) Dr. L. Beale, "On the Anatomy of the Liver of Man and Vertebrate Animals." 
glycerine, or in gelatine and glycerine. The most im. portant points in any such injections are shown if the preparation be dried and mounted in Canada balsam. The specimen must, in the first place, be well washed and floated upon a glass slide with a considerable quantity of water, which must be allowed to flow off the slide very gradually. The specimen may then be allowed to dry under a glass shade, in order that it may be protected from dust. The drying should be effected at the ordinary temperature of the air; but it is much expedited if a shailow basin filled with sulphuric acid be placed with it under a bell-jar."

Chemical Re-agents. - The following chemical re-agents and preservative fluids are recommended for microscopic uses : ${ }^{1}$

1. Alcohol, principally for the removal of air from sections of wood and other preparations; also as a solvent for certain colouring matters.

2. Ether, chiefly as a solvent for resins, fatty and other essential oils, \&c. ; also useful for the removal of air.

3. Solution of Caustic Potass, as a solvent for fatty matters; also of use occasionally in consequence of its action upon the rest of the cell-contents and thickening layers. This solution acts best upon being heated.

4. Solution of Iodine (iodine one grain, iodide of potassium three grains, distilled water one ounce) for the coloration of the cell-membrane and of the cell-contents.

5. Concentrated Sulphuric Acid, employed chiefly in the examination of pollen and spores.

6. Diluted Sulphuric Acid (three parts acid, one part water), for the coloration of cells previously immersed in the iodine solution. The preparation is first moistened with the iodine solution, which is afterwards removed with a hair pencil, and a drop of sulphuric acid added by means of a glass rod; the preparation is then immediately covered with a piece of glass. The action of the sulphuric acid and iodine, as well as that of the iodised chloride of zinc solution, is not always uniform throughout the whole

(1) A set of 12 test-bottles, packed in a small box, is supplied by $\mathrm{Mr}$. Matthews of Portugal Street, Lincoln's Inn Fields, the price of which is only a few shillings. 
surface of the preparation. The colour is more intense where the mixture is more concentrated; it frequently happens that many spots remain uncoloured. The colour changes after some time, the blue being frequently changed into red after twenty-four hours.

7. A Solution of Chloride of Zinc, Iodine, and Iodide of Potassium. A drop of this compound solution, added to a preparation placed in a little water. produces the same colour as iodine and sulphuric acid. This solution, which was first proposed and employed by Professor Schultz, is more convenient in its application than iodine and sulphuric acid, and performs nearly the same services, while it does not, like the sulphuric acid, destroy the tissues to which it is applied. It is prepared by dissolving zine in hydrochloric acid; the solution is then saturated with iodide of potassium: more iodine is to be added if necessary, and the solution diluted with water.

8. Nitric Acid, or what is better, chlorate of potass and nitric acid, as an agent to effect the isolation of cells. The mode of employing this agent, also discovered by Professor Schultz, is as follows:-

The object, a thin section of wood, for instance, is introduced, with an equal bulk of chloride, or chlorate of potass, into a long and moderately wide tube, and as much nitric acid as will at least cover the whole.

The tube is then warmed over a spirit-lamp; a copious evolution of gas takes place, upon which the tube is removed from the flame, and the action of the oxidising agent allowed to continue for two or three minutes. The contents of the tube are then poured into a watch glass with water, from which the slightly cohering particles are collected and placed in a tube, and again boiled in alcohol as long as any colour is communicated.

They are again boiled in a little water. The cells may now be isolated under the simple microscope by means of needles. The boiling with nitric acid and chlorate of potass should never be carried on in the same room with the microscope, the glasses of which may sufier injury from the vapours. The same remark applies to all chemical vapours.

Thin sections of vegetable tissue are warmer for half a 
minute, or a minute, in a watch-glass: boiling is here unnecess.ury. The section is taken out, and treated with water in another watch-glass.

9. Oil of Lemons, or any other essential oil, a drop of which will be found of value in the investigation of pollen and spores.

Lastly may be enumerated a pretty strong solution of Carbonate of Soda and also of Acetic Acid; which latter, however, is more especially useful in the investigation of animal tissues.

To the above may be added a test for protein compounds. This test is composed of sugar and sulphuric acid, and is thus employed:-A thin section or portion of the tissue to be examined is placed in a drop of simple syrup, this is then removed by means of a hair pencil, and a drop of the diluted sulphuric acid added; the red colour usually does not appear until after the lapse of about ten minutes.

In making thin sections of tissues, it is reconmended that, in those objects the consistence of which differs in different parts, the section should be carried from the harder into the softer portion; also, in making a thin section of a very minute yielding substance, to enclose it between two pieces of cork, and to slice the whole together. It is also useful sometimes to saturate the object with mucilage, which is to be allowed to dry slowly; in this way very delicate tissues may be sliced, or otherwize divided without injury, and with great facility.

Some of the above re-agents musc be used with caution, as it is not unusual for them to assume crystalline forms while under the microscope. Without a knowledge of th is fact, and a perfect recognition of crysialline forms, error's in microchemical research must occur. For example, if a drop of liquor potassæe be allowed to evaporate on a slip of glass, crystals appear, chiefly of six-sided tables, precisely like cystine; when in quantity, they are often crowded together as the cystine plates are, and sometimes exhibit a similar. nucleus-like borly in their centres. This peculiarity of crystallization does not arise from the presence of impurities; perfectly pure potash often exhibits the same phenomenus.

The form of the crystals of acetate of potash varics according to the strength of the acid out of which it 
crystallizes, and if formed out of strong acid, very much resembles that of the crystals of uric acid; when mixed up with otlier forms, long dagger-like or lascet-shaped crystals are seen, which might well deceive.

We may also notice in this place what Majendie pointed out, that in certain albuminous mixtmes, iodine loses the property of colouring starch blue. This difficulty must be got rid of before iodine can be said to be an infallible test in micro-chemistry.

Collecting Objects.-Mr. G. Shadbolt contributes the following useful hints for collecting objects for microscopical examination :-

" livers, brooks, springs, fountains, ponds, marshes, bogs, and rocks by the sea-side, are all localities that may be expected to be productive; some being more prolific than others, and the species obtained differing, of course, in genera], to a certain extent, according to the babitat. On considering the natme of some of the places indicated, it will be apparent that, in order to spend a day in collecting with auy comfort, it will be necessary to make some provision for keeping the feet dry, for which a pair of India-rubber goloshes will answer, or better still, a pair of waterproof fishing-boots; but without one or other the work is liy no means pleasant.

"A dozen or two of small bottles made of glass-tubing, about half an inch in diameter, and without necks, and from one to two inches in length, are the most convenient depositories for the specimens, if intended ultimately for mounting; and it is advisable also to take two or three wide-mouthed bottles of a larger size, holding from one to two fluil-ounces, an old iron spoon, a tiu hox, some pieces of linen or calico, two or three inches square, a piece of string, a slip or two of glass, with the edgres ground, such as are used for mounting objects; and lastly, a good and pretty powerful hand-magnifier. Two Coildington lenses, mounted in one frame of about balf an inch, and one-tenth of an inch in focal power, are specially couvenient.

"Swanscombe Salt-marsh will be found well worth a visit; and it can be reached by stearn-boat or railway from London-bridge to Nortlufleet. On quitting the railway station, make towards the alnushouses on the top of 
the hill; and arriving at the road, turn to the left, descend the hill, and cross a sort of bridge over a somewhat insignificant stream. Continue along the main-road a little firther, to a point where it begins to ascend again, and diverges to the left towards the railway; here quit it, taking your course along an obscure road, nearly in a dircet line with the main one; passing a windmill on the right hand, and contiming until you arrive at another still more obscure road, turning off to the right; which road appears as if made of the mud dredged from the bottom of the river, and partially hardened. This is Swanscombe Salt-marsh; and the road just described leads towards Broad Ness Beacon. On either side is a sort of ditch; one containing salt or very brackish water, the other filled with a sort of black mud, about the consistence of cream, the surface being in parts of a slaty grey, with little pitches here and there of a most brilliant brown colour, glistening in the sunshine, and presenting a striking contrast to the sombre shade. By carefully insimuatiug the end of one of the slips of glass under this briliant brown substance, and raising it gently, it can be examined with the Coddingten; and it will probably be formd to consist of myriuds of specimens of Pleurosigma (navicula of Ehrenberg) angulatum, or balticum, or some other species of this gemus. The iron spoon is now useful, as by its aid the brown stratum, with little or no mud, can be skimmed off and bottled for future examination. On the surface of the water in the other diteh may be noticed a floating mass of a dark olive colour, which to the touch feels not unlike a lump of the curd of milk, and consists of Cyclotella menighiniana, and a surivella or two embedded in a mass of Spirulina hutchinsia; and another mass of fioating weed, which feels harsh to the touch, proceeding from a quantity of a synedra, closely investing a filamentons alga; and elsewhere Melosira nummuloides (gallionella of Ehrenberg).

"In a trench by the seawall, as it is termed, is a mass of brown matter of a shade somewhat different to any hitherto observed, adhering to some of the parts of the trench, being partially submerged, and having a somewhat tremulous motion on agititng the water. This is a 
species of Schizonema; and it consists of a quantity of gelatinous hollow filaments filled with an immense number of bright-brown shuttle-shaped bodies, like ver'y minute navicule.

"It is not necessary to be particular alout collecting the specimen free from mud, as the filaments are so tough that the mud can be readily washed away by shaking the whole violently in a bottle of water, and pouring off the mud, without at all injuring the specimen. The $A m p h i$ porium alatum communicates a somewhat frothy appearance to the otherwise clear water, and to get any quantity of this requires a little management; but by skimming the surface with the spoon, and using one of the larger sottles, an abundance may readily be obtained. Between the sea-wall and the river the marsh is intersected in every direction with a number of meandering creeks, being in some places eight to ten feet deep, though in others quite shallow; but it is exceedingly difficult to make one's way amongst them, and I have never found them so prolific any where, on the few occasions of my visiting the place, as in the parts more away from the influence of the tide. It will be observed, that the brilliant brown colour, of a deep but bright cimmamon tint, is one of the best indications of the presence of diatomacece; and though this is by no means universal, the variation is most frequently dependent upon the presence of something which qualifies the tint. The peculiarity of the colour is due to the endochrome contained in the frustule; and this must in general be got rid of before the beantiful and delicate marking can be made out. But it is highly advantageous and instructive to view them in a living state; and this should be done as soon as possible after reaching home with all specimens procured from salt-water localities, as they rapidly putrefy in confinement, and emit a most disgusting odour, not unlike that arising from a box of iuferior congreve-matches.

"Washing in fresh water, and then immersing in creosote water, preserves many of the species in a very naturallooking manner; but they are killed by the fresh water, and the endochrome becomes much condensed in the Pleurosigmata and some other species. The addition of 
spirits quite spoils the appearance of the frustules, as it dissolves the endochrome.

"There is another salt-marsh a little farther down the same railway, at Higham, which it would be well to explore. The most favourable months for procuring diatomaceo, are April, May, September, and October; but some species are found in perfection as early as February, and many as late as November, and a few at all times of the year. There is a piece of boggy ground near Keston, beyond Bromley, in Kent, where the river Ravensbourne takes its rise, where many interesting species of desmidacece and other fresh-water algæ may bo procured. From Bromley, walk on towards Keston, passing near Hayes Common and Bromley Common on the right. Continue for about another half-mile along the road, and then turn to the right hand; pass the reservoirs, and approach au open space where there is a bog of about a quarter of a mile in extent; and tending towards the right, make your way amongst heaths, ferns, mosses, and the beautiful Drosera rotundifolia (sun-dew), to the lower part of the little stream rippling through a sort of narrow trench in the Sphagnum, \&c. By working your way up the stream, you avoid the inconvenience which would otherwise be experienced of the water being rendered turbid, in consequence of having to tread in the boggy ground. In the centre of the little stream may be observed something of a pale pea-green colour flickering about in the current, which, on your attempting to grasp, most likely eludes you, and slips through the fingers, from being of a gelatinous nature. It consists of a hyaline substance, with a comparatively small quantity of a bright green endochrome, disposed in little branches, and this is the Draparnaldia glomerata. Another object is a mass of green flameuts, rather harsh to the touch, and very slippery. When viewed with a lens of moderate power, each filament is seen to be surrounded with several bands of green dots, looking like a ribbon twisted spirally, and may be recognised as a species of Zygnema. In various parts there are other Zygnemacece, as spirogyra, mougeotia, mesocarpus, and many more.

"Keeping up the stream, and occasionally diverging a 
little on either side of it, amongst the miniature vays and pools formed by the sphagnum, on looking straight down iuto the water we shall probahly see at the bottom a little mass of jelly of a bright green, studded with numerous brilliant bubbles of oxygen-gas. This is the genera] appearance of most of the desmidacece, as Micrasterias, Euastrum, Closterium, Cosmarium, \&e. The spoon is also a handy tool in this case, though, by practice, the finger will do nearly as well; the ehief difficulty arises when the specimen is brought to the surface of the water, it not being easy to get it out without losing a cousiderable portion of it. Little pools in the bog, made by the footsteps of cattle, are particularly good spots to find desmidiece, many species being in a very contracted space. The most prolific bog is at Tunbridge Wells, near a house known as Fisher's Castle, not far from Hurst Wood. There is also a good one at Esher, at a spot called W'est-End. It must not be imagined that nothing can be obtained in this department of botany without going some distance from town; but assuredly only commoner and fewer species can be met with nearer home. At the West India Docks are Synedra fasciculata, Gomphonema curvata, Diatoma elongatum, Diatoma vulgare, Surirella ovata, \&c.; and at this same place a few objects, not of the botauical class, as Spongilla fluviatilis, Cordylophora lacustris, Alcyonella, stagnorum, \&c., are obtainahle in abundance in the autumn. In the oruamental water in St. James's Park may be found. Cocconema lanceolatum, and other species of this genus, Gomphonema cristatum, \&c. Epping Forest, about the neighbourhood of Leytonstone, Snaresbrook, Wanstead, and $W^{\prime}$ oodford Bridge, are also capital localities for the flamentous algæ, especially the last-named, where Nitella translucens and Chara vulgaris abound." 1

On the north side of the Serpentine, Hyde Park, especially near the bridge, may be found :-

Cymbelia maculata.

Gomphonema cristatum.

Scenedesmus quadricauda. obliquus.

Ankisirodesmus faicatus.

Pediastrum Heptactys.

Cocconema lanceolatum.

Ampliora uvalis.
Cocconeis placentula.

Uvella hyaliua.

Gallionella nummuloides.

Euastrum elegans.

Pixidula operculata.

Cladophora glomerata and Sphaero. plea crispa, with many olbes algæ.

(1) "Quarterly Journal of Microscopical Sciense." 
Mr. Wheeler, of Tollington Road, Holloway, N., has furnished us with the following list of 100 interesting and popular objects, in book-case complete :-

Tests:

P'eurusigma angu!ata.

" strigosa.

" formosum.

Scales of Podura.

Haï Lepisma.

" mouse.

" Larva of Dermestes.

Triceratium from Thames mud. Arachnoidiscus.

Fos. infusoria from Guano.

Fos. infusoria, Barbadoes.

" Upper Bann, Ireland.

" lslind of M all].

"Tuscany.

Infusoria from Thames mud.

Spicules of Gorgonia.

" sponge. fresh water.

Gemmules of sponge.

Section of shell-Pinna.

" " crab.

" ". llalintis.

" spine of Echinus.

" , Cidaris.

Xantlidia in flint.

Moss arate.

Limestore.

Fossil tooth of shark.

$"$ fish.

Fossil bone of whale.

$\begin{array}{ll}" & \text { reptile. } \\ & \text { elk. }\end{array}$

Fossil wood (Exoten).

Section's of coal.

(Endogen).

Simple cellular tissue.

Stellate tisiue.

Fibro-celiular tissue.

Spiral vessels.

Hairs from leaf of Deutzia.

Seeds of a fern.

Sections of fir.

", $\quad \begin{aligned} & \text { mahogary. } \\ & \text { clematis. }\end{aligned}$

Petal of geranium.

Leaf insect.

Flea.

Parasite of peacock.

Skin of caterpillar.

Wing of a butterfiy.
Scales of ditto.

t'roboscis of blow-fly.

Siomach of ditto.

Foot of ditto.

Spiracles of Dytiscus.

Foot of Ophion.

Proboscis of motl.

Tran. sects. of human hairs.

$$
\text { " hairs of elephant. }
$$$$
\text { "wlialebone. }
$$

Feather of bird.

Trans, sects. of human bone. " bone of bird.

" $\quad$ " $\quad$ reptile.

Blood of bird.

" fish.
" reptile.

Opaque :-

Gold dust.

Fossil shells.

Pollens.

Fern spores.

Neelle antimony.

A vanturine.

Polariscope :-

Selenite.

Starch.

Hairs from leaf.

Fimbryo oysters.

Rhinoceros hom.

Hoof of horse.

Agate.

Sandstone.

Sulphale of Cadmium.

Salicine.

Tartaric acid.

Carbonate of lime.

Anatomical :-

Section of cartilage, shoving the formation of the hone-cells.

Muscle of mamnalia.

$\begin{array}{ll}" & \text { reptile. } \\ " & \text { bird. } \\ \text { nfish. }\end{array}$

Injections of :Human lung.

" intestine.

"skin.

" kidney.

" stomach.

" muscle.

" section of finger. 
Mr. Collins has introduced a very complete Mounting. Case, which will prove useful to microscopists, especially so to those who devote a good deal of attention to the preparation of specimens. A place is here found for everything: the little box contains :- Shadbolt's turntable, brass table, spirit-lamp, pipettes, spring clips, wooden clips, tweezers, tin cells, balsam, marine glue, asphalte, turps, gold size, thin glass covers, glass slips, and five extra bottles. The price of this neat and convenient case is $30 s$. Another box, more particularly adapted for anatomical purposes, includes a neat injecting apparatus.

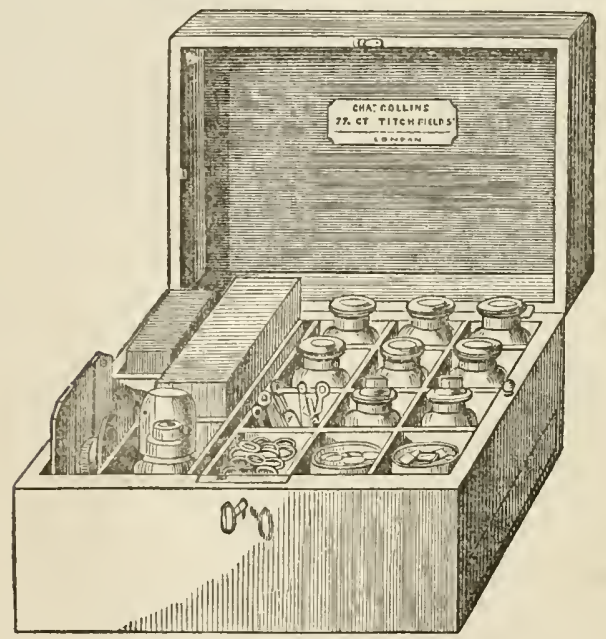

Fig. 143.-Collins' Mounting Cabinet.

A modification of Dr. Beale's neutral-tint glass for drawing is made by Mr. Collins, who supplies three glasses of different shades, which drop into a dovetail, so that they can be more easily cleaned and efficiently adapted to the power used; with his more complete instruments a prism is also supplied. 


\title{
P A R T II.
}

\begin{abstract}
THE VEGETABLE KINGDOM-VITAL CHARACTERISTICS OF CELLS-THE P.ROTOCOCCUS PLUVIALIS - OSCILIATORIE-FUNGI-ALG E-DESMIDACE $E-$ MOSSES-FERNS-STRUCTURE OF PLANTS-STARCH-ADULTERATION OF ARTICLES USED FOR FOOD-PREPARATION OF VEGETABLE STRUCTURES,
\end{abstract} ETC.

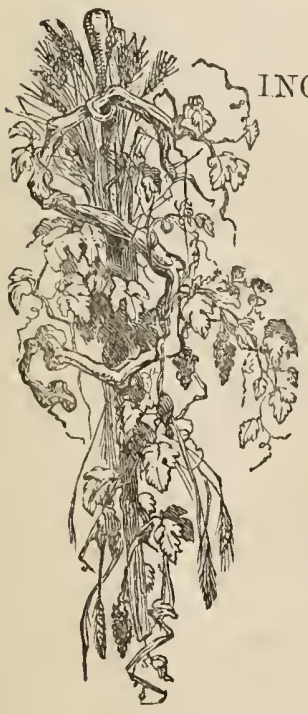

CE the introduction of the achromatic microscope, we have obtained nearly the whole of the valuable information we possess of the minute structure of plants. Indeed in no department of nature has microscopic investigation been more fertile of results than in that of the vegetable kingdom. Tlie humblest tribes of plants have had for microscopists an attraction,unequalled by that of any other department of nature,-from the time of our countryman Robert Brown, down to the present day. Although Brown had observed and recorded certain facts in the physiology of vegetable life, it was Professor Schleiden's labours that brought to light the great truth, "that the life-history of the individual cell is the first important and indispensable basis whereon to found a true physiology of the life-history of plants, as well as that of the higher orders of creation." The first problem 
which this observer set himself to solve, was, How is the cell oriyinated? It is not here desirable to go deeply into this most interesting inquiry; the object proposed is to give a slight sketch of the formative processes of plant life, chicfly in its relation to the earlicst, or cell condition. Almost every day brings forth a new discovery by which old land-narks estailished with a view to tire separation, arrangement, and classification of the vegetable and animal kingdoms become unsettled; the lowest forms of life, vegetable and animal, approach so near to each other, that we camnot with certainty always discriminate them, and say where the one ends and the other begins. The boundary assigned to the vegetable kingilom is, perhaps, too limited, and our definition of a vegetable organism requires to be enlarged: of this any one who will be at the trouble to study the microscopic forms of life must feel perfectly convinced. At the present day, the only generally applicable rule that can be applied to listinguish animals from vegetables is the dependerce of the animal for nutriment upon organic compounds already formed, which it takes into the interior of its body; this at once distinguishes it from the plant, which only possesses the power of obtaining its alimentary matter, by absorption, from the inorganic elements by which it is surrounded. Although there appear to be certain exceptions to this rule, yet it almost universally prevails.

Kützing maintains, that every organic being is constituted of vegetable and animal elements, and according as the one or the other prevails, the being becomes an animal or a vegetable: in the first stages of the development of superior beings, and permanently in those of inferior rank, the two elements are equally balanced. "If nature," writes Humboldt, "had endowed us with microscopic powers of sight, and if the integuments of plants were transparent, the vegetable kingdom would by no means present that aspect of immolility and repose under which it appears to our senses." And so with regard to the instruments of motion in the higher classes of creation, the muscles of animals very soon disappear as we descend in the scale to the simplest forms of life; neveriheless, wo cannot deny animality to those minute crea- 
tures-as the Amøba-in which we are quite unable to distinguish either muscles or any other distinct organs. Hence there is always some danger of believing that to be simple which in reality only seems to be so, and which the minuteness or transparency of organization may only conceal from our limited power of vision.

Plants and animals, if seen at the earliest stage of existence, present themselves to our eyes as an aggregation of transparent cells. Everything prior to the appearance of the cell may, in the actual state of our microscopical knowledge, be considered as not fully and certainly demonstrated; and therefore it is incumbent upon us to take our starting-point from the simple cell, which is the same, in respect to its principal characters, in animals and vegetables. The external coating of a cell is nearly or quite solid and transparent, and with no indication of structure; while in its interior is found a liquid or solid substance, with a nucleus either adhering to its wall or within its cavity. A nucleolus can sometimes be demonstrated within the nucleus; and (a state common to all living cells) an incessant mutual interchange of materials is going on between the fluid contents and matter external to the cell, by a process termed osmose, or diffusion, which causes a perpetual variation in its relative condition. Chemical reagents give a manifestly different result in the animal and vegetable cell, hence we may conclude that there is an important difference in their chemical composition. The vegetable cell has an extremely fine delicate membrane lining the inner wall, to perceive which we must have recourse to reagents, and then we find the apparently simple cellwall made up of two layers, each differing in composition and properties. The inner layer has received the name of primordial utricle, and its composition has been shown to be albuminous; agreeing in this respect with the formative substance of animal tissues. The external layer is regarded as the cell-wall, although it takes uo part, essentially, in the formation of the cell ; it is composed of cellulin, a material allied to the cellulose of vegetable tissues. The contents are more or less coloured: the internal colouring substance is termed endochrome; when green it is called chlorophyll. 
The successive changes in the cell contents furnish other very important characteristics, such as the disappearance and re-absorption of the nucleus; this occurs in every cell at some period of its existence; in the cells of the higher plants, the inner membrane, or primordial utricle, entirely disappears. The Algie, and some few unicellular plants, form an exception to the rule. In the animal, the enlargement of the cell-wall takes place in a uniform manner, whereas in the plant this is effected by a deposition of successive layers on its inner surface, in the shape of continuous rings, spiral bands, or other intermediate forms. Then the wall not only insreases in size, but appears to possess a power of separating and appropriating certain substances, as lime, silica, lignine, \&c., which form the so-called cuticle. In animals as well as in plants, new cells are formed within the old cells; but in the former, this process of a new formation begins in the extracellular fluid, while in the latter it is mostly endogenous. Multiplication of vegetable cells is effected by three different modes: 1st, Many nuclei appear in the maternal cell floating together with granular matter; around each collects a minute vesicle, this gradually increasing fills the maternal cell, which is eventually absorbed. $2 \mathrm{~d}$, The internal substance of the cell divides into two or more portions, each being furnished with a nucleus. $3 \mathrm{~d}$, In the third mode of multiplication, the wall itself of the maternal cell becomes gradually constricted, and divides into two portions.*

* "In most cells, especially when young, a minute, rounded, colourless body may be seen, either in the midule or on one side, called the nucleus. This is very distinct in a cell of the pulp of an apple: and within this nucleus is often to be seen another smaller body, frequently appearing as a mere dor, called the nucleolus.

"The nucleus Is imbedded in a soft substance, which fills up the entire cell; this is the protoplasm (protos, first, plasma, formative substance). As it is very transparent, it is readily overlooked; but it may usually be shown distinctly by adding a little glycerine to the edge of the cover with a glass rod, when it contracts and separates from the cell-walls. The protoplasm in some cells is semi-solid, and of uniform consistence, while in others it is liquid in the centre, the outer portion being somewhat firmer, and immediately in contact with the cell-wall. In the latter case it forms an inner cell to the cell-wall, and is called the primordial utricle. The terms 'protoplasm' and 'primordial utricle' are however used by some authors symonymously.

"The protoplasm is the essential portion of the cell, and it forms or secretes the cell-wall upon its outer surface in the process of formation of the cell, consid rea as a whole. It is also of different chemical composition, from the cell wall being allied in this respect to animal matter."-Griffiths. 
Taking for our examination the more simple organisms among vegetables, we shall find numbers which present, in their earliest as well as in their permanent state, the cell in its simplest condition, and its reproduction a bare repetition of the same thing. Unicellular plants, then, in the strictest sense, are represented only by those in which the whole cycle of life is completely shut up in the one cell ; the first reconstruction or division being at once the commencement of a new cycle, in which, consequently, the whole vegetative life is run through in the same cell where the propagation also appears.
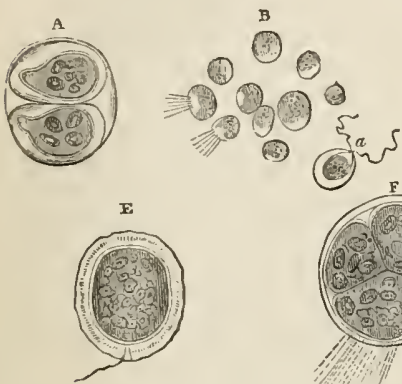

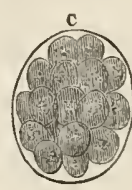

G
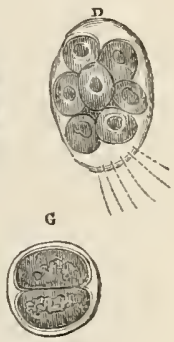

Fig. 144.-Cell Development. (Protococcus pluvialis.)

Protococcus phuvialis, Kützing. Hamatococcus pluvialis, Flotorv. Chlamidocrecus versatilis, A Braun. Chlamidococcus pluvialis. Flotow and Brann.

A, division of a simple cell into two, each primordial vesicle having developed a cellulin envelope around itself; B, Zoospores, after their escape from the cells; c, division of an encysted cell into segments: $D$, division of another cell, with vibratile filaments projecting from cell-wall; E, an encysted cell ; r, division of an enejsted cell into four, with vibratile filaments projecting; $a$, division of a Joung cell into two.

The most widely distributed of these single-cell plants is the Palmoglaea macrococca, of Kützing, which spreads itself as a green slime over damp stones, walls, \&c. If a small portion be scraperd off and placel on a slip of glass, and examined with a half or quarter-inch power, it will be seen to consist of a number of ovoid cells, having a transparent structureless envelope, nearly filled by a granular matter of a greenish colour. - At certain periods this mass divides into two parts, and ultimately the cell becomes two. Sometimes the cells are united end to end, just as we see 
them united in the actively-growing yeast plant; but in this case the growth is accelerated, apparently, by cold and damp. Another plant belonging to the same species, the Protococcus pluvialis, is found in every pool of water, the spores of which must be always floating in the air, since it appears after every shower of rain.

Unicellular plants occur in the series of Fungi and Algce, which have many and very varied correspondence in morphological respects. The unicellular Algæ-that is to say, Algæ, the contents of which, containing already organized particles, are inclosed in a single, semifluid envelope, anil this again in a cell-membrane, often consisting of several layers of different kinds; and many, moreover, possess the power of dividing into several secondary cells, for the most part equivalent to the primary cell. To this species of unicellular plant belongs Protococcus pluvialis. That this is the case is clearly seen in the still form of this plant, which is most distinctly characterised by its cell-membrane, a more or less thick though always colourless envelope. It never, however, secretes true thickening layers on the surface. Although this cellmembrane exhibits all the optical characters of one composed of cellulose, it is impossible to demonstrate the presence of that principle by means of iodine and sulphuric acid; it is not coloured by those reagents even after the contents of the cell have been expressed.

The contents vary much in consistence, colour, solid and fluid constituents; the red and green portions of which appear to be of equal physiological importance. The green colour is removed by ether, on the evaporation of which solvent there remain green as well as colourless drops. Dilute sulphuric acid at first renders the colour paler; but its prolonged action produces a bright green hue, which gradually becomes more and more intense, and often almost a blue-green. Hydrochloric acid has a similar effect; a tinge of brown is produced by nitric acid. Carbonate of potash scarcely affects the green colour; it is gradually but totally destroyed by caustic potash, the contents at the same time swelling and becoming trensparent.

The change of colour from green to red in Euglena 
appears to be a process very nearly allied to that which takes place in Protococcus, if it be not illentical with it. The red substance of Prot. pluvialis is not always of an oily aspect; it only becomes so in more alvanced age. And according to Cohn's researches, this oily material i. much more generally distributed than has been supposed, among the lower Algæ; occurring in many true brown spores, such as of Edogonium, Spirogyra, Vaucheria, \&c.

When still or motile cells of Protococcus are brought in contact with a very weak solution of iodine, they become internally, in most parts, of an intense violet or blue colour. With respect to the solid constituents of the Protococcus cell contents, they may be distinguished into chlorophyll vesicles, colourless or green particles, amylaceous granules, and nuclens. The motile form of Protococcus consists, as it were, of two cells, one within the other, both of which, however, differ essentially from the common vegetable cell : the external having a true cell-membrane and fluid contents ; the other, or internal one, with denser, muco-gelatinous coloured contents, but without a true cell-wall. Cohn called the external transparent vesicle the "enveloping" cell," and the internal coloured one the "primordial cell." The term "primordial sae, or utricle," can only be applied to its peripheral layer, and not to that together with the contents.

The form of Protococcus (fig. 144) presents a perfect analogy between the primordial cell and the nucleus of the common plant-cell. The filaments which proceed from the central mass to the peripheric cell-wall, are tubular, giving passage to the red molecules from the central mass. These filaments, however, which proceed from the outer wall of the primordial cell towards the inner surface of the enveioping cell, correspond morphologically to the so-termed mucous filaments by which the cytoblasts are commonly retained in the centre of their cells. That they also correspond chemically with these, is proved by the fact that they are rendered more distinct by iodine, and that they can be made to retract by means of reagents; and in fact they exhibit, in the course of development, peculiarities which characterise them as consisting of protoplasm. 
The existence of delicate threads passing from the central mass to the enveloping eells, and the appearance occasionally of little particles having molecular motion, serve to show that the contents of the enveloping cell are less of a gelatinous consistenee, than of a fluid nature. And the continuity of the primordial cell-wall with the filaments proves it is surrounded only with a layer of protoplasm, and is not inclosed in a dense membrane of cellulose. The most distinctive characteristic of the primordial cell, and what appears to constitute its most essential importance in the life of the cell in general, but particularly in that of the zoospore, consists in its boing the contractile element of the vegetable organism-that is to say, that from an intrinsic activity it possesses the faculty of altering its figure, without any corresponding change in volume.

'The Protococcus pluvialis has true motile organs, namely, two long vibratile cilia arising from the primordial cell (fig. 144, B, a), which, passing through two openings in the enveloping cell, move about in the water. These organs, draring the life of the cell, move so rapidly, that it is then diffieult to pereeive them; they are only recognisable by the currents they produce in the water. But when the motion is slackened they are evident enough. They are also rendered very distinct by iodine. They are always placed upon the extreme point of the conical elongation, on the anterior end of the primordial cell, and in such a manner as to appear to be immediate continuations of its substance ; and as that process itself consists of protoplasm, it is evident that the eilia must be regarded also as composed of the same substance. They resemble, in some respects, the so-called proboseis of certain Infusoria, such as Euglena and Monads, and do not differ very materially Irom the non-vibratile, retractile filaments of Acineta and Aetinophrys.

It is only that portion of the vibratile filaments beyond the enveloping cell that exhibits any motion, the portion within the outer cell being always motionless, and in that part of their course the filaments appear to be surrounded with a sheath. This seems to be the ease, not only from the gruater thickness at that part, but also from the cir- 
cumstarice that when, passing from the cell form into the still condition, the cilia disappear, the $\mathrm{V}$-shaped, or forked internal portions remain visible. And it is then, also, that the openings through the enveloping cell-wall become, for the first time, visible.

Perhaps the most remarkable of all the numerous aspects presented by Protococcus pluvialis, is the form of naked zoospores named by Flotow Hamatococcus porphyrocephalus. These are extraordinarily minute globules, consisting of a green, red, and colourless substance in unequal proportions. The colourless protoplasm in them, as in all primordial cells, constitutes the outermost delicate boundary; the red substance is for the most part collected towards the anterior end in minute spherules; the granular green substance occupies more the under part, while the middle is usually colourless.

Propagation depends upon a division of the cell contents, particularly of the colourless or coloured protoplasm, or of the primordial sac. This body, without any demonstrable influence of a nucleus, is capable of subdividing into a determinate number of portions. Each of these acquires a globular figure, and in the next place surrounds itself with an envelope of protoplasm, and then represents a visible organism, which after the reabsorption of the parent cell-membrane, is capable of existence as an independent reproductive indıvidual. Besides these, which are the most usual modes of propagation-viz. that of the still-cells into two, and of the motile into four, secondary cells-there are a number of others which may be considered as irregular, and in which forms are produced which do not re-enter the usual cycle until they have gone through a series of generations. Sometimes, under certain circumstances, the cell-contents of the still form separate into eight or more portions, which become naked zoospores of small size (fig. 144 B.) It is not quite clear what becomes of this form of motile zoospores, but there seems reason for believing that they occasionally develop an enveloping cyst, and thus become encysted zoospores, and at other times secrete a cellulin tissie, and become still-cells; but mosi of them probably perish without any further change. They would thus correspond 
with the smaller motile spores observed by Thuret and A. Braun in other Algæ (the Fucoid, \&c.), associated with the larger germinating spores, themselves deprived of the germinative faculty.

It appears that both longitudinal and transverse division of the primordial cell may take place; but that the vibratile cilia of the parent cell retain almost to the last moment their function and their motion after the primordial cell inclosed by it has long been detached as a whole, and become transformed into the independent secondary cells (fig. 144, G).

The most striking of the vital phenomena presented by this organism is that of periodicity. Certain forms-for instance, encysted zoospores, of a certain colour, appear in a given infusion, at first exclusively, then they gradually diminish, become more and more rare, and finally disappear altogether. After some time their number again increases, and reaches as before to an incredible extent; and this proceeding may be repeated several times. Thus, a glass which at one time presented only still forms, contained at another nothing but motile ones. The same thing may be observed with respect to segmentation. If a number of motile cells be transferred from a larger glass into a small vessel, it will be found, after the lapse of a few hours, that most of them have subsided to the bottom, and in the course of the day they will all be observed to be on the point of subdivision. On the following morning the divisional generation will have become free; on the next, the bottom of the vessel will be found covered with a new generation of selfdividing cells, which again proceed to the formation of a new generation, and so on. This regularity, however, is not always observed. The influence of every change in the external conditions of life upon the propagation is very remarkable. It is only necessary to pour water from a smaller into a larger and shallower vessel, or one of a different kind, to at once induce the commencement of segmentation in numerous cells. The same thing occurs in other Algæ; thus the Vaucheria almost always develop zoospores, at whatever time of year they may be brought from their natural habitat into a room. Light is conducive 
to the manifestation of vital action in the motile zoospores, and they always seek it, collecting themselves at the surface of the water, and at the edges of the vessel.

But in the act of propagation, on the contrary, and when about to pass into the still condition, the motile Protococcus cell seems to shun the light; at all events it then seeks the bottom of the vessel, or that part of the drop of water in which it may be placed, furthest from the light. Too strong sunlight, as when it is concentrated by a lens, at once kills the zoospores. A teinperature of undue elevation is injurious to the development of the more vigorous vital activity, that is to sily, for the formation of the zoospores; whilst a more moderate warmth, particularly that of the vernal sun, is singularly favourable to it. Frost destroys the motile, but not the still zoospores. *

Stephanosphara pluvialis is another variety of freshwater algæ, first observed by Cohn. It consists of a hyaline globe, containing eight green primordial cells, arranged in a circle (see Plate 1, No. $24 d$ ). The globe rotates, somewhat in the same manner as the volvox, by the aid of projecting ciliæ, two of which are seen to proceed from each cell and pierce the transparent envelope. Every cell divides first into two, then four, and lastly eight young cells, each of which divides into a great number of microgonidia, and are seen to have a motion within the globe, and ultimately escape from it. Under certain circumstances each of the eight cells is observed to move about in the interior of the mother-cell ; eventually they escape, lose their cilia, form a thicker membrane as at $b$, for a time become motionless, and sink to the bottom of the vessel. If the vessel be permitted to become thoroughly dry, and again water is poured into it, motile Stephanosphæra reappear : from which circumstances it is probable that the green globes are the resting spores of the plant. When in its condition of greatest activity its division into eight is perfected during the night, and early in the morning the young family escapes from the cell, soon to pass through similar changes. It is calculated that in

\footnotetext{
"On the "Natural History of Protococcus ptrvvialis," by F. Cohn, translated by G. Busk, F.R.S. for the Ray Society.
} 
eight days, under farourable circumstances, 16,777,216 families may be formed from one resting-cell of Stephanosphæra. In certain of the cells, and at particular periods, the remarkable amaboid bodies (Plate 1, No. 24 c), have been noticed. There is a marked difference between Stephanosphæra and Chlamydococcus, "for, while in the latter the individual portions of a primordial cell separate entirely from one anotber, each developing its own enveloping membrane, and ultimately escaping as a unicellular individual; in the former, on the other hand, the eight portions remain for a time united as a family." *

The simplest forms of vegetable life are met with in the Confervoids, which are as interesting as they are instructive to the microscopist. The confervæ consist of unbranched filaments composeủ of cylindrical cells, placed end to end; their reproductive process is carried on by zoospores produced from the cell contents. The freshwater genera are principally of a yellowish green colour; sometimes presenting a striated appearance, which has given rise to a supposition that their filaments are spiral. They are indeed plentifully distributed both in fresh and salt-water.

Oscillatoriacea.-The study of the structure of the OscilIntorice is particularly interesting, from the fact that we may not unreasonably expect to find in it a key to the singular motion from which they received their generic name, and which now, for more than a century, has formed an object of curiosity and interest to the microscopist, withont having received, as yet, a satisfactory explanation.

The following different tissues are observable in the true Oscillatorice:-1, An outer inclosing sheath; 2, A special cell-membrane, with its contents; and 3 , The axis, or pith, of the filament.

"The filaments of certain species are inclosed in sheaths or continuous tubes, never showing any cross-markings corresponding to the striæ of the filament; they are clearly composed of a lind of cellulose, although they remain unaffected by iodine. In other species, these tubes are

* See an interesting paper by F. Currey, F.R.S. Journal of Microscopica' Science, vol. vi. 1858, p. 131: also by Mir Wm. Archer, rol. v. 1865, p. 116 
absent, or have not yet been observed; when present, they will be found projeeting on one or buth sides of

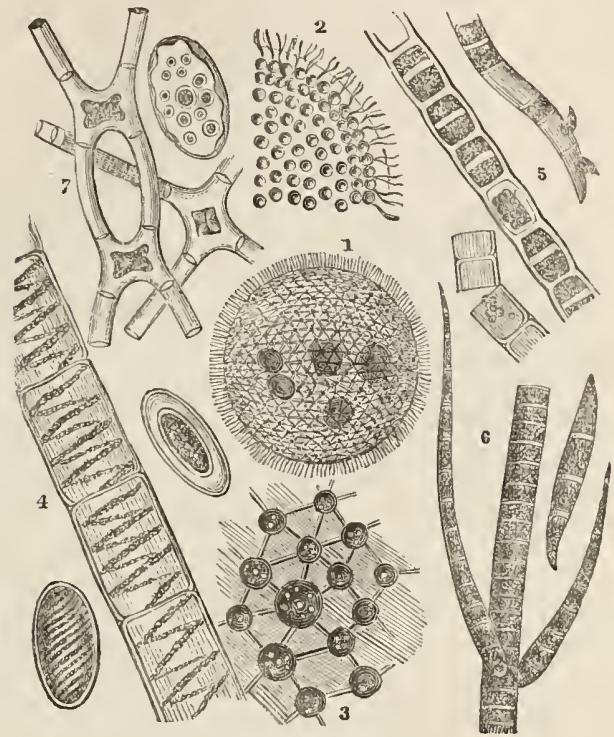

Fig. 145.-Conferve.

1, Volvox globator. 2, A section of volvox, showing the ciliated margin of the cell. 3, A portion more highly magnified, to show the young volvocina, with their nuclei and thread-like attachments. 4, Spirogyra, near which are spores in different stages of development. 5, Conferva floccosa. 6, Stigeoclonium protensum, jointed filaments and single zoospores. 7, Staurocarpus gracilis, conjugating filaments and spores.

the filament, being somewhat longer than the latter. Filaments inclosed in sheaths never, or but slightly, exhibit their peculiar motion, although they may be seen sliding in them, backwards and forwards, or leaving them altogether.

The filaments themselves have been supposed to consist wholly of protoplasm; this view, however, is scarcely correct, since the protoplasm is enclosed in a cell-membrane. The ccllulin coat always shows cross-markings corresponding to the strix when such are observable 
in the filament, and which divide it into distinct jcints or cells.

The presence of this cell-membrane may be best de. monstrated by breaking up the filaments, either by moving the thin glass cover, or by cutting through a mass of them in all directions with a fine dissecting knife. On now

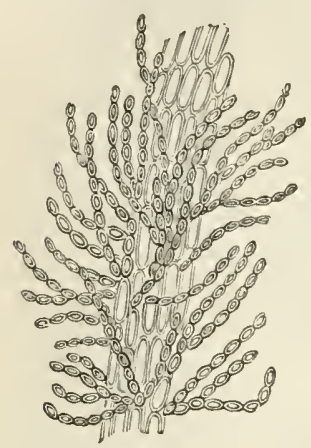

Fig. 146.-Mesoglia rermicularis, composed of strings of cells co. hering end to end. examining the slide, in most instances many detached empty pieces of this cell-membrane, with its striæ, will be found, as well as filaments partly deprived - of the protoplasm, showing in those places the empty, striated cellulin coat. On the application of iodine all these appearances become unmistakably evident; the greater portions of the filament turning brown or red, while the empty cells, with their striæ, remain either unaffected, or at most present a slight yellowish tint, as is frequently the case with cellulose when old.

With regard to the contents of the cell, the protoplasm (or endochrome) is coloured in the Oscillatoriae, and is deposited within it in the form of circular bands or rings around the axis of the cylindrical filament; iodine turns them brown or red, and syrup and dilute sulphuric acid produce a beautiful rose colour. As to their mode of propagation, nothing pusitive is known. If kept for some time they gradually lose their green colour-those exposed to the sun, much sooner than others less exposed; the stratum eventually becoming brown, sinks to the bottom of the vessel, and presents a granular layer, embodying great numbers of filaments in all stages of decay.*

The movenents of the Uscillatoria are indeed very singular, so much so that it is in rain to attempt to explain them as altogether dependent on physical causes, and equally so to show that they are due to a sarcode or animal

* Dr. F. d'Alquen, "On the Structure of the Oscillatorix," Journal of Microscopical Science, vol, iv. p. 245 . 1856. 
membrane. Their motion is not less lively than that of the Bacteria, which Dujardin and Ehrenberg placed amorg infusional animalcules. To observe the movements of the filaments, the very uppermost surface ought to be brought into focus, leaving the margins rather undefined, bearing in mind that the filament is not a flat but a cylindrical body. Certainly, with regard to its movement, or the mechanism by which it is effected, nothing positive is known.

The Bacillaria paradoxa is by far the most interesting specinien of the genus; the movements of which are very remarkable, and so little understood that it is rightly called paradoxica!

The Marine Confervoid AIgæ present a general appearance which might at first sight be mistaken for plants very much higher in the scale of organization. In the Ulvaceæ, the frond has no longer the form of a filament, but assumes that of a membranous expansion of the cell. These cells, in which zoospores are found, have an in. creased quantity of green protoplasm accumulated towards one point of the cell-wall; and the zoospores are observed to converge with their apices towards the same point. In some genera, which seem to be closely related in form and structure to the Bryopsidea, we notice this important difference, that the zoospores are developed in an organ specially destined to this purpose, which presents pesuliarities of form, distinguishing it from every other part of the branching tubular frond. In the genus Derbesia, distinct spore cases are seen, a young branch of which, when destined to become a sporecase, instead of elongating indefinitely, begins, after having arrived at a certicin length, to swell out into

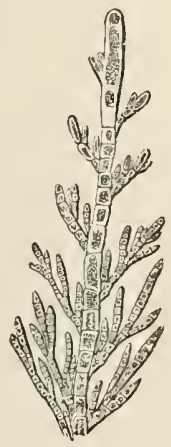

Fig. 147. - Sphacelaria cirrhosa, with spores borne at the sides of the branchlets.

an ovoid vesicle, in the cavity of which a rapid accumulation of protoplasma takes place. This is then separated from the rest of the plant, and becomes an opaque mass, surrounded by a distinct membrane. After a time a 
division of the mass takes place, and a number of pyriform zoospores, each of which is furnished with a crown of ciliæ, are set free.

In many families of the olive-coloured Algæ, reproduction by zoospores is the general rule; they differ, however, in the arrangement of their cilia. These organs, which are always two in number, are usually of nnequal length, and emanate not from the beak, but from a reddishcoloured point in its neiglıbourhood. The shortest is directed backwards, and seems to serve during the motion of the spoze as a rudrler. The longest, directed forwards, is closely applied to the colourless beak. Ectocarpus is one of the simplest forma of olive-coloured Algæ, consisting of branching filaments, the extremity of any of which is liable to become converted into a sporangium, by the absorption of the septa of the terminal cells. The zoospores are arranged in regular horizontal layers. In many genera a peculiarity exists, the signification of which is not yet completely understood-namely, that of a double fructification. The ovoidal sporangia contain numerons zoospores. In the genus Cutleria (fig. 150), there is seen another feature of interest: the appearance of two kinds of organs, which seem to be opposed to each other as regards their reproductive functions. The sporangia not only differ from those of other genera, but the frond consists of olivecoloured irregularly-divided flabelli, on each side of which tufts (sori) consisting of the reproductive organs, intermixed with hair-like bodies, are scattered. The zoospores are divided by transverse partitions into four cavities, each of which is again bisected by a longitudinal median septum. When first thrown off they are in appearance so much like the spores of Puccinia, that they may be mistaken for them; they are, however, about three times larger than those of the other olive-coloured algæ.

The fruit of most olive-green Sea-weeds is enclosed in spherical cavities under the epidermis of the frond, termed conceptacles, and may be either male or female. The zoids are bottle-shaped, each possessing a pair of cilia ; the transparent vesicle in which they are contained is itself inclosed in a second of similar form, and we have no certain evidence of the function performed by the 
antheridia. In monœcious and diøcious Fuci, the female conceptacles are distinguisherl from the male by their olive colour. The spores are leveloped in each in the interior of a perispore, which is borne on a peclicle emanating from the inner wall of the conceptacle. They rupture the perispore at the apex; at first the spore appears simple, but soon after a series of changes take place, consisting in a splitting of the endochrome into six or eight masses, which become spheroidal sporules. A budding-out occurs in a few hours' time, and ultimately elongates into a cylindrical tube. The Vaucherice present a double mode of reproduction, and their fronds consist of branched tubes, much resembling in general character that of the Bryopsidece, from which

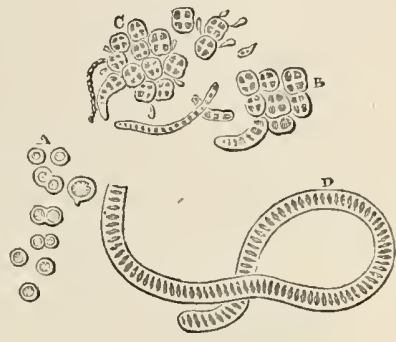

Fig. 148.-Development of Ulvo.

A, isolated cells of spores, B and c, clustering of the sume. $D$, cells in the filamentous stage. indeed they differ only in respect of the arrangement of their contents, chlorophyll. In that most remarkable plant Saprolegnia ferox, which is structually so closely allied to Vaucheriae, though separated from them by the absence of green colouring matter, we find a corresponding analogy in the processes of its development. In the process of the formation of its zoospores, we have an intermediate step between that of the Algæ and a class of plants usually placed among Fungi. Cohn has shown us that Pilobolus is structually more closely allied to the former class than to that of the latter. Pilobolus has a somewhat remarkable ephemeral existence; the spore germinates about mid-day, the plant grows till evening, re-opens during the night, and in the morning the spore-case bursts and the whole disappears, leaving behind scarcely a trace of its former existence.

Red Sea-weeds, Floridece, present great varieties of structure, although comparatively little is known of their re- 
productive processes ; it will, however, be sufficient for our purpose to notice the three leading forms. The first form, to which the term polyspore has been applied, is that of a gelatinous or membranous pericarp or conceptacle, in which an indefinite number of sporidia are contained. This organ may be either at the summit or base of a branch, or it may be concealed in or below the cortical layer of the stem. In some cases a number of sporidiumbearing filaments emanate from a kind of membrane at the base of a spheroidal cellular perisporangium, by the rupture of which the sporidia formed from the endochrome of the filaments make their escape. Other changes have been observed; however, they all agree in one particular, namely, - that the sporidium is developed in the interior of a cell, the wall of which forms its perispore, and the internal protoplasmic membrane en-

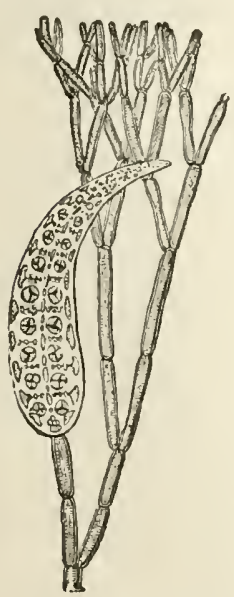

Fig. 149.-Dasya Kutzingiana, with seed vessel and two rows of tetrisspores. Magnified 50 diameters. doclirome, the sporidium itself, for the escape of which the perispore ruptures at its apex.

The second form is more simple, and consists of a globular or ovoid cell, containing a central granular mass, which ultimately divides into fonr quadrate-shaped spores, which when at maturity escape by rupture of the cell-wall. This organ, called a tetraspore, takes its origin in the cortical layer. The tetraspores are arranged either in an isolated manner along the branches, or in numbers together; in some instances the branches which contain them are so modified in form that they look like special organs, and have been called stichidia; as, for example, in Dasya (fig. 149). Of the third kind of repraluctive organ a difference of opinion exists as to the signification of their antheridia; although always produced in precisely the same situations as the tetraspores and polyspores, they are "agglomerations of little colourless cells, either 
united in a bunch as in Griffithsia, or enclosed in a transparent cylinder, as in Polysiphonia, or covering a kind of expanded disc of peculiar form, as in Laurencia." According to competent observers, these cellules contain spermatozoids. Nägeli describes the spermatozoid as a spiral fibre, which, as it escapes, lengthens itself in the form of a screw. Thuret does not coincide in this view; on the contrary, he says that the contents are granular, and offer no trace of a spiral filament, but are expelled from the cells by a slow motion. The antheridia appear in their most simple form in Callithamnion, being reduced to a mass of cells composed of numerous little bunches which are sessile on the bifurcations of the terminal branches. Are not these spirai filaments closely allied to Oscillatoriacece? The spores are simpler structures than the tetraspores, and mostly occupy a more important position. They are not scattered through the frond, but grouped in definite masses, and generally enclosed in a special capsule or conceptacle, which may be mistaken for a tetraspore case. The simplest form of the spore fruit consists of spherical masses of spores attached to the wall of the frond, or irnbedded in its subetance, without a proper conceptacle ; such a fruit is called a favellidium, and occurs in Halymenia; the same name is applied to the fruits of similar structures not perfectly immersed,. as

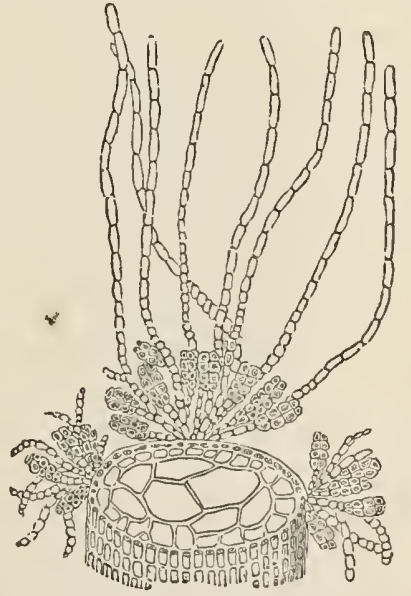

Fig. 150.-Cutleria dichotoma. Section of a lacinia of a frond, showing the stalked eigh chambered oosporanges growing on tufis with intercalated hairs Magnified 50 dismeters. those of Gigartina, Gelidium, \&c., where they form tubercular swellings on the lobes. In some, the tubercles present a pore at the summit, through which the spores find 
exit; when such a fruit is wholly external, as in Ceramium (see Plate II. Nos. 27 and 37) and Callithamnion, it is called a favella. The characteristic of Delesseria, No. 39, the coccidium, either occurs on lateral branches, or is sessile on the face of the frond, and consists of a case of angular spores attached to a central wall. The ceramidium is the most complete form of the conceptacular fruit: this is enclosed in an Jvate case, with an apical spore, containing a tuft of pear-shaped spores arising from the base of the cavity.

The general external appearance of the Red Sea-weeds is very varied. They are exquisite objects for the Microscope; we have figured several interesting varieties in Plate II., each showing peculiarities of fructification. Their beautiful leaf-like fronds are either simple, lobed, or curiously pinnate or feathered. The Floridex of warmer clinates exhibit most elegantly formed reticulated fronds, as may be seen on reference to the late Dr. Harvey's last great work, "Plyycologia A ustralica."

In the plant which results from the germination of the aggregate zoospores of Vaucheria, a genus of Siphonaceæ (Plate I. fig. 23), Kaisten has observed that on those filaments which come in contact with the atmosphere, are formed organs of a peculiar structure, which have the appearance of nipple or egg-shaped buddings-out of the cell-wall, distributed *in pairs along the whole course of the older filaments; one elongates and curves round to meet its fellow, which is seen to swell out into a globular form; finally conjugation takes place, preceded, however, by the conversion of the green contents of the tubular organ into oil globules. If the filaments be gathered at a favourable period, and cultivated in a vessel of water well exposed to the light, the blind ends, or ramifications of the filaments, are found densely filled with green contents, appearing to be almost black; if these ends be watched early in the morning, a remarkable series of changes is seen to occur in them when about to produce gonidia, and, ultimately, they escape in a peculiar way from the filament. The admirable essays of Unger, Nägeli, and Pringsheim on the process of their reproduction might be consulted with advautage. 
A fresh-water alga of singular beauty and interest to the microscopist is the Volvox globator. This little cell so well known to the older observers as the globeanimalcule, or revolving-cell, is represented in fig. 145 , Nos. 1, 2, 3, and Plate I. No. 15. These revolving globulas bodies were for a very long time classed with the lower forms of animal life, and there remained for the microchemical investigator of the present time to settle the perplexing question, and assign to them a place among plants.

Leeuwenhöek first perceived the motion of what he termed globes, "not more than the 30th of an inch in

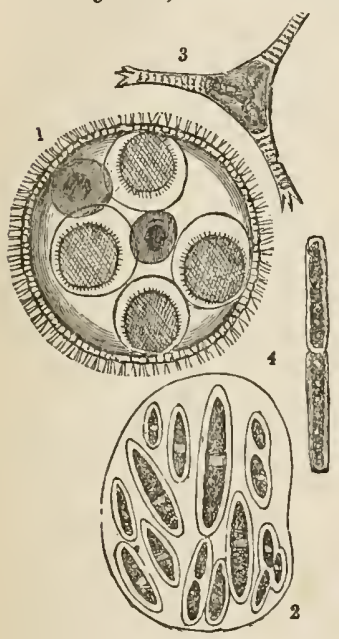

Fig. 151 . diameter, rolling through water; and judged them to be animated." These globes are studded with innumerable minute green spots, each of which is seen to he a perfect cell, about the 3,500 th part of an inch in size, with a nucleus and two active cilia attached. The whole are bound together by threads forming a beautiful net-work. Within the globe busy active nature is at work carefully providing a continuance of the species; and from six to twerty little brightgreen spheres have been found enclosed in the larger transparent case. As each Jittle cell arrives at maturity, the parent cell enlarges, and ultimately bursts asunder,

1. Volvox, just before the young burst forth, showing the vesicle wnich encloses eaeh. 2, Parent cell of Closteriun. 3, Docidium clavitum. 4, Staurastrum gracilis. launching torth its offspring to seek an independent exl. tence. Buth older and younger spheres possess openings through which the water frecly flows, affording food and air to the wonderfully constructed little being.

Dr. Carpenter believes, "The Volvocinece, whose vegetable nature has been made known to us by observation of cer- 
tain stages in the history of their lives, are but the motile forms (Zoospores) of some other plants, whose relation to them is at present unknown." Professor Williamson, having carefully examined the Volvox globator, says:"That the increase of its internal cells is carried on in a manner precisely analogous to that of the algæ; that between the outer integument and the primordial cell-wall of each cell, a hyaline membrane is secreted, causing the outer integument to expand; and as the primordial cellwall is attached to it at various points, it causes the internal colouring-matter, or endochrome, to assume a stellate form (see Plate I. No. 15), the points of one cell being in contact with those of the neighbouring cell, these points forming at a subsequent period the lines of communication between the green spots generally seen within the fullgrown Volvox." Cilia can be distinctly seen on the outer edge of the adult Volvox; by compressing and rupturing one, they may even be counted. Professor Busk has been able to satisfy himself, by the addition of the chemical test iodine, of the presence of a very mimute quantity of starch in the interior of the Volvox, which he considers as conclusive of their vegetable character. A singular provision is made in the structure of the gemmules, consisting of a slender elastic filament, by which each is attached to the parent cell-wall : at times it appears to thrust itself out, as if in search of food; it is then seen quickly to recover its former nestling-place by contracting the tether. It is impossible not to recognize the great similarity between the structure of Volvox, and that of the motile cell of Protococcus pluvialis. The influence of re-agents will sometimes cause the connecting processes of the young cells as in Protococcus, to be drawn back into the central mass, and the connecting threads are sometimes seen as double lines, which seem like tubular prolongations of a consistent membrane. At other times they appear to be connected by star-like prolongations to the parent cell, Plate I. No. 15, presenting an almost identical appearance with Pediastrum pertusum. Mr. Busk says that the body designated by Ehrenberg Spharosira volvox is an ordinary volvox in a different phase of development; its only marked feature of dissimilarity being that a large propor- 
tion of the green cells, instead of being single, are very commonly double or quadruple ; and the groups of ciliated cells thus produced, form by their aggregation discoid bodies, each furnished with a single cilium. These clusters separate themselves from the primary sphere, and swim forth freely under the forms which have been designated Uvella and Syncrypta by Ehrenberg. According to Mr. Carter, however, Sphcerosira is the male or spermatic form of Volvox globator. Dr. Braxton Hicks believes that he has seen the young volvox pass into an amœboid state; he observes:- "Towards the end of autumn the endochrome miss of the volvox increases to nearly double its ordinary size, but instead of undergoing the usual subdivision, so as to produce a macro-gonidium, it loses its colour and regularity of form, and becomes an irregular mass of colourless protoplasum, containing a number of brownish granules." (Plate I. No. 16.)

The final change and ultimate destination of these curious amœboid bodies have not as yet been made out; but from Dr. Hick's previons observation, marle on similar bodies developed from the protoplasmic contents of the cells of the roots of mosses, "which in the course of two hours become changed into ciliated bodies," he thinks it very probable that this is designedly the way in which these fragile structures are enabled to retain life, and to resist all the varied external conditions, such as damp, dryness, and rapid alternations of heat and cold. ${ }^{1}$

(1) We have had volvox under the meroscope for several months, towards the end of summer and throughout the autumn, and made more than a hundred examinations, without having once seen the remarkable change described by Dr. Hicks in the Quturterly Jour. Micros. Science, vol. viii. p. 96, 1862. Nevertheless, as Mr. Archer observes :- "If this reasoning be correct, then coutractility, ameboid contractility - for I can find no more comprehensive and expressive single adjective - must be accepted as an inherent quality or characteristic, occasionally more or less vividly evinced, of the vegetable cell-contents, and this in common with the animal; in other words, that the nature of the protoplasm in each is similar, as has indeed, as is well known, been urged before on grounds not so strong; thus reserving Siebold's doctrine, tha' this very contractility formed the strongest distinction between animals and plants, as he assumed it to be present in the former and absent in the latter of the two kingdoms of the organic world. Therefore, an organism whose known structural affinities, and whose mode of growth and of ultimate fructification point it out as truly a plant, but of which, however, certain cells may for a time assume a contractile, even a locomotive, quasi-rhizopodous state, must not by any means on this latter account alone be assumed as even temporarily belonging to the animal kingdom, or as tending towards a mutation of its vegetable nature. And from this it of course follows that an organism whose structural affinitics and reproduction are unknown, but which may possibly present an actively 
Desmidiacece.-A remarkably beautiful family of confervoid algæ, the most distinctive characteristics of the species being their bilateral symmetry. Each frustule is, however, a perfect unicellular plant, with a homogeneous structureless membrane, enclosing a cellular skeleton filled with chlorophyll. Four modes of reproduction have been observed in the desmids, and many points still remain to be clearecl up. Braun remarks of the products of conjugation, "that they do not pass, like the swarming-cells of the Palmellacece and the reproductive cells of the Diatomacex, directly and by uninterrupted growth into the primary generation of the new vegetative series, but persist for a long time in a condition of rest, during which, excepting as regards inperceptible internal processes, they remain wholly unchanged. To distinguish these from the germ-cell (gonidia) I shall call them seed-cells (spores). Certain early conditions observed in Closterium and Euastrum, namely, families of unusnally small imlividuals, enclosed in transparent, colourless vesicles, render it even probable that in certain genera of this family a number of individuals are produced from one spore, by a formation of transitory generations occurring already within the spore." 1

contractile, even locomotive nower, need not on this latter account be assumed as therefore necessarily an animal. In the former category fall the Volvocinaceæ and Rhisidinm; in the latter category Euglena and its allies, the so-called Astasiæan Infusoria, suggest themselves; and these must of course wait until their reproduction and history are better known before we can feel satisfied as to their true position: yet it seems hichly probable that these will presently, if they do not even now, take their place amongst adnitted plants.

"Several writers have, indeed, from time to time, put forward the (now, I think, generally accepted) vicw that the protoplasm of the vegatable and the sarcode of the animal cell are identical in nature; and, in seeking for analogies as regards contractility in the vegetable protoplasm as compared with the animal, and as demonstrative thereof, special attention has been directed to several of the now familiar phenomena displayed by certain vegetable cells. Such are the vibratory movements of ciliæ, and drawing in of these, the circulatory movenents of the cell contents, as in the liairs of the Tradescantia, \&c., the contractile vacuole in Gonium, Volvox, \&c., and so forth. But while these are, I think, unquestionably to a considerable, but wore limited extent, manifestations of the same plienomennn, it seems to me that nowe of these cases present so exact an analogy, strongly as they may indicate it, with the rhizopodous contractility as do the amoboid bodies of Stephanospliwra, of Volvox, of the Moss-radicles, and of Rhisidium. The amœboid bodies of Stephanosphæra seem to display this rhizopodous contractility in greatly the most marked or exaggerated degree, as their vigorous and energetic power of locnmotion indicate : in them, and infleed in those of Volvox, the Moss, and Rlisidium, the pseudopodal processes and their mode of protrusion and withdrawal, the flow of the granules, and the locomotion of the whole body, were in all respects analogous to the similar phenomena evinced by a true amœba."-Wm. Archer, Quarterly Journ. Micros. Science, vol. v. p. 185.

(1) "The Phenomenon of Rejurenescence in Nature." 
Reproduction both by conjugation and subdivision variously modified, is common to all the families of Desmidiaceæ; and in the Zygnemacer, which have a close relation to them, the phenomena of conjugation are very well known. In Staurocarpus we have those remarkable quadrate spores formed in the cross branch, produced by conjugation. In Spirogyra the union of two cells belonging to the opposite filaments takes place by the expansion of one side of each, so as to form a papilla or short rounded-off tube (see fig. 145). The ends of the two projections then come into contact, become slightly flattened, then pressed together, and finally united. The double wall formed by their union dissolves, or is broken through, so that a free passage is established between the two cells. Upon this, the whole of the chlorophyll, previously arranged round the inside of each of t] e cells, becomes a confused mass, which soon forms itself either in the cavity of one of them, or in the connecting canal, into a globular or oval spore invested with the colourless cellulnse membrane shown in one of our drawings of Penium (Fig. 155). In Closterium conjugation takes place in a somewhat similar manner, represented at No. 25, although it is quite clear that if the formation of germs by conjugation were the only provision for the reproduction of a species, all must disappear, inasmuch as the conjugation and consequent destruction of a pair of Closteria for the formation of one new plant will ultimately destroy the species.' Another mode, however, that of subdivision, appears to be designed as an effectual safeguard against such a possible extinction. Mr. Lobb has observed this process take place in Micrasterias denticulata. (Plate II. fig. 30), in the course of three hours and a half. 'The small hyaline hemisphere, put forth in the first instance from each frustule, enlarges with the flowing in of the endochrome ; it then undergoes progressive subdivision at its edges, first into three lobes, then into five, then into seven, then into thirteen, and finally at the time of its separation, aequires the characteristic notched outline of its type, being only distinguishable from the older half by its smaller size. ${ }^{2}$

(1) In certain species of Closterium the act cf conjugation gives origin to two sprorangize.

(2) E. G. Lobb, Trans. Micros. Soc. N.S. vol. i., 1861. 
Desmidiacece.-The once disputed question relating to the vegetable nature of these cells received much valuable elucidation from Mr. Ralfs, who gave to the world th

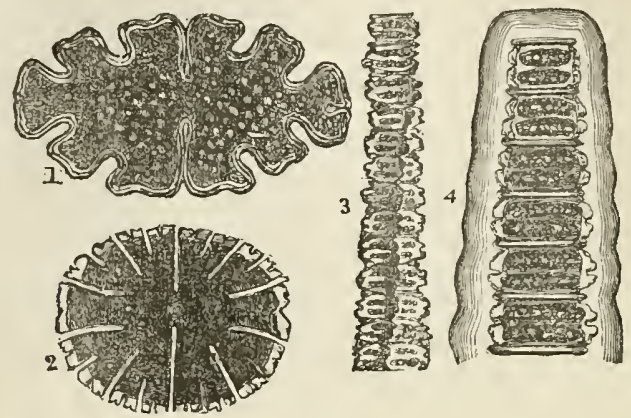

Fig. 152.

1, Euastrum oblongum. 2, Micrasterias rotata. 3, Desmidium quadrangulatum. 4, Didymoprium Grevillii.

results of his laborious researches in his excellent work on The British Desmidiece, published in 1848; and the conclusions arrived at by this painstaking author have been generally accepted by men of science. 'The interest which has so long attached to this topic will warrant us in devoting some space to its consideration; and we avail ourselves for that purpose of Mr. Ralfs' labours, with a recommendation to those of our readers who would wish to familiarise themselves more completely with this peculiar species, to consult the pages of the book above referred to.

Desmidiacece are grass-green in colour, surrounded by a transparent structureless membrane, a few only liaving their integuments coloured; they are all inhabitants of fresh water. Their most obvious peculiarities are the beauty and variety of their forms and their external markings and appendages; but their most distinctive character is their evident division into two or more segments. Each cell or joint in the Desmidiacece generally consist of two symmetrical valves or segments; and the suture or line of junction is in general well marked. The multiplication of the cells by repeated transverse division is full of interest, 
both on account of the remarkable manner in which it takes place, and because it unfolds the nature of the process iu other families, and furuishes a valuable addition to our knowledge of their structure and physiology.
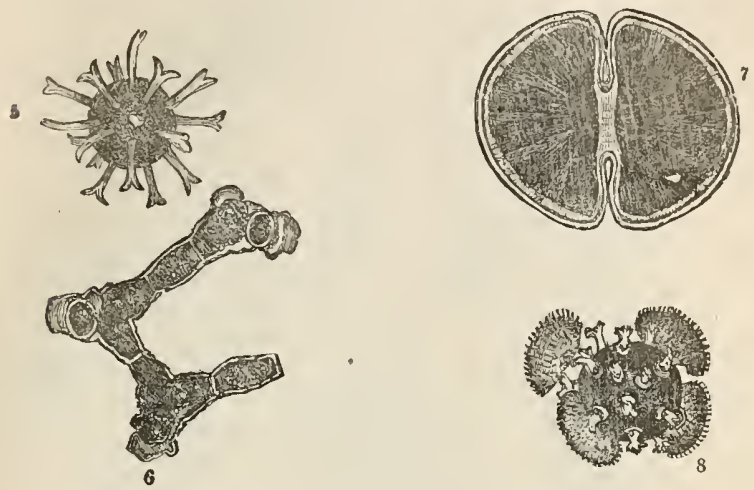

Fig. 153.

5, Micrasterias, sporangium of. 6, Didymoprium Borreri. 7, Cosmarium Ralfsii. 8, Staurastrum hirsutum.

The compressed and deeply-constricted cells of Euastrum offer most favourable opportunities for ascertaining the manner of their division; for although the frond is really a single cell, yet this cell in all its stages appears like two, the segments being always distinct, even from the commencement. As the conuecting portion is so small, and necessarily produces the new segments, which cannot arise from a broader base than its opening, these are at first very minute; though they rapidly increase in size. The segments are separated by the elongation of the counecting tube, which is converted into two roundish hyaline lobules. These lobules increase in size, acquire colour, and gradually put on the appearance of the old portions. Of course, as they increase, the original segments are pushed further asunder, and at length are discomnected, each taking with it a new segment to supply the place of that from which it has separated.

It is curious to trace the progressive development of the new portions. At first they are devoid of colour, and 
hive much the appearance of condensed gelatine; but as they increase in size, the internal fluid acquires a green tint, which is at first very faint, but soon becomes darker;

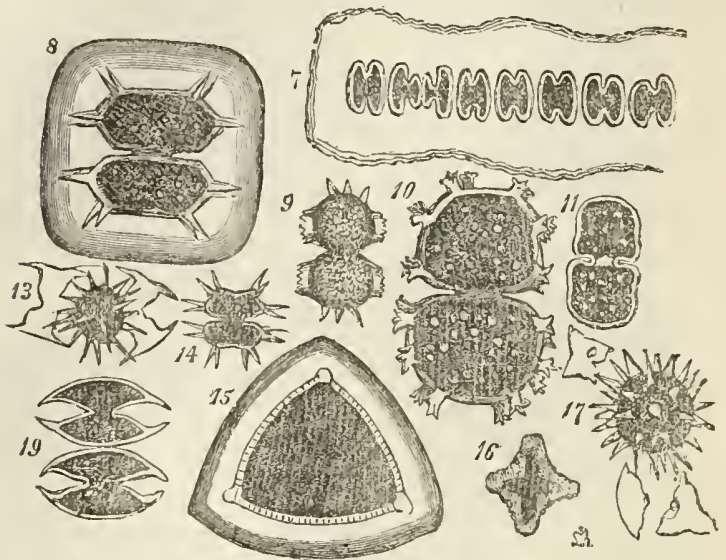

Fig. 154.

7. Sphorozosma vertebratum. 8, 9, Xanthidice. 10, X. armatum. 11, Cosmarium erenatum. 13, 17, Sporangia of Cosmarium. 14, X. fasiculatum. 19, Arthrodesmus convergens. 15, Staurastrum tumidum. 16, Staurastrum dilitatum.

at length it assumes a granular state. At the same time the new segments increase in size, and obtain their normal figure; the corcring in some species shows the presence of puncta or granules. Iu Xanthidium and Staurastrum the spines and processes make their appearance last, beginning as mere tubercles, and then lengthening until they attain their perfect form and size, armed with setre; but complete separation frequently occurs before the whole process is completed. This singular process is repeated again and again, so that the older segments are united successively, as it were, with many generations. When the cells approach maturity, molecular movements may be at times noticed in their contents, precisely similar to what has been described by Agardh and others as occurring in Confervce. 'This movement has been aptly termed a swarming. All the Desmidiacece are semi-gelatinous. In some the mucus is condensed into a distinct and well-defined 
hyaline sheath or covering, as in Didymoprium Grevillii and Staurastrum tumidum; in others it is more attenuated, and the fact that it forms a covering is discerned

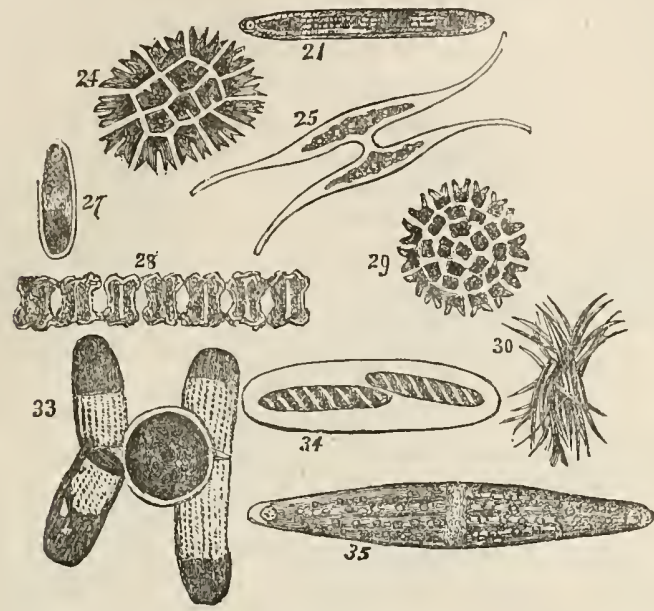

Fig. 155.

il, Penium. 24, Pediastrum bircdiatum. 25, Closterium, showing conjugation or self-division. 27, Penium Jenneri. 28, Aptogonum desmidium. 29, Pediastrum pecticum. 30, Ankistrodesmus falcatus. 33, Conjugation of Peniur margaritaceun. 34. Spirotemia. 35, Closterium.

only by its preventing the contact of the coloured cells. In general its quantity is merely sufficient to hold the fronds together in a kind of filmy clond, which is dispersed by the slightest touch. When they are left exposed by the evaporation of the water, this mucus becomes denser, and is apparently secreted in larger quantities, to protect them from the effects of drought. Meyen states, "that the large and small granules contain star'ch, and were sometimes even entirely composed of it ;" and " in the month of May he observed many specimens of Closterium in which the whole interior was granulated; these grains gave with iodine the beautiful blue colour, indicative of the presence of starch." 1

(I) The test for starch can be easily applied, and so remove any doubt that. may exist. It is only necessary to bear in mind that unless granular matter 
"Did we trust solely to the eye, we should indeed be very liable to pronounce these variable and beautiful forms as belonging to animals rather than vegetables. All favours this supposition. Their symmetrical division into parts; the exquisite disc-form, finely cut and toothed Micrasterias; the lobed Euastrum; the Cosmarium, glittoring as it were with gems; the Xanthidium, armed with spines; the scimitar-shaped Closterium, embellished with strix; the Desmidium, resembling a tape-worm ; and the strangely iusect-like Staurastrum, sometimes furnished with arms, as if for the purpose of seizing its prey;-all these characteristics appear to a superficial observer to belong rather to the lowest forms of animal, than vegetable life." Another indication Dr. Bailey adduced, by rendering apparent their power of motion; taking a portion of mud covered with Closteria, and placiug it in water exposed to light; after a time, it will be seen that if the Closteria are buried in the mud, they work their way to the surface, and cover it with a green stratum: this is no doubt owing to the stimulus light exerts upon all matter, althongh at first appearing very like a voluntary effort. Another is atforded by their retiring beneath the surface when the poc ${ }^{-}$, dry up. Mr. Ralfs states that he has taken advantage of this circumstance to obtain specimens less mingled with foreign matter than they would otherwise have been.

During the summmer of 1854 the Rev. Lord S. G. Osborne drew our attention to the economy of an interesting specimen of this family, the Closterium Lunula; after many careful investigations he came to the conclusion that the merabrane of the endochrome, both on its inner and outer surface, is ciliated.

In the Closterium Lumula, we have ascertained that the best view of its circulation is obtained by the use of strong daylight, or sunlight transmitted through coloured glass, or such a combination of tinted glass as that

be seen in the interior of the cell, starch cannot be present. A small quantity of diluted tineture of iodine may be applied, removing the free iodine by the aid of heat, occasionally adding a little water to facilitate its removal. This also will assist in the removal of the brownish stain which at first obscures the characteristic purple tint; and then, by applying the highest power of the microscope, the peculiar colour of the purple iodide ot starch will in general be perceived. 
proposed by Mr. Rainey, and adapted to a 1-4th achromatic condenser; with which must be used a 1-8th object-glass. 'The Gillett's condenser, or parabolic reflector, will do equally well if used with a 1-8th objective. In diagram A, fig. 156, a splecimen of the C. Lunula, as seen

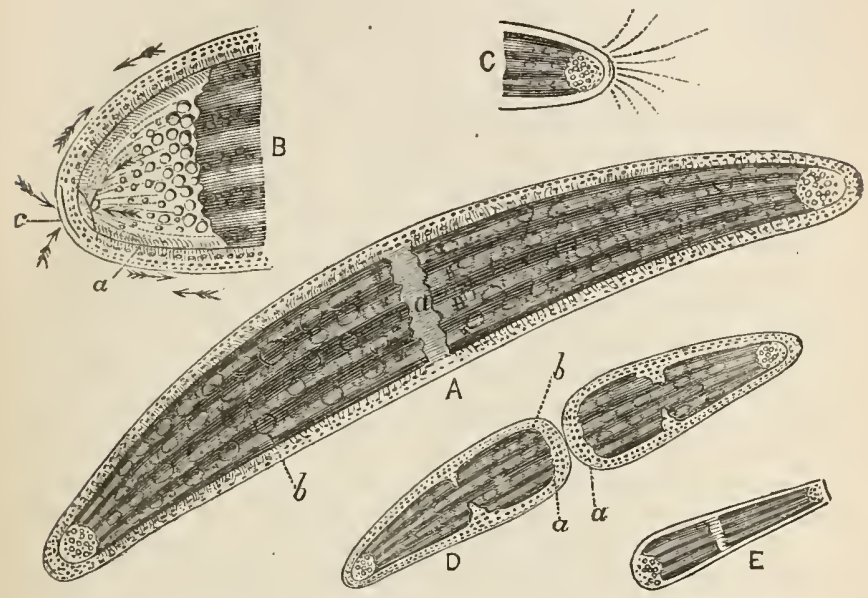

Fig. 156.-Closteria Lunula.

with the above arrangement of microscopic power, and a deep eye-piece, the cilia are in full action along the edge of the membrane which encloses the endochrome; and also, but not so distinctly, along the inside of the edges of the frond itself. Their action is precisely the same as that in the branchiæ of the mussel: there is the same wavy motion; and as the water dries up between the glasses in which the specimen is enclosed, the circulation becomes fainter, and the cilia are seen with more distinctness.

In diagram A, a line is drawn at $b$ to a small oval mark; these exist at intervals, and more or less in number over the surface of the endochrome itself, beneath the membrane which invests it. These seem to be attached by small pedicles, and are usually seen in motion on the spot to which they are thus fastened; from time to time they 
break away, and are carried by the circulation of the fluid, which works all over the endochrome, to the chambers at the extremities; there they join a crowd of similar bodics, each in action within those chambers; when the spccimen is a bualthy one.

The circulation, when made out over the centre of the frond, for instance at $a$, is in appearance of a wholly different nature from that seen at the edges. In the latter, the matter circulated is in globules, passing each other, in distinct lines, in opposite directions ; in the circulation as seen at $a$, the streams are broad, tortuous, of far greater body, and passing with much less rapidity. To see the centre circulation, use a Gillett's illuminator and the 1-Sth power; work the fine adjustment so as to bring the centre of the frond into focus, then almost lose it by raising the objective; after this, witl great care, work the milled head till the dark body of the endochrome is made out; a hair's-breadth more adjustment gives this circulation with the utmost distinctness, if it is a good specimen. It will be clearly seen, by the same means, at all the points where the spaces are put; and from them may be traced, with carc, down to both extremities.

The endochrome itself is evidently so constructed as to admit of contraction and expansion in every direction. At times the edges are in semi-lunar curves, leaving uninterrupted clear spaces visible between the green matter and the investing membrane; at other times, the endochrome is seen with a straight margin, but so contracted as to leave a well-defined transparent space along its whole edge, between itself and the exterior cuse. It is interesting to keep changing the focus, that at one moment we may see the globular circulation between the outer and inner case, and again the mere sluggish movement between the inner case and the endochrome.

At $B$ is given an enlarged sketch of one extremity of a $C$. Lunula. 'The arrows within the chamber pointing to $b$, denote the direction of a very strong current of fluid, which can be detected, and occasionally traced, most distinctly; it is acted upon by cilia at the edges of the chamber, but its chief force appears to come from some impulse given from the very centre of the endochrome. 
The fluid is here acting in positive jets, that is, with an almost arterial action; and according to the strength witl which it is acting at the time, the loose floating bodies are propelled to a greater or less distance from the end of the endochrome; the fluid thus impelled from a centre, and kept in aetivity by the lateral cilia, causes strong eddies, which give a twisting motion to the free bodies. The line $-a$, in this diagram, denotes the outline of the membrane which encloses the endochrome; on both sides of this cilia may be detected. The circulation exterior to it passes and repasses it in opposite directions, in three or four distinct courses of globules; these, when they arrive at $-c$, seem to encounter the fluid jetted through an aperture at the apex of the chamber; which disperses them. so much, that they appear to be driven, for the most part, back again on the precise course by which they had arrived. Some, however, do enter the chamber ; oceasionally, but very rarely, one of the loose bodies may be seen to escape from within, and get into the outer eurrent, it is then earried about until it becomes adherent to the side of the frond.

With regard to the propagation of the C. Lunula, we have never seen anything like conjugation; but we have repeatedly seen what the reverend gentleman has so well described-increase by self-division.

Observe the diagram $\mathrm{D}$; but for the moment suppose the two halves of the frond, represented as separate, to just overlap each other. Having watched for some time, the one half may be seen to remain passive; the other has a motion from side to side, as if moving on an axis at the point of juncture : the separation then becomes more and more evident, the motion more active, until at last with a jerk one segment leaves the other, and they are seen as drawn. It will be observed, that in each segment the endochrome has already a waist; but there is only one chamber, which is the one belonging to the one extremity of the original entire frond. The globular circulation, for some hours previous to subdivision, and for some few hours afterwards, runs quite round the obtuse end of the endochrome - $a$, by almost imperceptible degrees; from tho end of the endochrome symptoms of 
an elongation of the membranous sac appear, giving a semi-lunar sort of chamber; this, as the endochrome elongates, becomes more defined, until it has the form and outline of the chamber at the perfect extremity. The obtuse end $-b$ of the frond is at the same time elongating and contracting; these processes go on ; in about five hours from the division of the one segment from the other, the appearance of each half is that of a nearly perfect specimen, the chamber at the new end is complete, the globular circulation exterior to it becomes affected by the circulation from within the said chamber; and, in a few hours more, some of the free bodies descend, become exposed to, and tossed about in the eddies of the chamber, and the frond, under a 1-6th power, shows itself in all its beautiful construction. $\mathrm{E}$ is a diagram of one end of a $C$. didymotocum, in which the same process was noticed.

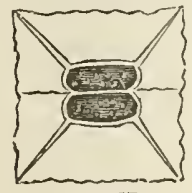

Fig. 157 .

The Euastrum Didelta is well worthy of attention, as well as many other species, the Tanthidium Penium, Docidium, \&c.

The Arthrodesmus Incus has a very beautiful hyaline membrane stretching from point to point, cut at the edges, something like the Micrasterias. This is represented at fig. 157 .

The Mode of Finding and Taking Desmidiacece.-As the difficulty of obtaining specimens is very great, it will materially assist the efforts of the microscopist to know the method adopted by Mr. Ralfs, Mr. Jenner, and Mrr. Thwaites. "In the water the filamentous species resemble the Zygnemata; but their green colour is generally paler and more opaque. When they are much diffused in the water, take a piece of linen, about the size of a pocket handkerchief, lay it on the ground in the form of a bag, and then, by the aid of a tin box, scoop up the water and strain it throngh the bag, repeating the process as often as may be required. The larger species, Euastrum, Micrasterias, Closterium, \&c., are generally situated at the bottom of the pool, either spread out as a thin gelatinous stratum, or collected into finger-like tufts. If the finger be gently passed beneath them, they will rise to the surface in little masses, and with care may he removed and 
strained through the linen as above described. At first nothing appears on the linen except a mere stain or a little dirt; but by repeated fillings-up and strainings a considerable quantity will be obtained. If not very gelatinous, the water passes freely through the linen, from which the specimen can be scraped with a knife, and transferred to a smaller piece; but in many species the fluid at length does not admit of being strained off without the employment of such force as would cause the fronds also to pass throngh, and in this case it should be poured into bottles until they are quite full. But many species of Staurastrum, Pediastrum, \&c., usually form a greenish or dirty cloud upon the stems and leaves of the filiform aquatic plants; and to collect them requires more care than is necessary in the former instances. In this state the slightest touch will break up the whole mass, and disperse it through the water : for securing them, let the hand be passed very gently into the water and beneath the cloud, the palm upwards and the fingers apart, so that the leaves or stem of the inverted plant may lie between them, and as near the palm as possible; then close the fingers, and keeping the hand in the same position, but concave, draw it cautiously towards the surface; when, if the plant has been allowed to slip easily and equably through the fingers, the Desmidiacere, in this way brushed off, will be found lying in the palm. The greatest difficulty is in withdrawing the hand from the surface of the water, and probably but little will be retained at first; practice, however, will soon render the operation ensy and successful. The contents of the hand should be at once transferred either to a bottle, or, in case much water has been taken up, into the box, which must be close at hand; aud when this is full, it can be emptied on the linen as before. But in this case the linen should be pressed gently, and a portion only of the water expelled, the remainder being poured into the bottle, and the process repeated as often as necessary."

When carried home, the bottles will apparently contain only foul water; but if it remain undisturbed for a few hours, the Desmidiacea will sink to the bottom, and inost of the water may then be poured off. If a little filtered rain-water be added occasionally, to replace what has been 
drawn off, and the bottle be exposed to the light of the sun, the Desmidiacece will remain unaltered for a long time.

Fungi.-This interesting class of cellular flowerless plants are chiefly microscopic, many requiring a high magnifying power to determine their peculiarities of structure. They abound in damp places, among decaying and decayed vegetable and animal matters, everywhere, and in almost every place. The structure of all Fungi exhibits a well defined separation into two parts, a mycelium (thallus) jointed and branched, forming a kind of cottony filamentous mass, and a reproductive spore or fruit, which, although exceedingly minute, differs somewhat in appearance under the microscope. The "spawn" used for planting mushroom beds is composed of mycelium, and may be readily obtained for examination (fig. 188, No. 19). The dust-like powder of any of the moulds or mildew when sprinkled on a slip of glass and kept under a bell glass over water, will soon throw out filaments and spores in all directions.

De Bary's observations show that resting-spores are not peculiar to the algæ; for he found them in two genera of fungi, and Tulasne ascertained their production in Peronosporce, many of which are parasitic, as $P$. parasitica, a species found on the cabbage and turnip leaf, as well as on the shepherd's-purse, Capsella bursapastoris. For the growth of $P$. infestans, the potato mould, the exclusion of light seems to be needful, and it is easy to conceive how the spores, washed down to the tuber during heavy rains, throw out germinating threads, which easily penetrate the thick cuticle of the potato, and quickly produce a murrain.

The Rev. MI. J. Berkeley, our English authority on Fungi, says :- "The genus Cystopus comprises those parasitic fungi amongst the Uredines which are remarkable for their white spores. Till the resting-spores of the different species were ascertained, it was almost impossible to find good distinctive characters : one species at least, Cystopus candidus, is to be found everywhere on the common shepherd's-purse, and often accompanied by Peronospora parasitica. It is also frequent on the cruciferæ: the acrospores, or gonidia, which spring from the swollen threads of the myeelium, form necklaces, as in oidium, the joint 
of which give rise to zoospores, as first observed by Prevost, in $180 \mathrm{~T}$. Like those of Peronospora, they move about in water by means of two lash-like appendages, and there germinate. When resting on the leaves of a plant, they make thoir way by means of a germinating thread into its subjacent tissues, and throw out little suckers. The branched mycelium gives off sporangia and antheridia, exactly as in Peronospora; when ripe, the sporangia are strongly warted. They fall, doubtless, with the leaves to the ground, where they remain till a fitting season arrives for their development. The provision made for the rapid development of these parasites and for the preservation of their species is truly marvellous, and sufficiently accounts for the difficulty of extermination and their aprarently sudden dispersion, especially in wet weather."

De Bary's observations on the germination of Uromyces appendiculatus are interesting, inasmuch as they show that the sporidia produce a mycelium, from which springs in succession-1st, spermogonia ; 2dly, peridia, producing chains of orange-coloured fruit, or, in other words, an Ecidium; and 3dly, the original fruit of Uromyces, accompanied by the more simple fruit commonly called uredo, and now called uredo-stylospores. The germination of the fruit produced by the peridia, as well as that of the uredo-stylospores, produces, according to De Bary, 1st, Uredo-stylospores, and $2 \mathrm{~d}$, the original Uromyces. spores. Thus we see the Uromyces-spores passing through the generations of promycelium, sporidia, and myceliumthe latter producing successively the two different products, spermogonia and recidia, and ultimately the original fruit of Uromyces, accompanied by the Uredo. The spermatia, or contents of the spermogonia, never germinate; but we find the fruit of the rcidia, and also of the Uredo, reproducing first the Uredo itself, and subsequently the original fruit of Uromyces. Other interesting points, noticed bv the same author, are, "that not only has each species of parasite a certain special nutrient plant, but that in certain Uredines with multiple fruit and alternate generations each sort of reproductive organ buries its germ in a difterent nutrient plant; and that the vegetation of the para. site is the cause of the disease." 
De Bary has also carried out a series of experiments which go far to satisfy him that the sporidia of Puccinia graminis germinate on the leaves of Berberis, and that the Ecidium of the Berberis (Plate I. No. 22) is a stage in the cycle of development of Puccinia. Thus, whilst in most Uredines the entire development is carried out upon one and the same nutrient plant, the alternate generations in Puccinia graminis require a change of host. This is a state of things well understood now in the animal kingdom in the Tænix and Trematoda, but Puccinia graminis is, we believe, the first of the parasitic fungi in which it has been particularly ascertained. Another point of interest is a confirmation of the supposed injurious effect of the proximity of Berberis to corn, which has been denied. De Bary further shows that Mucor mucedo (the common mould) has three, if not four, different forms of fruit; and that the mould called Thamnidium by Link, or Ascophora elegans by Corda, and the mould described by Berkeley as Botrytis Jonesii, and made into a new genus by Fresenius, under the name of Chatocladium, are only varieties of the fruit of Mucor mucedo. Also that yeast, Achyla, Saprolegnia, and Entomophthora or Empusa, are identically the same as Mucor mucedo, consequently that a large reduction is needed in the genera of the mucorini.

The main interest, however, of De Bary's paper on the fructification of the Ascomycetes, consists in observations on Erysiphe Cichoracearum, \&c., in which the author traces the origin of the perithecium, from its earliest state up to the formation of the single ascus and spores. $\mathrm{He}$ notices two cells as being always present and visible from the earliest period, one of which he conjectures may be the female, and the other the antheridium or male organ. He says that the cell, by the division of which the ascus and its coating are formed, only develops itself when it has been in contact with the antheridium; and he considers it very probable that impregration is effected by such contact, and that the perithecium of Erysiphe (excepting the outer wall) is the product of such impreg nation.

De Bary's paper on parasitic fungi was, it appears, undertaken with a view to contribute to the solution of 
the question as to their origin; and he concludes that endophytes are not produced from the metamorphosed substance of diseased plants, but that they originate from germs which penetrate healthy plants and develop a mycelium. In the course of his investigations he notices the occurrence in the genus Cystopus of organs similar to those long since discovered by Tulasne in Peronospora, which have been called Oogonia. He observes that ramifications perform the functions of antheridia, or male organs; and he proceeds to describe the production by the oospores (or impregnated contents of the oogonia) of active zoospores, similar to those produced by the ordinary spores of Cystopus. Dr. De Bary states that these zoospores, after remaining active for three or four hours, lose their cilia and power of motion, assume a cellulose covering, and germinate. He adds that the germ-filaments entor readily by the stomates and leaves of the nutrient plant, but that those filaments only become developed which enter the stomates of cotyledons. In Peronospora the development of the antheridia, oogonia, and oospores is said by De Bary to be the same as in Cystopus; and he gives particulars of the mode of germination of the conidia, and remarks on the growth of the parasite, which will be more profitably studied in the paper itself.

Parasitic fungi, vegetable blights as they are commonly called, have of late years become objects of earnest attention, on account both of the enormous damage done to our growing crops, and also of the many curious facts in their history which have been brought to light. Corn-blights consist chiefly of nildew, Puccinia, smut, bunt, rust, or red-robin, Uredo. Oidium is a common mildew; $\mathrm{Bo}$ trytis another; Acidium forms a kind of rust infecting pear-trees, the peridia of which form a very pretty object for the microscope. (Plate I. No. 22, Escidium Berberidis.) In the full-grown condition they appear as little cups filled with reddish-brown powder (spores), and may be detected in their earliest stages by the deformities they produce in the structure of the plants infested, or by pale or reddish spots on the green surface, arising from the presence of the fungus beneath. They are common on the coltsfoot, the berberry, gooseberry, buckthorn, nettle, \&c. Plate I. No. 19, 
sepresents a vertical section of a leaf of black-currant, infested with Ecilium grossularice; its spermogonia aro seen on the surface, and the perithecia below. The family Sphariacei (No. 3, Plate I.), common enough on most herbaceous stems, first seem to be little black spots, $a$; when examined more closely are found to resemble little brownish bottles, $b$, filled with rolls of spores. Other instructive specimens are-

Cystopus candidus (Uredo olim), Crucifer White-rust; conidia equal, globose; membrane equal, ochraceous; oospores sub-globose, epispore yellowish-brown, with irregular obtuse warts; warts solid. On shepherd's purse, cabbage, and other Cruciferæ: receptacle consisting of thick branched threads; conidia concatenate, at length separating; oospores deeply seated on the mycelium.Phyllactinia guttata (Olim Trysiphe). Plate I. No. 9. Hazel Blight; amphigenous; mycelium web-like, often evanescent; conceptacles large, scattered, hemispherical, at length depressed; appendages hyaline, rigid, simple; sporangia 4-20, containing 2-4 spores. On leaves of hawthorn, hazel, ash, elm, \&c.-Aregma (Phragmidium) bulbosum. Plate I. No. 20. Bramble Brand; hypogynous, with a dull red stain on the upper surface; spores in large tufts, 4-septate, termiual joint apiculate; peduncles incrassated, and bulbous at the base.-Puccinia variabilis, Variable Brand; sori amphigenous, minute, roundish, surrounded by the ruptured epidermis, nearly black; spores variable, obtuse, cells often subdivided; peduucle very short. On leaves of dandelion.-Puccinia buxi, Box Brand. Plate I. No. 17. Sori sub-rotund, convex, and scattered; spores brown, oblong, rather strongly constricted, lower cell slightly attenuated; peduncle very long. On both surfaces of box leaves: spores uniseptate, supported on a distinct peduncle. Plate I. No. 18.-Trichobasis (Uredo olim) senecionis, Groundsel-rust; spots obliterated; sori solitary or regularly crowded; sub-rotund and oval, on the under surface, surrounded by the ruptured epidermis; spores sub-globose, orange. On various species of groundsel: spores free; attached at first to a short peduncle, which at length falls away.

It appears that at particular periods of the year the 
atmosphere is, so to speak, more fully charged with the various spores of fungi than it is at others. The spores of the moulds aspergillus, penicillium, and puccinia are perhaps the most widely distributed bodies, and towards the end of the hot weather, or about autumn time, they are very abundant. Among those who have taken them at this period of the year, we must ever associate the name of the Rev. Lord Godolphin Osborne, who first experimented in this direction during the cholera visitation of 1854 . He exposed prepared slips of glass, slightly moistened with glycerine, over cesspools, gully-holes, \&c., near the dwellings of those where the disease appeared, and caught what he termed aërozoa-chiefly minute germs and spores of fungi. A drawing made from one of these glasses (Plate I. No. 13), exhibits spores almost identical with those found on the human skin, \&c.

From the year 1854 to the present time we have amused ourselves by catching these floating atoms, and, so far as we can judge, they are found everywhere, and in and on every conceivable thing, if we only look close enough for them. Even the open mouth is an excellent trap; of this there is ample evidence, since we find on the delicate membrane lining the mouth of the sucking, crying infant, and on the diphtheritic sore throat of the adult, the destructive plant Oidium albicans. The human or animal stomach is invaded, and in a certain deranged condition we find the Sarcina ventriculi, with its remarkable-looking quaternate spores, its torulæ, \&c., seriously interfering with the functions of this organ. ${ }^{1}$ Torula diabetica is another of these destructive products found in the human bladder.

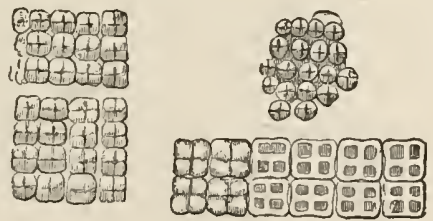

Fig. 158.-Sarcina ventriculi.

(1) What part do the fungi, or bacteria, play in the production of that fearful scourge of the human race, cancer? is a question not unfrequently asked since 
It is now more than a quarter of a century since Pro fessor Owen first pointed out the vegetable nature of a diseased growth found in the lungs of a Flamingo he was dissecting. Soon after, Bassi discovered the vegetable character of a disease which caused great devastation among silkworms; and, about the same time, Schönlein, of Berlin, was led to the detection of certain cryptogamic vegetable formations in connexion with skin diseases.

The Favis fungus is perhaps best known from its having been the first to attract the attention of Schönlein. It is commonly called cupped ringworm, or honeycomb scall, but it is very rarely seen in this metropolis. The crust is of a dingy yellow colour, and almost entirely composed of the Achorion, mixed with epithelial scales and broken hairs. When the fungus once establishes itself, so fearful are its ravages, that in a very short space of time the whole of the cutaneous surface, with the exception of the palms of the hands and soles of the feet, becomes covered with it. As the spores penetrate the hair-follicles they destroy the sheaths of the hairs, which shrivel up and lose their colouring matter, and then break off, leaving the surface bald.

Upon comparing the fermentation of the achorion fungus with that of good healthy yeast, it will be seen to be almost identical. In the first place, it is as actively

in the first edition of this book.(1854) we expressed a belicf in "the fungoid origin of cancer." Subsequent examinations of diseased structure more or less tend to confirm this view; it appear's" that in this disease we have superadided to a fungoid growth "degraded germinal matter"-which, by its entrance into the circulation, produces a terment and blood poisoning. 'The circular animal cell degenerates, is converted into the ovoid or elongated vegetable cell, and ultimately the structure, or some organ it may be, is changed into that remarkablelooking caudate body, the typical cancer cell. This in some respects bears the most perfect resemblance to certain spores of fumgi, and to the yeast torulæ. As might be expected, its form is modifled and its character more or less changed by the peculiar kind of nourishment and condensed tissue in which it is deposited and grows; its powers of growth are, so to speak, perverted and degraded, and then, as we see in other instances, it soon obtains a powcr of indefinite multiplication, and destroys, not only the vitality of the organ, but the individual. Ml. Davaine belieres he has traced splenic disease in sheep to the entrance into the blood of bacterium-like bodies, and fungi ; a zymotic disease is caused by the ferment, and by the rapid growth of the fungi the life of the animal is quickly sacrificed to the destroyer.

To mount specimens of fungi, separate them, and add a drop or two of spirit: when this has evaporated, add a drop of glycerine solution, or balsan dissolved in chloroform, and put on a glass cover. If the balsam renders the asci too trausparent, use gelatine: no cells are required. 
carried on by the former as by the latter. There is, however, just a slight difference in the size of the spores. or cells (Plate I. Nos. 7, 8, 11), those from yeast being the larger and more clearly spherical, with a greater number of reproductive spores, - that is, cells with a single, clear, nucleated cell in their interior,-while others are filled with a darker granular matter, having only a slight tendency to coalesce or become filamentous; those from achorion are for the most part ovoid, and very prone to coalesce and produce elongated cells or torulæ. With reference to the slight difference in size, we must look upon this as a matter of very little importance; for to the presence of light in the one case, and its almost total exclusion in the other, this difference. no doubt. is almost entirely due. It would be more trustworthy if comparisons of this kind could be made at the same stage of development; for be it remembered that yeast obtained from a brewery is in a more favourable state, inasmuch as it is stopped at a certain stage of growth or development, and then set to begin its fermentation over again in fresh supplies of a new pabulum, which give increased health and vigour to the plant; while, on the other hand, the achorion, or Favus fungus, is obtained and used in an exhausted state from an already ill-nourished or starved-out soil. Neither can we attach much importance to differences in size and form of the spores, for even this occurs in yeast ferment; and although the ovoid is most frequently seen in achorior, it is equally common to yeast when exhausted. This is strikingly exhibited in Plate I. No. 8, a drawing made from a drop of exhausted yeast taken from porter; here we have oval and elongated cells with torulæ. To ensure success in these and similar experiments, the fungus or yeast should be left floating on the surface of liquids; the process is either carried on very slowly, or is entirely arrested by submersion.

Turpin and others, in their experiments on yeast, noticed that the cells become oval and bud out in about an hour after being added to the wort (fig. 159); but this change depends as much upon temperature and density of the solution as upon the quality of the yeast. It is a well-ascertained fact that when yeast is added to distillery wash, which is 
worked at a higher temperature than brewers' wort, fermentation commences earlier, and the yeast-cell grows to a much larger size. It is, indeed, forced in this way much as a plant in a hothouse is, and then obtains to greater perfection in a shorter time. It will, however, be seen that it sooner becomes exhausted; and now, if we take a portion of this yeast and add it to barley wort, and at the same lime keep it in a temperature of from $60^{\circ}$ to $65^{\circ}$ Fahr., it ferments languidly, and small yeast-cells are the product. If the yeast is allowed to stand in a warm place for a few days, it partially recovers its activity, but never quite. With such a yeast there is always a good deal of torulæe mixed up with the degenerated cells, and sometimes a filamentous mass, which falls to the bottom of the vessel; from this stage it readily passes to that of must and miliew, and then becomes a wasteful feeder or destroyer.

With yeast already in a state of exhaustion, we have seen a crop of fungus produced in the head of a strumous boy, seven years of age, who was much out of health, and had suffered from eczema of the eyelids, with impetigo. On placing portions of the broken hairs on a glass slip, and moistening with a drop of liquor potassæ, spores and torula were seen in abundance; represented in Plate $\mathrm{I}$. No. 14.

In another experiment we took portions of penicillium and aspergillus moulds, and added these to sweetwort, and stood them by in a warm room. On the second day afterwards in one of the solutions, and the third in the other, fermentation had fairly set in; the surface of the solution was covered with a film, which proved to be well-developed ovoid spores, filled with smaller granular spores (conidia): Plate I. No. 8. Ou the sixth day the cells changed in form and were more spherical. Again removing these to another supply of fresh wort, the results obtained were quite characteristic of exhausted yeast ferment.

Extreme simplicity of structure characterises all moulds or mildews. Their reproductive organs are somewhat more complex, and both in penicillium and aspergillus the mycelium terminates in a club-shaped head, bearing upon 
it smaller filaments with small bead-like bodies upon the apex, piled one upon the other, or, more properly speaking, strung together; these, again, are surmounted by larger spores of a discoid shape filled with granular matter, and others which are quite empty. Those of the aspergillus are apparently without granular matter or nuclei, and are more highly refractive. The puccinia are elub-shaped, the very rapid growth of the spores and spawn of which appears to exert a specific and peculiarly exhaustive action over the tissues of the plant on which it feeds. Plate I. No. 12, represents a portion of the mould taken from a saccharine solution.

The yeast plant, in its most perfect condition, is chiefly made up of globular vesicles, measuring, when fully grown, about the $\frac{1}{2500}$ th of an inch in diameter. The older cells are filled with granular or nucleated matter; the nucleus rapidly increases, and nearly fills up the parent cell, which then becomes ovoid, and ultimately the young cell buds out and is separated from the parent. Some-
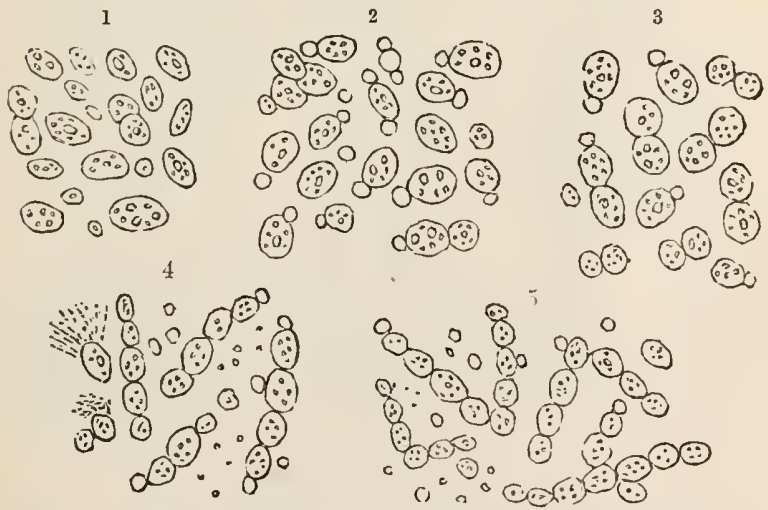

Fig. 150.-A diagrammatic representation of the development of the Ycast Plant.

No. 1, Fresh Yeast: No. 2, one hour after adding it to wort; No. 3, three hours; No. 4, eight hours; No. 5 , third day, after which jointed filaments are produced.

times other and smaller ceils are formed within the young one before it leaves the parent globule. This process goes 
on most rapidly until the supply of food becomes ex hausted; the vesicles, it would appear, derive their nourishment by the process of osmose, sucking in, as it were, certain portions of the organic fluid and chemically decomposing it, appropriating a part of its nitrogen and throwing off the carbonic acid. If, however, it be placed

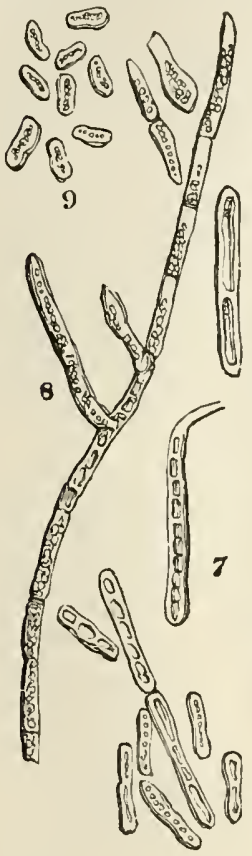

8

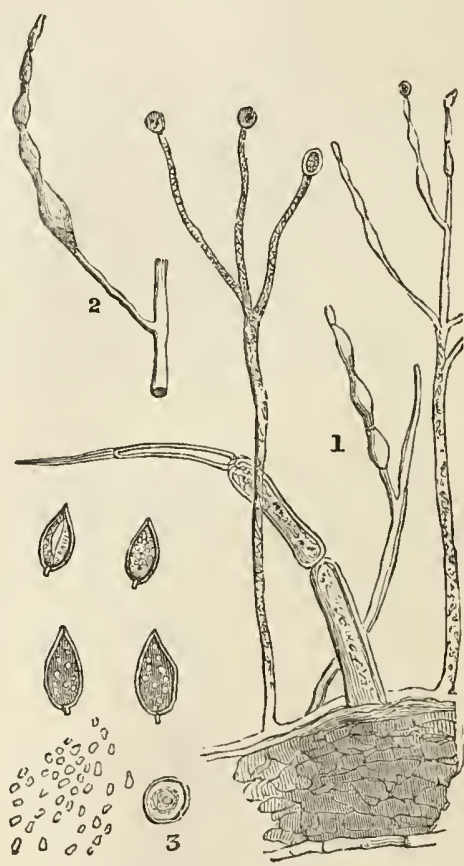

Fig. 160.-Fungoid growths.

1, Section from a Tomata, showing sporangiæ growing from cuticle. 2, A portion of same, detached, to show the mode of burlding out from the upper part of a brauch, 3, Vertical and lateral views of spores with oospores turned out. 6, 7, and 8, Different stages of growth of Mycodernza cerevisice. 9, Torula diabetica.

in any adverse condition, it becomes surrounded by layers of condensed material, resulting from the death of the 
germinal matter; ultimately a mere trace of life remains, which, taking the form of an impalpable powder, is free tc be driven hither and thither with every breath of air.

From these facts we may conclude that it matters little whether we take yeast, achorion, or penicillium spores; the resultant is the same, and depends much more on the food or nourishment supplied, whether the pabulum contains more or less of a saccharine, albuminous, or nitrogenous material, lactic acid, \&c., together with light and temperature; whether we have a mould (green or blue), an achorion, or yeast fungus produced. Diversity of form in the cells, as well as quality and quantity of their material contents, are certainly due to, and in a manner regulated and controlled by that beautiful law of diffusion, which admits, separates, sifts, and refines the coarser from

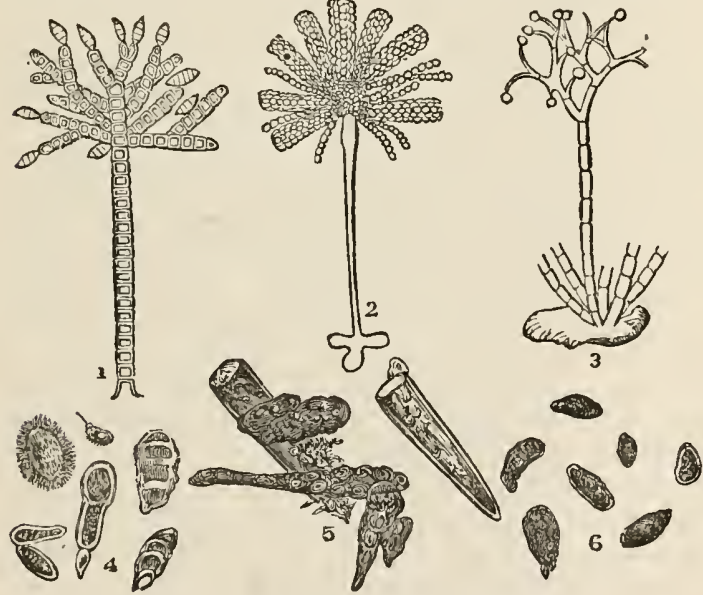

Fig. 161.-Fungl. (Magnified 200 diameters.)

1, Brachycladium penicillatum, growing on the stem of a plant. 2, Aspergillus glaucus, growing on cheese, \&c. 3, Botrytis; the common form of mould on decaying vegetable substances. 4, Spharia, fungi caught cver a sewer (foul air). 5, Fungi growing on a pumpkin. 6, Fungi caught in the air at the time of the cholera visitation, 1854.

the finer, the lighter from the denser particles, through the porous structure of the cell-wall. 
We cannot cunclude this brief notice of the fungi with. out adding a few words upon that curious group of subterranean plants, which instead of producing their spores at the summit of a basidium, or extremity of a simple filament, produces them in the interior of a vesicle or pouch, called a theca or ascus. Of this species the best known example is the truffle (Tuber cibarium).

It is, perhaps, not very generally known that the curiously formed, irregular mass, so much esteemed for its delicious taste, and songht after as a luxury, the truffle, is in truth a species of mushroom; more properly speaking, a subterranean puff-ball, or fungus. Its existence, entirely removed from the action of light, is an anomaly even among plants of the fungus kind; for light, although not in a large degree necessary to the fungus, is almost always indispensable to its full development. It would, therefore, be most difficult to discover, if it were not for a peculiar and penetrating odour, which dogs are taught to recognise; and by the aid of these useful animals its presence is detected hidden beneath the soil.

Tulasne and others have pointed out that these fungi present two essentially different types. In the one, $H y$ menogastrece, the internal fleshy mass presents a number of irregular cavities, lined by a membrane analogous to that which clothes the gills of the Agaric, and the superficial cells produce at their free extremities three or four spores, or seeds, which become detached, and eventually fill up the cavities. The other type, Elaphemyca, Tuberacea, comprising those of the truffle kind, and as may be surmised by the scientific name assigned to them-Tuber nibarium,-are plants characterised from the underground root presenting a fleshy mass, the outer surface of which constitutes the common envelope, peridium, while the numerous narrow sinuous cavities are lined and in part filled up by filamentous tissue, mingled with cells of a peculiar form, and terminating in spores.

A section from the fleshy-looking mass cut very thin (Plate I. No. 2), and viewed under a power of 250 diameters, is found to be chiefly composed of cellular substance, the interspaces of which are filled up by jointed filaments, homologous to the mycelium or spawn 
of other fungi,-in the mushroom, as an example, it is the mushroom spawn, - while the reins, the reproductive parts, contain in their cellular tiss' e minute ovoid capsules, with two or more globular yellowish seeds; this curious structure having all the parts of uutrition and reproduction enclosed internally, instead of externally, as in other fungi.

Truffles are produced in this way: the mycelium quickly decays and allows the fungoid body to grow on in an isolated condition. About September the ground becomes covered with numerous white cylindrical articulated filaments, not visible singly to the unassisted .eye, but by their immense numbers and rapid growth readily seen, and found traversing the soil in every direction. These white flaxen threads are continuous with other flocculent filaments of the same nature. In the young truftles the external layer is gradually consolidated, and in a short time the destruction of the flocculent filaments are complete and lost in the young plant, which is soon isolated in the soil, and then the outer or cortical coat hardens, and ultimately has the appearance of a small nut. Thus, like other fungi, truffles are reproduced by spores, which give origin to filamentous mycelium and seed-vessels, the source of numerous offspring. Groups of spores are pretty objects; their stellate appearance reminds one of the Xanthidiæ ; the mass of the full-grown plant at particular seasons is almost wholly made up of these bodies, which are of a yellowish-brown colour.

In these plants we have a double system of laminated filaments ; one set arising from the cortical tissue absorbing the surrounding moisture and serving to transmit this to the cells in which the spores are formed, being therefore the organs of nutrition; the others white and opaque, terminating externally also, but conveying air to all parts of the body, and bringing the whole into contact with sporigenous cells.

The spores are developed freely in the vesicular cells destined to produce them. They are limited in number in each vesicle; less than two is never seen in one vesicle; the hexagonal basket-work arrangement of each seed appears to close with a lid, and ten or twelve short spines projert out from every point. 
Leneath the external dark-coloured reticulated membrane is a second integument, smooth and transparent, masily separated by maceration, although it resists the action of chemical agents, and is not coloured by iodine. The simple cavity of the internal spore is filled with minute granular particles and fatty globules, suspended in a fluid probably albuminous, as well as the various chemical salts found by Riègel, and upon which its peculiar flavour depends.

"Two new British fungi" are figured and described by the Rev. M. J. Berkeley, in vol. xxv. p. 431, Linnean Soc. Trans. Peziza pygmaea, Plate I. No. 4, is a remarkably interesting specimen of a genus which presents much variety in form. The description given of it is, " that it is about $\frac{1}{4}$ inch high, the stem often splitting or branching out into several divisions, each of which is terminated by a minute cup, giving the plant the appearance of a Ditiola, or a Tympanis. Each cup produces other smaller cups on its surface; the branched and young cups resemble the genus Solenia: in a specimen found at Wimbledon, the mass of secondary cups gave the plant almost the appearance of a small Gyromitra." The proliferous form is shown at Plate I. No. 5. The colour of the mature plant is a bright apricot, whitish and tormentose at the base of the stem. Found in swampy places, rotten gorse, \&c. at Ascot, Wimbledon, \&c. Peziza belongs to the Ascomycetous fungi; the genus contains numerous species, and many of them are brightly coloured, as in the very pretty $P$. bicolor, Plate I. No. 1. Tulasne says that some of them have a secondary fructification, consisting of stylospores. They are mostly found growing on trunks of trees, dead wood, \&c.

We now pass to the examination of Lichens; in these plants, as in the Fungi, the germination of the spore consists in the emission of a hollow filament from some part of its surface. This filament, which is simply an extension of the spore-membrane, branches repeatedly, and spreads over the surface on which the spore has been sown; at the same time it divides by numerous septa which occur at irregular intervals. By the intertwining of the resurtant ramifications, a stroma is formed, to which 
the term hypothallus is applied, and which constitutes the vegetative system of the future lichen. So far the development is the same as that of the fungi ; but at a longer or shorter period after the formation of the hypothallus, we may observe upon its surface a whitish layer of spheroidal cellules, intimately united with each other as well as with the filaments from which they take their origin. This layer is the groundwork for a second formation of globular cells, and these are only to be distinguished from the first by the chlorophyll which they contain. They are called gonidia, and are peculiar to Lichens. Such is the formation of the most simply organized of the class, as the Verrucarice, the receptacles (apothecia) of which closely resemble those of a Sphceria, and are found upon the surface of the hypothallus. In the more complicated foliaceous Lichens, as Parmelia, the mature thallus is made up of tro kinds of tissues, the medullary and the cortical. The corticular portion forms the layers, an inferior and superior, and consists of thick-walled cells, closely adherent to each other; from the surface of the inferior layer are given off numerous root-like appendages, on either side of which, or rather $\epsilon$ mbedded in its cortical substance, are the gonidia, which form a green tissue. Of the spore-like organs, spermatia and stylospores, there are three varieties, to which the terms apothecia, spermogonia, and pyenides have been applied. The most common form of the apothecium is that of the disc, which may be plane, convex, or cup-shaped. This form is that which characterises the Gymnocarpous Lichens. In the Angiocarpece the organ is closed upwards, its superior surface becoming internal, so as to form a conceptacle like that of the Pyrenomycetes; the form, however, of which is subject to much variation.

The reproductive organs of Lichens, as in Fungi, are of five kinds.-1, Sporules, which are formed by the construction and subsequent separation of the extremity of a simple cylindrical filament; 2, Spermatia with their supporting pedicles; 3, Stylospores with their styles; 4, Thecæ or asci; 5, Basidia with their basidiospores. As regards the complexity of their form and structure they may be taken in the order in which they are here placed; 
but, of the last-mentioned, it should be stated that they are almost solely found in Fungi, which have really no other reproductive organ. The spores present many points of difference, both in number and character, in different genera and species, and for this reason are most interesting microscopic objects. We would direct the reader's attention to an interesting and valuable paper, from the pen of Dr. Lauder Lindsay, in the Linnean Soc. Trans., vol. xxv. p. 493, "On the Lichens of New Zealand" (the country par excellence of certain Lichens). The paper is very beautifully illustrated, showing chiefly the minute or microscopic anatomy of the reproductive organs of the species examined, especially the character of their spores and spermatia.

A vertical section of Parmelia stellati is given in Plate I. No. 26: it belongs to an extensive genus of Gymnocarpous open-fruited Lichens, found growing upon trees, palings, stones, walls, \&c. The emission of the ripe spores of the Lichens is a curious process, and not unlike that which is seen to take place in some of the Fungi, as in Pezizce, Sphcerice, \&c. If a portion of the thallus be moistened and placed in a common phial, with the apothecia turned toward one side, in a few hours the opposite surface of the glass will be found covered with patches of spores, easily perceptible by their colonr; or if placed on a moistened surface, and one of the usual glass slips laid over it, the latter will be covered in a short time. As to the powvers of dissemination of these lowly organized plants, Dr. Hicks's observations lead to the conclusion that the gonidia of Lichens have greater powers in this direction than has been generally supposed. He found by placing a clean sheet of glass in the open air during a fall of snow, and receiving the melting water in a tube or bottle, that he obtained large quantities of what has been looked upon as a "nnicellular plant, commonly called 'Chlorococcus,' the cells of which may remain in a dormant condition for a long time during cold weather, but upon the return of warmth and moisture they begin to increase by a process of subrivision, into two, four or eight portions, which soon assume a rounded form and burst the parent cell-wall open; these secondary cells 
soon begin to divide and subdivide again, and this process may go on without much variation even for years. The phenomena described may also be watched by taking a portion of the bark of a tree on which the Chlorococcus has been deposited, and placing it under a glass to keep it in a moderately moist atmosphere; the only difference being a change in colour, which is caused by the growth of the fibres, as may be seen on microscopical examination. And this," Dr. Hicks says, "is an instructive point, because it will be found that the colour varies notably according to the Lichen prevalent in its neighbourhood." $1 \mathrm{He}$ thinks there can be no doubt that what has been called Chlorococcus, is nothing more than the gonidia of some Lichen; and that under suitable conditions, chiefly drought and warmth, the gonidium often throws out from its external envelope, a small fibre, which, adhering and branching, ultimately encases it and forms a "soridium." "The soridia also remain dormant for a very long time, and do not develop into thalli unless in a favourable situation; in some cases it may be for years. It will be easily perceived that the soridium contains all the elements of a thallus in miniature ; in fact, a thallus does frequently arise from one alone, yet, generally, the fibres of neigh bouring soridia interlace, and thus a thallus is matured more rapidly. This is one of the causes of the variation of appearance, so common in many species of Lichens, and is more readily seen towards the centre of the parent thallus. When the gonidia remain attached to the parent thallus, the circumstances are, of course, generally very favourable, and then they develop into secondary thalli, attached more or less to the older one, which, in many instances, decays beneath them. This process being continued year after year, gives an apparent thickness and spongy appearance to the Lichen, and is the principal cause of the various modifications in the external aspect of the Lichens which caused them formerly to be misclassified." 2

(1) "For instance, where the yellow Parmelia is found, the Chlorococcus will assume a yellow tinge in its soridial stage. Viewed by transmitted liglit, they are also opaque balls, with irregular outline."

(2) "Contributions to the Knowledge of the Develonment of the Gonidia of Lichens." By J. Braxton Hicks, M.D. dic., Quarterly Journal of Microscopica, Science, vol, viii. 860, p. 238.9 
The little group of Ilepaticee or Liverworts, which is intermediate between Lichens and Mosses, presents numerous objects of interest for the microscopist. These plants are produced by dust-like grains called spores, and minute cellular nodules called gemmoe or buds. The gemmæ of Marchantia polymorphia are produced in elegant membranous cups, with a toothed margin growing on the upper surface of the frond, especially in very damp court yards between the stones, or near rumning water, where its lobed fronds are found covering extensive surfaces of moist soil. At the period of fructification, these fronds send up stalks, which carry at their summit round shield-like or radiating dises. Besides which, it generally bears upon its surface a number of little open basket-shaped "conceptacles" which are borne upon the surface of the frond, as in fig. 162, and may be found

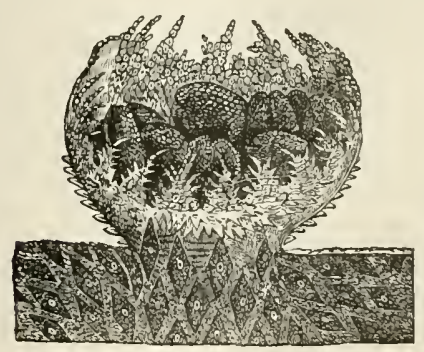

Fig. 162,-Gemmiparaus Conceptacle of Marchantia polymorphia, expanding and rising from the surface of a frond. in all stages of development. When mature it contains a number of little green round or oblong discs, each composed of two or more layers of cells; the wall is surmounted by a glistening fringe of teeth, whose edges are them. selves regularly fringed with minute outgrowths. The cup seems to be formed by a development of the superior epidermis, which is raised up and finally bursts and spreads out, laying bare the seeds. The development of this structure presents much analogy to that of the sori of Ferns.

Muscacece, Mosses, are an interesting form of vegetable life, Linnæus called them servi,--servants, or workmen,as they seem to labour to produce vegetation in newlyformed countries, where soil is not yet formed. They also fill and consolidate bogs, and form rich mould for the growth of larger plants, which they protect from the 
winter's cold. The common, or Wall Screw-moss, fig. 163, growing almost every where on old walls and other brick-work, if examined closely, will be found to have springing from its base numerous very slender stems, each

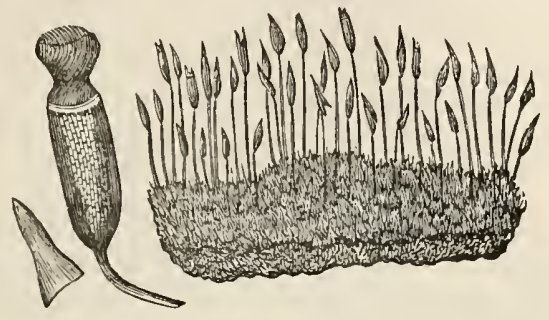

Fig. 163.-Screw MIoss.

of wh.ch terminates in a dark brown case, which encloses its fruit. If a patch of the moss is gathered when in this state, and the green part of the base is put into water, the threads of the fringe will uncoil and disentangle themselves in a most curious and beautiful manner; from this circumstance the plant takes its popular name of Screw-moss. The leaf usually consists of either a single or a double layer of cells, having flattened sides, by which they aahere one to anotner. The leaf-cells of the Sphagnum bogmoss, fig. 179, exhibit a very curious departure from the ordinary type; for instead of being small and polygonal, they are large and elongated, and contain spiral fibres loosely coiled in their interior. Mr. Huxley pointed out, that the young leaf does not differ from the older, and that both are evolved by a gradual process of "differentiation." Mosses, like liverworts, possess both antheridia and pistillida, which are engaged in the process of fructification. The fertilized cell bo-

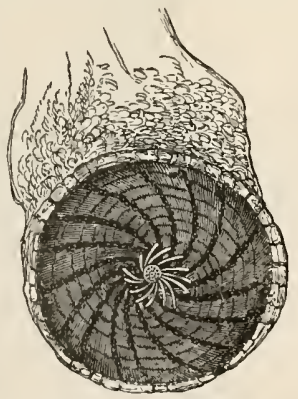
comes gradually developed into a conical body elevated 
upon a foot stalk; and this at lengtlı tears across the walls of the flask-shaped body, carrying the higher part upwards as a calyptra or hood upon its summit, while the lower part remains to form a kind of collar round the base. These spore-capsules are closed on their summit by opercula or lids, and their mouths when laid open are surrounded by a beautiful toothed fringe, termed the peristome. This fringe is shown in fig. 164 in mouth of capsule of Funaria, with its peristome in situ. The fringes of teeth are variously constructed,

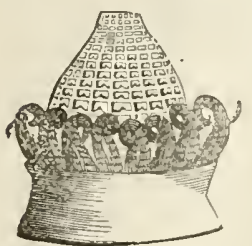
Fig. 165.-Doubie Peristrme of is termed the columella; and at the Neckera Antipyrtica.

time of maturity, the interior of the capsule is almost entirely occupied by spores.

It may here be mentioned, that all mosses and lichens are more easily detached from the rocks and walls on which they grow in frosty weather than at any other

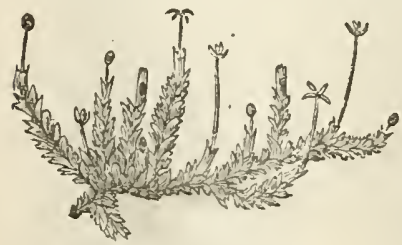

Fig. 166. -Scale-MLoss. season, and consequently they are best studied in winter. One of the commonest, Scale-moss, fig. 166 (Jungermannia bidentata), grows in patches, in moist, shady situations, near the roots of trees: see Plate II. Nos. 35 and 36. The seedvessels are little oval bodies, which if gathered when unexpanded, and lrought into a warm room, burst ander the eye with violence the moment a drop of water is applied to them, the valves of the ressel taking the shape of a cross, and the seeds distending in a cloud of brown dust. If this dust be examined with the 
microscope, a number of curious little chains, looking something like the spring of a watch, will be found among it, their use being to scatter the seeds; and if the seed-vessel be examined while in the act of bursting, these little springs will be found twisting and writhing about like a nest of serpents. The undulating Hair-moss (Polytrichum undulatum), fig. 167, is found on moist shady banks, and in woods and thickets. The seedvessel has a curious shaggy cap ; but in its construction it is very similar to that of the Serewmoss, except that the fringe around its opening is not twisted.

Equisetacece.- The history of the development of the Equisetaceæ (horse-tails) corresponds in some respects with that of Ferns. The spore-case of this solitary genus is a most interesting object under the microscope; they have apparently only one coat, for the outer coat splits up into four thread-like processes (elaters), clubbed at their free ends. While the spore remains on the

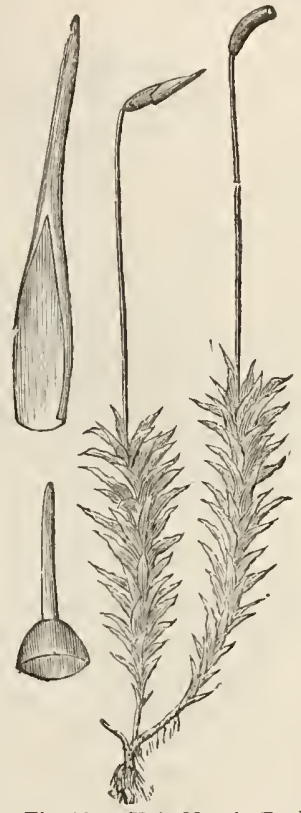

Fig. 167.-Hair-Moss in Fruit. sporange, these fibres are rolled round the spore, as seen in fig. 170 , G; but by gently shaking the fruit spike, the spores are discharged, the coiled fibres immediately umroll, as at F, their elasticity causing them to spring about in a most curious manner. In a few minutes this motion apparently ceases, but if breathed upon they again unroll and dart about with wonderful elasticity.

Ferns.-In the Ferns we have an intermediate state, somewhat between mosses and flowering plants; this wot'i $\dot{a}$ not apply to the reproductive apparatus, which is formec upon the same type as that of Mosses; and, furthermore, it is to be observed, that Ferms do not form 
buds like other plants, but that their leaves, or fronds as they are properly called, when they first appear, are rolled up in a circinate form, and gradually unfold, as in fig. 168 .

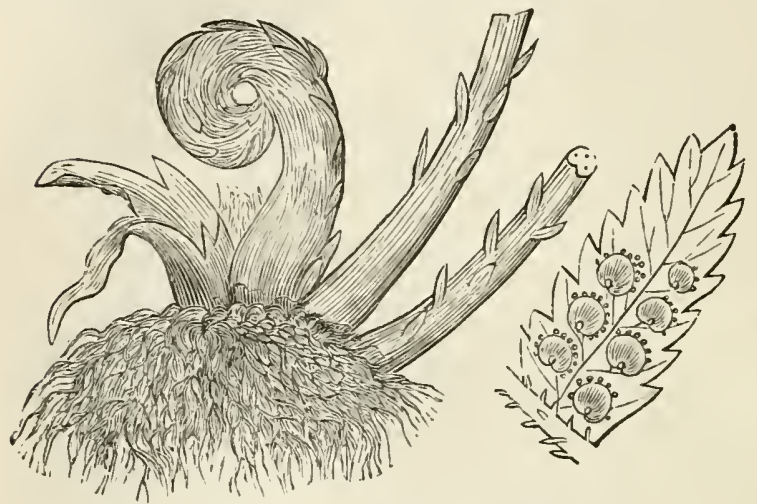

Fig. 168.-Male Fern. A portion of leat with sori.

Ferns have no visible flowers; and their seeds are produced in clusters, called sori, on the backs of the leaves. Each sorus contains numerous thecæ, and each theca encloses almost innumerable sporanges, with spores or seeds. There are numerons kinds of ferns, all remarkable for some interesting peculiarity; but it is their spores which are chiefly sought for by the microscopist.

The first account of the true mode of development of Ferns from their spores was published in 1814, by Nägeli, in a memoir entitled Moring Spiral Filuments (spermatic flaments) in Ferns, wherein he amnounced the existence of the bodies now called antheridia; but, mistaking the archegonia for modified forms of the antheridia, he was led away from a minute investigation of them. If he had followed the development of the prothallia further, he would have detected the relations of the nascent embryo, which would probably have put him on the right track. As it was, the remarkable discovery of the moving spiral filaments occupied all his attention, and caused him to fall 
into an error in certain important respects; for example, he has represented what is undoubtedly an archegonium filled with cellules, sperm-cells, which, he states, "emerged from it as from the antheridia." This description is not quite correct.

The reproduction of ferns had, until within the last few jears, been a vexed question among botanists. The riddle was at length solved by the labours of Count Suminski, who discovered that it is in the structures developed from the spores in germination that the pistillidia and antheridia of ferns are to be songht. The nature of the phenomena by which the propagation of ferns is effected, is as follows. In all the different species of ferns, the spores are contained in brown dots, on lines collected on the under surfaces, or along the edges of the fronds. Each of the spore-cases is surrounded by an elastic ring, which when the time arrives for the spores to be set free, makes an effort to straighten itself, and in so doing causes the sporecase to which it is attached to split open, and the spore dust to be dispersed. Very soon after these spores have begun to germinate, a flat plate-like expansion, somewhat resembling a

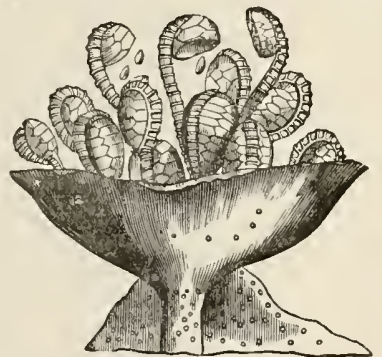

Fig. 169.-Sorus of Deparia prolifera. heart in form, shows itself. This expansion gradually thickens, the tube from which it had sprung withering away. So far, observes Mr. Henfrey, there is nothing very remarkable in the development of these plants from their spores, but the succeeding phenomena are exceedingly curious. The main particulars are thus described by him : "At an early period of the expanding growth of the leaflike product of the spore, termed the prothallium or germ-frond; a number of little cellular bodies are found projecting from the lower surface, which, if placed in water when ripe, burst and discharge a quantity of microscopic filaments, curled like a corkscrew, and furnished with vibrating hair-like appendages, by the motion of 
which they are rapidly propelled through the water. The cellular bodies from which these are discharged are termed the antheridia of the ferus, and are in their physiological zature the representatives of the pollen of the flowering plants. At a somewhat later period other cellular bodies of larger size and more complex structure are found in small numbers about the central part of the lower surface of the prothallium on the thickened portion, situated between the notch and the part where the radical filaments arise. These, the pistillidia or archegonia of the ferns, are analogous to the ovules or nascent seeds of flowering plants, and contain, like them, a germinal vesicle, which becomes fertilized through the ageney of the spiral filaments mentioned above, and is then gradually developed into an embryo plant possessing a terminal bud. This bud begins at once to unfold and push out leaves with a circinate vernation, which are of a very simple form at first, and rise up to view beneath the prothallium, coming out at the notch; single fibrous roots are at the same time sent down into the earth, the delicate expanded prothallium withers away, and the foundation of the perfect fern plant is laid. As the bud unfolds new leaves, the root stock gradually acquires size and strength, and the leaves become larger and more developed; but it is a long time before they assume the complete form characteristic of the species."

These ubservations on Ferns have acquired vastlyincreased interest from the subsequent investigations of Hoffmeister, Mettenius, and Suminski, on the allied Cryptogams, and, above all, from Hoffmeister's observations on the processes occurring in the impregnation of the Conifers. Not only have these investigations given us a satisfactory interpretation of the archegonia and antheridia of the Mosses and Liverworts, but they have made known and co-ordinated the existence of analogous phenomena in the Equisetacece, Lycopodiacece, and Rhizocarpece, and shown, moreover, that the bodies described by Mr. Brown in the Conifers, under the name of "corpuscles," are analogous to the archegonia of the Cryptogams; so that a link is hereby formed between these groups and the higher Howering plants. 
The fruits, Gi sori, of Ferns afford a very beautiful variety of objects for the microscopist, and they possess an advantage in requiring little or no preparation-nothing more being necessary than that of taking a portion of a frond, place it on a glass-slip under the microscope, and throwing a condensed light upon it by the aid of the side reflector. Even germination may be watched by simply employing gentle heat and moisture. Take, as Hofimeister directs, a frond of a Fern whose fructification is mature, lay it upon a pieee of glass covered with fine paper, and place the spore-bearing surface downwards upon this ; in the course of a day or two this paper will be found to be covered with a fine browuish dust, which consists of the liberated spores. These must be carefully collected, and spread out npon the surface of a smooth fragment of porous sandstone, and then placed in a saucer, the bottom of which is before covered with water; a glass tumbler being inverted over it to ensure the requisite supply of moisture, and prevent rapid evaporization. Some of the prothallia soon germinate; if the cup be kept only slightly moist for some time, and then suddenly watered, a large number of antheridia and archegonia quickly open, and in a few hours thie surface of the larger prothallia will be covered with moving antherozoids. If sections of these be made, that is, the canals laid open, with a power of 200 or 300 diameters we may occasionally see antherozoids in motion.

Characes.-Chara vulgaris is the plant in which the important fact of vegetable circulation was discovered; Fig. 170, No. 1, is a portion of the plant of the natural size. Every knot or joint may produce roots; but it is somewhat remarkable, that they always proceed from the upper surface of the knot, and then turn downwards; so that it is not peculiar that the first roots also should rise upwards with the plant, come out of the base of the branch, and then turn downwards.

Mr. Varley noticed :- "The ripe globules spontaneously open; the filaments expand and separate into clusters." "These tube-like filaments are divided into numerous compartments, in which are produced the most extraordinary objects ever observed of vegetable origin, Fig. $170 \mathrm{~A}$. At first they are seen agitating and moving in their 
cells, where they are coiled up in their confined spaces, every cell holding one. They gradually escape from their

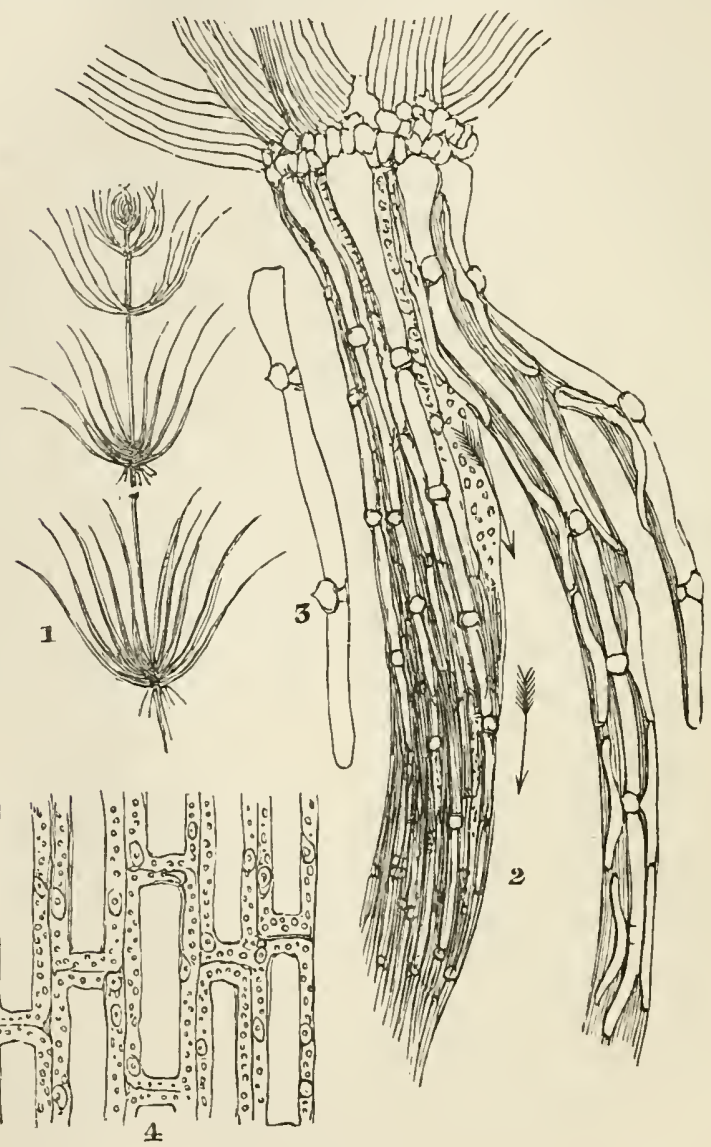

Fig. 170.

1, Branch of Chara vulgaris. 2, Magnified view: the arrows indicate the courme taken by the granules in the tubes. 3, A limb of ditto, with buds at jcints. 4. Portion of a leaf of Vallisneria spiralis, with cells and granules. 
cells, and the whole field soon appears filled with life. They are generally spirals of two or three coils, and never become straight, though their agitated mction alters their shape in some degree. At their foremost end is a filament so fine as only to be seen by its motion, which is very rapid and vibratory, running along it in waves: and of a globule be forcibly opened before it is ripe, the filaments will give little or no indication of life."

They swim about freely for a time, but gradually getting slower and slower; in about an hour they become quite motionless. Unger described these moving filaments in Sphagnum (bog-moss) as Infusoria, under the name of
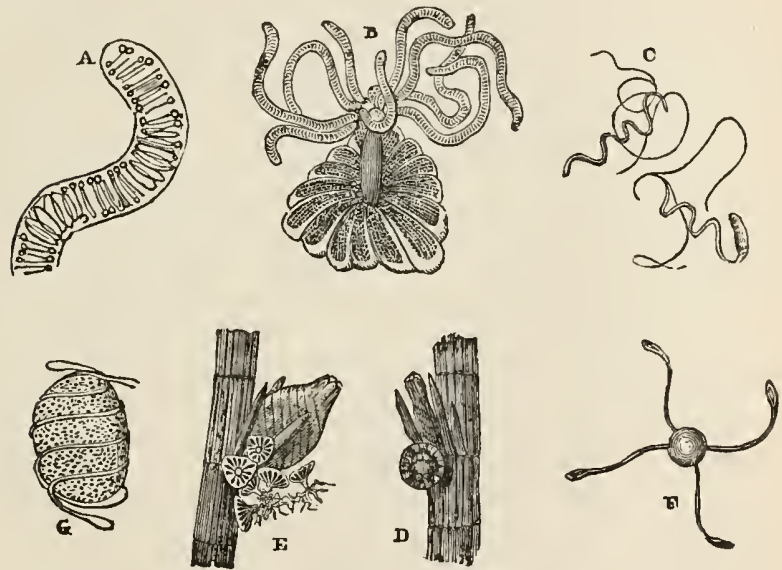

Fig. 171.-Antheridia of Chara jragilis, \&.c.

A Portion of filament dividing into Phytozoa, "antheroids." B, A valve, with its group of antheridial filaments, composed of a series of cells, within each of which an antherozoid is formed. c, The escape of the mature antherozoids is shown. D, Antheridium, or globule, developed at the base of nucule. E, Nucule enlarged, and globule laid open by the separation of its valves. F, Spores and elaters of Cquisetum. G, Spores surrounded by elaters of Equisetum.

Spirillum; and consequently they have been the cause of much controversy. Schleiden, very properly denying their animal nature, says:-"They are nothing more than fibre 
in an early stage of development." The Characeæ are all aquatic plants of filamentous structure. Some authors have divided the species into two genera, Nitella (simple tubes) and Chara (cortical tubes). The circulation in the ordinary tubes or cells consists in the movement of the gelatinous protoplasmic sac, seen, as one mass, slowly passing up one side, across the ends, and down the opposite side, not perpendicularly, but in an oblique or spiral course, as indicated in the figure. The Characce multiply by gemmæ, produced at the articulations of their stems.

Mr. H. J. Carter, in a paper of great interest, published. January, 1857, on the "Development of the Root-cell and its Nucleus, in Chara Verticillata," describes a structureless cell-wall, and a protoplasm composed of many organs. "This," he says, "is surrounded by a cell, the "protoplasmic sac,' which is divided into a fixed and rotatory portion; these again respectively enclose the nucleus ' granules,' and axial fluid ; while small portions of irregular shaped granular bodies are common to both. If we take the simple root-cell about the eighteenth hour after germination, when it will be about half-an-inch long, and 1-600th of an inch broad, and place it in water between two slips of glass for microscopic observation, under a magnifying power of about four hundred diameters, we shall find, if the circulation be active and the cell-wall strong and healthy, that the nucleus, which is globular, gradually becomes somewhat flattened, having several hyaline vacuoles of different sizes; the change goes on gradually until it appears of more elongated form, growing fainter on its ontline, and then entirely disappears, leaving a white space corresponding to its capsule or cell-wall, with a faint remmant of some structure on the centre. Subsequently, this space becomes filled up with the fixed protoplasm, and after an hour or two the nucleus reappears a little behind its former situation, but now reduced in size, and with its nucleolus double, instead of single as before; each nucleolus being about one-fourth part as large as the old nucleolus, and hardly perceptible. Meanwhile a faint septum is seen obliquely extending across the fixed protoplasm. a little beyond the nucleus; 
and if iodine be applied at this time, the division is seen to be confined to the protoplasm, as the latter, from centraction, withdraws itself from each side of the line where the septum appeared, and leaves a free space which is bounded laterally by an uninterrupted continuation of the protoplasmic sac. In this way changes go on until its shape is altered and it bccomes converted into a bunch of rootlets. Thus the new cells are never entirely without a nucleus, which would appear to exert some influence upon their development, for as soon as the only two new cells which the root-cell gives off are formed, the old nucleus becomes effete.

"Now as to the office of the nucleus, nothing more is revealed to us in the development of the roots of Chara, than that, so long as new cells are to be budded forth, the mucleus continues in active operation, but when this censes it becomes effete; while the rotation of the protoplasm and subsequent enlargement of the cell, \&c., which are much better exemplified in the plant-stem than in the root-cell, go on after the nucleus ceases to exist. Hence the development of the root-cells of Charc affords us nothing positive respecting the functions of this organ; and therefore, if we wish to assign to it any uses in particular, they must be derived from analogy with some organism in which there is a similar nucleus whose office is known. If for this purpose we may be allowed to compare the nucleus of Chara with that of the rhizopodous cell, which inhabits its protoplasm, we shal, find the two identical in elementary composition; that is, both consist at first of a 'nuclear utricle,' respectively enclosing a structureless homogeneous nucleolus; the latter, too, in both, is endowed with a low degree of movement.

"After this, however, the nucleolus of the Rhizopod cell becomes granular and opaque; and when, under circumstances favourable for propagation, a new cell-wall is formed around the nuclear utricle,-or this may be an enlargement of the nuclear utricle itself,-I do not know which; the granular substance of the nucleolus becomes circumscribed, and shows that it is surrounded by a spherical, capsular cell; the granules enlarge, separate, pase 
through the spherical capsule into the cavity of the nuclear utricle; a mass of protoplasm makes its appearance, and this divides up into monads, or, as I first called them, 'gonidia.' 1

"The movement of the rotating protoplasm in the Characece is also very slow; for, when it is viewed in the long internodes of Nitella with a very low power, or even with the naked eye, it seems hardly to move faster than the foot of a Gasteropod; still there is no positive evidence that it moves round the cell after the manuer of the latter, although it would appear to possess the power of movement per sc. Hence the question remains undecided, viz. whether it moves round the cell by itself, or by the aid of cilia disposed on the inner surface of the protoplasmic sac, in like manner to those which appear to exist in the abdominal cavity of Vaginicola crystallina, and which have been seeu in Closterium lunula."2

The stems and arms of Chara are tubular, and entirely covered with smaller tubes, the circulation can mostly be observed in these, as shown at Fig. 170. Any ordinary cutting to obtain sections would squeeze the tube flat, and spoil it and the lining; it is, therefore, better to avoid this, by laying the Chara on smooth wood, just covered with water; then, with a sharp knife, make suddenly a number of quick cuts across it, and so obtain the various sections required. Wet a slip of glasi, and turn the wood over so as just to touch the water, and the sections will fall from the wood on to the glass, ready for the microscope.

"The Chara tribe is most abundant in still waters or ponds that never become quite dry ; if found in running water, it is mostly met with out of the current, in holes or side bays, where the stream has little effect, and never on any prominence exposed to the current. If the Chara could bear a current, its fruit would mostly be carried on and be deposited in whorls; but it sends out from its variono joints very long roots into the water, and these would by agitatiou be destroyed, and then the plant

(1) Ann. and Mag. Nat. Hist. vol. xvit. 1856.

(2) In Plate I. No. 27, is represented the Hoss gonida, assuming the amoboid form as described by Dr. Hicks in the Linnean Trans. 1862. 
decays; for although it may gruw long before roots are formed, yet when they are produced their destruction involves the death of the plant. In order, therefore, to preserve Chara, every care must be taken to imitate the stilluess of the water by never shaking or suddenly turning the vessel. It is also important that the Chara should be disturbed as little as possible; and if requisite, it must be done in the most gentle manner, as, for instance, in cutting off a specimen, or causing it to descend in order to keep the summit of the plant below the surface of the water.

Similar care is requisite for Vallisneria; but the warmest and most equal temperature is better suited to this plant. It should be planted in the middle of the jar in abont two inches deep of mould, which has been closely pressed; over this place two or three handfuls of leaves. then gently fill the jar with water. When the water requires to be changed, a small portion is sufficient to change at a time. It appears to thrive in proportion to the frequency of the changing of the water, taking care that the water added rather increases the temperature than lowers it.

The natural habitat of the Frog-bit, another waterplant of great interest, is on the surface of ponds and ditehes; in the autumn its seeds fall, and become buried in the mud at the bottom during the wiuter; in the spring these plants rise to the surface, produce flowers, and grow to their full size during summer. Chara may be found in many places around London, the Isle of Dogs, and in ditches near the Thames bank.

Anacharis alsinastrum.-This remarkable plant is so unlike any other water-plant, that it may be at once recognised by its leaves growing in threes round a slender stringy stem. The watermen on the river have already named it "Water-thyme," from a faint general resemblance which it bears to that plant. In 1851 the Anacharis was noticed by Mr. Marshall and others in the river at Ely, but not in great quantities. Next year it had increased so much, that the river might be said to be full of it.

The colour of the plant is deep green; the Jeaves are 
nearly half an inch long, by an eighth wide, egg-shaped at the point, and beset with minute teeth, which cause them to cling. The stems are very brittle, so that whenever the plant is disturbed, fragments are broken off. Its powers of increase are prodigions, as every fragment is capable of becoming an independent plant, producing roots and stems, and extending itself indefinitely in every direction. Most of our water-plants require, in order to their increase, to be rooted in the bottom or sides of the river or drain in which they are found; but this is independent altogether of that condition, and actually grows as it travels slowly down the stream after being cut. The specific gravity of it is so nearly that of water, that it is more disposed to sink than float. A small branch of the plant is represented, with a Hydra attached to it, in a subsequent chapter.

Mr. Lawson pointed out the particular cells in which the current or circulation will be most readily seenviz. the elongated cells around the margin of the leaf and those of the midrib. On examining the leaf with polarised light, these cells, and these alone, are found to contain a large proportion of silica, and present a very interesting appearance. A bright band of light oncircles the leaf, and traverses its centre. In fact, the leaf is set, as it were, in a framework of silica. By boiling the leaf for a short time in equal parts of nitric acid and water, a portion of the vegetable tissue is destroyed, and the silica rendered more distinct, without changing the form of the leaf. ${ }^{1}$

It is necessary to make a thin section or strip from the leaf of Vallisneria, for the purpose of exhibiting the circulation in the cells, as shown in fig. 170, No. 4. Among tho cells granules, a few of a more transparent character thau: the rest, may be seen, having a nucleolus within.

The phenomenon of cell rotation is seen in other plants besides those growing in water. The leaf of the common plantain or dock, Plantago, furnishes a good example; the movement being seen both in the cells of the plant, and hairs of the cuticle torn from the midribs. The Spiderwort will be noticed further on.

(1) See also a paper in Vol. IV. Microscopical Journal of Science, un the Ci. culation in the Leap of Anacharis, by $\mathrm{Mr}$ F H. Wenham 
Structure of Plants. -The development of cells in plants takes place in all cases in essentially the same way, but the form of the result is subject to a number of inportant modifications. Most elementary works on Botany enter so fully into this interesting question, that it is quite unnecessary to do more than refer very briefly to the more important structural peculiarities of plants. Cell. division, as we have seen, is the universal formative process by which vegetative growth is effected, and free-cell. formation occurs only in the production of cells connected with reproduction. In the lower classes of plants, especially in aquatic genera, we can observe the process of celldivision in all its details; but in the higher, knowledge of this kind is only accessible by dissection.

As long as the cell retains its primordial utricle, it is capable of producing new cells, and organized forms of assimilated matter, like starch, chlorophyll, \&c. in its contents. This is the case in all nascent tissues, but it ceases to be so at various periods in different parts of the vegetable organization. In all woody tissues, in all pitted and spiral-fibrous cells it disappears early ; the secondary deposits of the ligneous character being formed apparently from the watery cell-sap. In herbaceous organs, such as leaves, in the cells of the cellular plants generally, the primordial ntricle remains. "This explains why the power to form adventitious buds exists not only in the cambrium layer of the higher plants, but, under certain conditions, even in the leaves, and why germination or propagation by little cellular bulbels, or isolated cells detached from the vegetative organs, is so common among the cellular plants, and in the Mosses and Liverworts, where parenchymatous tissues so greatly predominate."(Henfrey.)

The tissues of plants, properly so called, consist of collections of cells of uniform character, permanently combined together by more or less complete union of their outer surfaces. Tissues are of many kinds, according to the form of the cells, the character of the cell-membrane, and the manner in which the cells are connected together. The milk-vessels found in connexion with certain celis appear to be formed out of the intercellular passages, and not by 
fusion of celis ; hence they do not constitute a true cellular tissue.

Vascular tissue is formed by the fusion of perpendicular rows of cells; by the absorption of their contiguous walls they become converted into continuous tubes of more or less considerable length. Then we have a combination of tissues destined for particular purposes in the economy of the plant, divided into three primary systems-the Cellular, the Fibro-vascular, and Cortical. The 1st, Cellular, forms the great mass of the living structure of plants; and it is in this system that the vital processes of vegetation are chiefly carried on. The $2 d$, Fibro-vascular, forms all the woody structures, which in all cases are composed of a quantity of conjoined portions of cellular and vascular tissue arranged in a peculiar manner; differing in their modes of growth in different classes of plants, and which in consequence present considerable differences in the structure of their mature stems. The $3 \mathrm{~d}$, Cortical, also termed the epidermal, exists in the form of a simple flat layer of cells united firmly together by their sides, and forming a continuous coat over the surface of a plant. Such a layer clothes all the organs of plants above the Mosses, and, as stemis grow olcler, the epidermal layer gives place to the bark or rind. Stomata are orifices between the meeting angles of the epidermal cells; most abundant usually on the lower surface of leaves, often wanting on the upper surface. On the leaves of aquatic plants they are only found on the upper surface, and are absent where the leaf touches the water.

Hairs and scales of all kind depend on the development of the epidermal cells. Simple hairs are merely single epidermal cells produced in a tubular filament, and when cell-multiplication occurs in them they present a number of joints; see hairs of nettle, fig. 18S, No. 2. Thorns, such as those of the rose, are aborted branches, in which the cells become thickened by woody secondary deposits. In leathery or hard leaves, and in the thick tough leaves of succulent plants, such as the aloes, the secondary layers acquire great thickness. The aerial roots of the Orchidace exhibit a curious structure, the growing extremities being clothed by a whitish cellular tissue composed of several 
layers of cells with a delicate spiral fibrous deposit on their walls. This layer forms a kind of coat over the real epidermis of the root, and is known by the name of the Velamen radicum. The young shoots of Dicotyledonous trees and shrubs are clothed with epidermis-like herbaceous plants ; but, before the close of the past season of growth, in most cases the green colour gives place to brown, which is owing to the formation of a layer of cork from the outer layers of cortical parenchyma. Cork is composed of tubular thin-walled cells containing only air ; and sometimes these intercellular passages occupy a considerable space, and communicate in all directions, forming a system of air-spaces in the tissue. In addition there is the secretory system, consisting of glands, simple and compound, mill-vessels, and canals filled with resins, oils, \&c.

Much of the physiological history of plant life has yet to be made out: the mode in which the circulation and the formation of wood are carried on is by no means a settled question. Mr. Herbert Spencer observes : 1_ "That the supposition that certain vessels and strings of partially united cells, lined with spiral, annular, reticulated, or other frameworks, are carriers of the plant juices, is objected to on the ground that they often contain air; as the pressure of air arrests the movements of blood through arteries and veins, its presence on the ducts of stems and patioles is assumed to unfit them as channels for sap. On the other hand, that these structures have a respiratory office, as some have thought, is certainly not more tenable, since the presence of air in them negatives the belief that their function is to distribute liquid. The presence of liquid in them equally negatives the belief that their function is to distribute air. Nor can any better defence be made for the hypothesis which I find propounded, that these parts serve 'to give strength to the parenchyma.' In the absence of any feasible alternative, the hypothesis that these vessels are distributors of sap claims reconsideration." To obtain data for an opinion on this vexed question, Mr. Spencer instituted a series of experiments on the absorption of dyes by plants. His first experiments were failures, and it was only

(1) Linnean Soc. Trans. vol. xxy. page 405. 
after trying experiments with leaves of different arres and different characters, and with undeveloped axes, as well as with axes of special kinds, that it became manifest that the appearances presented by ordinary stems, when thus tested, are in a great degree misleading. "If an adult shoot of a tree or shrub be cut off, and have its lower end placed in an alumed decoction of logwood, or a dilute solution of magenta, ${ }^{1}$ the dye will, in the course of a few hours, ascend to a distance, varying according to the rate of evaporation from the leaves. On making longitudinal sections of the part traversed by it, the dye is found to have penetrated extensive tracts of the woody tissue; and, on making transverse sections, the openings of the ducts appear as empty spaces in the midst of a deeply-coloured prosenchyma. It would thus seen that the liquid is carried up the denser parts of the vascular bundles, neglecting the cambium layer, the central pith, and the spiral vessels of the medullary sheath." This, however, is found to be only partially true. "There are indications that while the layer of pitted cells next the eambium has served as a channel for part of the liquid, the rest has ascended the pitted ducts, and oozed out of these into the prosenchyma around. This is seen, if, instead of allowing. the dye time for oozing through the prosencliyma, the end of the shoot be just dipped into the dye and taken out again, we find that, although it has become diffused to some distance round the ducts, it has left tracts of wood between the ducts mentioned. Again, if we use one dye after another, a shoot that has absorbed magenta for an hour and then placed for five minutes in the logwood decoction, transverse sections taken at a short distance from the end of the shoot, show the mouths of the ducts surrounded by dark stains in the midst of the much wider red stains. The behaviour of these corresponds perfectly with the expectation that a liquid will ascend capillary tubes in preference to simple cellular tissue, or tissue not differentiated into continuous

(1) "These two dyes have affinities for different components of the tissues, and may be advantageously user in different cases. Magenta is rapidly taken up by woody matter and secondary deposits, while logwood colours the cell-membranes, and takes bit reluctantly to the substances seized by magenta. By trying botb of them on the same structure, we may guard ourselves against any error arising from relative combination." 
canals. Experiments with leaves bring out parallel facts ; and this, then, is confirmed, that in ordinary stems the staining of the wood by an ascending coloured liquid is due, not to the passage of the coloured liquid up the sub-

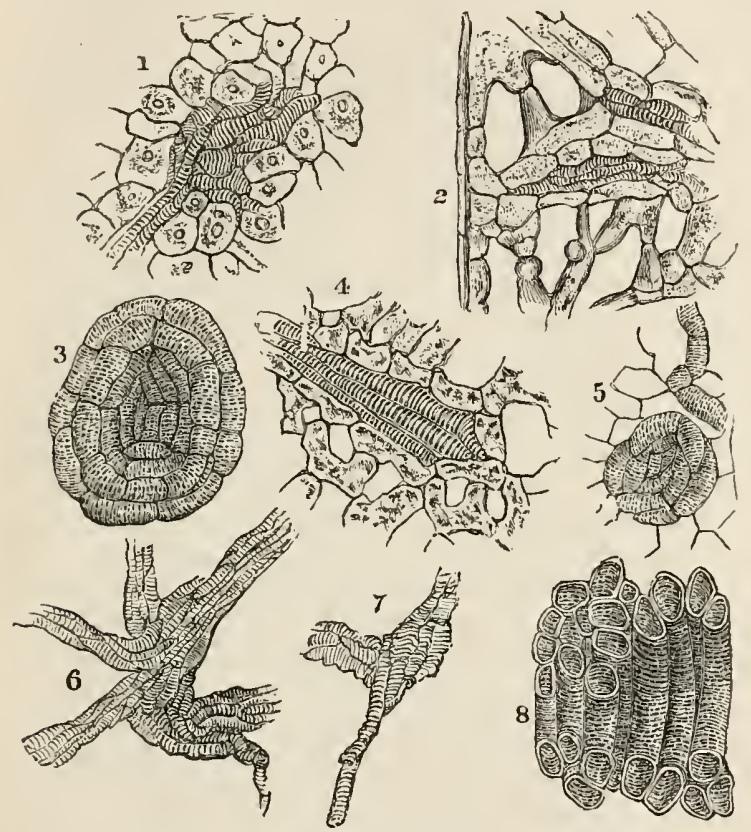

Fig. 172.-Termination of Vascular System (after Spencer).

1. Absorbent organ from the leaf of Euphorbia neriffolia. The cluster of fbrous cells forming one of the terminations of the vascular system is here embedded in a solid parenchyma.

2. A structure of analogous kind from the leaf of Ficus elastica. Here the expanded terminations of the vessels are embedded in the network parenchyma, the cells of which unite to form envelopes for them.

3. End view of an absorbent organ from the root of a turnip. It is taken from tlie outermost layer of vessels. Its funnel-shaped interior is drawn as it presents itself when looked at from the outside of this layer, its narrow end being directed towards the centre of the turnip.

4. Shows on a larger scale one of these absorbents from the leaf of Panax Lessonii. In this figure is clearly seen the way in which the cells of the network parenchyma unite into a closely-fitting case for the spiral cells.

5. A less-developed absorbent, showing its approximate connexion with a duct. In their simplest forms, these structures consist of only two fenestrated cells, with their ends bent round so as to meet. Such types occur in the cen- 
tral mass of the turnip, where the vascular system is relatively imperfect. Besides the comparatively regular forms of these absorbents, there are forms composed of amorphous masses of fenestrated cells. It should be added that both the regular and irregular kinds are very variable in their numbers: in some turnips they are abundant, and in others scarcely to be found. Possibly their presence depends on the age of the turnip.

6. Represents a much more massive absorbent from the same leaf, the surrounding tissues being omitted.

7. Similarly represents, without its sheath, an absorbent from the leaf of Clusia flava.

8. A longitudinal section through the axis of another such organ, showing $j$ ts annuli of reticulated cells when cut through. The cellular tissue which flls the interior is supposed to be removed.

stance of the wood, but to the permeability of its ducts and such of its pitted cells as are united into regular canals; and the facts showing this at the same time indicate with tolerable clearness the process by which wood is formed, for what in these cases is seen to take place with dye may be fairly presumed to take place with sap."

Taking it, then, as a fact that the vessels and ducts are the channels through which the sap is distributed, the varying permeability of their walls, and consequent formation of wood, is due to the exposure of the plant to intermittent mechanical strains, actual or potential, or both, in this way. "If a trunk, a bough, shoot, or a petule is bent by a gust of wind, the substance of its convex side is subject to longitudinal tension, the substance of its concave side being at the same time compressed. This is the primary mechanical effect. The secondary is when the tissues of the convex side are stretched, they also produce lateral compression of them. In short, that "the formation of wood is due to intermittent transverse strains, such as are produced in the aerial parts of upright plants by the action of the wind." Thus the subject is most ingeniously worked out, and the results of many very interesting and instructive experiments are recorded by the author of the paper.

"In the course of experiments on the absorption of dyes by leaves, it happened that, in making sections parallel to the plane of a leaf, with the view of separating its middle layer, containing the vessels, I came upon some structures that were new to me. These structures, where they are present, form the terminations of the vascular system. They are masses of irregular and imperfectly united fibrous cells, such as those out of rich vessels, 
are developed; and they are sometimes slender, sometimes bulky-usually, however, being more or less club-shaped. In transverse sections of leaves, their distinctive characters are not shown; they are taken for the smaller veins. It is only by carefully slicing away the surface of a leaf, until we come down to that part which contains them, that we get any idea of their nature. Fig. 172, No. 1, represents a specimen taken from a leaf of Euphorbia neriifolia. Occupying one of the interspaces of the ultimate venous network, it consists of a spirally-lined duct or set of ducts, which connects with the neighbouring vein a cluster of half-reticulated, half-scalariform cells. These cells have projections, many of them tapering, which insert themselves into the adjacent intercellular spaces, thus producing an extensive surface of contact between the organ and the embedding tissues. A further trait is, that the ensheathing prosenchyma is either but little developed or wholly absent; and consequently this expanded vascular structure, especially at its end, comes immediately in contact with the tissues concerned in assimilation. The leaf of Euphorbia neriifolia is a very fleshy one; and in it these organs are distributed through a compact, though watery, cellular mass. But in any leaf, of the ordinary type, which possesses them, they lie in the network of parenchyma, composing its lower layer; and wherever they occur in this layer its cells unite to enclose them. This arrangernent is shown in No. 2, representing a sample from the Caoutchouc-leaf as seen with the upper part of its envelope removed; and it is shown still more clearly in a sample from the leaf of Panax Lessonii, No. 4. Nos. 6 and 7 represent, without their sheaths, other such organs from the leaves of Panax Lessonii and Clusia fava. Some relation seems to exist between their forms and the thicknesses of the layers in which they lie. Certain very thick leaves, such as those of Clusia flava, have them less abundantly distributed than is usual, but more massive. When the parenchyma is developed not to so great an extreme, though still largely, as in the leaves of Holly, Aucuba, Camellia, they are not so bulky; and in thinner leaves, like those of Privet, Elder, \&c., they become longer and less conspicuously club-shaped. 
"Some adaptations to their respective positions seem implied by these modifications; and we may naturally expect that in many thin leaves these free ends, becoming still narrower, lose the distivictive and suggestive characters possessed by those shown in the drawings. Relations of this kind are not regular, however. In various other genera, members of which I have examined, as Rhus, Viburnum, Griselinia, Brexia, Botryodendron, Pereskia, the variations in the bulk and forn of these structures are not directly determined by the spaces which the leaves allow; obviously there are other modifying causes. It should be added that while these expanded free extremities graduate into tapering free extremities, not differing from ordinary vessels, they also pass insensibly into the ordinary inosculations. Occasionally, along with numerous free endings, there occur loops ; and from such loops there are transitions to the ultimate meshes of the veins.

"These organs are by no means common to all leaves. In many that afford ample spaces for them they are not to be found. So far as I have observed, they are absent from the thick leaves of plants which form very little wood. In Sempervivum, in Echeveria, in Bryophyllum they do not appear to exist; and I have been unable to discern them in Kalanchö̈ rotundifolia, in Kleinia ante-euphorbium, and ficoides, in the several species of Crassula, and in other succulent plants. It may be added that they are not absolutely confined to leaves, but occur in stems that have assumed the functions of leaves. At least I have found, in the green parenchyma of Opuntia, organs that are analogous, though much more rudely and irregularly formed. In other parts, too, that have usurped the leaf-function, they occur, as in the phyllodes of the Australian acacias. These have them abundantly developed; and it is interesting to observe that here, where the two vertically-placed surfaces of the flattened-out petiole are equally adapted to the assimilative function, there exist two layers of these expanded vascular terminations, one applied to the inner surface of each layer of parenchyma.

"Considering the structures and positions of these organs, as well as the natures of the plants possessing them, may we not form a shrewd suspicion respecting their function? 
Is it not probable that they facilitate absorption of the juices carried back from the leaf for the nutrition of the stem and roots? They are admirably adapted for performing this office. Their component fibrous cells, having angles insinuated between the colls of the parenchyma, are shaped just as they should be for taking up its contents, and the absence of sheathing tissue between them and the parenchyma facilitates the passage of the elaborated liquids. Moreover, there is the fact that they are allied to organs which obviously have absorbent functions. I am indebted to Dr. Hocker for pointing out the figures of two such organs in the Icones Anatomica of Link. One of them is from the end of a dicotyledonous root-fibre, and the other is from the prothallus of a young fern. In each case a cluster of fibrous cells, seated at a place from which liquid has to be drawn, is connected by vessels with the parts to which liquid has been carried. I bave met with another such organ, more elaborately constructed, evidently adapter to the same office, in the common turnip-root. As shown by the end view and longitudinal section in Nos. 3, 5, and 8, this organ consists of rings of fenestrated cells, arranged with varying degrees of regularity into a funnel, ordinarily having its apex directed towards the central mass of the turnip, with which it has, in some cases at least, a traceable connexion by a canal. Presenting as it does an external porous surface terminating one of the branches of the vascular system, each of these organs is well fitted for taking up with rapidity the nutriment laid by in the turnip-root, and used by the plant when it sends up its flower-stalk. The cotyledons of young beans furnish other examples of such structures, exactly in the places where, if they be absorbents, wo may expect to find them. Ansid the branchings and inosculations of the vascular layer running through the mass of nutriment deposited in each cotyledon, there are found conspicuous free terminations that are club-shaped, and which prove to be composed, like those in leaves, of irregularly-formed and clustered fibrous cells, some of them diverging from the plane of the vascular layer, dipping down into the mass of starch and albumen which the young plant has to utilize, and which thess structures can have no other function but to take up." 
To return to cell-development; we found the cell changing in its outward form, the transparent membranous cell wall becoming thickened, and spontaneous fissure taking place; and thus is formed a series of connected cells variously modified and arranged, according to the conditions under which they are developed and the functions which they are destined to exercise. The typical form, as we have
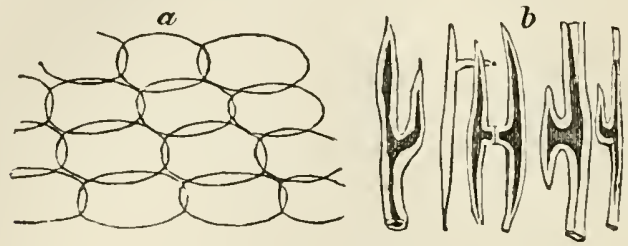

Fig. 173. - $a$, elementary cells; $b$, branched cellular tissue.

before observed, of the vegetable cell is spheroidal; but when developed under pressure within walls, or denser

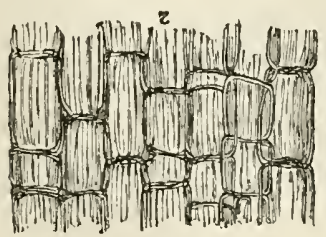
tissues, it takes other shapes; as the oblong, lobed, square, mismatical, cylindrical, fusiform, muriform, stellate, filamentous, \&c.: and is then termed Parenchyma, and the cells woven together are called cellular tissue. In pulpy fruits

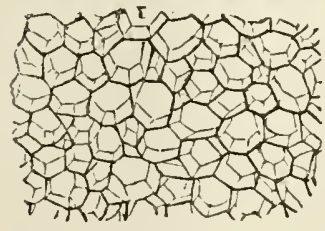

Fig. 174.

1, A transverse section of stem of Equisetum, showing the lexagoDal slape of cells. 2, A vertical section of elongated cell. the cells may be easily separated one from the other: a thin transverse section of a strawberry is represented at fig. 188, No. 15 : within the cells are smaller cells, commonly known as the pulp. Fig. 173, $a$, is the elementary form of oval cells or vesicles, passing on to the formation of branched cellular tissue, $b$. Remarkable specimens of the filamentous tissue may be seen in fig. 188, No. 19, the circular elongated cells from the Ifushroom; only another and more closely connected growth of mucedinous fungi, commonly called mushroom spawn. 
Fig. 175, in the stellate tissue cut from the stem of a rush, we have the formative network dividing into ducts for the purpose of giving strength and lightness to the stem of the plant. These ducts may undergo other transformations ; the cell itself become gradually changed into a spiral continuous tube or duct, as seen in fig. 198;

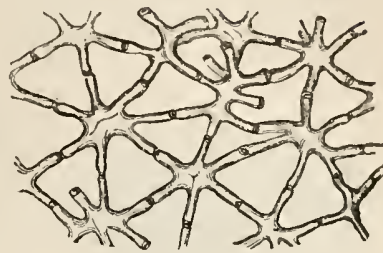

Fig. 175.-Steilate tissue, from stem of $a$ Rush. these are sometimes formed by the breaking down of the partitions; in the centre of which we may have a compound spiral duct, resembling portious of tracheæ from the silkworm.

Another important change occurs in the original cell,it is that of its conversion into woody fibre. Common woody fibre (Pleurenchyma) has its sides free from definite markings. In the coniferous plants, the tubes are furnished with circular dises; these discs are thought to be contrivances to enable the tubules of

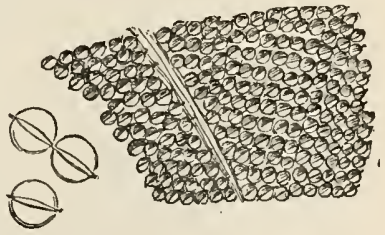
the woody tissues to dis- Fig. 176. - A section of stem of Clematis, charge their contents from with pores, highly snagnified, to shore the line which passes round them. one to the other, or into the cellular spaces. Plants having aromatic secretions are furnished with glands; these form a series of interesting objects, and such as the sage-leaf should be mounted as opaque specimens. A large central gland is seen in a section of a leaf from Ficus elastica, India-rubber-tree, fig. 177, No. 2. Professor Quekett observes, "The nature of the pores, or discs, in conifers, has long been a subject for controversy; it is now certain that the bordered pores are not peculiar to one fibre, but are formed between two contiguous to each other, and always exist in greatest numbers on those sides of the woody fibres parallel to the medullary rays. They are hollow; their shape biconvex; and in their centre is 
a smali circular or oval spot, fig. 176: the latter may occur singly, or be crossed by another at right angles,
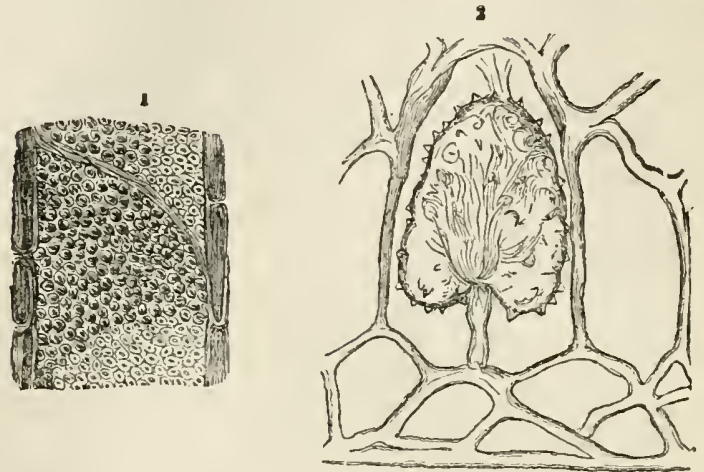

Fig. 177.

1, Vertical section of root of Alder, with outez wall. 2, A vertical section of a leaf of the lndia-rubber tree, exhibiting a central gland.

which gives the appearance of a cross, as in fig. 204, Nos. 3 , 4 , a vertical section of fossil wood, remarkable for having three or four rows of woody tissue occupied by large pores without central markings.'

We now pass to the milk, lacticiferous ducts or tissue,-

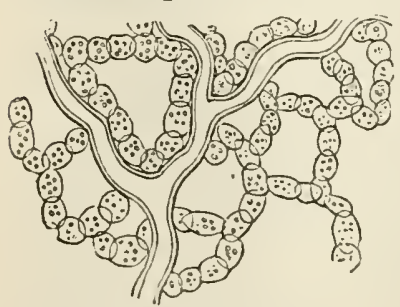

Fig. 17s. - Lacticiferous tissue. the proper vessels of the old writers. These ducts convey a peculiar fluid, sometimes called latex, usually turbid, and coloured red, white, or yellow ; often, however, colourless. It is supposed they carry latex to all the newly-formed organs, which are nourished by it. The fluid becomes darker after being mounted for specimens to be viered under the microscope. This tissue is remarkable from its resemblance to the earliest aggregation of cells, the yeastplant, and therefore has some claim to being considered the stage of development preceding that of the reticu- 
lated ducts seen in fig. 178. In a section from tho India-rubber-tree, fig. 177 , No. 2 , a network of these lactiferous tubes will be found filled with a brownish or
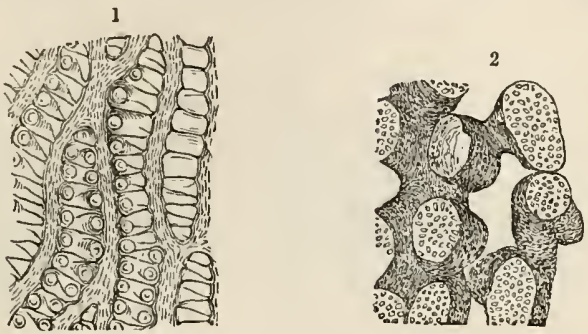

Fig. 179.

1, A portion of the leaf of Sphagnum, showing ducts, vascular tissue, and spiral fibre in the interior of its cells. 2, Porous cells, from the testa of Gourdseed, communicating with eacb other, and resembling ducts.

granular matter; that in fig. 178 is an enlarged view of this tissue from the wood of an exugen, taken near the root.

1

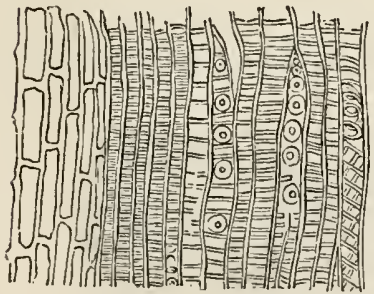

2

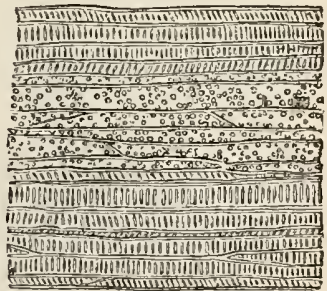

Fig. 180.

1, Reticulated ducts.

2, A vertical section of Fern-root.

In many plants external to the cuticle, there exists a vely delicate transparent pellicle, without any decided traces of organisation, though occasionally somewhat granular in appearance, and marked by lines that seem to be impressions of the junction of the cells in contact with each other. In nearly all plants, the cuticle is perforat 
by minute openings termed Stomata, which are bordered by cells of a peculiar form, distinct from those of the cuticle. In Iris germanica, fig. 181, each surface has nearly 12,000

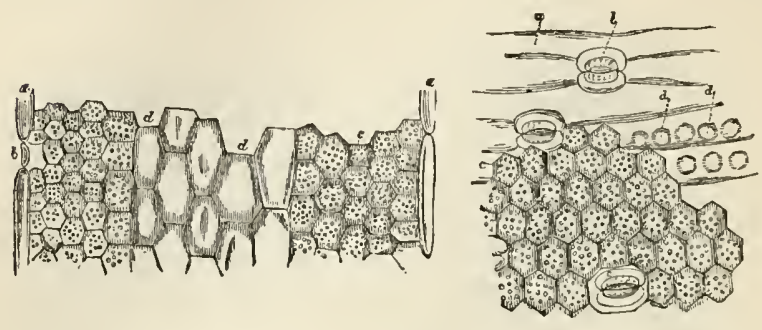

1. Fig. 181.

2.

1, Portion of a vertical section of the Leaf of the Iris: $a, a$, elongated cells of the epidermis; $b$, stomata cut through longitudinally; $c, c$, cells of the parenchyma; $d, d$, colourless tissue of the interior of the leaf. 2 , Portion of leaf of Iris germanica, torn from its surface; $a$, elongated cells of the cuticle; $b$, cells of the stomata; $c$, cells of the parenchyma: $d$, impressions on the epidermic cells; $c$, lacunæe in the parenchyma.

stomata in every square inch; and in Yucca each surface has about 40,000 .

The structure of the leaf of the common Iris shows a central portion, formed by thick-walled colourless tissue, very different from ordinary leaf-cells or from woody fibre.

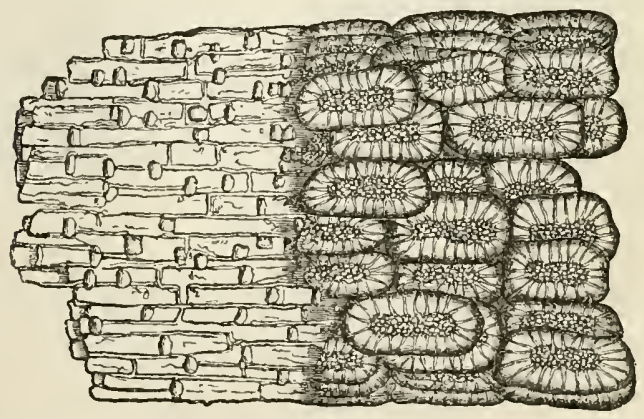

Fig. 152.-A portion of the epidermis of the Sugar-cane, showing the two kinds oj cells of which it is composed. (Magnified 200 diameters.) 
Variously-cut sections of leaves should be made, and slices taken parallel to the surfaces at different distances, for the purpose of microscopic examination.

Among the cell-contents of some plants, are beautiful crystals called Raphides: the term is derived from paфıs, a needle, from the resemblance of the crystal to a needle. They are composed of the phosphate and oxalate of lime ; there is a difference of opinion as to their use in the economy of the plant.

Mr. Gulliver has insisted upon the value of Raphides as characteristic points in many families of plants.

$\mathrm{He}$ observes that doubts as to the value of raphides as natural characters and as to their importance in the vegetable economy at all will be entertained by those who do not clearly distinguish between raphides and sphæraphides. Schleiden asserts that the "needle-formed crystals, in bundles of from twenty to thirty in a cell, are present in almost all plants," and "that inorganic crystals are rarely met with in cells in a full state of vitality."

He further states that so really practical is the presence or absence of raphides, that by noticing them he has been able to pick out pots of seedling Onagracer, which had been accidentally mixed with pots of other seedlings of the same age, and at that period of growth when no botanical character before in use would have been so readily sufficient for the diagnosis.

If we examine a portion of the layers of an onion, fig. 133, No. 1, or a thill section of the stem or root of the garden rhubarb, fig. 183, No. 4, we shall find many cells in which, either bundles of needle-shaped crystals, or masses of a stellate form occur, not strictly raphides.

Raphides were first noticed by Malpighi in Opuntia: and subsequently described by Jurine and Raspail. According to the latter observer, the needle-shape or acicular are composed of phosphate, and the stellate of oxalate of lime. There are others having lime as a basis, in combination with tartaric, malic, or citric acid. These are easily destroyed by acetic acid, and are also very solublo in many of the fluids employed in the conservation of objects; some of them are as large as the 1-40th of an inch, others are as small as the 1-1000th. They occur in all 
parts of the plant; in the stem, bark, leaves, stipules, petals, fruit, root, and even in the polien, with somo exceptions. They are always situated in the interior of cells, and not, as stated by Raspail and others, in the

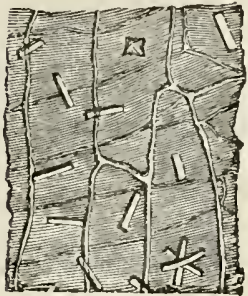

1

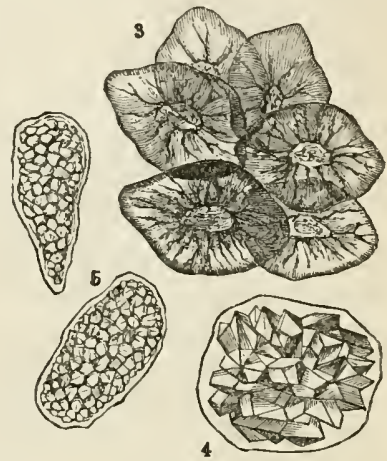

Fig. 183.

i, A section from the outer layer of the bulb of an Onion, showing crystals of carbonate of lime. 3, Cells of the Pear, showing Sclerogen, or gritty tissue. 4. Cells of garden Rhubarb, filled with raphides. 5, Cells from same, filled with starch-grains.

intercellular passages. ${ }^{1}$ Some of the containing cells become much elougated; but still the cell-wall can be readily traced. In some species of Aloe, as, for instance, Aloe verrucos $\alpha$, with the naked eye we are able to discern small silky filaments: when magnified, they are found to be bundles of the acicular form of raphides, which no doubt act the part of a stay or prop to the internal soft pulp.

In portions of the cuticle of the medicinal squillScilla maritima-several large cells may be observed, full of bundles of needle-shaped crystal. These cells, however, do not lie in the same plane as the smaller ones belonging to the cuticle. In the cuticle of an onion every cell is occupied either by an octahedral or a prismatic crystal of oxalate of lime: in some specimens the octahedral form predominates; but in others from the same plant the

(1) "As an exception, many years ago they were discovered in the interior of the spiral vessels in the stem of the grape-vine; but with some botanists this rould not be considered as an exceptional case, the vessels being regarded as -longated cells."-Quekett. 
crystals will be principally prismatic, and are arranged as if they were beginning to assume a stellate form. Some plants, as many of the cactus tribe, are made up almost entirely of raphides. some instances every cell of the cuticle contains a stellate mass of crystals; in others the whole interior is full of them, rendering the plant so exceedingly brittle, that the least touch will occasion a fracture; so much so, that some specimens of Cactus senilis, said to be a thousand years old, which were sent a few years since to Kew from South America, were obliged to be packed in cotton, with all the care of the most delicate jewel-

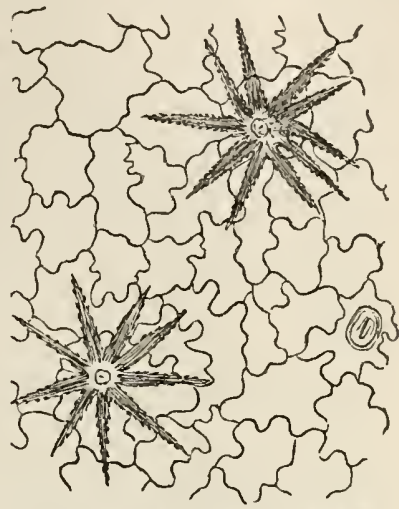

Fig. 184.-Siliceous cuticle from under surface of leaf of Deutzia scabra. lery, to preserve them during transport.

Raphides, of peculiar figure, are common in the bark of many trees. In the Hiccory (Carya alba) may be observed masses of flattened prisms having both extremities pointed. In vertical sections from the stem of Elocagnus angustifolia, numerous raphides of large size are embedded in the pith. Raphides are also found in the bark of the apple-tree, and in the testa of the seeds of the elm; every cell contains two or more very minute crystals.

In figs. 184 and 185 we have other representations of the crystalline structure

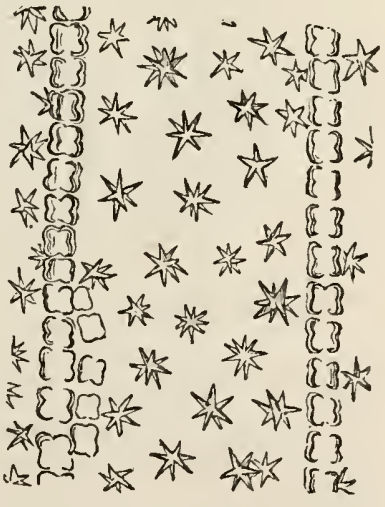

Fis. 185.-Siliceous cuticle of Grass (Pharus cristatus). 
of plants, in sections taken from grass, and the leaf of Deutzia scabra. This insoluble material is called silica, and is abundantly distributed throughout certain orders of plants, leaving a skeleton after the soft vegetable matters have been destroyed: masses of it, having the appearance of irregularly-formed blackened glass, will always be found after the burning of hay or straw; which is caused by the fusion of the silica contained in the cuticle combining with the potash in the regetable tissue, thas forming a silicate of potash (glass). To display this siliceous structure, it is necessary to cut very thin slices from the cuticle, and mount them in fluid or Canada balsam.

In the Grammacea, especially the canes; in the Equisetum hyemale, or Dutch rush; in the husk of the rice,

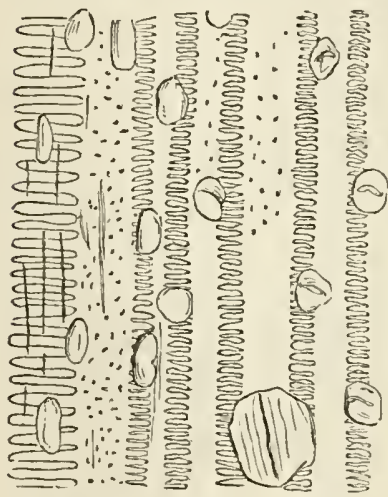

Fig. 1se-Portion of the husk of Wheat, showing siticeous crystals. wheat, and other grains,silica is abundantly found. In the Pharus cristatus, an exotic grass, fig. 185, we have beautifully-arranged masses of silica with raphides. The leaves of Deutzia, fig. 184 are remarkable for their stellate hairs developed from the cuticle, of both their upper and under surfaces; forming most interesting and attractive objects when examined under the microscope with polarised light. See Plate VIII. No. 173.

Silica is found in all $R u$ biacece; both in the stem and leaves, and if present in sufficient thickness, depolarises light. This is especially the case in the prickles, which all these plants have on the margin of the leaves and the angles of the stem. One of the order Compositce, a plant popularly known as the "sneezewort," (Archillae ptarmica) has a large amount of silica in the hairs found on the donble serratures of its leaves; commonly said to be the 
cause of its errhine properties when powdered and used as sunff. It is in the underlying or true epidermis, that the silica occurs. This membrane is permeable by fluids, not by means of pores, but by endosmotic force.

The most generally-distributed and conspicuous of the cell-contents is Starch; at the same time it is one of great value and interest, performing a similar office in the economy of plants as that of fat in animals. It occurs in all plants at some period of their existence, and is the chief and great mark of distinction between the regetable and animal kingdoms. Its presence is detected by testing with a solution of iodine, which chauges it to a characteristic blue or violet colour. ${ }^{1}$ Being insoluble in cold water, it can be readily washed away and separated from other matters contained

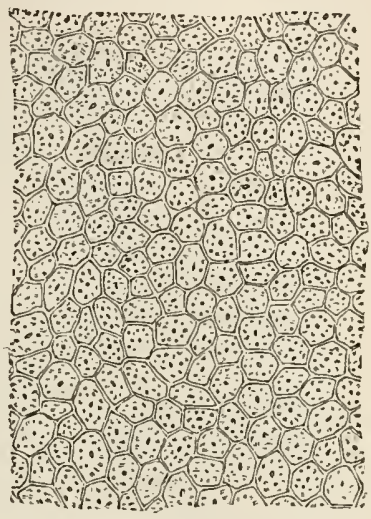

ig. 187. - Section of a Cane; with cellwatls of silica, and internal pores fitled with granular natter.

in the cellular parts of full-grown plants. It is often found in small granular masses in the interior of cells, shown in fig. 183 from the garden-rhubarb. Starch-grains are variable in size: the tous-les-mois, fig. 188, No. 5, are very large; in the potato, No. 14, they are smaller; and in rice, No. 6, they are very small indeed. Nearly ail present the appearance of coucentric irregular circles ; anc most of the granules have a circular spot, termed the hilum, around which a large number of curved lines arrange themselves : better seen under polarised light. Plate VIII. No. 167.

Leeuwenhöek, to whom we are indebted for the earliest notice of starch-granules, enters with considerable minuteness into a description of those of several plants-such as wheat, barley, rye, oats, peas, beans, kidney-beans, buckwheat, maize, and rice; and very carefully describes experiments made by him in order to investigate the structure of starch-granules. Dr. Reissek regards the

(1) This is not a test for starch when combined with albuminous matters. 
granule as a perfect cell, from the phenomena presented during its decay or dissolution, when left for some time in water. Schleiden and others, after examining its expansion and alteration under the influence of heat and of sulphuric acid, considered it to be a solid homogeneous structure.

Professor Busk agrees with M. Martin in believing the primary form of the starch-granule to be "a spherical or ovate vesicle, the appearance of which under the microscope, when submitted to the action of strong sulphuric acid, conveys the idea of an unfolding of plaits or rugæ, which bave, as it were, been tucked in towards the centre of the starch-grain." 1 The mode of applying the concentrated sulpburic acid is thus described by Mr. Busk :- "A small quantity of the starch to be examined is placed upon a slip of glass, and covered with five or six drops of water, in which it is well stirred about; then with the point of a slender glass-rod the smallest possible quantity of solution of iodine is applied, which requires to be quickly and well mixed with the starch and water; as much of the latter as will mnst be allowed to drain off, leaving the moistened starch behind, or a portion of it may be removed by an inclination of the glass, before it is covered with a piece of thin glass. The object must be placed on the field of the microscope, and the $\frac{1}{4}$-inch object-glass brought to a focns close to the upper edge of the thin glass. With a slender glass-rod a small drop of strong sulphuric acid must be carefully placed immediately upon, or rather above the edge of the cover, great care being necessary to prevent its running over. The acid quickly insinuates itself between the glasses, and its course may be traced by the rapid change in the appearance of the starch-granules as it comes in contact with them. The course of the acid is to be followed by moving the object gently upwards; and when, from its diffusion, the re-agent begins to act slowly, the peculiar changes in the starch-granules can be more readily witnessed. In pressing or moving the glasses, the starch disc becomes torn, and is then distinctly seen, especially in those coloured blue, to

(1) Professor G. Busk, F.R.S., on the Structure of the Starch-granule; Quarterly Journal of Microscojical Science, April, 1853. 
consist of two layers, an upper and a lower one; and the collapsed vesicular bodies of an extremely fine but strong and elastic membrane." Mr. Busk believes the hilum to be a central opening into the interior of the ovate vesicle.

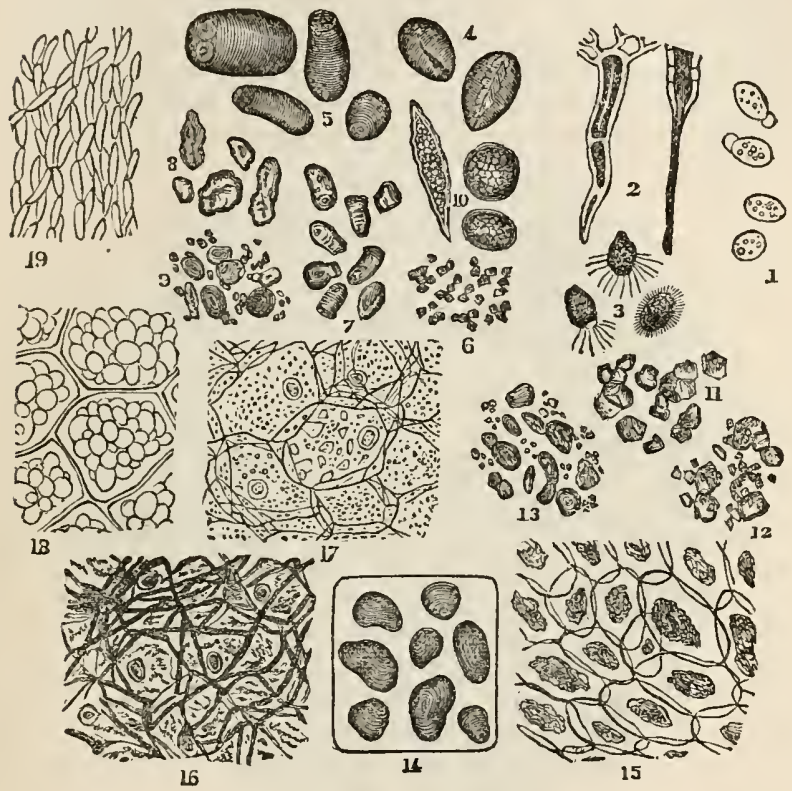

Fig. 188.

1, Nucleated Cells. 2, Stinging-nettle Hairs, Urtica Dioica. 3, Ciliated spores of Conferve. 4, Starch grains, broken by the application of heat. 5, Starch from Tous-les-mois, 6. Starch from Rice. 7, Starch from Sago. 8, Imitation Bago-starch. 9, Wheat-starch. 10, Rhubarb-starch, in isolated cells. 11, Maize-starch. 12, Oat-starch. 13, Barley-stareh. 14, Potatostareh. 15, Section of Strawberry, cells ovoid, containing granular matter. 16, Section of Potato, with stareh destroyed by fungoid disease. 17, Potato, with nearly all stareh-grains absent. 18, Section of Potato, cells filled with healthy starch. (These starches are grouped for comparison.) 19, Mushroom spawn, elongated cells.

Nitric acid communicates to wheat-starch a fine orangeyellow colour; and recently-prepared tincture of guaiacum gives a blue colour to the starch of good wheat-flour. 
Pure wheat-flour is almost entirely dissolved in a strong solution of potash, containing twelve per cent. of the alkali; but mineral substances used for the purpose of adulteration remain undissolved.

Wheat-flour is frequently adulterated with various substances; and in the detection of these adulterations, the microscope, together with a slight knowledge of the action of chemical re-agents, lends important assistance. It enables us to judge of the size, shape, and markings on the starch grains, and thereby to distinguish the granules of

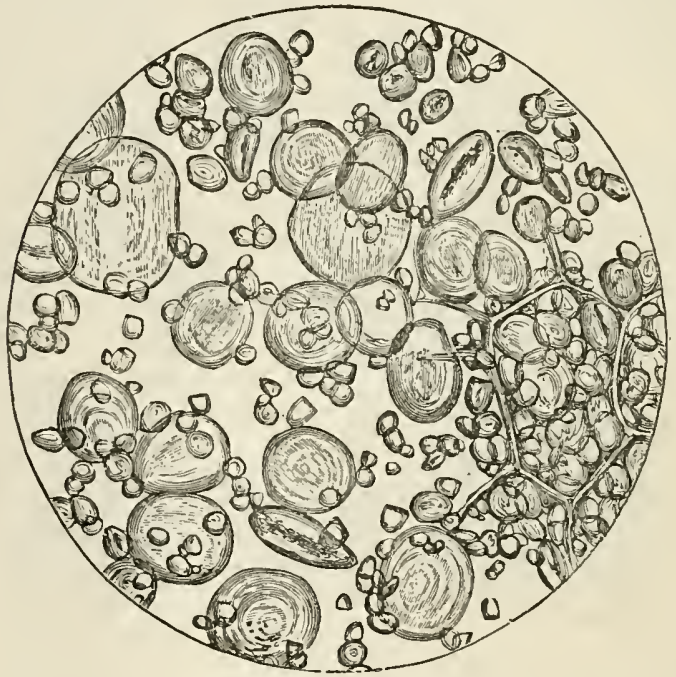

Fig. 189. - Wheal-Flour Starch-granules, with a small porlion of its cellulose. (Magnified 420 diameters.)

one meal from that of another. In some cases the microscopic examination is aided by an application of a solution of potash. Thus we may readily detect the mixture of wheat-flour with either potato-starch, meal of the pea or bean, by the addition of a little water to a small quantity of the flour, then, by adding a few drops of a solution of potash (made of the strength one part liquid potash to three parts of water), the granules of the potato- 
starch will immediately swell up, and acquire three or four times their natural size; while those of the wheat-starch are scarcely affected by it ; if adulterated with pea or bean meal, the hexagonal tissue of the seed is at the same time rendered very obvious under the microscope. Polarised light will be of use as an additional aid ; wheat-starch presents a faint black cross proceeding from the central hilum, whereas the starch of the oat shows nothing of the kind.

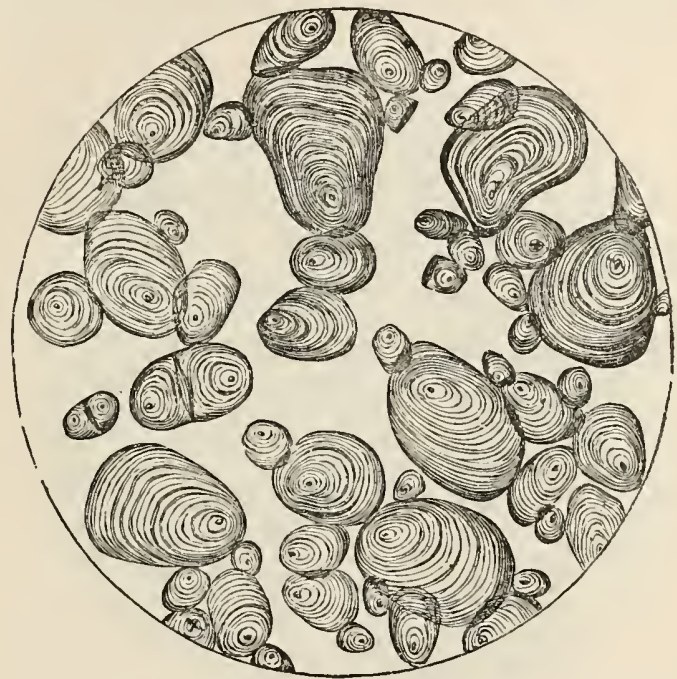

Fig. 190.-Potato Starch-yranules, said under the name of British Arrow-root, used to adulterate flour and bread. (Magnified 240 diameters.)

The diseases of wheat and corn are readily detected under the microscope; some of which will be seen to be produced by a parasitic fungus, and by an animalcule represented in another place: all are more or less dangerous when mixed with articles of food.

Adulteration of bread with boiled and mashed potatoes, next to that by alum, is, perhaps, the one which is most commonly resorted to. The great objection to the use of potatoes in bread, is, that they are made to take the place 
of an article very much more nutritious. This adulteration cau be instantly detected by means of the microscope. The cells which contain the starch-corpuscles are, in the potato, very large, fig. 190 ; in the raw potato they are adherent to each other, and form a reticulated structure, in the meshes of which the well-defined starch-granules are clearly seen; in the boiled potato, however, the cells separate readily from each other, each forming a distinct article: the starch-corpuscles are less distinct and of an altered form.

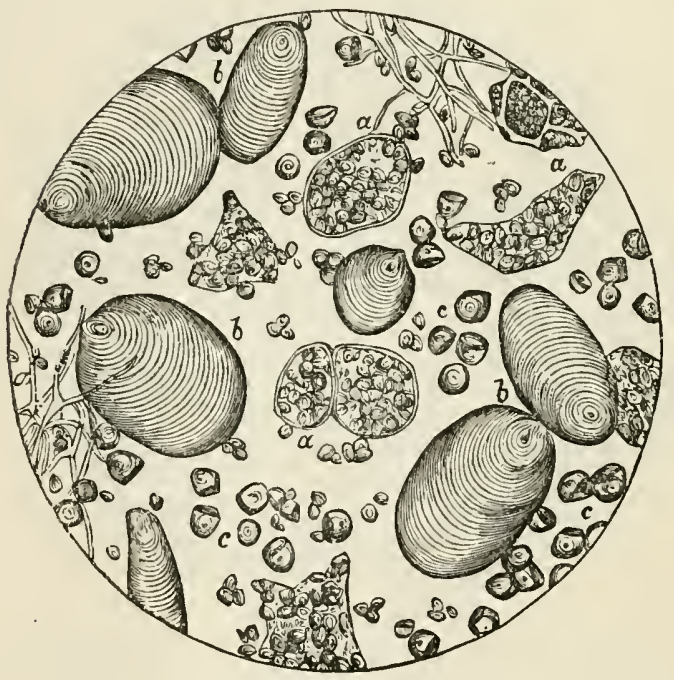

Fig. 191.-Adulterated Cocoa, sold under the name of Homocopathic Cocoa. (After Hassall.)

$a a a$, granules and cells of cocoa; $b b b$, granules of Canna-starch, or Tous-lesmois; $c$, granules of Tapioca-starch.

Adulteration with alum and "stuff." - This adulteration is practised with a twofold object : first to render flour of a bad colour and inferior quality white and equal, in appearance only, to flour of superior quality ; and secondly to enable the flour to retain a larger proportion of water, 
by which the loaf is made to weigh heavier. By dissolving out the alum in water and then re-crystallising it under the microscope, this adulteration is readily detected.

Before leaving the subject of starch, allnsion may be made to the prevalent and destructive epidemic amongst potatoes, which is a disease of the tuber, not of the haulm or leaves. "Examined in an early stage, such potatoes are found to be composed of cells of the usual size; but they contain little or no starch: this will be seen upon reference to Nos. 16 and 17, fig. 188. Hence it may bo

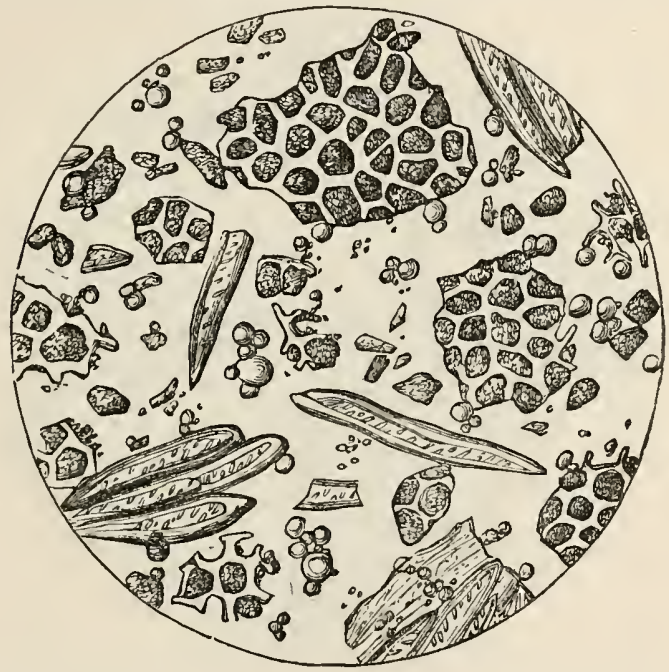

Fig. 192.-Structure and Character of genuine Ground Coffee. (After Hassall.)

inferred, that the natural nutriment of the plant being deficient, the haulm dies, the cells of the tuber soon turn black and decompose; and fungi developed as in most other decaying vegetable substances.

"This will undoubtedly explain the most prominent symptom of the potato-disease, the tendency to decomposition; and is a point in which the microscope confirms the result of chemical experiment: for it has been found 
that the diseased potatoes contain a larger proportion of water than those that are healthy. A want of organizing power is evidently the cause of this deficiency of starch ; but we fear the microscope will never tell us in what the want of this organising force cousists." 1

The adulteration of articles of food and drink has long been a matter of uneasy interest, and of strong, though vague, misgiving. Accum's Death in the Pot, between thirty and forty years ago, awoke attention to the subject; which has since been more or less accurately explored by

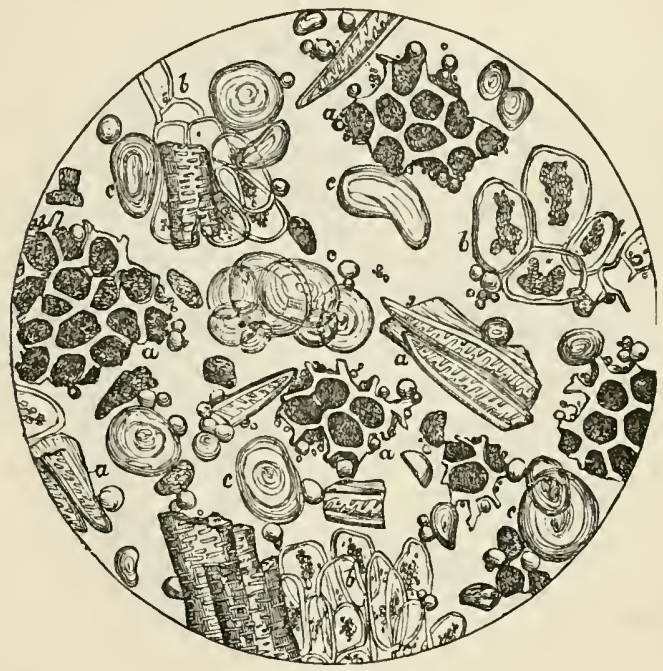

Fir. 193.-Sample of Coffee, adulterated with both Chicory and Roasted Wheat. (After II assall.)

a $a$, small fragments of coffee; $b b b$, portions of chicory; $c c c$, starch-gramules of wheat.

Mitchell, Normandy, Chevalier, Jules Garnier, and Harel; and has at length derived a siugularly lucid exposition from Dr. Hassall's researches, whose report of these inquiries fills between 600 and 700 closely printed pages

(1) Professor Quekett's Histology of Vegelables. We would refer the reader to a curious work on Fungi, by Arimini, an Italian botanist, 1759 . 
of a large octaro, replete with details of the fraudulent contaminations commonly practised by the people's purvcyors, at the people's expense of health and pocket. ${ }^{1}$

"In nearly all articles," said Dr. Hassall, before a committee appointed by the Iouse of Commons to inquire into these adulterations, "whether food, drink, or drugs, my opinion is that adulteration prevails. And many of the substances employed in the adulterating process were not only injurious to health, but even poisonous." The microscope was the effective instrument in the work of

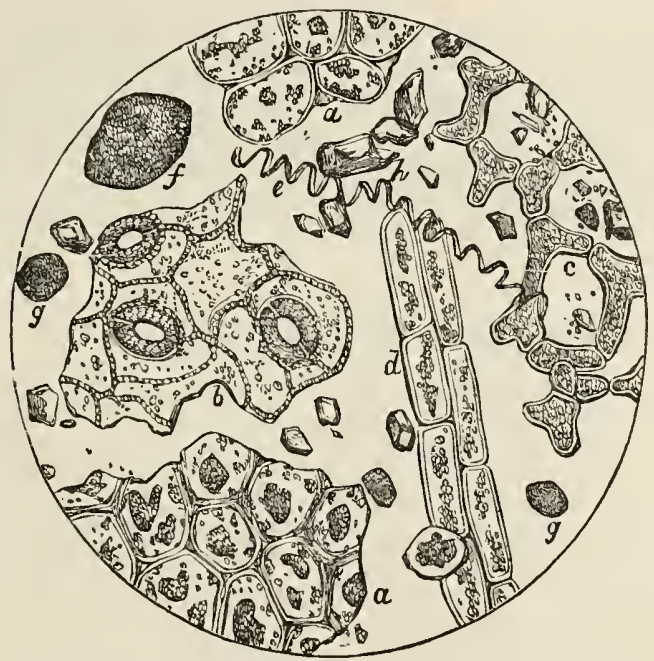

Fig. 19.1, - Tea adullerated with foreign teaves. (After Hassall.)

$a$, upper surface of leaf; $b$, lower surface, showing cells; $c$, chlorophyll cells; $d$, elongated cells found on the upper surface of the leaf in the course of the veins ; $e$, spiral vessel; $f$, cell of turmeric; $g$, fragment of Prussian blue; $h_{\text {, }}$ particles of white powder, probably China clay.

detection. Less than five years ago, it would, we are told, have been impossible to detect the presence of chicory in coffee : in fact, the opinion of three distinguished chemists was actually quoted in the House of Commons to that effect;

(1) Food and its Adulterations; comprising the Reports of the Analytical Sanitary Commission of the Lancel, for the years 1851 to 1854 inclusive. By Arthur
dill Hassall, M.D. 
whereas by the use of the microscope the differences of structure in these two substances, as in many other cases, can be promptly discerned. Out of thirty-four samples of coffee purchased, chicory was discovered in thirty-one; chicory itself being also adulterated with all manner of compounds. There is no falling back either upon tea or chocolate; for these seem rather worse used than coffee. Tea is adulterated, not only here, but still more in China; while as to chocolate, the processes employed in corrupting the manufacture are described as "diabolical." "It is

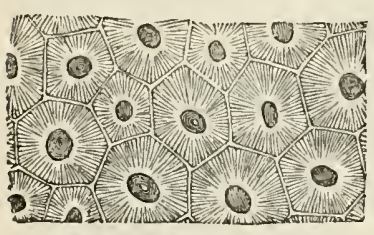

1

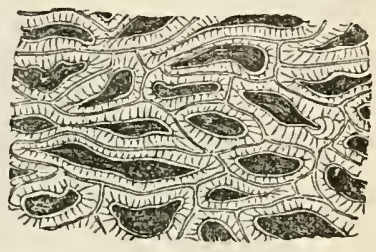

2

Fig. 195.

1, Radiating cells from the outer shell of the Ivory Nut. 2, Section of a Nut, slowing cells with small radiating pores.

often mixed with brick-dust to the amount of ten per cent., ochre twelve per cent., and peroxide of iron twentytwo per cent., and animal fats of the worst description. In this country, cocoa is sold under the names of flake, rock, granulated, soluble, dietetic, homœopathic cocoa, \&c., fig. 191. Such names are evidently employed to disguise the fact that they are compounded of sugar, starch, and other substances.

To return to the subject more immediately before us-

Sume of the plants belonging to the Orchidex-Commelinea-particularly Tradescantia virginica (Spiderwort), portions of the epidermis, and the jointed hairs of the filament, form interesting microscopic objects. The surface of the latter is marked with extremely fine longitudinal parallel equidistant lines or strix, whose intervals are equal, from about $\frac{1}{15000}$ to $\frac{1}{20000}$ of an inch. It might therefore in some cases be used as a micrometer or test object. The nucleus of the joint or cell is very distinct as well as regular in form; and by gentle pressure 
is easily separated entire from the joint. It then appears to be exactly round, nearly lenticular, and its granular matter is either held together by a coagulated pulp not visibly granular, or, which may be considered equally probable, by an enveloping membrane. The analogy of this nucleus to that existing in the various stages of development of the colls in which the grains of pollen are formed in the same species, is sufficiently obvious.

In the joint of the same, when immersed in water, being at the same time freed from air, and consequently made more transparent, a circulation of very minute granular matter is visible. This requires at least a $\frac{1}{4}$ power to show it well. The motion of the granular fluid is seldom in one uniform circle, but in several apparently independent currents: and these again, though often exactly longitudinal, and consequently in the direction of the striæ of the membrane, are not unfrequently observed forming various angles with these strix. The smallest of the currents appear to consist of a single series of granules. The course of these currents seems often in some degree affected by the nucleus, towards or from which many of them occasionally tend or appear to proceed. They can hardly, however, be said to be impeded by the nucleus, for they are occasionally observed passing between its surface and that of the cell; a proof that this body does not adhere to both sides of the cavity, and also that the number and various directions of the currents cannot be owing to partial obstructions arising from the unequal compression of the cell.

Filower-buds. - "In the very early stage of the flowerbud, while the anthera are yet colourless, their loculi are filled with minute lenticular grains, having a transparent flat limb, with a slightly convex and minutely granular semi-opaque disc. This disc is the nucleus of the cell, which probably loses its membrane or limb, and, gradually enlarging, forms in the next stage a grain also lenticular, and which is marked either with only one transparent line dividing it into two equal parts, or with two lines crossing at right angles, and dividing it into four equal parts. In each of the quadratures a small nucleus is visible; and, even where one transparent line is only dis- 
tinguishable, two nuclei may frequently be found in each semicircular division. These nuclei may be readily extracted from the containing grain by pressure, and after separation retain their original form. In the next stage, the greater number of grains consist of the semicircular divisions, naturally separated, but now containing only one nucleus, which has gradually increased in size. In the succeeding stage the grain apparently consists of the nucleus of the former stage, but considerably enlarged, and having an oval form, and a somewhat granular surface. This oval grain, continuing to increase in size, and in the thickening of its membrane, acquires a pale yellow colour; and is now the perfect grain pollen."

In the whole tribe of Orchideæ an abundance of raphides may be found in almost every part of the cellular tissue. The crystals are usualiy cylindrical in form, and so arranged as to disguise their true character, often causing them to be overlooked : by making use of a dark-ground illuminator, or polarised light, this is not likely to occur. The cause of the disease known as "spot" in Orchids, of which several kinds have been noticed by cultivators, has been traced by Mr. Berkeley, in one instance, to the oceurrence of a minute parasitic fungus, belonging to the genus Leptothyrium. A description of the disease, with illustrations, giving the general appearance of the diseased leaves, and a magnified figure of the parasite, appears in the 1st part of the new series of the Journal of the Horticultural Society.

The Colouring matter of Flowers.-M. F. Hildebrands, having carefully investigated the colouring matters of flowers, has arrived at the following conclusion respecting them, and their distribution in the tissue of the several orgaus.

1. That the colour of flowers is in constant connexion with the cell contents, never with the walls of cells.

2. Blue, violet, rose, and (if there be no yellow in the flower) deep red are due, with little exception, to a cellfluid of corresponding colour.

3. Yellow, orange, and green, are usually associated with solid, granular, or vesicular substances in the cells. 
4. Brown or gray, and in many cases bright red and orange (apparently uniform to the naked eye), are found to be compounded of other colours, as yellow, green, or orange with violet, or green and red; bright red and orange in like manner of blue-red with yellow or orange.

5. Black, excepting in the bean, is due to a very deeplycoloured cell-fluid.

6. All the cells of an organ are rarely uniformly coloured.

7. The colour usually resides in one or in a few of the outer layers of cells.

8. The coloured cells are but exceptionally covered by a layer of uncoloured ones.

9. Combinations of colour are occasioned by diverselycoloured matters in the same or in adjacent cells.

The Woody tissue of plants is not without its interest, it consists of elongated transparent tubes of considerable strength : some are almost entirely made up of this tissue. It is by far the most useful, and supplies material for our linen, cordage, paper, and many other important articles in every branch of art. This tissue, remarkable for its toughness, is termed fibre, the outer membrane of which is usually structureless. In Flax and Hemp, in which the fibres are of great length, there are traces of transverse markings, and tubercles at short intervals. In the rough condition, in which it is imported into this country, the fibres have been separated, to a certain extent, by a process termed hackling. It is once more subjected to a repetition of
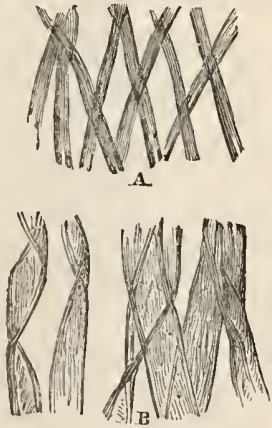

Fig. 196. hackling, maceration, and bleach- A, Fibres of Silk. B, Fibres ing, before it can be reduced to the white silky condition required by the spinner and weaver, when it has the appearance of structurelcss tubes, fig. $197 \mathrm{~B}$. China-grass, New Zealand flax, and some other plants, produce a similar material, but are not so strong, in conse- 
quence of the outer membrane containing more lignine. It is important to the manufacturer that he should be able to determine the true character of some of the textures of articles of clothing, and this he may readily do with the microscope. In liuen we find each component thread made up of the longitudinal, rounded, unmarked fibres of flax; but if cotton has been mixed, we recognise a flatteued, more or less twisted band, as in fig. $196 \mathrm{~b}$, having a very striking
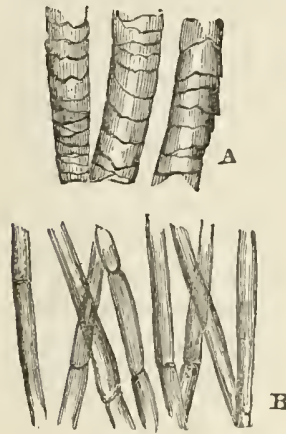

Fig. 197.

A, Wool of Sheep. B, Filaments of Flax. resemblance to hair, which, in reality, it is ; since, in the condition of elongated cells, it lines the inner surface of the pod. 'These, again, should be contrasted with the filaments of silk, fig. $196 \mathrm{~A}$, and also of vool, fig. $197 \mathrm{~A}$. The latter may be at once recoguised by the zigzag transverse markings on its fibres. The surface of wool is covered with these furrowed and twisted fine cross lines, of which there are from 2,000 to 4,000 iu an inch. On this structure depeuds its felting property. In judging of fleeces, attention should be paid to the fineuess and elasticity of the fibre,- the furrowed and scaly surface, as shown by the microscope,-the quantity of fibre in a given surface, the purity of the fleece, upon which depend the success of the scouring and subsequent operations.

In the mummy-cloths of the Egyptians, flax only was used, whereas the Peruvians used cotton alone. By recent improvements introduced iuto the manufacturing processes, flax has been reduced to the fineness and texture of silk, and made to resemble other materials.

Silk is secreted from a pair of long tubes endiug in a pore of the under-lip of the silkworm. Each thread is made of $t$ wo filaments coming from these, and they are glued together by a secretion from a small gland near. The quality of the silk depends on the character and difference of the two secretions.

All woody fibre is made up of elongated cells, generally 
more or less pointed at both extremities, and having their walls strengthened by internal deposits. Occasionally, however, the fibre is short, as in the Clematis, Elder, \&c.; it is marked with pores or dots, from a deficiency of the internal deposits at these points.

Vascular tissue consists of cells, more or less elongated, joined end to end, or overlapping each other, in which either a spiral fibre, or a modification of the same, has been deposited; hence, if the spiral be perfect, it is called a true spiral vessel ; if interrupted, or the fibre breaks up into rings, it is termed annular; if the rings are connected together by branching fibres, so that a network is produced, the vessel is called reticulated; if the vertical fibres are short, and equidistant, the ressel is said to be

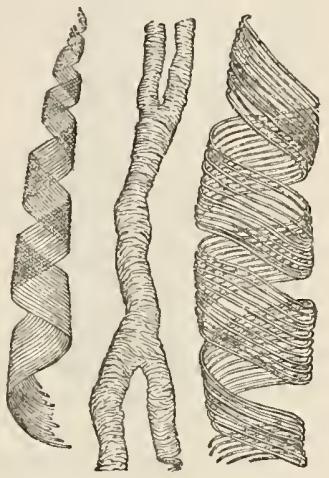

Fig. 193.-Simple and compound spiral ressels. scalariform, from its resemblance to a ladder. Spiral vessels have been also termed trachece, from their resemblance to the air-tubes of insects, as in fig: 198 .

Under thic head other membranaceous tubes are included. in which the arrangement of the fibre has been considerably modified in its deposition, Elongated tubes or ducts, with porous walls, come under the bead of vascular tissue; they somewhat differ from the spiral varieties, inasmuch as they cannot be unroled without breaking. It is a curious fact, that mostly the spiral coils from right to left; and it has been suggested that the direction of the fibre may determine that in which the plant coils round an upright pole. The Hop has left-handed spirals, and is a left-handed climber, which would therefore appear to support this theory. The nature of the fibre, and the development of the tissue, have been frequently the subject of dispute between botanists.

The late Mr. Edwin Quekett gave much attention to this 
subject; and published an excellent paper in the Micra scopical Society's Transactions, 1840, which contains the results of his observations.

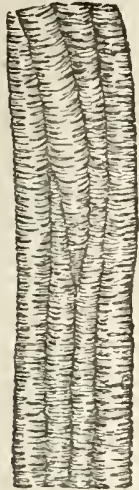

1

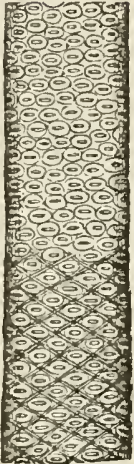

2

Fig. 199.

1. Interior cast of the siliceous portion of spiral tubes of the Opuntia. 2, Vertical section of Elm, showing spiral fibre.

In order to watch the development of the membranous tubes of plants, no better example can be chosen than the

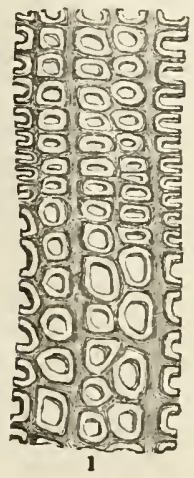

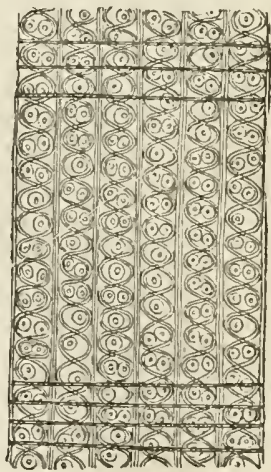

2

Fig. 200.

1, A transterse section of Taxus baccata (Yew), showing the woody fibre 2, Vertical section of the Yew, exhibiting pores and spiral fibres. 
young flower-stalk of the long-leek (Allium porrum), in the state in which this vegetable is usually sent to marl ct; it is then most frequently found to be about an inch or
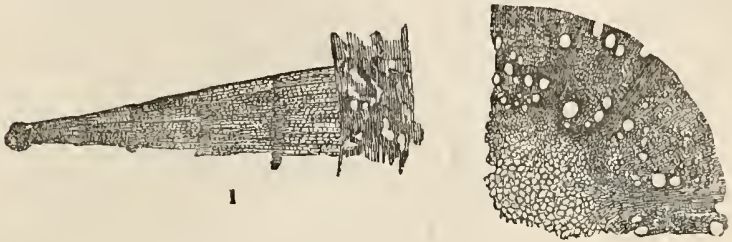

2

Fig. 201.

1, Portion of transverse section of stem of Cedar, showing pith, wood, and bark.

2. Portion of transverse section of stem of Clematis, showing medullary rays:

more in length, and from a quarter to half an inch in diameter. This membrane occurs very low down amidst the sheathing bases of the leaves; and from having to lengthen to two or three feet, and containing large vessels, it forms a very fit subject for ascertaining the early appearances of the vascular tissue.

To examine the development of vessels, it is necessary to be very careful in making dissections of the recent plant; and it will be found useful to macerate the specimen for a time in boiling water, which will render the tissues more easily separable. When the examination is directed in search of the larger vessels, it will be found that at this early stage they present merely the form of very elongated cells, arranged in distinct lines; amongst which some vessels, especially the annular, will be found matured, even before the cytoblasts have disappeared from the cells of the surrounding tissue.

As development proceeds, the versels rapidly increase in length, till they arrive at perfection. No increase in diameter is perceptible after their first formation. At this period, in the living plant the young vessels appear full of fluid, which is apparently, as remarked by Suhleiden, of a thick character, and wnich he has designated vegetable jelly ; by boiling which, or by the addition of alcohol, the contents, or at least the albuminous portion, become coagulated. From this circumstance, every cell appears 
to enclose another in a shrivelled condition; this state is sometimes so far extended, that a thick granular cord is all that can be seen of the contents.

"The period of growth at which the laying down of fibre commences, determines the distance between the several coils; for instance, when it is
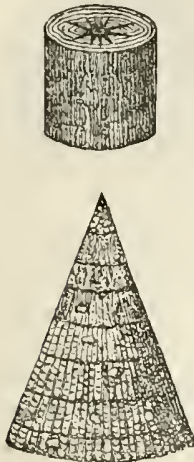

Fig. 202. $-A$ section from the stem of a coniferous plant, with a transverse cutting magnified, to show the zones of annual growth, annual rings.

possess vascular tissue.

first formed, the coils are quite close, scarcely any perceptible trace of membrane existing between them. In the anmular vessel, the development of the cell and the adherence of the granules to each other are conducted in the same manner; the deposit showing a tendency towards the spiral direction, by the presence of a spire connecting two rings, or by a ring being developed in the middle of a spiral fibre. The annular vessel is the first observed in the youngest parts of plants, and when foumd alome indicates a low degree of organisation; as shown by its occurrence in Sphagnum, Equisetum, and Lycopodium, which plants, in the ascending scale of vegetation, are almost the first that

"It will be found that spiral fibre occurring with rings marks a higher step in the scale of organising power"; the true spiral more so; and the reticulated and dotted mark the highest; this being the order in which these several vessels are placed in herbaceons exogens proceeding from within outwards, the differences of structure of the several vessels being indices of the vital energy of the plant at the several periods of its development. In those vessels in which the amnular or spiral character of the fibre is departed from, some curious modifications of the above process are to be observed, as in the reticulated vessels met with in the common balsam (Balsamina hortensis). The primary formation of fibre in these vessels is marked by the teudency of the granules to take a spiral course, when it happens that some one of the granules becomes 
enlarged by the deposition of new matter around it. This becomes a point originating another fibre or branch, which becomes developed by the successive attraction of granules into bead-like strings, taking a contrary direction to the original fibre, forming a cross-bar, or ramifying, thereby causing the appearauce by which the vessel is recognised.

"In the exogenic vessel, the development of fibre proceeds in the same manner as in the last example; but the vessels will be seen to be dotted with a central mark, usually of a red colour, which, when viewed under high power, may be thought to resemble a minute. garnet set in the centre of each dot. This red colour is owing to the dot being somewhat hollowed or cupped, aud the centre only thin membrane. These vessels are best seen in the young shoots of the Willow. In the endogenic vessel the connecting branches are given off beneath each other, so that the ojots, which are rounded, are arranged in longitudinal rows; but in the acrogenic, or scalariform, in which the vessels are generally angular, and present distinct facets, the branches come off in the same line, corresponding generally to the angles of the vessel; the spaces left between are linear instead of round."

Mr. E. Quekett affirms, in opposition to the views entertained by Mirbel, Richard, and Bischoff, "that the dots left in these several vessels are not holes, neither do they consist of broken-up fibre, but are the membranous tubes, unsupported by internal deposit; and on account of the extreme tenuity of the tissue, and the minute space between the fibres, the light in its transmission becomes decomposed, and appears of a greenish-red hue. The structure of the dot is best seen by examining the broken edge of any such vessels, when it will be found that the fracture has been caused by the vessel giving way from one dot to another, so that the torn edge of the membrane can be observed in each dot."

\section{PREPARATION OF VEGETABLE TISSUES.}

The proper mode of preparing and preserving vegetable tissues is a matter of some importance to the microscopist; we therefore propose to add a fer general directions for the studeut's guidance. 
Vegetable tissues are best prepared for the microscope by making thin sections, either by maceration, by tearing between the thumb and the blade of a knife, or by dissection.

The spiral and other vessels of plants require to be dissected out under a simple magnifying-glass. Take, for instance, a piece of asparagus, and separate with the needle-points the vessels, which require to be finished under a magnifying-glass, in a single drop of distilled water. When properly done, keep in spirits of wine and Tater until mounted.

Vascular tissue requires both maceration and dissection for its separation. The cuticle or external covering of plants is a highly interesting structure; it is best seen in the pelargonium, oleander, \&c. ; and may be mounted dry or in Canada balsam.

Cellular tissue is best seen in fine sections from the pith of elder, pulp of peach, pear, \&c. The petals of flower's are mostly composed of cellular tissue, and their brilliant colours arise from the fluid contained within the cells. In the petal of the anagallis, or scarlet chickweed, the spiral vessels diverging from the base, and the singular cellules which fringe the edge, are very interesting. The petal of the geranium is one of the most beautiful objects for microscopic examination. The usual way of preparing it is by immersing the leaf in sulphuric ether for a few seconds, allowing the fluid to evaporate, and then putting it up dry. Dr. Inman of Liverpool suggests the following method: "First peel off the epidermis from the petal, which may be readily done by making an incision through it at the end of the leaf, and then tearing it forwards by the forceps. This is then arranged on a slip of glass, and allowed to dry; when dry, it adheres to the glass. Place on it a little Canada balsam diluted with turpentine, and boil it for an instant over the spirit-lamp; this blisters it but does not remove the colour ; then cover it with a thin slip of glass, to preserve it. Many cells will be found showing the mamilla very distinctly, and the hairs surrounding its base, each being slightly curred and pointed towards the apex of the mamilla. It is these hairs and the mamilla which give the velvety appearance to the petal."

Fibro-cellular tissue is found readily in Sphagnum or 
bog-moss, and in the elegant creeper Coboea scandens. In some orchidaceous plants the leaves are almost entirely composed of it. A modification of this form of tissue is found in the testa of some seeds, as in those of Salvia, Collomia grandiflora, \&c.

The curious and interesting sporules of ferns, when ripe, burst, and are dispersed to a distance; so that they should be gathered before they come to maturity, and mounted as opaque objects. The development of ferns may be observed by placing the seeds in moistened flannel, and keeping them at a warm temperature. At first a single cellule is produced, then a second; after this the first divides into two, and then others follow; by which a lateral increase takes place.

Pollen-grains from most flowers are very interesting objects; the darker kinds show best when mounted in dark cells, and viewed as opaque; objects more trans-
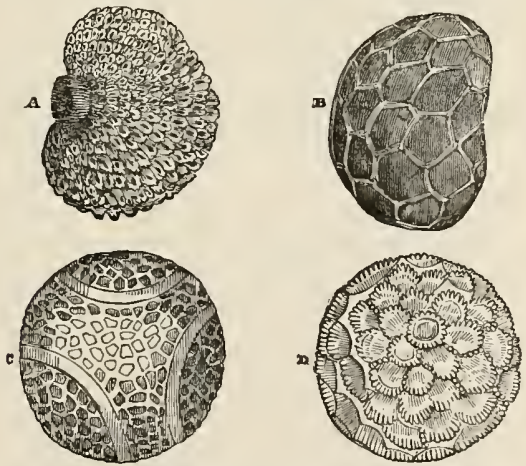

Fig. 203. - Pollen grains and seeds.

A, Seed C: Clove-pink. B, Poppy seed. c. Pollen of Passion flower (Passiftora carulea). D, Pollen of Cobcea siandens.

parent should be mounted in fluid, to show internal structure. The prettiest and most delicate forms are found in Amarantacea, Cucurbitacex, Malv acea, and Passiflorea; others are furnished from the Convolvulus, Geranium, Campanula, Hollyhock, and some other plants. The curious peculiarities of a few are shown in fig. 203 . 
Many of the smaller kinds of secds will reward the microscopist; use only a low power; that of Caryophyllum (clove-pink), is regularly covered with curiouslyjagged divisions; every one of which has a small bright, black hemispherical knob in its middle, represented in fig. 160, A.

The seeds of the carrot are remarkably formed, having some resemblance to a star-fish, with its long radiating processes. The seeds of umbelliferous plants have peculiar receptacles for essential oil, in their coats, termed vitto; various points of interest may be noted as occurring in the iesta, envelopes of seeds, such as the fibre-cells of Cobora, and the stellate cells of the Siar-anise.

All plants are provided with hairs; and a few, like insects, with hairs of a defensive character. Those in the Urtica dioica, commonly called the Stinging-nettle, are elongated hairs, developed from the cuticle, nsually of a conical figure, and containing an irritating fluid; in some of them a circulation is visible: when examined under the microscope, with a power of 100 diameters, they present the appearance seen at fig. 188, No. 2. At No. 3, same figure, are represented a few interesting ciliated spores from Conferver.

The circulation of the fluid-contents of vegetable cells may be examined at the same time with the Chlorophyll globules, by selecting for the purpose the transparent water-plants Chara, Nitella, Anacharis, and Vallisneria, or the hairs of Groundsel and Tradescantia. The circulation of the sap in plants growing in water is termed by botanists Cyclosis.

Fossil plants. - We detect in some of the primordial fossils a noticeable likeness to families familiar to the modern algæologist. The cord-like plant, Chorda filium, known as 'dead men's ropes,' from its proving fatal at times to the too adventurous swimmer who gets entangled in its thick wreaths, had a Lower Silurian representative, known to the palæontologist as the Palceochorda, or ancient chorda, which existed, apparently, in two species,a larger and a smaller. The still better known Chondrus crispus, the Irish moss, or Carrageen moss, has, likewise, 
its apparent, though more distant representative, jn Chondritis, a Lower Silurian alga, of which there seems to exist at least three species. The fucoids, or kelpweeds, appear to have also their representatives in such plants as Fucoides gracilis, of the Lower Silurians of the Malverns ; in short, the Thallogens of the first ages of vegetable life, seem to have resembled, in the group, and in at least their more prominent features, the Algce of the existing time. And with the first indications of land we pass direct from the Thallogens to the Acrogens, -from the sea-weeds to the fern-allies. The Lycopodiacece, or club-mosses, bear in the axils of their leares minute circular cases, which form the receptacles of their spore-like seeds. And when, high in the Upper Silurian system, and just when preparing to quit it for the Lower Old Red Sandstone, we detect our earliest ter-

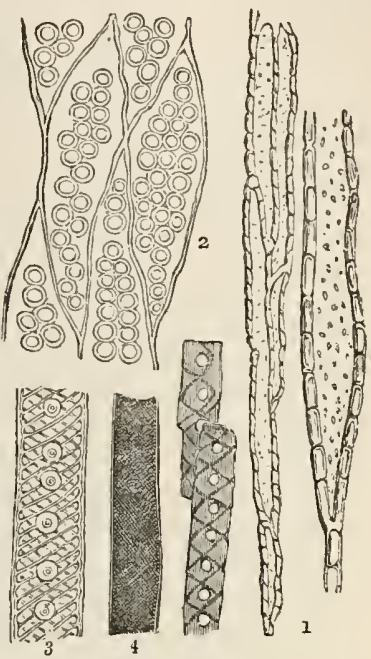

Fig. 204.

1, Woody fibre from the root of the E.der, exhibiting small pores. 2, Woody fibre of fossil wood, showing large pores. 3, Woody fibre of fossi wood, hordered with pores and spira: fibres. 4, Fossil wood taken from coal restrial organisms, we find that they are composed exclusively of those little spore-receptacles.

"The existing plants whence we derive our analogies in dealing with the vegetation of this early period, contribute but little, if at all, to the support of animal life. The ferns and their allies remain untouched by the grazing animals. Our native clu'b-mosses, though once used in medicine, are positively deleterious; horsetails (Equisetacece), though harmless, so abound in silex, which wrap them round with a cuticle of stone, that they are rarely cropped by cattle; while the thickets of fern which cover nur hill and dell, and seem so temptingly rich and green 
in their season, scarce support the existence of a single creature, and remaiu untouched, in stem and leaf, from their first appearance in spriug, until they droop and wither under the frosts of early winter.

"It is not until we enter into the earlier Tertiaries that we succeed in detecting a true dicotyledonous tree; on such au amount of observation is this order determined, that wheu Dr. John Wilson, the Parsee Missionary, submitted to me specimens of fossil woods which he had picked up in the Egyptian Desert, in order that, if possible, I might determine their age, I told him that if they exhibited the coniferous structure, they might belong to any geologic period from the times of the Lower Old Red Sandstone downwards; but if they manifested in their tissue the dicotyledonous character, they could not be older than the times of the Tertiary. Ou submitting them in thin slices to the microscope, they were found to exhibit the peculiar dicotyledonous structure as strougly as the oak or chestnut. And Lieutenant Newbold's researches in the deposit in which they occur has since demonstrated, on stratigraphical evidence, that it belongs to the comparatively modern formations of the Tertiary.

"The flora of the coal measures was the richest and most luxuriant, in at least individual productions, with which the fossil botanist has formed any acquaintance. Never before or since did our planet bear so rank a vegetation as that of which the ntimerous coal seams and inflammable shales of the carboniferous period form but a portion of the remaius, - the portion spared, in the first instance, by dissipation and decay, and in the second by the denuding agencies. Almost all our coal,- the stored-up fuel of a world,--forms but a comparatively small part of the produce of this wonderful flora. Yet, with all this siugularly profuse vegetation of the coal measures, it was a flora unfitted, apparently, for the support of either graminiverous bird or herbirorous quadruped. Nor does the flora of the Oolite seem to have been in the least suited for the purposes of the shepherd or herdsuan. Not until we enter on the Tertiary periods do we find floras amid which man might liave profitably laboured: nay, there are whole orders and families of plants, of the very first importance to man, 
which do not appear until late in even the Tertiary ages. The true grasses scarce appear in the fossil state at all. For the first time, amid the remains of a flora that seems to have had its few flowers, - the Oolitic ages,-do we detect, in a few broken fragments of the wings of butterflies, decided traces of the flower-sucking insects. Not, however, until we enter into the great Tertiary division do these become numerous. The first bee makes its appearance in the amber of the Eocene, locked up hermetically in its gem-like tomb, -an embalmed corpse in a crystal coffin,-along with fragments of flower-bearing herbs and trees. Her tomb remains to testify to the gradual fitting up of our earth as a place of habitation for a creature destined to seek delight for the mind and eye, as certainly as for the proper senses, and in especial marks the introduction of the stately forest trees, and the arrival of the deliciouś flowers." 1

\footnotetext{
"Sweet flowers! what living eye hath viewed Their myriads? endlessly renewed;

Wherever strikes the sun's glad ray,

Where'er the subtile waters stray,

Wherever sportive zephyrs bend

Their course, or genial showers descend.'
}

(1) Hugh Miller's Testimony of the Rocks.

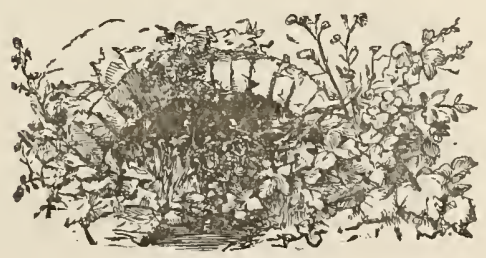




\title{
CHAPTER II.
}

\author{
DIVISION OF ANIMAL KINGDOM.
}

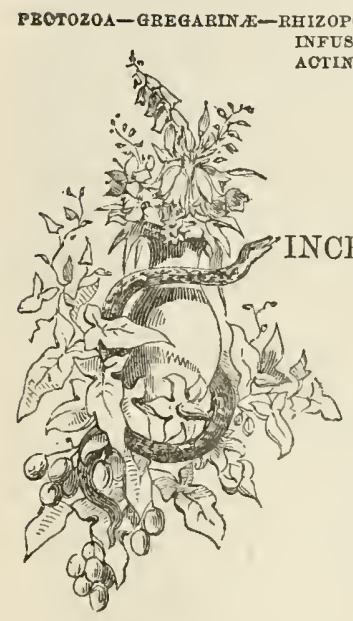

our very limited space forbade more than a cursory glance at the many and varied points of beauty and arrangement displayed in every part of the vegetable kingdom; so in the present division is it necessary to be equally brief in noticing the wonders displayed by the help of the micioscope, in the world of animal life. In the cour'se of remarks made upon the early condition of vegetable life, we drew attention to the difficulties presented in all attempts to mark out the boundary line between vegetables and animals, and to define where the one ends, and the other begins.

Upon reviewing the different characters by which it has been attempted to distinguish the special subjects of the botanist and zoologist, we find that animals and plants are not two natural divisions, but are specialised members of one and the same group of organised beings. When a certain number of characters concur in the same organism, its title to be regarded as a "plant," or an "animal," may be readily recognised; but there are very numerous living beings, especially those that retain the 
form of nucleated ceils, which manifest the common organic characters, but are without the true distinctive superadditions of either kingdom.

The animal kinglom is conveniently arranged under the following primary groups, sub-kingdoms, or departments : -Protozoa, Celenterata, Annulosa, Mollusca, and Vertebrata. These are again divided into classes, classes into orders, orders into families, families into genera, and genera into species. In the first-named department, the Protozoa, is included a vast number of creatures of the simplest organisation, classified as follows:-1. Gregarinidæ, 2. Rhizopoda, 3. Polycystina, 4. Thalassicollidæ, 5. Spongidæ, 6. Infusoria. It is not unusual to place Rhizopoda and its typical form, Amøba, in the front rank; and as the presence of a mouth characterises the Infusoria, the remaining part of the Protozoa are frequently designated by a collective name, Astomata.

The first-named, Gregarinida, form a group of the very simplest structural character, and any one of them, setting minor modifications aside, may be said to consist of a sac, composed of a more or less structureless membrane, containing a soft semi-fluid substance, in the midst of which is a circular vacuole or vesicle, having in its centre a more solid particle or nucleus. Professor Huxley appends to this description the obvious, but highly important reflection, that its statements are all true concerning the ova of any of the animals much higher in the scale. ${ }^{1}$ The Gregarinidce inhabit the bodies of other animals, and they multiply by becoming encysted, and dividing into a multitude of minute objects, called pseudo-navicelloe, from their resemblance in shape to the ship-like diatoms (naviculce). When a young pseudo-navicella escapes, it behaves somewhat like an amœba, and, if it chance to get swallowed by an appropriate host, it grows into the parent form. The whole life-histury of these creatures is not known, as they have not been traced into the exhibition of sexual properties ; and it is therefore possible that their position in the scale may not be exactly defined.

In the course of the numerous investigations made of the flesh of animals dying during the year 1866 from the sattle-plague, it was noticed that large quantities of peculiar bodies infested the muscular structures, more especially

(1) Huxley's "Lectures on the Elements of Comparativs Anatomy." 
that of the heart, and it was supposec that these had some share in the production of the disease; but upon making further investigations this has been found not to be so, and since the same bodies are known to be generally distributed throughout the ultimate fibres of animals dying from other diseases, the only interest that can attach to the discovery is, that it promises to add some valuable facts to our knowledge of the remarkable group of Protozoa, the Gregarina. The Gregarines observed in the flesh of oxen, and described by Dr. Beale, have elongated spindle-shaped sacs, containing granular reniform bodies arranged horizontally, and apparently capable of multiplying by division. The investing sac is covered with minute, motionless, hair-like bodies. No nucleus is present in the sac; but the reniform granular masses are stated by Dr. Cobbold to possess nucleoli. The structure thus presented is not far removed. from that of many Gregarina, particularly of the larger individuals occurring in the earthworm, though the hairlike processes sometimes observable on these are considered as extraneous by Dr. Leiberkiihn. The compacted reniform masses may be considered as the results of a process of segmentation, similar to that by which the pseudonaviculæ are formed. The bodies thus described are by no means peculiar to diseased cattle; they are met with in the healthy muscles of the ox, sheep, pig, deer, rat, mouse, mole, and perhaps other animals. Gregarina, in various stages, are represented in Plate III. figs. 53, 54,55 , and 56 . Miescher, in 1843, described such bodies, taken from the muscles of a mouse, and a very good account of them, obtained from the muscles of a pig, is given by Mr. Rainey, in the " Philosophical Transactions," for 1857, though he erroneously regarded them as the young stage of cestoid entozoa. They have been described under a variety of titles, such as worm-nodules, egg-sacs, eggs of the fluke, young measles, "corpuscles produced by muscular degeneration," \&c. When considered in connexion with the minute cysts described by Gabler, Virchow, and Dressler, from the human liver, they have an especial interest; and the observations of Lindemann on the psorospermial sics obtained from the hair of a peasant at 
Niscluney-Novgorod, and in the kidneys of a patient who died from Bright's disease, bear very strongly on the nature of these bodies. The people of Novgorod are believed to get these parasites from washing in water in which Grega. rince abound. ${ }^{1}$ The most interesting inquiry which is placed before us by these various facts is whether, as Professor Leuckart has observed, "the psorospermiæ" (and we may add the "spurious entozoa" of cattle, and even many so-called Gregarince) are to be considered as the result of a special animal development, or whether they are the final products of pathological metamorphosis.

It appears, from the reseaches of M. Claparède and others, that some of the unilocular forms do present very curious, elongated, and active forms, which, from their movements and general appearance, might he mistaken for nematodes. Dr. Joseph Leidy has, in the "Transactions of the Philadelphia Society, 1853," denied the fact that the Gregarinoe are unicellular animals, on the following grounds:-In the examinations of some new species of Gregarince which he has described, and also in the G. Blotharum of Siehold, he discovered that the membrane enclosing the granular mass of the posterior sac was double. He observes: "Within the parietal tunic of the posterior sac is a second membrane, which is transparent, colourless, and marked by a most beautiful set of exceedingly regular parallel longitudinal lines."

M. Leiberkühn contributed a very elaborate paper on the Gregarina of the earthworm. He does not express any very decided opinion upon the two questions which have been discussed by Leidy and Bruch, but derotes the principal part of his memoir to the development and reproduction of the Gregarinæ. With regard to the development of Gregarinæ into a filaria-like worm, which Bruch thought probable, M. Jeiberkühn says but little, but, nevertheless, has proved beyond doubt that the nematodes

(1) Many vague and improbable statements appeared at one time on a supposed discovery of Gregarine in the human hair. Upon a more careful exam:nation being made by competent persons, the foreign body proved to be a wellknown vegetable fungus, often found associated with a disease, or dirts neglected stato of the skin. It is very well known that Gregarines are never lound on free or exposed surfaces. 
of the earthworm are developed from egrgs, whence they emerge, not as Gregarinæ, but as true nematodes. The transformation of two Gregarinæ, after a process of encysta tion, into navicula-like bodies, has been fully described by Bruch ; but Leiberkühn has more carefully illustrated the changes that go on, and has endeavoured to trace the existence of the pseudo-naviculæ after they have keen expelled from the cyst. In the perivisceral cavity of the earthworm he found large numbers of small corpuscles, exhibiting amœba-like movements, and likewise pseudonaviculæ, containing granules, formed from encysted Gregarine. He imagines that these latter bodies burst, and that their contained granules develope into the amœoiform bodies which subsequently become Gregarinæ. M. Leiberkiihn shortly afterwards published another paper, in which he adopts the same view, that the amœbiform corpuscles of the blood of fish are Gregarines. But few physinlogists will feel disposed to agree with him, in considering these bodies as parasites.

Mr. E. Ray Lankester has contributed a valuable and exhaustive paper on this subject; ${ }^{1}$ he observes: "I have made careful examination of more than a hundred worms, for the purpose of studying these questions, but have sacceeded in arriving at no other conclusion than that certain forms of these may be the products of encysted Gregarinæ. The G. Lumbrici is one of those forms which are unilocular, and are met with most frequently among Annelids. It consists of a transparent contractile sac (which bas not hitherto been demonstrated to be formed hy more than a single membrane), enclosing the characteristic granules and vesicle. The vesicle is not always very distinct, and is sometimes altogether absent; occasionally it contains no granules, sometimes several, one of which is generally nucleated. In some of these cysts a number of nucleated cells may be seen, developing together from the enclosed Gregarina, which gradually become fused tngether and broken up, until the entire mass is converted into these nucleated bodies, which are then evident in different stages of development, assuming the form of a

(1) E. Ray Lankester, "On the Gregarinidæe found in the common Earthnorm "-Micros. Trans. vol. iii. p. 83. 
double cone, like that presented by some species of Diatomacee, whence their name pseudo-naviculæ. At length the cyst contains nothing but pseudo-naviculæ, sometimes enclosing granules, which gradually disappear, and finally the cyst bursts. Encystation seems to take place much more rarely among the bilocular forms of Gregarinæ than in the unilocular species found in the earthworm and other Annelids."

In the Gregarince the food is taken in indiscriminately at every point of the surface of the body by imbibition. The food most likely is in the fluid state. In Spongilla, also, this is probably the case. But it is generally agreed that in Amoeba, Actinophrys, and agastric In $n^{2} u s o r i a$, only solid alimentary particles are taken as food. The simplest animal is indeed far more complex than is implied in the word unicellular, and it can be clearly proved that there are fery points in common between a simple cell and a socalled unicelluiar protozoon. The system of contractile vesicles and dependent sinuses, so general in the least organized protozoon, is unknown in the history of cells. Fluid absoption by the surface is the normal method of feeding in these low types of animal life. This absorptive faculty is an inherent property of the substance of which they are composed. It attracts certain aliments, as gelatine attracts water. Tissue, distinguished by the same character, prevails throughout the entire class of the Protozoa. Although the Gregarince mostly inilabit the intestines of invertebrate animals, they are often found in the alimentary canal of the Vertebrata. In this class they appear to be represented, however, by very closely allied organisms, the Psorospermiae. Miuller gave this lastmentioned name to some very singular minute bodies he discovered within sacs upon the skin and gills, and in the internal organs, of many fishes. These animals are generally of a cylindrical or somewhat elliptical form, although sometimes a sort of head appears to be produced by the constriction of the anterior extremity of the body, and this head-like portion is occasionally furnished with a curious soft process and lobes. They are very sluggish in their movements, although a few possess true cilia. Their surious mode of development, with other points in the 
history of these minute parasites, are well worthy of investigation.

The Rhizopoda appear as creatures of a low type of organization, and are considered, with the former, to hold a medium state between animals and vegetables. Almost all of them live in water; it would be a fruitless search to look for distinct internal organs, as the small bladder looking spaces enclosed within their substance,-believed by Ehrenberg to be stomachs,-present only the appear: ance of transparent gelatinous cells, or rather moving spaces, within the sarcode envelope, and may be regarded as the earliest dawn of a circulatory system.

The term Rhizopoda is derived from the Greek, and
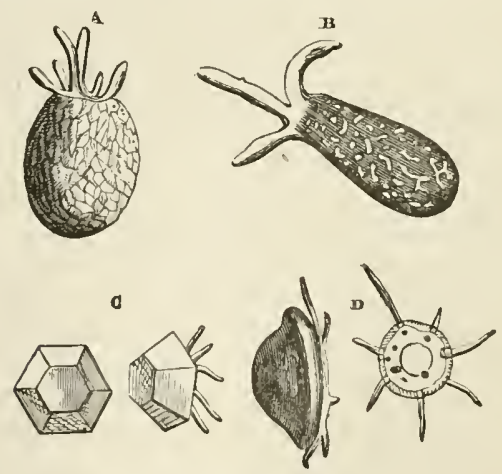

Fig. 205.-Simple Rhizopods.

A, Diflugia proteiformis. B, Diflugia oblonga. C, D, Arcella acuminata and dentata.

means " root-footed," - the body is composed entirely of gelatinous matter, sarcode,-motion being effected by the extension of portions of the substance into processes, which, as in fig. 205 , is seen to partake of various forms.

Lobosa. - In the deposit formed at the bottom of freshwater ponds, we may often meet with a singular minute gelatinous body, which constantly changes its form even under our eyes; and moves about by means of finger-like processes, called pseudopodia, which it appears to have tho power of shooting out from any part of its substance. 
This shapeless mass is well known to microscopic observers under the name of the Proteus (A moeba diffuens, fig. 206), which, from the continual changes of shape it presents, is
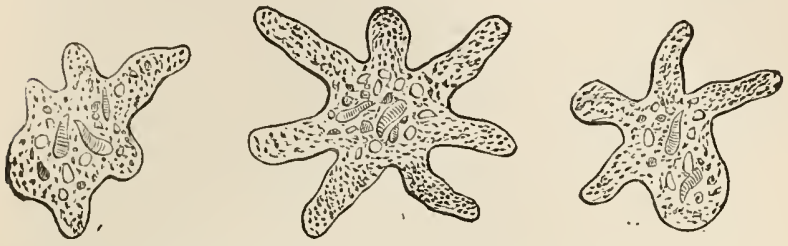

Fig. 206.-Amaba difluens, or Proteus, in diderest forms.

honoured with the name of a fabled god, who could be either animal, vegetable, or mineral in his nature. This curious animal presents us with the essential characters of the large class Rhizopoda in their simplest form. It appears to be of an exceedingly voracious disposition, seizing upon any minute aquatic animals or plants that may come in its way, and appropriating them to the nutrition of its own gelatinous body. The mode in which this tender and apparently helpless creature effects this object is very remarkable. The gelatinous matter of which it is conposed is capable, as we have seen, of extension in every direction; accordingly, when the $A$ moeba meets with anything that it regards as suitable for its support, the substance of the creature, as it were, grows round the object until it is completely enclosed within its body. The substances swallowed (if such a term be admissible) by this hungry mass of jelly are often so large, that the creature itself only seems to form a sort of gelatinous coat enclosing its prey.

Professor Ecker believes in an exact similarity of contractile substance between that of the lower animal forms, such as the Rhizopoda, and that observed in the Hydra. He says : "The properties of this substance, in its simplest form, are seen in the Amoeba, the body of which, as is known, consists of a perfectly transparent albumen-like homogeneous substance, in which nothing but a few granules are imledded, and which presents no trace of 
further organization. This substance is in the highest degree extensible and contractile; and from the main mass are given out, now in one part and now in another, perfectly transparent rounded processes, which glide over the glass like oil, and are then again merged in a central mass. There is no external membrane. In the body of the A mœba there occur, besides the granules, clear spaces with fluid contents, which are sometimes unchangeable in form, and sometimes exhibit rhythmical contractions."

Belonging to the family is the very curious Acineta of Ehrenberg, Actinophrys sol, "sun-animalcule." This creature consists of a jelly-like contractile substance, or sarcode, with tentacular filaments radiating from the central mass, in such a manner as to have suggested the name for the species. It abounds in pools where Desmidiacece are found in many parts around London; they are ravenous feeders, not only upon the Desmidiacece, int also upon all kinds of minute spores and arimalcules. (Plate III. fig. 66.) It was on examining some beautiful Desmidiacea that my attention was arrested by the curious appearance of two or three very small Actinophrys floating very lightly upon the surface of the water, in the form of a ball, with their delicate tentacular filaments perfectly erect all over their bodies ; in fact, they seemed to be floating upon these delicate filaments. ${ }^{1}$

The most beautiful forms of the Rhizopoda are found among those possessing a calcareous covering, as the Polythalamia, Rosalina, Faujasina, \&c. ; their systematic arrangement is founded upon their shells, which exhibit a very great diversity in form. Out of these forms, it would appear that the labours of various naturalists in the last hundred years have made us acquainted with nearly 2,000 recent and fossil Foraminifera; and although the observations of Dr. Carpenter ${ }^{2}$ tend to show the probability that very many of these supposed species are merely varieties, still the number is sufficiently great to prove the importance and interesting nature of the inquiry.

(1) Weston, Junrn. Mficros. Science, vol. iv. New series, p. 116; Claparède, Ann. Nat. His. Secund series, vol. xv. p. 211.

(2) Carpenter's "Introduction to the Study of the Foraminifera," published by the Ray Soc. 1862 . 
Dr. Schultze acknowledges the difficulties attending the study of the Rhizopoda, and insists, very properly, upon the necessity of viewing them in all positions, and under different modes of illumination and of preparation, in order to arrive at a due conception of their astonishing conformation. When the shells of Foraminifera are dissolved in dilute acid, an organic basis is always left after the removal of the calcareous matter, accurately retaining the form of the shell with all its openings and pores. The earthy constituent is mainly carbonate of lime ; but Dr. Schultze has satisfied himself of the presence of a minute amount of phosphate of lime in the shells of recent Orbiculina adunca from the Antilles and of Polystomella strigilata from the Adriatic.

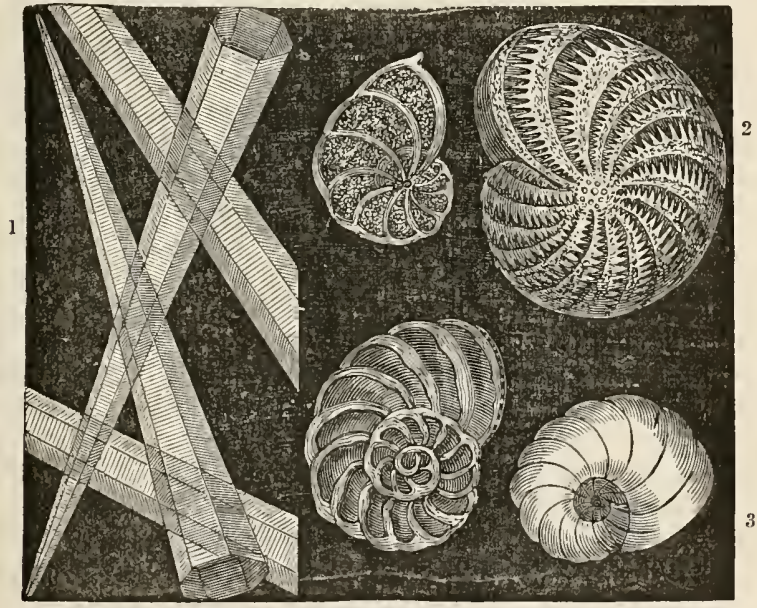

Fig. 207.

1, Separated prisins from outer layer of Pinna shell. 2.2Skeletons of Foraminifera from limestone. 3, Recent shell of Polystomella crispa: viewed with dark-ground illuminator.

The solitary Rhizopoda, furnished with a horny shell or capsule, forming a case for the animal, is nearly the only representative of the Arcellidae. In the Areella, from which the family derives its name, the shell is somewhat of a bell-shape, with a very large round opening. In 
Englypha it is of an oval or flask-like form, with the opening at the smaller end, and the shell appears as though formed of a sort of mosaic of small horny pieces. In Diffugia, Fig. 205, A B, the shell is often globular. Rhizopods which never develop more than one chamber or loculus are classed as Monothalamia. ${ }^{1}$

The Polythalamia, or Multilocular Rhizopods, in their earliest state, are unilocular; but, as the animal increases, successive chambers are added in a definite pattern for each family of the order. They all inhabit the sea, and frequently occur in such great numbers, that the fine calcareous sand which constitutes the sea-shore in many places consists almost entirely of their microscopic coats. At former periods of the earth's history, they existed in even greater profusion than at present; and their fragile shells form the principal constituent of several very important geological formations. Thus the chalk appears to consist almost entirely of the shells of these animals, either in a perfect state, or worn and broken by the action of the waves; they occur again in great quantities in the marly and sandy strata of the Tertiary epoch.

In the Stichostegidee the chambers are placed end to end in a row, so as form a straight or but slightly curved shell. In the second family, the Enallostegidce, the chambers are arranged alternately in two or three parallel lines; and as the construction of the shell is always commenced with a single small chamber, the whole necessarily acquires a more or less pyranidal form. The third family, the Helicostegidce, presents us with some of the most beautiful forms that it is possible to meet with in shells. They commence by a small central chamber; and each of the subsequent chambers, which are arranged in a spiral form so as to give the entire shell much the aspect of a minute flattened snail, is larger than the ono preceding it. It is in this family that we find the nearest approach, in external form, to the large chambered shells of the cephalopodous mollusca, of which the Nautilus pompilius is an example. The fourth family, the Entomostegida, stand in the same relation to the preceding as the Enallostegidce to the

(1) Diffugia and Ancella form a connecting link between the naked forms, moeba, Actinophrys, \&c. and the shell-bearing Rhizopods, Lagena striata, \&e. 
Stichostegidae; that is to say, the chambers are also arranged in a spiral form, but in a double series. A fifth family includes those shells in which the chambers are arranged round a common perpendicular axis in such a manner that each chamber occupies the entire length of the shell. The orifices of the chambers are placed alternately at each end of the shell, and are furnished with a curious tooth-like process. The Mitiola serve as an example of this family. Every handful of sea-sand, every shaking of a dried sponge, and the contents of the stomachs of most Lamellibranch molluses, oyster and mussel, are pretty sure to exhibit a considerable admixture of these minute calcareous, or occasionally silicious, Foraminifera.

It is considered that the fossil shells, termed $\mathrm{Num-}$ mulites, found in great quantities in the chalk and lower tertiary strata, are also to be regarded as members of this class ; in a fossilized state, whole mountains consist almost entirely of their shells. The late Professor Quekett had an opportunity of examining a few living specimens, which, he says, "are composed of a sarcode element, built up into a series of chambers with calcareous material."

The great Pyramid of Egypt, covering eleven acres of ground, is based on blocks of limestone consisting of Foraminifera, Nummulites, or stone coin, and other fossil auimalcules. Nummulites vary in size from a very minute object to that of a crown-piece, and many appear like a snake coiled up in a round form. A chain of mountains in the United States, 300 feet high, seems wholly formed of one kind of these fossil-shells. The crystalline marble of the Pyrenees, and the limestone ranges at the head of the Adriatic gulf, are composed of small Nummulites. Vast deposits of Foraminifera have been traced in Egypt and the Holy Land, on the shores of the Red Sea, Arabia, and Hindostan, and, in fact, may be said to spread over thousands of square miles from the Pyrenees to the Himalayas.

The fossilized Foraminifera in the Poorbandar limestone, although occasionally reaching the twenty-fifth, do not average more than the hundredth part of an inch in diameter; so that more than a million of them may be computed to exist in a cubic inch of the stone. They may 
be separated into two divisions-those in which the cells are large, the regularity of their arrangement visible, and their bond of union consisting of a single constructed portion between each; and those in which the cells are minute, not averaging more than the 900th part of an inch in diameter, the regularity of their arrangement nor distinctly seen, and their bond of union consisting of many thread-like filaments. To ascertain the mineral composition of the amber-coloured particles or casts, after having found that it was mostly carbonate of lime with which they were surrounded, they were placed for a few moments in the reducing flame of a blow-pipe, and it was observed that on subsequently exposing them to the influence of a magnet, they were all attracted by it. Hence, in a rough way, this rock may be said to be composed of carbonate of lime and oxide of iron.

By far the greater number of Furaminifera are marine. They are found in most seas, and in those of the tropics they increase both in size and variety, forming extensive deposits.

During the Canadian Geological Survey large masses of what appeared to be a fossil organism, the Eozom Canadcnse, were discovered in rocks situated near the base of the Laurentian series of North America. Dr. Dawson, of Montreal, referred these remains to an animal of the foraminiferal type; and specimens were sent by Sir W. Logan to Dr. Carpenter, requesting him to subject them to a careful examination. As far back as $1858 \mathrm{Sir}$ W. Logan had suspected the existence of organic remains in specimens from the Grand Calumet limestone, on the Ottawa river, but a microscopic examination of one of these specimens was not successful. Similar forms being seen by Sir William in blocks from the Grenville bed of the Laurention limestone were in their turn tried, and ultimately revealed their true structure to Dr. Dawson and Dr. Sterry Hunt.

The masses of which these fossils consist are composed of layers of serpentine alternating with calc-spar. It was found by these observers that the calcareous layers represented the original shell; and the siliceous layers the flesh, or sarcode, of the once living creature. These 
results were arrived at through comparison of the appearance presented by the Eozoon with the microscopic structure which Dr. Carpenter had previously shown to characterise certain members of the foraminifera. The Eozoon not only exceeded other known foraminifera in size, to an extent that might have easily led obscrvers astray, but, from its apparently very irregular mode of growth, its general external form afforded no help in its identification, and it was only by careful examination of its minute structure that its true character could be ascertained. Dr. Carpenter says :- “The minute struc. ture of Eozoon may be determined by the microscopic examination either of thin transparent sections, or of portions which have been subjected to the action of dilute acids, so as to remove the calcareous shell, leaving only the internal casts, or models, in silex, of the chambers and other cavities, originally occupied by the substance of one animal."

Dr. Carpenter found the preservation of minute structure so complete that he was able to detect "delicate pseudopodial threads, which were put forth through pores in the shell wall, of less than $\frac{\text { ? }}{1000}$ th of an inch in diameter" (see Plate III. figs. 64, 65); and in a paper read at the meeting of the Geological Society he stated that he had detected Eozoon in a specimen of ophicalcite from Cesha Lipa in Bohemia, in a specimen of gneiss from near Moldau, and in a specimen of serpentine limestone sent to Sir C. Lyell by Dr. Giimbel, of Bavaria, all these being parts of the great formation of "fundamental" gneiss, which is considered by Sir Roderick Murchison as the equivalent of the Laurentian rocks of Canada. There can be little doubt that a rich field of research is now opened to those who will undertake the examination of rocks of various ages, which present the appearance of analogous structure; as it is, the microscope has been the means of demonstrating the existence of animal life at a very ancient geological date; and, in the words of Sir W. Logan, "we are carried back to a period so far remote that the appearance of the so-called Primordiai Fauna may be considered a comparatively modern event." Recent Foraminifera present symmetrical shells, of 
minute size for the most part, and consisting, as we have already seen, either of olle, two, or more connected chambers. A jelly-like mass, or "sarcode," occupies the chambers and their connecting passages; and, protruding itself both from the external aperture of
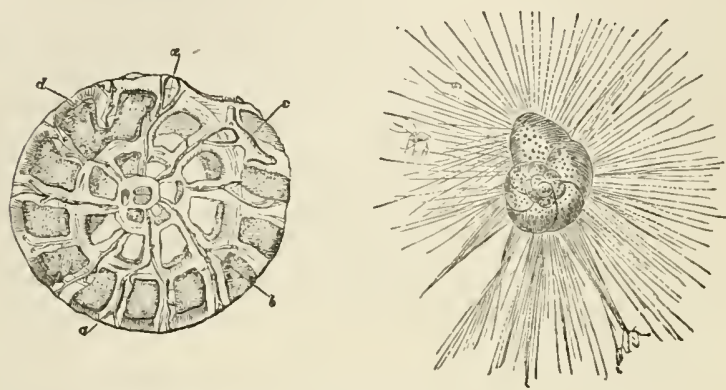

Fig. 208.

1, Section of Faujasina: $a$, radiating interseptal canals; $b$, their internal bifurcations; $c$, a transverse branch; $d$, tubular wall of the chambers. 2, Rosalina ornata, with its pseudopodia protruded.

the last chamber, and in many cases from the sorretimes numerous perforations in the shell-walls, extends itself not only over the surface of the shell, but also into radiating contractile threads or pseudopodia, and into gemmule-like masses, which latter become coated over with calcarcous matter, and thus form additional segments of the animal. ${ }^{1}$

"Foraminifera, indeed, are to be compared witl the other lowest orders of animals and of plants in the study of their specific relations. In these several low forms of crcatures we have comparatively few species, but extremely numerous individuals, with an enormous range of

(1) Among the more important works on Foraminifera, refcrence may be made to D'Orbigny's Foraminiferes fossiles du Bassin, Tertiaire de Vienno (Autriehe); Schultze, Ueber den Organismus der Polythalamien, 1854; Carpenter's and Williamson's Researches on the Foraminifera, Phil. T'rans. 1856. Also an excellent jajer by Mr. W. R. Parker, in the Annals of Natural History, April, 185T. Slecimens of Foraminifera may be obtained for examination fron the shaking of dried sponges; but if required alive they must be dredged for, 0 r pitked off the fronds of living seaweeds, over the surface of which they are seen to move by the aid of a lens. 
variety. In the higher orders of plants and animals the specific forms are more definite, there being a more complex organization, harmonizing with the special habits of each creature; and the individuals of each species are less

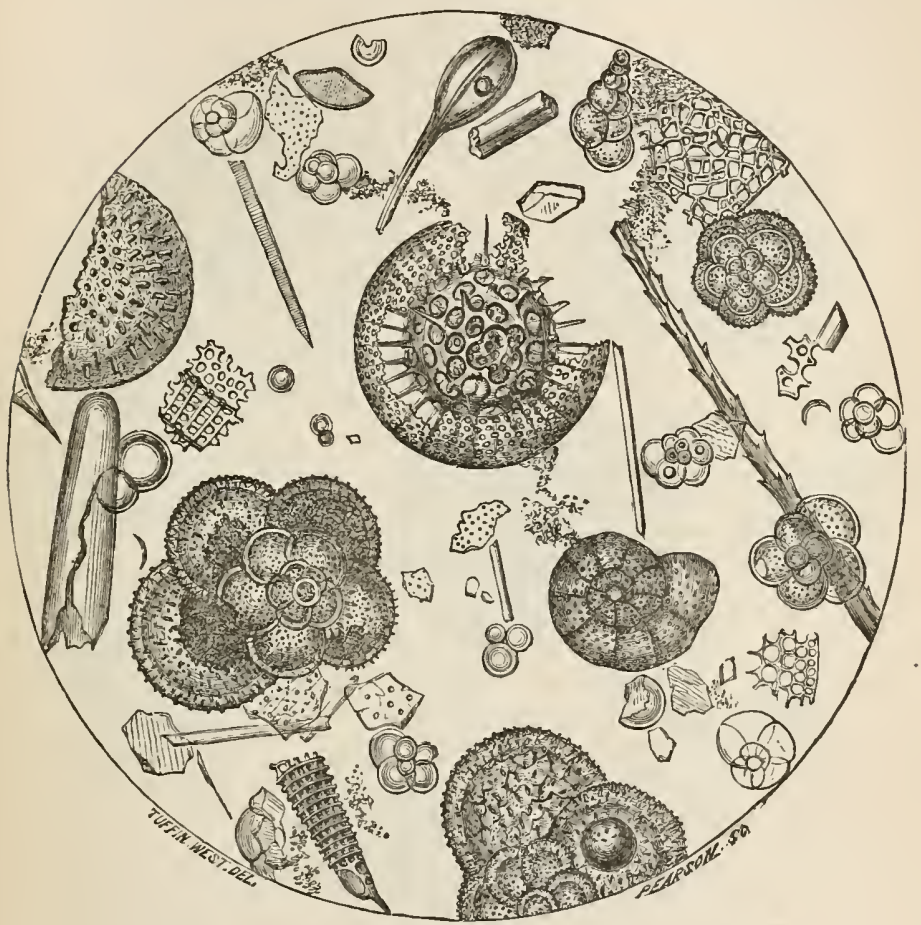

Fig. 209.-Foraminifera taken in Deep-sea Soundings. (Atluntic.)

numerous than is the case in the Protozoans and Protophytes."

These lowly organized Foraminifera, having great simplicity of structure, more easily adapt themselves to varying external conditions than the more complex and specialized higher animals. 
In the deep-sea soundings, portions of many beautiful Diatoms, figured and described by Professor W. Smith, in gatherings from the Bay of Biscay, near Biarritz, are Melosira cribrosa, marine, orbicular, cellulate,

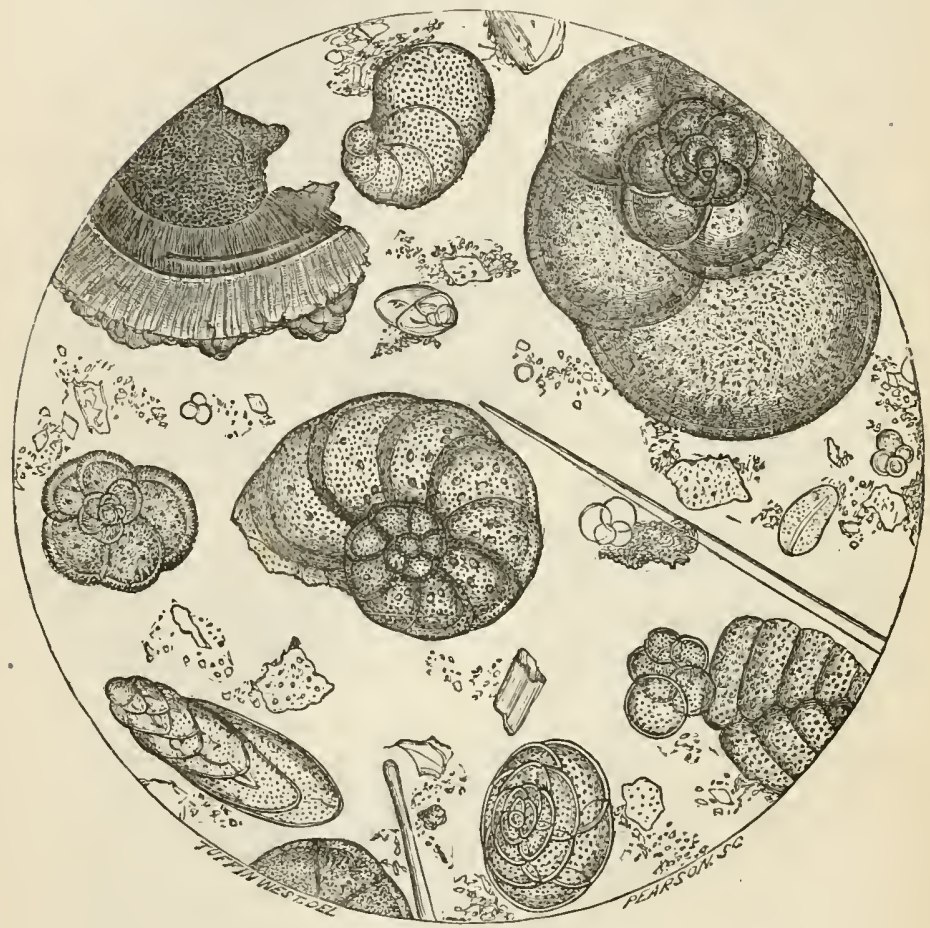

Fig. 210.-Foraminifera taken in Deep-sea Soundings. (Atlantic.)

cellules, all equal and hexagonal. He writes: "In Dccember, 1853, I received isolated frustules of this species, collected on the coast of Normandy, under the above name, from M. de Brébisson; and I have since detected the same in a gathering from the Black Sea. In no case have I seen the frustules in a recent state, and do not know 
whether they ever form a lengthenod filament. As this is the only circumstance that would justify their separation from Coscinodiscus, to which the separated valve would otherwise seem to belong (Synop. British Diatomacea, vol. i. p. 22), their position in Melosira must rest upon the authority of my accurate correspondent."

In figs. 209 and 210 are represented many of the beautiful forms brought up with soundings made in 1856, for the purpose of ascertaining the depth of the Atlantic, prior to the laying down of the electric telegraph wirs from England to America; these specimens were taken from a depth of 2,070 fatloms.

Major S. R. J. Owen, while dredging the surface of mid-ocean-Indian and Atlantic oceans-found attached to his nets a few interesting forms of Rhizopods, belonging to the two genera Globigerina and Pulvinulina, which always make their appearance on the surface of the ocean after sunset. ${ }^{1}$

"Many of the forms," writes this observer, "have hitherto been claimed by the geologist, but I liave found them enjoying life in this their true home, the siliceous shells filled with coloured sarcode, and sometimes this sarcode in a state of distension somewhat similar to that found projecting from the Foraminifera, but not in such slender threads. There are no objects in natme more brilliant in their colouring or more exquisitely delicate in their forms and structure. Some are of but one colour, crimson, yellow, or blue; sometimes two colours are found on the same individual, but always separate, and rarely if ever mixed to form green or purple. In a globular species, whose shell is made up of the most delicate fretwork, the brilliant colours of the sar'code shine through the little perforations very prettily. In two specimens of the triangular and square forms (Plate III. figs. 44, 45, and $46)$, the respective tints of yellow and crimson are vivid and delicately shaded. In one the pink lines are concentric; while another is of a stellate form (fig. 43), the points and uncoloured parts being bright clear crystal, while a beautiful crimson ring surrounds the central por1867 . 
tion. One globular species appears like a specimen of the Chinese ball-cutting-one sphere within another; but it is of a marked and distinct kind.

"The shells of some of the globular forms of these Polycystina, whose conjugation I believe I have witnessed, are composed of a fine fretwork, with one or more large circular holes; and I suspect the junction to take place by the union of two such apertures. That the figures of these shells become elongated, lose their globular form after death, and present a disturbed surface is seen in some of the figures represented near the bottom part of Plate III." Major Owen proposes to make Orbulina a subgenus of Globigerina. The internal chambers of the former are in form remarkably like those of the latter, and like them also they present themselves with varying surfaces, some free from, while others are covered with, spines. Those without internal chambers have been known as Orbulina universa, fig. 78, Plate III. while figs. 75 and 76 , althongh members of the same family, have been separated; but he wishes to see all united under the name of "Glubigerina universa."

The minute siliceous shells of Polycystina present wonderful beauty and variety of form; all are more or less perforated, and often prolonged into spines or other projections, through which the sarcode body extends itself into pseudopodial prolongations resembling those of Actinophrys. When seen besporting themselves in all their living splendour, their brilliancy of colouring, says Major Owen, "renders them objects of unusual attraction." We have endeavoured to give some idea of the colour of the living forms in Plate III. Nos. 43 to 52. The same observer believes that they wish to avoid the light, "as they are rarely found on the surface of the sea in the day time; it is after sumset, and during the first part of the night especially, that they make their appearance."

The Polycystina appear to have most affinity with the Foraminifera. Thirty four genera and about two hundred and nincty species have been described. They are most abundant in the fossil state; and are very plentiful in the rocks of Bermuda, in the tripoli of Richmond, Virginia, in the marls of Sicily, and other places. Their minute shells 
form beautiful microscopic objects for the binocular; they must be mounted dry, and viewed either with the dark ground illuminator, or by condensed light.

\section{SPONGIADE.-SPONGES.}

The term Porifera, or Canal-bearing Zooplyte, was applied by Professor Grant to designate the remarkable class of organized beings known as sponges, which are met with in every sea, growing in great abundance on the surface of rocks.

Ellis, in the course of his investigations, was astounded by discovering that sponges possessed a system of pores aud vessels, through which sea-water passed, with all the appearance of the regular circulation of fluids in animal bodies, and for the seeming purpose of conveying animalcules to the animals for food.

The description given of sponges by Dr. Johnston is, that they are "organized bodies growing in a variety of forms, permanently rooted, uumoving aud irritable, fleshy, fibro-reticnlar, or irregularly cellular ; elastic and bibulous, composed of a fibro-corneous axis or skeleton, often interwoven with siliceous or calcareous spicula, and containing an organic gelatine in the interstices and interior canals; and are reproduced by gelatinous granules called gemmules, which are generated in the interior, but in no special organ. All are aquatic, and with few exceptions mariue." " Our author continues :- "Mr. Johu Hogg, in a letter to me dated June 25, states that the green colour of the fresh-water sponge (Spongilla fluviatilis) depends upon the action of light, as he has proved by experiments which showed that pale-coloured specimens became green when they were exposed for a few days to the light and full rays of the sun; while, on the contrary, green specimens were blanched by being made to grow in darkness or shade."

The living sponge, when highly magnified, exhibits a reticulated structure, permeated by pores, which united into cells or tubes, ramify through the mass in every direction, and terminate in larger openings. In most

(1) See Dr. Johnston's History of British Sponges, aLd Mr. Bowerbank's revisiun of the class, in the publications of the Ray Socie Jy. 
sponges the tissue is strengthened and supported by spines, spicula, of various forms; and which, in some species, are siliceous, and in others calcareous. The minute pores, through which the water is imbibed, have a fine transverse gelatinous network and projecting spicula, for the purpose of excluding large animals or noxious particles; water incessautly enters into these pores, traverses the cells or tubes, and is finally ejected from the larger

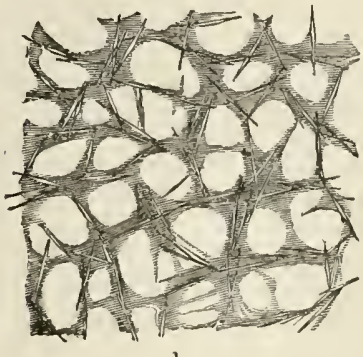

1

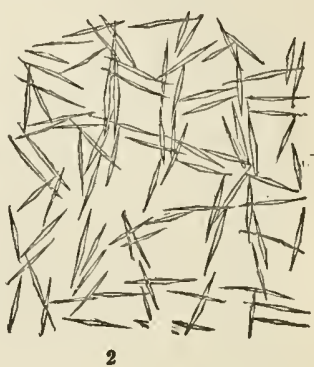

2

Fig. 211.

I, A portion of Sponge, Ialichondria simulans, showing siliceous spicula imbedded in the sarcode matrix. 2, Spicula divested of its matrix.

vents. But the pores of the sponge have not the power of contracting and expanding, as Ellis supposed; the water is attracted to these openings by the action of instruments of a very extraordinary nature (cilia), by which currents are produced in the fluid, and propelled in the direction required by the economy of the animal.

Mr. Bowerbank, in a paper on the "Structure and Vitality of Spongiada," states that sponges cousist principally of sarcode, strengthened sometimes by a siliceous or calcareous skeleton, having remarkable reparative and digestive powers, and consequently a most tenacious vitality; so much so, that having cut a living sponge into three segments, and reversed the position of the centre piece, after the lapse of a moderate interval, a complete junction of the parts became effected, so as to render the prerious separation indistinguishable.

Professor Grant's careful and laborious researches, have fually classed sponges in the animal series of the creation. 


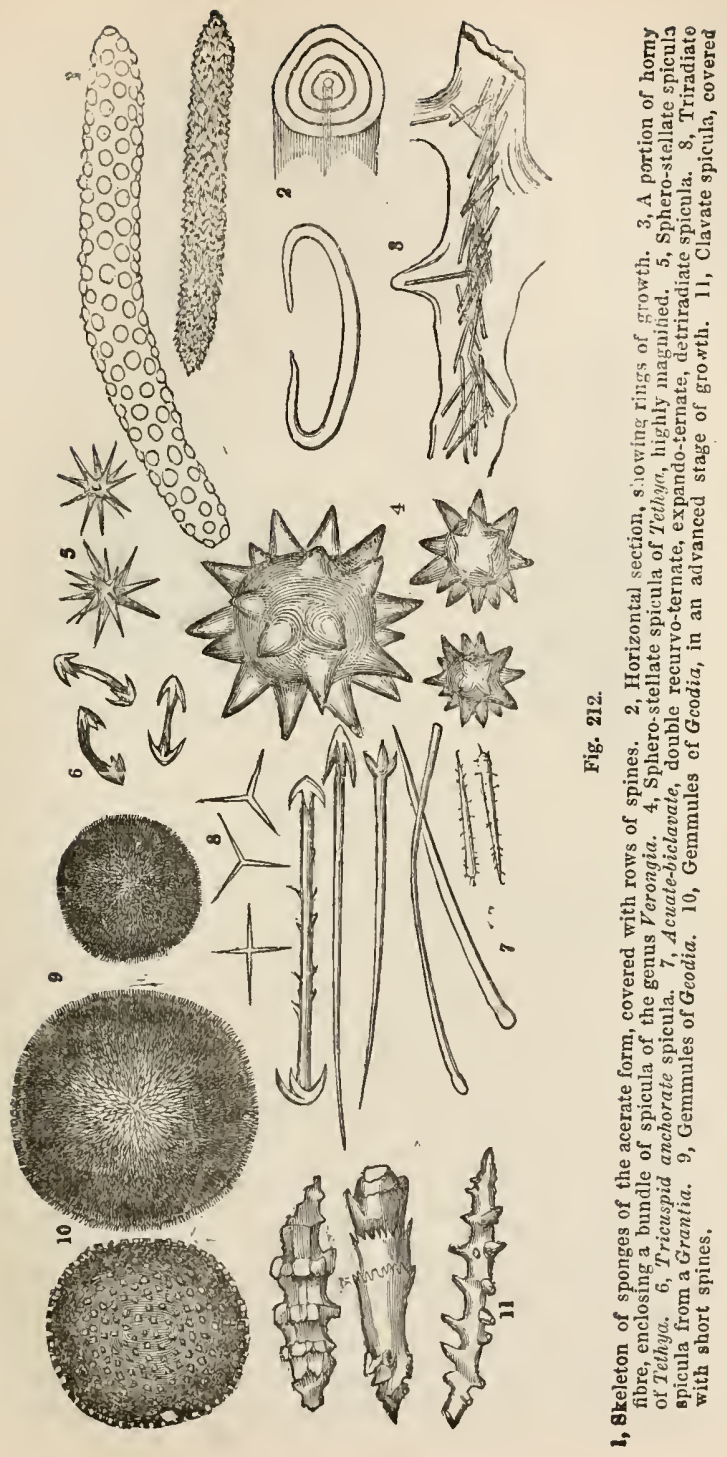


He ascertained that the water was perpetually sucked into the substance of the sponge, through the minnte pores that cover its surface, and again expelled through the larger orifices. His own account is so very interesting, that we cannot resist giving, in his own words, the results arrived at in these investigations:- " Having placed a portion of live sponge (Sponyia coalita, fig. 1, No, 213) in a watch-
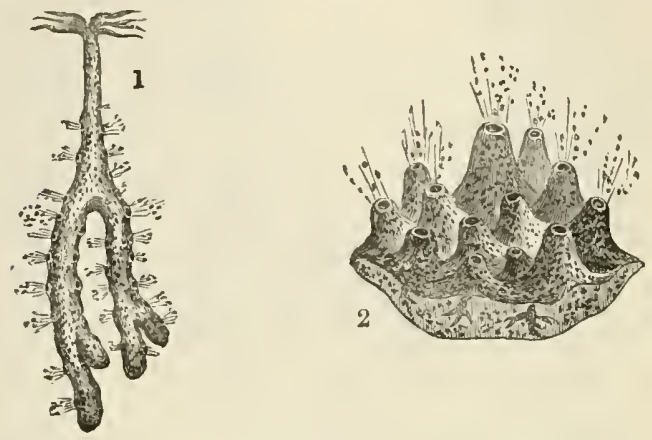

Fig. 213.

1. Spongia coalita. 2, Spongia panicea.

glass with some sea-water, I beheld for the first time the splendid spectacle of this living fountain, better represented in No. 2, vomiting forth from a circular carity an impetuous torrent of liquid matter, and hurling along in rapid succession opaque masses, which it strewed everywhere around. The beauty and norelty of such a scene in the animal kingdom long arrested my attention; but after twenty-five minutes of constant observation, I was cbliged to withdraw my eye from fatigue, without having seen the torrent for one instant change its direction, or diminish the rapidity of its course. In observing another species (Spongia panivea), I placed two entire portions of this together in a glass of sea-water, with their orificcs opposite to each other at the distance of two inches; they appeared to the naked eye like two living batteries, and soon covered each other with the materials they ejected. I placed one of them in a shallow vessel, and just covered 
its surface and highest orifice with water. On strewing some powdered chalk on the surface of the water, the currents were visible to a great distance; and on placing some pieces of cork or of dry paper over the apertures, I could perceive them moving, by the force of the currents, at the distance of ten feet from the table on which the specimen rested."

Dr. N. Lieberkiihn, in his valuable contributions to the History of the Development of the Spongillae, observes that with regard to the skeleton of $S$. fluviatilis, the spicules are not united at the base by a siliceous material, as stated by Meyen, but by a substance destructible by heat. The spicules are usually arranged in aggregate bundles, which meet point to point at an obtuse angle, and project slightly above the surface of the sponge. Minute portions of the gelatinous substance exhibit under the microscope amoeba-like movements, respecting which it is unknown whether they are vital phenomena, as supposed by Dujardin, or referable to a process of decomposition. ${ }^{1}$

The living spongillce are often seated, not immediately npon the wood, stone, \&c. upon which they may be growing, but separated from it by a pseuliar dark-brown substance often several inches thick. This mass is composed chiefly of the remains of the dead sponge, empty gemmulc-cases with their amphidises, various forms of siliceous spicules, \&c.; and occasionally there may be

(1) The motile phenomena hitherto observed in sponges are connected with larger or smaller portions of the external integument, and of the exbalent tubules, or with isolaterl cells. When the exhalent tubules of Spongilla contract, their walls become shortened and thickened, and the previously smooth surface uneven, from the presence of the spherical contracted cells, whose outlines at the same time are rendered very distinct, whilst they were before invisible, or at most here and there perceptible. Other motile phenomena are witnessed when a Spongilla with external membrane and exhalent canals is produced from a cut-off portion. The fragment thus cut off may be so thin as to consist of only a single layer of retieular parenchymatous fibres. The interstitial rounded, oval, or irregular spaces, under these circumstances, become for the most part elosed, owing to the gradual increase in breadth of the trapeculæ, or civities may be left when their membranes are streteher over them only from the upper and under sides of the trabeculæ, which enclose a space between them, and may become portions of the outer membrane with exhalent canals. It cannot be determined with certainty to what extent this change of form is connected with any multiplication of cells. Lastly, movernents in the individual cells have been noticed, the globular cells becoming stellate, and the stellate ones globular in turn, but withont any locomotion. This phenomenon occurs, not ouly in the cells of the unilijured substance, bnt also in those which have been detached. - N. Leiberliulin, "On the Motile Phenomena in Sponges," Álcros. Journ. vol. iv. 1864, p. 189, and Journ. Micros. Science, vol. v. 1857, p. 2:2, "On the Development of the Spongills." 
found in it gemn.ules still retaining their brown colsus and contents capable of development.

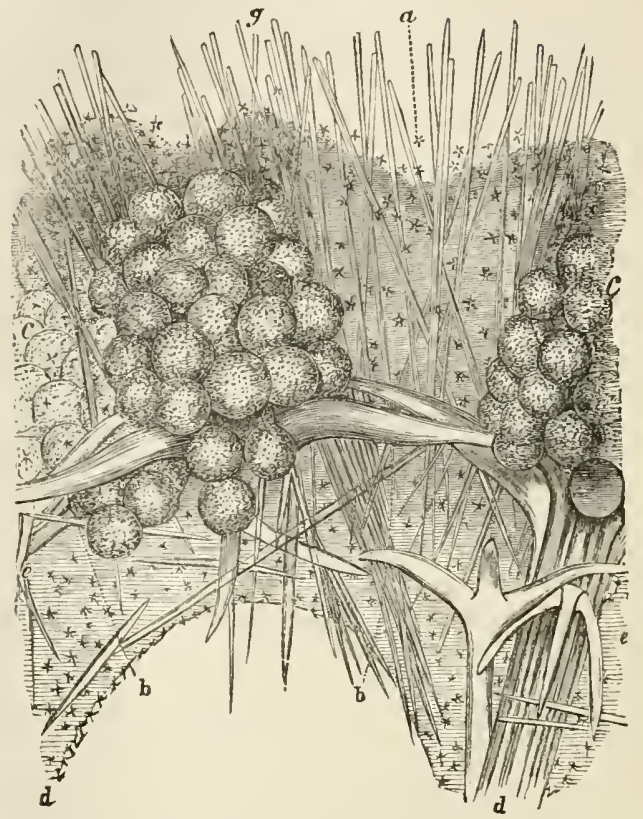

Fig. 214.-Geodia Barretti (Bowerhunk'.

A section at right angles to the surface, exhibiting the radial disposition of the fasciculi of the skeleton, and a portion of the dermal crust of the sponge, inagnified 50 diameters. $a$, intermarginal cavities; $b$, the basal diaphragms of the intermarginal cavities ; $c$, imbedded ovaria forming the dermal erust of the sponge ; $d$, the large patentoternate spicula, the heads of which form the areas, for the valvular bases of the intermarginal cavities; $e$, recurvo-ternate defensive and aggressive spicula within the summits of the intercellulsr spaces of the sponge; $f$, portions of the interstitial membranes of the sponge, crowded with minute stellate spicula: $g$, portions of the secondary system of external defensive spicula.(1)

The usual contents of the gemmules have been described by Meyen (Muller's Archiv. 1839, p. 83). In many in-

(1) See Bowerbank's Monograph of the British Spongiada, Ray Soc. p. 16 . 
stances Lieborkihn found that the globular arrangement no longer existed, the globules being replaced by granules exhibiting an active molecular motion. That the gemmules are formed from agglomerations of sponge-cells may be readily proved in the branched sponge containing smooth gemmules. Lieberkihn notices four kinds of gemmules characterised respectively by their cases or shells.

1. Those with smooth cases.

2. Those with stellate amphidiscs.

3. Those with amphidiscs, in which the discoid ex. tremities are entire, and not stellate.

4. Gemmules whose case, instead of amphidiscs, is furnished with minute, usually slightly curved siliceous spicules.

It would appear, therefore, that the "globules" of Meyen are nothing more than altered sponge-cells. The autumn is the most favourable season for observing the process of their formation.

In the journal of the Bombay branch of the Royal Asiatic Society for 1849, Surgeon H. J. Carter gives a very accurate account of fresh-water sponges found in the water tanks of Bombay. Of five species that he discovered, one was the Spongilla friabilis, the others he named Sp. cinerea, Sp. alba, Sp. Meyeni, Sp. plumosa.

Spongilla cinerea is stated to present on its surface a dark rusty, copper colour, lighter towards the interior, and purplish under water. It throws up no processes, but extends horizontally in circular patches, over surfaces two or three feet in circumference, or accumulates on small objects; and is seldom more than half an inch in thickness. It is found on the sides of fresh-water tanks, on rocks, stones, or gravel. The ova are spheraidal, about 1-63d of an inch in diameter, presenting rough points externally. Spicula of two kinds, large and small; large spicula, slightly eurved, smooth, pointed at both ends, about 1-67th of an inch in length; small spicula, slightly curved, thickly spiniferous, about 1-350th of an inch in length.

Spongilla friabilis.-Growing in circumscribed masses, on fixed bodies, or enveloping floating objects; seldom 
attaining more than two inches in thickness. From the other sponges it is distinguished by the smooth spicula which surround its seed-like bodies, and the matted structure.

Spongilla alba.-Its texture is coarse and open; structure reticulated. The investing membrane abounds in minute spicula; has seed-like spheroidal bodies about 1-30th of an inch in diameter, with rough points externally. The large spicula are slightly curved, smooth, pointed at each end, about 1-54th of an inch in length; the small spicula are slightly curved, thickly spiniferous, or pointed at both ends; the former, pertaining to the seed-like bodies, are about 1-200th of an inch in length; the latter, pertaining to the investing membrane, are more slender, and a little less in length; these last numerous small spiniferous spicula when dry present a white-lace appearance, from which $\mathrm{Mr}$. Carter gives them the name of alba.

Spongilla meyeni is massive, having large lobes, mammillary eminences, or pyramidal, compressed, obtuse, or sharp-pointed projections, of an inch or more in height; also low wavy ridges. Its seed-like bodies are spheroidal, about 1-47th of an inch in diameter, studded with little toothed discs.

Mr. Carter enters very minutely into the structure of "fresl-water sponge, which "-he believes--" is composed of a fleshy mass, supported on a fibrous, reticulated, horny skeleton. The fleshy mass containing a great number of seed-like bodies in all stages of development, and the horny skeleton permeated thronghout with siliceous spicula. When the fleshy mass is examined by the aid of the microscope, it is found to be composed of a number of cells, imbedded in and held together by an intercellular substance.

"In the development of the sponge-cell of Spongilla, a set of large granules make their appearance at a very early period, and increase in number and size until they form a remarkable feature. At this time they are about 1-10,000th of an inch in diameter, of an elliptical shape, and of a light amber colour by transmitted light; they are the colour bearing granules or cells, and give the 
colour of chlorophyll to this organism when it becomes green. The trausparent intercellular substance of Spongilla has a polymorphism equally great with the fully developed cells. This, however, can only be satisfactorily seen when the new sponge is growing ont from the seedlike body, at which time it spreads itself over the glass in a transparent film, charged with contracting vesicles of different sizes, and in various degrees of dilatation and contraction. How this substance is produced so early, it is difficult to conceive, since it seems to come into existence independently of the development of the sponge-ovules, which are seen imbedded in it, and there undergoing their transformation into sponge-cells. The spicula, too, are developed syuchronously with the advancing transparent border, from little glairy globules about the size of the largest ovules, which send ont a linear process on each side, and thus gradually grow into their ultimate forms. The only way of accounting for the early appearance of this intercellular-substance is to consider that it is a development from some remuants of the original protoplasm; and perhaps possesses also the power of producing new sponge-cells, as we see the protoplasm in Vorticella and the roots of Chara prodncing new buds, independently of the cell-nucleus.

"The cells of the investing membrane are characterised by their uniformly granular composition and colourless appearance. They are nucleated, possess the contracting vesicle singly or in plurality, and are spread over the membrave in such numbers, that it seems to be almost entirely composed of them; while they are of such extreme thinness, and drawn out into such long digitated forms, that they present a foliated arrangement, not unlike a compressed layer of multifidous leaves, ever moving and changing their shapes. The apertures are circular or elliptical holes in the investing membrane in the cells. Throngh these apertures the particles of food are admitted into the cavity of the investing membrane. The Parenchyma consists of a mass of gelatinous substance, in which are embedded the smooth spicules and ovi-bearing cells, and through which pass the afferent and efferent oanals. The ovi-bearing cells do not inurst and allow their con- 
tents to become indiscriminately scattered throngh tho gelatinous mass in which they are imbedded, but each becomes developed separately in the following way :-the ovules and granules of the ovi-bearing cells subside into a granular mass by the former losing their defined shape and passing into small mono-ciliated and unciliated sponge cells; this mass then becomes spread over the interior surface of the ovi-bearing cell, leaving a cavity in the centre, into which the cilia of the monociliated spongecells dip and keep up an undulating motion; meanwhile, an aperture becomes developed in one part of the cell which communicates with the adjoining afferent canal, and thus the ovi-bearing cell passes into an ampullaceous spherical sac. The cilia may now be seen undulating in the interior ; and if the Spongilla be fed with carmine, this colouring matter will not only be observed to be entirely confined to the ampullaceous sacs, but wheu the Spongilla is torn to pieces and placed under a microscope, particles of the carmine will be found in the interior of the monociliated and unciliated sponge cells, proving that of such cells the ampullaceous sac is partly composed. This sac then must be regarded as the animal of Spongilla, as much as the Polype-cell is regarded as the animal of the Polype, and the whole mass of Spongilla as analogous to a Polypidom.

"The united efforts of all the ciliaterl sponge-cells in the ampullaceous sac are quite sufficient to produce a considerable current, and thus catch the particles of food as they pass through the afferent canals. Thus we find Spongilla composed of a number of stomachal sacs imbedded in a gelatinous substance permeated with spicules for its support, and an apparatus for bringing them food, as well as one for conveying away the refuse, while the nourishment abstracted by the process of digestion common to Rhizopodous cells (e.g. A moba), no doubt passes through the intercellular gelatinous substance into the general development of the mass; and if right in comparing the ampullaceous sacs to the stomachal cavities of the simplest polypes, are we not further justified in drawing a resemblance between the ciliated sponge-cells and those which line the stomach of Cordylophora, of Otostoma, and many 
of Ehrenberg's Allotreta, together with those in the stomach of the Rotifera and Planaria?

“The 'swarm-spore,' described by M. N. Lieberkiihn, appears to be a ciliated form of the seed-like body, and the same as the 'gemmule' described by Grant; but this I have not yet been able to see. The formation of the seed-like body, however, now that we know the structure of the ampullaceous sacs, seems very intelligible, for we have only to conceive an enlargement of the small sponge-cells lining the interior, with the addition of ovules to them respectively, and the spieule-bearing sponge-cells of the cortical substance supplying the spicular erust to the exterior, to have a globular capsule thus composed, with a hilum precisely like the seed-like body - a conjecture which seems to derive support from the fact, that in some iustances, when Spongilla is begin. ning to experience the want of nourishment, these sacs, small as they are, assume a defined, rigid, spherieal form, from their pellicle becoming hardened and encrusted with extremely minute spicules." 1

Clionce.-Not the least wonderful circumstance connected with the history of sponges, is the power possessed by certair species of boring into substances, the hardness of which might be considered as a sufficient protection against such apparently contemptible foes. Shells (both living and dead), coral, and even solid rocks, are attacked by these humble destroyers, gradually broken up, and, no doubt, finally reduced to such a state as to render substances which would otherwise remain dead and useless in the economy of nature available for the supply of the necessities of other living creatures.

These boring sponges constitute the genus Cliona of Dr. Grant. They are branched in their form, or consist of lobes united by delicate stems; they all bury themselves in shells or other ealcareous objects, preserving their communication with the water by means of perforations in the outer wall of the shell. The mechanism by which a creature of so low a type of organisation contrives to produce such remarkable effects is still doubtful, from the great difficulties which lie in the way of eoming to any satis-

(1) Ann. of Nat. II ist., July, 1857 . 
factory conclusions upon the habits of an animal that works so completely in the dark as the Cliona celata-it will probably long remain so. Mr. Hancock, to whom we are indebted for a valuable memoir upon the boring sponges, published in the Annals and Magazine of Natural History, attributes their excavating power to the presence of a multitude of minute siliceous crystalline particles adhering to the surface of the sponge; these he supposes to be set in motion by some means analogous to ciliary action. In whatever way this action may be produced, however, there can be no doubt that these sponges are constantly and silently effecting the disintegration of submarine calcareous bodies - the shelly coverings, it may be, of animals far higher in organisation than they; nay, in many instances they prove themselves formidahle enemies even to living mollusca, by boring completely through the shell. In this case the animal whose domicile is so unceremoniously invaded, has no alternative but to raise a wall of new shelly matter between himself and his unwelcome guest; and in this manner generally succeeds at last in barring him out.

Skieletons of Sponges. - The skeletons of sponges, which give shape and substance to the mass of sarcode that constitutes the living animal, is best made out by cutting thin slices of sponge submitted to firm compression, and viewing these slices mounted upon a dark ground, or backed up with black paper.

The skeletons of sponges are composed principally of two materials, the one animal, the other mineral ; the first of a fibrous horny nature, the second either siliceous or ealcareous. The fibrous portion consist of a network of smooth, and more or less cylindrical, threads of a lightyellow colour, and, with few exceptions, always solid; they frequently anastamose, and vary considerably in size; when developed to a great extent, needle-shrped siliceous bodies termed spicula (little spines) are formed in their interior; in a few cases only one of these spicula is met with, but most commonly they occur in bundles. In some sponges, as those belonging to the genus IIalichondria, the same horny kind of matcrial is present in greater or less abundance; but its fibrous structure has become obscure: 
the fibres, however, in these cases are represented ky siliceous neelle-shaped spicula, and the horny matter serves the important othine of binding them firmly together, as

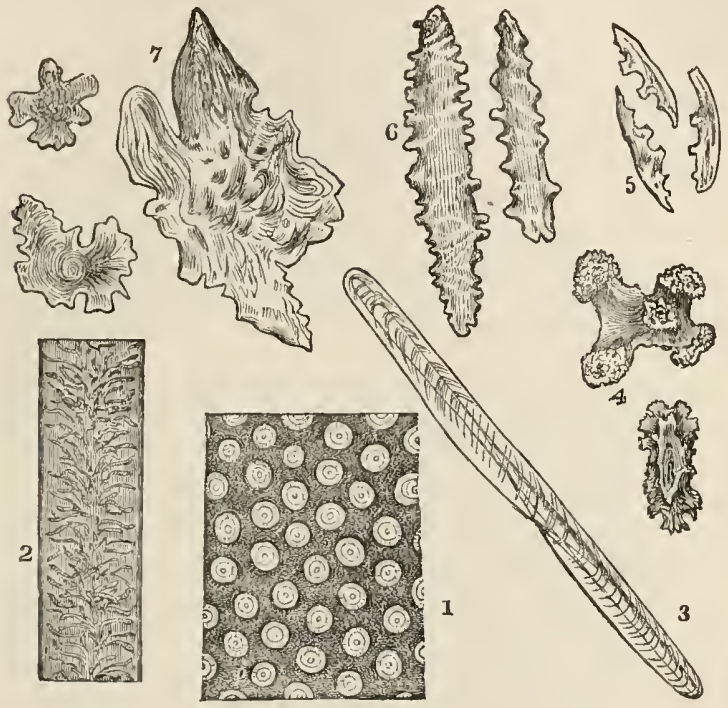

Fig. 215.

1. Transverse section of a branch of Myriapore. 2, A section of the stem of Virgularia mirabilis. 3, A spiculum from the outer surface of a Sea-pen. 4, Spienla from crust of Isis hippuris. 5, Spicula from Gorgona elongatu. 6, Spicula from Alcyonium. 7, Spicula from Gorgonix umbraculum.

shown in fig. 213, No. 1. There are, however, some remarkable exceptions to this rule, one, Dictyochalix pumiceus, described by Mr. S. Stutchbury, in which the fibrous skeleton is composed of threads of silex quite as transparent as glass ; another, the Hyalonema, Glass-rope.

The mineral portion, as before stated, consists of spicula composed either of silica or carbonate of lime; the first kind is the most common and likewise most variable in shape, and presents every gradation in form, from the acuate or needle-shaped to that of a star. The calcareous spicula, on the contrary, are more simple in their form, 
being principally acicular, but not unfrequently branched or even tri- or quad-radiate: the two kinds, the siliceous and calcareous, according to Dr. Johnston, not having hitherto been detected co-existent in any native sponges.

The spicula exhibit a more or less distinct trace of a central cavity or canal, the extremities of which are closed, or hermetically sealed; in their natural situation they are invested by an animal membrane, sarcode, which is not confined to their exterual surface; but in many of the large kinds, as pointed out by Mr. Bowerbank, its presence may be detected in their central cavity, by exposing them for a short time to a red heat, when the animal matter will become carbonised, and appear as a black line in their interior.

Many authors have described the spicula as being crystalline, and of an angular figure, and have considered them analogous to the raplides in plants; but it requires no great magnifying power to prove that they are always round, and, according to their size, are made up of one or more concentric layers, as shown in fig. 212 , No. 2. 'The epicula occupy certain definite situations in sponges; some are peculiar to the crust, others to the sarcode, others to the margins of the large canals, others to the fibrous network of the skeleton, and others belong exclusively to the gemmules. Thus, for instance, in Pachymatisma Johnstonia, according to $\mathrm{Mr}$. Bowerbank, the spicules of the crust are simple, minute, and fusiform, having their surfaces irregularly tuberculated, and their terminations very obtuse; whilst those of the sarcode are of a stellate form, the rays varying in number from three to ten or twelve.

Silica, however, may be found in one or more species of sponge of the genus Dysidea, not only in the form of spicula, but as grains of sand of irregular shape and size, evidently of extraneous origin, but so firmly surrounded by horny matter as to form, with a few short and slightlycurved spicula, the fibrous skeleton of the animal. In these sponges the spicula are of large size, and are disposed in lines parallel with the masses of sand.

Most of the sponges of the earlier geological periods had 
tubular fibres; but in all existing species, with one or two exceptions, they are solid. These tubular fibres are very commonly filled with portions of iron, which accounts for the colour of many of the remains in flint.

The Moss-agates, found among the pebbles at Brighton and elsewhere, are flints containing the fossilised remains of sponges. The coloured fibres seen in the Green-jaspers of the East are of the same character. There is reason to believe that most flints were originally sponges; those from chalk even retain their original form. Recent sponges from the Sussex coast present forms precisely similar to some chalk flints, but it is from sections made sufficiently thin to be transparent, for examination under the microscope, that we learn their true nature and origin.

Every horny sponge, whilst living, is invested with a coating of jelly-like substance, which can only be preserved by placing the sponge in spirit and water immediately after its removal from its place of growth. Spicula are not exclusively confined to the body of sponges, but occasionally form the skeleton of the gemmules, and are situated either on the external or internal surface of these bodies. A good example of the former kind occurs in the common fresh-water sponge (Spongilla fluviatilis), represented in fig. 216 , No. 1, and No. 3 . The spicula are very minute in size, and are disposed in lines radiating from the centre to the circumference, the markings on the outer surface of the gemmules being the ends of spicula. In all the young gemmules the spicula project from the outer margin as so many spines; but in process of growth the spines become more and more blunt, until at last they appear as so many angular tubercles. Turkey sponge (Spongia officinalis) is brought from the Mediterranean, has a horny network skeleton rather fine in the fibres, solid, small in size, and light in colour. In some larger specimens there is a single large fibre, or a bundle of smaller ones. In Halichondria simulans the skeleton is a framework of siliceous needle-shaped spicula, arranged in bundles kept together by a thick coat of horny matter. Other species of Malichondria have siliceous spicula wointed at both extremities-acrate (fig. 212, No 2); 
while the spicula of some are round at one end, and pointed at the other-acuate; some have spicula round at one end, the former being dilated into a knob-spinulate.
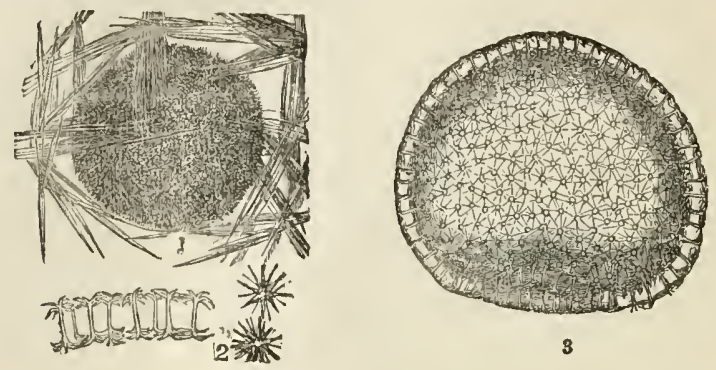

Fig. 216.

1. Gemmule of Spongilla fluviatilis, enclosed in spicula. 2, Birotulate spicula, from Fluviatilis. 3, Gemmules of Spongilla fluviatilis, after having been immersed in acid, to show coating of birotulate spicula.

Among the genus Grantia, Geodia, and Levant sponge, are found spicula of a large size, radiating in three directions-triradiate. In the Levant specimen, a central communicating cavity can be distinctly seen. Some Smyrna sponges, and species of Geodia, have four raysquadriradiate. Some spicula in P. Jolnstonia and Geodia have as many as ten rays-multiradiate. In some species of Tethya and Geodia the spicula consist of a central spherical body, from which short conical spines proceedstellate spicula. (Fig. 212, Nos. 4 and 5.) Spicula having both extremities bent alike-bicurvate-have been obtained from Trieste sponge. Some South Sea sponges have spicula twice bent, and have extremities like the flukes of an anchor-bicurvate anchorate; sometimes the flukes have three pointed ends. (Fig. 212, No. 6.) The gemunules in fresh-water sponges are generally found in the oldest portions near the base, and each one is protected by a framework of bundles of acerate spicula of the flesh, as shown in fig. 212 , No. 9 ; but in many marine species, Geodia and Pachymatisma, they are principally confined to the crust. In the fresh-water sponges, the amount of nnimal matter in the gemmules is considerable; but in 
Pachymatisma, Geodia, and many other marine species, a very small quantity only is ever to be found, the substance of each gemmule being almost entirely composed of minute siliceous spicula; if they be viewed when taken fresh from the sponge, and also after removing the animal matter by boiling in acid, a slight increase in transparency is the only perceptible difference of appearance noticed.

Hyalonema, "Glass-Rope" Sponge.-A bundle of from 200 to 300 threads of transparent silica, glistening with a satiny lustre like the most brilliant spun glass; each thread is about eighteen inches long, in the middle the thickness of a knitting needle, and gradually tapering towards either end to a fine point; the whole bundle coiled like a strand of rope into a lengthened spiral, the threads of the middle and lower portions remaining compactly coiled by a permanent twist of the individual threads; the upper portions of the coil frayed out, so that the glassy threads stand separate from each other. The spicules on the outside of the coil stretch its entire length, each taking about two and a half turns of the spiral. One of these long needles is about one-third of a line in diameter in the centre, gradually tapering towards either end. The spirally twisted portion of the needle occupies rather more than the middle half of its entire length. In the lower portion of the coil, which is embedded in the sponge, the spicule becomes straight, and tapers down to an extreme tenuity, ultimately becoming so fine that it is scarcely possible to trace it to its termination.

"Many spicules of the awl-shaped and simple cross types, especially short spicules, are met with within the siliceous coil to its very centre, and, in cases where the coil has been brought home without the sponge, such needles can be shaken out from the interstices of the threads. The spicules of Hyalonema are marked in their character, and all the forms are found in all specimens of the sponge imbedding the characteristic bundle of enormous spicules; so that there can be no reasonable doubt of the specific identity of the sponge in all cases.

"Within the round apertures on the surface of tho 
sponge there is usually a brownish orange-coloured mem. brane, which Schultze found presented the marked characters of a minute parasitic polyp, probably alcyonarian, which inhabited the oscula and their passages during the life of the sponge.

"The glassy wisp of Hyalonema is certainly very remarkable, but it is not entirely without analogy. Hyalonema seems to represent the extreme form of a little group of sponges, including, with probably a few other forms, Euplectella (Alcyonellum) speciosa (Qnoi and Gaimard), and $\boldsymbol{E}$. cucumer (Owen). The last-named is an oval sponge with siliceous spicules, in form and character somewhat like the spicules of Ifyalonema. From one end of the sponge a tuft of long siliceous threads, resembling in structure those of the Japan sponge, twine round a stone or other foreign body. Dr. Bowerbank isolated one of the spines of Euplectella, three inches long." See Intellectual Observer, March, 1867.

\section{INFUSORIA.}

The term Infusoria is applied to a certain class of animals because they were first discovered in water whero vegetable matter was decomposing, the infusion was considered necessary for their production. Now, however, it is an established fact, that they are in a healthier state of existence when taken from pure streams and clear ponds than from putrid and stagnant waters. A little bundle of hay, or sage leaves, left for about ten days in a mug containing some pure rain-water, caught before entering a butt, produces the common wheel-animalcules, which are found adhering to the sicies of the mug near to the surface of the water. The only use of the vegetable matter seems to be to facilitate in some way the development of the ova of animalcules which find their way into the water. It was at one time thought an indispensable condition that air be admitted to the infusion : but even this element is not absolutely needed in the case of infusorial life; and the appearance of living organisms at all under the circumstances, has been regarded as important evidence in favour of the doctrine of spontaneous generation. 
The astronomer turns his telescope from the carth, and ranges over the rast vault of heaven, to detcct and delineate the beantiful objects of his pursuit. The naturalist turns his microscope to the earth, and in a drop of water finds a wondrous world of animated beings, more numerous than the stars of the milky way; and these he classifies into genera and families, and catalogues in his history of the invisible world.

The Infusoria are a mighty family, as they frequently, in countless myriads, cover leagues of the ocean, and give to it a beautiful tinge from their vivid hue. They are discovered in all climes, have been found alive sixty feet below the surface of the earth, and in the mud brought up from a depth of sixteen hundred feet of the ocean. They exist at the poles and the equator, in the fluids of the animal body, and plants, and in the most powerful acids. A brotherhood will be found in a little transparent shell, to which a drop of water is a world; and within these are sometimes other communities, performing all the functions granted them by their Creator, and eagerly pursuing the chase of others less than themselves.

The forms of the Infusoria are endless; some changing their shape at pleasure, others resembling globes, eels, trumpets, serpents, boats, stars, pitchers, wheels, flasks, cups, funnels, fans, and fruits.

The multiplication of the species is effected in some by spontaneous division or fissuration, in others by gemmation or budding, as well as a true sexual process. The first step in the process by which infusorial animals are eliminated, is the formation of globular corpuscles or cells, which, by their aggregation in some cases, and individual evolutions in others, give birth to organisms which subsequently appear.

The Infusoria have wo night in their existence; they issue into life in a state of activity, and continue the duration of their being in one ceaseless state of motion; their term is short, they have no time for rest, and therefore have but one day, which ends only with their death and decomposition. Nevertheless, they appear to love that which promotes life, - the light of heaven; but others, born in the bowels of the earth, and whn never 
partook of the blessing, like the ignorant among mankind, have their own contracted round of unenlightened joys, perform their mechanical duties, and expire hidden and unknown.

On examining the structure of infusorial animalcules, some are found to have a soft yielding skin, so elastic as to stretch when food or other circumstances render it necessary, returning again to its previous condition as the cause of distension ceases; these are designated illoricated, which signifies shell-less. Others are termed loricated, from being covered with a shell, which is beautifully transparent, and flexible like horm. When the delicate and soft substance in which the functions of life perform their allotted duties perishes, the coat that protected it from injury during its hours of existence remains as a token of the past labours of nature; this covering consists mostly of siliceous material or of lime united with oxide of iron, destructible in some instances either by chemical agents or by fire.

Some of these minute beings have apportioned to them setce, or bristles; these stiff hairs, attached to the surface of their bodies, do not rotate, but are movable, and appear to be a means for the support of their bodies, as aids in climbing over obstacles that present themselves, or as feelers. Others are possessed of unci, or hooks, projecting from the under part of the body, which are capable of motion; and by their means the animalcule can attach itself to anything that lies in its way. Some, again, have styles, which are a kind of thick bristle, jointed at the base, possessing a movement, but not rotary ; they are in the shape of a cone, large at their base, and delicate at their summit. Many, also, can extend and withdraw their bodies at pleasure, in a similar manner to the snail or leech.

One of the most interesting and important organs possessed by infusorial animalcules is scientifically known by the term cilium, which is the Latin word for eyelash, the plural being cilia. Its appearance is that of a minute delicate hair.

The cilium is not only useful in the act of progression, but also as an assistance in procuring food; the two duties 
being performed at the same time, the motion of the organs that propels it forward causing a current to set towards the mouth, which carries with it the prey on which the animal feeds. From the cilia being found in the cills of the young tadpole, the oyster, and mussel, it would appear that they are serviceable as organs of respiration, by imbibing oxygen, and emitting the carbonic acid generated in the blood duriug its circulation through the body; they are also believed to be the medium of taste and touch. It is not only at the mouth, but over the whole body that cilia are discovered; and it is now satisfactorily shown that cilia exist also in the internal organs of man and other vertebrated animals; aud are agents by which many of the most important functions of the animal economy are performed. They vary in size from the 1000 th to the 10,000 th of an inch in length. These minute organs would often be invisible, were it not from the water being coloured when placed under a microscope ; then the little currents made by the action of the cilia are easily perceived; and when the water is evaporated, the delicate tracing of their formation may be observed on the glass. They are differently placed, and vary in quantity in the numerous species of Infusoria. In some they are in rows the whole length of the body, in others on the base; many have them over the whole of the body; sometimes they fringe the mouth, form bands around projections on the body; and many have but two projecting from the mouth, as long as the body of the creature. Ehremberg says they are fixed at their base by the bulb moving in a socket, in a similar manner to a man's outstretched arm; and by their moving round in a circle, they form a cone, of which the apex is the bulb. Poison, galvanism applied to the animal, even death, will not immediately stop the motion of the cilia; they continue moving some hours afterwards, even longer than nervous or muscular action can be sustained, until the fluids dry up, and they stiffen.

Very little is known of the muscular attachment of cilia in Infusoria; but the motive power must be derived from muscular structure in all. Now in the wheel-animalcules the cilia are in circular rows; and each revolves around 
its bulb, giving a singular appearance, seeming to move together like a wheel upon its axle, whence their name Rotifer; in a few of these muscles can be traced. The cilia must not be mistaken by the young microscopist for the stiff hairs and bristles found on some, and serving, as before stated, for the purpose of locomotion in crawling or climbing.

If the roof of the mouth of a living frog be scraped with the end of a scalpel, and the detached mucous membrane placed on a glass slide, and examined with a power of 300 diameters, the eiliated epithelium-cells will be well seen. When a number of these are collected together, the movement is effected with apparent regularity; but in detached scales it is often so violent, that the scale itself is whirled about in a similar manner to an animalcule provided with a locomotive apparatus of the same description, and has frequently been mistaken for such. The animals commonly employed for the examination of the cilia are the oyster and the mussel; but the latter are generally preferred.

To exhibit the movement to the best advantage, the following method must be adopted:-open carefully the shells of one of those molluses, spilling as little as possible of the contained fluid; then with a pair of fine scissors remove a portion of one of the gills (branchia); lay this on a slide, or the tablet of an animalcule cage, and add to it a drop or two of the fluid from the shell; by means of the needle-points separate the filaments one from the other, cover it lightly with a thin piece of glass, and it is ready for examination. The cilia may then be seen in several rows beating and lashing the water, and producing an infinity of currents in it. If fresh water instead of that from the shell be added, the movement will speedily stop; hence the necessity of the caution of preserving the liquid contained in the shell. To observe the action of any one of the cilia, and its form and structure, some hours should be allowed to elapse after the preparation of the filaments as above given, their movements then will have become sluggish. If a power of 400 diameters be used, and that part of the cilia attached to the epithelium scale marefully watched, each one will be found to revolve $s$ 
quarter of a circle, whereby a "feathering movement" is effected, and a current in one direction constantly produced. In the higher animals, the action of the cilia can only be observed a very short time after death. In a polypus of the nose, when situated at the upper and back part of the Schneiderian membrane, the cilia may be beautifully seen in rapid action some few hours after its removal ; but in the respiratory and other tracts, where ciliated epithelium is found, it would be almost impossible ever to see it in action, unless the body were opened immediately after death. In some animals it may be seen in the interior of the kidney, as first made known by Professor Bowman in the expanding extremity of the small tube surrounding the network of blood-vessels forming the socalled Malpighian body. In order to exhibit the ciliary action, the kidney should have a very thin slice cut from it; and this is to be moistened with the serum of the blood of the same animal. The vascular and secreting portions of the organ may then be seen with a power of 250 diameters, and also the cilia in the expanded extremity of each tube, as it passes over to surround the vessels; the epithelium of the tubes themselves is of the spheroidal or glandular character.

The infusorial and invisible atoms of life have various periods allotted to them for the enjoyments of existence ; some accomplish their destiny in a few hours, others in a few weeks. The watchful devotee in this branch of science has traced an animalcule through a course of existence extending to the old age of twenty-three days. The vital spark flies instantaneously in general; but in those of a higher organisution there is a spasmodic convulsion, as if the delicate and intricate machinery rendered life $s n$ axraisite, that the parting with the "heavenly flame" was reluctant and painful. The most surprising circumstance attendant on the nature of some of the Infusoria is that of apparent death. When the water or mud in which they have sported in the fulness of buoyant health becomes dried up, they lie an inanimate speck of matter; but after months, nay, years, a drop of water being applied, their bodies will be resuscitated, and in a short time their frames become active with life. Leeuwenhoek kept some in a hard and dry condition, 
and restored them to life after a sleep of niore than twenty-one months. Professor Owen saw an animalcule that had been entombed in a grave of dry sand four years reborn to all the activity of life. Spallanzani tried the experiment of alternate life and death, and accomplished it in some instances on the same olject fifteen times, after which nature was exhausted, and refused further aid in this miraculous care of those minute objects of her wonderful works.

Naturalists consider the phosphoric light of the marise animalcule to be the effect of vital action. The sparks are intermittent like the fire-fly; they measure from the 12,000 th to the 100 th of an iuch in size. Captain Scoresby found that the broad expanse of waters at Greeuland was nearly all discoloured by animalcules, and computed that of some species one hundred and fifty millions would find ample room in a tumbler of water. The phosphorescence of the sea is eloquently portrayed by Darwin, in his Voyage of the Beagle. Mr. Gosse thus describes the luminous appearances presented by a closer inspection of these minute animalcules: "Some weeks afterwards I had an opportunity of becoming acquainted with the minute animals, to which a great portion of the luminousness of the sea is attributed. One of my large glass rases of seawater I had observed to become suddenly at night, when tapped with the finger, studded with minute but brilliant sparks at various points on the surface of the water. I set the jar in the window, and was not long in discovering, without the aid of a lens, a goodly number of the tiny jelly-like globules of Noctiluca miliaris swimming about in various directions. They swam with an eren gliding motion, much resembling that of the Volvox globator of our fresh-water pools. They congregated in little groups, and a shake of the ressel sent them darting down from the surface. It was not easy to keep them in view when seen, owing rather to their extreme delicacy and colourless trausparency than to their minuteness. They were, in fact, distinctly appreciable by the naked eye, measuring from 1-50th to 1-30th of an inch in diameter." Noctiluca miliaris belongs to the highest class of the Protozoa, and with a power of about 200 diameters they are seen 
of various forms and stages of growth, represented in fig. $217 . .^{1}$

Ehrenberg included in his family of Infusoria, Rhizopoda, Unicellular, and other Algæ, embryonic forms, and Rotifers. Most naturalists, however, now admit that the

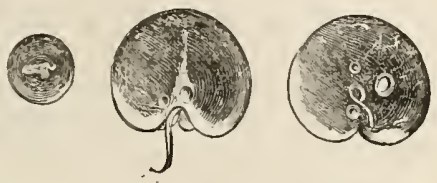

Fig. 217.-Noctiluca miliaris.

organization of the Rotiferæ is of a far higher nature than had been suspected by Ehrenberg; and some assert that their proper place in the classification of animals is the annulose sub-kingdom ; the true nature of many of the Infusoria is still a disputed question. In outward form they may be said to vary almost indefinitely ; but anatomicaliy their bodies should be regarded as consisting of three distinct structures. The cuticle or integument (" pellicula " of Carter), on which are borne the cilia and other locomotive apparatus ; the cortical layer or parenchyma of the body ("diaphane" of Carter); and the chyme mass, occupying the abdominal cavity, or interior of the body (sarcode or "abdominal mucus" of Carter), within which the particles of food rotate. The term "ventral" is usually applied to that side of the body on which the mouth is placed.

In the well-known Paramoecium we have the true and most widely distributed type of the Infusoria. The general structural character of this minute animal is common to the species; indeed, its structural features may be accepted as a fair definition of the whole group. The Paramacium is surrounded on its external covering by cilia, wbich are constantly in action, and enable it to move about in its watery element in a most remarkably active manner. At one point the body appears to be slightly

(1) See Gosse's Naturalist's Rambles: Huxley, Micro. Juurnal, 1855 
constricted, and here is a slit seen which opens into a little funnel-shaped cavity, leading to the gullet and stomach. The outer or cortical layer is composed of a denser material, which indicates a differentiation into cellular layers, while the internal substance is evidently composed of sarcode which exhibits, at two points in particular, the power of contracting and dilating: a process available both for the expulsion of digested food, and for aëration of the circulatory system. The whole systemic arrangement should be regarded as the very simplest form of respiratory and secretory mechanism. The several circular transparent bodies seen in the interior of these animals led Ehrenberg to denominate the group "Polygastrica" (many stomached). The remarkable powers of multiplication by fission and germination, as well as by a true sexual process, which these creatures exhibit, have attracterl the attention of all observers; within the last few years, Miuller, Balbiani, Stein, and others, have shown that the sexual organs of such animals as Paramcecium are those bodies which have hitherto been simply regarded as the "nucleus," and "nucleolus;" and ultimately it was seen that the Infusoria have a life history as wonderful as that of the higher classes of animals. Although Ehrenberg was the first to call attention to the importance of the "nucleus" in the reproductive process, it is to the observations of Balbiani that we are indebted for an explanation of its importance in the generative function: his investigations also derive additional interest from the very complete manner in which they have been carried out. As an instance, he states that in his examinations of Paramcecium aurelice, he could not look upon them as conclusive until he had succeeded in extracting uninjured some of the eggs from the parent body, and had subjected them to the action of the surrounding water, when he saw each egg resolve itself into two portions, the smaller being enclosed within the larger; then by employing re-agents, acetic acid and iodine, he produced the changes more rapidly ; and in this way again and again obtained abundant proofs of the truth of each observation. So much then for Dr. Balbiani's researches on the phenomena of reproduction among the Infusoria, which have added much valuabie 
information to our former meagre knowledge of these in. teresting forms of organic life.

Some Infusoria undergo a process of encystation before reproduction by fissure; that is, they become coated with a secretion of gelatinous matter, which gradually hardens so as to enclose the body in a "cyst." According to Stein, the process of encystation is sometimes followed by a remarkable succession of phenomena, such as have been observed to occur in the case of Vorticella microstoma. An old Vorticella loses or retracts its cilia, becomes encysted, and finally drops off its stalk. The cyst may either burst and discharge its contents, or become wholly changed into an Acineta-form body. The latter may subsequently develop a foot-stalk, assume the appearance of a Podophyra, or even that of the Acineta tuberosa, Plate III. No. 68. In either case, the band-like nucleus becomes transformed into a peculiar ovate body, somewhat like Nos. 71 or 73, the narrow end of which is provided with a circlet of vibratile cilia, and a mouth leading into an internal cavity, with a contractile vesicle in its interior. Relations, somewhat similar to those which connect Vorticella and Acineta, have been stated to exist between other families, as Aspidisca or Trichoda, and Oxytricha, Plate III. No. 71.

MoNadide.-Monads. These are amongst the smallest atoms of matter possessing the mysterious principle of life, discernible by the highest magnifying power of the microscope. Minute, however, as they are, no one can say that they do not derive their sustenance by preying on animals even less than themselves, as larger ones of the same

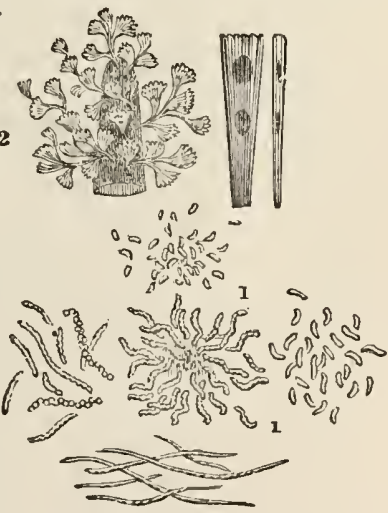

Fig. 218.

1, Vibrio spirilla. 2, Licmophora flabellata; near which an enlarged view of one is shown. species do upon them. 
Monads vary in their colours, some are red, green, or yellow, others nearly colourless. In shape they are round or oval (5 and 6, fig. 226), and possessed of immense astivity, having one or more cilia devoted to the purpose of locomotion. Monads have been claimed by the botanist, and accordingly placed among the Volvocinea, confervoid Alga. Ehrenberg described them as Infusoria. He says : "All true Infusoria-even the smallest monads, are organised animal bodies."

Vibrio.-Vibriones.-In this family Ehrenberg wrongly includes the well-known eels in paste and vinegar.

Vibrio spirilla, Trembling animalcules, when motionless are seen as very minute bodies; but when exerting the powers of locomotion they take a spiral form, like the threads of a fine screw, and by undulations wind themselves through the water with rapidity. Euch apparent hair is a collection of animals bound together by a pliant band; thus, as they are individually so small, little is known of their structure. Still they form very interesting objects to view ; their very minuteness claiming attention, while their activity and motions excite surprise. The species are numerous, as represented in fig. 218. They are almost invariably the first organisms found in decaying acetous and putrefying organic matters. When treated with iodine and then sulpluric acid, their jointed structure is rendered visible to the higest powers of the microscope, but not otherwise.

Astasian.-Astasia, signifying without a station, in contradistinction to those living in groups, is the term given to a kind of crimson-coloured animalcule, the 350 th of an inch in length, that exist in enormous numbers, and give the waters in which they live the appearance of their bodies. Ehrenberg describes several varieties of them.

The family Euglence of Dujardin in many particulars corresponds with that of the $\Lambda$ stasio of Ehrenberg; while Mr. Carter would refer Euglence to the vegetable and Astasice to the animal kingdom. "The power, however, to vary the figure can be no adequate charucter, for this is partaken by the gonidia of various algæ; the tapcring, hairlike prolongation, the existence of one, two, or more ciliary filaments, and the red speck, are also the well- 
known characteristics of some zoospores." The individuals are all free, and furnished with a hairlike appendage ; ova are perceptible in Astasia hamatodes, and probably exist in other species. From their varying colour, their apparent changes of form, and the rapidity of their motions, they are most interesting objects under the microscope. The immense number in which these Infusoria are sornetimes developed in a few days, and the blood-red colour they impart, have frequently been the cause of alarm and anxiety to persons residing in the vicinity of ponds which have become coloured by their swarming. Ehrenberg describes a species of Euglena, $E$. sanguinea, and he conjectures that the miracle in Egypt, recorded by Moses, of turning the water into blood, might have been effected by the agency of these creatures. Very lately, Mr. Sheppard ${ }^{1}$ met with another speeimen, probably belonging to this family, adhering to the submerged stones in a elear spring, between Ashford and Maidstone. His specimens were taken home in a piece of glazed paper, and upon opening them he found the paper "stained with lines of red, blue, and purple ;" and the whole "resembling elots of red jelly, or recently coagulated blood." Upon placing a small quantity on a glass side for viewing under the Microscope, "the colour appeared to be opaquered, looking like a small quantity of vermilion mixed with the water; but when held up to the light the red disappeared, and a pale transparent blue tcok its place."

Believing this colour to depend upon the presence of albumen mixed with the animal organisms, Mr. Sheppard placed a small quantity of the jelly-like substance in contaet with some white of egg diluted with water ; and "soon the whole became converted into magenta dye," the solution exhibiting the same colouring properties, namely, that of reflecting from its surface all the red and yellow rays, and transmitting the blue and violet." Mr. Browning, upon submitting speeimens to the micro-spectroscope, found that it gave a very marked band in the red-ray. The whole spectrum is, indeed, very remarkable, and, writes

(1) " $\Delta$ n example of the production of a coloured fluid possessing remarkable qualities by the action of monads (or some other microscopic organism) upon organized substances." By J. B. Sheppard, M.R.C.S.-Trans. Micros. Soc. Juy, 1867, p. 64 . 
Mr. Sorby, "it is the only blue solution in class C (of which blocd is the type) that gives this particular spectrum." . The fluid emits a most pungent and disagreeable odour when the bottle in which it is kept is uncorked.

The common form of the Euglence is represented in Plate III. No. 67, a contracted, $b$ elongated. Oxytricha are larger and the body more elongater ; their movements are more impulsive, alteruately creeping, running, and climbing. In all the species digestive vacuoles are evident; and they multiply by self-division, as well as ova. Ehrenberg counted ten cilia anteriorly, and four or five setæ posteriorly. The species is found both in fresh and brackish water. Plate III. No. 70 , represents a side view of O. gibba, No. 71, O. Pellionella. In the genus Glaucoma, Nos. 73 and 74, Ehrenberg saw "indications of an alimentary canal." Dujardin places Glausoma among Paramrecia; the body is oval and covered with cilia; mouth large, with vibratory valves; increase takes place by self-division. A re-examination of all the enumerated species of Infusoria is quite necessary before we can come to any safe conclusion as to their true affinities, especiaily as many appear to be ouly larval forms of life.

"The question of how far individuals belonging to the same species may vary is one more intimately connected with that department of Zoology which treats of the distribution of animals than their development. For it can be readily shown that animals are capable of becoming modified to an indefinite extent by the physical conditions under which they are placed, and, indeed, that one species may be, so to speak, made to pass into that of another; so that many of the apparently dissimilar animal forms found on the earth may be more correctly viewed as varietias of the same species, the differences between them being due to the external agencies to which each has respectively been subjected."

The remarkable manner in which the Infusoria make their appearance in fluids, and the seeming inexplicable phases in their existence, led some early observers to start a "spontaneous generation" theory of life; but the researches of M. Pasteur and others have completely exploded this viow of the formation of living organisms. The order 
in which these minute creatures appear in vegetable infusions has been made the subject of carcful inquiry. Mr. Samuelson, whose researches on this point were carried on in conjunction with Dr. Balbiani of Paris, and confirmed by him, tells us that when a carefully prepared infusion of vegetable matter in distilled water is exposed to the air, the Protozoa which first appear in it are Amobce: these in a few days disappear, and are succeded by ciliated infusoria, such as Kolpoda, Cyclidium glaucoma, and sometimes Vorticella, and these in their turn by what we have looked upon as higher forms, Oxytrichum, Euplotes, lerona, \&c. Mr. Samuelson thinks that Monads are but the larval condition of the ciliated infusoria, and he noticed the constant occurrence of Monads belonging to the species Circomonas fusiformis, or acuminata of Dujardin, \&c., in pure distilled water after a certain exposure to the air, and this without the previous admixture of vegetable matter of any kind in the water. The same results were obtained upon shaking rags, from various and distant parts of the world, over the distilled water; in all cases in about three weeks he invariably obtained forms of ciliated infusoria. "The fusiform body of the Circomonas bears a long whip-like cilium. t its anterior end, and a short seta at its caudal extremity : chis finally drops off, and when exposed to excessive heat and light, the animal is transformed into an Amcba."

Mr. Samuelson's results do not very materially differ from our own, save in one or two particulars. The succession of generations do not take quite the same course, and the animal and vegetable bodies generally appear simultaneously, or so soon after each other that it is at times difficult to decide the priority of appearance; but our experiments have been chiefly confined to collections of rain and distilled water, voithout the addition of vegetable matter of any kind. We are, however, quite agreed as to the very extensive distribution of these infusorial germs, and their great tenacity of life. With regard to the supposed purity of rain-water, at no time can it be taken without the numerous matters floating in the air being brought down with it; and, consequently, within a few hours after it is caught, Protococcus pluvialis, Amoba, and Circomoncs may always be found in vast numbers. It is somewhat 
remarkable that the purest snow-water, caught in a clear glass vessel, and allowed to remain well corked, will, in the course of two or three weeks, be found to contain $A m a b a$ and Circomonas, but it rarely presents other forms of animal life; the vegetable matter then completes its growth very slowly, gradually passes to Confervæ, and for a time no other change is seen to take place; so that it is painfully apparent that the atmosphere in which we live and move and have our being is something more than a mixture of gases, as apparently determined by chemical analysis.

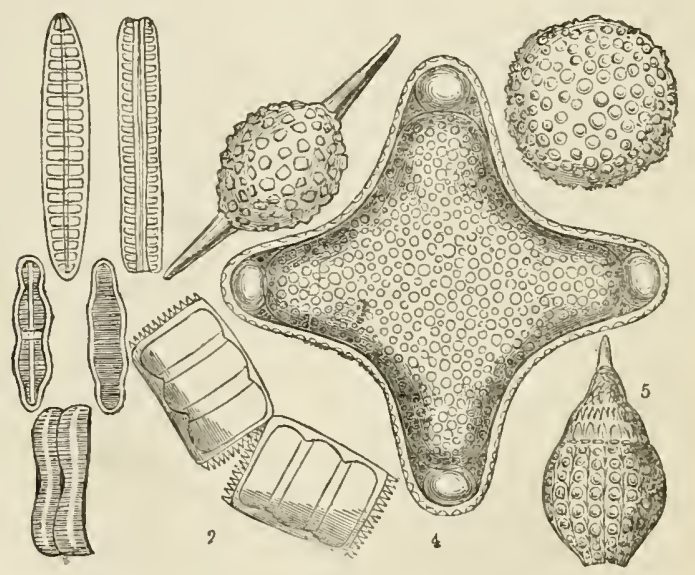

Fig. 219.

1, Achnanthidium coarotatum. 2, A. lineare. 3, Tryblionella gracillis. 4, Amphitetras antediluviana. 5,6 , and 7 , Orthosira spinosa. Front view, with globular and oval forms. (Fossil lnfusoria from Springfield, Barbadoes.)

Ehrenberg's "Polygastric Infusoria" have indeed undergone a complete revision : some have been degraded to the vegetable kingdom, as the Desmidiacece, Volvocinece, \&c., whilst others have been advaneed a step higher in the animal series; none having reeeived so much attention from microscopists, or excited so much controversy, as the Desmidiacece and Diatomacea. The first of these we have already disposed of in our remarks on the vergetable kingdon, where we must be coritent to leave then for the present: 
rot so the Diatomaceo, which offer many interesting stuructural characteristics of sufficient importance to warrant our keeping them in the animal division. They are most striking objects under the microscope, from the very peculiar beanty and variety of their forms, and from their bilateral symmetry, external markings, and indestructible siliceous skeletons; so that we believe they wonld be more correctly placed in a median, or Molluscian sub-kingdom. Appearing everywhere with the first-born of life, and wherever matter is found in a condition fit for their development and nourishment, these marvellous indestructible creatures have been preserved and brought down to us, in forms unchanged, from the remotest periods of our globe's history; and supplying, as they do to the microscopist, some of the most valuable test-objects, - the Gyrosigma. Grammatophora, Fragilaria, Ripidophora, Pleurosigma angulatum, with many others, -it cannot be a matter of surprise that considerable attention should have been directed to them, and an earnest inquiry instituted into their nature and structure.

"Comparing," says Kützing, "the arguments which seem to indicate the vegetable nature of Diatomacece with those which favour their animal nature, we are of necessity led to the latter opinion. If we suppose them to be plants, we must admit every frustule, every Navicula to be a cell. We must suppose this cell with walls penetrated by siliea, developed within another cell of a different nature, at least in every case where there is a distinct peduncle, or investing tube. In this siliceous wall we must recognise a complication certainly unequalled in the vegetable kingdom. It would still remain to be proved that the eminently nitrogenous internal substance corresponded with the generic substance, and that the oil globules could take the place of starch. The multiplication would be a simple cellular reduplication; but it would remain to be proved that it takes place, as in other vegetable cells, either by the formation of two distinct primitive utricles, or by the introflection or constriction of the wall itself. Finally, there would still remain unexplained the external motions and the internal clianges; and we nust prove the accumulated observations on the 
exterior organs of motion to be false, by a clearer line of argument than has hitherto been adopted by those who aro opposed to this view. But again, admitting their animal nature, much would remain to be investigated, both in their organic structure and their vital functions; excepting this, so far as we know, we have only one difficulty to overcome, that of the probably ternary nonazotiscd composition of the external gelatinous substance of the peduncles and investing tubes. But as the presence of nitrogen is not a positive character of animal nature,

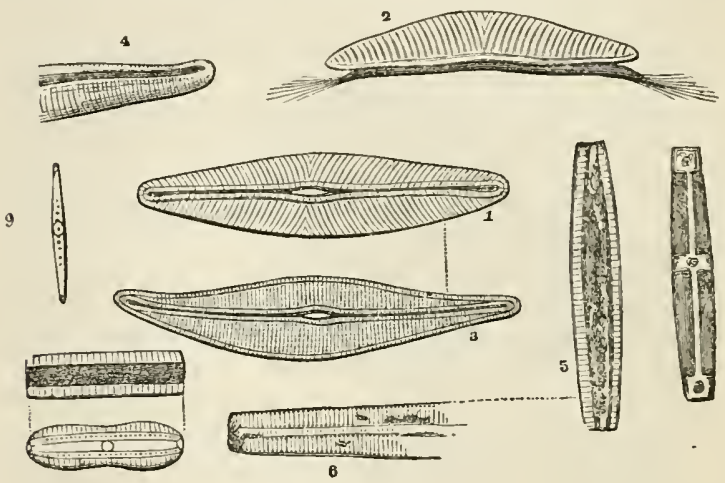

Fig. 220.

1, Cymbella Ehrenbergii. 2, Side view of the same, showing an arrangement of the sarcode and pseudopodia. 3, Pleurosigmata lanceolatum. 4, Lateral view of a portion of the same. 5, 6, and 7, Pinnularia. 8, Diatoma vilgare. 9 , Nitzschia varvula.

so the absence of it is not a proof of vegetable. And, in order that the objection should really have some weight, it would be well to demonstrate that this substance is isomeric with starch. For then, supposing all the arguments in favour of the animal nature of Diatomacca were proved by new and more circumstantial observations, this peculiarity, if it deserve the name of objection, might still be regarded as an important discovery. We should then have in the animal, as well as in the vegetable kingdom, a ternary substance similar to that forming the bases of the vegetable tissue." 
Diatomacea, brittleworts, siliceous Bacillaria, are organisms composed of two symmetrical plates or valves, narrow or wand-like, navicular-as a miniature boat or "littlo ship;" hence their name, Navicula. A rectangular or prismatic figure is, however, the typical form of this family, and the angles of junction of the valves are as a rule, acute. Deeply notched frustules, such as we see in the Desmids, Micrasterias denticulata, Docidium pristida, Plate II. Nos. 30 and 31 , do not occur, and the production of spines and tubercles is rare among the Diatoms. Each individual Diatom is enclosed by a soft organic matter (sarcode); the internal portion is yellowish or orange-brown in colour. In the discoid forms two portions are commonly distinguishable, viz. the disc and margin-or rim, and these present different markings, with an occasional central prominence, called an umbo or boss. Great variety of outline may prevail in a genus, so much so, that no accurate definition can be safely laid down: thus in the genera Navicula, Pinnularia, the frustules are in one aspect boat-shaped, and in another oblong with truncated ends, prismatic. Mr. Brightwell thus describes and explains the transitions of form produced by a change in position of the frustules of the genus Triceratium. ${ }^{1}$ "The normal view of the frustule may be represented by a vertical section of a triangular prism. If the frustule be placed upon one of its flat sides, we look down upon its ridge and obtain a front view of its two other sloping sides. If it be placed upon one of its ridges, we have a front view of one of its flat sides, generally broader than long, and of its smooth or transparent suture or connecting membrane. If the frustule be progressing

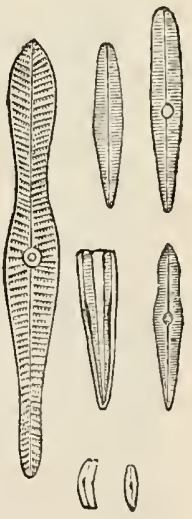

Fig. 221.-Gompho nema elongatum and capitatum. towards self-division, it is then often considerably longer than broad, and when nearly matured for separation, presents the appearance of a double frustule." So with re- 
gard to the beautiful Surirella constricta, the side view is so longer bacillar, but the breadth of the valve is very considerable, and when about to undergo subdivision it becomes square-shaped. The distinctive character, bowever, of this genus, in addition to the presence of canaliculi, is derived from the longitudinal line down the centre of each valve, and the prolongation of the margins into "alæ." The sudden cliange in appearance presented to the eye as the frustule is seen to roll over, is very remark: able. As a rule, therefore, we must examine all specimens in every aspect, to accomplish which very shallow cells should be selected, say of 1-100th of an inch deep, and covered with glass 1-250th of an inch thick. A good penetrating objective must be used, and careful illumination obtained. The examination of living specimens should be conducted during very bright weather, the mirror directed towards a white cloud, or even sumlight: with coloured glasses to protect the eyes from injury. The Diatomaceæ are perhaps more widely distributed than any other class of infusorial life; they inhabit fresh, salt, and brackish water; many grow attached to other bodies by a stalk (Plate II. No. 33), Licmophora and Achnanthes; while others, as the Pleurosigma, No. 40, swim about perfectly free in the water.

There are a considerable number of Diatomaceæ which, while in the young state, are enclosed in a muco-gelatinous sheath; while others are attached by a stipes or stalk to Algæ. Ehrenberg recognised a tribe of compound Diatoms, with a double lorica, and introduced them into his great family of Bacillaria, under the name of Lacernata or Navicula. Silex enters largely into the composition of their valves, but, being in combination with organic substances, it does not depolarize light. In several genera silex is very deficient, and the wall of the frustule of great delicacy. Mr. Brightwell, speaking of the lorica or siliceous covering of the Triceratium, states "that the valves are resolvable into several distinct layers of silex, dividing like thin divisions of talc, and frequently of such exquisite delicacy as to be difficult of detection." Nägeli speaks of a mucilaginous pellicle on the inside of ths organic layer as a sort of third tunic; and, as Meneghini 
truly observes, "An organic membrane ought to exist, for the silica could not become solid except by crystallizing or lepositing itself on some pre-existing substance." The surface of the frustules is generally very beautifully sculptured, and the markings assume the appearance of dots (puncta), stripes (striæ), ribs (costæ), pinnules (pinna), of furrows and fiue lines; longitudinal, transverse, and radiating bands; canals or canaliculi; and of cells or areolæ; whilst all present striking varieties and modifications in their form, character, and degree of development. Again, the fine lines or striæ of many frustules are resolvable into rows of minute dots, such as occur in Pleurosigma, \&c.

The nature of the markings on the Diatom valves is one of considerable interest, and has excited much attention, and attempts have been made to produce them artificially. On the addition of sulphuric acid to a mixture of powdered fluor spar and sand, an immediate evolution of fluoride of silicon takes place, as is shown by the white fumes. This whiteness is due to the presence of minute particles of silex arising out of the decomposition of the fluoride by the moisture contained in the atmosphere ; and it a solid body be ex-

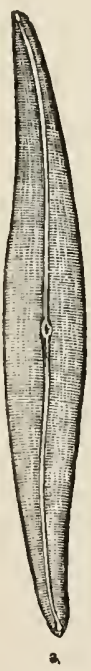

Fig. 222.
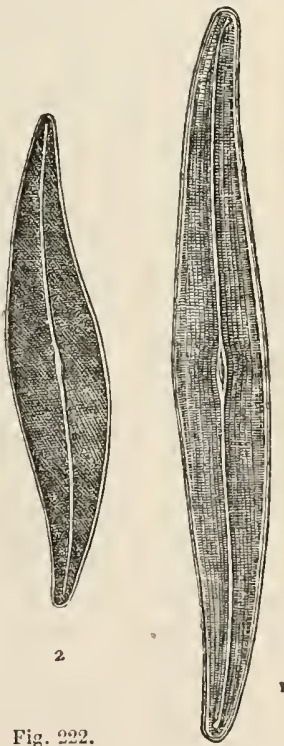

1, Pleurosigma attenuatum. 2, Pleurosigma an gulatum, magnified 250 diameters. 3, Pleuro. sigma Spencerii, magnified 350 diameters. posed to these vapours, a portion of the silex will be deposited on it, in the form of a fine white powder, con- 
sisting of thin walled vesicles filled with air. If some of the deposit be crushed between two pieces of glass, and examined with a power of about 300 diameters, a marking will be perceived on the outer or convex surface of many of these vesicles, similar to that of many Diatomacex, such as Pleurosigma, Coscinodiscus, \&c. Rounded elevations, more or less hexagonal at the base, and more or less regularly arranged, cover the surface of the siliceous pellicle, and not unfrequently this kind of marking is so regular as to give the fragments exactly the appearance of portions of diatomaceous valves.

This remarkable circumstance attracted the attention of Professor Max Schultze, who devoted a great deal of time to the investigation of the subject, and has recorded in a voluminous paper ${ }^{1}$ the results of his observations. $\mathrm{He}$ says, "The appearances presented under the microscope by the siliceons pellicles were such as to suggest that they were due possibly to crystallisation. The minute elevations on the surface, when viewed on the side, often appear sharply acuminate, so as readily to convey the impression that they are formed by minute crystals of silex; and this impression is strengthened at first sight by their sharply defined hexagonal basis, when viewed vertically. The circumstance, again, that these elevations are sometimes rounded at the summit and circular at the base, might be attributed to the accidental interference of free hydrofluosilicic acid, \&c. But experiments to eleminate the action of this agent showed that it had nothing to do with the variety of appearance in the elevations.

"NIost of the species of the diatomacex are characterised by the presence on their outer surface of certain differences of relief, referable either to elevations or to depressions disposed in rows. The opinions of microscopists with respect to the nature of this marking are divided. Whilst in the larger forms, and those distinguished by their coarser dots, the appearance is manifestly due to the existence of thinner spots in the valve, we can not so easily explain the cause of the striation or punctation in Pleurosigma angulatum and similar finely-marked forms. In these it

(1) "Verhandl. d. Natur Hist. Vereins der Preussisch. Rheinland, u. Test phal." Jahr xx. p. 1. Micros. Jour. Science, vol. iit. p. 120. 
often seems as if the appearance were due to pyramidal elevations, with hexagonal bases, standing in regular rows, exactly like those observed in the siliceous vesicles above mentioned.

"In any case, it seemed likely, since the marking just noticed is essentially alike in many different species of diatoms, that its ultimate causes were to be sought, less, perhaps, in any organic formative process, than in the deposition of silex under the same laws as those by which its deposition in the other case is regulated. Were this ultimate cause shown to be crystallisation the question would be solved. But that the secretion of amorphons silex in diatoms, as in these pellicles, is undeniable, on account of their specific gravity being about $2 \cdot 2$, the specific gravity of crystallised silex being $2 \cdot 6$."

But further investigation rendered the correctness of this assumption (that the markings are due to crystallisation) doubtful in the highest degree, and it soon became quite certain that neither in the artificial siliceous pellicles nor in the diatom valves are the peculiar forms essentially due to a crystalline structure.

Mr. Charles Stodder, of Boston, says, "I have reasons for thinking that neither party has the true explanation of the structure. $\mathrm{My}$ opinion is that the exterior of the shell is smooth, or nearly so, and that the borders of the hexagons, or other shaped areolæ, and costæ of the Costate forms, are internal projections from the outer plate, as on the under side of the leaf of the Victoria Regia, intended to give strength to the cell with the smallest quantity of material. This will explain the trace of the hexagons seen on the inner plate of Heliopelta, as only the projecting wall of the areolæ would come in contact with the inner plate. Dr. Griffiths reasoned that the areolæ were depressions, because they were the thinnest parts of the shell; the facts are correct, but the inference may not be, as there is another explanation of the phenomena." See directions for the illumination of test-objects, page 174.

Movements of Diatoms. - The researches of Professor Max Schultze, of Bonn, published 1865, appear to throw some light on the vexed question of the movements of the 
Diatomacece. This author is of opinion that a sarcode substance envelopes the external surface of the diatom, and its movement is due to this agent exclusively. $\mathrm{He}$ prefers the $P$. angulatum, Plate 11 . No. 38, for examination to the larger $P$. balticum, because the transverse markings on its frustule do not impede to so great an extent the observation of what is going on within. When you have a living specimen of $P$. angulatum under the microscope, it always has its broad side turned to view, with one long curved "raphe" uppermost, and the other in contact with the glass on which it is placed; at the central part is seen the thickened "umbilicus," Plate 2, No. 40. Within the siliceous frustule is the yellow colouring matter, or "endochrome," which fills the cavity more or less completely, and is arranged in two longitudinal masses, to the right and left of the raphe. In the broader part of the frustule these bands of endochrome describe one or two complicated windings. It is only possible in those specimens in which the bands are narrow properly to trace their foldings, and ascertain that only two exist, siuce an examination of frustules richer in endochrome has led to the impression that there are tliree or four of these bands. "The next objects which strike the eye on examining a living Pleurusigma are highly refractive oil-globules. These are four in number; one pair near either end of the the Diatom. They are not, however, all in the same place, one globule of each pair being nearer the observer than the other; their relative position is best seen when a view of the narrow side of the frustule ean be obtained, so that one raphe is to the left and the other to the right. The blue-black colour, which is assumed by these globules after the Diatom has been treated with hyperosmic acid, demonstrates that they consist of oleaginous matter. The niiddle of the cavity of the frustule is occupied by a colourless finely granular mass, whose position in the body is not so clearly seen in the flat view as in the sicle riew. Besides the central mass, the conical carities at either end of the siliceous shell are seen to be filled with a simllar granular substance, and two linear extensions from each of the three masses are developed, elosely underlying that part of the shell which is beneath the raphæ; so that 
in the side view they appear attached to the right and left edges of the interior of the frustule. This colourless granular substance carries in its centre, near the middle part of the Diatom, an imperfectly developed nucleus which it is not very easy to see, but may be easily demonstrated by the application of acid. The colourless substance is what, in other Diatoms, Schultze shows to be Protoplasm, or vegetable sarcode, and which contains numerous small refractive particles. On adding a drop of hyperosmic acid, these are coloured blue-black, and prove to be fat. It is, however, exceedingly difficult to determine the exact limitations of the protoplasm, on account of the highly refractive character of the siliceous shell, and the obstruetion presented by the bands of endochrome.

After a short distance, the protoplasm reappears, and is contracted into a considerable mass within the conical terminations of the frustule. Schultze observed in this part of the protoplasm a rapid molecular movement such as is known to occur in the Closterium, and further, a current of the granules of the protoplasm along the raphe. Pleurosigma angulatum "crawls," as do all Diatoms possessing a raphe, along this line of suture. To erawl along, it must have a hixed support. He believes free swimming movements are never to be observed in this or in any other Diatom. Accordingly, Schultze invariably found that the raphe is in contact with either the glass slip or the glass cover, between which the Diatom is placed, or is in apposition with some foreign body of considerable size. Schultze repeated the experiments of Siebold, and observed, as he and also IVenham had done long before, that particles of foreign matter stick to the raphe as though it were covered with some glutinous material, and are carried slowly along by the action of a current. This he observed in many Diatomacex, and found invariably that foreign particles adhered only to the raphe, or what corresponded to it. "There is obviously," says Schultze, "but one explanation; it is clear that there must be a band of protoplasm lying along the raphe, which causes the particles of colouring matter to adhere, and bives rise to a gliding movement For there is but one phe- 
nomenon which can be compared with the gliding motion of foreign bodies on the Diatomacex, and that is, the taking up and casting off of particles by the pseudopodia of the rhizopoda, as observed, for instance, on placing a living Gromia or Miliolina in still water along with powdered carmine. The nature of the adhesion and of the motion is in both cases the same in all respects. And since, with Diatoms as unicellular organisms, protoplasm forms the principal part of the cell body (in many cases two distinctly moving protoplasms), everything suggests that the external movements are referable to the movements of this protoplasm." It is quite evident to those who have studied the movements of the Diatoms that they are surrounded by a sarcode structure far more delicate in its character than that of the Amoba. Six years before Schultze's observations were published, the fact appeared in a third edition of this work, page 307 ; thereiu it is stated that "The act of progression rather favours the notion of contractile tentacular filaments,-pseudopodia, - as the organs of locomotion and preheusion. The hyaline or sarcode covering is of so transparant a nature that as yet no microscopic power has enabled us to assign its precise boundary and attachments. ${ }^{1}$ Some observers would deny a membranaceous (hyaline) covering of any kind to Diatoms, which we have certainly seen." Professor Smith, of America, has satisfactorily traced a sarcode covering in Pinnularia. The defining power, penetration of the objective, and mode of illumination, is everything in such investigations.

It was in 1841 Messrs. Harrison and Sollitt, of Hull, discovered the beautiful longitudinal and transverse strice (groovings) on the Pleurosigma hippocampus. A curved graceful line runs done the shell, in the centre of which is an expanded oval opening. Near to the central opening

(1) Referring to the strength and vigour of the movements of the Diatomaceæ, Dr. Donkin (Quart. Jour. Mic. Sci. vol. V1. new series, p. 26), observed a species Bacillaria cursoria, discovered by himself, push away " A. arenaria, a species at least six times their own size;" and Mr. Barkas states that he has seen them "push away particles of foreign matter, and that with the greatest apparent ease, at least one hundred times larger than all the frustules combuned and what is more remarkable still, is that they not only push the accumulated particles away when they are in their direct line of motion, but, if they merely touch them in passing, they drag them after them as though they were literally held by some magnetic attraction, or strong cement." 
the dots elongate crossways, presenting the appearance of small short bands. The Pleurosigma angulatum (fig. 223), was first discovered in the Humber; the lines upon its surface resemble the most elegant tracery, which are resolvable into raised minute dots. The markings are seen to be longitudinal, transverse, and oblique.

In the vicinity of Hull many very interesting varieties of Diatomacece have been found, the beanty of the varied forms of which are such as to delight the microscopist;
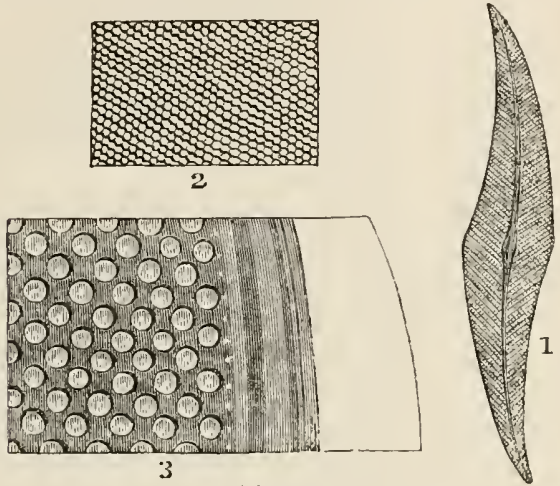

Fig. 223.

1, Plexrosigma angulatum. 2, Portion of the same, magnified 1200 diameters. 3 , Portion of $P$. formosum, magnified 5500 diameters.

at the sanie time some of them are highly useful, as forming that class of test objects which are best calculated above all others for determining the excellence and powers of object-glasses. It has been shown by Mr. Sollitt that the markings on some of the shells are so fine as to range between the 30,000th and 130,000 th of an inch; the Pleurosigma strigilis having the strongest markings, and the Pleurosigma acus the finest.

As to the value of the Diatomacere as test-objects, it is generally admitted, and since Mr. J. D. Sollitt first proposed the use of their shells for this purpose, we append his measurements of the lines :-

Amphipleura Pellucida, or Acus, 130,000 in the inch, cross lines. Sigmoidea, 70,000 in the inch.

Naricula Rhomboides, 111,000 in the inch, cross lines. 
Pleurosigma Fasciola, fine slell, 86,000 in the inch, cross lines. " ," strong shell, 64,000 in the inch, cross lines.

" Strigosum, 72,000 in the inch, diagonai lines.

" Angulatum, 5l,000 in the inch, diagonal lincs.

"Quadratum, 50,000 in the inch, diagonal lines.

" Spencerii, 50,000 in the inch, cross lines.

" Attenuaturn, 42,000 in the inch, cross lines.

" Balticum, 40,000 in the inch, cross lines.

", Formosum, 32,000 in the inch, diagonal lines.

" Strigilis, 30,000 in the inch, cross lines.

\section{Mr. Norman, of Hull, has favoured us with the follow- ing :-}

\section{HINTS FOR COLLECTING DIATOMACEE.}

These minute forms are found in all waters, but the most interesting species are those found in salt water, especially shallow lagoons, salt water marshes, estuaries $0_{\mathrm{c}}^{c}$ rivers, pools left by the tide, \&c.

Their presence in any quantity is always shown by the colour they impart to the aquatic plants and sea-weeds they are found attached to, and if found on the mud, which is very frequently the case, they impart to it also a yellowish brown colour approaching to black brown, if in sreat numbers. This brownish pellicle, if carefully renoved with a spoon (without disturbing the mud) will be found very pure. Capital gatherings of Diatomaceæ might be obtained by carefully scraping the brown coloured layer from mooring posts, and piles of wharfs and jetties.

In elear running ditches the plants and stones have often long streamers of yellowish brown slimy matter attacbed to them, which is generally entirely Diatomaceous.

When found in large quantities on the mud the layer is often covered with bead-like bubbles of oxygen. This often detaches them from the bottom and buoys them to the surface, where they form a dense brown scum, which is blown to leeward in large quantities, and presents the creneral appearance of dark-coloured yeast. In this form it may be collected in abundance, often quite free from particles of sand and other impurities. Good and rare species have been obtained from the stomachs of oysters, scallops, and other shell-fish inhabiting deep water. The sea-cucumbers (Holothuridx) found so frequantly in southern latitudes contain many species. These animals 
might be simply dried and preserved just as found, and the contents of the stomach afterwards obtained by dissection.

Noctilucx, which are the cause of phosphorescence in the sea, are Diatom feeders, and might be caught in largo quantities in a fine gauze towing-net, and preserved. The Asciclians found attached to oyster shells and stones from leep water have yielded excellent gatherings. The Salpæ often noticed in warm latitudes floating on the surface of the sea, and assuming chain and other like forms, should be bottled up for examination. These Salpæ are well known Diatom feeders. Deep-sew soundings ought to bo preserved, especially from great depths, and are often exclusively Viatomaceous. Sea-weed from rocks ought to be preserved, especially the smaller species, and if covered with a brown furriness, so mueh the better. Very rare species have been found in immense quantities in the ARCTIC and ANTARCTIC regions, by melting the "pancake ice," often found discoloured by these minute beings. The sea is often observed to be covered by brownish patches. The discoloured water (or "spawn" as it is called) should be collected, filtered through cotton wool, and the brown residue preserved. When a fine impalpable dust is observed to be falling at sea, it ought to be eolleeted from the folded sails and other places where it lodges. This may yield Diatomacer, which from the method of collecting would be highly interesting to examine. The roots of the various species of Mangrove (Rhizaphora), which form impenetrable barriers along the salt water rivers and estuaries in the tropical parts of Africa, Australia, the Eastern Archipelago, \&c., are found frequently covered with a brown mucous slime very rich in Diatomacex. When the Diatomaceæ are collected from any of the above-mentioned sourees, they may be at once transferred to sinall bottles, or the deposit may be partially dried and wrapped up in pieces of paper or tinfoil. When placed in bottles, a few drops of spirits added will keep them nice and sweet. In all cases it is essential to keep the gatherings separate and distinct, and that the locality whence obtained be written on each package.

The collector will probably find that, notwithstanding 
every care, his specimens are mixed with much foreign matter, in the form of minute particles of mud or sand, which impair their value, and interfere with observation, especially with the higher powers of his instrument. These substances the student may remove in various ways: by repeated washings in pure water, and at the same time, profiting by the various specific gravities of the Diatoms and the intermixed substances, to secure their separation; but, more particularly, by availing himself of the tendency which the Diatomacece generally have to make their way towards the light. This affords an easy mode of separating and procuring them in a tolerably clean state; all that is necessary being to place the gathering which contains them in a shallow vessel, and leave them undisturbed for a sufficient length of time in the sunlight, and then carefully remove them from the surface of the inud or water. The simplest method of preserving the specimens, and the one most generally useful to the scientific observer, is simply to dry them upon small portions of talc, which can at any time be placed under the microscope, and examined without further preparation; and this mode possesses one great advantage, -that is, that the specimens can be submitted without further preparation to a heat sufficient to remove all the cell-contents and softer parts, leaving the siliceous epiderm in a transparent state.

On Cleaning Diatomaceous Deposits. - "The first point to be ascertained is the nature of the material which binds the mass together. In the generality of deposits, this seenis to be aluminous or earthy matter, often mixed with some siliceous material which renders the action of acids of little avail. When the bulk of the deposit is clayey matter, the best plan is to place the lumps broken quite small into a vessel and pour on a few ounces of hot water, rendered thoroughly alkaline with common washing soda. This plan frequently answers, causing the lumps to swell, gradually separating into layers, and finally falling asunder into a pulpy mass. The strong soda ley must now be removed by repeated washing, and afterwards boiling in a flask with pure nitric acid; the whole must afterwards be transferred to a large stoppered vessel and 
violently shaken, in order to break up the minuto frag. ments of dirt, and set free the siliceous Diatoms. After shaking, allow the vessel to stand for half an hour or more according to the size and density of the valves: the Diatoms having subdivided, the dirty water is drawn off by a syphon, and fresh water added, and the shaking repeated. The whole secret depends upon getting rid of the impurities by this violent shaking and washing; when quite free from all impurities the material may be transferred to a test tube, washed in distilled water, and finally mounted." 1

Fossil Infusoria.-Startling and almost incredible as the assertion may appear to some, it is none the less a fact, established beyond all question by the aid of the microscope, that some of our most gigantic mountain-ranges, such as the mighty Andes, towering into space 25,250 feet above the level of the sea, their base occupying so vast an area of land; as also our massive limestone rocks, the sand that covers our boundless deserts, and the soil of many of our wide-extended plains; are principally composed of portions of invisible animalcules. And, as Dr. Buckland truly observes: "The remains of such minute animals have added much more to the mass of materials which compose the exterior crust of the globe than the bones of elephants, hippopotami, and whales."

The stratum of slate, fourteen feet thick, found at Bilin, in Austria, was the first that was discovered to consist almost entirely of minute flinty shells. A cubic inch does not weigh quite half an ounce; and in this bulk it is estimated there are not less than forty thousand millions of individual organic remains! This slate, as well as the Tripoli, found in Africa, is ground to a powder, and sold for polishing. The similarity of the formation of each is proved by the microscope; and their properties being the same, in commerce they both pass under the name of Tripoli : one merchant alone in Berlin disposes annually of many hundred tons weight. The thickness of a single shell is about the sixth of a human hair, and its weight the hun-

(1) G. Norman, Esq. Micros. Journ. vol. iv. p. 238. For other methods of cleaning and preparing Diatoms, see Smith's Symopsis of the British Diatomacece; also Quar. Journ. Micros. Science, vol. vii. p. $16 T$, and vol. i. N. S. 1861. p. 143. 
dred-and-eighty-seven-millionth part of a grain. The well-known Turkey stone, so much used for the purpose of sharpening razors and tools; the Rotten-stone of commerce, a polishing material; and the pavement of the quadrangle of the Royal Exchange, are all composed of infusorial remains.

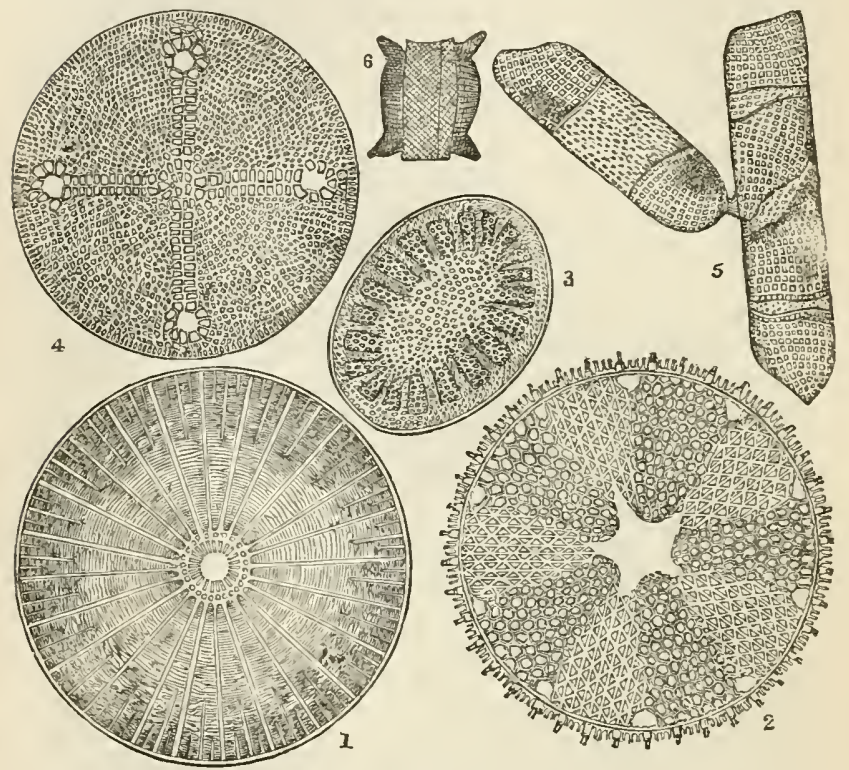

Fig. 224.

1, Shell of Arachnoidiscus. 2, Actinucyclus (Bermurla). 3. Coccencis (Algoa B 1y).

4. Cuscinodiscus (Bermuda.) 5, Isthmia enervis. 6. Zygoccros rhombus.

The bergh-mehl, mountain-meal, in Norway and Lapland, has been found thirty feet in thickuess ; in Saxony, twentyeight feet thick; and it has also been discorered in Tuscany, Bohemia, Africa, Asia, the Sonth Sea Islands, and Sonth America; of this, almost the entire mass is composed of flinty skeletons of Diatomacece. That in Tuscany and Bohemia resembles pure magnesia, and consists entirely of a shell called campilodiscus, about the 200th of an inch in size. 
Darwiu, writing of Patagonia, says : "Here along the coast, for hundreds of miles, we have our great tertiary formation, inclnding many tertiary shells, all apparently extinct. The most common shell is a massive gigantic oyster, sometimes a foot or more in diameter. The heds composing this formation are cevered by others of a peculiar soft white stone, including much gypsum, and resembling chalk; but really of the nature of pumicestone. It is highly remarkable, from its being composed, to at least one-tenth of its bulk, of Infusoria; and Professor Ehrenberg has already recognized in it thirty marine forms. This bed, which extends for five hundred miles along the coast, and probably runs to a considerably greater distance, is more than eight hundred feet in thickness at Port St. Julian." Ehrenberg discovered in the rock of the voleanic island of Ascension many siliceous shells of fresh-water Infusoria; and the same indefatigable investigator found that the immense oceans of sandy deserts in Africa were in great part composed of the shells of animalcules. The mighty Deltas, and other deposits of rivers, are also found to be filled with the remains of this vast family of minute organization. At Richmond in Virginia, United States, there is a flinty marl many miles in extent, and from twelve to twenty-five feet in thickness, almost wholly composed of the shells of marine animalcules; for in the slightest particles of it they are discoverable. On these myriads of skeletons are built the towns of Richmond and Petersburg. The species in these earths are chiefly Naviculce; but the most attractive, from the beauty of its form, is the Coscinodiscus, or sieve-like disc, found alike near Cuxharen, at the mouth of the Elbe, in the Baltic, ncar Wismar, in the guano, and the stomachs of our oysters, scallops, and other shell-fish. Another large deposit is found at Andover, Connecticut; and Ehrenberg states "that similar beds occur by the river Amazon, and in great extent from Viryinia to Labrador." The chalk and flints of our sea-coasts are found to be principally shells and animal remains. Ehrenberg computes, that in a cubic inch of chalk there are the remains of a million distinct organic beings. The Paris basin, on bundred and eighty miles long, and averaging ninety in 
breadth, abounds in Infusoria and other siliceous remains. Ehrenberg, on examining the immense deposit of mud at the harbour of Wismar, Mecklenburg-Schwerin, found one-tenth to consist of the shells of Infusoria; giving a mass of animal remains amounting to 22,885 cubic feet in bulk, and weighing forty tons, as the quantity annually deposited there. How vast, how utterly incomprehensible, then, must be the number of once living beings, whose remains have in the lapse of time accumulated? In the frigid regions of the North Pole no less than sixtyeight species of the fossil Infusoria have been found. The guano of the island of Ichaboe abounds with fossil Infusoria, which must have first entered the stomachs of fish, then those of the sea-fowl, and became ultimately deposited on the islands, incrustating its surface; whence they are transported, after the lapse of centuries, to aid the fruitiou of the earth, for the benefit of the present race of civilized man. The hazy and injurious atmosphere met with off Cape Verd Islands, and hundreds of miles distant from the coast of Africa, is caused entirely by a brown dust, which upon being examined microscopically by Ehrenberg, was found chiefly to consist of the flinty shells of Infusoria, and the siliceous tissue of plants: of these Infusoria, sixty-four proved to belong to fresh-water species, and two were denizens of the ocean. From the direction of the periodical winds, this dust is reasonably supposed to be the finer portions of the sands of the desert of the interior of Africa.

The deposit of the beneficent Nile, that fertilises so large a tract of country, has undergone the keen scientific scrutiny of Ehrenberg; and he found the nutritive principle to consist of fossil Infusoria. So profusely were they diffused, that he could not detect the smallest particle of the deposit that did not contain the remains of one or more of the extensive but diminutive family that once revelled in all the enjoyment of animal existence. It is very remarkable that at Holderness, in digging out a submerged forest on the coast, numbers of fresh-water fossil Diatomaceec have been discovered, although the sea flows over the place at every tide.

Sode of Preparing Fossil Infusoria.-Before entering 
on further details of the fossil Infusoria, we would first state how they may be prepared for microscopic examination. A great many of the infusorial earths may be mounted as objects without any previous washing or preparation; some, such as chalk, however, must be repeatedly washed, to deprive the Infusoria of all impurities; whilst others, by far the most numerous class, require either to be digested for a long time, or even boiled in strong nitric or hydro-chloric acid, for the same purpose. Place a small portion of the earth to be prepared in a testtube, or other convenient vessel, capable of bearing the heat of a lamp; then pour upon it enough diluted hydrochloric acid to about half fill the tube. Brisk effervescence will now take place, which may be assisted by the application of a small amount of heat, either from a sand-bath or from a lamp : as soon as the action of the acid has ceased, another supply may be added, and the same continued until no further effect is produced. Strong nitric acid should now be substituted for the hydro-chloric, when a further effervescence will take placc, which may be greatly aided by heat; after two or three fresh supplies of this acid, distilled water may be employed to neutralise all the remains of the acid in the tube ; and this repeated until the water comes away perfectly clear, and without any trace of acidity. The residuum of the earth, which consists of silica, will contaiu all the infusorial forms; and some of this may be taken up by a dipping-tube, laid on a slide, and examined in the usual manner. Should perfect specimens of the Coscinodiscus, Gallionella, or Navim cula be present, they may be mounted in Canada balsam; if not, the slide may be wiped clean, and another portion of the sediment taken, and dealt with in the same way, which, if good, after being dried, may be mounted in Canada balsam.

Dr. Redfern adopts an excellent mode of isolating Naviculce and other test-objects. He says: "Having found the methods ordinarily employed very tedious, and frequently destructive of the specimens, I adopted the following plan. Select a fine hair which has been split at its free extremity into from three to five or six parts, and having fixed it in a common needle-holder by passing it 
through a slit in a piece of cork, use it as a forceps under a two-thirds of an inch objective, with an erecting eyepiece. When the split extremity of the hair touches the glass-slide, its parts separate from each other to an amount proportionate to the pressure, and on beilg brought up to the object, are easily made to seize it, when it can be transferred as a single specimen to another slide without injury. The ohject is most easily seized when pushed to the edge of the fluid on the slide. Hairs split at the extremity may always be found in a shaving-brush which has been in use for some time. Those should be selected which have thin split portions so closely in contact that they appear single until touched at their ends. I have also found entire hairs very useful, when set in needle-holders, in a similar manuer; any amount of flexibility being given to them by regulating the length of the part of the hair in use." Professor Smith, of Kenyon, U.S. contrived a very ingenious "Mechanical finger" for picking up and arranging diatoms and other minute objects.

Professor J. W. Bailey, of New York, has enriched the Museum of the College of Surgeons with several valuable specimens of the skeletons of Infusoria; among them is a fresh-water Bacillaria, named Meridion circulare, which Professor Quekett, in the Historical Catalogue, describes as " cousisting of a series of wedge-shaped bivalve siliceous lorica, arranged in spiral coils; when perfect, and in certain positions, they resemble circles; each lorica is articulated by two lateral surfaces." It is asserted that they ercep about wheu free from the stalk-plate. (Fig. 225, No. 16.) Cocconema lanceolata have two lanceolate flinty cases that taper towards their ends, one of which is attached to a little foot. Each lorica has a line marked in its centre, aud transverse rows of dots on both sides: Ehrenberg says there are twenty-six rows in the one-hundredth of a line. (Fig, 22\%, No. 14.) Achranthes Longipes have at the margins two coarse convex pieces roughly dotted, and two inner pieces firmly grooved; the inside seens filled with green matter. At one cornor they are affixed to a jointed pedicle, which in many specimens contains green granules. Iu a specimen of a fossil Eunotia, found in some Bermuda earth, the flinty case is in four parts; it is of a half- 
lnnceolate shape, and a little indented on both margins; two of them have curved rows of dots, and the other two are partly grooved with finer rows. Ehrenberg says they have four openings, all on one side (fig. 225, No.13),

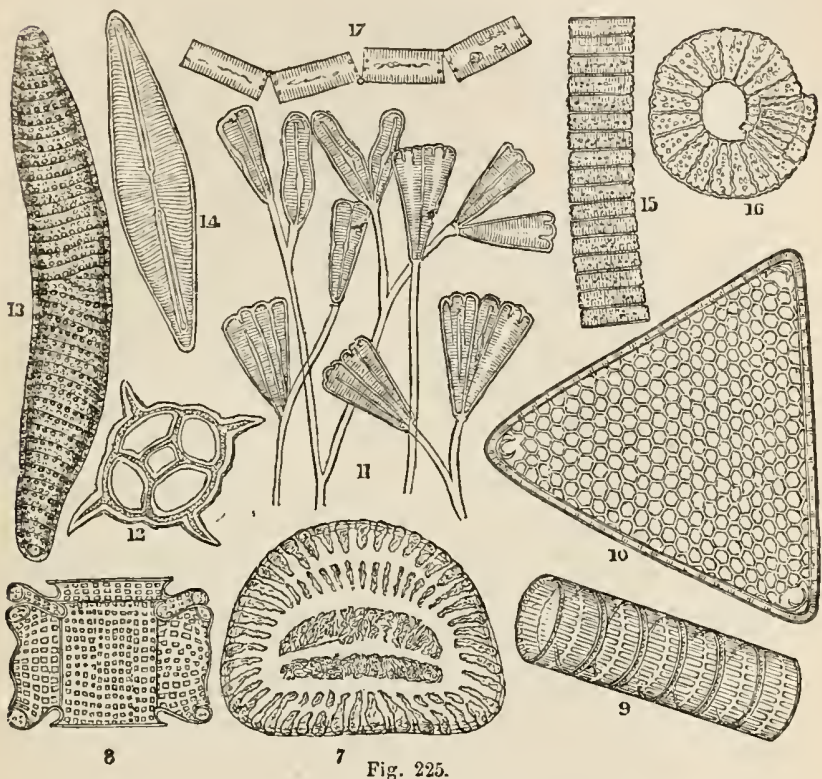

7, Campilodiscus clypeus. 8, Billin'phia. 9, Galionelia sulcata. 10, Tricerotium, found iu Thames nud. 11, Gomphonema geminalum, with their stalklike attachments. 12, Diclyocha fibula. 13, Ennolia. 14, Cosconema. 15, Fragilaria pectinalis. 16, Meridion circulare. 17, Dialoma flucculosum.

presenting a row of dots varying very much in number; minute striæ in some cases extend from each dot towards the middle of the lorica; and on the circumference there are two of these dots. The spirals and the individual lorica are very fragile, and therefore easily separated from each other. Of a glistening whiteness is the ribbon-liko flinty case of Fragilaria pectinalis, which consists of many bivalve segments: on the articulating surface there are small grooves, represented in fig. 225 , No. 15 . A sin- 
gular class of objects are Diatoma flocculosum, being rather oblong-looking, and joined to each other at opposite corners : they are sometimes grooved on each side. (Fig. 225, No. 17.) The "Swollen Eunotia" is generally about from the 11th to the 200th of an inch in length: a groove, widest in the centre, and tapering off to the ends, passes along its centre on both sides; it has curved lines proceeding from it. So wonderfully close are these lines or ribs, that as many as eight of them have been counted in the space of the 1200 th of an inch. 'They are usually found when alive adhering to a branch of some weed that forms the green coating over stagnant waters. They propagate by self-division; a slight line running down the centre niarks where the separation will occur, on each becoming perfectly developed as a distinct creature; and thus they grow and separate, filling the earth with their flinty shells.

Gallionella sulcata is found in many parts of North America; it somewhat resembles the cylindrical box for spices, which was at one time so common among good housewives; scientifically, it is described as consisting of chains of cylindrical bivalve loricx, having their outer surfaces marked or furrowed with longitudinal striæ; short joints may occasionally be seen, having their ends uppermost, the depth of the furrows being shown on the margin; within the margin is a thin transparent rim liaving radiating strix. Sometimes as many as forty will be found joined together. (Fig. 225, No. 9.) The Gallionella received its designation from a celebrated French naturalist named Gaillon, it is often termed the Box-chain Animalcule, and when the flinty case is seen lying on its face, it much resembles a coin. These living infusoria are found in almost all waters, and are stated to be so rapid in their growth, that one hundred and forty millions will by self-division be produced in twenty-four hours. A species named the Striped Gallionella was discovered by Dr. Mantell near London; the same species is also found in the ocean. Sometimes the chains are three inches long; their size is from the 14 th to the 400 th part of an inch.

Professor Quekett, in the catalogue we have referred to, describes an "earth from Bohemia, particularly rich in 
fossil specimens of Navicula viridis, which consists of four prismatic loricæ, two ventral and two lateral ; the former having round, the latter truncate extremities; and both provided with two rows of transverse markings and dots, longer and more marked on the ventral than on the lateral surfaces. The specimens having their ventral surfaces uppermost, exhibit a longitudinal marking in the centre, with a slight dilatation or knob at each extremity; this marking is interrupted in the middle of the lorica, and a diamond-shaped spot is left; if one of the lateral loricæ be examined, two of the same spots will be seen, one on each side ; they are of triangular figure, and appear to be thicker parts of the shell, described as holes by Ehrenberg." Four smaller triangular spots may be observed in the same lorica, one being situated at each corner; these also havi been considered as openings by Ehrenberg: their length varies considerably; some exceed the 100 th, whilst others are even smaller than the 1000th of an inch. Isthmia enervis (fig. 224, No. 5) is usually found attached to sea-weed ; it is in three parts; and of a trapezoid shape, the centre part appears like a band passing over, and is bounded by broad straight lines: its outer surface is covered with a network of rounded reticulations, arranged in parallel lines. Among the most remarkable is Amphitetras antediluviana; this is of a cubical or box-like figure, and consists of three portions, the one in the centre being in the form of a band, as shown at fig. 225, No. 8, and the two lateral ones having four slightly projecting angles, with an opening into each. When viewed in detached pieces, the central one is like a box, and the two lateral portions resemble the cover and bottom. The former may be readily known, as consisting merely of a square frame-work with striated sides; but both the latter are marked with radiating reticulations. When recent, they are found in zigzag chains, from their cohering only by alternate angles. In some instances, as in Biddulphia, and Isthmia, two young specimens may be found within an old one. Cocconeis is marked with eight or ten lines proceeding from the inmer margin to the centre; between which are dotted furrows, with the earlier Epot in the centre of each. (Fig. 224, No. 3.)

Campilodiscus clypeus is oval, and curved in opposite 
ways at the long and short diameters. On the margin there are two series of dots, sometimes joined; and on the oval centre there are also dots about the margin, while the middle is nearly plain. (Fig. 225, No. T.) Actinocyclus has a round bivalve flinty case, with unmerons cells formed by radiating paritions; very often every alternate cell only is on the same plane. The specimen in the Museum of the College of Surgeons is exquisite in its markings; it was found in some Bermuda earth, and has a beantifullyraised margin, and a five-rayed star in the centre; the number of cells is ten, five being on one plane and five on another. One set has the usual hexagonal reticulations crossed with diagonal lines, the other has the same lines, with a much smaller series of triangular retieulations, so disposed that they appear to form with each other parts of very small circles. One valve from this specimen is represented in fig. 224 , No. 2 .

As well as the beautiful shell of the Coscinodiscus, found both in a fossil and recent state, there is one of exquisite elegance and richness, of the genus Arachnoidiscus, so named from the resemblance of the markings of the shell to the slender fibres of a spider's web. (Fig. 224, No. 1.) This is found in the guano of Ichaboe, and also in earth from the United States, as well as among sea-weed from Japan, and the Cape of Good Hope. Mr. Shadbolt believes: "These shells are not, strictly speaking, bivalves, although capable of being separated into two corresponding portions; but are more properly multivalves, each shell consisting of two discoid portions, and two annular valves exactly similar respectively to one another." (See MFicroscopical Society's Transactions, for an excellent paper on these shells by Mr. Shadbolt.)

Artists who design for art-manufacturers might derive many useful hints from the revelations of the microscope, as evidenced in the arrangement of the shell last noticed, and in that of the genus Coscinodiscus; a very handsome object, the shells of which are marked with a network of cells in a hexagonal form, arranged in radiating lines or circles, and varying from 1-200th to 1-800th of an inch in dianeter. A specimen found in Bermuda earth has on one of its valves two parallel rows of oval cells that form a kind of cross; 
which gradually enlarge from the centre to the margin; the angles of the cross are filled up with bexagonal cells as previously noticed. (Fig. 224. No. 4.)

The unskilled manipulator may for some time endeavour to adjust a slide, having a piece of glass exposed not larger in size than a pea, on which he is informed an invisible oiject worthy his attention is fixed, before he is rewarded by a sight of Triceratium farus, extracted from the mud of the too-muddy Thames. The lexagonal markings, cells, are beautiful, and at each corner there is a curved projecting horn or foot. (Fig. 225, No. 10.) In Bermuda earth there is a small species found, which has its three margins curved; and also a curious species, which resembles the triradiate spiculum of a sponge.

It is remarkable how, in these minute and obscure organisms, we find ourselves met by the same difficulties concerning any positive laws governing the formation of generic types, as in larger and more complex forms of animal and vegetable life. It appears as if we could carry our real knowledge little beyond that of species; and when we attempt to define kinds and groups, we are encountered on every side by forms, which set at nought our definitions.

Man even uses infusorial remains as food; for the bergmehl, or mountain-meal found in Swedish Lapland, and which, in periods of scarcity, the poor are driven to mix with their flour, is principally composed of the flinty shells of the Gallionella sulcatu, Navicula viridis, and Gomphonema geminatum. Dr. Trail, on analysing it, found it to consist of 22 per cent of organic matter, 72 of silica, 5.85 of alumina, and 0.15 of oxide of iron. This would seem to be the same substance described by M. Laribe the missionary, and put to a similar use in China: "This earth," he says, "is ouly used in seasons of extreme dearth."

Xanthidia.-In conjunction with the skeletons of the former species it will be as well to offer a few remarks upon animals long classed with Infusoria, and but rarely found except in the fossil state. There is every reason to believe that the Xanthidia, double-bar animalcules, are sporangia of Desmidiacra. In proof of this it call be shown that 
their skeletons are composed of a horny substance, and not of silica, as was once supposed.

The name Tantlidia is derived from a Greek word signifying yellow, that being their prevailing hue. They are found plenteously in a fossil state, imberded in flint, as many as twenty being detected in a piece the twelfth of an inch in diameter; in fact, it is rare to find a gun-flint without them. When living they may be described as having a round transparent shell, from which proceed spikes varying in size and shape. One kind, found by Dr. Bailey in the United States, was of an oval form, the 288th of an iuch in length; and another species circular, found by the late Dr. Mantell, at Clapham: both were of a beautiful green colour. Specimens of Branched Xanthidium, found in flint by Dr. Mantell, were from the 300th to the 500th of an inch in diameter. Mr. Ralfs says: "That the orbicular spinous bodies so frequent in flint, are fossil sporangia of Desmidiacece, cannot, I think, be doubted, when they are compared with figures of the more recent forms. Indeed, the late Dr. G. Mantell, who, in his Medals of Creation, without any misgiving, had adopted Ehrenberg's ideas concerning them, changed his opinion; and in his last work regards them as having been reproductive bodies, although he is still uncertain whether they are of vegetable origin."

The fossil forms vary as much as recent Sporangia, in being smooth, bristly, or furnished with spines, some are simple, and others branched at the extremity. Sometimes, a membrane may be traced, even more distinctly than in recent specimens, either covering the spines, or entangled with them. Writers lave described the fossil forms as having been siliceous in the living state; but Mr. Williamson informs us that he possesses specimens which exhibit bent spines and torn margins; and this wholly contradicts the idea that they were siliceous before they were embedded in the flint. In the present state of our knowledge, it would be somewhat premature to identify the fossil with recent species; it is better, therefore, at least for the present, to retain the names bestowed on the former by those observers who have doscribed them. 
Near to Sydden Spoint, and the Round Down Cliff, on the Dover beach, Mr. H. Deane cut out a piece of pyrites with the adherent chalk, which, on examination, "exposed to view bodies similar to, if not identical with, Xanthidia in flints; he clearly recognised $X$. spinosum, ramosum, tubiferum, simplex, tubiferum recurvum, malleoferum, and pyxidiculum, together with easts of Polythalamia, and other bodies frequently found in flints. In shape they are somewhat flattened spheres, the greater part of them having a remarkable resemblance to gemmules of sponge, with a circular opening in the centre of one of the flattened sides. The arms or spines of all appear to be perfectly closed at the ends, even including those which have been considered in the flint, specimens decidedly tubiferous; showing that if the arms are tubes, they could afford no egress to a ciliated apparatus similar to those existing among Zoophytes. On submitting them to pressure in water between two pieces of glass, they were torn asunder laterally, like a horny or tough cartilaginous substance; and the arms in immediate contact with the glass were bent. Some specimens, put up after several weeks' maceration in water, were so flaccid, that, as the water in which they were suspended evaporated away, the spines or arms fell inclined to the glass. These circumstances alone seem clearly to disprove the idea of their being purely siliceous. The casts of the Polythalamia, portions of minute crustaceans, dre. appeared also to be, like the Xanthidia, some modification of organic matter; and in the case of the Polythalamia, the bodies are so perfectly preserved, that in some the lining membranes of the shells are readily distinguishable."

Mr. Wilkinson, who examined recent Xanthidia found in the Thames mud, and slime, on piles and stones at Greenhithe, said that, in his opinion, they are not siliceous, but of a horny nature, similar to the wiry sponges, which Mr.. Bowerbank deseribes as being very difficult to destroy without the action of fire. He also met with a peculiarity in a $X$. spinosum, which he has never seen in any other species; it was in a pieee of a gun-flint. There appeared, as it were, a groove or division round the circumference, similar to that formed by two cups when placed 
on each other, so as to make their rims or upper edges meet.

The other fossil Infusoria, found most abundantly in the chalk and flint of England, are the Rotalia, or wheel-shaped, and the Textularia or woven-work animalcules; the latter having the appearance of a cluster of eggs in a pyramidical form, the largest being at the base, and lessening towards the apex.

We must here bring to a close this short notice of some of the marvellous creations in the invisible world, every glimpse inspiring awe, from the immensity, variety, beauty, and minuteness of its organised habitants. Immensity, in its common impression on the mind, hardly conveys the idea of the myriads upon myriads of Infusoria that have lived and died to produce the tripoli, the opal, the flints, the bog-iron, the ochres, and limestones of the world.

Professor Owen beautifully explains the uses of this vast amount of animalcule life:- "Consider their incredible numbers, their universal distribution, their insatiable voracity; and that it is the particles of decaying vegetable and animal bodies which they are appointed to devour and assimilate. Surely we must, in some degree, be indebted to these ever-active, invisible scavengers, for the salubrity of the atmosphere and the purity of water. Nor is this all; they perform a still more important office in preventing the gradual diminution of the present amount of organised matter upon the earth. For when this matter is dissolved or suspended in water, in that state of com. minution and decay which immediately precedes its final decomposition into the elementary gases, and its consequent return from the orgauic to the inorganic world, these wakeful members of nature's invisible police are everywhere ready to arrest the fugitive organised particles, and turn them back into the ascending stream of animal life. Having converted the dead and decomposing particles into their own living tissues, they themselves become the food of larger Infusoria, and of numerous other small animals, which in their turn are devoured by larger animals; and thus a food, fit for the nourishment of the highest organised beings, is brought back, by a short route, from the extremity of the realms of organised matter. 
These invisible animalcules may be compared, in the great organic world, to the minute capillaries in the microcosm of the animal body; receiving organic matter in its state of minutest subdivision, and when in full career to escape from the organic system, turning it back, by a new route, towards the central and highest point of that system."

Such, then, seem to be some of the purposes for which are created the wonderful invisible myriads of infusorial animalcules. In the words of Holy Writ: "All these things live and remain for ever for all uses; and they are all obedient. All tlings are double one against another ; and $\mathrm{He}$ hath made nothing imperfect. One thing estab. lisheth the good of another; and who shall be filled with beholding His glory ?"

Vorticellid E. - We now come to a family, which includes some of the most beautiful of living infusorial animalcules, and in which we meet with phenomena more curious than any yet witnessed, and perhaps as wonderful as any that will be presented to our notice, in the natural history of the higher classes of animals. The family of Voricellida, bell-animalcules, are characterised by the possession of a fringe of rather long cilia, surrounding the anterior extremity, which can be exerted and drawn in at the pleasure of the creatures. Some are furnished with a horny case for the protection of their delicate bedies, whilst others are quite naked.

The genus Vorticella, from which the name given to the family is derived, consists of little creatures placed at the top of a long flexible stalk, the other extremity of which is attached to some object, such as the stem or leaves of an aquatic plant. This stem, slender as it is, is nevertheless a hollow tube, through the entire length of which runs a muscular thread of still more minute dianeter. When in activity, and secure from danger, the little Vorticella stretches its stalk to the utmost, whilst its fringe of cilia is constantly drawing to its mouth any luckless animalcule that may come within the influence of the vortex it creates; but at the least alarm the cilia vanish, and the stalk, with the rapidity of lightning, draws itself up into a little spiral coil. But the Vorticella, is not wholly condemued to pass a sort of vegetable existeuce, 
rooted, as it were, to a single spot by its slender stalk; its Creator has foreseen the probable arrival of a period in its existence when the power of locomotion would become

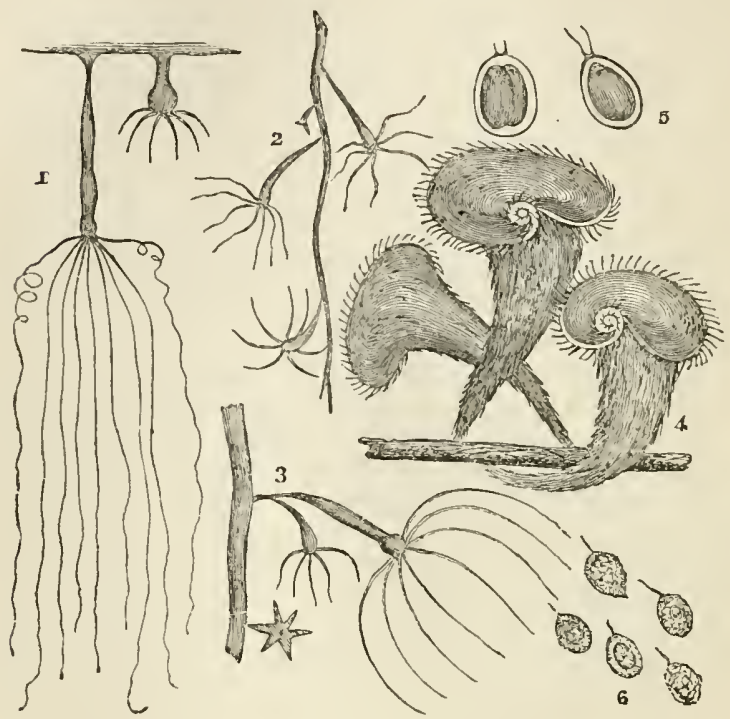

Fig. 220.

1, 2, 3, IIydre in various stages of development. 4, A group of Stentor poly. morphus, many-shaped Stentor. 5, Englena. 6, Monads.

necessary, and this necessity is provided for in a manner calculated to excite our highest admiration. At the lower extremity of the body of the animal, at the point of its junction with the stalk, a new fringe of cilia is developed; and when this is fully formed, the Vorticella quits its stalk, and casts itself freely upon its world of waters. The development of this locomotive fringe of cilia, and the subsequent acquisition of the power of swimming by the Vorticella, is generally connected with the propagation of the species, which, in this and some of the allied genera, presents a series of most curious and complicated phenomena. 
The Vorticella possess means of propagation which is denied to other Infusoria, with the exception of a few, although we meet with the same in other forms of animal life. The mode of reproduction referred to is called gemmation; it consists in the production of a sort of bud, which gradually acquires the form and structure of the perfect animal. In the Vorticella, these buds, when mature, quit the parent stem after developing a circlet of cilia at the lower extremity, and fix themselves in a new habitation in exactly the same manner as those individuals produced by the fissuration of the bell.

At an earlier or later period of their existence, the Vorticellae withdraw the dises surrounded by cilia which forms the anterior portion of their bodies, and contracting themselves into a ball, secrete a gelatinous covering, which gradually solidifies, and forms a sort of capsule, within which the animal is completely inclosed. By this process the little animal is said to become encysted; and at this point of its history it is seen to be more complicated. Sometimes its further progress commences by the breaking up of the nucleus into a number of minute oval discs, which swim about in the thin gelatinous mass inter which the substance of a parent has become dissolved. The body of the parent animal, inclosed within the cyst, now becomes apparently divided into separate little sacs or bags, some of which gradually acquire a considerable increase in size, and at length break through the walls of the cyst. After a time one of these projections of the internal substance bursts at the apex; and through the opening thus formed the gelatinous contents of the cyst, enclosed embryos, are suddenly shot ont into the water, there to become diffused, giving rise to new generations. From the name Acineta given to them by Ehrenberg, who described them as a new genus, they are denominated Acineta-forms.

But the final object of this singular metamorphosis still remains to be described. The nucleus, which at the change of the encysted animalcule into the Acineta-form was still distinctly observable, becomes entirely and altogether converted into an active young Vorticella, acquiring an ovate form, with a circlet of cilia round its narrower 
extremity, and presenting at the opposite end a distinct rnouth. Within this young animal, whilst still inclosed in the body of its parent, we see a distinct nucleus, and the usual contractile space of the full-grown creature. When mature, the offspring tears its way through the membranes inclosing the Acineta, which, however, immediately close again. The latter continues protruding and retracting its fllaments, and soon produces in its interior a new nucleus, which in its turn becomes metamorphosed into a young Vorticella.

The same faculty of enclosing themselves in a cyst is

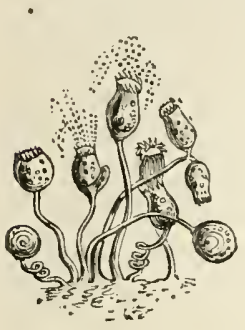

Fig. 227.

Vorticella microstoma. said to be made use of by the Vor . ticella, as a means of self-preservation if the water in which they have been living dries up. When the animal is thus encased, the mud at the bottom of the pool may be baked quite hard in the sun without doing it the least injury ; and in this state the creatures are often taken up by the wind with the dust which it raises from the surface of the parched ground, and borne along to great distances, so as to cause their appearance in most unexpected loocalities (they are frequently found in roof gutters), where the first shower of rain calls them back to active life.

Conochilus vorticella, ${ }^{1}$ belonging to the family Acistina, Plate III. No. 80, is one of the most remarkable and interesting Rotifers met with. It is found in compound groups of a whitish globular form in shallow ponds about London. On Hampstead Heath a good supply is often obtained throughout the summer months. The group consists of from twenty to thirty, or more, animals, of about twice the size of the full-grown volvox, and, like the latter, can be readily seen actively rolling about when the collecting-bottle is held up to the light. The colony is attached to a centre disc, resembling a wheel with its naves and spokes. The foot-stalk is three or four times the length of the body, and has a somewhat spiral form, which it contracts at pleasure, drawing the body down in an instant close to the axis, although it docs not appear to

(1) Commonly called molvor. but this is an error, as it clearly does not belong to the Volvocinie. 
have the power of retracting itself perfectly within the hyaline membrane. The hyaline membrane is at certain periods of the year so very translucent that it cannot be made out; later in the season it is found studded with parasitic desmids, when its gelatinous form is readily seen. The body is oroid or cup-shaped, and the mouth is surrounded by long cilia, which are always in rapid motion. When the animal becomes alarmed it instantly retracts, and then has the appearance of a small round ball. On the frontal plane four thickish conical erect papillæ are placed, each furnished with one or more spines or setes; very near their base, rather behind, and between the division of the eiliary band, are the very minute visual organs. The jaws, it is said, are furnished with teeth, but these we have not been able to make out, chiefly owing to their disposition to break up in a short time after being placed in confinement. The stomach is oval, and two ovoid bodies are observed near the termination of the œsophagus ; below these the ova-sack encloses a single ovum of a dark colour. The ovum is surrounded by spinous processes, or cilia, and when first thrown off it lodges for a time in the hyaline membrane; but, when set free, moves slowly about. A few minutes after being placed in the glass-cell the colony become uneasy, break themselves off one after the other, and swim away to die. They were formerly classed among Volvocinece, but bear no resemblance to them, except in their roll through the water; and are more properly placed among the Rotifers.

Acineta tuberosa, l'late III. No. 68.-The researches of Stein are said to prove that the several members of this family are simply a developmental phase of Vorticellina; this view, however, is controverted by Lachmann, Claparede, and others who have witnessed the reproduction of Acinetoe from parent forms. A. tuberosa has a triangularshaped body and three obtuse tubercles or horns, each furnished with tentacula. Many other forms of this genus are well known; but, notwithstanding the diversity in construction, Stein declares their tubular ramificd processes to be morphologically and physiologically identical with ordinary tentacula. Vaginicola crystallina he puts forward as one of the best illustrations to be obtained of 
the conversion of an encysted Vorticellina into an Acineta. $\mathrm{He}$ also consider's Actinophrys Sol and Podophrya fixa (Ehr.) to be the acinetiform representatives of Vorticella microstoma.

Stentors, Trichodina, and a few others are included by some author's in the Vorticellina. Stentors (fig. 226, No. 4) are exclusively found in fresh water, between or upon water plants in still running waters. Some are colourless, others green, black, or clear blue. "It is," says M. Dujardin, "in the Stentors where we can view the several supposed interual organs isolately, and that new observations will make known their real nature."

Rotiferce comprise animals which were placed by Ehrenberg in nine genera; named Ptygura, Acistes, Conochilus, Megalotiocha, Lacinularia, Tubicolaria, Limnius, Melicerta, and Cephalosiphon. As, however, each of these genera contain but a single species, Mr. Gosse proposes to reduce the nine to two; thus, Ptygura, Ecistes, Tubicolaria, Limnias, Melicerta, and Cephalosiphon, as they seem to be only so many species of one genus, might constitute one, and Megalotrocha, Lacinularia, and Conochilus, another. Mrr. Gosse also wishes to coustruct a family to be called Melicertoda; in this he would include two genera, Melicerta and Megalotrocha, degrading some of the present genera to form species of Melicerta, and others, to constitute three species of Megalotrocha. Each group will be readily distinguished-the former by the circumstance that the individuals are solitary; in the latter they are, in adult life, agcregated in a common envelope-spherical masses, composed of many animals radiating from a central point. These compound masses are either free or fixed. In the genus Melicerta, or tube-dwellers proper, the front on upper part of the body is capable of being turned in upon itself, concealed with purse-like folds, and of being expanded, at the will of the animal, into a disc form, which is usually much wider than the diameter of the body; this again is either flat or in the form of a shallow funmel. Its outline will form either a simple circle, as in $M . p t y$ gura and $M$. occistes, two circles united at one point, as in Limnias (Plate III. No. 72), or four sinuous lobes, more or less developed, as in M. cephalosiphon, M. tubicslaria, and 
34. ringeris, in each of which there is, according to Professor Huxley, a double edge to the disc, of which the subordinate one is placed on the under-side, and a little within the line of the principle one. The former is fringed with minute cilia, whose vibratile waves form well-marked movements, which run evenly along the margin. The eggs are usually laid within the case.

To the genus Melicerta Mr. H. Davis ${ }^{1}$ adds one, if not two, new species, which he has named, provisionally, $M$. longicornis and $M$. intermedius. The two peculiarities of these new forms are the remarkable length of the antennæ, and the construction of tube-dwellings for the purpose of concealing themselves and their eggs. (Plate III. No. 69.) Ecistes longicornis: each animal lives in a separate semi-transparent cylindrical sheath (urceolus), into which it entirely withdraws on the approach of danger. The footstalk is long, and firmly attached to the bottom of the tube. Two or three ova are concealed in the lower part of the urceolus. The trocbal disc is large, and completely surrounded by a ciliary wreath. The antennæ, two, are very long, well placed below the disc, and terminating in a small brush of setæ. The tube, Mr. Davis believes, is built up in the same way as that of Melicerta.

Mr. Slack describes, in the "Intellectual Observer," a tubicolous Rotifer, discovered in the stems of the Anacharis, which shows an affinity with Ecistes, Limnias, and Melicerta, but differs in some points, especially in the antenur; this, named by Mr. Gosse Cephalosiphon, displays a single antenna of extraordinary length and versatile power. Like Melicerta and Limnias, it shows no visible construction below the dise, whereas in Excistes this is a conspicuous feature.

"The animal inhabits a case slightly trumpet-shaped, generally of greater length and slenderness compared with those of its allies, standing erect on the pond-weed. It is irregular and floccose in outline, very opaque, and of a deep umber brown by transmitted light, but of a much lighter hue by reflected light. It is composed, doubtless, of an excretion from the skin as the foundation layer,

(1) "On two new species of the genus Elcistes," by Henry Davis, F.R.M.\&. Micros. Soc. Trans. April 1867. 
thickened and rendered opaque by the addition of the dark material, which I conjecture to be the fxeal pellets sucessively discharged in process of growth. Contrary to the rule in the allied senera, the petaloid disc is made to open by the bending forward of the head towards the ventral aspect, and its widest nargin is the dorsal one. Immediately behind the dise are two minute lateral hornlike points, which project from the head, and curve towards each other. These are sometimes visible both in a frontal and a lateral view, and with the disc closed or open; but at other times the closest scrutiny fails in discerning them. Behind these, in the median line, there is an organ which is never concealed: it is the single antenna, which stands up perpendicularly from the occiput to a great height (being almost half as long as the body, exclusive of the foot), and generally arches over the front, but is eapable of vigorous and sudden movements to and fro, and from side to side. It is cvidently tubular throughout ; either a simple tube with thick walls, or else, if the walls are thin, furnished with a slender piston which runs through its length.

"The Ceplualosiphon is very lively and active in its motions. It is very ready to protrude from its case, and not at all prone to retire upon ordinary alarms, such as a jar upon the instrument, that would send the Floscularia or the Stephanoceros into its retreat in an instant. It is very curious to see it protruding: the long antenna is first thrust out, and jerked to and fro as a feeler, exploring the surrounding water for safety. The entire height of an average specimen, in its ordinary state of extension, is $1-33 \mathrm{~d}$ of an inch; of which the foot is 1-50th, the body 1-200th, and the antemne 1-400th of an inch.

The rotating or wheel-animalcules occupy the most conspicuous place among infusorial aniuals. They require water for their development, although they are indwellers occasionally of the cells of mosses and damp weeds. They do not possess many stomachs, but cne, and generally have teeth and jaws to supply its warts. They can elongate and contract their bodies, and scme species have their extremity prolonged to a tail, or rather a foot, or a forked process, by means of which they 
fix themselves to extraneous substances; while the cilia is in rapid motion, this prevents the anterior portion of the body being drawn in by the force of the rotatory

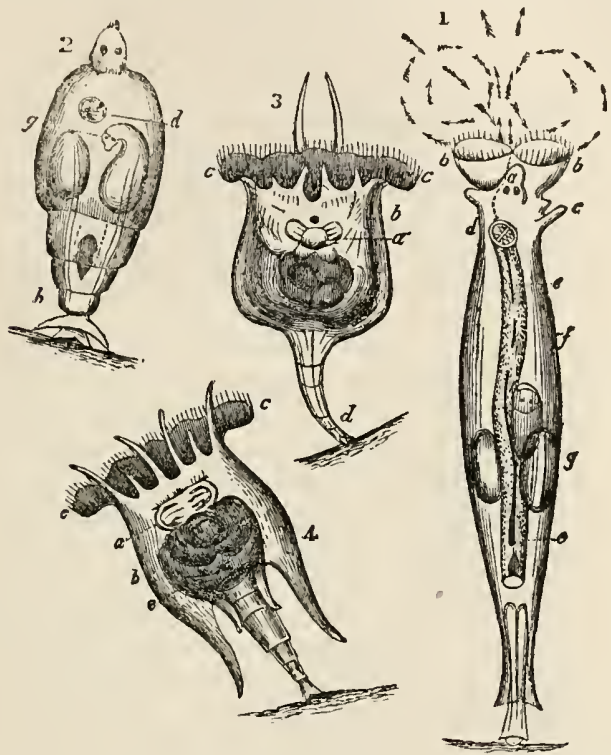

Fig. 228.

1, The common Wheel-Animalcule, Rotifer vulgaris, with its cilia or rotatort $b$, protruded; $c$, its horn; $d$, cesophagus ; $e$, gut : $f$, outer case; $g$, eggs. 2 , Thi same in a contracted state, and at rest: at $g$ is seen the development of the young. 3, Pitcher-shaped Brachionus : $a$, its jaws; $b$, shell; $c$, cilia, or rotators : $d$. tail 4. Baker's Brachionus: $a$, the jaws and teeth; $b$, the shell; $c$, the eilia : $e$, the stomach.

action. They multiply by eggs; a few have been seen to bring forth their young alive. In the atmosphere the eggs have been discovered whirling along by the force of the wind to some resting-place, where, when circumstances admit, they spring into active life, and fulfil their appointed destiny. The eggs are of an oval form, and some ten, twenty, or thirty may be seen in an animal, of a brown colour, others are of a deilicate pink and deep golden yellcr. In those of a light colour, the young are somctimes 
seen with their cilia in active vibration. Ehrenherg aecurately described the upper part of a common wheel-animaleule, with the cilia, jaws, teeth, eyes, \&e., as seen under a magnifying power of 200 diameters, and represented in fig. 228, No. 1. The small arrows indieate the direction of the currents produced by the cilia $b$, turning on their base. At the will of the animal a change is made in the direction in which the wheels appear to revolve, these it has the power of withdrawing, with the quickness of thought; a cluster of hairs appears at the extremity, that do not revolve, and certainly differ from the cilia: as they are usually protruded when the ereature is moving from place to place, their function has been imagined to be that of feelers.

'The red spots, very generally believed to be the eyes of the Rotiferce, are mostly of a bright red colour; and the number and arrangement of these organs vary. In some species there have been discovered as many as eight, often placed on either side of the head, in a row, circle, or cluster, and in some they take a triangular shape. The Rotiferce delight in the sunshine; and when the bright lnminary is hidlen behind clouds, the animals sink to the bottom of the water, and there remain. When the water of their haunts is becoming much evaporated, they rise to the top, and give a bright-red tint to it; but whon canght and placed in a jar, their beantiful colour fades in a few days. Locomotion is performed by swimming, the rotatory action of the crowns of cilia inpelling it forward; in other instances it bends its body, then moves its tail up towards the head, with the two processes that serve as feet near the tail ; it then jerks its head to a further distance, again draws up its tail, and so proceeds on its journey. Another peculiarity is that of drawing in the head and tail until nearly globular, and remaining in this condition fixed by the sucker; at other times they become a complete ball, and are rolled about by every agitation of the water.

The body of the wheel-animalcule is of a whitish colour; its form is indicated in the engraving. The tube for respiration appears to allow of water passing to the inside. On the food being drawn by the currents to the sup part. 
of the wheels, it passes through a funnol as it were to the month, which is situated rather lower down, and whero the food is crushed by teeth placed on the plates of the jaw, with a hammer-like action ; from this point it passes through the alimentary canal for the sustenance of the animal.

Brachionæa.-Ehrenberg's genus Brachionus, "Spinebearing animalcules," helonging to the Rotijerce, are truly interesting, from their very perfect and complex organisation. Some are entirely enclosed in a horny covering, others only partially covered. Their structure, so beautiful and symmetrical, has always made them favourites with those who delight in microscopical studies.-Brachionus striatus, "Striped shell animalcule" (No. 3, fig. 228), of an elegant, jug-like form, has the transparent coat or carapace, striated and scalloped out at the upper part; through which the citroncoloured inhabitant protrudes itself. Two hornlike processes are appended to its under-side. As occasions require, it sinks firmly and seeurely within its crystal home, which is sufficiently transparent to permit a view of its organisation. Its progress is effected by means of ciliary processes. - Brachionus Pala, or Anurce Cervicornis, "Bent horn animalcule," is possessed of double rotatory organs, and four long processes, which project above the external coat. It measures the 90 th part of an inch.-Brachionus Ovalis, "Egg-shaped brachionus," is remarkable for the strength of its transparent coat, which is beyond that of other horny creatures. Its projecting tail, as well as head, is at pleasure withdrawn into its very Fig. 229.
strong case.-Brachionus Dentatus, 1, Brachionus ovalis, closel. "Toothed brachionus." This active,

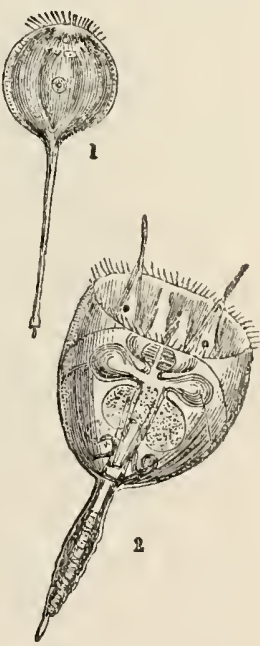

Fig. 229. 2, Cilia displayed. bright pink-eyed little creature, the 90 th part of an inch in size, is apparently enclosed in a two-valved shell, having 
each 海d indented so as to form two pair of teeth. Mr. Pritchard says:-"In addition to the rotatory organs for supplying it with food, I have observed it attached to a stem of conferve, and abrading it with its teeth fixed in the bulbous œsophagus, which, during the operation, oscillates quickly; the rotatory cilia at the same time move rapidly, which makes it highly probable that they perform some office connected with the organs of respiration, as their motion seems altogether unnecessary while the creature is feeding in this manner."-Brachionus Bakeri, "Baker's brachionus," (fig. 228, No. 4), is a curious and beautifully-formed animal. At the points of a halfcircle are situated the rotatory organs and cilia, between which rise some long spines, each side of the shell proceeding to a point in the lower part, while a square seems taken out of its body, forming thus two spines; from the central part of the body projects a long tail. The eggs are sometimes attached to these spines, and in other instances are seen in the ovisac.

Notommata Aurita, the "Eared notommata."-The anatomy of this animal, a genus of Rotifero, family Hydatincea, has been most lucidly explained and illustrated by Mr. P. H. Gosse, in the Microscopical Society's Transactions.

Mr. Gosse states, that his specimens were found in a jar of water obtained in the autumn from a pond near Walthamstow, the jar having stood in his study-window through the winter; and from a swarm in the succeeding February he selected one the 70th of an inch in length when extended, but its contractions and elongations rendered its size variable.

"Its form, viewed dorsally, is somewhat cylindrical, but it frequently becomes pyriform by the repletion of the abdominal viscera. Viewed laterally, the back is arched gibbous posteriorly, with the head somewhat obliquely truncate, the belly nearly straight. The posterior extremity is produced into a retractile foot, terminating in two pointed toes; this, both in function and structure, is eertainly analogous to a limb, and must not be mistaken for the tail, which is a minute projection higher up the hody. When not swimming or rotating, the head assumes a rounded outline, displaying through the transparent 
integument an oval mark on each side, within which a tremulous motion is perceived; but at the pleasure of the animal a semi-globular lobe is suddenly projected from each of these spots by evolution of the iutegument. These projections have suggested the trivial name of aurita. Each lobe is crowned with a wheel of cilia, the rapid rotation of whose waves forms the principal source of swift progression in swimming. The protrusions of these lobes are evidently eversions of the skin, ordinarily concealed in two lateral cavities. They may be protruded by pressure, and are then seen to be covered with long but firm and close-set cilia, which are bent backward, and move more languidly, as death approaches. The whole front is also fringed with short vibratile cilia, which extend all along the face, as far as the constriction of the neck. The whole body is clear and nearly colourless; but its transparency is much hindered by the net-work of dim lines and corrugations that are everywhere seen, particularly all abont the head."

Mr. Gosse, throwing a little carmine into the water, saw the jaws working slightly, the points opening a little way, and then closing; the rods of the hammers were drawn tcwards the bottom for opening, and upwards for closing. A lit.tle mass of pigment was soon accumulated beneath the tips of the jaws, which spread itself over a rounded surface, but did not pass farther; nor did an atom at this time go into the stomach.

After entering into further minute details of the little animal, he observes: "They possess organs that many others do not, and want some that others possess. They prove that the minutencss of the animals of this class does not prevent them from having an organisation most elaborate and complex, and therefore it justifies the belief that the Rotiferce should occupy a place in the scile of animal life much higher than that which has been commonly assigned to them."

Like most of the class, this Notommata is predatory. Mr. Gosse once saw one eagerly nibbling at the contracted body of a sluggish Rotifer vulgaris; the mouth was drawn obliquely forward, and the jaws were protruded to the food, zo as to touch it. It did not appear, however, to do the 
rotifer much damage. It appeared chiefly to feed on monads.

Flosculariea. - The Stephanoceros, "Crowned animaicule." This beautiful little creature is about the 36 th of an inch in leugth; and is enclosed in a transparent cylindrical flexible case, over which it protrudes five long arms in a graceful manuer, which, touching at their points, give a form from which it derives its name. These arms are furnished with several rows of short cilia, and retain the prey brought within their grasp until it can be swallowed. The case is attached to the animal on the part we may term the shoulders; so that when it shrinks down in its transparent home, the case is drawn inwards. To the bottom of its home it is secured by an elongation of the body; and this part, as well as the body, contracts instantly on the approach of danger, the arnis coming close together are also withdrawn. Its mouth differs a little from the common wheel-animalcule; it has two distinct sets of teeth, with which it tears and crushes its food. The eggs of the Steplanoceros, after leaving the animal's body, remain in their crystal-like shell until hatched; and Dr. Mantell from close observation found, that about eighty hours elapsed before their organs were all developed and fitted for use.

Limnias Ceratophylli, "Water-nymph," is of this family, being about the 20 th of an inch in size, and is enclosed in a white transparent cylindrical case, one-half the length of the animal; which, being glutinous, becomes of a brownish colour, from the adhesion of extraneons matter. Its rotatory apparatus is divided into two lobes, possessing vibrating cilia, as well as a singular projecting angular chin. In the rows of little eggs in the body of the parent, may clearly be distinguished most of the young organs in a state of activity. From its fondness for hrrnwort, it is often called by the name of that plant. (Plate III. No. 72.)

Floscularia Ornata, "Elegnnt floscularia," is a beautiful type of the family, and has its rotatory organs dividerl into several parts; when it contracts itself into a small compass, its transparent covering becomes wrinkled. This creature is an interesting olject, as its internal structure can be seen through the translucent sheath that constitutes 
its dwelling. The little beings are very rapacious, althongh but the 108th part of an inch in size. Floscularia Proboscidea, "Horned floscularia," has six lobes, fringed with cilia shorter than in the preceding species. Its name is derived fiom a peculiar kind of horn or proboscis, also having cilia placed in the centre of the lobes. The eggrs cast off by the parent enclosed in a sheath, are very pretty objects for microscopic observation. In fact, the tinted case, the light ethereal frame of the tiny animal, the variously coloured fond, \&c., in the stomach, combine in rendering it singularly interesting.

Melicerta Ringens, "Beaded melicerta."-Of all the Melicerta, or "Honey floscularia," this is the most beautiful. Its crystalline body is first enclosed in a pellucid covering, wider at the top than the bottom, of a dark yellow or reddish-brown colour, which gradnally becomes encrusted with zones of a variety of shapes, glned together by some peculiar exudation that hardens in water: it is these little pellets, appearing as rows of beads, give the name to the animal. Mr. Gosse furnishes an excellent account of the "architectural instincts of Melicerta ringens," which is not only truly surprising, but full of interest. He writes:"This is an animalcule so minute as to be with difficulty appreciable by the naked eye, inhabiting a tube composed of pellets, which it forms and lays one by one. It is a mason who not only builds up his mansion brick by brick, but makes his bricks as he goes on, from substances which he collects around him, shaping them in a mould which he carries upon his body.

"The animal, as it slowly protrudes itself from its ingeniously-formed mansion, appears a complicated mass of transparent flesh, involved in many folds, displaying at one side a pair of hooked spines, and at the other two slender, short blunt processes projecting horizontally. As it exposes itself more and more, suddenly two large rounded discs are expanded, around which, at the same instant, a wreath of cilia is seen performing its surprising motions. Often the animal contents itself with this degree of exposure; but sometimes it protrudes farther, and displays two other sinaller leaflets opposite to the former, but in the same plane, margined with cilia in like manner. The 
appearance is not uulike that of a flower of four unequal petals; from which circumstance Linnæus gave it the name ringens, by which it is still known."

Below the large petals on the ventral aspect, and just above the level of the projecting respiratory tubes, is a small circular disc or aperture, within the margin of which a rapid rotation goes on. This little organ, which seems to have hitherto escaped observation, Mr. Gosse can compare to nothing so well as to one of those little circular ventilators which we sometimes see in one of the upper panes of a kitchen-window, running round and round, for the cure of smoky chimneys. The gizzard, or muscular bulb of the gullet, is always very distinct, and its structure is readily demonstrated. It consists of two sub-hemispherical portions, or jaws, each of which is crossed by three developed teeth, which are succeeded by three or four parallel lines, as if new teeth might grow from thence. The teeth are straight, slender, swelling towards their extremity, and pointed. These armed hemispheres work on each other, and on a V-shaped or tabuliform apparatus beneath, common to most of the Rotifers, but in this genus very small.

The pellets composing the case are very regular in form and position : in a fine specimen, about the 1-28th of an inch in length when fully expanded, of which the tube was the 1-36th of an inch, Mr. Gosse counted about fifteen longitudinal rows of pellets at one view, which might give about thirty-two or thirty-four rows in all.

In November, 1850, Mr. Gosse found a fine specimen attached to a submerged moss from a pond at Hackney; this he saw engaged in building its case, and at the same time discovered the use of the curious little rotatory organ on the neck. When fully expanded, the head is bent back at nearly a right angle to the body, so that the dise is placed nearly perpendicularly, instead of horizontally; the larger petals, which are the frontal ones, being above the smaller pair. Now, below the large petals (that is, on the ventral side) there is a projecting angular chin, which is ciliated; and immediately below this is the little organ in question. It appears to form a small hemispherical cup, and is capable of some degree of projection, as if on a short 
redicle. On mixing carmine with the water, the course of the ciliary current is readily traced, and forms a fine spectacle. The particles are hurled round the margin of the disc, until they pass off in front through the great sinus, between the larger petals. If the pigment be abundant, the cloudy torrent for the most part rushes off, and prevents our sceing what takes place; but if the atoms be few, we see them swiftly glide along the facial surface, following the irregularities of outline with beautiful precision, dash round the projecting chin like a fleet of boats doubling a bold headland, and lodge themselves, one after another, in the little cup-like receptacle beneati. Mr. Gosse, believing that the pellets of the case might be prepared in the cup-like receptacle, watched the animal, and presently had the satisfaction of seeing it bend its head forward, as anticipated, and after a second or two raise it again; the little cup having in the meantime lost its contents. It immediately began to fill again ; and when it was full, and the conteuts were consolidated by rotation, aided probably by the admixture of a salivary secretion, it was again bent down to the margin of the case, and emptied of its pellet. This process he saw repeated many times in succession, until a goodly array of dark-red pellets were laid upon the yellowish-brown ones, but very irregularly. After a certain number were deposited in one part, the animal would suddenly turn itself round in its case, and deposit some in another part. It took from two and a half to three and a half minutes to make and deposit a pellet.

Melicerta may be found in clear ponds, mill-ponds, and other places through which a current of water gently tlows. It a portion of water-weed be brought home and placed in a small glass zoophyte-trough, and carefully examined with a magnifying power of about fifty diameters, a few delicate looking projections of a reddish brown colour will probably be seen adhering to the plant; these are the tubular cases of melicerta, which, after a short period of rest, will throw out little animals of one-twelfth of an inch in length. ${ }^{1}$

(1) For further information, see Gosse, Trans. Micros. Soc. vol. iii. 1852, p. 58; Slack's Marvels of Pond Life, 1861 ; and Pritchard's History of Infusorin Ath Edition, 1861. 
Porypifel21.- The chief characteristic of this vast race of animals is, that their mouths are surrounded by

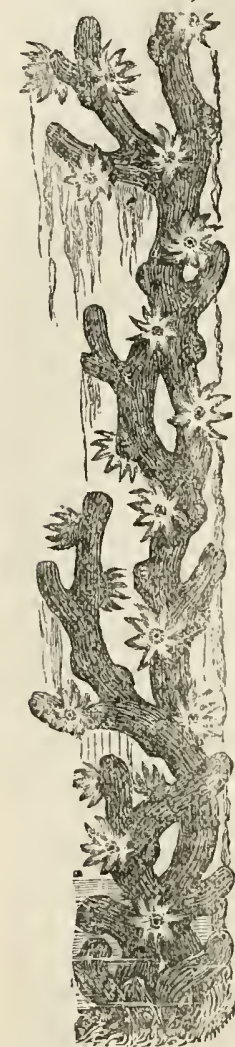

Fir. 230.-1stcroid Zoophytes. ladianng tentacula, arranged somewhat like the ray of a flower; and hence the term Zoophyte. So plant-like: indeed, are thenr forms, that the early observers regarded them as vegetating stones, and invented nuany theories to explain their growth.

They belong to a sub-kingdom termed Coelenteruta, now divided and subdivided by Professor Huxley into the following :-

$$
\begin{gathered}
\text { Septa, xic., } \times 5 \text { or } 6 . \quad \text { Septa, \&c., } \times 4 . \\
\text { Simple sofl-bodied. }
\end{gathered}
$$
1. Acrinid D.
Aclinea, Minyas.
1. BEROIDE.
Cydippe, Cestrm.

Compound-Skelelon spicular.
2. ZOANTIIDE.
2. ALCYONID 3.. Zounlihus. Alcyonium.

Compound-Skeleton sclerobasir.
3. Ansipathibe. Anlipathes.
3. GORgONidn. Gorgonia, 1sis, Curallium.

Compound and Simple-Skeleton thecal-continuous
4. Perforata.
Poriles, Madrepora.
4. TUві Рон п्. Tubipora.

5. TA BULATA.

5. RUGosa.

Millepora, Seriatopora. $\begin{aligned} & \text { Stauria, Cyullanonia. } \\ & \text { Cyathophylium. }\end{aligned}$

6. Aporosa.

Cyallina, Oculina.

Cystiphyllam.

Astraa, Fungia.

Opposed to all our common ideas of auimal life is this singular portion of creation. If we cut a limb off a tree, or sever that of an animal, these parts will wither and decompose, by passing into other forms of matter. Cut a tree across its niddle, and its natural symmetry is irreparably disfigured; slit it down its centre, aud it is destroyed: all animals so treated suffer instant death, with the exception of the polype tribe; for they will put forth new limbs, form a new head or tail, and if slit, become two separate perfect creatures. 
The seas which wash our shores swarm with beantiful forms of minute Polypes, having nearly the same organisation as the IIydra, but which are protected by an external horny integument. This peculiar covering sends forth shoots or buds, which are developed into new polypes, thus producing a compound animal; but the exercise of this gemmiparous faculty is prevented by a horny defence from effecting any other change than that of adding to the general size, and to the number of tentacles, prehensile fingers, and digestive sacs; yet the pattern according to which new polypes, and branches of polypes, are developed, is fixed and determinate in each species, and there consequently results a particular form of the whole compound animal, by which the species can be readily recognized

Cordylophora, a fresh water group of zoophytes, has been made the subject of an admirable memoir by Professor Alhman. (See Phil. Trans. 1853, "On the Anatomy and Physiology of Cordylophora.") In the interesting and pretty group, Corynidce, the tentacles are not arranged in a single transverse series, as in Hydra, but are usually scattered more or less irregularly over the surface of the polype - while in Tubularia there are two transverse rows of tentacles, an upper shorter, and a lower longer. The reproductive organs, which in Hydra are extremely simple, attain, in many of the Corynidce and Tubulariadce, to the condition of zooids, sometimes becorning detached, and swimming about frecly before discharging their products. These free zooids have always the form of a bell or disc, from whose centre a pyriform or oval body-either a closed sac or an open mouthed polype-is suspended; and they have been regarded as distinct animals, and grouped together with other forms of Hydrozoa, under the head of Medusce. The bell possesses considerable contractility, and at each contraction the water which fills its cavity is forced out, and the bell itself is thereby propelled in the opposite direction. In Tubu. laria a more completely medusiform body is developed, but is never detached.

In the Sertulariadce, the nnmerous digestive zooids, or polypes, developed from the original germ, remain always attached; but their most remarkable feature is the posses- 
sion of a "cell," surrounding the base of each polype, and usually capable of receiving it when retracted. The development of this cell at once distinguishes it from the not altogether dissimilar group, the Diphydoe and Physophoridce. In some Sertulariadae (Sertularia dynamena), the margins of the cells are converted into membranous valves; and in the genus Plumuluria, we find special orwans of offence.

The Diphydce are among the most remarkable and beautiful inhabitants of the ocean, to whose warmer regions, they, like the Physophoridee, are principally confined. They are free swimmers in the adult state, and probably, at all times of their existence ; but while actively locomotive by means of the contractions of the natatorial organs with which they are provided, they possess no special supporting apparatus, or "float," such as that developed in the Physophoridce. The tentacle of the Diphydae is a long filiform process of the peduncle, capable of great elongation and contraction; the terminal filament of which is closely beset with minute thread-cells, so that the whole must constitute a very efficient weapon of offence. Three genera of the Physophoridce are particularly worthy of notice: the Physalia, whose air-vesicle may attain the length of eight or nine inches, while its formidable tentacles hang down for as many feet, inflicting instantaneous death upon the smaller animals, and giving rise to no small amount of pain and irritation, even in man; and the Velella and Porpita, in which the texture of the air-vesicle is so exceedingly firm, as to give it the appearance of an internal shell, while its cavity is subdivided into numerous chambers. Originally, however, the "shell" of the Velella is a perfectly simple air-vesicle, like that of any other Physophorid. In the very peculiar genns Lucernaria,lovely "Lamp-polype," with little knobbed tentacles-we have a IIydrozoon, in which the polype occupies the centre of an expanded disc, the two presenting essentially the same structure and relations as in the Medusiform zooids of other divisions. In fact, a Lucernaria is in all essential respects comparable to an Aurelıa, or other Medusa fixed by the middle of the upper surface of its disc.

Mr. Husley prefers the term Lucernariadae to that of 
Medusce; and he does so "from the fact that the MedusideE of authors consists of two groups, the Naked-eyed (Gymnophthulmata) and the Covered-eyed (Stegnnophthalmata). Some of which, the Aurelia, is but a derivative zooid form of an animal, essentially resembling Lucernaria; while so far as regards the Naked-eyed Medusoe, we have no evidence that any genus except Aiginopsis, is other than the reproductive zooid of one of the Hydridce or Sertulariadce. It seems better, therefore, to avoid the term Medusa, as the denomination of an ascertained group, reserving it merely to denote the medusiform creatures of whose origin we are ignorant, but whose structure entitles them to a provisional place among the Lucernariadce. As our knowledge increases, we shall be able to arrange those Medusae which are the zooids of Hydridoe, Sertulariadae, Diphydae, or Physophoridce, under their respective groups, while the rest will form the sub-sections of the Lucernariadce; the first consisting of such forms as Aurelia and Rhizostoma, zooids developed by fission from fixed Lucernariadce; the second consisting of such forms as Eginopsis, free Lucernariadce, developed at once from the ovum, without any fixed state."

The Actinozoa are those Coelenterata in which the stomach is a sac suspended within and entirely distinct. from the body, from whose parietes it is separated by a portion of the general cavity of the body, which may receive the special denomination of "perivisceral cavity." The stomach communicates freely by an inferior aperture with the general cavity. A rough conception of the relations between the Actinozoa and the Hydrozoa may bo obtained by supposing the walls of the natatorial disc of a Lucernaria to become united with those of its central polype; it would then become, to all intents and purposes, an Actizozoon. As the Hydra is the type of the Hydro$z o a$, so the "Sea-anemone" ( $A$ stinia) is considered to be the type of the Actinozoa. As in the Hydrozoa, the body of the Actinia is essentially composed of two layers, a superficial layer, composed of polygonal cells, frequently detached and renewed again, beneath which lies a granular layer; whilst in the deep dermal layer two sets of muscular fibres are found,--a superficial circular, and a deep 
longitudinal set: both are flattened, and exhibit no transverse striation. In some Actinice, such as $A$. mesembryanthemum, bright blue sacs are placed at the edges of the oral

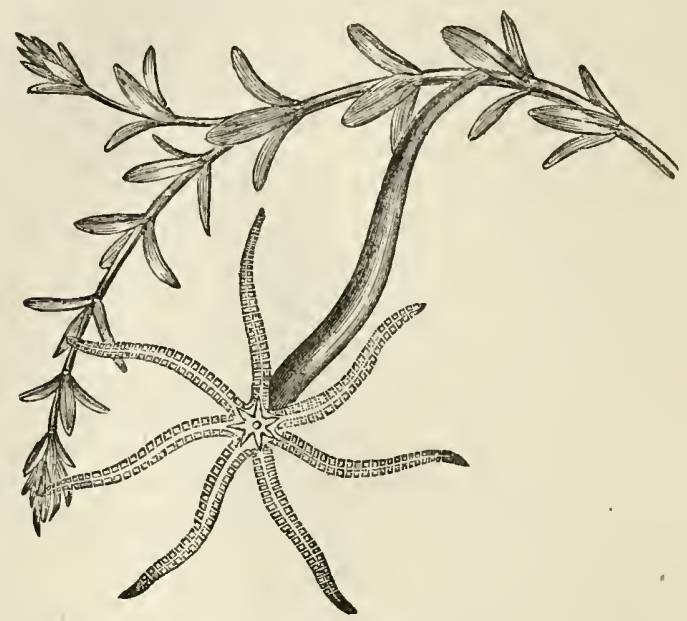

Fig. 231.-Hydra, with tentacles displayed and magnified, adhering to a statk of. Anacharis alsinostrum.

disc, while in A.gemmacea, A. sessilis, \&c. clear spots are scattered over the integument, which have been regarded as apertures or tubercles; M. Hollard, however, states, that these are imperforate Ampulloe, possessing a kind of bilabiate mouth, surrounded by a sphincter-like arrangement of muscular fibres. Any foreign body introduced into these ampullæ is seized and forcibly held; or if the finger be placed within reach, it gives the sensation of a very fine rasp passing over it. The margins of the radiate tentacles of the young animal are surrounded by cilia; the gastric epithelium is likewise ciliated, and doubtless secretes a powerfully solvent fluid. The majority of the Actinia are oviparous, the young being developed from ova within, and evacuated by the mouth: they are also capable of multiplication by budding, and occasionally by fission. while their power of restoring themselves after muti 
lation appears to be as great as that possessed by the IIydra. ${ }^{2}$

The great majority of the Actinozoa exhibit a structure closely corresponding with that of the Actiminos; but from the manner in which they grow up into compound masses of associated Zooids, produced by gemmation or by fission, they stand in nearly the same relation to Actinia, as the compound Hydrozoa to Hydra.

Sir John Dayell believes that Actinice conquer their prey by mere strength; this is doubtless the case, as from experience we find nothing like a stinging property belonging to the tentacles; nor are there any poison vesicles attached thereunto. The tentacles appear to be armed with rows of spines, which give a clinging and slight rasping sensation when the finger is thrust against them; and by the same means they are able to obtain a firm hold of any smaller animal that falls within their reach. Animals with a hard case or shell seem to escape from their clutches without having sustained the smallest injury. The same remarks apply to the Hydra; they have neither the power to sting or benumb their prey, as asserted by many authorities. It has been said that certain minute organs found in polypes, and variously styled thread capsules, filiferous capsules, or urticating cells, are stinging organs. This thread Agassiz likens te a lasso thrown by the polype to secure its prey. Mr. Lewes writes:- "On a survey of the places where these 'urticating cells' are present, I stumbled upon an unlucky fact, and one likely to excite our suspicion. They are present in a few jelly-fisls which urticate, in Actinioe which urticate, and in all polypes, which, if they do not urticate,

(1) The Author's aquarium affords at this time (1856) a curious illustration of increase both by budding and fissuration, in a beautiful $A$. dianthus. In the first case an offset was seen to protrude; it resembled a small bud near the foot; this increased until it attained to a perfect animal of a considerable size, when it became detached. In another, the animal, after having remained for several weeks firmly adherent to the side of the glass, with a part of its disc out of the water, by a great effort tore itself away, leaving six small pieces behind, attached to the glass. For some days these pieces served only to mark so many spots, but in about a week rudimentary tentacles were observed to be sprouting out from each piece, which went on rapidly increasing, and ultimately six perfect animals resulted therefrom. The repair of the marginal portion of the disc of the parent animal was completed in a few days, and it suffered no injury whatever from its selfmutilation. This furnishes a proof, if one were wanting, of the hydrozoid character of this class of animals. 
are popularly supposed to do so, and at any rate possess some peculiar power of adhesion. In all these cases, organ and function may be said to go together; but the cells are also present in the majority of jelly-fish which do not urticate, in Eolids which do not urticate, and in Planarice which do not urticate. Here, then, we have the organ without any corresponding function; urticating cells, but no urtication. It thus appears that animals having the cells, have none of the power attributed to the cells; and that even in those animals which have the power, it is only present in the tentacles, where the cells are much less abundant than in parts not manifesting the power; the conclusion, therefore, presses on us, that the powor does not depend upon these cells. When at rest, and in an ordinary natural state, the animal is never seen to dart out these threads, nor upon capturing his prey; it is only when some force is used to dislodge him from some spot to which he has securely attached himself, that he presses or squeezes out these threads; more for the purpose of compressing himself into a closer and smaller mass, to add to the difficulty of detaching him.

"Actinice do not effect their preparation of nutriment by chemical means; that is, they do not, in the strict sense of the term, digest, but simply derive nourishment by mechanical pressure, exerted upon any particle of food that they may draw into their stomachs. This has been proved by experiments made after the manner of Reaumur. A small piece of meat having been put into a quill, and allowed to remain in the stomach of the Actinia sufficiently long, and then withdrawn, no solvent fluid is found to have acted upon it. When placed under the microscope the muscle fibres are not at all disintegrated, and the striæ are as perfect as before the experiment, with the exception of a pulpiness and loss of colour, as in any ordinary mechanical maceration.

"Light has been thrown upon the reproductive system of the Actinice by M. Jules Haime, in the Annales des Sciences Naturelles, 1854, $4^{\text {ieme }}$ série, tom. i.; which contains accurate and detailed descriptions and plates of the disposition of ova and spermatozoa in the Actinioe. To find the ovaries it is only necessary to take a live animal, 
and with a rapid, but not deep incision, lay open the envelope from the outside; a series of convoluted bands will bnlge through the opening, but if these are quickly brushed aside, certain lobular or grape-like masses will be perccived, darker in colour, and almost entirely hidden by these bands, but growing from the wall of the envelope. They do not appear to have a fixed locality, as they may be found near the base, about the centre, or close to the disc ; it is, therefore, sometimes necessary to make three or four incisions before detecting them; once seen, they will be easily distinguished from the convoluted bands, although very difficult to remove them without removing some of the bands."

The manner in which the aggregation of zooids, constituting a compound coral, is developed from the primarily solitary Actinozoic embryo, exercises an all-important influence upon the form of the Corallum. Sometimes, as in most Turbinolida, neither gemmation nor fission ever take place. In the Oculinida, gemmation alone occurs, and in these and other Actinozoa, the development of buds takes place either from the base or from the sides of a corallite, or from the cœnosarc. Certain of the extinct Rugosa, however, exhibit calicular gemmation, the buds being developed from the oral disc or cup, which can hardly have retained any active vitality. The massive coralla of some Cyathophyllide, thus standing like inverted pyramids, all the buds supported upon the narrow surface of the primary zooid, have a very singular and striking aspect. The Beroidae appear at first to differ very widely from the type of structure which prevails among the other Actinozoa, but a close examination of any of their forms suffices to demonstrate the justice of the conclusion advocated by Frey and Leuckart, as to their essential identity with Actinia. The Cydippe, which abounds mpon our own coasts, affords, from its small size and extreme transparency, an excellent subject for the student of $\mathrm{Be}$ roida. It is impossible here to enter into the varieties of form presented by the Beroida, and which chiefly arise from the development of lateral lobes, a process which is carried to so great a length in Cestum that the body becomes ribbon-like. The Beroidce and the Actinido 
inhabit the shallower and even aeeper parts of all seas; Alcyonidae and Gorgonidce are found at considerable depths; Corallium and Gorgonia abound both in cold and warm latitudes. The Perforata and Tabulata almost exclusively haunt the shallower parts of warm seas, but the Turbinolido extend into very cold regions. The whole group of the Rugosa is now extinct, only one genus, Holocystis, having survived even the palæozoic period. Not only heat, but light, and probably rapid and effectual aëration, are essential conditions for the activity of the reef.building Actinozoa. Different species of corals exhibit great differences as to the rapidity of growth, and the depth at which they flourish best; and no one must be taken as evidence for another in these respects. Certain species of Perforata, Madreporida, and Poritida, appear to be at once the fastest growers, and those which delight in the shallowest waters. The Astrceidce among the Aporosa, and Seriatopora among the Tabulata, live at greater depths, and are probably slower of inerease. The most careful accounts of the structure of corals extant, are from the pens of M. M. Milne Edwards and Haime, published at various times in the Annales des Sciences $\Lambda^{\top}$ aturelles, in the Mémoires $d u$ Museum, and in the publications of the Paloontographical Society; and by Mr. Darwin, whose beautiful work on Coral Reefs will amply repay perusal.

With these brief introductory observations, the principal part of which have been derived from Professor Huxley's lectures, we proceed to direct the reader's attention to some of the more interesting generic forms.

HYDRA, FRESH-WATER POLYPE. - In polypes of this family the body generally consists of a homogeneous aggregation of vesicular granules, held together by a sort of glairy intercellular substance, and capable of great extension and contraction; so that the creature can at pleasure assume a great variety of forms, exteuding its body and tentacles until the latter become so fine as to be almost invisible, and again retracting itself until it acquires the appearance of a small gelatinous mass. The tentacles which surround the anterior extremity are imegular in uumber; they are capable of extension to a very great 
length when seeking for prey; and on conning in contact with any object floating through the water, they immediately twine round it, and convey it to the month. In some genera the tentacles appear to be tubular, the internal cavity being continuous with that of the stomach. The mouth is situated in the centre of the circle of tentacles, and leads directly into a simple digestive cavity.

Hydree are found in ponds and rivulets, adhering to the leaves of aquatic plants, or twigs and sticks that have fallen into the water. When stretched out, they resemble pieces of hair, from a quarter to three-quarters of an inch in length. Some are of a light-green colour, and others brown or yellow; that is, the five varieties found in England. It received its name from its several long arms being supposed to resemble the fifty-headed water-serpent called Hydra, which was destroyed by Hercules in the lake of Lerna, as we are informed in fabulous history. Leeuwenhoek, in 1703, first drew attention to the Hydra; and in 1739, M. Trembley from the Hague, more accurately described its habits.

Polypes are not vegetarians; M. Trembley fed IIydra on minced fish, beef, mutton, and veal; they are voracious and active in seizing worms and larvæ much larger than themselves, which they devour with avidity. They carefully and adroitly bring their food towards their mouth; and when near, pounce upon it with eagerness. To make up for the want of teeth, the mouth enlarges to receive the food brought to it by the arms that have twined around the sacrifice. The red worm that tinges the mud of the Thames appears to be the dainty dish they like best to have set before them. Dr. Mantell saw the lasso of a polype thrown over two worms at the same time; yet they could not escape, and lost all power of motion.

Dr. Johnston states: "Sometimes it happens that two polypes will seize upon the same worm, when a struggle for the prey ensues, in which the strongest gains, of course, the victory; or each polype begins quietly to swallow his portion, and continues to gulp down his half, until the mouths of the pair near, and come at length into actual contact. The rest that now ensues, appears to prove that they are sensible of their untoward position, from which 
they are frequently liberated by the opportune break of the worm, when each obtains his share; but should the prey prove too tough, woe to the unready! the more resolute dilates the mouth to the requisite extent, and deliberately swallows his opponent; sometimes partially, so as, however, to compel the discharge of the bait; while at other times the entire polype is engulfed! But a yolype is no fitting food for a polype, and his capacity of endurance saves him from this living tomb; for, after a time, when the worm is sucked out of him, the sufferer is disgorged with no other loss than his dinner."

The orgau of prehension, which is called the hasta, consists of a sac opening at the surface of the tentacle, within which, at the lower portion, is placed a saucer-shaped vesicle, supporting a minute ovate body, which again bears a sharp calcareous piece called the sagitta, arrow. This can be pushed out at the pleasure of the animal, serving to roughen the surface of the tentacle, and afford a much firmer lold of its living prey. The polype increases rapidly: a portion of the body swells, a young one puts forth its head from the part, its arms begin to grow, it then is industrious in catching food; its body, communicating with that of its parent and participating in the fears and actions of its progeuitor, is finally cast off to wander the world of waters. Sometimes, ere yet free from parental attachment, it has two generations on its own hody. Four or five offspring are thus produced weekly. But the most extraordinary circumstance in respect to this creature is thus described by M. Trembley : "If one of them be cut in two, the fore part, which contains the head and mouth and arms, lengthens itself, creeps, and eats on the same day. The tail part forms a head and mouth at the wounded end, and shoots forth arms nore or less speedily, as the heat is favourable. If the polype be cut the long way throngh the head, stomach, and body, each part is half a pipe, with half a head, half a mouth, and some of the arms at one of its ends. The edges of these half pipes gradually round themselves and unite, heginning at the tail end; and the half mouth and half stomach of each becomes complete. A polype has been cut lengthways st seven in the morning, and in eight hours afterward 
each part has devoured a worm as long as itself." Still more wonderful is the fact, that if turned inside out, the parts at once accommodate themselves to their new condition, and carry on all their functions as before the accident. Indeed, this animal seems so peculiarly endowed with the germs of vitality in every part of its body, that it may be cut into ten pieces, and everyone will become a ncw, perfect, living animal. This seems bordering on the vegetable kingdom, in which it is common to propagate by means of slips from the mature shrub.

The best known of the British species are Hydra vulgaris, Common polype, H. viridis, Green polype, H. Fusca, Brown polype, $H$. verrucosa, and $H$. lutea.

Every reflecting person who reads even the slight sketch we have given of this polype must be struck witl astonishment at a creature so primitive in structure, possessing the actions, sensations, and powers of higher organised beings. The stomach is but one simple structureless membraue or cell, the external surface-cells forming a kind of double skin, the inside a mere wall of cells runuing crosswise, possessed of a velvet-like surface, and red or brown coloured grains held together by a glutinous substance. This singular formation, with some of the functions of animal life, has led to mauy learned surmises and discussions tending to the most important results in the science of physiology.

Tubulariade.-The Tubular or Vaginated Polypes are of an arborescent appearauce; the animals live near the ends of branches, and are found attached to stones, seaweeds, and shells." The Tubularia indivisa, "Iudivided tubes," are found on shells, with a living head resembling a fine scarlet cluster of blossoms. Ellis says, "they seem part of an oat-straw with the joints cut off." At the summit protrudes the scarlet-coloured polypes, well furnished with tentacula, and connected witl a pinkish fluid that fills the tubes. It was in these that Dr. Roget discovered the singular peculiarity of a circulation, similar to that seen in many plants. He says, "In a specimen of the Tubu-

(1) These are grouped with Hydroida, and at the head of the family stand Van Beneden's IIydractinia; and Gaertner's Coryne. 
laria indivisa, when magnified one hundred times, a current of particles was seen within the tubular stem of the polype, strikingly resembling, in steadiness and continuity of its stream, the vegetable circulation in the chara. Its general course was parallel to the shightly spiral lines of irregular spots on the surface of the tube, ascending on the one side, and descending on the other; each of the opposite currents occupying one-half of the circumference of the cylindric cavity. At the knots, or contracted parts of the tube, slight eddies were noticed in the currents; and at ench end of the tube the particles were seen to turu round, and pass over to the other side.

"The particles carried by it present an analogy to those of the blood in the higher animals of one side, and of the sap of vegetables on the other. Some of them appear to be derived from the digested food, and other's from the melting down of parts absorbed; but it would be highly interesting to ascertain distinctly how they are produced, and what is the office they perform, as well as the true character of their remarkable activity and seemingly spontaneous motious; for the hypothesis of their individual vitality is too startling to be adopted without good evidence."

Respecting the singular property of the head dropping off, Tubularia indivisa, Sir J. G. Dalyell observes, "The head is deciduous, falling in general soon after recovery from the sea. It is regenerated at intervals of from ten days to several weeks, but with the number of external organs successively diminishing, though the stem is always elongated. It seerns to rise within this tubular stem from below, and to be dependent on the presence of the internal tenacious matter with which the tube is occupied. A head springs from the remaining stem, cut off very near the root; and a redundance of heads may be obtained from artificial sections. Thus, twenty-two heads were produced through the course of 150 days from three sections of a single stem."

Included in this family are the $T$. ramous, $T$. ramea, the branched pipe-coralline, with its dark brown stem terminating in clusters of red and yellow polyps; and the 
Hermia Glandulosa of Dr. Johnston, who says-"I found the name in Shakspeare :

'What wicked and dissembling glass of mine,

Made me compare with Hermia's sphery eyne ?"

The fancy that the glands which surround the heads were the guardians of the animal, its "sphery eyne," suggested the name here adopted. These polypes are adherent by a tubular fibre, and creep along the surface of the object on which they grow; they are seldom an inch in height, irregularly branched; the stem filiform, tubular, horny, sub-pellucid, wrinkled, and sometimes ringed at intervals, especially at the origin of the branches, each of which is terminated with an oval or club-shaped head of a reddish colour, and armed with short scattered tentacula,tipped with a globular apex. The ends of the branches are not perforated, but completely covered with a continuation of the horny sheath of the stem. The animal can bend its armed hands at will, or give to any separate tentaculum a distinct motion and direction ; but all its movements are very slow.

The beautifulo little Coryne coryne. Coryne Stanridia, slender stauridia, "Slender coryne," and magnified 200 diameters.

(fig. 232 , No. 1), is thus described by Mr. Gosse: "It was found by me adhering to the footstalk of a Rhodymenia, about which it creeps in the form of a white thread; by placing both beneath the microscope, this thread appeared cylindrical and tubular, perfectly trausparent, withont wrinkles, but permeated by a central core, apparently celSular in texture, and hollow; within which a rather slow 
circulation of globules, few in number, and remote, is perceived. It seuds off numerous branches; the terminal head of which is oblong, cylindrical, rounded at the end. At the extreme point are fixed four tentacula of the usnal form, long, slender, and furnished with globular heads; one of which is shown at No. 2, detached, and more highly magnified. It is much infested with parasites : a vorticella grows on it, and a sort of vibrio; the latter in immense numbers, forming aggregatcd clusters here and there; the individuals adhering to each other, and projecting in bristling points in every direction. These animalcules vary in length; some being as long as 1-80th of an inch, with a diameter of 1-7000th of an inch. They are straight, equal in thickness throughout, and marked with distinct transverse lines; they bend themselves about with considerable activity, and frequently adhere to the polype by one extremity, while the remainder projects freely."

Some of this family attain a considerable size; the Corymorpha nutans, one of the most beautiful of the group, attains a length of four inches and a half. of the beauty of its appearance, Forbes, who discovered it in the British seas, speaks in the following terms: "When placed in a vessel of sea-water, it presented the appearance of a beautiful flower. Its head gracefully nodded (whence the appropriate specific appellation given it by Sars), bending the upper part of its stem. It waved its long tentacula to and fro at pleasure, but seemed to have no power of contracting them. It could not be regarded as by any means an apathetic animal, and its beauty excited the admiration of all who saw it." The general colour of the creature is a delicate pink, with longitudinal lines of brownish or red dots. The tentacles are very numerous and long, and of a white colour; and the ovaries, which are situated immediately above the circle of tentacles, are orange. Most of the Tululariadce inhabit the sea; one species, the Cordyloplora lacustris, is found in the dock of the Grand Canal, Dublin, in water which is perfectly fresh.

Sertulariade. - This interesting and beautiful family of pclypes derive their name from their plant-like appearance, and are readily attainable on our own sea-shores. 
Linnæus made a large genus of them; but Lamarck considerably reduced his classification. There are seventeen British species, which Dr. Fleming proposes to divide into two groups, with stems simple or compound.

The tentacles of Sertularia are abundantly supplied with cilia; the cells are pitchershaped, arranged alternately, or in pairs obliquely, not exactly opposite, on the stem and branches of the polypidom, which is horny. Lamouroux classed with this family Thoa; of which there have been several kinds found i. Great Britain. The name is supposed to be derived from the Greek word for sharp; but we think, with Dr. Johnston, that it more probably is a mis-spelling of Thoe, one

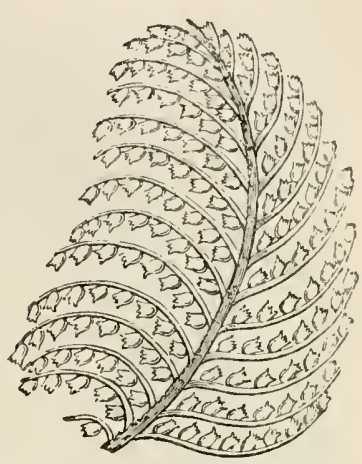
of the Nereids, nymphs of the sea. They are generally of a brown and yellow colour, branched, and from an inch and a half to six inches in height.

Sertularic pumila. - This is parasitic, and spreads its brown-coloured shoots over various fuci and sea-shells; but rarely attains more than half an inch in height. Stewart says:- "This species, and probably many others, in some particular states of the atmosphere, emits a phosphorescent light in the dark. If a leaf of the fucus serratus, with Sertularia upon it, receives a smart stroke in the dark, the whole is most beautifully illuminated, every denticle seeming to be on fire."

On the south-eastern coast of England the most common kind found is the Sertularia setacea, or operculata, SeahairCoralline: it reaches from six inches and upwards in height, and grows in tufts, like bunches of hair. The stem and branches seem composed of separate pieces, fitting accurately into each other, and terminate in a star-like head, from which radiate the tentacles. Mr. Lister was observing a living specimen, when a littlo 
globular animalcule swam rapidly by one of the expanded polypes; the latter immediately contracted, seized the globule, and brought it to the mouth or central opening by its tentacula; these gradually opened sgain, with

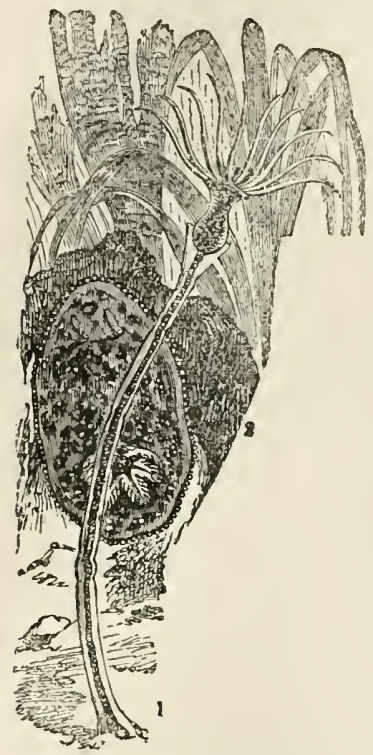

Fig. 234. the exception of one, which remained folded, with its extremity on the animalcule. The mouth instantly seemed filled with cilia, which, closing over the prey, was carried slowly down its stomach; here it was imperfectly seen, and soon disappeared.

Appertaining to this family are Dr. Fleming's Thuiarea, so named by him from their resembiance to a cedar-tree; some kinds look more like a knobbed thornstick with a bottle-clearer at the top; others resemble a fir-tree. Antennularia are so called from their resemblance to a lobster's antenna. They are plentiful on the north-eastern coast of England and the coast of Ireland, brown in colour, and covered with I, Plumularia pinnata, Fenther poly ps hair-like little branches; and 2, Doris tuberculata, Sea slug. as the hairy process is continued up its jointed stem, it is sometimes denominatea Sea-beard. Dr. Hassall's Coppinia is another very interesting species.

The Plumularia, so named from their shoots and offsets being plumose, are an extensive and beautiful family. Professor Grant thus describes the Plumularia falcata: "The Sickle Coralline is common in the deeper parts of the Frith of Forth; its vesicles are very numerous, and its ova are in full maturity at the beginning of May. The ova are large, of a light-brown colour, semi-opaque. nearly spherical, composed of minute transparent granuler. 
ciliated on the surface, and distinctly irritable. There are only two ova in each vesicle; so that they do not require any external capsules, like those of the Campanularia, to allow them sufficient space to come to maturity. On placing an entire vesicle, with its two ova, under the microscope, we perceive through the transparent sides the cilia vibrating on the surface of the contained ova, and the currents prodnced in the fluid within by their motion. When we open the vesicles with two needles, in a drop of sea-water, the ova glide to and fro through the water, at first slowly, but afterwards more quickly, and their cilia propel them with the same part always forward. They are highly irritable, and frequently contract their bodies so as to exhibit those singular changes of form spoken of by Cavolini. These contractions are particularly observed when they come in contact with a hair, a filament of conferva, a grain of sand, or any minute object ; and they are likewise frequent and remarkable at the time when the ovum is busied iu attaching its body permanently to the surface of the glass. After fixing themselves, they become flat and circular, and the more opaque parts of the ova assume a radiated appearance; so that they now appear, even to the naked eye, like so many minute greycoloured stars, having the interstices between the rays filled with a colourless transparent matter, which seems to harden into horn. The grey matter swells in the centre, where the rays meet, and rises perpendicularly upwards surrounded by the transparent horny matter, so as to form the trunk of the future zoophyte. The rays first formed are obviously the fleshy central substance of the roots; and the portion of that substance which grows perpendicularly upwards, forms the fleshy central part of the stem. As early as I could observe the stem, it was open at the top; and when it bifurcated to form two branches, both were open at their extremities ; but the fleshy central matter had nowhere developed itself as yet into the form of a polype. Polypes, therefore, are not the first formed of this zoophyte, but appear long after the formation of the root and stem, as the leaves and flowers of a plant."

Attached to fucus and shells in abundance on the southern coast of England, may be found the Plumularia cristata. It 
is affixed by a horny, branching, interlacing, tubular fibre to the object on which it grows. At different parts there nre plumose shoots, usually about an inch in height. The cells are of a yellow colour, set in the stalk, of a bellshape, and are compared to the flower of the lily of the valley; the rim is cut into eight equal teeth; the polype minute and delicate, tentacles ten and annulate, with a mouth infundibuliform in shape.

"Each plume," says Mr. Lister, in reference to a specimen of this species, "might comprise from 400 to 500 polypes;" "and a specimen," writes Dr. Johnston, " of no unusual size, before me, has twelve plumes, with certainly not fewer cells on each than the larger number mentioned: thus giving 6000 polypes as the tenantry of a single polypidom! Now, many such specimens, all united too by a common fibre, and all the offshoots of one common parent, are often located on one sea-weed, the site then of a population which nor London nor Pekin can rival."

Plumularia pinnata, "Feather polyp," (represented magnified in fig. 234, No. 1,) is as remarkable for the elegance of its form, as its likeness to the feather of a pen. It serves not among the denizens of the deep the same purpose as its earthly prototype; nature writes her works in hieroglyphics formed by the objects themselves. It is plumous, and the cells in a close row, cup-like, and supported on the under side by a lengthened spinous process.

An interest pervades the valuable work of Dr. Johnston, arising from the circumstance that the plates and woodcuts which adorn the volume are, with few exceptions, engraved from drawings made for it by Mrs. Johnston, who alsn engraved several of them ; and the Doctor states, he conld not have undertaken the history without sucl assistance. From this devotion too, and understanding of the subject, it was natural, when an opportnnity presented itself, to write in the catalogue of Zoophytes a lasting memorial of his "colleague :" and thus is written the graceful compliment of the beantiful Plumularia Catharina: "Catharine's Feather," whose stem is plumous, pinnæ opposite, bent inwards; cells distant, campanulate, with an even margin; vesicles scattered, pear-shaped, smooth. 
Found in old shells, corallines, \&c., in deep water; in Frith of Forth and in Berwick Bay, by Dr. Coldstream.

The sub-families, Campanularia and Laomedea, are also frequently found on our shores; they possess a simple circle of cilia on their feelers or arms, with pitchershaped cells on stalks that branch, twist, or climb on an axis.

Campanularia volubilis, "Twining polype," is the commonest of the family : it is parasitical, and infests the antennæ of crabs; its stem is filiform, and at the end of its slender branches are situated the cells containing the polypes. The polype itself is sleuder when protruded, somewhat like Plumularia pinnata, and becomes dilated at the base into a sort of foot which spreads over the diaphragm; widening again at the top, where it fills the mouth of the cell, and gives origin to about twenty slender tentacula, set in two or three series. From the central space, which is surrounded by tentacles, a large fleshy nouth protrudes, somewhat funnel-shaped, with lips, endowed with the power of protrusion and contraction; these appear to be very sensitive. Mr. Gosse found the species in great abundance round Small-mouth Caves.

The Campanularia gelatinosa and its beautiful bellshaped cells, out of which the animals protrude, giving the semblance of a green flower on a delicate pink stalk. It is indeed an interesting object, and currents may be seen in its tubes. Dr. Johnston says, "On Saturday, May 29th, 1837, a specimen of Campanularia gelatinosa was procured from the shore; and after having ascertained that the polypes were active and eutire, it was placed in a saucer of sea-water. Here it remained undisturbed until Monday afternoon, when all the polypes had disappeared. Some cells were empty, or nearly so; others were half-filled with the wasted body of the polype, which had lost, however, every vestige of their tentacula. The water had become putrid, and the specimen was therefore removed to another vessel with pure water, and again set aside. On examining it on the Thursday, June lst, the cells were evidently filling again, although no textacula were visibly protruded : but on the afternoon of Friday, June 2d, every cell had its polype, complete, and 
displayed in the greatest perfection. Had these singular facts been known to Linnæus, how eagerly and effectively would he have impressed them into the support of his favourite theory! Like the flowers of the field, the heads, or 'flores,' of these polypidoms expand their petaloid arms, which after a time fall, like blighted blossoms off a tree; they do become 'old in their youth,' and, rendered hebetous and unfit for duty or ornament by age or accident, the common trunk throws them off, and supplies its wants by ever-young and vigorous growths. The phenomena are of those which justly challenge admiration, and excuse a sober scepticism, so alien are they to all we are accustomed to observe in more familiar organisms. Faithful observation renders the fact undeniable ; but besides that, a reflection on the history of the Hydra might almost have led us to anticipate such events in the life of these Zoophytes. 'Verily, for mine own part,' observes Baker, 'the more I look into Nature's works, the sooner am I induced to believe of her even those things that seem incredible."

ActiNIADE.-All persons accustomed to wander by the sea-shore must have admired the livid green, dark littlo jelly-masses adhering to the rocks, - called Actinia, from a Greek word signifying a ray,-and left in some little pool by the ebbing tide, living as they do principally within high and low water mark, and expanding their broad surfaces and fringing feelers to the finger of inquisitive youth, so often thrust into the centre, to feel the effect of the suction and rasping, as the poor animal draws itself up in the form of a little fleshy hillock.

Some few years ago it might have been necessary to explain what we meant by an Actinia, or "Sea-anemone " thanks to the universal distribution of aquaria, this beautiful class of animals is no longer unfamiliar to the world. Nevertheless, much as people read, and hear, and write, and observe in the matter, we do not hesitate to say that the natural arrangement of these animals is as little known in the world of naturalists, as their very existence was a short time ago to the world at large. A familiar instance of this position may be given in a few words. Dr. Johnston (Hist. Brit. Zooph.) describes three distinct 
Actinia, under the names of $A$. troglodytes (the Cavedweller), $A$. viduata, and A. Anguicoma (the Snakylocked). Mr. Gosse, in his Devonshire Coast, makes $A$. viduata synonymous with $A$. anguicoma; and gives a drawing and a description of an anemone which he calls anguicoma, and which closely resembles undoubted specimens of Johnston's A. troglodytes, Many objections might be taken to Mr. Gosse's description of species, which he makes out from the number of their tentacles, although found in company with each other, and, as he justly remarks, are of "the same size and form."

Of the voracity of the actinia many remarkable statements have been made known; it may nevertheless be kept in the aquarium for many months, if supplied with

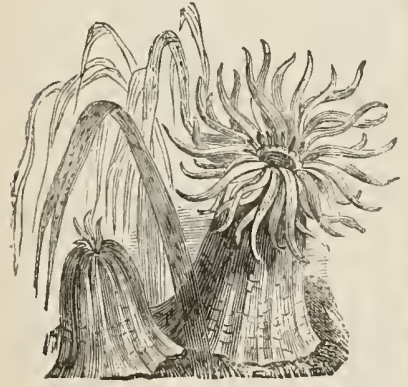

1

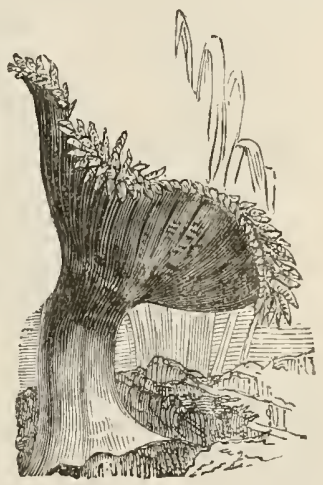

2

Fig. 235.

1, Actinia rubra Sea marigold, near which is one shown retracted. 2, Actinia bellis, Daisy sea-auemone (side view).

water containing particles of organic matter. Although the several structures of actinia admit of being resolved into two foundation membranes, an ectoderm and an endoderm, yet each of these, more especially the former, manifests a tendency to differentiate into secondary layers, so that several apparently distinct tissices are recognisable in the body of the fully-formed animal. Both membranes have their free surfaces more or less covered with cilia 
and the margin of the disc is furnished with a series or white or bright blue specks, which some observers believe to be rudimentary organs of vision; but they are rather to be regarded as sac-shaped prolongations of the outer layer, A transverse section of the

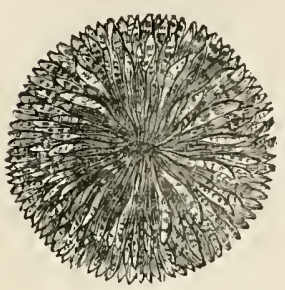

Fig. 236.-Actinia bellis, scen from above with its crown of tentacles fully expanded.

body of the actinia exhibits two concentric tubes, the outer being constituted by the body-wall, and the imer by the digestive sac. The wide space which intervenes between these tubes is divided by a number of radiating partitions, or "mesenteries," arriving at definite intervals from the inner surface of the body wall. To the face of the mesenteries are attached the reproductive organs, which occur $\mathbf{s}$ thickened bands of a reddish tint, at certain periods filled with ova. The animal has the power of effecting considerable alterations of form, as well as of locomotion; although if well supplied with food it attaches itself so firmly as not to be removed without laceration of its base.

Allied to the family Actinia are those laminated, inverted pyramidal looking bodies, Fungia, commonly called "Sea-mushrooms," often found in great variety. The colour of the polypidom is white, of a flattened round shape, made up of thin plates or scales, embedded in a translucent jelly-like substance, and within which is a large polype; the foot-stalk, by means of which the animal is attached to the rock whereon it lives, is of a calcareous nature. Ellis says: "The more elevated folds or plaits have borders like the denticulated edge of needlework-lace. These are covered with innumerable oblong vesicles, formed of a gelatinous substance, which appear alive under water, and may be observed to move like an insect. I have observed these radiating folds of the animal, which secrete the lamellæ, and which shrink between them when the animal contracts itself on being disturbed. They are constantly moving in tremulous undulations; but the resicles appeared to me to be air- 
vessels placed along the edges of the folds, and the vesicles disappeared when the animal was touched."

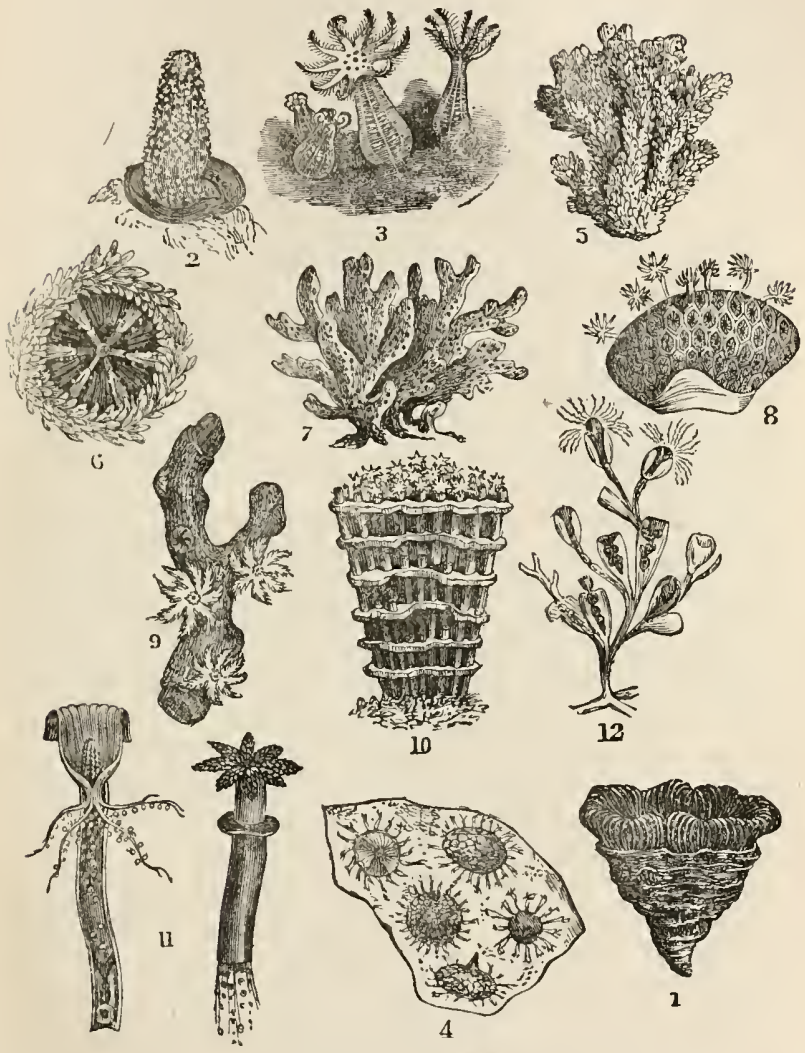

Fig. 237.-Aetinozow

]. Fungia agariciformis. 2, Aleyonium, Cydonium Mulleri. 3, Cydonium, polypes protruding and tentacles expanded, others closed. 4, Zoantharic viewed from above. 5, Madrepore Abrotanoide. 6, Madrepore, cell slightly magnified, showing intemal structure. 7, Corallidoe; Coral, 8, Coral, polypes protruding from the cells. 9, Gorgonia nobilis, polypes expanded. 10, Tubipora musica. 11, a tube of same, with polype expanded, and one cut longitudinally to show internal structure. 12, Sertularia, polypes protruded; and others witldrawn into polypicioms. 
Madreporide. - Madrepores, Mother pores, "tree corals," differ from other corals in not having a small skeleton, but one inducted by numbers of small cells for the residence of the living animal : these are very visible in the Madrepore muricata, when the polype is dead and decomposed; but most distinct in the Oculina ramea, as they are-situated at the apparently broken stumps that branch from the trunk of the skeleton (fig. 237, No. 5). Every branch is seen to be covered with multitudes of small pits or dots, scarcely visible to the unassisted vision; but, when viewed under the microscope, are found to be cells of the most beautiful construction, remarkable alike for their mathematical regularity and the exquisite fineness of the materials employed in their composition. A magnified drawing of a cell is given at No. 6. The living polypes are exquisite objects for a low power ; their varying colours adding to the richness of the hues covering the bed of the ocean.

Asteroides.-A group of Zoophytes received the name of Asteroida from the polypes presenting the form of a star. The fleshy mass is supported by hard calcareous spicula; some having thick branching processes, performing the part of the skeleton in the human frame. This central internal support is usually denominated the axis. The fleshy mass, or covering, is possessed of sensation, and is ramified by vascular tubes and canals for the sustenance of the animal, and carrying on its vital functions. Included in this genera are Gorgoniada, Pennatulida, Alcyonidce, Isidoe, and Tubiporidce.

The term coral, or corallum, is restricted to the hard structures deposited in the tissues or by the tissues of the Actinozoa. The whole of this class, however, which are thought to possess a framework called a "coral," are not coralligenous. The Ctenophora, and several species, as the soft-bodied non-adherent Zoantharia, deposit no corallum. There are two kinds of such structure, one called the "sclerobasic" corallum, a true tegumentary excretion, formed by the successive growths from the outer surface of the ocderon; and another, the "scleroaermic" corallum. deposited within the tissues of the animal. Two principal modifications of form distinguish the sclerobasis: in 
some it constitutes a free axis, virgate or primately divided and varying in thickness; in others it is attached, simple or branched, and plant-like, as in Gorgonidac, from which circumstance the name of "Sea-shrubs" has been applied to them. In the Gorgonia we have, in addition to the basal corallum, a deposition of tissue secretions, sclerodermic spicules appear within the substance of the investing membrane, and when the animal is dried, and the soft parts washed away, a thin layer of calcareous spicules is seen adhering to the horny sclerobasis. M. Valenciennes made out five kiuds of spicules, or sclerites, which he severally designates capitate, fusiform, massive, stellate, and squamous. These spicules form interesting objects for the microscope, mounted dry or in balsam. "The parts of a typical coralite are these : first, an outer wall, or 'theca,' somewhat cylindrical in form, terminating distally in a cup-like excavation, or 'calice,' and having its central axis traversed by a columella. The space between this and the theca is divided into loculi, or chambers, by a number of radiating vertical partitions, the septa. These do not, in certain instances, quite reach the columella, but are broken up into upright pillars or pali, arranged in one, two, or more circular rows termed 'coronets;' all of which are best brought into view by transverse section." Longitudinal division of a corallite shows certain modifications and changes in the partitions, or dissepiments ; and the septa are seen to be covered with "styliform or echinulate processes," which meet to form "synapticulæ or transverse props, extending across the loculi like the bars of a grate." Nevertheless, there is no difficulty in recognising the close resemblance that sucn an organism presents to the typical Actinia, and they have accordingly been classed with the Actinozoa.

The Gorgonidat are permanently fixed, as are many other corallitic actinozoa, and multiply by continuous gemmation. As to their muscular system, most of them appear to be well endowed in this particular. Pennatulida possess so much muscular contractibility, that Mr. Darwin relates, that on the south coast of America he observed "a Sea-pen which, on being touched, forcibly drew back into the sand some inches of its compound, polypi-covered 
mass." The muscular fibre, however, is wanting in those distinct transverse strix, so fully developed in the muscle of the higher orders of animals.

The ova of the compound Actiniæ are of a rounded form, often brilliantly coloured, and their embryos, by a series of gradual changes, finally assume the appearance and condition of the parent. Milne Edwards, to whom we are indebted for most of our knowledge of the reproductive processes of actinozoa, insists on the necessity of distinguishing between that of gemmation and fissuration, the polype-bud at first being no more than a protuberance from the parent "enclosing a cæcal diverticulum of the somatic cavity." Both simple and composite Fungidae occur, and multiply by lateral gemmation.

The Gorgonidae differ from all other Alcyonaria in having an erect branching cænosare so firmly rooted that they are reputed to rival oaks in size; but it is doubtful whether they ever attain to a height of more than five or six feet.

Pennatulida. - This family derives its name from penna, a quill, and the spicula closely resemble a pen, one of which is represented in fig. 239, No. 1. The polypes are fleshy white, provided with eight rather long retractile tentacula, beautifully ciliated on the inner aspect with two series of short processes, and strengthened moreover with crystalline spicula, there being a row of these up the stalk: the series of smaller processes are ciliated. The mouth, in the centre of the tentacula, is somewhat angular, and bounded by a white ligament, a process from which encircles the base of each tentaculum, and thus seems to issue from an aperture. The ova lie between the membraneous part of the pinnæ; they are globular, of a yellowish colour, and by a little pressure can be made to pass through the mouth.

Dr. Grant writes: "A more singular and beautiful spectacle could scarcely be conceived than that of a deep purple Pennatula phosphorea, with all its delicate transparent polypes expanded and emitting their usual brilliant phosphorescent light, sailing through the still and dark abyss, by the regular and synchronous pulsations of the minute fringed arms of the polypes." The power of 
locomotion is doubted by other writers, and the pale blue light is said only to be emitted when under the influence of some degree of irritation.

Alcyonaria.-Actinozoa, in which each polype is furnished with eight primately fringed tentacles. Corallum selerobasic or spicular.

Alcyonium digitatum, "Fingered Alcyoninm" (Fig. 237, No. 2).-The French call it Main de Mer, "seahand," the Germans Diebshand, "thief's hand." Sometimes they are very small; but when larger are named by the fishermen Cows'-paps, and Dead Men's Hands. The mass, at first repulsive, when placed in sea-water gradually expands into delicately pellucid polypes, with erowns of beautiful tentacula. The cells occupied by the polypes are placed at the terminations of canals which run through the polypidom, and which, by their union with each other, serve to naintain a communication between the individual polypes constituting the mass. The rest of the polypidom is made up of a transparent gelatinous substance, containing calcareous spicula, and pervaded by numerous small fibres, which form a sort of irregular network. Alcyonidae are always attached to submarine bodies. The species already mentioned is exceedingly common round our coasts; so much so that, as Dr. Johnston says, "scarce a shell or stone can be dredged from the deep that does not serve as a support to one or more specimens."

The ova, as Professor Grant remarks, placed uncler the microscope, and viewed by transmitted light, appear as opaque spheres surrounded by a thin transparent margin, which increase in thickness as the ova begin to grow, and such of the ova as lie in contact unite and grow as one ovum. A rapid current in the water immediately around each ovum, drawing along with it all the loose particles and floating animalcules, is distinctly seen moving with an equal velocity as in other ciliated ova; and a zone of very minute vibrating cilia is quite perceptible, surrounding the transparent margin of all the ova.

Tubiponide. - To this family belongs the handsome Tubipora musica, "Organ-pipe Coral" (fig. 237, No. 10), the polypidom of which is composed of parallel tubes, united by 
lateral plates, or transverse partitions, placed at regular distances; in this manner large masses, consisting of a congeries of pipes or tubes, are formed. When the animals are alive, each tube contains a polype of a beautiful brightgreen colour, and the upper part of the surface is covered with a gelatinous mass, formed by a confluence of the polypes. This species occurs in great abundance on the coasts of New South Weles, of the Red Sea, and of the Molucca Islands, varying in colour from a bright red to a deep orange. It grows in large hemispherical masses, from one to two feet in circumstance, which first appear as small specks adhering to a shell or rock; as they increase, the tubes resemble a group of diverging rays, and at length other tubes are produced on the transverse plates, thus filling up the intervals, and constituting one uniform tubular mass; the surface being covered with a green Heshy substance beset with stellate polypes.

Mr. Dana, who devoted much time to the examination of the corals of the Pacific, thus writes of their diversities of form and character:- "Trees of coral are well known; and, although not emulating in size the oaks of our forests -for they do not exceed six or eight feet in height-they are gracefully branched, and the whole surface blooms with coral polypes in place of leaves and flowers. Shrubbery, turfts of rushes, beds of pinks, and feathery mosses, are most exactly imitated. Many species spread out in broad leaves or folia, and resemble some large-leaved plant just unfolding; when alive, the surface of each leaf is covered with polype flowers. The cactus, the lichen, clinging to the rock, and the fungus in all its varieties, have their numerous representatives. Besides these forms imitating vegetation, there are gracefully-modelled vases, some of which are three or four feet in diameter, made up of a network of bianches and branchlets and sprigs of flowers. There are also solid coral hemispheres like domes among the vases and shrubbery, occasionally ten or even twenty feet in diameter, whose symmetrical surface is gorgeously decked with polype-stars of purple and emerald green."

Nothing can be more impressive than the manner in which these diminutive creatures carry out their stupendous undertakings, which we denominate instinct, intelli- 
gence, or design. Commencing betimes from a depth of a thousand or fifteen hundred feet, they work upwards in a perpendicular direction; and on arriving at the surface form a crescent, presenting the back of the arch in that direction from which the storms and winds generally proceed : by which means the wall protects the busy nillions at work beneath and within. 'These breakwaters will resist more powerful seas than if formed of granite; rising as they do in a mighty expanse of water, exposed to the utmost powers of the heavy and tumultuous billows that eterually lash against them.

As we glance at the map of the world, and think of the profusion of fragrant vegetation and delicious food almost spontaneously produced on the lovely sunny islands of the broad Pacific, how startling does it seem, when we are told that these islands, bearing on their bosoms gardens of Eden, are entirely formed by the slow-growing corals, which, rising up in beautiful and delicate forms, displace the mighty ocean, defy its gigantic strength, and display a shelly bosom to the expanse of day! The vegetation of the sea, cast on its surface, undergoes a chemical change; the deposit from rains aids in filling up the little gaping catacomb, the fowls of the air and the ocean find a resting place, and assist in clothing the rocks; mosses carpet the surface, seed brought by birds, plants carried by the oceanic currents, animalcules floating in the atmosphere, live, propagate, and die, and are succeeled, by the assistance their remains bestow, by more advanced vegetable and animal life; and thus generation after generation exist and perish, until at length the coral island becomes a paradise filled with the choicest exotics, the most beautiful birds and delicious fruits, among which man may indolently revel to the utmost desire of his heart.

ACALEPHE. - In great variety of form and colour, swimming freely about the waters of the ocean, are found in abundance the beantiful Acalephoe. Some of them have a remarkable stinging property, from which circumstance they derive their name of Sea-nettles; others, from their gelatinous nature, are known as Sea-jelly, or Jellyfish.

These interesting animals were furst arranged in three 
orders : A. stabiles (fixed), A. liberce (free), and $A$. hydrastatice (hydrostatic). Cuvier classed then in two orders:

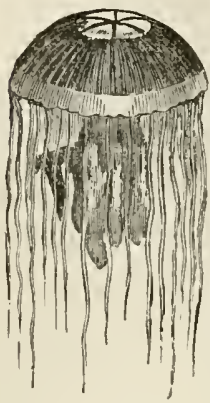

Fig. 23s - Jelly-fish. A. simplices and A. hydrostatica. They are now, however, divided differently, and arranged in groups according to the peculiar mode by which they effect their locomotion. A very interesting point of connexion between this class and the preceding is the interchange of form. Some of the Zoophyta, as the Tubulariade and the Campanulariado, give birth to a progeny which are in every respect Naked-eyed Medusa; while, on the other hand, the young of the Medusce are in their earlier stages stationary polypes.

The Medusce spread on the surface of the water a beallful jelly-like mass, in form resembling an umbrella; and, by a continual contraction and opening out of this, they swim freely about (Plate IX. $c, d, c, h$ ). They are all more or less' phosphorescent. The Beroe, one of the family Ctenophora, propel themselves with active ciliated arms. The Physalide have an organ common to fishes,-swimming bladders,-by filling or emptying which they rise or sink, and move along in their watery home.

The Medusoid family, Lucernarida, has, from a mistaken view of its organization, been referred to the class Actinozoa: Milne-Edwards has, however, placed it in a sub-class, under the name of Podactinaria. In the Lucernarida the body is cup-shaped, about an inch in height, terminating in a short foot-stalk. Round the distal margin of the cup arise a number of short tentacles, which are disposed in eight or nine turfts; in Carduella they form one continuous series. Their free extremities appear sucker-like, and the whole organism is semi-transparent, of a gelatinous consistence, and variously coloured. The cup, viewed from above, presents in its centre a fourlobed mouth, which is seen to form the free extremity of a distinct polypite, occupying the axis of the entire hydrosoma. Its gastric region exhibits a number of tubular filaments, arranged in vertical rows dipping into 
the digestive cavity. The space between the polypite-wall and the inner surface of the cup is equally divided. A circular sinus has its course beneath the insertion of the tentacles. By means of a band of muscular fibres which transverse its margin, and another set which radiate towards the polypite, the distal extremity of the cup can be folded or drawn inwards. It has been observed to detach itself, and swim in an inverted position by the slowly repeated movements of its cup-like umbrella, thus resumbling Pelagia, a more active and permanently free member of the same order.

Three families of the beautiful Lucernaridæ; all of which are at once distinguishable by their umbrella, may be defined as follows :-

Family 1, Lucernaridæ. Reproductive elements developed in the primitive hydrosoma, without the intervention of free zoöids. Umbrella with short marginal tentacles and a proximal hydrorhiza. Polypite single. Family 2, Pelagidæ. Reproductive elements developed in a free umbrella, which either constitutes the primitative hydrosoma, or is produced by fission from an attached Lucernaroid. Umbrella with marginal tentacles. Polypite single. Family 3, Rhizostomiå. Reproductive elements developed in free zoöids produced by fission from attached Lucernaroids. Umbrella without marginal ten. tacles. Polypites numerous, modified, forming with the genitalia a dendriform mass depending from the umbrella.

For a further description of this interesting species see Professor Müller's paper, Journal of Microscopical Science, vol. iii. p. 265; or, Professor Greene's Manual of the Coelenterata.

The flat circular horny disc forming the skeleton of Propita gigantea, to the naked eye exhibits both radiating and concentric markings; and, when examined with a powrer of 40 diameters, its upper surface is found to be furrowed, and two rows of small projecting spines oceur upon the ridges between the furrows, the ridges being the radiating fibres above noticed. The under-surface, or that to which the greater portion of the soft parts of tine animal are attached, is more deeply furrowed; and plicæ or folds of the mantle fit accurately into the furrows, from 
which they can easily be removed by the application of a gentle force. The concentric markings have in all cases small scalloped edges; they occur at certain regular intervals, and are so many indications of the lines of growth. In the centre there is a circular depression; and between its circumference and that of the first concentric marking there are eight flattened radii. If the under-surface be examined with a power of 100 linear, the ridges will all be found to have small jointed tubular processes like hairs projecting from them. In no part of this horny tissue is there a trace of a cellular or a reticular structure.

Wonderfully beautiful as are these creatures in form and colour, the amount of solid matter contained in their tissues is incredibly small. The greater part of their substance appears to consist of a fluid, differing little, if at all, from the sea-water in which the animal swims; and when this is drained away, so extreme is the tenuity of the membranes which contained it, that the dried residue of a jelly-fish, weighing two pounds, which was examined by Professor Owen, weighed only thirty grains. The transparency of the tissues render the whole of the Acalephae delightful objects for the microscope. ${ }^{1}$

The Echinodermata belong to the division Annuloida, the most familiar of examples of which are star-fishes and sea-urchins. The labours of that distinguished comparative anatomist and physiologist of Berlin, Johannes Miuller, have made us better acquainted with the structure and development of these remarkable animals than with those of most classes of the animal kingdom. The series of feet which protrude along certain fixed lines from the body of an Echinoderm have received the name of "ambulacra;" and hence, says Mr. Huxley, "we may distinguish their system of vessels as the ambulacral vascular system. The existence of an ambulacral vascular system has as yet been demonstrated only in the following orders:-Echinidea, Ophiuridea, Crinoidea, Asteridea, and Holothuridea, with which the fossil Cystidea and Blastoidea are inseparably connected. I therefore limit the Echinodermata to the

(1) See an excellent paper in the Transactions of the Microscopical Society. "On the Anatomy of Two Species of Naked-eyed Medusæ," by G. Busk, Esq. ; also Professor Forbes' works on this family. 
very natural group formed by these orders. A more or less complete calcareous skeleton is always developed within the Echinoderms, resembling that of the Actinozod, not only in this respect, but also in consisting of detached spicula. In this form the skeleton remains in the Holothuridea, but in the other Echinoderms, the spicula coalesce into netirorks, which may become consolidated into dense plates by additional deposits. It is by the different shape and arrangement of these plates that the diversity exhibited by the skeletons of different Echinodermata is produced."

Asteridea.-Star-fishes have been divided according to the mode of locomotion into Spinigrades, moving by means of spines; Cirrhigrades, by suckers; and Pinnigrades, by fins or pinnæ. Of the last-named division we have only one British genus, Comatula. At the very extremity of each ray is an organ like an eye, having spinous appendages, which are termed the eyelids. It is doubtful, however, whether these parts have really any visual endowment; no proof of their possessing the faculty of sight has ever been advanced, and, from what we know of the nature of this sense generally in the lowest forms of animal life, we should be disposed to consider that the organs in question must serve some other as yet unknown purpose.

The species Ophiura and Ophiocoma, Plate IV. Nos. 88 and 91 , may be easily recognised by the great length and tenuity of their rays, and their excessive fragility. The whole surface, both of disc and rays, is covered by scales, which are so closely approximated as to give an almost perfectly smooth surface. These scales are arranged in definite and often in very beautiful patterns, and in some species the primary scales are edged or encircled by series of circular bosses or tubercles, giving a reticulated appearance to the disc and rays. Ophicoma rosula has its spines tipped with curious anchor-shaped processes, which are supposed to facilitate the motion of the creature. In Ophiura they are very short, and not apparent without careful inspection; while in Ophiocoma they are so long as to give quite a bristly, sinous appearance to the animal, being sometimes, in fact, very much longer thar. the 
breadth of the rays. A striking species is the Palmipes membranaceus, the "Bird's-foot Sea-star," which is almost as thin as parchment, and might, as Professor Forbes says, be readily mistaken for the torn-off skin of some bulkier species. Its surface is covered with a number of raised tubercles, and very closely-set fasciculi of short and sharp spines. Asterias aurantiaca and Luidia fragilissima present a surface-structure very different from any of the species previously noticed, their tuberculated epidermis being so closely set with upright spines as to be almost wholly invisible. These spines are arranged in a radiated or rosette-shaped manner, and have a roughened surface. A portion of the ray of Luidia forms a microscopic object of exquisite beauty. A single spine is given in Plate IV. No. 89 .

The cirrhigrade star-fishes are furnished with certain curious appendages, the use of which is at present very imperfectly understood. These are the "pedicellarice" and "madreporiform tubercle." The latter is a rounded, cushion-like eminence of considerable size, situated on the disc, mostly very much out of the centre. It is irregularly fissured in a radiate manner, and is not at all unlike the animal from which it derives its name. Various conjectures have been made as to the use of this tubercle. Forbes looks upon it as being merely the analogue of the stalk which exists in the young condition of the crinoid star-fishes. The pedicellarice (Plate IV. Nos. 93 and 94) are pincer-like organs irregularly scattered over the surface of the animal, and which have distinct characters in the different species. They were supposed to be parasitic creatures, but are now generally admitted to be true epiderinic appendages. They are in a constantly active motion during the life of the star-fish, and grasp firmly anything which is brought between their blades. Their nearest analogues are the birds'-head processes which occur in certain zoophytes. The Pedicellarice of Echinus are pariially covered with ciliated epithelium: they are also placed upon a stalk, the lower portion of which encloses a calcareous nucleus, whilst the other portions are enft, and spirally retractile.

The Feather-star (Comatula rosacea) is perhaps th $\_$most 
interesting of the British star-fishes, and quite unique in the gracefulness of its form and the exquisite beauty of its colouring; its life-history is not only remarkable, but it possesses the additional interest of being the only living representative in our seas of the group of organisms so familiar to us in the fossil state as Encrinites. The delicate structure of this species renders it impossible to exhibit it satisfactorily in a dry or mounted state. The central cup-shaped body gives off five rays, which divide so near the base as to appear like ten. These are furnished throughout their length with membranaceous pinnæ. Tubularia Dumortierii, Plate IV. No. 92, appears rather to belong to Comatula than Tubularia. A description of this interesting polype will be found in Johnston's Brit. Zoophytes, p. 53.

Professor Wyville Thompson, in a paper on "SeaLilies" (see Intellectual Observer, August, 1864), says :"Comatula rosacea, the most common British species, is found abundantly in Lamlash Bay, in Arran and Strangford Loughs, in Dulkey Sound, in Kirkwall Bay, and generally distributed in deep water all round the British and Irish coasts. In general structure it resembles very closely the head of Neocrinus decorus; it has, however, no stem, but in the position of the stem, and forming the base of the cup, there is a hemispherical plate covered with rows of cirrbi, exactly like the stem-cirrhi on the stalked forms. When at rest it holds on to a stone or weed, and spreads out its beautiful feathery crimson arms, like the petals of a flower. At other times it swims rapidly through the water by graceful impulses of its arms. In spring, the hundreds of ovaries dotted over its pinnules are turgid with eggs, and if at this time it is captured, and placed with some sea-weed in a tank, bunches of bright orange-coloured eggs hang in clusters around, giving the delicate pinnatic arms the appearance of the fronds of some wonderfully graceful fern in rich fructification.

"The phases passed through by the young before they come to resemble their parents in form and mode of lifo are of extraordinary beauty, and most instructive in determining the true zoological relation which the free crinoids bear to their fixed ancestors. At first a minute, almost 
invisible, pale yellow germ escapes from the egg; this, if placed under the microscope the first day after its birth, has a very definite form, but not the least like a star-fish. Four bands of long vibratile cilia guard the body at different points, and by their motion the little animal whirls about in the water. About the end of the second day two rows of five each of delicate calcareous trellised plates may be seen, making a kind of five-sided basket. A dark mass now collects within the trellised basket, and the rings are united together by little bundles of rods, till they form what looks like a joined pillar supporting the basket. Gradually the plates enlarge and distort the outer wall; and the stem-like series of joints lengthen, stretching out the narrow end with it. The old mouth disappears, the gelatinous wall settles round the little living skeleton, a round sucker appears, and the animal fixes itself upright to a sea-weed or a stone at the bottom of the tank. Five leaf-like valves, each supported by one of the upper tier of plates, now open on the top of the wider extremity, and the little creature looks when these valves are open much like a microscopic wine-glass, and when closed like a tulip bud."

Echinida. ${ }^{1}$-Sea-urchins are found in abundance upon our sea-shores, lurking among the rocks, where they entrap their prey. Their spines and suckers are used as feet, or as a mode of progression, even to the climbing of rocks, in order to feed upon corallines and zoophytes: they march along with ease where apparently no footing could be found, or dig holes with their spines to bury themselves in the sand, to escape pursuers, or hide from observation. The

(1) Description of Plate 9 :-

ASTEROIDA.

a, Astrophyton scutatum.

n, Ophiocoma rosula.

Sudibranchitata Gastropoda.

$b$, Doris pinnatifida-back and side view.

ACALEPHAE.

c, Equorea Forbcsina.

d, Medusæ Bud.

$e$, Thaumantias coryneles.

$h$, Cydippe pyleus.
Echinordes.

$f$, Echinus (A Young Sea-urebin).

$g$, Echinus sphora.

Tunicata.

$i$, Ascidice.

$k$, Botryllus violaceus, on a Fucus

Crustacea.

$l$, Corystes cossivelaunus.

m, Eurynome aspera.

o, Pagurus Prideauxii.

p, Ebalia Permantii. 


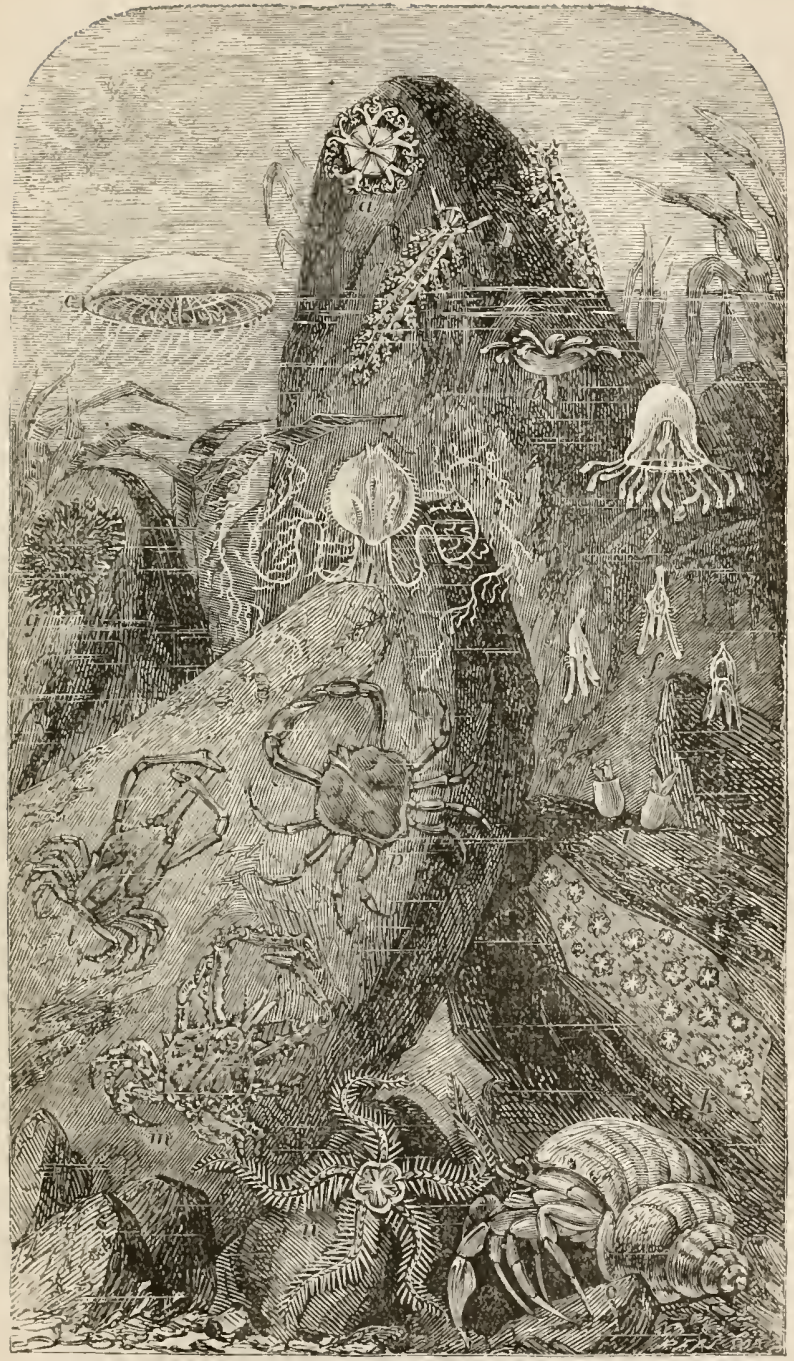

PLAIE IX. Asteroidea, Echinidea, Crustacea, \&c.

K K 2 

Echinodermata, ${ }^{1}$ sea-urchins, or sea-eggs, derive their name from these curious spinous processes.

In most Echinidea all the feet are expanded into sucker discs, at their extremities, and are here strengthened by a calcareous plate or plates; but in the Echino-cidaris and some others, the feet of the oral portion of the ambulacra only have this structure, while those of the apical portion are pectinated, flattened, and gill-like. Müller distinguishes four kinds of feet in the Spatangoidea,-simple locomotive feet, without any sucking disc ; locomotive feet, provided with terminal suckers, and containing a skeleton; tactile feet, whose expanded extremity is papillose ; and gill-like feet, triangular, flattened, with more or less pectinated lamellæ.

In the Clypeastrodea the petaloid portions of the ambulacra possess branchial feet, interspersed with delicate locomotive sucker feet, provided with a calcareous skeleton. In the Ophiuridea and Crinoidea the feet are tentaculiform; and there are no vesicles at the bases of the feet, while in the Asteridea they are well developed, but simpler than those of the Echinider. The madreporic canal is, in the Asteridea, strengthened by a remarkable calcareous framework, which has given rise to the notion that it is filled with sand, and to the name "sand-caual," which has been applied to it. The canal terminates in the madreporic tubercle, which is always placed interradially on the antambulacral surface of the star-fish.

In some, Holothuridea, the feet are scattered over the whole ambulacral region, as well in the inter-ambulacra as in the ambulacra. In others, Psolus, the feet are developed only from three of the five ambulacra; while in the Synaptae and Chirodatce there is only a circlet around the mouth.

Many star-fishes, and Synapta among the Holothuridea, have the curious habit of breaking themselves up into fragments when taken; Muiller has pointed out the very curious fact, that in Synapta, at any rate, this act may be prevented by cutting through the oral nervous circle. The nervous circle in the Echinus surrounds the cesophagus near the mouth, and is enclosed by the alveoli, between which the

(1) Derived from echinos, a soine, and derma, skin 
ambulacral nerves pass to reach it. In the Asteridea, the circle lies at the extreme limit of the soft membrane, which surrounds the mouth, and may be readily exposed by cutting away the hard inter-ambulacral oral lips. In the Holothuridea it lies immediately beneath the perisoma of the oral disc. The only known organs of sense in the Echinodermata are the pigmented "eye-spots," developed in commexion with the ends of the ambulacral nerves, and on the oral nervous circle in many Holothuridea.

The great majority of the Echinodermata commence their existence as free-swimming larvæ covered with cilia, but a great difference exists in their further course, according as they belong to the Asteridea, the Holothurdea, and the Crinoidea on the one hand, or to the Echinidea and Ophiuridea on the other. Of the development of the Crinoidea we know very little, beyond the observations of Mr. Thompson, that the larva of the Comatula is provided with several transverse bands of cilia, almost like that of a Holothuria, and that the development of the Echinoderm. commences while the larva is still free. At a later period, the young Comatula is seated upon a long, jointed stem, so as to resomble a Pentacrinus; and it becomes detached from this stem, in assuming its adult condition.

Mr. Huxley, after mature examination of this class of animals, says, "he can see no reason for retaining them amongst the Radiata of Cuvier, but, on the other hand, thinks them properly placed among the Anmuloida."

The skeleton of the Echinoderms generally consists of an assembiage of plates, or joints, of calcareous matter. The minute structure of which presents a reticulated character, and the solid parts are usually composed of a series of super-imposed laminæ or scales. The openings, or areolæ, in one layer being always placed over the solid cell-walls of the layer beneath it, the spines are situated on the external surface of the shell; they are generally of a conical figure, and are articulated with the tubercles by a balland-socket joint. When a thin transverse section of one of these spines is examined with the naked eye, it appears to be made up of a series of concentric layers, varying considerably in number; not, however, with the size of the spine, but with the distance from the base at which 
the section was made: when a section taken from the middle of the spine is examined with a power of fifty diameters, it will be seen that the centre is occupied by a reticulated structure; around the margin of this may bo observed a series of small structureless spots, arranged at equal distances apart (Fig. 240, No. 1): these are the ribs or pillars, and indicate the external surface of the firstlayer deposited; passing towards the margin, other rows of larger pillar's may be seen, giving it a beautiful indented appearance; all the other parts of the section are occupied by the usual reticulated tissue. In the greater number of spines the sections of the pillars present no structure, in others they exhibit a series of concentric rings of successive growth, which strongly remind us of the medullary rays of plants; occasionally they are traversed by reticulated structures, as represented in Fig. 246, No. 1. When a vertical section of a spine is examined, it is found to

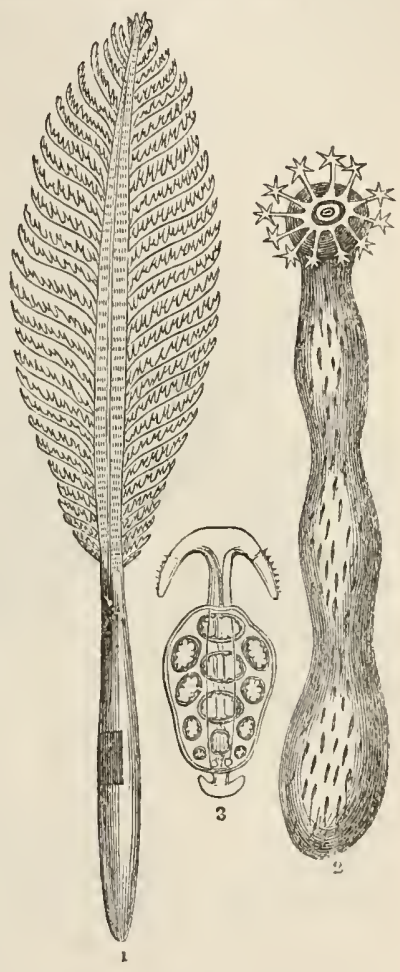

FIg. 239.

be composed of cones 1, Polynidom of Pennatula phosphorea. 2 placed one over the other, Synupta Chirodota. 3, Anclior-shaped. the outer margin of each cone being formed by the series of pillars. In the genus Lichinus the number of cones is considerable, while in that of Cularis there are seldom more than one or two; so that 
from these species transverse sections may be made, having no concentric rings, and in which only the external row of pillars cau be seen.

"The skeleton of the Echinodermata contains very little organic matter. When it is submitted to the action of very dilute acid, to dissolve out the calcareous matter, the residuum is very small in amount. When obtained, it is found to possess the reticular structure of the calcareous shell (Fig. 240, No. 1); the meshes or areolæ being bounded

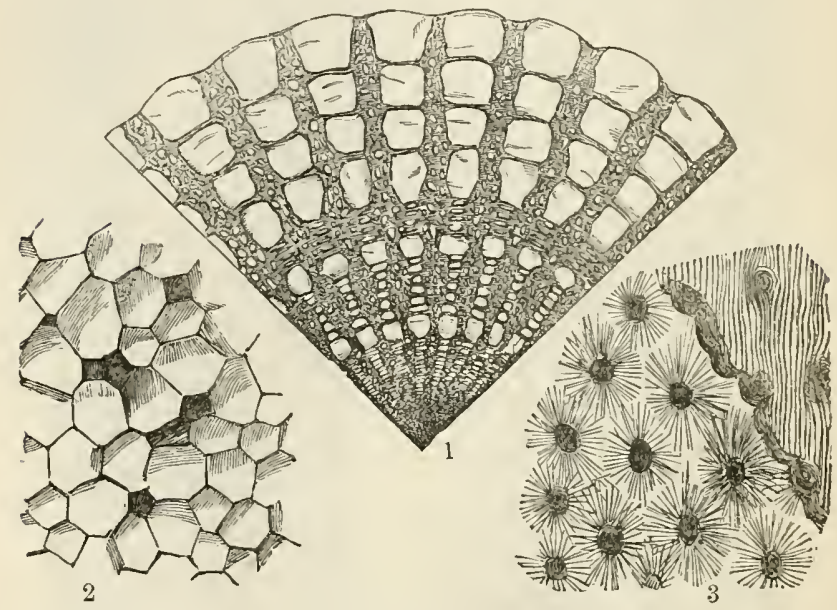

Fig. 240.

1, Section of spine of Echinus, exhibiting reticulated structure, the calcareous portion having been dissolved out by acid. 2, Transverse section of shell of the Pinna ingens. 3, Horizontal section of shell of Terebratulata rubicunda, showing its radiating perforations.

by a substance in which a fibrous appearance, intermingled with granules, may be discerned under a sufficiently high magnifying power, as originally pointed out by Piofessor Valentine. 'This tissue bears a close resemblance to the areolar tissue of higher animals; and the shell may probably be considered as formed, not by the consolidation of the cells of the epidermis, as in the Mollusca, but by the calcification of the fibro-areolar tissue of the true skin. 
This calcification of areolar or simply fibrous tissue, ny the deposit of mineral substance, not in the meshes of areolæ, but in intimate union with the organic basis, is a condition of much interest to the physiologist; for it presents us with an example, even in this low grade of the animal kingdom, of a process which seems to have an important share in the formation and growth of bone, namely, in the progressive calcification of the fibrous tissue of the periosteum membrane covering the bone." 1

From their peculiarity of structure they may be said to be almost imperishable. Their shells exist abundantly in all our chalky cliffs, innumerable specimens of which may be obtained, exhibiting the same wondrous forms and characters as those which now frequent our shores.

The Crinoidea, "Sea-lilies," - so called from the resemblance which many of them present to the lily,-were exceedingly abundant in former ages of the world; and their remains often for'm the great bulk of large masses of rock, fig. 241. These animals were all supported upon a long stalk, at the extremity of which they floated in the waters of those ancient seas, spreading their long arms in every direction in search of the small animals which constituted their food. Each of the arms, again, was feathered with a double series of similarly jointed appendages; so that the number of separate calcareous pieces forming the skeleton of one of these animals was most enormous. It has been calculated that one species, the Pentacrinus briareus, must have been composed of at least 150,000 joints; and "as each joint," according to Dr. Carpenter, "was furnished with at least two bundles of muscular fibre,-one for its contraction, the other for its extension, -we have 300,000 such in the body of a single Pentacrinus - an amount of muscular apparatus far exceeding any that has been elsewhere observed in the animal creation." A furrow runs along the inside of the arms, which is covered with a continuation of the skin of the disc; and from this the ambulacra are protruded, as in other Echinodermata.

In the family of Ophuridea, so called from the resemblance of their arms to a serpent's tail, (Gr. ophis, a snake,

(1) Dr. Carpenter, Cyclopadia of Anatomy and Physiolsgy. 
oura, a tail); the body forms a roundish or somewhat pentagcual disc, furmished with five long simple arms, which have no furrow for the protrusion of the ambulacra.

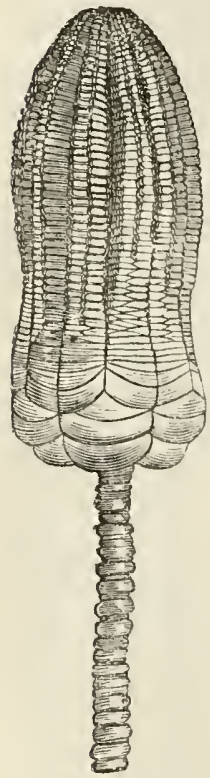

l'ig. 241.-Encrinus, sira-lily.

Ophiuridea are exceedingly plentiful in al? our seas, and their remains occur in al! the more recent marine strata of the earth's crust. 'They are more commonly called Sand Stars, or Brittle Stars.

"However much the famnas of the various geologic periods may have differed from each other, or from the fauna which now exists, in their aspect and character, they were all, if I may so speak, equally underlaid by the great leading ideas which still constitute the master types of animal life. Ancl these leading ideas are four in number. First, there is the star-like type of life,-life embodied in a form that, as in the corals, the sea anemones, the sea urchins, and the star fishes, radiates outwards from a centre; second. there is the articulated type of life,-life embodied in a form composed, as in the worms, crustaceans, and insects, of a series of rings united by their edges, but more or less moveable ou each other ; third, there is the bilateral or molluscan type of life, - life embodied in a form in whick. there is a duality of corresponding parts, ranged, as in the cuttle fishes, the claws, and the snails, on the sides of a central axis or plane; and fourth there is the vertebrate type of life-life embodied in a form in which an internal skeleton is built up into two cavities placed the one orer the other, the upper for the reception of the nervous centres, central and spinal,-the lower for the lodgment of the respiratory, circulatory, and digestive organs. Such have been the four central ideas of the faunas of every sncceeding generation, except perhaps the earliest of all, that of the Lower Silurian System, in which, so far as is yet kwown, only three of the number existed,the radiated, articulated, and molluscan ideas or type 
That Omnipotent Creator, infinite in His resources-who, in at least the details of His workings, seems never yet to have repeated Himself, but, as Lyell well expresses it, breaks, when the parents of a species have been nnoulded, the dye in which they were cast,-manifests Himself, in these four great ideas, as the unchanging and unchange. able

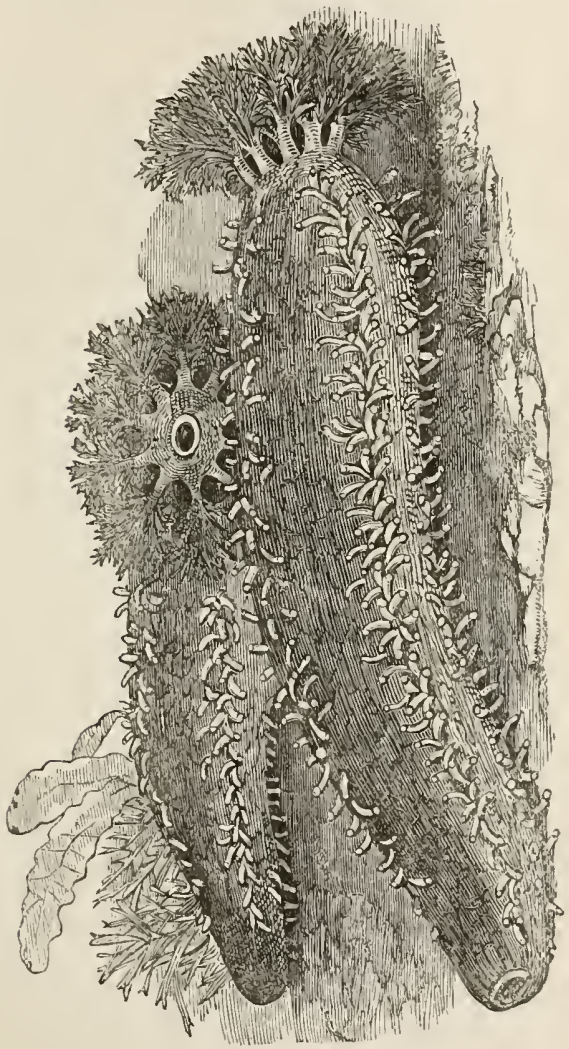

Fig. 242.-Holothuridea, Sea-cucumbers.

One. They serve to bind together the present with the past, and determine the unity of the authorship of a wonder- 
fully complicated design, executed on a groundwork broad as time, and whose scope and bearing are deep as eternity." 1

The Synaptidce are characterised by a total absence of ambulacra, the motions of the animals being assisted by peculiar anchor-like processes which project from the skin, and roughen the surface of the animal. The spiculum represented in fig. 239 is serrated on the convex edge, and, Dr. Herepath says, apparently belongs to S. Galliermii, but that the drawing of the animal near it is singularly inaccurate, although taken from Professor Forbes' work; and that the oral tentacles are imaginary developments of S. digitata. See Herepath, "On the Genus Synapta," Quar. Journ. Micros. Science, 1865, p. 1. The spicules are beautiful objects for polarised light. (Plate IV. No. 87 , shows a side view of one set in the skin.)

Holothuridea, "Sea-cucumbers." - In this family the body acquires a slug-like form. The radiate structure is in fact scarcely recognisable in these animals, except in the arrangement of the tentacles which surround the mouth. The body is always more or less elongated, with the mouth at one end and the anal opening at the other; the calcareous deposit in the skin is reduced to scattered granules; and in one family the ambulacra are entirely wanting.

The integument consists of a number of minute reticulated plates, set closely on the substance of the skin. The forms of the plates are various, as well as the spicula set in them. The Australian seas furnish many varieties : Plate VIII. Nos. 171 and 172 , are representations of plates and spicula under polarised light. Objects of this class are also well suited for black-ground illumination.

The structure of the spines and other solid parts of the skeleton of Echinodermata can only be displayed by making thin sections, in the way described for cutting bone, at page 209. Their peculiar texture requires that certain precautions should be taken to prevent the section from breaking whilst being reduced to a desirable thinness, and to prevent the interspaces of the network from being clogged by the particles abraded in the reducing 
process. In mounting the specimens, liquid balsam must be employed, and only a very gentle heat should be applied; and if, after it has been mounted, the section should be found too thick, it will be easy to remove the glass cover and reduce it further, care being taken to harden the halsam, as directed in preparing bone sections.

\section{PRESERVATION OF THE POLYPIDOMS OF ZOOPHYTES.}

The following excellent and simple plan for preserving zoophytes as fluid preparations, so as to retain the polypes and their tentacular arms in situ, was adopted by the late Dr. Golding Bird. For this purpose a lively specimen should be chosen, and then plunged into cold pure water $;^{1}$ the polypes are killed almost immediately, and their tentacles often do not retract: proper-sized specimens should then be selected, and preserved in weak alcohol. Little phials about two inches long should be procured, made from thin flat glass tubes, so as to be half an inch wide, and about a quarter of an inch, or even less, from back to front. The specimens should be fixed to a thin platinum wire, and then placed in one of these phials (previously filled with weak spirits), so as to reach half-way down. When several are thus arranged, they should be put on a glass cylinder, and removed to the air-pump. On pumping out the air, a copious ebullition of bubbles will take place; and many of the tentacles previously concealed will emerge from their cells. After being left in vacuo for a few hours, the bottles should be filled up, closely corked, and tied over, like anatomical preparations in general. For all examinations with a one or two-inch object-glass, these bottles are most excellent, and afford cheap and useful substitutes for the more expensive and difficultly-managed cells. In this manner, specimens of the genera Membraniporce, Alcyonida, and Crisiades, \&c., exhibit their structure most beautifully.

A few dozen of these little bottles hardly occupy any room, and would form a useful accompaniment to the microscopist by the sea-side. Any one visiting the caverns

(A) A small quantity of gin thrown into distilled water answers the purpose better than pure water, and specimens may be put up in the same. The animais are nearly always preserved in their po'ypidoms by using this fluid to kill then. 
in St. Catherine's Island at Tenby, might reap a harvest which would afford amusement and instruction for many weeks. These caverns are so rich in zoophytes and sponges, that they are literally roofed with the Laomedece, Grantioe, and their allies; whilst the elegant Tubularioe afford an ornament to the shallow pools on the floor; and the walls are wreathed with the pink, yellow, green, and purple Actinice.

When these objects are examined by polarised light, most interesting results are produced. For this purpose, let a piece of selenite be placed on the stage of the microscope, and the polarising prisms arranged so that the ray transmitted is absorbed by the analyser. If a specimen of Sertularia operculata be placed on the selenite stage, and examined with a two-inch object-glass, the central stem is shown to be a continuous tube, assuming a pink tint throughout its whole extent. The cells appear violet in colour; their pointed orifices are seen much more distinctly than when viewed with common light. The vesicles are paler than the rest of the object; and their lids, which so remarkably resemble the operculum of the theca of a moss, are beautifully distinct, being of an orange-yellowish colour. This zoophyte is often covered with minute bivalve shells, distinguished by the naked eye from the vesicles only by their circular form ; and these, when present, add much to the beauty of the specimen, presenting a striated structure, and becoming illuminated with most. beatitiful colours. 


\section{CHAPTER III.}

WiZIZOA - MOLLUSCA - GASTEROPODA - BRACHIOPODA - CONCHIFERA -
OEPHALOPODA-PTEROPODA-TUNICATA-CRUSTACEA-ENTOMOSTRACAANNULOSA-CIRRIPEDA-ENTOZOA-ANNELIDA.

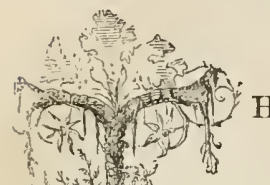

HE term Mollusc, derived from mollis, soft, is one which at once indicates a chief structural characteristic of the class of animals about to occupy our attention. The body of most of the molluscous sub-kingdom is soft and fleshy; and all except the Tunicata and a few Pteropoda are covered or protected by a hard calcareous shell. The shell is of two kinds; first of an epidermal character, being formed upon the surface of a filmy cloak-like organ called a mantle, answering to the true skin of othes animals; and next of a dermal character, being concealed within the substance of the mantle, and frequently moulded into a great diversity of forms, and coloured with various tints.

The molluscs belonging to the class Gasteropoda have become a large and important section of the animal series, presenting very many objects of great interest for the microscopist. Of the large family of molluses, the only species which bears any resemblance in structure to the Polyzoa, which now form a portion of the molluscous division, is the Brachiopoda, and this is confined to a resemblance in internal structure. 
The Polyzoa were placed by Dr. Johnston under the head Ascidioida; in the generality of works they are named Bryozoa, and the individual, Bryozoon; derived

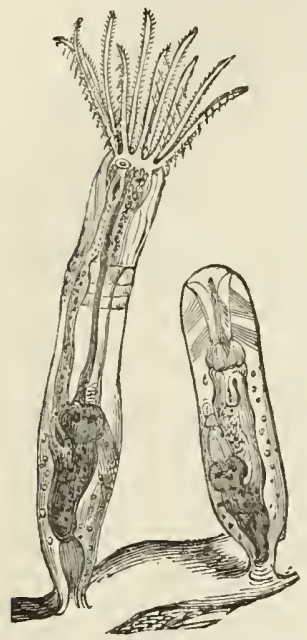

Fig. 243. - Bryozonon Bowerbankia, "Bowerbank Bryozoon," showing its internal stmucture; another animal withdrawn into its cell.

in the respet some bling in this respect some of the Actinozoa, and ure protected by membranaceous coverings or polypido: Protrusion and retraction are performed by two sets muscles, one acting on the body of the animal, the othr

(1) Mr. Gosse, in his Manual of Marine Zoolngy, adopts the idea, now peetty general, that the Polyzoa belong to the Molluscous division, in spite of their external resemblances to Polypes, and he places them among Molluses. In this, perhaps, he has thought more of systematic views on classification than of the student's convenience. It seems to us quite clear that, without adopting De Blainville's principle of classifying animals according to their eivelope as the best principle of scientific classification, we should adopt it in works of reference like the present, since the external characters are necessarily tliose most immediately recognised by the student; and in the case of the Polyzoa, they are so remarkably similar in external characteristics to the hydroid polypes, that they were alwajs classed with them, until the profounder investigations of Van Beneden, Allman, and others, revealed the resemblancss betwen the internas cheracteristice of the Polyzos and those of Molluscs. 
upon its cell. The oral extremity is surrounded by a circle of long tubular tentacles covered w:th cilia ; at each of these feelers or arms there is an aperture, the one at the base communicating with a canal that passes round the edge of the oral aperture or mouth. The food passes down a long gullet, that contracts during the process of swallowing. At the end of this is an orifice that opens into what appears to be a gizzard, having two bodies opposite to each other, with a rongh surface, as if for the comminution of food, moved by muscular fibres. Those of the species without this gizzard have a digestive stomach that secretes a coloured fluit. From the upper part of the stomach near the entrance from the gizzard arises an intestine, having a narrow opening surrounded by cilia that proceeds upwards, ending in an orifice near to the tentacles, from which the refuse food is ejected.

Their cells are of various shapes, and from one, a family of millions come, budding forth from its sides; and though the living matter disappears, the catacombs exist for the foundation of their families, branching out and enduring for ages.

Bryozoon Bowerbankia received its name from Dr. Arthur Farre, in honour of the well-known microscopist, Mr. Bowerbank. A magnified representation of the animal is seen in fig. 243. "When fully expanded, it is about onetwelfth of an inch in length. In its retracted state, it is completely enclosed in a delicate horny cell, sufficiently transparent to admit of the whole structure of the contained animal being seen through its walls. The cells are connected together by a cylindrical creeping stem, upon which they are thickly set, sessile, ascending from its sides and upper surface. The animal, when completely expanded, is seen to possess ten arms of about one-third the length of the whole body; each arm being thickly ciliated on either side, and armed at the back by about a dozen fine hair-like processes, which project at nearly right angles from the tentacles, remaining motionless, while the cilia are in constant and active vibration."

Notamia, Back-cell, so named from the cells being exactly opposite, and united back to back with a thick partition, and having a joint above and below each pair. In some 
species of the Flustro the interior of the cell is protected by a lid which bears some appearance to the head and beak of a bird, and hence it is termed the bird's-head process. This has been made the subject of investigation by many naturalists. George Busk, Esq., F.R.S., 1 contributed to the Transactions of the Microscopical Society, 1849, an admirable paper on the Notamiabursaria, "Shepherd's-purse Coralline," (represented in fig. 244, Nos. 1 and 3), which adds to our knowledge of this curious process. He says: "This most beautiful pearl-

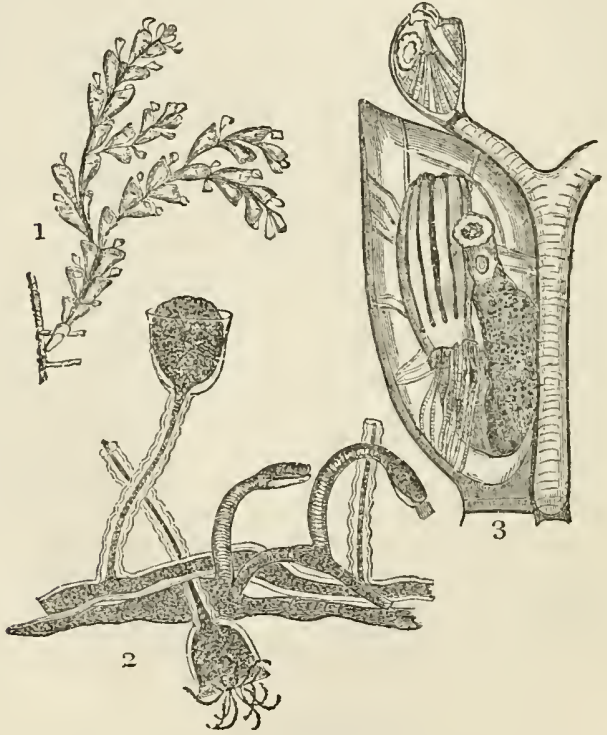

Fig. 244.

1, Notnmia tursaria, Shepherd's-purse Coralline. 2, Anguinaria spatulala, Snake Corallinc, growing with the Campanularia inlegra. 3, The Shepherd's purse Animalcule withdrawn into its cup, and the internal organisin showu greatly magnified.

coloured coralline adheres by small tubes to fuci, from whence it changes into flat cells; each single cell, like "(1) Mr. Busk has added to the description here given of this bird's-hesd frocess in the Quarterly Journal of Microscopical Science, for January 1854. 
the bracket of a shelf, broad at top and narrow at the bottom: thesc are placed back to back in pairs, one above another, on an extremely slender tube that seems to run through the middle of the branches of the whole coralline. The cells are open at top. Some of them have black spots in them; and from the top of many of them a figure seems to issue out like a short tobacco-pipe, the small end of which seems to be inserted in the tube that passes through the middle of the whole. The cells in pairs are thought by some to have the appearance of the small pods of the plant "Shepherd's Purse," by others the shapo of the seed-vessel of the Veronica, Speedwell.

"The polypidom of this bryozoon, like those of most of its congeners, may be said to consist of a radical portion, by which it is affixed to the objects upon which it grows, and of a celliferous portion or branches, upon which the polypes themselves are lodged. The radical portion in the present species consists of a central discoid body of a nearly circular form, and of branches radiating from the periphery of the disc, which thence exhibits something of the aspect of the body of an ophiura. The radical tubes or branches springing from the margin of the disc are usually five or six in number, and they are civen off at pretty regular distances apart; but besides these radical tubes, one or more celliferous branches are not unfrequently seen to arise immediately from the upper surface of the discoid portion.

"The central disc, and the radical tubes arising from it, exhibit a sinilar structure, and are formed of a thick, firm, apparently horny envelope, containing a coarse granular matter, of a yellowish-white colour, and which in some portions of the tubes assumes the form of distinct irregularly-globular masses, of nearly uniform sizc. The central disc is subdivided into distinct compartments by septa of considerable thickness, and each radiating branch arises from one of these distinct compartments; so that there appears to be no communication between one radical branch and another. The radical branches give off at irregular distances secondary branches, which ultimately become celliferous. Each of these secondary branches, however, arises from a distinct compartment, as it were, of 
the tube from which it springs. This compartment is formed, like those of the central disc, by a thick septum, which shuts off the origin of the secondary branch from the main cavity of the primary one."

The larger, or polypiferous cells, Mr. Busk proposes to term cells, and the smaller tobacco-pipe-shaped organs cups; the latter being usually above the former throughout the polypidom, "excepting immediately below each fork, where the cup is invariably absent above one of the cells of the pair from between which the fork springs. The polype-cells are several times larger than the cups, and their walls are much thinner; in fact, sufficiently transparent to allow of the contents of the cell being pretty well seen, withont any preparation, even during the life of the animal. In shape they are inversely conical, and the outer and upper angle is usually produced into a prominent, sharp point. From the internal and upper augle arises the tubular prolongation going to form the next cell or cup, as the case may be, in succession. They are entirely closed at the top, contrary to what is stated by previous observers; and, as has been shown, there is no. connexion whatever between the cell and the cup placed immediately above and behind it. The aperture of the cell is on the anterior face, and towards the upper margin; it is of a crescentic form, and placed obliquely, as it were, across the upper and internal angle of the cell, with the convexity of the curve directed upwards and inwards. The lips of the aperture are strengthened by thin bands of horny material; and, under favourable circumstances, indications of short muscular fibres, for the purpose of opening or closing the aperture, may be seen."

Tho shell, which Mr. Busk believes to be entire at the bottom, though closed only by a very delicate membrane, contaius an ascidoid polype of the usual typical form of that class. "It has ten tentacles, and no gizzard. Tro sets of muscular fibres at least may be distinguished as appertaining to the polype. The most important of these are the retractor muscles, which, arising from the bottom of the cell, in the form of long, somewhat flattened, transversely striped, isolated fibres, about the one tenthousandth of an inch in width, are inserted, some of them 
at the base of the teutacles, and others lower down the body of the polype."

When we consider the minuteness of the delicate little sprig which is the natural size of this polype, we cannot but wonder at the triumphs of the microscope in giving such precise details as Mr. Busk relates of the Notamia bursaria. Its beautiful and perfect organisation, the careful provision for the safety and engagements of this minute being, make us awe-stricken at the power of Divine intelligence.

The Halodactylus, better known as Alcyonidium, is remarkable among the marine forms of Polyzoa, for the large size of its tentacular crowns; these when expanded are distinctly visible to the unassisted eye, and present a spectacle of great beauty when viewed with the binocular microscope. Its polyzoary has a spongy aspect very much resembling that of the Alcyonian zoophyte: when however the animals are expanded, they are at once seen to be widely different, as the plumose turfts which then issue from the surface of Halodactylus give it the appearance of a beautiful downy film. The opacity of its polyzoary renders it unsuited for the examination of anything more than the tentacular crown.

Lepralia, "Sea-scurf,"-from the Greek for marine leprosy, -is the name given to this family of the Celleporidce by Dr. Johnston.

Lepralia nitida, found attached to shells, is thus described: "Crust spreading circularly, closely adherent, rather thin, greyish white, calcareous; cells contiguous, in radiating rows, large, subalternate, ovate, ventricose, silvery, the walls fissured with six or seven cross slits which are on the mesial line; aperture subquadrangular, depressed, terminal; anterior to it there is often found a globular, pearly, smooth, oviferous operculum, with a round even aperture. The remarkable structure of the cells renders this one of the most interesting species under the microscope. There is sometimes an appearance of a spine on each side of the lower angle of the mouth, which is merely the commencement of the walls of the next cell."

$L$. coccinea, $L$. variolosa, $L$. ciliata, $L$. trispinosa, and L. immersa, are the other British species. 
The family Cellularia, little-cells, have a curious and wonderful provision of nature for their: protection, an operculum, a lid or cover over the apertures of each cell. Cellularia ciliata is parasitical, branching, calcareous, white and tufted; grows about half an inch in height, and the oblique aperture is armed on the outer edge with four or five long hollow spines. The operculum is pearly, and near the base there is that singular appendage, described as the bird's-head process. Its beauty and transparency render it a fitvourite object with microscopists.

The Cellularia (now Bugula) avicularia are very aceurately described by Mrr. Gosse, from his own observations upon specimens secured on the Devonshire coast, during a residence there. He says: "Well does it deserve the name of Bircl's-head Coralline, given to it by the illustrious Ellis; for it presents those curious appendages that resemble vultures' heads in great perfection. All my specimens were most thickly studded with them; not a cell withont its bird's head, and all see-sawing, and snapping, and opening their jaws with the most amusing activity ; and what was marvellous, equally so in one specimen from whose cells all the polypes had died away, as in those in which they were still protruding their lovely bells of tentacles. The stem ascends perpendicularly from a slender base, which is attached to the rock, or to the cells of a Lepralia growing from the rock. The central part of the spine is most expanded, the diminution above and below being pretty regular; during life, the usual colour is a pale buff, but the cells become nearly white in death. When examined microscopically it is, however, that the curious organisation of this zooplyyte is discovered, especially when in full health and vigour, with all the beautiful polypes protruded and expanded to the utmost, on the watch for prey. It seems to me as poor a thing to strain one's eyes at a microscope over a dead and dry polypidom, as it does to. examine a shrivelled and blackened flower out of a herbarium; though I know well that both are often indispensable for the making out of technical characters. But if you want to get an insight into the structure and functions of these minute animals, or if you would be charmed with the perception of beauty, or delighted with new 
and singular adaptations of merns to an end,-or if you desire to see vitality under its most unusual, and yet most interesting phases, - or if you would have emotions of adorincr wonder excited, and the tribute of praise elicited to that mighty Creator who made all things for His own glory,- then take such a zoophyte as this, fresh from the clear tide-pool, take him without inflicting injury ; therefore detach with care a minute portion of the surface-rock, and drop your prisoner, with every organ in full activity, into a narrow glass cell with parallel sides, filled with clear sea-water, and put the whole on the stage of the microscope, with a power of not more than 100 linear, at least, for the first examination. I greatly mistake if you will not confess that the intellectual treat obtained is well worth-ay, ten times more than worth-all your trouble."

CRisraD 2 , signifying a separation; applied to a parasitical family. Crisia cornuta, "Goat's-horn Coralline" of Ellis, having cells linked in a single series; the same remark applies to C. chelata, "Bull's-horn Coralline ;" the latter look like a number of shoes fitting close to the ancle, joined by the toe-part to the heel of others. Ellis says: "This beautiful coralline is one of the smallest we meet with. It rises from tubuli growing upon fuci, and passes from thence into sickle-shaped branches, consisting of single rows of cells, looking when magnified like bull's horns inverted, each one arising out of the top of the other. The upper branches take their rise from the fore-part of the entrance of a cell, where we may observe a stiff, short hair, which seems to be the beginning of a branch. The opening of each cell, which is in the front of its upper part, is surrounded by a thin circular rim; and the substance of the cells appears to consist of a fine transparent shell or coral-like substance."

Crisia eburnea, "Tufted-ivory Coralline," attains the height of an inch, and displays its beautiful white, bushy tufts, with often a dash of light-red intermingled. Its cells are loosely aggregated and cylindrical, with bent tubular orifices free; while the Crisia aculeata have cells closely aggregated, cylindrical, nearly straight, with long slender spines springing from the margin of every cell, giving it a delicate and pretty appearance.

Eucratiada. - We select from this family a specimen of 
great interest, the Anguinaria,-from the Latin anguis, a snake. This, and also Notamia, belong to the class Polyzoa. An account of the Anguinaria spatulata, "Snake-head - Coralline," appeared in the Transactions of the Microscopical Society, by Mr. Busk, who corrects the errors of other observers. The polype is parasitical upon fuci, and is not unfrequently associated with other kinds on the same plants, as in fig. 244, No. 2, on Campanularia. The A. spatulata "as a whole, consists, like all its congeners, of two distinct portions, one usually termed the radical, and another which constitutes the proper polype cells. In the present instance, the arrangement of these parts is in some respects very peculiar and curious; but it will be found upon strict examination to accord accurately with the universal type."

"In the radical tubes, and on the dorsal or" upper surface of the dilated extremity of the polype-cell, represented at No. 2, this earthy matter is deposited in the form of minute angular or rounded particles, presenting faint traces of a linear arrangement; but in the main body of the polype-cell, or the upright portion, the calcareous material is arranged in beautifully regular rings, giving that part of the zoophyte a peculiarly elegant appearance under the microscope. This calcareous ingredient is sufficiently abundant to render the contents of the radical tubes and polype-cells indistinct; and to obtain a satisfactory view of these parts it is necessary to remove the earthy matter by some weak acid. When this is done, it will be found that the contents of the radical portion are coarsely granular, and the wall rather thicker than those of the proper polype-cell. The latter contains an ascidian polype, which has about twelve tentacles, and no gizzard." The polype, as far as Mr. Busk has observed, is always lodged in the upright portion of the cell; but the long retractor muscular fibres arise near the commencement of the horizontal portion of the cell, from its upper wall, and nearly at one point.

The expanded portion of the cell, besides the special muscles of the aperture, contains other muscular fibres, in all respects resembling those described by Dr. Farre, as conducing to the extrusion of the polype in Bowerbankia, 
and which are also very distinct in the Notamia; but which, in the present instance, would seem to have for their chief function the drawing-up or corrugation of the membraneous portion of the polype-cell. These muscular fibres have a distinct central nucleus or thicker portion, as is the case in the analogous muscles in some other polypes.

(EsCHARIDE. - This interesting family justly deserves the great attention many naturalists have bestowed upon it. Linnæus named it Flustra, from the Saxon word flustran, to weave; it is commonly called a Seamat, and resembles fine network spread over stones, rocks, shells, and marine plants. This network, when submitted to the powers of the microscope, is found to be a cluster of cells, in each of which dwwells an animal, that protrudes its feelers when searching for food, and sinks into its little home when tired, or alarmed by approaching danger.

Dr. Grant estimates that a single Flustra has as many as four hundred millions of cilia on these restless tentacles. The tentacula vary from ten to twolve; the general organization consists of a gullet, a gizzard, a stomach,

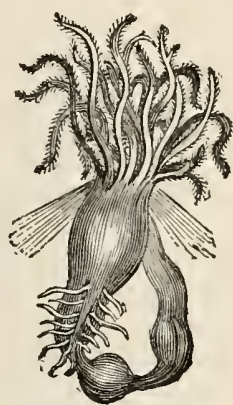

Fig. 245.-Escharc cervicornis, Sea-moss prolype: the animal is represcnted out of its polypidom. and intestines, the body itself being quite transparent. When collected together in clusters they take the form of: a delicate minute tree, having cells in all parts, and of various colours. Lamouroux says: "When the animal has acquired its full growth, it protrudes from the opening of its cell a small globular body, which it fixes near the aperture, and, as it increases in size, soon assumes the form of a new cell; it is yet closed, but through the transparent membrane that covers its surface the motions of a polype may be detected; the habitation at lengtle bursts, and the tentacles protrude; eddies are produced in the water, and conduct to the polype the atoms necessary for its subsistence. The aperture of the cells is formed by a semicircular lid, convex externally and concave internally, which folds down when 
the polype is about to advance fram the cell. The opening of this lid in the $F$.truncata, where it is very long, appears through the microseope like the opening of a snake's jaws; and the organs by which this motion is effected are not perceptible. The lids of the cells open and shut in the Flustrce without the slightest perceptible synchronous motion of the polypes."

In the formation of their stony skeletons, the animals appear to take a most insignificant part; they are principally secreted by the integuments or membranes with which they are invested, in like manner as the bones and nails in man are secreted by tissues designed for that purpose, and acting slowly and imperceptibly. From an analysis of the stony corals, it appears that their composition is very analogous to that of shells. The porcellaneous shells, as the cowry, are composed of animal gluten and carbonate of lime, and resemble, in their mode of formation, the enamel of the teeth; whereas the pearly shells, as the oyster, are formed of carbonate of lime and a gelatinous or cartilaginous substance, the earthy matter being secreted and deposited in the interstices of a cellular tissue, as in bones. In like manner, some corals yield gelatine upon the removal of the lime, while others afford a substance in every respect resembling the membranons structure obtained by an analysis of the nacreous (pearly) shells. A recent elaborate analysis of between thirty and forty species of corals, by an eminent American chemist (Mr. B. Silliman), has shown, contrary to expectation, that they contain a much larger proportion of fluorine than of phosphoric acid.

Flustra foliacea, the broad-leaved Horn-trrack of Ellis, is about four inches high, and of a brown colour. The cells are small, in alternating rows; and sometimes covered by a lid opening downwards. Hook says: "For curiosity and beauty, I have not, among all the plants or regetables I have yet observed, seen any one comparable to this sea-weed." Flustra truncata is abundant in deep water, and grows to a height of about four inches; it is of a delicate yellow colour, and bushy. This is the narrowleaved Horn-wrack of Ellis ; for it must not be forgotten that the older writers regarded the whole grenera as plants. 
Flustrc chartacea.-Ellis states: "The cells of this sea mat are of an oblong square figure, swelling out a little in the middle of each side. The openings of the cells are defended by a helmet-like figure; from hence the polypeshaped suckers extend themselves. This sea-mat is of a slender and delicate texture, like a semi-transparent paper, of a very light straw-colour. 'It was first found on the coast of Sussex, adhering to a shell. I have since met, on the same coast, about Hastings, in the year 1765, with several specimens whose tops are digitated, and others that were very irregularly divided."

The Flustra carbasea grow out in a leaf-like manner, gradually widening to the end : they are found on shells of a yellowish-brown colour; on one of the sides the cells are both large and smooth. The animals have about twentytwo arms or feelers, which, says Dr. Grant, after a most careful examination of these polypes, "are nearly a third of the length of the body; and there appear to be about fifty cilia on each side of a tentacle, making 2200 cilia on each polype. In this species there are more than eighteen cells in a square line, or 1800 in a square inch of surface; and the branches of an ordinary specimen present about ten square inches of surface; so that a common specimen of the $F$. carbasea presents more than 18,000 polypes, 396,000 tentacles, and 39,600,000 cilia.

"They are very irritable, and frequently observed to contract the circular margin of their broad extremity, and to stop suddenly in their course when swimming; they swim with a gentle gliding motion, often appear stationary, revolving rapidly round their long axis, with their broad end uppermost, and they bound straight forward, or in circles, without any other apparent object than to keep themselves afloat till they find themselves in a favourable situation for fixing and assuming the perfect state. The transformation of the ova, from that moving, irritable, free condition of animalcules, to that of the fixed and almost inert zoophytes, exhibits a new metamorphosis in the animal kingdom not less remarkable than that of many reptiles from their first aquatic condition, or that of insects from their larva state."

Flustra avicularis.-This is another of the little beaties 
of the deep, found usually on old shells, an inch in height, spreading itself fan-like, and of an ashy colour, deeply divided in a dichotomous mamner into narrow, thin, plane segments, truncate at the end, formed of four or five series of oblong cells, capped with a hollow, globose, pearly, operculum seated between the spines, of which there is one on either side of the circular aperture. The opercula are so numerous, that they give to the upper surface the appearance of being thickly strewn with orient pearls; the undersurface is even and longitudinally striated, the number of strixe corresponding to the number of rows in which the cells are disposed. Dr. Johnston describes, amongst many other British species, $F$. membranacea, " a gauze-like incrustation on the frond of the sea-weed, spreading irregularly to the extent of several square inches."

Dr. Perceval Wright discovered on the western coast of Ireland a new genus of Alcyonidae, which he named after the well-known naturalist Mr. Harte, Hartea elegans, Plate IV. No. 86 . This polype is solitary, the body cylindrical, and fixed by its base to the rock; it has eight ciliated tentacles, which are knobbed at their base and most freely displayed. It is a very beautiful polyzoon of a clear white colour, and when fully expanded stands three-quarters of an inch high. ${ }^{1}$

The fresh-water Polyzoa are peculiarly interesting objects for microscopic observation, from the very beautiful manner in which they display their ciliated tentacula, set upon a crescentic, horseshoe-shaped "lophopore." The arrangement of the latter appendage has been the cause of separating the fresh-water Polyzoa, from their marine allies, into a sub-class; the former being named Hippocrepa (horseshoe-like), and the latter Infundibulata (funnellike). ${ }^{2}$ The most striking form among the Hippocrepa is the Cristatella Mucedo-a wandering Polyzoon, capable of moving freely through the water. It may be met with during a great part of the summer in our ponds and streams, amidst the stems and leaves of aquatic plants (Plate IV.

(1) On a new genus of Alcyonido. By Dr. E. Perceval Wright. Micros. Journ. Scienec, vol. v. p. 213 . 1865.

(2) The reader is referred to a valuable treatise on the structure and classifiation of this group by Professor Allman, Monograph of the British Freshwater Polyzoa, published by the Ray Society, $185 \pi$. 
No. 97). It is always regarded as one of the most exquisite specimens of the class Polyzoa; should there be any diffeulty in finding the animal itself, the eggs, met with late in the summer or autumn, should be carefully stored in an aquarium without fish. The eggs or "statoblasts," No. 95, are small, dark, circular bodies, about the size of a pir's head, surrounded by a series of minute hooked spines; the animal conceals them among tangled masses of decayed grasses and confervæ. Polyzoa are known to live upon Desmids and Algæ; and to keep them alive the tank must be freely supplied with such kinds of food. Cristatella Mucedo is rarely found in the same spot a second day, it wanders about apparently in search of food; it is, therefore, provided with a contractile dise or foot, and by means of this it creeps about not far from the surface of the water, for it delights to display its beautiful crests of tantacula (about eight in number), in the broad light of day or sunlight; in this respect Cristatella also differs from most Polyzoa. Below the external margin is a series of tubular chambers visible through the translucent membrane ; and the cænæcium or common dermal system is of a light yellow colour, often concealing several dark, brownish-looking eggs.

Lophopus crystallinus is a finer Polyzoon than the former, and displays beautiful plumes of transparent tentacles arranged in a double horseshoe-shaped series. They at times abound in slow running streams, adhering to the stems of water-plants. When first removed from the water they resemble masses of the ova of one of the water snails, and have often been mistaken for them. On putting one of those jelly-like masses into a glass trough with some of the clear water from the stream, delicate tubes will be cautiously protruded, and then the beautiful fringes of cilia are soon brought into play. The organization of L. Crystallinus is simple, although it is provided with organs of digestion, circulation, respiration, and generation. A nervous ${ }^{1}$ and muscular system are also tolerably well

(1) It has been demonstrated by Fritz Müller that the Polyzoa possess a nervous system:- "The nervous system of each branch consisting of-lst, a considerable sized ganglion sitarted at its origin; $2 \mathrm{~d}$, of a nervous trunk running the entire length of the branch, at the upper part of which it subdivides into branches, going to the ganglia of the internodes arising at this part; and $3 \mathrm{~d}$, of 
developed. It increases both by budding and by ova, both of which conditions are shown in Plate IV. No. 98. The ova are gencrally seen enclosed in the transparent case of thie parent. In Lophopus and most other freshwater genera, such as Cristatella, Plumatella, and Alcyonella, the neural margin of the lophopore is extended into two triangular arms, giving it the appearance of a deep crescent.

Alcyonella is a genus of fresh-water polyzoa, found usually about the autumnal period of the year in the several Docks at the East end of London, adhering to floating pieces of timber. It assumes the form of an irregular sponge-like mass, with an aggregation of membranaceous tube-like openings covering the surface. From these openings, the polypes are seen to project, the grouths of which are encircled with a single series of filiform ciliated tentacles, which keep the surrounding water in active motion. The polypidom seen in water has the appearance of a blackish-green sponge.

Trembley gave an interesting account of the family of Alcyonella; and Mr. J. Newton Tomkins favours us with the following observations on the development of the Alcyonella stagnorum (fluviatella) :-

"The ova now under examination ( $\frac{1}{2}$-inch obj. A. eyepiece-100 lin. diam., Wollaston's condenser), are the products of some healthy specimens of Alcyonella stagnorum given me by Mr. Lloyd, and sketched in full activity in September 1856. Soon after this period their movements decreased in energy, numerous ova were detached, which floated to the surface of the water of the jar. in which they were confined, and in the course of a very few weeks no trace remained of the parent animals, except a spongy mass of an almost gelatinous character, which still exists, though devoid of definite form, and appears composed of a mass of broken and disorganized cells.

"In November, with a view of preserving the water in a normal condition, I introduced a sprig of Anacharis

a rich nervous plexus resting on the trunk, and connecting the ganglia just mentioned, as well as the basal ganglia of the individual polypides." For further account, sec paper ia the Micros. Journ. vol. i. New Series. p. 330. 
Alsinastrum, and finding it grew freely, but soon covered with a filamentous confervoid growth, threw in two small water-snails, which are there still. About January last, the ova, which till then had floated on the surface of the water, began to sink and attach themselves to the leaves of the Anacharis and elsewhere. Latterly, they have all subsirled to the bottom of the jar, where they lie in company with a quantity of decajed vegetable matter, spawn of the Limnous, \&c. 'They are of a light-brown colour, ovoid in shape, longest diameter 0089 , shortest diameter 0172. The outer rim seems built up of cells of oblong shape, but necessarily ill-defined, owing to their being observed by light transmitted through two surfaces; the inner or central portion also cellular, but from the convexity of the object, more easy to determine as to its true nature, formed of larger hexagonal-shaped cells. Seen by higher power ( $\frac{1}{4}$-in. obj. A. eye-piece-220 lin. diam.), these central cells, besides being unmistakably hexagonal in form, have each a distinct dark nucleus in the centre : this, however, may be an optical fallacy, due to their peculiar position on a curved surface. No movement yet visible, April 25, 1857."

Plumatella Repens, Plate IV. No. 99, so named from its feather-like crown of tentacles, is a well-known fresh-water Polyzoon, found in ponds and rivulets attached to aquatic plants, generally choosing the under-surface for the purpose of avoiding the strong light. It is a very elegant variety, rather timid, withdrawing on the least disturbance of the water, and not again venturing to display its bealltiful plume until all is once more perfectly quiet. Professor Allman says of it:- "Except in the condition of the dermal systeni the structure of Plumatella differs in no essential point from that of Alcyonella. This system, however, in the coalescence of the tubes into a cornmon mass in Alcyonella, while they remain totally distinct in Plumatella, presents us with a difference of suficient importance to justify the placing the two forms in separate generic groups. The number of known speries are twelve, of which nine are British. The cænæcium consists of a linear-branched series of tubula. cells of membranocorneous consistence, which is terminated by the orifice 
aestined for the egress of the polypides. The tentacula are about sixty in number, long, and ciliated on either side. The statoblasts are ovoid, of a dark-brown colour without marginal spines, and contained within the poly-

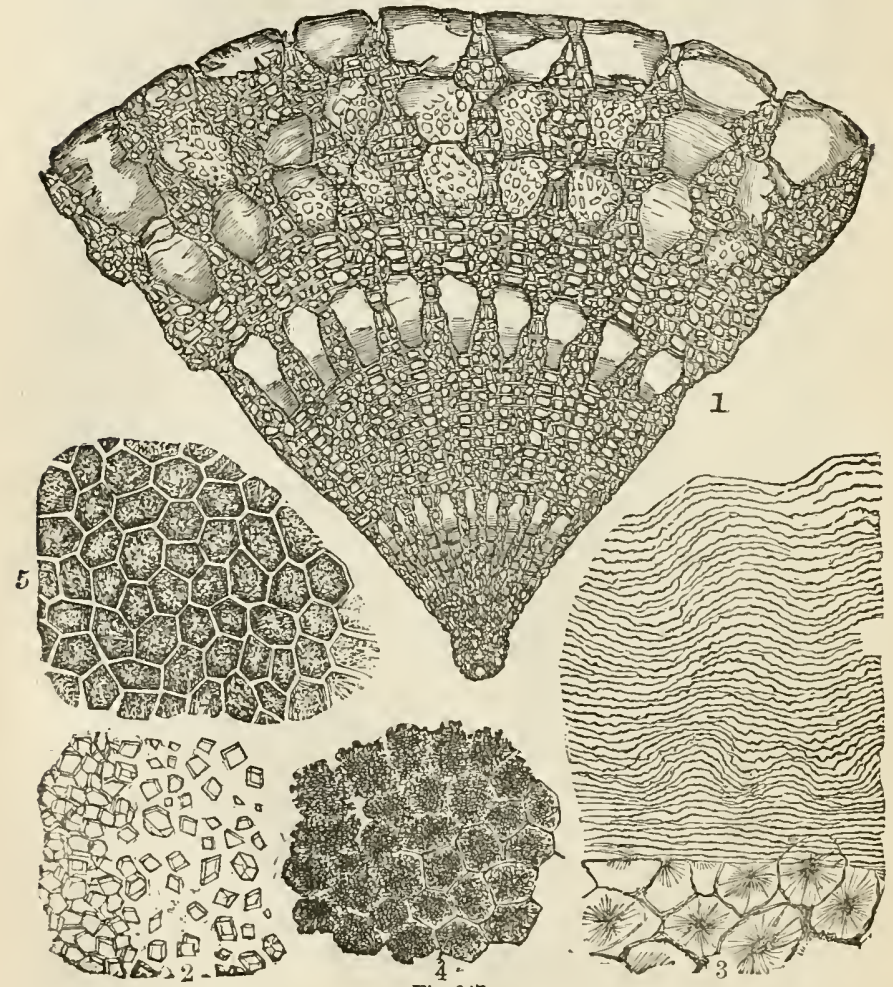

Fig. 247.

1. Portion of a transverse section of the spine of an Echinus. 2, Crystals of carbonate of Lime, froin the surface of shell of Oyster. 3, Horizontal section of shell of Haliotis splendens, with stellate pigment in the interior. 4, Portion of shell of a Crab, showing granules beneath the articular layer, 5, Another portion of the same shell, showing its hexagonal structure.

pidom, through the transparent walls of which they can be readily seen. It occurs in greatest perfection during the 
summer and towards autumn, often obtaining to a considerable size." Most of the species may be found in the ponds around London, and few objects are capable of affording greater pleasure than these Polyzoa when examined in a living state under a moderate power, and with a darkground illuminator. The withdrawal of the Polyzoa from the Radiate sub-kingdom, and their location among Mollusca, was a step in the right direction; while the important division of the molluscan sub-kingdom by Milne-Edwards into primary sections of the Mollusca and Molluscoida, the latter including the Tunicata and the Polyzoa, is all that can be desired in the systematic location of the Polyzoa.

Shell of Mollusca.-The simplest form of shell occurs in the rudimentary oval plate of the common Slug, Limax rufus; it is imbedded in the shield situated at the back and near the head of the animal.

When a molluscous or conchiferous shell is composed of a single piece, it is then termed univalve; when of two pieces, bivalve. The bivalve Mollusca exhibit no trace of any distinct head; whilst in the univalve this part of the body is well-marked, and usually furnished with special organs of sense (tentacles, eyes, nerves, \&c.).

The older naturalists recognised a group of multivalve shells, or shells composed of several valves, the majority of which belonged to the Cirrhopod order of: Crustacea, and were regarded as Mollusca by earlier observers. The Pholades, however, which in other respects are true bivalve Mollusca, are furnished with a pair of accessory plates in the neighbourhood of the hinge; whilst the Chitons, a small but singular group of Molluses nearly allied to the univalve limpets, have an oval shell composed of eight movable plates, which gives them a great resemblance to enormous woodlice; and they have been regarded as forming a sort of transition towards the articulated division. Those Mollusca not furnished

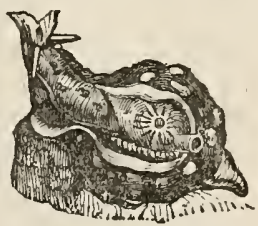
Fig. 248. - Aplysia, sea-
hare. with a shell, or having only a small calcareous plate en- 
closed within the mantle, are called Nudibranchiata; an example of this family is seen in fig. 248, Aplysia; but it is remarkable that most of them are provided with a small shell when they first quit the egg. In the shell-bearing or Testaceous Mollusca, this embryonic shell, which often difiers greatly in shape and texture from the shell of the mature animal, is, however, a commencement of the latter ; additions being constantly made to the free edge by the secretion of calcareous matter at the margin of the mantle. The delicate membranaceous part of the mantle, which lines the internal portion of the shell inhabited by the animal, has also the power of secreting a thin layer of shelly matter upon its inner surface. This is frequently of a pearly lustre; and in many bivalves a new layer of this substance is deposited at the time when the size of tho shell is increased by additions to its margins,-for it must be observed that the formation of new shell is not constantly going on, but appears to be subject to periodical interruptions, as indicated by lines on the surface of tho shell; which are called lines of growth. In many cases, the margin of the mantle, instead of being even, presents lobes of tubercles; these produce corresponding irregularities,-ribs, tubercles, or spines, - on the surface of the shell.

Dr. Bowerbank says, "Shell is developed from cells that in process of growth have become hardened by the deposition of calcareous matter in the interior." This earthy matter consists principally of carbonate of lime, deposited in a crystalline state; and in certain shell, as in that of the common Oyster (fig. 247, No. 2), from the animal-cell not having sufficiently controlled the mode of deposition of the earth particles, they have assumed the form of perfect rhomboidal crystals.

The shell of the genus Pinna, "Wing-shells," is composed of a series of hexagonal cells filled with transparent calcareous matter, seen in fig. 240, No. 2, the cuter layer of which can be split up into prisms, like so many basaltic columns; as at No. 1.

Organs of sense are possessed by some of this class in an advanced state of development. Iz the Seallop (Pecten), for example, eyes occur in great numbers, placed among 
the tentacles on the borders of the mantle. In other genera, the eyes are differently placed, in Pinna on the fore part of the mantle, and around the siphon-orifices in Pholas and Solen. In the Cockle (Cardium) the short siphons are surrounded with an extraordinary number of tentacles, capable of protrusion, each of which bears a pretty little eye; these are beautiful objects under the microscope. Cockles are able to perform vigorous leaps by means of a well developed foot, which they possess ; in other species the foot is grooved; and being associated with a gland which has the power of secreting a glutinous substance, the latter is drawn out into slender threads, with a sucker-like or flattened extremity, by which they attach themselves to rocks. The grooved foot is then withdrawn, and the thread hardens into an elastic sort of cord, called a byssus. It is by an aggregation of these threads that the common Mussel moors itself securely. The hinge of the shell is formed of variously shaped dentations; those under the beak are called car. dinal teeth ; those on either side are lateral teeth.

The Pholadida are a series of animals remarkable for their destructive boring propensities. The Teredo, shipworm, is well known for the damage it does to the bottoms of ships, especially in the tropical seas. Others of this family give a preference to sandstone, and even the most compact marble has been found bored through by them.

Mr. J. Robertson says :- "Having, while residing here (Brighton), opportunities of studying the Pholas dactylus, I have endeavoured during the last six months to discover how this molluse makes its hole or crypt in the chalk,--by a chemical solvent? by absorption? by ciliary currents? or by rotatory motions ? My observations, dissectious, and experiments set at rest controversy in my mind. Between twenty and thirty of these creatures have been at work in lumps of chalk in sea water in a finger glass and a pan, at my window, for the last three months. The Pholas dactylus makes its hole by grating the ehalk with its rasp-like valves, licking it up when pulverized with its foot, foreing it up through its principal or branchial siphoni, and squirting it out in oblong nodules. The crypt protects the 
Pholas from Conferva, which, when they get at it, grow not merely outside, but even within the lips of the valves, preventing the action of the siphons. In the foot there is a gelatinous spring, or style, which when taken out has great elasticity, and which seems the mainspring of the motion of the Pholas dactylus."

Tunicata.-The most remarkable group of animals belonging to this order are the Ascidians. The cell of the Polyzoon is represented in the Ascidian by a test or tunicfrom which they derive their name-of a membraneots or cartilaginous consistence, and often including calcareous spicules,-having two orifices, within which is another envelope, distinguished as the mantle. Few microscopic spectacles are more interesting than the sight of the circulation along this network of muslin-like fabric, and that of the ciliary movement by which the cireulating fluid is kept moving. In the transparent species, such as Clavelina and Perophora, this movement is seen to great advantage. The animals are found very commonly adhering to the broad fronds of fuci, or on pieces of shell, near low water-mark. They thrive in tanks, and multiply both by fissuration and budding. Two species are figured in Plate IX. $i$ and $k$, Botryllus violaceus belonging to the family Didemnians, the zooids of which are often arranged in the beautiful stellate clusters seen in the plate. ${ }^{1}$

Pteropoda. - The most prominent character of this class is the possession of two broad muscular fins, one on either side of the neck, somewhat resembling the expanded wings of a butterfly, whence Cuvier gave them the name of Pteropoda, "wing-footed." In Clio, the anatomy of which has been carefully investigated, there is a very curious apparatus developed for seizing its prey. On each side of the mouth are three fleshy warts, covered with minute red specks. Under the microscope, these specks, numbering about three thousand on each tentacle, are seen to be transparent cylinders, each containing in its cavity twenty stalked dises, and forming so many adhesive suckers.

(1) For information respecting the Compound Ascidians, see the admirable monograph of Milne-Edwards, Art. Tunicata in the Cyclop. Anatomy and Physiology, Huxley in Phil. Trans. for 1851, or Journ. Micros. Soc. vol. iv. 1856; also Prof. Allman, same jommal, vol vii. 1859. 
The Oyster is the type of the tribe Ostracea, all of which are Acephalus, that is, animals without a distinct head. The gills, or breathingr apparatus, form what is commonly called the beard of the oyster. The creature is attached by strong muscles to its shell. The mouth of the oyster is a mere opening in the body, without jaws or teeth; its food consists of nourishing substances suspended in the water, and which are drawn into the shell when it is open by means of cilia. Oysters attach one of their valves to rocky ground, or some fixed substance, by a mucilaginous liquid, which soon becomes as hard as the shell itself. They spawn some time in May ; and their growth is so rapid, that in three days after the deposition of the spawn, the shell of the young oyster is nearly a quarter of an inch broad; in three months it is larger than a shilling. The spawn is a very interesting object for microscopic examination, especially with polarised light. The young fry is represented in fig. 254 ; some with cilia protruded.

In the stomach of the Oyster, and in the alimentary canal, myriads of living Paramcecium and other Infusoria are found swimming in great activity; swarms of a conglomerate and ciliated living organism, somewhat resembling the Volvox globator, and so extremely delicate in their structure that they require a good objective to define them.

Pearls are usually met with in the Meleagrina Margaritifera, "Pearl Oyster," which, however, does not belong to the family Ostracea. They are likewise found in the Mussel known as Mya Margaritifera, and an inferior kind in many Mussels of the rivers of Great Britain; and, at one time, the pearl-fishery of "Treland was justly celebrated. Naturalists somewhat differ in their opinions as to the mode in which pearls are formed. Some think that they are produced by particles of sand getting into the stomach ; the animal, to prevent the roughness of these particles from injuring its delicate structure, covers them over with a secretion from a gland, and, by continual additions, they gradually increase in size. Mussels, in which artificial pearls were said to have been formed by the Chinese, have frequently found their way to this country. 
It is now, however, very generally admitted to be a diseased condition. Pearls are matured on a nucleus, consisting of the same matter as that from which the new layers of shell proceed at the edge of the Miussel or Oyster. The finest kinds are formed in the body of the animal, or originate in the pearly-looking part of the shell. It is from the size, roundness, and brilliancy of pearls that their value is estimated.

The microscope discloses a difference in the structure of pearls : those having a prismatic cellular structure have a brown horny nucleus, surrounded by sinall imperfectly-

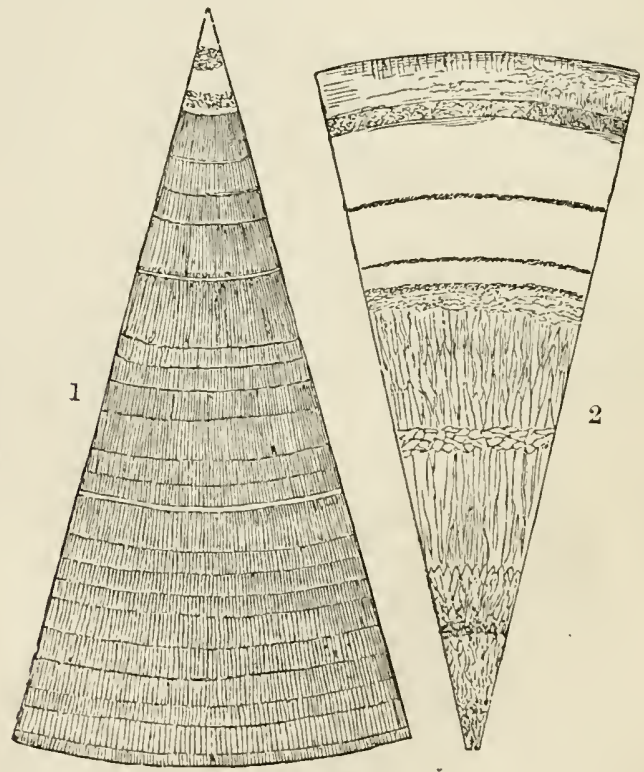

Fig. 249.

1, A transrerse section of a Pearl from Oyster, showing its prismatic structure. 2, A transverse section of another Pearl, showing its central cellular strnc:ture, with outside rings of true pearly matter. (Magnified 50 diameters.)

formed prismatic cells; there is also a ring of horny matter, followed by other prisms, and so on, as represented 
in fig. 249; and all transverse sections of pearls from Oysters show the same successive rings of growth or deposit.

In a segment of a transverse section of a small purple pearl from a species of Mytilus (fig. 250), all trace of prismatic structure has disappeared, and only a series of fine curved or radiating lines is seen. This pearl consists of a beautiful purple-coloured series of concentric laminæ; many of which have a series of concentric zones, and are of a vellow tint. The most beautiful sections for microscopic examination are obtained from Scotch pearls.

Erachiopoda, "Lamp-shells," or, as the name literally

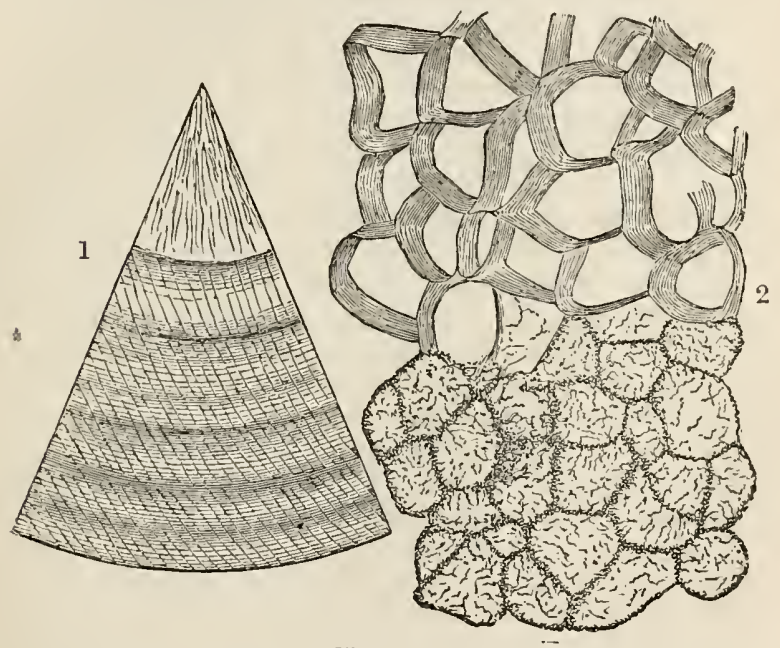

Fis. 250.

1, A transverse section of a small Pearl from a species of Mytilus, 2, Horizuntal section of same Pearl magnified 250 dianeters, to show prismatic strusture and transverse striæ.

signifies, arm-footed, is intended to express a most remarkable characteristic of these animals, the presence of a pair of arms, often of great length, rolled up in a spiral form, and believed by Cuvier to replace the foot in other bivalves. Professor Owen has shown that these organs are tubes closed at each end, and contain a fluid, which by 
the contraction of the circular muscular fibres of which the walls of the tube are composed, is propelled from the base to the extremity, thereby unrolling, as he believes, the spiral coils. One side of each arm is fringed with a vast number of long filaments: these are ciliated. The shell is opened by a peculiar process, which has given to the Terebratula the name of Coach-spring Shell. In the shell there are minnte openings surrounded by a series of radiating lines : these at first appear like dark oval spots ; but in a vertical section they are seen to be perforations or tubes running obliquely from the inner to the outer surface of the shell, and having a series of radiating lines on the edge, as in fig. 240, No. 3. The outer layer has been removed, to show a radiating structure around the perforations. Dr. Carpenter fully describes Terebratula in the Philosophical Magazine, 1854.

Not less curious than beautiful is the internal layer of many kinds of bivalves, which present an iridescent lustre, the whole surface being varied with a series of grooved lines running nearly parallel to each other. The well-known gorgeously coloured univalve, the Ear-shell, Haliotus splendens, has been ascertained to consist of numerous plates, resembling tortoise-shell, forming a series of hexagonal cells, in the centre of which the stellate pigment is deposited (fig. 246, No. 3), alternating with thin layers of pearl, or nacre; and this exhibits, when highly magnified, a series of irregular undulating folds, represented in the upper portion of the section. The iridescent lines are often extremely pleasing; and if a piece be submitted to the action of diluted hydrochloric acid, until the calcareous portion of the nacreous layers are dissolved out, the plates of animal matter fall apart, each one carrying with it the membraneous residuum of the layer of nacre that belonged to its inner surface. But the nacre and membrane covering some of these horny plates remain undisturbed; and their folded or plaited surfaces, although divested of calcareous matter, exhibit iridescent hues of the most gorgeous description. If the membrane be spread out with a needle, and the plates unfolded to a considerable extent, the iridescence is no longer seen; a fact which clearly demonstrates that the beautiful colours 
presented by the nacreous portions of shells, commonly called mother-of-pearl, are produced solely by the disposition of single membraneous layers in folds or plaits, lying more or less obliquely to the general surface.

In the Chitonida, Coat of mail Shells, the shell consists of eight transverse plates, imbedded in the mantle ; in the Limpets, the ordinary form is that of a cone. The arrangement of the teeth is somewhat remarkable.

The majority of Gasteropoda are furnished with a shell, denominated spirivalve. The cause of this spiral arrangement is said to be owing to the shape of the body of the animal inhabiting the shell, which, as it grows, enlarges its shell principally in one direction; thus, of course, making it form a spire, modified in shape according to the degree in which each successive turn surpasses in bulk that which preceded it. It would rather appear that this is principally owing to the ciliary motion imparted to the early stage of the embryo ; the first deposit of calcareous matter forming the axis, the tube continues to rotate upon its axial pillar or columella, as it is called; and by reason of some other peculiar vital tendency, the shell is gradually deposited in a series of cells ; thus enlarging its conical form, and winding obliquely from right to left. Every turn around the axis is termed a whorl; and when the columella is hollow, it is said to be umbilicated. In the spirivalve-shelled Gasteropoda, we find a difference in structure between that part of the mantle which envelopes the viscera, and which is always concealed within the cavity of the shell, and the portion placed around its aperture.

The moutlis of most Gasteropoda consist of a strong muscular cavity, and a crescentic-shaped tooth-bearing membrane, armed with sharp points, and separated by semi-circular cutting spaces, admirably adapted for the division of the food upon which they feed. Most of them are beautiful objects for the microscope.

Professor Huxley very properly objects to the use of the commonly accepted term tongue for the tooth-bearing membrane of the mollusca, and more appropriately designates it " the odontophore."

"The odontophore consists essentially of a cartilaginous 
strap, which bears a loug series of transversely-disposed teeth. The ends of the strap are connected with muscles attached to the upper and lower surface of the hinder extremities of the cartilaginous cushions ; and these muscles, by their alternate contractions, cause the toothed strap to work backwards and forwards over the end of the pulley formed by its anterior end. The strap consequently acts

1
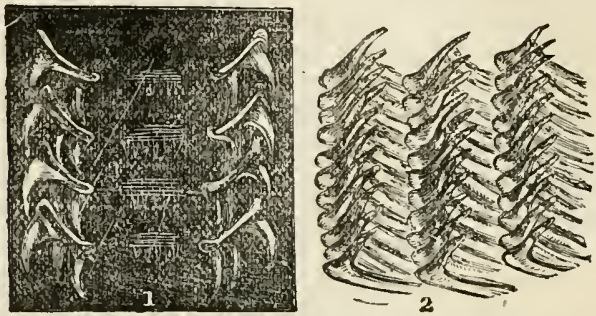

Fig. 251.

1, Palate of Buccinum undatum, common whelk, seen under polarised light. 2, Palate of Doris tuberculata, Sea-slug.

after the fashion of a chain-saw (rather of a rasp,) upon any substance to which it is applied, and the resulting wear and tear of its anterior teeth are made good by the incessant development of new teeth in the secreting sac in which the hinder end of the strap is lodged. Besides the chain-saw-like motion of the strap, the odontophore may be capable of a licking or scraping action as a whole."1

In the constant growth of the band we observe the development of new teeth. In some the teeth on the extreme part of the band differ much, both in size and form, from those in the median line: so much, that if at any time one portion be separated from the other and then examined, it might be supposed to belong to another species.

Since the investigations of Professor Loven, of Stockholm, into the lingual dentition of the glossophorous Mollusca, various observers have studied the subject with great advantage to our knowledge of the affinities of those animals. Although the patterns or types of the lingual membranes are, on the whole, remarkably constant, yet

(I) Elcments of Comparative A natomy, p. 36. 


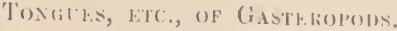
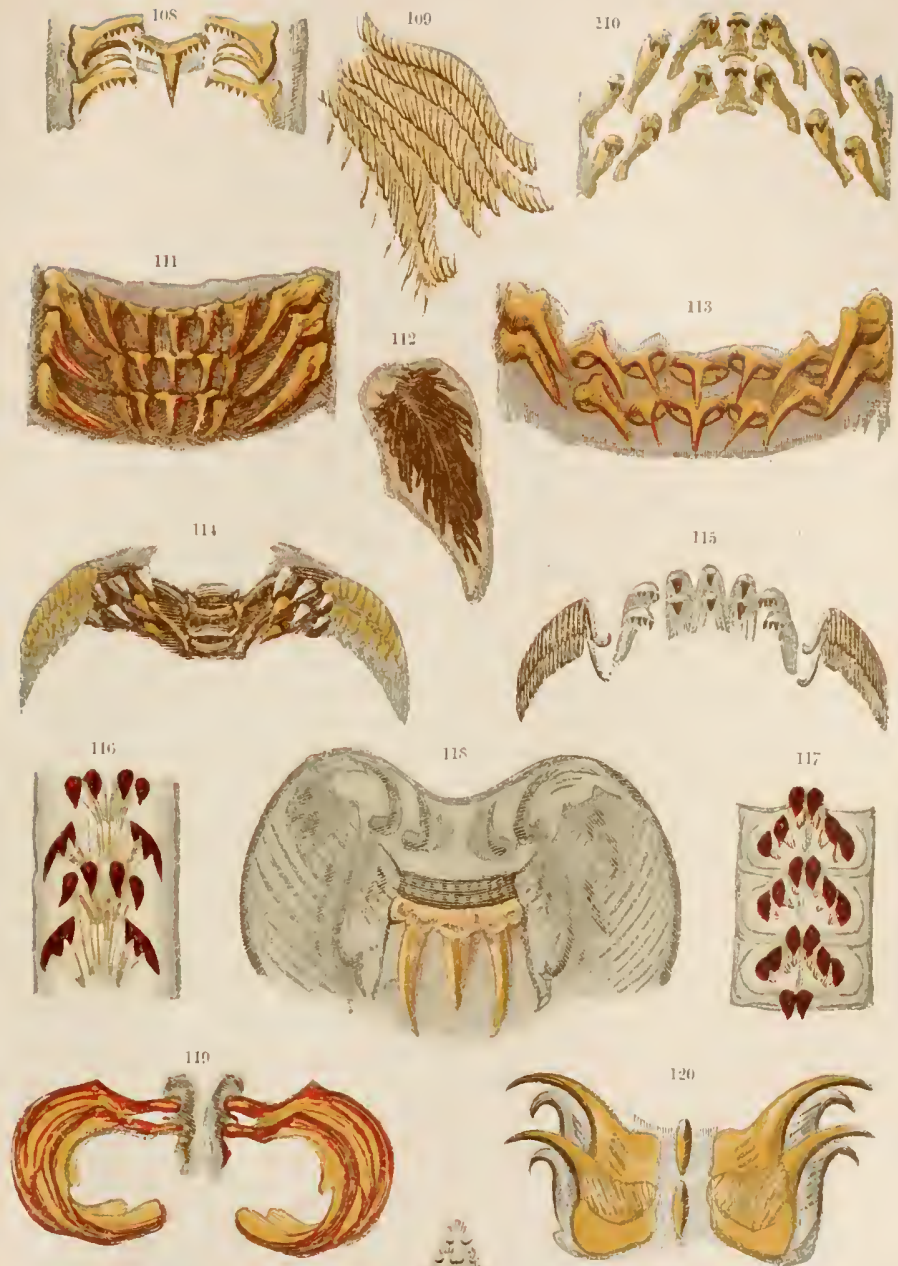

their systematic value is not uniform; and therefore tha attempts to remodel the arrangement of the Gasteropoda by their peculiarities of dentition have not become so complete a success as was at first expected. Some, how. ever, hold a different opinion; and Dr. J. E. Gray writes : - "One result of the study of these papers (Loven's, On the Tongues of Mollusca) and the examination of the tongues of several molluses has been to establish more firmly the theory which I have long entertained, that no species of gasteropodus molluscous animal can be properly placed in the system unless we are enabled to examine the animal, the shell, the operculum, and the structure of its tongue; and as none of these parts but the shell can be examired in the fossil species, their position in the various genera must be always attended with more or less uncertainty." 1

Dr. Troschel has laboured much in this field of investigation, and in his valuable work on the subject attempts a classification of the principal types by their lingual dentition. The union under one formula of so many creatures widely äiffering in anatomy, habits, and shell structure, clearly indicates that, if the lingual ribbon contains generic characters, they have not yet been ascertained. At the same time, it does present differences which may offer collateral evidence in cases otherwise difficult of discrimination. It does not help us to separate carnivorous from phytophagus animals; but it seems possible to make use of it as a mark between species; for, in all, there is a distinct difference between the tongues even of the most closely allied. Thus, amongst other changes, it has been found necessary to remove the Proserpinadoe from the neighbourhood of the Cyclophorida, to which they were formerly supposed to be nearly related, and to place them in a more natural position near the Neritidce. That these investigations are of value is also shown by the light which has been shed on the true position of Aporrhais, supposed by so great a naturalist as Forbes to be akin to the Cerithiidae, but which is shown by its dentition to belong to the Strombida. ${ }^{2}$

(1) Annals of Nat. Hist. Ser. ii. vol. x. p. 413.

(2) See a paper on the subject in the Trans. Linn. Soc. 1567. 
The relations of the freshwater operculata are as varied as those of the land. Ampullaria seems to find its nearest marine relative in Natica, an opinion which seems confirmed by the form of the shell. A TVest Indian species found on the trees of the forests of those islands, and placed by Lamarch in the Helicina, would rather appear to belong to Neritina. The several peculiarities of their teeth, especially that of $H$. nemoralis, with its numerous uncini, its sub-opaque trapezoid laterals, which seem heretofore to have been overlooked, confirm the belief in its close relationship to Neritina. The horny mandibles of the Mollusca may be deserving of some attention with a view to the elucidation of their affinities. In Cyclotus translucidus the mandible is divided into two portions by a median articulation, and it is covered with fine denticulations in regular rows, somewhat like that of Velutina, Plate V. No. 109. In most of the inoperculata, the mandible is horse-shoe-shaped, and striate or corrugate. In 1 mpullaria, the same organ is beak-shaped, like the upper mandible of Octopus or Loligo.

"The lingual band, we should premise, has been, for convenience of description, divided into longitudinal areas, which are crossed by many rows of teeth. There are five, distinguishable by the different characters of the teeth they bear; but the characteristics are not always present. The teeth are consequently named median, lateral, and uncini, although the latter are not necessarily more hooked than the others. The areas bearing the uncini have been called pleurce. Since each row is a repetition of all the rest, the system of teeth admits of easy representation by a numerical formula, in which, when the uncini are very numerous, they are indicated by the sign $\infty$ (infinity), and the others by the proper figure. Thus, $\infty \cdot 5 \cdot 1 \cdot 5 \cdot \infty$, which represents the system in the genus Trochus, signifies that each row consists of one median, flanked on both sides by five lateral teeth, and these again by a large number of uncini. When only three areas are found, the outer ones are to be considered as the pleurr, inasmuch as there is frequently a manifest division in the membrane between them and the lateral areas."

Most of the Cephalopod molluses are provided with 
strong, well developed teeth; they are all animal feeders. Loven describes those of the cuttle-fish (Sepia officinalis, Plate V. No. 111), as like Pteropoda, formula of teeth, $3 \cdot 1 \cdot 3$. The Sepia is also furnished with a retractilo proboscis, and a prehensile spiny collar, apparently for the purpose of holding its prey while the teeth are employed in drilling or abrading it. In the Squid (Loligo, No.113), the medians, broad at the base, approach the tricuspid form. with a prolonged acute central cusp; while the uncini aro much prolonged and slightly curved. The lingual band increases in breadth towards the hinder part, in some instances to twice the diameter of the anterior. The band, when mounted dry, forms a fine object for the blackground illuminator, or side reflector. The lingual band of Octopus tuberculatus differs slightly with Sepia.

The Nudibranchiata have become more attractive since the publication of the valuable and beautifully-illustrated monograph of Messrs. Alder and Hancock. The nudibranchs are without a shelly covering, slug-like in their appearance, and most voracious feeders, greedily devouring zoophytes, sponges, \&c. (Plate IX. b.) They possess the remarkable property of restoring lost parts; their powers of endurance are great, so that they may be kept alive for some time in a small glass jar of sea-water. While keeping a specimen of the Piplida in confinement, the Rev. Mr. Lowe noticed on several occasions a display of a brilliant phosphorescence. Many of the genus Dorididee and Eolidida are infested with parasitic Entromostraca, which either live freely on the surface, under the skin, or adhere to the branchire of the animals. Oncidoris bilamellata (the Sea-lemon) belongs to the Dorididæ; its mouth is provided with a narrow band of strong hooked teeth (Plate V. No. 120 ), which in some species are serrated; all are provided with mandibles, consisting of two horny plates uniting near the fore part. The median row of teeth are small and inconspicuous; the band is represented by the formula 2.1 2. A portion of the mandible of Aplysia hybrida (the Sea-hare) is shown in Plate V. No. 112.

Patella radiata (the Rock-limpet).- The band of this molluse, No. 116, may be readily distinguished from the common limpet of our coasts; the remarkably long ribbon- 
like membrane, which lies folded up in the abdominat cavity, is furnished with numerous rows of strong, nearly opaque, dark brown tricuspid teeth. The teeth of A cmoex (No. 117) are differently arranged; their formula is $3 \cdot 1 \cdot 3$. Chitonidoe are said to be near relatives of the Patellidce; the mouths of all are furnished with mandibles.

Testacella maugei, belonging to the Pulmonifera, is sluglike in its appearance, and, curiously enough, is subterranean in its habits, chiefly feeding on earth-worms. During winter and in dry weather it forms a kind of cocoon, and thus completely encloses itself in an opaque white mantle, which effectually protects it from atmospheric influences. Its lingual membrane is large, and covered with about fifty rows of divergent teeth, which gradually diminish in size towards the median row; each tooth is barbed and pointed, broader towards the base, and furnished with an articulating nipple set in the basement membrane. A few rows are represented slightly magnified, Plate V. No. 121. Their formula is $00 \cdot 1 \cdot 0.0$.

Cymba olla (the Boat-shell) belongs to the species Velutinidæ, formula, $0 \cdot 1 \cdot 0$, or $1 \cdot 1 \cdot 1$. The lingual band, No. 118, is nasrow and ribbon-like in its appearance, with numerous trident-shaped teeth set on a strong muscular membrane. The end of the strap and its connexion with the muscles at the hinder extremity of the cartilaginous cushion is shown in the drawing. The blueish appearance seen in the Plate is due to a selenite film and polarised light. Scapander ligniarius (the Boatman shell).--Tho band (Plate V. No. 119), is narrow, but the teeth are bold and of extraordinary size; their formula is $1 \cdot 0 \cdot 1$. This molluse is said to be without eyes. Pleurobranchus plumula belongs to the samo family; its teeth are simple, recurver, and convex, and arranged in numerous divergent rows; the medians of which are largest. The mandible (Plate V. No. 122), presents an exceedingly pretty tesselated appearance, and the numerous divergent rows have tricuspided denticulations. Velutina lavigata (the Velvety shell), formula $3 \cdot 1 \cdot 3$. - The teeth (Plate V. No. 108) aro small and fine; medians recurved, with a series of delicate denticulations on either side of the central cusp, which is much prolonged: 1st laterals, denticulate, with 
outer cusp prolonged; $2 d$ and $3 d$ laterals, simple curved. or hooked-shaped. The mandible, No. 109, divided in the centre, forms two plates of divergent denticulations.

Haliotis luberculatus (the Ear-shell), is a well-known beautiful shell much used for ornamental purposes. The lingual band, Plate V. No. 114, is well developed. Tho medians are flattened out, recurved obtuse teeth; 1st laterals, trapezoidal or bean-like ; uncini numerous, about sixty, denticulate, the few first pairs are prolonged into strong pointed cusps. Turbo marmoratus (the Top-shell). After the outer layer of shell is removed, it presents a delicate pearly appearance. The lingual band, No. 123, closely resembles Trochus; it is long and narrow, the median teeth are broadest, with five recurved laterals, and numerous rows of uncini, slender and hooked. A singlo row only is represented in the plate. Cyclotus translucidus, a family of operculate land-shells, belongs to tho Cyclostomatidoe. The teeth shown at No. 110, formula $3 \cdot 1 \cdot 3$, are arranged in slightly divergent rows on a narrow band; they are more or less subquadrate, recurved, with their central cusps prolonged. Cistula catenata, one of the family Cyclophoridce; its band, No. 115, formula $2 \cdot 1 \cdot 2$, shows teeth resembling those of Littorina, and should certainly be separated from Cyclophorida. It would also seem that the teeth of Cyclostomatidae point to a near alliance with the Trochidae; but this question can only be determined by an examination of several. species, when it may, perhaps, be decided to give them rank as a sub-order. They are numerous enough; the West Indian islands alone furnish us with 200 species.

Professor William Thompson, in his paper "On the Dentition of British Pulmonifera," Ann. Nat. IIis. vol. vii. 1851 , pointed out that the length of the lingual band, and number of rows of teeth borme on it, vary greatly in different species. The rows, however, being closely set are usually very numerous; but it is among the Pulmonifera we meet with tho most astonishing instances of large numbers of teeth Limax maximus possesses 26,800 , distributed through 180 rows of 160 each; the individual teeth mensuring only one 10,000th of an inch. 
Helix pomatia has 21,000, and its comparatively dwarfed congener, H. obvoluta, no less than 15,000 . When it is remembered that these estimates refer to series of forms, curiously carved and sculptured, the total area sustaining them not measuring in most of the molluses half an inch in length, we must be filled with admiration at the marvellous creative power bestowed upon the organization of these lowly-creeping creatures.

The Preparation of Teeth of Mollusca.-The method of preparing the lingual membranes of Mollusca is as follows :-The animal having been taken from its shell, pin down the muscular foot to a piece of cork, pour water upors it, and let the water be changed as often as it becomes turbid. Then with a dissecting microscope and a good bull's eye condenser cut open and expose to view the floor of the mouth; pin back the cut edges thronghout its whole length, and work out the dental band with knife and forceps. When the band is detached place it in a watch-glass, and again clean it well by repeated washings and a camel's hair brush : then place it in weak spirit and water, where it must remain for a few days before mounting. If the membrane is dense and fatty it must be soaked for a time in liquor potassa, and when removed carefully washed. The best fluids for mounting are glycerine, weak spirit and water, Rimmington's glycerine-jelly, or Goadby's solution. Canada balsam renders them so very pellucid that the finer teeth are completely lost in it.

Thread-cells.-These curious appendages, so commonly met with in the Actinozoa, and in the tentacles surrounding the mouth of the Medusæ, are also seen in some species of Mollusca.

These prehensile threads, now generally termed "urticating organs," were discovered in 1835, in the Hydra, by Corda and by Ehrenberg. About the same time they were found by R. Wagner in the Actiniæ, who at first regarded them as zoosperms. Subsequently, however, he recognised their identity with similar organs in the Medusæ, and gave them the name of urticating organs. Since then numerous observations have shown that these organs exist in the entire class of polypes; in that of the Hydra, Medusæ, as well as in the Synaptæ, many Turbellariæ, 
some Annelids, and lastly among the Mollusca, the Eolidæ in particular.

Max Schultze has divided these organs into two categories; one including those of a rod-like form, or the bacillar, which are found pretty generally in the Turbellariæ; and the other containing the urticating capsules armed with a long filament. But the researches of otler observers, including Dr. Bergh, have shown that this distinction is unimportant.

Dr. Bergh has devoted murh attention to the urticating filaments or cnida of the Mollusca, which are far less well known than those of the Cælenterata. The existence of sacs with enida-that is to say, the existence of true urticating batteries-is then at the present day a wellestablished fact as regards the typical forms of the Eolidx, i.e. in the genera Eolidida, Montagna, Facelina.

In every case the urticating batteries are planted at the extremities of the papillæ above the hepatic lobe. The sac opens to the exterior by a minute pore situated at the summit. Its walls are muscular, a circular layer of fibres being the most considerable element. The interior is filled with urticating cells, together with cysts full of closelypacked filaments and free filaments. The genus Pleurophyllidium, according to Dr. Bergh, is the only mollusc besides the Eolidæ in which cnida are met with, and it is to be remarked that in their anatomical conformation these animals appear to approach very closely to the Eolidæ.

The use of these crida is still involved in doubt. Mr. Lewes, however, has shown that they do not serve to paralyse the animals upon which Actiniæ feed.

Ova of Mollusca. - It is interesting to watch the development of the spawn of the Mollusca under a low magnifying power. The ova of the Limnaeus is usually found adhering to the surfaces of stones, pieces of weed, or other matters in the water; they are always contained in a long ribbonlike delicate ova-sac of a curious and beautiful form. The mass of eggs deposited by the Doris resembles a frill of lace of great beauty. In Aplysia the spawn resem. bles long strings of vermicelli, of varying tints through-

(1) On Urticating Filaments in the Mollusca, bv Dr. Bergh. Journ. MIicros. Sci. vol. ii. p. 274.1862. 
out the different parts of the thread. The Limnceus stag. nalis deposits small sacs, containing from fifty to sixty
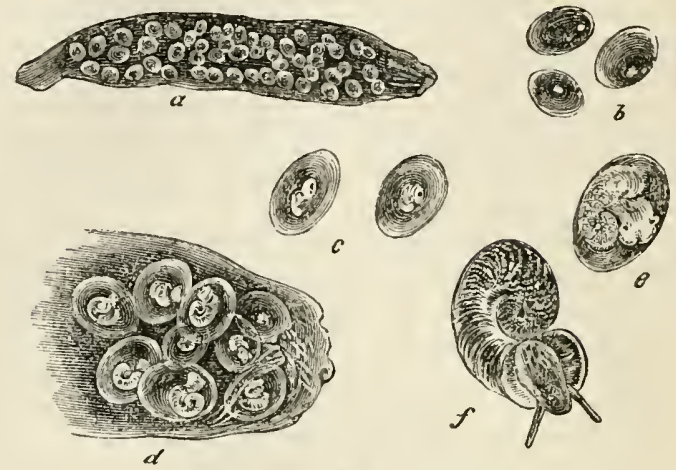

Fig. 252.-Limnaa slagnalis.

ova; one of which is represented at $a$, fig. 252. When examined soon after they are deposited, the vesicles appear to be filled with a perfectly clear fluid; at the end of twenty-four hours a very minute yellow spot, the nucleus, or germ, may be seen near the side of the cell-wall. In about forty-eight hours afterwards, this small germ has a smaller central spot rather deeper in colour, which is the nucleolus. On the fourth day the nucleus has changed its position, and is enlarged to double the size: a magnified view is given at $b$; upon viewing it more closely, a transverse fissure or depression is seen; this on the eighth day most distinctly divides the small mass into the shell and soft part of the future animal, $c$. It is then detached from the side of the cell, and moves with a rotatory motion around the cell-interior; the direction of this motion is from the right to the left, and is always increased whon the sunlight falls upon it. The increase is gradual up to the sixteenth day, when the spiral axis can now be made out as at $d$; it presents a striking difference in appearance to the soft parts. On the eighteenth day, these changes are more distinctly visible, and the ova crowd down to the mouth of the ova-sac; by using a higher magnifying power, a minute black speck, the future eye, is seen protruded 
with the tentacles, at $e$. Upon closely observing it, a fringe of cilia is noticed in rnotion near the edge of the shell. It is now apparent that the rotatory motion first observed must have been in a great measure due to this; and the current kept up in the fluid contents of the cell by the ciliary fringes. For days after the young animal has escaped from the egg, this ciliary motion is carried on, not alone by the fringe surrounding the mouth, but by cilia entirely surrounding the tentacles themselves,

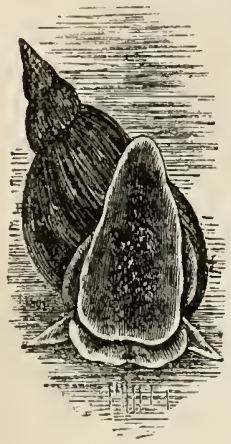

Fig. 253.-Limnceus stagnalis. which whips up the supply of nourishment, and at the same time the proper aeration of the blood is effected. Whilst in the ova, it probably is by this motion that the cell-contents are converted into tissues and shell. From the twenty-sixth to the twenty-eighth day, it appears actively engaged near the side of the egg, using all its force to break through the cell-wall, which at length it succeeds in doing; leaving the shell in the ovasac, and immediately attaching itself to the side of the glass-vase, to recommence its ciliary play, and appears in the advanced stage represented at $f$. It is still some months before it grows to the perfect form represented at fig. 253, where the animal is drawn with its sucker-like foot adhering closely to the side of the glass-vase. One of these snails may deposit from two to three of these ova-sacs a week; producing, in the course of six weeks or two months, from 900 to 1,000 young, thus supplying food for fish.

The shell itself is deposited in minute cells, which take up a circular position around the axis; on its under-surface a hyaline membrane is secreted. The integument expands, and at various points an internal colouring matter or pigment is deposited. The increase of the membrane goes on until the expanded foot is formed, the onter edge of which is rounded off and turned over by condensed tissue in the form of a twisted wire; this encloses a net-work of small vassels filled with a fluid in constant and rapid motion. The course of the blood or fluid, as it passes 
from the heart, may be traced through the larger branches to the respiratory organs, consisting of branchial-fringes placed above the month; the blood may also be seen returning through other vessels. The heart, a strong muscular apparatus, is pear-shaped, and enclosed within a pericardium or enveloping membrane, which is extremely thin and pellucid. Affixed to the sides of the heart are muscular bands of considerable strength, the action of which appears very like the alternate to-and-fro motion occasioned by drawing out bands of India-rubber, and which, although so minute, must be analogous to the muscular cords of the mammal heart; it beats or contracts at the rate of about sixty times a minute; and is placed rather far back in the body, towards the axis of the shell. The nervous system is made up of ganglia, or nervous centres, and distributed throughout the various portions of the body.

The singular arrangement of the eye cannot be omitted; it appears at an early stage of life to be within the tentacle, and consequently capable of being retracted into it. In the adult animal, the eye is situated at the base of the tentacle; and although it can be protruded at pleasure for a short distance, it seems to depend much upon the tentacle for protection as a coverlid-it invariably clraws down the tentacle over the eye when that organ needs protection. The eye itself is pyriform, somewhat resembling the round figure of the human eye-ball, with its optic-nerve attached. In colour it is very dark, having a central pupillary-opening for the admission of light. The tentacle, which is cylindrical in the young animal, becomes flat and triangular in shape in the adult. The young animal is for some time without teeth; consequently, it does not very early betake itself to a vegetable sustenance: in place of teeth it has two rows of cilia, as before stated, which drop off when the teeth are fully formed. The lingual band bearing the teeth, or the "tongue," as it is termed, consists of several rows of cutting spines, pointed with silica.

It is a fact of some interest, physiologically, to know that if the young animal is kept in fiesh water alone, without vegetable matter of any kind, it retains its cilia, but arrest of development follows, and it acquires no gastric teeth, and never attains perfection in form or sizu. 
If, at the same time, it is confined within a narrow cell, or space, it grows only to such a size as will enable it to move about freely; thus it is made to adapt itself to the necessities of a restricted state of existence. Some young animals in a narrow glass-cell, at the end of six months, were alive and well, and the cilia retained around the tentacles in constant activity; whilst other animals of the same brood and age, placed in a sitnation favourable to growth, attained their full size, and produced young, which grew in three weeks to the size of their elder relations.

Should any injury occur to the shell, or a portion of it become broken off, the calcareous deposit is quickly resumed, in order to replace the lost part; the cells being apparently only half the size of those originally deposited. This may be thought to afford some proof of the statement made by Professor Paget,- - "that, as a rule, the reparative power in each perfect species, whether it be higher o1 lower in the scale, is in an inverse proportion to the amount of change through which it has passed in its development from the embryonic to the perfect state. And the deduction to be made from them is, that the powers for development from the embryo are identical with those exercised for the restoration from injuries; in other words, that the powers are the same by which perfection is first achieved, and by which, when lost, it is recovered. Indeed, it would almost seem as if the species that have the least means of escape or defence from mutilation were those on which the most ample power of repair has been bestowed,- - an admirable instance, if it be only generally true, of the beneficence that has prepared for the welfare of even the least of the living world, with as much care as if they were the sole objects of the Divine regard."

The primordial cell-wall of the cell does not appear to enter into the formative process of the embryo-the cellcontents alone nourishing the vital blastema of the nucleus. A gradual cycle of progressive development once set up, goes on, until the animal is sufficiently matured to break through the cell-wall and escape from the ova-sac. At the same time, it may be inferred, that all this is in some measure aided by the process of endosmose; and that cer. 
tain gases or fluids are drawn into the interior, and thus aid in the supply of nourishment for the growth of the animal. The cell-wall appears to bear the same relation to the future perfect animal that the egg-shell of the chick does to it; it is, in fact, but an external covering to a certuin amount of gaseous and fluid nuatter, used for placing the germ of life in a more farourable state for development, assisted, as it is, by an increase of temperature, usnally the resultant of a chemical action, set up or once begun in an organism and a medium. "The ovum destined to become a new creature originates from a cell, enclosing gemmules, from which its tissues are formed, and nutriment is assimilated, and which eventually enables the animal to successively renew its organs, through a series of metamorphoses that give it permanent conditions, not only different, but even directly contrary to those which it had primitively."

Cephalopoda.-Molluscous animals without a foot, or a distinct head, and covered with fleshy arms, bearing sucker-like discs. The Cuttles and Squids form the principal groups of this class, only a few species of which are found on our shores. These molluses are the nearest approach of all invertebrate animals to the vertebrate forms; and their organs of sense appear to be highly developed. ${ }^{1}$ Cuttle-fish bone, cut in thin sections, or broken into small fragments, are interesting microscopic objects : the peculiarities of structure are best seen when small pieces are detached with a sharp knife. In the living state these creatures have the power of suddenly changing the colour of their skins.

Structure of Shell. - We may exhibit the structure of shell by using an acid solvent in the following manner. If a sufficient quantity of hydrochloric acid, considerably diluted with water (say one part acid to twenty-four of water), be poured upon a shell contained in a glass vessel, it will soon exhibit a soft floating substance, consisting of innumerable membranes, which retain the figure of the shcll, and afford a beautiful and popular object for th,

(1) In the Cephalopoda ve have the first indication of a true internal skeleton, in the form of a broal ftattened cartilage which protects the centrich (optic) ganglia of the nervous systen. 
uicroscope. In analysing shells of a finer texture than such as are generally submitted to the test of experiment, the greatest circumspection is necessary. So much so, that M. Herissant, whose attention was particularly devoted to the subject, after placing a porcelain shell in spirits of wine, added, from day to day, for the space of two months, a single drop of spirits of nitre, lest the air, generated or let loose by the action of the hydrochloric acid on the earthy substance, should tear the net-work of the fine membranaceous structure. This gradual operation was attended with complete success, and a delicate and beautifully reticulated film, resembling a spider's web in texture, rewarded the patience of the operator ; the organization of which film, from its extreme fineness, he was not, however, able to delineate. In shells of peculiar delicacy, even five or six months are sometimes necessary for their complete development; but in others of a coarser texture the process is soon completed. Sections of shells are usually mounted in Canada balsam, or in shallow cells with glycerine.

Mr. George Rainey pointed out the remarkable fact that many of the appearances presented by the shell or hard structures of animals, and which had been usually referred to cell-development, are really produced by the physical laws which govern the aggregation of certain crystallizable salts when exposed to the action of vegetable and animal substances in a state of solution. Mr. Rainey gives a process for obtaining artificially a crystalline substance which closely resembles shell in its chemical structure.

"The chemical substances to be employed in the production of the artificial calculi are, a soluble compound of lime, and carbonate of potash or soda, dissolved in separate portions of water ; and some viscid vegetable or animal substance, such as gum or albumen, mixed with each of these solutions. The mechanical conditions required to act in conjunction with the chemical means are, the presence of such a quantity of the viscid material in each solution as will be sufficient to make the two solutions, when mixed together, of about the same density as that of the nascent carbonate of lime, and a state of perfect rest of the fluid in which the decomposition is going on, so that the newly-formed compound may be interfered with 
as little as possible in its subsidence to the sides and bottom of the vessel. This will require two or three weeks, or longer, according to the size and completeness of the calculi. But I have not found that they increase at all after six weeks."

Mr. Rainey shows ${ }^{1}$ the analogy or identity of his artificially formed crystals with those found in natural products both in animals and vegetables, chiefly confining himself to the structure and formation of shells and bone, pigmental and other cells, and the structure and development of the crystalline lenses, which he contends are all formed upon precisely the same physical principles as the artificial crystals. Take, for instance, the calculi found in the body : these cannot be distinguished from the crystals of artifically formed carbonate of lime. Again, the shell of the crustaceans; the resemblance between these and the artificial products is, in some respects, more complete than in that of calculi. All the appearances in shells can be best observed by merely cleaning them in water, and examining them in glycerine, grinding being unnecessary and injurious. Polarised light is indispensable; as in the young hermit-crab, at the part where the calcareous and membranous portions of the shell are continuous, the circular forms of globular carbonate are so delicate that no evidence whatever of its presence can be detected under powerful lenses, and with the best illumination, until polarised light is brought to bear upon the specimen. To obtain the most satisfactory results in the investigation of the process of calcification of animal tissues, it is indispensably necessary that the parts examined should be in the earliest stages of the process, and before the calcifying membrane is entirely covered with the globular coalescing deposit. 'The usual plan of examining shells in thin vertical sections is entirely useless, unless it be simply to see the number and arrangement of their layers; the part of the section in such specimens, in which the calcifying process ought to be best seen, being entirely ground off. This part, being the softest, can only be preserved in the process of grinding by extreme care, and by keep-

(1) G. Rainey, "On the Mode of Formation of Shells, Bone, dc. by a process of Molecular Coalescence." 1858. 
ing the lower edge of the section always thicker than the upper.

Dr. Carpenter describes the shell of the Crab and Lobster as being composed of three layers, viz. the epidermis or cuticle, the rete-mucosum or pigment, and the corium. The epidermis is of a horny nature, being generally more or less brown in colour, and under the highest magnifying powers presenting no trace of structure (fig. 247, No. 2); it invests all the outer parts of the shell, and has in many instances large cylindrical or feather-like hairs developed from certain portions of its surface. The rete-mucosum, or pigment cells, consist of either a series of hexagonal cells, forming a distinct stratum, or of pigmental matter diffused throughout a certain thickness of the calcareous layer. (Fig. 247, No. 5.) In the Crab and Lobster it is very thin, but in the Crayfish it occupies in some parts more than one-third of the entire thickness of the shell; when examined by the microscope, this portion appears to be composed of a large number of very thin laminæ, which are indicated by fine lines taking the same direction on the surface of the shell, the number of lines being the greatest in the oldest specimens; these layers, even in the Crayfish, are covered by a thin stratum of very minute hexagonal cells, without any trace of cell matter in their interior. The corium is the thickest layer of the three, being the one on which the strength of the shell depends, in consequence of the calcareous material deposited in it. (Fig. 247, No. 4.) When a vertical section of the shell of the Crab is examined, it is found to be traversed by parallel tubes, resembling those in the dentine of the human tooth; these tubes extend from the inner to the outer surface of the shell, and are occasionally covered by wavy lines, probably those of growth, shown in a portion of No. 3, fig. 247. If a horizontal section of the same shell be made, so that the tubes be divided at right angles to their length, the surface will clearly exhibit their open ends, surrounded by calcareous matter. In Shrimps and very small Crabs, the deposition of the calcareous matter takes place in concentric rings like those of agate; and occasionally small centres of ossification, somewhat like Pinna, with radiating striæ, are met with in the Shrimp. If the calcarcous 
portion of the shell be steeped in hydrochloric acid, a distinct animal structure or basis is left behind, and the characters of the part will be very accurately preserved. The calcareous matter, like that of bone, generally presents a more or less granular appearance, as at No. 4, and so angular in figure as to resemble certain forms of rhomboidal crystals : No. 2 is a section from the outer brown shell of the Oyster. The beauty of all such structures is much increased if viewed with polarised light on the selenite stage. ${ }^{1}$

Crustacea.-The skeletons of Crustacea are external to the soft parts; in a great number of species the shell is thin and membranous, in others it is of a horny material, thickened with calcareous matter, having a distinct seriss

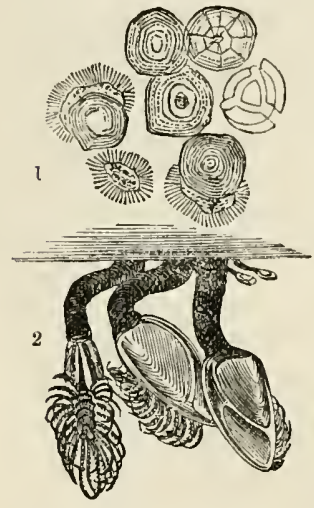

Fig. 254. of pigment cells of a stellate fignre, all supplying beautiful objects for microscopic examination and polarised light. The Astacus, Crayfish, may be taken as the type of that large and important group of Crustacea to which the term Podophthalma, Stalk-eyed, is applied.2

Cirrhopoda or Cirripedia, when mature, attach themselves to rocks and other objects. The Barnacle (fig. 254) and Acornshell are the best known examples of this order; they generally select floating objects to 1, Young fry of the Oyster, a dwell upon; and bottoms of portion of then with cilia protruded. 2, Body and cirri of Raruacles.

ships have been covered by them to such an extent as even to impede their progress through the water. The soft bodies of these animals are enclosed in a case composed of five calcareous plates; from this circumstance they weres grouped with the multivalve shells of the older concholo-

(1) See Prof. Huxley's article on the "Tegumentary Organs," Cyclop. Anat. and Physio. vol. v. p. 487.

(2) Some valuable information will be found on the minute structure of shells in Prof. Williamson's paper, "On some Histological Features in the Shells of the Crustacea," Journ. Mitros. Scien. vol. viii. p. 35, 1860. 
gists. Their limbs are converted into tufts of jointed cirri, and protrude through an opening in the mantle which lines the interior of the shell. The cirri, twelve in number, are covered with cilia, which, when the animal is alive, are in continual motion. The intestinal canal is complete, and the nervous system exhibits the usual series of ganglia, characteristic of the articulate type. The head is marked only by the position of the mouth, and is armed with a pair of jatrs, if we may so term the shells.

Balanidae, "Sea-acorns," a sessile species, whose curious little habitations may constantly be met with upon the rocks of the sea-shore, and not unfrequently upon many species of marine shells. The shell forms a short tube, and is usually composed of six segments securely united together. The lower part of the tube is firmly fixed to the object on which the Balanus has taken up its abode; whilst the superior orifice is closed by a movable roof, composed of from $t w o$ to four valves, between which the little tenant of this curious domicile protrudes his delicate cirri in search of nourishment. In the young state the Balanidce freely swim about, and somewhat resemble the following group, Entomostraca.

Entomostraca, or Water-fleas, undergo a series of remarkable changes from the moment of their escape from the egg to the attainment of their fully matured form. And it is of the highest interest to remark that, in obedience to a law which, if not miversal, is at any rate widely prevalent in the animal kingdom, these temporary or larval forms are themselves closely analogous to the prefect forms of groups still lower in the scale of existence, so that many of them in their early forms were formerly, before their life-history was known, either classed as distinet species, or placed in a position very far from that which they are now seen to occupy. The embryo of the Shore-crab (Carcinus monas) before, and for a short time after, its liberation from the ovum, presents both in size and general outline a strong resemblance to Entomostraca. In this transition stage it was assigned to a distinct genus under the name of $Z$ oea ; and having undergone a still further transfurmation was called Megalopa. In this latter stage it 
puts on somewhat the appearance of the Lobster crab (Galathea), and after another step attains its true crabform, being the highest development of which it is capable. These changes are not produced gradually, but by a succession of "moults," the animal becoming at times sluggish, casting its hard covering, and reappearing in a new guise. The after growth of a crustacean is carried on by the system of moulting; the hard caleareous case of the animal preventing its growth in any other mode. And as in the higher orders of Crustacea, so also amongst the Entomostraca, transformations of this kind constantly take place. Cyclops quadricornis, when first born, is totally unlike its parents, being of an ovoid shape, having only two short antennæ and two pairs of feet; in three moults the animal reaches its perfect form, with its two pairs of antennæ, five pairs of feet, and body divided into several distinct rings or segments.

The animals comprising the order Ostracoda are generally

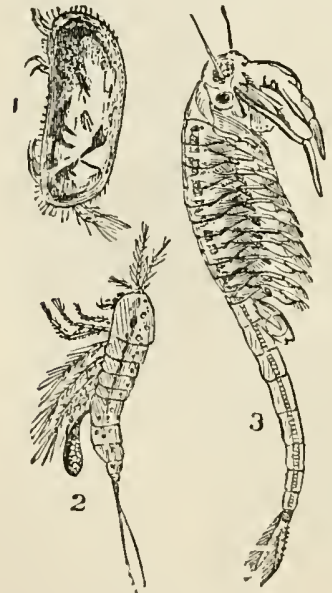

Fig. 255.

1, Cypris. 2, Polyphemus, Cyclops. 3, Branchiopus stagnalis. of very minute size; the body, which strongly resembles that of the Copepoda, is always enclosed in a little bivalve shell, the feet and antenne being protruded between the lower edges of the valves. These little shells so closely resemble those of minute bivalve Mollusca, that those of some of the larger species have aetually been deseribed by conchologists as the eovering of animals belonging to that class. The antennæ are often curiously branehed; and the hinder extremity is usually prolonged into a sort of tail, which is seen in constant action when the animal is in motion. In Cypridina, the bolly is entirely enclosed by a shell, of which the genus Cypris (fig. 255) is an example: 
and in Daphnia, "Water-fleas," the head is protruded beyond the shell. In Polyphemidce the head is large, and almost entirely occupied by an enormous eye, giving the creatures a most singular appearance; the Monoculus is a well-known example of this group. Another family, not provided with a shell or carapace, called Branchiopoda, from the name of the typical genus, Branchiopus stagnalis (fig. 255), is often found after heavy rains in cart-ruts and other small pools.

Daphinia pulex is found commonly in fresh water, and is scarcely inferior to its marine relative, Talitrus locusta, in agility. The Corophium longicorne, remarkable for its long antennæ, is not less so for its singular habits. It is found at Rochelle, where it burrows in the sand, and wages constant war with all other marine creatures of moderate size that come in its way.

Dr. Baird has followed up the successive generations in Daphnia pulex; so far as the fourth change in the Daphnia born from the ordinary ova, and so far as the third in those born from the ephippial egrss. These ephippia, or "winter eggs," require a few words of explanation. 'They are, in fact, eggs covered with envelopes of more than usual hardness and thickness, being enabled to withstand an excess of cold, which would surely prove fatal to the parent. This observer found, upon examining ponds which had been filled up again by the rain after remaining two months dry, numerous specimens of the Cyclops quadricornis in all stages of growth. Dr. Baird, in his "Natural History of British Entomostraca," 1850, tells us that they have many enemies.

"The larva of the Corethra plumicornis, known to microscopical observers as the skeleton larva, is exceedingly rapacious of the Daphnia. Pritchard tells us they are the choice food of a species of Nais; and Dr. Parnell states that the Lochlevin trout owes its superior sweetness and richness of flavour to its food, which consists of small shell-fish and Entomostraca." These animals abound both in fresh and salt water. Artemia are formed exclusively in salt water, in salt marshes, and in water highly charged with salt. "Myriads of these Entomostraca are to be found in the salterns at Lymington, in the open tanks or reservoirs where the brino 
is deposited previous to boiling. A pint of the fluid contains about a quarter of a pound of salt, and this concentrated solution destroys most other marine animals." During the fine days in summer Artemice may be observed in immense numbers near the surface of the water, and, as they are frequently of a lively red colour, the water appears tinged with the same hue. ${ }^{1}$ There is nothing more clegant than the form of this little animal. Its movements are peculiar. It swims almost always on its back, and by means of its tail it runs in all directions, its feet being in constant motion. They are both oviparous and ovoviviparous, according to the season of the year. At certain periods they only lay eggs, while during the hot summer months they produce their young alive. In about fifteen days the eggs are expelled in numbers varying from 50 to 150 . As is the case with many of the Entomostraca, the young present a very different appearance from the adult animals; and they are so exactly like the young of Chiroceghalus, that with difficulty they can be distinguished the one from the other. The ova of other species are furnished with thick capsules, and imbedded in a dark opaque substance, presenting a minutely cellular appearance, and occupying the interspace between the body of the animal and the back of the shell. This is called the ephippium. ${ }^{2}$ The shell is often beautifully transparent, sonetimes spotted with pigment: it consists of a substance known as chitine, impregnated with a variable amount of carbonate of lime, which produces a copious effervescence on the addition of a small quantity of acid, and when boiled it turns red, like the lobster. Their shells vary in structure. Sometimes they consist of two valves united at the back, and resembling

(1) It is a curious fact that salt-water when highly concentrated frequently assumes a red colour, and that this should have been attributed to the presence of the Artemia salina, as in the case of fresh-water noticed elsewhere found coloured red by a species of Paramacium. The cause of this red colour, which was well known to take place in the salt marshes and reservoirs of salt-water at Montpellier, was made the subject of a very grave discussion in France. some maintained that the colour was caused by the presence of Artcmice, while others declared that it arose from vegetable matter, either Hcmatococcus or Protococcus. M. Joly, came to the conclusion, after many careful examinations, that the red colour depends upon the presence of myriads of monads, and that the Artemice living upon these partook of the same red hue, and thus the water appeared to be of the same colour.

(2) See paper on "Reproduction in Daphnia," by Sir John Lubbock, Philos. Trans. $185 \%$, p. 79. 
the bivalve shell of a mussel ; others are simply folded at the back, so as to appear like a bivalve, but are really not so; or they consist of a number of rings or segments. The body of the Cypris presents a reticulated appearance, resembling that of cell structure. All the Entomostraca are best preserved in a solution of ehloride of calcium.

Anvulosa.-Articulata. The animals composing the sub-kingdom Articulata are characterised by having the body enclosed in a tunic, or integument, consisting of a series of rings, segments, or joints, "articulated" together by a flexible membrane.

The Annulosa are divided, by Professor Huxley, into two nrincipal groups, the Arthropoda and the Annuloida. The Arthropoda, comprising Insecka, Myriapoda, Crustacea, and Arachnida, possess a definitely segmented body; the segments being provided with appendages, the anterior of which are so modified as to subserve the functions of sensation and manducation. They have almost always a heart, communicating with the general cavity of the body, for propelling the true corpusculated blood which that eavity contains. The nervous system consists of a longer or a shorter chain of ganglia.

Nothing can be more variable than the characters of the budy, the appendages, and the nervous system among the rest of the Annulosa, which are included under the Anmuloida; nevertheless, theie are two features in which they all agree; firstly, they possess a remarkable system of vessels, either ciliated, or deprived of eilia, and containing a fluid very different from the true blood which fills the general eavity of the body or perivisceral space ; secondly, in no annuloid animal has any true heart been hitherto discovered. Contractile vessels belonging to the system just referred to abound, but no organ comparable in structure to the heart of other animals has yet been found in any of the Annuloida.

The Annuloida, as thus defined and limited, fall into two parallel series; in one of which, for the most part, diœcious forms predominate, as the Annelida, while of the latter, the Trematoda may be regarded as the typical exanple; on the other hand, the Echinodermata and 
Rotifera, the Taniadae and the Nematoidea, may be considered as the most aberrant groups of their respective series.

Under the head Annelida, Mr. Huxley includes the errant and tubicular Amnelids of Cuvier, and the Gephyrea of De Quatrefages; he thinks that the Terricola-the Earthworms and Naides-should be separated from the Scoledoe of Milne Edwards, and brought into the same group. So far as external structure is concerned, the genus Polynöe is, perhaps, the best fitted to serve as the type to which other Annelida may be referred: the commonest form of the genus being the P. squamata. ${ }^{1}$ The best developed branchix among the Annelids are possessed by the Amphinomida, the Ennicida, the Terebellida, and the Serpulida. The branchire in the three former families are ciliated, branched plunies or tufts attached to the dorsal surface of more or fewer of the segments. In the last they are exclusively attached to the anterior segments of the body, and present the form of two large plumes, each consisting of a principal stem, with many lateral branches; this stem is itself supported on a kind of cartilaginous skeleton.

The teeth in a great number of the Annelida are very curious and distinctive. In the Polynüe there are four, planted in the muscular wall of the proboscis. In the Nereis there are two powerful teeth working horizontally, besides minute accessory denticles. In Syllis there is a circle of sharp teeth, surrounding a triangular median tooth. In Glycera there are a pair of teeth; but the most complex arrangement of teeth is that presented by the Ennicida. The tubicular Annelids possess neither proboscis nor teeti.

Many Annelids pass through a larval condition, in which the body exhibits mere indications of segments, and the appendages are entirely absent; locomotive function being performed by a circlet of cilia, disposed around the anterior part of the body. There is a large group of very remarkable orgauisms, observes Mr. Huxley, the minute "wheel animalcules," Rotifera, whose whole

(1) Consult a valuable paper on this genus in Müller's Archiv. 1857. Also Huxley's "Elements of Compratire Anatomy." 
organization demonstrates, not merely their annulose nature, but their position among the Annuloida, and which exhibit precisely the same indistinct segmentation, the same gcneral absence of appendages, and whose means of locomotion are in like manner confined to one or two ciliated circlets at the anterior part of the body. The connexion between the Annelidu and the Rotifera is further illustrated by such remarkable forms as the Polyophthalmus of De Quatrefages, a true Annelid, which, nevertheless, possesses on each side of the head a ciliated lobe, capable of being voluntarily protruded and retracted, and presenting a close resemblance to the trochal disc of a Rotifer. Hydatina senta has been so well and accurately described by Dr. Cohn, ${ }^{1}$ and others, that it may be taken as the typical form of the Rotifera. The trochal disc in the species, undergoes great changes of form. In Hydatina, it is circular, and its margin is skirted by two distinct continuous bands of cilia, the one immediately in front of, the other behind the mouth. In Brachionus the ciliated circlet fringing the edges of the trochal disc is horseshoe-shaped, but the circlet is produced into three lobes or processes, which stand out perpendicularly to the surface of the trochal disc. In Stephanoceros it fringes the edges of a number of tentaculiform processes, into which the trochal disc is produced, so as to give the whole animal somewhat the appearance of a Polyzoon.

The Tuibellaria, a group of serpent-like worms, for the most part the inhabitants of fresh and salt waters, a few only being found in damp siuations on land, are characterised by the ciliation of the entire surface of the body. In their internal organisation they approximate in many respeets to the Trematoda, while in others they exhibit a ccrtain affinity with the other great group of parasitic Annuloida, the Nematoidea. Polycelis lavigatus, one of the Dendrocoela so well described by De Quatrefages in his Monograph on the Marine Planaria, may be most advantageously selected as a type of the group. The Nemertida have engaged the attention of the same learned anthority, the ova of which undergo a remarkable kind of

(1) Ueber die Fortflawzung der Raderthiere, Von Dr. F. Cohn, in Breslau. 1855. 
metamorphosis. The embryo has at first a ciliated noncontractile, oval body, exhibiting no structure but a semilunar superficial cleft, provided with raised edges. After a time, a small actively-contractile, vermiform creature, resembling the parent, escapes from the interior of the larval form, which it leaves behind like a cast skin. The semilunar cleft becomes the mouth of the imago, and is only part of the larva carried away. The Gordiacei ${ }^{3}$ best enable us to connect the Turbellaria with the very puzzling group Nematoidea, and the structure of several species belongiug to the two genera which compose the group Mermis and Gordius, have recently been made the subject of two elaborate monographs by Meissner. ${ }^{2}$

The Gordiacei are excessively elongated, thread-like worms, plentiful enough in Thames mud, and, as Von Siebold discovered, partake both of the free habit of the Nemertida, and of the parasitic nature of the Nematoidea. The young Mermis, for instance, is parasitic upon insects, inhabiting the perivisceral cavity of the larva or of the imago.

The Nemertida seem to differ from their close allies the Turbellaria, in possessing a vascular system distinct from and added to the water-vessels. In the Hirudinido, Leeches aud Earthworms, a system of vessels homologonous with the pseud-hæmal system exists, and, in addition a series of more or less coiled tubules lie in the perivisceral cavity, and open, by pores, on the ventral surface of the body. These organs have beeu regarded sometimes as secretory, sometimes as respiratory apparatus; but all that we know about them in reality is that they are tubular, and are more or less richly ciliated within, and that, in some cases (Nais, Lumbricus), they present at their internal extremities a ciliated aperture, whereby they freely communicate with the perivisceral cavity.

There remains, however, yet another system of vessels in the Annuloida-the ambulacral vessels of the Echinodermata. These are frequently termed "water-vessels," and, indeed, if we regard the structure with reference to

(1) Huxley, General Natural History.

(2) Beitrüge zur Analomie und Physiologie von Mermis Albicaus, v. Zeitschrit für Wiss, Zoologie, Bd, v 1854; and Beiträge zur Anat.\& Physiol.des Gordiacen, Ibid Bd, vii. 1855 . 
their peculiar functions, they singularly rescmble true water-vessels; they open by an external pore, and are ciliated internally; they unite around the gullet, as do the water-vessels in some Trematoda, and are eventually shut off from such communication; the ambulacral vessels of the Holothuridae undergo precisely this change, and thus they facilitate our comprehensiou of a transition from the water-vessels of the Trematoda to the pseud-hæmal ressels of the Annelida. We may take it as an established fact that, whatever the functions of this varied vascular system and its contents in different classes of the Annuloida, they have nothing to do with the blood or true blood vessels. The latter are entirely absent in all the Annuloida at present known, the blood (improperly called "chyle-aqueous fluid") being simply contained in the perivisceral cavity and its processes. The development of the Nematoidea appears to take place without metamorphosis; the embryo assuming within the egg a form nearly resembling that of the adult. Encysted asexual nematoid worms are frequently found in various parts of the body of fishes; and the remarkable T'richina spiralis is the asexual state of a nematoid worm, encysted within the substance of the muscles of man. Zooid development is only known to occur in one nematoid, the Filaria Medinensis, Guinea-worm. Mr. Busk's careful observations have long siuce placed this fact beyond a doubt.

The Toniadoe and the Acanthocephala, like the Trematoda, entirely parasitic in their habits, differ from them in the total absence of mouth or digestive cavity. The T'aniadce, Tapeworms, are ribbon-like creatures, usually divided throughout the greater part of their length into segments, whose usual habitation is the intestinal cavity of vertebrate animals; and apparently of a carnivorous vertebrate, in fact, though capable of existence elsewhere, it, is there alone that they are able to attain their complete development. The anterior extremity of a tænoid worm is usually called the head, and bears the organ by which the animal attaches itself to the mucous membrane of the creature which it infests. These organs are either suckers or hooks, or both conjoined. In T'cnia, four suckers are sombined with a circlet of hooks, disposed around a median 002 
terminal prominence. The embryo passes through a similar course of development to the Trematoda; viz. four forms or changes: but the embryo itself is very peculiar, consisting of an oval non-ciliated mass, provided upon one face with six hooks, three upon each side of the middle line. The Treniadce are found in many other situations besides the alimentary canal : the eye, the brain, the muscular tissues, the liver, \&c. ; the following cystic worms are included in this genera, Cysticercus Anthocephalus, Coenurus, and 'T'. Echinococcus. Plate IV. No. 100, this figure shows an entire and full-grown Tania with rostellum and suckers, and then three succeeding segments, the last of which contains the ova, \&c. The water-vascular system is represented coloured with carmine. This parasite infests the human body more frequently than other varieties. This accurately-drawn figure is copied from Cobbold.

Von Siebold, Leuckart, and others, have shown, by many interesting experiments, such as feeding puppies with $C y s-$ sicercus pisiformis, that in the course of a few weeks these entozoa are transformed into fully formed Tania serrata, again, rabbits fed with the embryo of the Trenia, the enibryo bore their way, by means of hooks, through the walls of the intestine, until they reach some blood-vessel : by the current of blood they are carried into the liver, and here Lenckart has traced their further development. The embryos grow to the 1-16th of an inch in length, and become elongated, so as almosis to resemble an Ascarid in form; they then make their way to the surface of the liver, and pass out into the peritoneal cavity.

In like manner, Cysticercus fasciolaris is rapidly developed within the liver of white mice; Cysticercus cellulosce seen in the muscles of the pig fed with the Tania solium, produces the diseased state of pork familiarly known as "measly pork:" If a lamb is the subject of the feeding experiment with Tania serrata, the final transformation will be very different; within a fortnight, symptoms of a disease known as "staggers" are manifested, and in the course of a few wecks, the Conums cerebralis will be found iransformed and developed within the brain. Von Siebold pointed out the bearing of this fact upon the important 
practical problem of the prevention of "staggers." Others of the same family of parasites are quite as remarkable, in giving a preference to the alimentary canal of fishes. 'The Echinorhynchus is developed in the canal of the Flounder, Tricenophorus nodulus in the liver of the Salmon, attaining a more perfect development in the alimentary canal of the Perch and Pike. Another, found in the Stickleback, becomes changed in the intestines of water birds, which devour these fish; and thus, by careful and repeated observations with the microscope, the connexion existing between the Cystic and Cestoid Entozoa have been most satisfactorily established.

The Fluke belongs to the order Trematoda, which signifies that they are internal parasites, suctorial worms, or helminths; they are all usually visible to the naked eye, although a fow of the smallest scarcely exceed 1-100th of an inch in length; many are larger, and the species best known, Fasciola hepatica, attains to an inch or more in length. The Fluke shown in Plate IV. No. 103, is coneshaped: it is the Amplistome conicum of Rudolphi. This parasite is common in oxen, sheep, and deer, and it has also been found in the Dorcas antelope. It almost invariably takes up its abode in the first stomach, or rumen, attaching itself to the walls of the interior. ${ }^{1}$ In the full-grown state it never exceeds half an inch in length; in our plate it is represented magnified. On closer inspection it will be seen that the animal is furnished with two pores or suckers, one at either extremity of the body, the lower being by far the larger of the two. By means of the latter the Amphistome anchors itself to the papillated folds of the paunch, or first stomach, as this organ is improperly called.

In the figure the oral sucker at the anterior end, or head, leads into a narrow tube, forming the throat or oesophagus, and this speedily divides, or rather widens out, into a pair of capacious canals. These cavities are correctly regardecl

(1) The larval condition of Amphistoma in all probability lives in or upon the body of snails. This we infer from the eircumstance that the larva, cercario, of a closely allied species, the Amphistoma subclavatum, which is known to infest the alimentary canal of frogs and newts, have been also found on the surface of the body of the Planorbis by ourselves, whilst Van Beneden discovered the larvæ in a species of Cyclas. The cercarice, larvæ, are taken, it is supposed, by the cattle while drinking. They then attach themselves to the walls of tho stomach, where they soon complete their further stages of developinent. 
as together constituting the stomach; but they are cæcal, that is, closed below, having no other outlet than the entrance above mentioned. The water-vascular system, artificially coloured in the plate, or rather the vessels thus named, bear a very striking resemblance to that of arteries or veins; and the centrally-placed pouch, shown in the figure, might very easily be taken to represent the heart. This large cavity gives origin to two primary trunks, which pass forward along the inner sides of the digestive cæca; in their passage they send off secondary branches, which divide and sub-divide until we arrive at a series of minute capillary ramifications; the latter, according to Blanchard, terminating in small oval-shaped sacs or lacunæ.

"It should be further observed, that the surface of the Amphistome, though quite smooth to the naked eye, is clothed with a series of minute tubercles, which may be readily brought into view under a half-inch object glass. Beneath the cuticle we find a layer of cellules forming the true skin; and beneath this, again, there are two, if not three, layers of muscular fibre; an anterior longitudinal series and an inner circular set being readily distinguishable. The substance of the body is traversed by bands of cellular parenchyma or connective tissue, which here and there form thickened sheaths for the support of the various delicate organs above described." The reproductive organs of flukes possess the greatest amount of interest both from an anatomical and physiological point of view. They are produced from eggs, which are found in large numbers is the ova-sac; varying in size from 1-150th to 1-250th of an inch.

"The large fluke (Fasciola hepatica) is not only of frequent occurrence in all varieties of grazing cattle, but has likewise been found in the horse, the ass, and also in the hare and rabbit, and in some other animals. Its occurrence in mian has been recorded by more than than one observer; the oral sucker forming the mouth leads to the short œesophagus, which very soon divides into two primary stomachal or intestinal trunks, which latter in their turn give off branshes and branchlets; the whole together forming that beautiful dendritic system of vessels which has often been compared to plant-venation. This remarkably- 
formed digestive apparatus is accurately represented in Plate IV. Nos. 106 and 107, Fasciola gigantea of Cobbold, which should be contrasted with the somewhat similarly racemose character of the water-vascular system. Let it be expresely noted, however, that in the digestive system the majority of the tubes branch out in a direction obliquely downwards, whereas those of the vascular system slope obliquely upwards. A further comparison of the disposition of these two systems of structure, with the same systems figured and described as characteristic of the Amphistome, will at once serve to demonstrate the important differences which subsist between the several members of the two genera, if we turn to the consideration of the habits of Fasciola hepatica, which, in so far as they relate to the excitation of the liver disease in sheep, acquire the highest practical importance. Intelligent cattle-breeders, agriculturists, and veterinarians have all along observed that the rot, as this disease is commonly called, is particularly prevalent after long-continued wet weather, and more especially so if there have been a succession of wet seasons; and from this circumstance they have very naturally inferred that the humidity of the atmosphere, coupled with a moist condition of the soil, forms the sole cause of the malady. Courdinating with these facts, it has likewise been noticed that the flocks grazing in low pastures and marshy districts are much more liable to the invasion of this endemic disease than are those pasturing on higher and drier grounds; a noteworthy exception occurring in the case of those flocks feeding in the salt-water marshes of our eastern shores." 1 Plate IV. No. 106, Fasciola gigantea: the anterior surface is exposed to display oral and ventral suckers, and the dendriform digestive apparatus injected with ultramarine; No. 107, shows the dorsal aspect of the specimen and the multiramose character of the water-vascular system, the vessels injected with vermilion.

One of the most remarkable of the Trematode helminths is Bilharzia hoematobra of Cobbold ; Distomia hoematobium of some other authors. Plate IV. No. 102. This genus

(1) Entozon: An Introduction to the Study of Helminthology. By T. Sprencer Cobbold, H.D. F.R.S. 1866. P. 148. 
of fluke, discovered by Dr. Bilharz in the human portal system of blood vessels, gives rise to a very serious state of disease among the people of Egypt. So common is the occurrence of this worm, that this physician expressed his belief that half the grown-up people are infested with it. Griesinger conjectures that the young of the parasite exists in the waters of the Nile, and in the fishes which abound therein. Dr. Cobbold thinks "it more probable that the larvæ, in the form of cercariæ, rediæ, and sporocysts, will be found in certain gasteropod moliusca proper to the locality." The anatomy of this fluke is fully descriled in Kuchenmeister's able work on Parasites, by Leuckart, and by Cobbold. 'The eggs and embryos of Bilharzia are peculiar in possessing the power of altering their forms in both stages of life; and it is more than probable that the embryo form has been mistaken for some extraordinary form of eiliated infusorial animalcule, their movements veing most quick and lively. The cannot fail to notice the curious leech-like form of the male animal, and, remarkable enough, he is generally found carrying the female about. The whip-like appendage seen in the figure is a portion of the body of the female. The disease produced by them is said to be more virulent in the summer months, which is probably owing to the prevalence of the cercarian larvæ at the spring time of the year.

Trichina spiralis.-This, the smallest of the helminths, measures only the 1-20th of an inch. The female is a little larger; it was discovered by Professor Owen in a portion of the human muscle sent to him from St. Bartholemew's Hospital, 1834. The young animal presents the form of a spirally-coiled worm in the interior of a minute ovalshaped cyst (Plate IV. No. 104), a very small speck scarcely visible to the naked eye. In the muscle it resembles a little particle of millet seed, more or less calcareous in its composition. The history of the development of Trichinæ in the human muscle is briefly that in a few hours after the ingestion of diseased flesh, Trichince, disengaged from the muscle, are found free in the stomach : they pass thence into the duodenum, and afterwards advance still further into the small intestine, where they become developed. From the third or fourth day, ova or 
spermatic cells art found, the sexes in the mean while becoming distinctly marked. Shortly afterwards the ova are impregnated, and young living entozoa are developed within the bodies of the female. The young have been noticed (by Virchow) under the form of minute Filarice, more especially in the serous cavities, in the mesenteric glands, \&c. Continuing their migrations, they penetrate as far as the interior of the primitive muscular fasciculi, where they may be found as early even as three days after ingestion, in considerable numbers, and so far developed that the young entozoa have almost attained a size equal to that of the full-grown Trichince. They progressively advance into the interior of the muscular fasciculi, where they are often seen several in a file one after the other. Behind them the muscular tissue becomes atrophied, and around them an irritation is set up, and from the commencement of the third week they are found sncysted. The sarcolenuma is now thickened, and the contents of the muscular fibres exhibit indications of a more active cell-growth; the cyst is the product of a sort of inflammatory irritation.

Professor Virchow draws the following conclusions:"1. The ingestion of pig's-flesh, fresh or badly dressed, containing Trichino, is attended with the greatest danger, and way prove the proximate cause of death. 2. The Trichino maintain their living properties in decomposed flesh ; they resist immersion in water for weeks together, and when encysted may, without injury to their vitality, be plunged in a sufficiently dilute solution of chromic acid for at least ten days. 3. On the contrary, they perish and are deprived of all noxious influence in ham which has been well smoked, kept a sufficient length of time, and then well boiled before it is consumed."

The Echinococcus found in cysts, chiefly in the human liver, is represented in Plate IV. No. 101. A large collection was taken from the liver of a boy who died in Charing-cross Hospital from accidental rupture of the liver. The cysts containing these parasites are always situated in cavities in the interior of the body. These cavities may be situated in any part of the tissues or organs of the body, but are more frequently found in the solid 
viscera, and especially in diseased livers. Fig. 256 represents the microscopical appearance of the contents of a cyst.

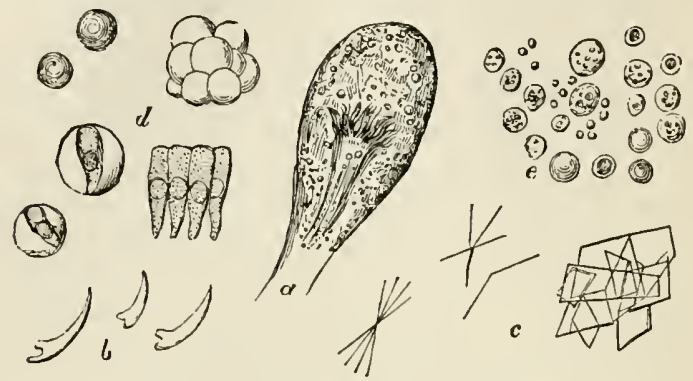

Fig. 256. - Cystic Disease of Liver. (Human).

$\not$, Cyst with an echinococcus enclosed. $b$, Detached hooklets from the head of Echinococcus, magnified 250 diameters. c, Crystals found in the cyst, cholesterine. $d$, Cylindrical epithelium, some enclosed in structureless globules. e, Puro-mucus, and fat corpuscles.

Mr. Busk, who has examined several of these cysts, says :- "When a large hydatid cyst,- - for instance, in the liver of the sheep,-very shortly after the death of the animal, is carefully opened by a very small puncture, so.as to prevent at first the too rapid exit of the fluid, and consequent collapse of the sac, its internal surface will be found covered with minute granulations resembling grains of sand. These bodies are not equally distributed over the cyst, but are more thickly situated in some parts than in others. They are detached with the greatest facility, and on the slightest motion of the cyst, and are rarely found adherent after a few days' delay. When detached, they subside rapidly in the fluid, and consequently will then be usually found collected in the lowest part of the cyst, and frequently entangled in fragments of the inner thin membraue. When some of these granulations are placed between glass under the microscope, and viewed with a power of 250 diameters, upon pressure being employed it will be seen, after rupture of the delicate enveloping membrane, that the Echinococci composing the graaulations are all attached to a common central mass by short pedicles; which, as well as the central mass, appear to be composed of a substance more coarsely granular by 
far than that of which the laminæ of the cyst are formed. This granular matter is prolonged beyond the mass of Echinococci into a short pedicle, common to the whole, and by which the granulation is attached to the interior of the hydatid cyst, as represented in No. 101. In specimens preserved in spirits, Echinococci of all imaginable forms and appearances are to be met with,-differenees owing to decomposition or to mechanical injury ; and in many cases no traces of them can be found except the hooklets or spines, - which, like the fossil remains of animals in geology, remain as certain indications of their source, and not unfrequently afford the only proof we can obtain of the true nature of the hydatid."1

The Echinorhynchi, or Acantho cephali, constitute a group of entozoa, with respect to whose development and life-history we are indebted to Prof. Leuckart of Giessen. Most observens, and in partieular Von Beneden and G. Wagner, have been disposed to assign to the Echinorhynci a simple metamorphosis, hardly perhaps more remarkable than that which has been shown to take place in some other of the Nematode worms. The latter observer goes so far even as to believe that the organization of the perfect animal may be discerned in the embryo. Leuckart instituted, in 1861, a series of experiments with the ova of Echinorhynchus Proteus, which is found parasitic upon the Gammarus Pulex. The ova "of E. Proteus resemble in form and structure those of the allied species. They are of a fusiform shape, surrounded with two menbranes, an external, of a more albuminous nature, and an internal, chitinous one. When the exys have reached the intestine, the outer of these membranes is lost, being in fact digested; whilst the inner envelope remains until ruptured by the embryo." 2

Anguillulce are very small eel-like worms, of which one species, ${ }^{3}$ Anguillula Aluviatilis, is found in rain-water amongst Confervce and Desmidiaceoe, in wet moss and moist earth, and sometimes in the alimentary eanal of the

(1) Microscopical Society's Transactions. 1st Series.

(2) Prof. Lenckart "On Echinorhynehus." Journ. Micro. Soc. vol. iii. p. 57. 1863.

(3) For the fullest information of marine, land, and fresh-water species, consult Dr. Bastian's "Monograph on the Anguillulidæ." Lin. Soc. Trans. vol. xxт. p. 75. The "Anguillula Aceti."-Popular Soience Review, January, 1563. 
Limneus, the frog, fish, \&c.; another species is met with in the ears of wheat affected with a blight termed the "cockle ;" another, the A. glutinis, is found in sour paste; and another, $A$. aceti, in stale, bad vinegar. If grains of the affected wheat are soaked in water for an hour or two before they are cut open, the eels will be seen in a state of activity when placed under the microscope. The paste-eel makes its appearance spontaneously in the midst of paste that is turning sour; but the best means of securing a supply for any occasion, consists in allowing any portion of a mass of paste in which they show themselves, to dry up, and then lay it by for stock; if at any time a portion of this is introduced into a little fresh made paste, and the whole kept warm and moist for a few days, it will be found to swarm with these curious little worms. A small portion of paste spread over one face of a Coddington lens is a ready way of showing them.

Planarice: a genus of the order Turbellaria. Some of the species are very common in pools, and resemble minute leeches; their motion is continuous and gliding, and they are always found crawling over the surfaces of aquatic plants and animals, both in fresh and salt water. The body has the flattened sole-like shape of the Trematode Entozoa; the mouth is surrounded by a circular sucker, this is applied to the surface of the plant from which the animal draws its nourishment. The mouth is also furnished with a long funnel-shaped proboscis, and this, even when detached from the body, continues to swallow anything presented to it.

"In imitation of the name bestowed on the trunk of the elephant, the extensile organ serving to imbibe the nutriment of many of the smaller animals is called a proboscis, whether it simply unfolds from the root, protrudes from a sheath, or unwinds from a regular series of volutions. But in none is the designation equally strict and appropriate as in the Planarice. There it is absolutely the organ of the elephant in miniature, with this exception, that it is neither annulated nor composed of segments. It is of surprising length, being little, if any, shorter when fully extended than the whole animal. It seems of greater consistency, barder, and tougher than the rest of the borly 
so as to admit insertion into decaying vegetaoles, and when stretched to the utmost the root becomes an apex of the slenderest cone." 1

Planaria multiply by eggs, and by spontaneous fissuration, in a transverse direction, each segment becoming a perfect animal. Professor Agassiz believes that the infusoral animals, Paranacium and Kolpoda, are nothing else than Planarian larva.

Hirudinida, the Leech tribe, are usually believed to form a link between the Annelida on the one hand, and the Trematoda on the other; but their affinities are closer connected with the latter than the former. 'Totally deprived of the characteristic setæ of the Annelida, and exhibiting no sectional divisions, they are provided with suckers so constantly possessed by the Trematoda, and present no small resemblance to them in their reproductive organs. On the other hand, in the arrangement of their nervous system and in their vascular system, the Hirudinidee resemble the Annelida. The head in most of these animals is distinctly marked, and furnished with eyes, tentacles, mouth, and teeth, and in some instances with auditory vesicles, containing otolithes. The nervous system consists of a series of ganglia running along the ventral portion of the animal, and communicating with a central mass of brain.

The medicinal leech puts forth strong claims to our atteution, on the ground of the services which it renders to mankind. The whole of the family live by sucking the blood of other animals; and, for this purpose, the mouth of the leech is furnished with an apparatus of horny teeth, by which they bite through the skin. In the common leech, three of these teeth exist, arranged in a triangular, or rather triradiate form, a structure which accounts for the peculiar appearance of leech-bites in the human skin. The most interesting part of the anatomy of the leech to microscopists is the structure of the mouth (fig. 257). "This piece of mechanism," says Professor Rymer Jones, " is a dilatable orifice, which would seem at first sight to be but a simple hole. It is not so ; for we find that just

(1) Sir John Dalyell's Osservations on some intercsting Phenomena exhibifod by suveral Species of Planaria. 1814. 
within the margin of this hole three beautiful little semi . circular saws are situated, arranged so that their edges

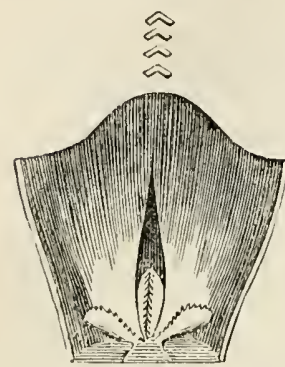

Fig. 257.-Mouth of Leech. meet in the centre. It is by means of these saws that the leech makes the incisions whence blood is to be procured, an operation which is performed in the following manner: No sooner is the sucker firmly fixed to the skin, than the mouth becomes slightly everted, and the edges of the saws are thus made to press upon the tense skin; a sawing movement being at the same time given to each, whereby it is made gradually to pierce the surface, and cut its way to the small blood-vessels beneath. Nothing could be more admirably adapted to secure the end in view than the shape of the wound thus inflicted, the lips of which inust necessarily be drawn asunder by the very contractility of the skin itself ; and that the enormous sacculated stomach, which fills nearly the whole body of the leech, was designed to contain its greedily devoured meal, there can be no reasonable question. The leech, in its nativo element, could hardly hope for a supply of hot blood as food; and, on the other hand, its habits are most abstemious, and it may be kept alive and healthy for years, with no other apparent nourishment than what is derived from pure water frequently changed; even when at large, minute aquatic insects and their larvæ form its usual diet."

In Clepsinida, the body is of a leech-like form, but very much narrowed in front, and the mouth is furnished with a protrusile proboscis. These animals live in fresh water, where they may often be seen creeping upon aquatic plants. They prey upon water-snails.

Tubicola. - The worms belonging to this series of branchiferous Annelida are all marine, and distinguished by their invariable habit of forming a tube or case, within which the soft parts of the animal can be entirely retracted. This tube is usually attached to stones or other submarine bodies. It is often composed of various 
foreign materials, such as sand, small stones, and the débris of shells, liued intermally with a smooth coating of hardened mucus; in others it is of a leathery or horny consistency; and in some it is composed, like the shells of Mollusca, of calcareous matter secreted by the animal. The Tubicola generally live in societies, winding their tubes into a mass which often attains a considerable size: a few are solitary in their habits. They retain their position in their habitations by means of appendages very similar to those of free worms, with tufts of bristles and spines; the latter, in the tubicular Annelids, are usually hooked; so that, by applying them to the walls of its romicile, the auimal is enabled to oppose a considerable resistance to any effort to draw it out of its case. In the best known family of the order (Sabellidce), the branchiæ are placed on the head, where they form a circle of plumes or a tuft of branched organs. The Serpulce form irregularly twisted calcareous tubes, and ofren grow together in large masses, when they secure themselres to shells and similar objects; uther species, Terebella, build their cases of sand and stones, appear to prefer a life of solitude. The curious little spiral shells seen upon the frouds of sea-reeds, are formed by an animal belonging to the family Spirorbis.

If the animals be placed in a vessel of sea-water, a very pleasing spectacle will soon be witnessed. The mouth of the tube is first seen to open, by the raising of an exquisitely-constructed door, and then the creature cautiously protrudes the anterior part of its body, spreading
out at the same time two beautiful fan-like expansions, of a
calcareos tube.

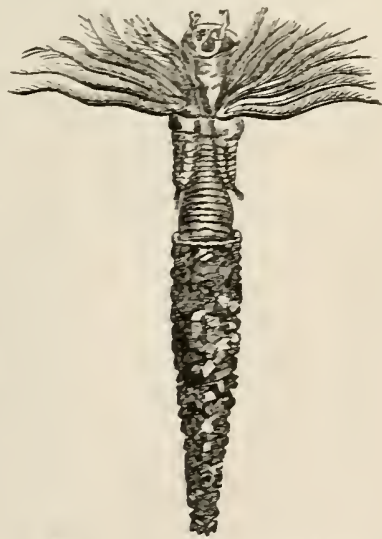
rich purple, or scarlet colour, which float elegantly in the surrounding water, and serve as branchial or breathing organs. 
The Serpula, if withdrawn from its calcareous tube (fig. 260 ), is found to have the lower part of the body composed of a series of flattened rings, and entirely destitute of limbs or other appendages. Its food is brought to its mouth by currents created by the cilia on the branchial tufts.

Some of the Annelids are without tubes or cells of any sort, and simply bury their bodies in the sand about the tidal mark. The Arenicola, lob-worm, is a well-known specimen of the class; its body is so transparent that the circulating fluids can be distinctly seen under a moderate magnifying power. Two kinds of fluids flow through the vessels, one nearly colourless, the other red; the vessels through which the latter circulate are looked upon as blood-vessels. A few also have not only no tubes but are free and active swimmers. Drs. Carpenter and Claparède, during a sojourn at Lamlash Bay, Arran, met with an interesting member of this class of Annelids, the Tomopteris onisciformis. It possesses a remarkable pair of "frontal horns," projecting laterally from the most anterior part of head, as well as pair of greatly elongated appendages, designated by those observers as "the second antennoe," in contradistinction to another and a shorter pair of appendages situated just in advance of them, the first pair being characteristic of the larval, and the second of the adult state of the annelid.

"The head also bears on its dorsal surface a pair of ciliated epaulettes, which extend over the edges of the bilobed nervous ganglion. These, at a certain stage of development, are fringed with long cilia both at their margins and their base; but as the cilia are only occasionally to be seen in activity, they may escape the attention of the observer. Cilia are likewise distinguishable on certain parts of that innermost layer of the general integument which forms the external boundary of the perivisceral space, and by their agency a special movement is imparted to the corpuscles of the fluid contained in the cavity."

The development of the caudal prolongation is peculiar, and this as well as other points of interest are given in the Lin. Soc. Trans. vols. xxii. and xxiii. pp. 335 
Ixskets' Egtis, HTC.

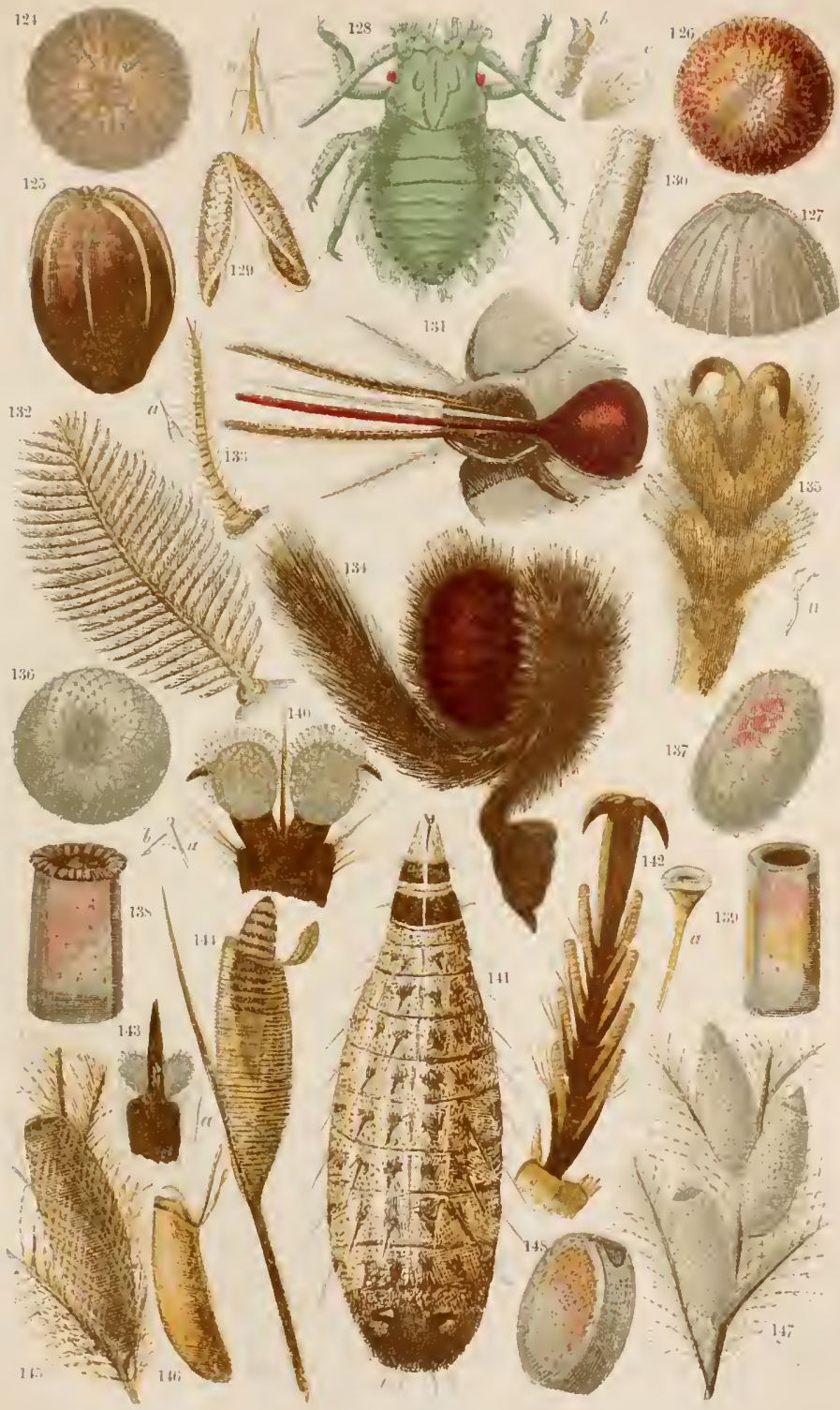



and 59. Dr. Carpenter believes that this creature "is a degraded form of the annelidan type-its nearest affinities being (as already pointed out by Drs. Leuckart and. Pagenstecher) the chætopod or setigerous Annelids. Every part of the characteristic organization of the type is here reduced to the extreme of simplicity. The alimentary canal passes in a straight line from one extremity of the body to the other, without either sacculations or glandular appendages. The nutritive fluid which transudes through its walls, and which finds its way into the perivisceral cavity, is distributed throughout the body solely by means of extensions of that cavity, through which it is propelled in part by the agency of cilia that clothe its walls. 'This fluid is obviously the homologue of the blood of higher animals, that we cannot but regard the existence of the type of structure before us (the wonderful transparency of the body not permitting the slightest doubt as to the absonce of anything resembling a dorsal vessel) as affording a further confirmation of the view of the so-called circulating apparatus of the higher Annelida, which regards their perivisceral cavity and its extensions as representing the proper sanguiferous system, and which looks upon the system of vessels containing coloured fluid as a special arrangement having reference rather to the respiratory functions. ${ }^{1}$ The extreme tenuity of the walls of the body and its appendages renders it unnecessary that any special provision should be made for the aeration of the nutritive fluid; and we accordingly find neither branchiæ nor any trace of what is commonly described as the sanguiferous system in Annelida. The centres of the nervons system would seem to consist solely of the cephalic ganglia-the absence of the ordinary longitudinal series being apparently related to the very incomplete segmentation of the body, and constituting a link of affinity to the Turbellarian Worms. The ocelli present a condition of extreme simplicity, yet the duplication of the corneale in each of them marks that tendency to repetition which so peculiarly distinguishes the Articulate type. It is, however, the extreme simplicity of its generative apparatus that con-

(1) See Prof. Iuxley's Lectures in Medical Times and Gazette. July 12 and 26, 1856. 
stitutes one of the chief points of interest in the organization of Tomopteris."

Not solely in this class, but in that of the Annelida generally, does much interest attach to the developmental period. Most of them come forth from the egg in a condition so closely resembling the cililated gemmules of polypes, that competent observers have been known to mistake them for animals of a lower class; fortunately a few hours' careful watching is sufficient to dispel the illusory belief, and the embryonic globular shapeless mass is seen soon to change its form; segmentation takes place, and the various internal organs beccme more and more developed; eye spots appear, and the young animal assumes the likeness of its parent.

The Actinotrocha, even in the adult state, in many particulars resembles the "bipinnarian larva of the star-fish." Its long body is surmounted by a head, or mouth, around which is placed a number of ciliated ventacula: they are not only employed for feeding purposes, but also for enabling it to swim about; and in this particular, according to Dr. A. Schneider and other competent authorities, it is quite remarkable. ${ }^{1}$ Dr. Carpenter tells us that he has captured these free-swimming Annelids among other marine animals by the careful use of the "stick-net." 


\section{CHAPTER IV.}

BUB-EINGDOM ARTICULATA,-INSECTA,-ARACHNIDA.

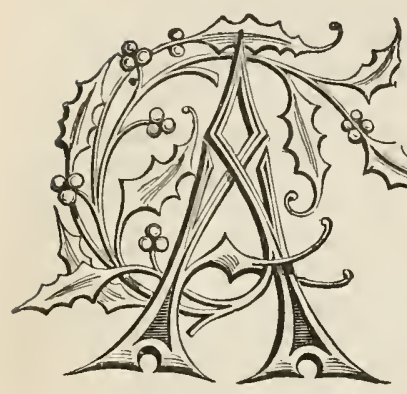

MONG the numerousobjects which engage the attention of the microscopist, the insect tribes in general are far

$y$ from being the least interesting; their curious and wonderful economy is a subject well deserving especial investigation. Earth, air, and water, teem with the various tribes of insects, many of which are invisible to the unassisted eye, but presenting, when viewed with the microscope, the most beautiful mechanism in their frame-work, the most perfect regularity in their laws of being, and exhibiting the same wondrous adaptation of parts to the creature's wants, which, throughout creation, furnishes traces of the love and wisdom that so strongly mark the works of God. ${ }^{1}$

"I caunot," says the learned Swammerdam, "after an attentive examination of the nature and structure of both the least and largest of the great family of nature, but allow the less an equal, perhaps a superior degree of dignity. Whoever duly considers the conduct and instinct of the one with the manners and actions of the other, must acknowledge all are under the direction and control of a superior and supreme Intelligence; which, as in the

(1) We commend to the reader the excellent Introduction to Entomology, by Kirby and Spence. Longmans. Art. "Insecta," Crclop. Ant, and Physio. 
largest it extends beyond the limits of our comprehension, escapes our researches in the smallest. If, while wo dissect with care the larger animals, we are filled with wonder at the elegant disposition of their limbs, the inimitable order of their muscles, and the regular direction of their veins, arteries, and nerves, to what a height is our astonishment raised w'nen we discover all these parts arranged in the least of them in the same regular manner! How is it possible but that we must stand amazed, when we reflect that those little animals, whose bodies are smaller than the point of the dissecting knife, have muscles, veins, arteries, and every other part common to large animals! Creatures so very diminutive that our hands are not delicate enough to manage, nor our eyes sufficiently acute to see them."

The Articulate sub-kingdom, Insecta, is divided and sub-divided into orders, families, genera, species, as for example :-

Lepidoptera; typical form, Butterfly, Moth.

Dipteria $;^{1}$ typical form, Fly, Gnat, \&c.

Aptera; typical form, Flea, Louse, Springtail.

Coleoptera; typical form, Beetle, Water Beetle, \&c.

Orthoptera; typical form, Locust, Grasshopper.

Neuroptera; typical form, Dragon-fly, May-fly.

Hymenoptera; typical form, Bee, Wasp, Ant.

Homoptera; typical form, Plant-louse (Aphis), Lantern-fly.

Hemiptera ; typical form, Water-scorpion, Water-boatman.

A rachnida; typical form, Spider, Scorpion, \&c. The Acarina belong to this genus, family Acarea, well known as "Mites" and "Ticks."

Insects are characterised by a simple breathing apparatus; by the division of the body into distinct regions or segments; when three, the middle one, the thorax, bears three pairs of jointed legs, and usually two pairs of wings; and by the possession of a single pair of jointed

(1) Comprised within the order Diptera, or two-winged flies, are several genera having no wings, the apterous and suctorial Pulex, and the apterous and pupiparous Episboscida: among the latter, we have the winged Hippobosca, or horse fly, and some others. 
antes.næ. The metamorphoses which all nndergo, before they arrive at the perfect state and are able to fulfil all the ends of their existence, are more curions and striking than in any other department of nature; and in the greater number of species the same individual differs so materially at the several periods of life, both in its internal and external conformation, in its habits, locality, and kind of food, that it becomes one of the most interesting investigations of the physiologist to ascertain the manner in which these changes are effected, to trace the successive steps by which that despised and almost nnnoticed larva that but a few days before lay grovelling in the earth, with an internal organization fitted only for the reception and assimilation of the crudest vegetable matter, has had the whole of its external form so completely changed, as now to have become an object ot admiration and delight, and able to "spurn the dull earth," and wing its way into the wide expanse of air, with internal parts adapted only for the reception of the purest and most concentrated aliment, which is now ren. dered absolutely necessary for its support, and the renovation increased energies demand.

The greater number of insects undergo a complete series of changes. They are for the most part oviparous, and their eggs assume a variety of forms, colour, \&c. as will be seen in Plate VI. On first leaving the egg, they assume a more or less worm-like appearance, known as larva, maggot, or caterpillar. The next stage is that of the nymplia, pupa, or chrysalis; this is succeeded by that of the perfect insect or imago. In some insects the changes are incomplete; the body, legs, and antennx are nearly similar, but wings are wanting. In others the pupa continues active, is of a large size, and acquires rudimentary wings; and some are without material alteration of structure, the change consisting in what is termed " zclysis," a casting off, or moulting only.

The hearls of insects present many points of interest; Plate VI. No. 134, shows the head of the Tortoise butterfly in profile, with large compound eye, palpi, and spiral tongue. No. 131 is a portion of the head with lancets, sic. of the Glossina morsitans, Tsetse-fly of Africa. In 
the mouths and tongues of insects, the most admirable art and wisdom are displayed ; and their diversity of form is almost as great as the variety of species. The mouth is usually placed in the fore part of the liead, extending somewhat downwards. Many have the mouth armed with strong jaws, or mandibles, provided with muscles of great

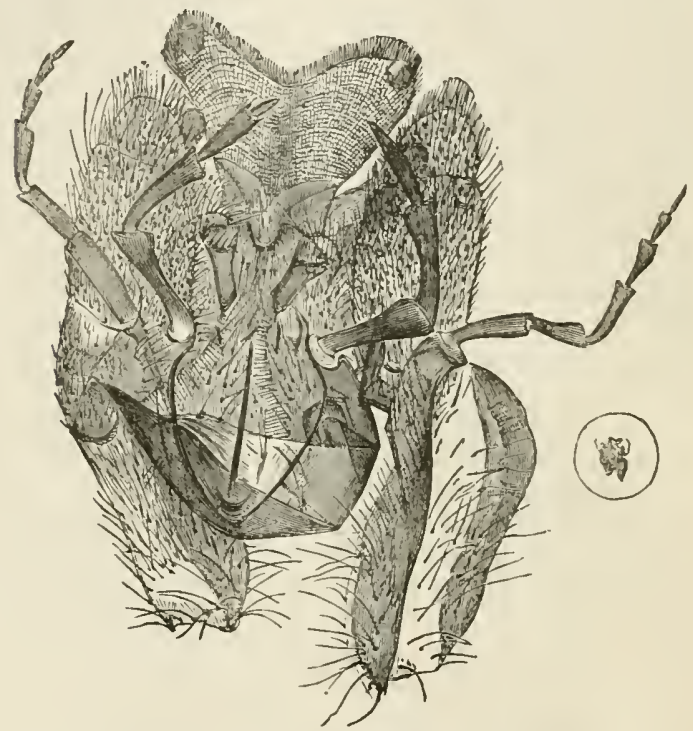

F'g. 259.-Under-surface of a Wusp's tongue, Feelers, \&c. (Within the circle the same is seen life-size.)

power, with which they bruise and tear their food, answering to the teeth of the higher animals; and in their various shapes and modifications serving as knives, scissors, augers, files, saws, trowels, pincers, or other tools, according to the requirements of each insect.

The tongrue is generally a compact instrument, used principally to extract the juices on which the insect feeds, varying greatly in its length in the different species. It is capable of being extended or contracted at the insect's 
pleasure; sometimes it is dexterously rolled up in a taper and spiral form, as in the Butterfly ; tubular and fleshy, as in the Wasp. In fig. 259, the under lip of the Wasp is represented with its brush on either side; above which are two jointed feelers (palpi labiales), the use of which is probably for the purpose of making an examination of the food before it is

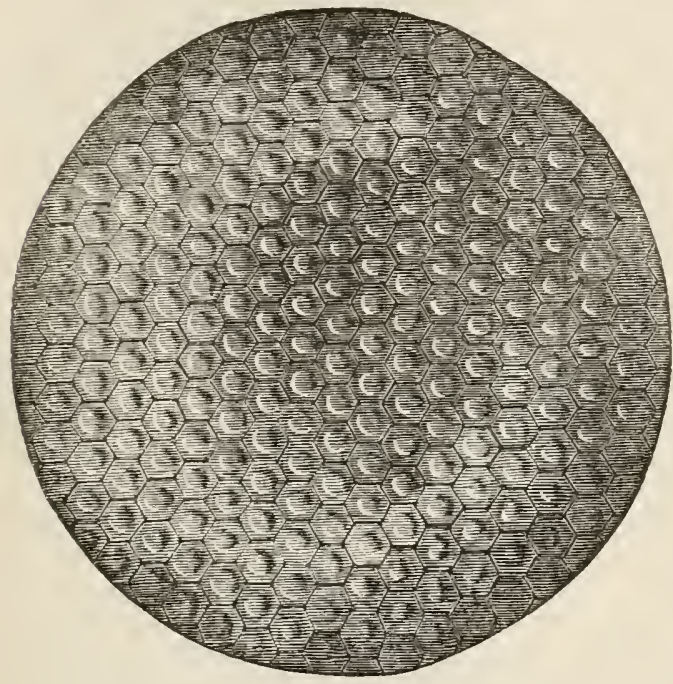

Fig. 260.-Eye of Fly (magnified 150 diameters.)

taken into the mouth, or that of cleaning the tongue. Near these feelers the antennæ or horns are placed, as curious in form as they are delicate in structure. The antennæ of the male generally differ from those of the female: some writers believe these are organs of smell or hearing; others that they are solely intended to add to the perfection of touch or feeling, increasing their sensibility to the least motion or disturbance. Apart from their use, they are the most interesting and distinguishing characteristics of insects, and appear to be often employed for the purpose of exanining every object they alight upon. 
The structure of the eye is in all creatures a most admirable piece of mechanism, in none more so than in those of the insect tribe. The eyes differ in each species; varying in number, situation, figure, simplicity of construction, and in colour. Fig. 260 represents a portion of the eye of the common Fly, drawn by the light of the sun upon a prepared photographic surface of wood realy for the engraver. Fig. 261 represents a side view of the eye when thrown down, showing

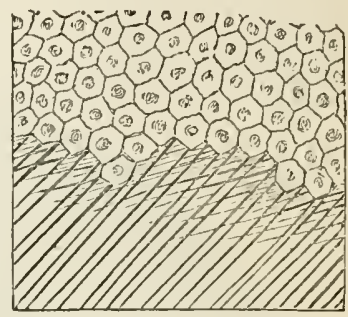

Fig. 261. the compound nature of the organ, with its series of cylindrical tubes; better seen in ${ }_{1 \sim}$. 262.

"On examining the head of an insect, we find a conple of protuberances, more or less prominent, and situated symmetrically one on each side. Their outline at the base is for the most part oval, eliiptical, circular, or truncated; while their curved surfaces are spherical, spheroidal, or pyriform. These horny, round, and naked parts seem to be the corner of the eyes of insects; at least, they a: $\theta$ with propriety so termed, from the analogy they bear to those transparent tunics in the higher classes of animals. They differ, however, from these; for, when viewed by the microscope, they display a large number of hexagonal facets, which constitute the medium for the admission of light to as many simple eyes. Under an ordinary lens, and by reflected light, the entire surface of one cornea presents a beautiful reticulation, like very fine wire gauze, with a minute papilla, or at least a slight elevation, in the centre of each mesh. These are resolved, however, by the aid of a compound microscope, and with a power of from $81)$ to 100 diameters, into an almost incredible number (when compared with the space they occupy) of minute, regular, geometrical hexagons, well defined, and capable of being computed with tolerable ease, their exceeding minuteness being taken into consideration. When viewed in this way, the entire surface bears a resemblance to that which might easily and artificially be produced by straining a 
portion of Brussels lace with hexagonal meshes over a small hemisphere of ground glass. That this gives a tolerably fair idea of the intricate carving on the exterior may be further shown from the fact, that delicate and beautiful rasts in collodion can be procured from the surface, by siving it three or four coats with a camel-hair pencil. When dry it peels off in thin flakes, upon which the impressions are left so distinct, that their hexagonal form can be discovered with a Coddington lens. This experiment will be found usefinl in exanining the configuration of the facets of the hard and unyielding eyes of many $o_{1}$ the Coleoptera, in which the reticulations become either distorted by corrugation, or broken by the pressure required to flatten them. It will be observed also, that by this method perfect easts can be obtained without any dissection whatever; and that these artificial exuvio-for such they really are - become available for microscopic investigations, obviating the necessity for a more lengthened or laborious preparation. The dissection of the cornea of an insect's eye is by no means easy. I have used generally a small pair of scissors, witl well - adjusted and
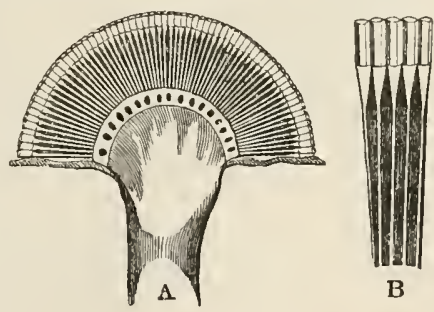

Fig. 262.

A, is a section of the eye of Melolontha vulgans, Cockchafer. B, a portion more highly magnified, showing tlie facets of the comea, and its transparent py ramids, surrounded with pigment. At $\mathrm{A}$ they meet, and form the optic nerve.

pointed extremities, and a camel-hair pencil, having a por. tion of the hairs cut off at the end, which is thereby flattened. The extremity of the colar handle should be cut to a fine point, so that the brush may be the more easily revolved between the finger and thumb; and the coloured pigment on the interior may be scrubbed off by this simple process. A brush thus prepared, and slightly moistened, forms by far the best forceps for manipulating these objects preparatory to mounting; as, if only touched with any hard-pointed substance, they will often spring from the table and be lost. 
"Each hexagon forms the slightly horny aase of an eye. Their margins of separation are often thickly set with hair, as in the Bee; in other instances naked, as in the Dragon-fly, House-tly, \&c. The number of these lenses has been calculated by various authors, and their multi-

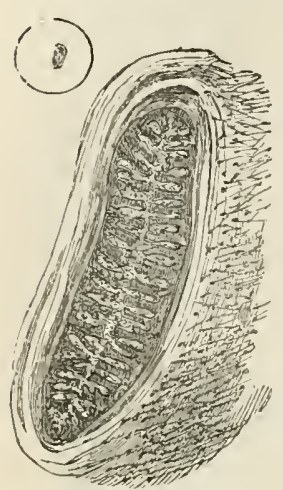

Fig. 263 - Breathing - arerture, spiracle, of silkworm. (The circle incloses the object about the natural size.) tude.cannot fail to excite astonishment. Hooke comnted 7,000 in the eye of a House-fly; Leeuwenhoek more than 12,000 in that of a Dragon-fly ; and Geoffry cites a ralculation, according to which there are 34,650 of such facets in the eye of a Butterfly." 2

The trunk is situated between the head and the abdomen; the legs and wings are inserted into it. The thorax is the upper part of the trunk; the sides and back of which are usually armed with points or hairs. The abdomen forms the posterior part of the body, and is generally made up of rings or segments, by means of which the insect lengthens or shortens itself. Running along the sides of the abdomen are the spiracles, or breathing apertures, fig. 263, communicating directly with the internal respiratory organs. Pure air being thus freely admitted to every part, and the circulating fluids

(1) 'Each of the eyelets, or 'ocelli,' which aggregated constitute the compound eye of a Bee, is itself a perfect instrument of vision, consisting of two remarkably formed lenses, namely, an outer 'corneal' lens and an inner or 'conical' lens. The 'corneal' lens is a hexahedral or six-sided prism, and it is the assemblage of these prisms that forms what is called the 'cornca' of the compound eye. This 'cornea' may easily be peeled off', and if the whole or a portion be placed under the inicroscope, it will be seen that each corneal lens is not a simple lens but a double convex compound one, composed of two planoconvex lenses of different densities or refiacting powers, joincd together by their plane surfaces. The effect of this arrangement is, that if there should be any aberration of the rays of light during their passage through one portion of the lens, it is rectifies in its transit through the other. It appears questionable whether the normal shape of these lenses is hexagonal, or whether this form is not rather a necessity of growth, \&c., that is, that tliey are normally round, but assume the hexagonal shape during the process of development in cousequence of their agglomeration." J. Samuelsun and B. Hicks, "On the Eye of the Bee," Journ. Micrca. Soc. vol. i. p. 51 .

(2) "Remarks on the Cornea of the Eye in Insects," by John Gurhar, 3. R.C.S. Journ. Micros. Science, p. 76, 2853. 
kept exposed to the vivifying influence of the atmosphere, the necessity for more complicated and cumbersome breathing organs is at once obviated ; and the whole body is at the same time rendered lighter. The spiracles are usually nine or ten in number, and consist of a horny ring, of an oval form. The air-tubes are exquisitely composed of two thin membranes, between which a delicate elastic thread or spiral fibre, is interposed, forming a cylindrical pipe, and keeping the tube always in a distended condition; thus wonderfully preserving the sides from collapse or pressure in their passage through the air, which otherwise might occasion suffocation. Fig. 264 represents the beautiful me-

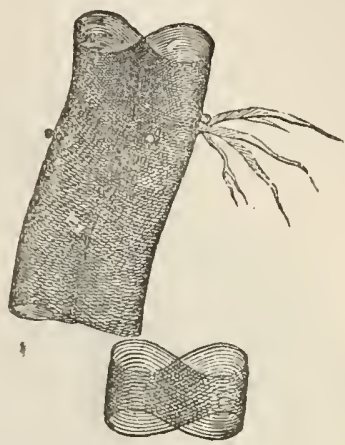

Fig. 264. - Magnified portions of the trachea of the Hydrophilus, show:ing spiral tubes and their arrangement.

chanism of a portion of the tracher of Hydropilus, showing the peculiar arrangement of the spiral tubes, which give it elasticity and strength.

The legs of insects are extremely curious and interest. ing ; each leg consists of -several horny cylinders, connected by joints and ligaments, inclosing within them sets of powerful muscles, whereby their movements are effected.

Feet of Flies, \&c.-_"The tarsus, or foot of the Fly, consists of a deeply bifid, membranous structure, pulvillus; anterior to the attachment of this part to the fifth tarsal joint, or the upper surface, are seated two claws, or 'tarsal ungues,' which are freely movable in every direction. These ungues differ greatly in their outline, size, and relative development to the tarsi, and to the bodies of the insects possessing them, and in their covering; most are naked over their entire surface, having however a hexagonal network at their bases, which indicates a rudimentary condition of minute scale-like hairs, such as are common on some part of the integument of all insects. Flexor and 
extensor muscles are attached to both ungues and the flaps; the flaps, corrugated or arranged on the ridge and furrow plan, are in some cases perfectly smooth on their superior surface, in others this surface is covered with minute scale-like hairs. The thickness of the flaps on the Blow-fly does not exceed the 1-2000th of an inch at the margin; thence they increase in thickness towards the point of attachment. Projecting from their inferior surface are the organs which have been termed 'hairs,' ' hair-like appendages,' 'trumpet-shaped hairs,' \&c. 'That

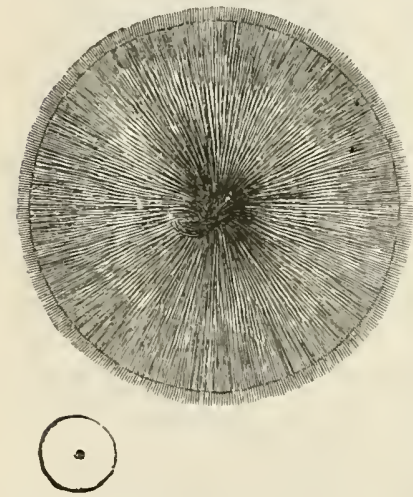

Fig. 265.-Sucker on the leg of a Waterbeetle. (The dot in circle shows the object natural size.)

these are the immediate agents in holding is now admitted by most observers, and it will be conveuient - to term them " "tenent" hairs,' in allusion to their office. Plate VI. No. 140, the under surface of left forefoot of Musca Vomitoria, is shown with tenent-hairs; $a$ and $b$ are more magnified hairs, $a$ from below, $b$ from the side. No. 142 is the left forefoot of Amara communis, showing under surface and form of tenent appendages, one of which is seen more magnified at $a$. No. 143, under surface of left forefoot, Ephydra riparia. This fly is met with sometimes in immense numbers on the water in salt marshes; it has no power of climbing on glass, which is explained by the structure of the tenent-hairs : the central tactile organ is also very peculiar, the whole acting as a float, one to each foot, to enable the fly to rest on the surface of the water; $a$ is one of the external hairs. No. 135, under surface of left forefoot of Cassida viridis (Tortoise-beetle), showing the bifurcate tenent appendages, one of which is given at $a$ more magnified. These, in the ground-beetles, are mets with only in the males, and are used for sexual purposes. The delicacy of the structure of these hairs in the fly, the 
bend near their extremity, in each of which supervenes an elastic membranous expansion, and from which a very minute quantity of a clear, transparent fluid is emitted when the fly is actively moving, explains its capacity for clinging to polished surfaces. It simply remains to add that the tubular nature of the shaft of the tenent-hairs on the foot of this insect has been surmised, although its minute size and homogeneity hardly admits of actual confirmation. At the root of the pulvillus, or its under surface, is a process, which in some instances is short and thick, in others long and curved, and tapering to its extremity (Scatophaga), setose (Empis), plumose (Hippoboscid $x)$, or, in one remarkable example (Ephydra), so closely resembling in its appearance the very rudimentary pulvillus with which it is associated. Just at the base of the fifth tarsal joint, on its under surface, there is present, in Eristalis, a pair of short, very slightly curved hairs, which point almost directly downwards. It became desirable to endeavour to ascertain how far the structure of these tenent-hairs agrees with that of true hairs, on which some valuable critical observations were made last year by Dr. Hicks. ${ }^{1}$

"Tenent-hairs are usually present in some modification or other, that it is really difficult to name a beetle which has not some form of them; the only one I yet know that seems to me really to possess nothing of the kind is a species of Helops, which lives on sandy heaths; I suppose the dense cushion of hairs on the tarsi here to be for the protection, simply, of the joints to which they are attached. I have detected them on the tarsal joints of species of Ephydra, and on the first basal tarsal joint of the Drone of the Hive-bee. A very rudimentary form of tenent-hairs is present on the under surface of some of the Tree-bugs (Pentatomidae), which have in addition a large, deeply-cleft organ at the extremity of the tarsus, which appears to be a true sucker.

"When walking on a rough surface, the foot represents what of a Coleopterous insect without any tenent appenlages. The ungues are always attached to the last joint of

(1) See Trans. Linn. Soc. vol. xxiii. p. 143. 
an insect's tarsus. They are not attached to the fifth tarsal joint of a Dipterous insect; neither are they attached to the fifth tarsal joint of a Hymenopterous insect, but to the terminal sucker, which again, in this great order, is a sixth tarsal joint, membranous, flexible, clastic in the highest degree, retractile to almost its fullest extent within the fifth tarsal joint-a joint modified to an extraordinary degree for special purposes.

"The plantula of Lucanus, with its pair of minute claws, at once occurred as a case strictly in point. The ungues are hairs modified for special purposes; and they have the structure of true hairs. The sustentacula of Epeira, the analogous structures on the entire under surface of the last tarsal joints in Pholcus, the condition of the parts in the hind limbs of Notonecta, in both its mature and earlier conditions, as well as in Sarcoptes, Psoroptes, and some other Acari, all contribute to the proof of this fact. The various orders of insects have, for the most part, each their own type of foot. 'Thus there is the Coleopterous type, the Hymeropterous type, the Dipterous type, the Homopterous type, \&c.; and so very distinctive, that in critical instances they will sometimes serve at once to show to which order an insect should be referred. Thus, amongst all the Diptera, I have as yet met with but one subdivision which presents an exception to the structure described. This exception is furnished by the Tipulida, which have the Hymenopterous foot. With hardly an exception, then, I believe the form of foot described will be found universal amongst the Diptera, and will be found amongst the members of this order alone. It may be desirable to add a few words on the best plan of conducting observations on these parts. Their action should be studied in living insects under the influence of chloroform, careful notes taken of appearances, and accurate drawings made. It is of the greatest advantage to preserve carefully all the parts examined: for this purpose Deane's medium or glycerine jelly suits very well; some of the delizate preparations, however, can only be kept satisfactorily in a solution of chloride of zinc. The old plan of soaking in caustic potash, crushing, washing, putting into spirits of wine (or pressing and drying first), and then into turpen- 
tine, and lastly into Canada balsam, is perfectly useless, except in rare instances where points connected with the structure of the integument have to be made out. Of course, the parts should be viewed from above, from below, and in profile, in order to gain exact ideas of their relations. The binocular microscope, however, promises to diminish vastly the difficulties which had until recently to be encountered, as by its use the parts may be clearly viewed just as they are, without preparation of any kind."I
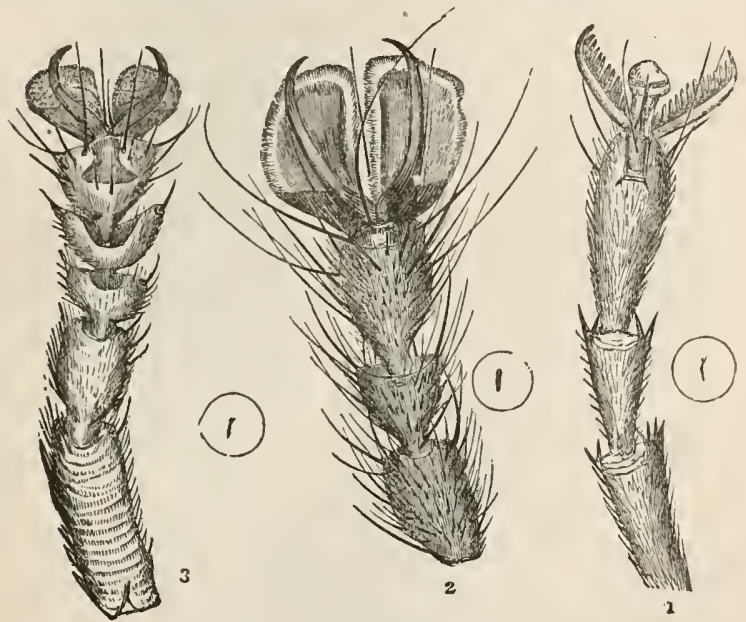

Fig. 266.

1, Foot and leg of Opñion. 2, Foot and leg of Flesh-fly. 3, Foot and leg of Drone-fly. (The small circles inclose objects about natural size.)

Fig. 267 represents the tongue and piercing apparatus of the Drone-fly. This remarkable compound structure, together with the admirable form and exquisite beauty of the apparatus, must strike the mind with wonder and delight, and lead the observer to reflect on the weakness and impotence of all human mechanism, when compared with the skill and inimitable finish displayed in the object before us. The barder structures which surround it have

(1) Tutfen West, Trans. Linn. Soc. vol. xxiii. p. 393. 


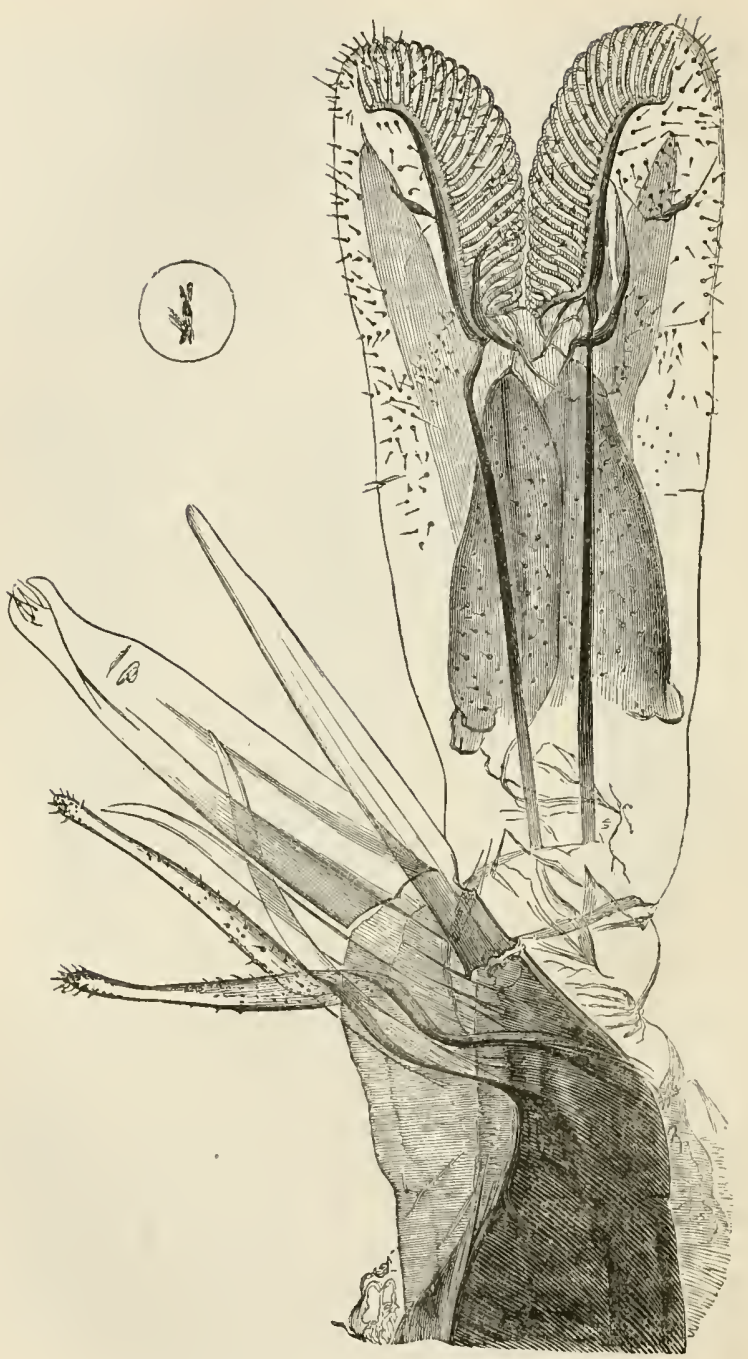

Fig. 267.-Tungue and Piercing Apparatus of Drone-fly. 
been removed for the purpose of bringing the several parts into view, and which consist of two palpi, or feelers, covered with short hairs, and united to the head by a ses of muscles; these feelers appear to be in frequent requisi. tion for guarding the other organs from external injury. The two lancets seen above them are formed somewhat like a cutlass, or the dissecting knife of the anatomist, and are purposely interided for making a deep and sharp cut, also for cutting vertically with a sweeping stroke. The other' and larger cutting instrument appears to be intended to enlarge the wound, if necessary; or it may be for the purpose of irritating and exciting the part around, thereby increasing the flow of blood to the part, being jagged or toothed at the extremity. The larger apparatus, with its three peculiar prongs, or teeth, is tubular, to permit of the blood passing through it and thence to the stomach; this is inclosed in a case which entirely covers it. The spongy tongue itself projects some distance beyond this apparatus, and is composed of a beautiful network of soft muscular spiral fibres, forming a series of absorbent tubes; and these are moved by powerful muscles and ligaments, the retractile character of which may be seen in the drawing of the proboscis of the Fly, fig. 268: by the aid of two hooklets placed in each side, he is enabled to draw in and dart out the tongue with wonderful rapiaity. Another set of muscles is seen at the root of the whole apparatus.

"In the organization of the mouth of various insects we have a modification of form, to adapt them to a different morle of use; as in the Muscidce, or common House-flies. When the food is easily accessible, and almost entirely liquid, the parts of the mouth are soft and fleshy, and simply adapted to form a sucking tube, which in a state of rest is closely folded up in a deep fissure, on the under surface of the head. The proboscis at its base appears to be formed by the union of the lacinia above and the labium below, the latter forming the chief portion of the organ, which is tenanted by dilated muscular lips. In the Tabanus these are exceedingly large and broad, and are widely.expanded, to encompass the wound made by the insect with its lancet-sbaped mandibles in the skin of the animal it attacks. On their outer surface they are fleshy and 
muscular, to fit them to be employed as prehensile organs; while on their inner they are more soft and delicate, but thickly covered with rows of very minute stiff hairs, directed a little backwards, and arranged closely together. There are very many rows of these hairs on each of the lips; and from their being arranged in a similar direction, they are easily employed by the insect in scraping or tearing delicate surfaces. It is by means of this curious structure that the busy House-fly often occasions much mischief to the covers of our books, by scraping off the white of egg and sugar varnish used to give them the polish, leaving traces of its depredations in the soiled and spotted appearance which it occasions on them. It is by means of these also that it teases us in the heat of summer, when it alights on the hand or face, to sip the perspiration as it exudes from and is condensed upon the skin. The fluid ascends the proboscis, partly by a sucking action, assisted by the muscles of the lips themselves, which are of a spiral form, arranged around a highly elastic, tendinous, and ligamentous structure, with other retractile additions for rapidity and facility of motion." 1

The beautiful form of the spiral structure of the tongue should be viewed under a magnifying power of 250 diameters, or a quarter-inch object-glass.

These insects are of great service in the economy of nature, their province being the consumption of decaying animal matter, which is found about in quantities so small as to be imperceptible to most people, and is not removable by ordinary means, even in the best-kept apartments, during hot weather. It was asserted by Linuæus, that three flies would consume a dead horse as quickly as a lion. This was, of course, said with reference to the offspring of such three flies; and it is possible the assertion may be correct, since the young begin to eat as soon as they are born. A single Blow-fly has been known to produce twenty thousand living larvæ (one of which is represented in Plate VI. No. 141), and in twenty-four hours each has increased its own weight above two hundred times; in five days it attains to its full size. When the larvæ are of full size,

(1) Mr. G. Newport, Cyclopadia of Anatomy and Physiolugy. 
they change into the pupa state, and remain in that state a few days; they then become flies, soon produce thou-

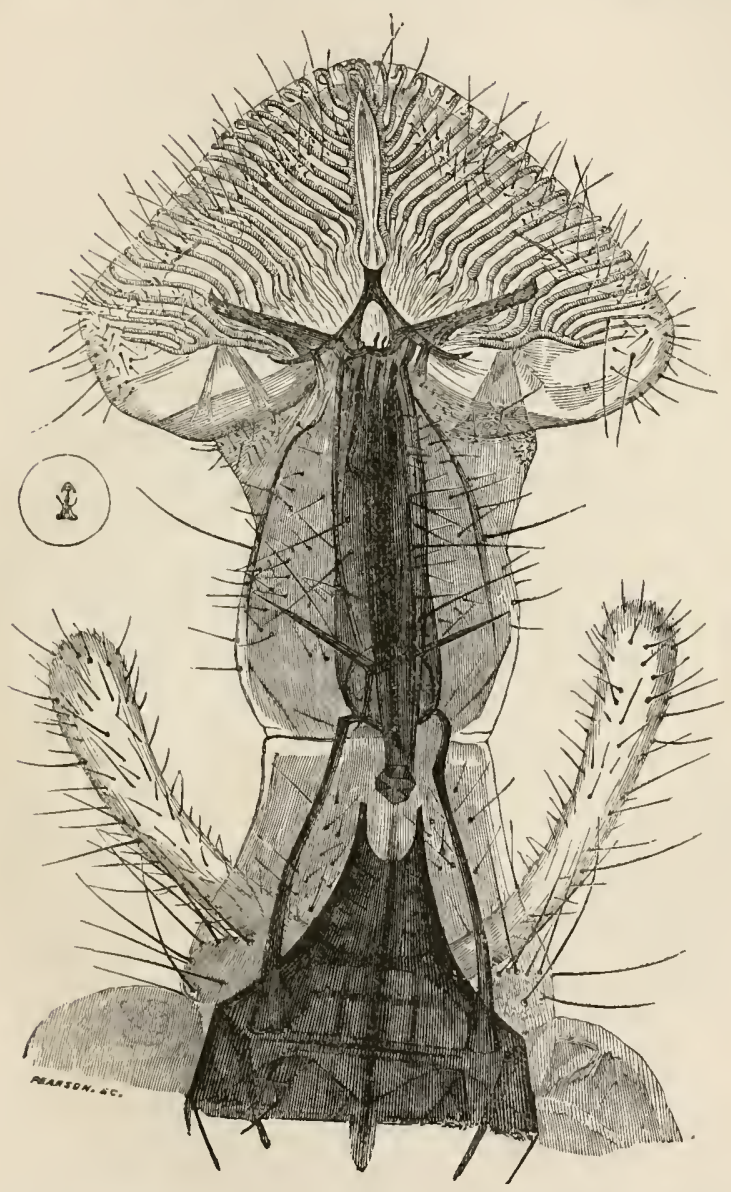

Fig. 268.-Proboscis of House-fly.-Drawn from a preparation by Toppisg. (Tho small circle in:loses the same about uatural size.) 
sands of eggs, larvæ, and flies, and this is repeated again and again until the whole brood is destroyed by winter's cold. ${ }^{1}$

The "Tsetse" fly of tropical Africa (Glossina morsitans): the moutl, proboscis, and piercing apparatus of one, viewed from the under-side, is represented in Plate VI. No. 131. The biting apparatus consists of four parts, of which two are lateral setose palpi ; if a horny case for the protection of the proboscis and its contained style can be so called. The palpi, althongh arising from two roots, when joined together and accurately embracing the proboscis, as they will do when the fly is at rest, appear as one only; but when the insect is in the act of piercing or sucking, they divide, and are thrown directly upwards. The palpi are furnished on the outer, or convex sides, with long and sharply pointed, dark-brown setæ or hairs; while the inner or concave sides which are brought in contact with the proboscis are perfectly smooth and fleshy. Three circular openings seem to indicate the tubular character of what in the common fly is a fleshy, expanded, and highly. developed muscular proboscis: in the Glossina it is a straight, horny-looking, red-coloured bristle, the apex of which is slightly dilated and rounded, and apparently barbed, while it expands a little towards the base, and there dilates into a large-sized fleshy bag, or muscular sac, filled with a red-coloured fluid. The proboscis is grooved on the under-side for the purpose of receiving a slender glassy style, acutely pointed, and equal to it in length. The style, nicely adapted to the groove, and taking its rise from the poison gland, is the penetrating instrument. A series of long and thin muscular bands embrace this gland, and their tendinous insertions are so arranged as to bring considerable force to bear upon its contents. Physiological science has made us familiar with the fact, that

(1) Dead fies may be constantly observed, ahont autumn, surrounded by a sort of halo, which, upon examination, is found to consist of the slores of a ungus. The abdomen is mucl distended, and the rings composing it are separated from each other, the intervals being occupied hy white prominent rones, constituted of a fungoid growth proceeding from the interior of the body. Further examination will slow that the whole of the contents or the body of the fly have been consumed by the parasitic growth, and that nothing remains but an empty shell, lined with a thin felt-like layer composed of the interlaced mycelia of the innumerable fungi.-See Dr. F. Cohn's observatious upon the parasite in vol. v. p. 154, Journ. Mieros. Sci. 1857. 
all fluid poiscns are enormously increased in their power for good or for evil, when injected beneath the skin; this knowledge prepares us to understand how it is that a drop from the poison gland of that wonderful little fly is sufficiently potent and virulent to destroy a large animal in a few minutes. Dr. Livingstone tells us that on one occasion he lost forty-three oxen in as many minutes, "when not more than a score of the "Tsetse' flies could be seen." The tongue is neither large nor well developed, but this is in a measure balanced by the mouth and lips; the latter are muscular, and capable of affording great assistance while the fly is in the act of sucking the blood of its victim. The wings are long and powerful ; the legs strong and muscular; the feet provided with the usual appendages, terminating in a pair of strong claws of a rather large size.

The wings of insects exhibit variety in form and structure, as well as beauty of colouring; the art with which they are connected to the body, the curious manner in which some are folded up, the fine articulations provided for this purpose, with the various ramifications by which the nourishing fluids are circulated and the wings strengthened, all afford a fund of rational investigation highly entertaining, and exhibiting, when examined under the microscope, beautiful and wonderful design in their forma tion. Take the Libellulidoe (Dragon-flies) as an example, whose wings, with their horny framework, are as elegant, delicate, and as transparent as gauze; often ornamented with coloured spots, which, at different inclinations of the sun's rays, show all the tints of the rainbow. One species (Calepteryx virgo) will be seen sailing for hours over a piece of water, all the while chasing, capturing, and devouring the numerous insects that cross its course; at another time driving away competitors, or making its escape from an elemy, without ever seeming tired or inclined to alight.

In fine weather, female Dragon-flies are seen to deposit their eggs upon the water, making a strange noise, as though they were beating the water; the cluster of eggs looks like a floating bunch of small grapes. The larvæ. when hatched, live in the water; and it is scarcely possible to fancy more strange-looking creatures. They aro 
short and comparatively thick, with movements heary and clumsy, and after shedding their skins become pupæ: still continuing to live in the water. The pupæ differ from the larvæ principally in having four small scales on their sides; these conceal their future wings. While the Dragonfly continues in its aquatic state, both as larva and pupa, it devours all the insects it can entrap; and as it only moves slowly, it is furnished with a very curious apparatus near its head, which it projects at pleasure, and uses as a trap. This apparatus consists of a yair of very large, jointed, movable jaws, which the insect keeps closely folded over its head, like a large mask, till it sees its prey; when it does, it ereens softly along till it is sufficiently near; it then darts out those long, arm-like jaws, and suddenly seizing its prey, conveys it to its mouth. When the Dragon-fly is about to emerge from its pupacase, it places itself on the brink of the pond, or on the leaf of some water-plant sufficiently strong to bear its weight, and there divests itself of its pupa-case. When the perfect insect first appears, it has two very 'small wings; these gradually increase, and in a short time two other wings appear. As soon as the wings are fully expanded, and have attained their beautiful gauze-like texture, the Dragon-fly begins to dart about, and then commences its work of destruction.

Equally rapid, exactly steered, and unwearied in its flight is the Gnat. The wings of a Gnat have been calculated, during its flight, to vibrate 3,000 times in a minute; these wonderful wings are covered on surface and edge with a fine down or hair. The alternations of bright sunshine and rain, so commonly seen in March, are extremely favourable to the appearance of Gnats. The first that appear are called Winter Midges (Trichocera hyemalis). As the spring advances, these Midges are succeeded by others somewhat different; and as the weather becomes warmer, the true Guats appear. The sting of the Gnat (Culex pipiens) is well known; although the insects themselves, so very rapid in their movements, are so much dreaded that very few people care to examine the delicacy and elegance of their forms. The sting is very curicusly contrived (see fig. 269), and inclosed in a sheath, folds up 
after one or more of the six lancets have pierced the flesh ; it will inflict a severe though minute wound, the pain of which is increased by an acrid liquid injected into it through a curiously-formed proboscis ; this latter is covered over with feathers or scales. A magnified view of one of these feathers is seen at No. 3. Another scale from a

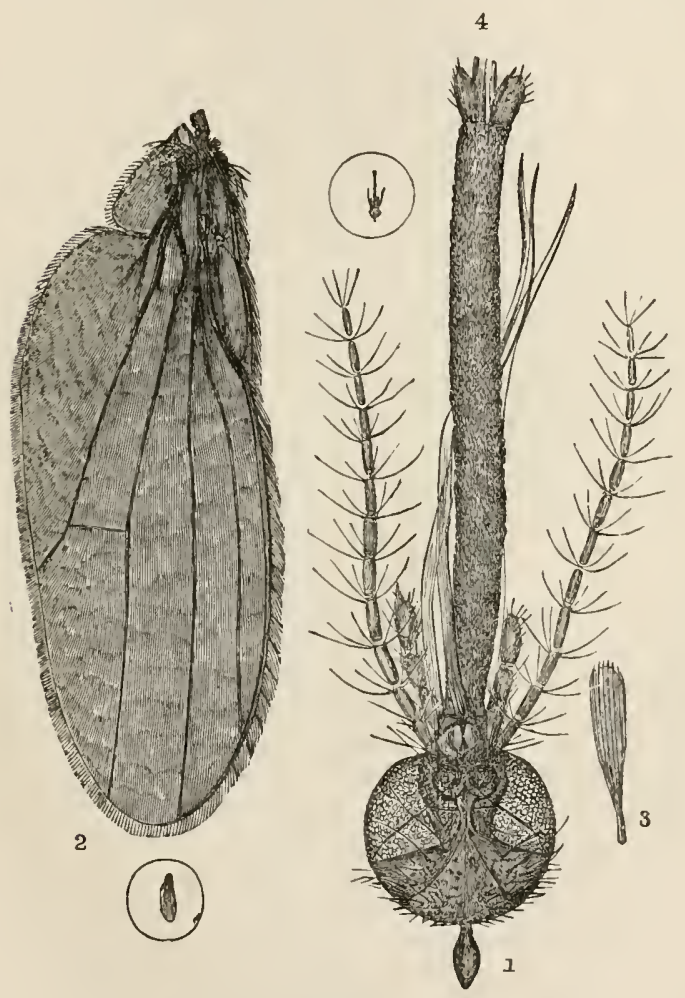

Fig. 269.

1. Head of Culex pipiens, Feinale Gnat, detached from the body. 2, Wing. 3, A Scale from the Proboscis. 4, Proboseis and Lancets. The reticulation on each side of the head shows the space occupied by the eyes. The feather or scale from proboseis is magnificd 250 diameters 
Gnat's wing is magnified 500 diameters, fig. 273 , No. 7. The proboscis is protected on either side by antennæ, or' feelers.

The metamorphosis of the larra of Corethra plumi. cornis, one of the Gnat tribe, has been carefully investigated by Professor Rymer Jones, F.I.S. ${ }^{1}$ This competent observer brings out many points of interest; one in particular deserves notice, namely, the use of the four remarkable-looking jet-black bodies situated in the body of the larva, two of which are placed in the thoracic region, and two near the centre of the posterior half of the body. These have hitherto puzzled all observers, but at length receive elucidation at the hands of Professor Jones, who had the good fortune to witness the metamorphosis. In form these bodies are more or less kidney-shaped, and to all appearance completely isolated in the body. Upon crushing the insect beneath the compressorum, they are found to be filled with air, and by their means the creature is enabled to rise and sink at pleasure. They are composed of a series of small vesicles, each of which has several coats: of these the outermost, when feebly magnified, seems of a uniform hue of black, but under a higher power is seen to be made up of many pigment cells, so as to give the organ a reticulated appearance; and it is only when this black pigment has been removed, together with a dull opaque membrane whereon the black patches rest, that the real air-sac is displayed. When thus denuled, the true walls of the air-sac appear to be composed of a dense membrane, possessing great refractive power, the effect of which upon transmitted light is extraordinary. When highly magnified, it is found to be entirely composed of numerous coils of a delicate fibre, similar to that which maintains the permeability of the trachea of ordinary insects, arranged in several superimposed layers, and having the appearance of being closed in on all sides. It is not until the larva thus constituted has arrived at its full size that the appearances described become complicated by intermixture with organs belonging to the pupa condition of the insect. At this period, however, the rudinients of

(1) See Trans. Micros. Soc. Oct. 1867, "On the Structure and Metamorphosis of the larva of Corethra pluinicornis." 
future limbs berin to show themselves under the form of transparent vesicles, which, as they enlarge, crowd the thoracic region of the body. The change from the larva to the pupa condition involves phenomera of the most startling character. The air-sacs, situated both in the thoracis region and in the hinder portion, burst and unfold themselves into an elahorate tracheal system, and a pair of ear-shaped tubes, of which not the slightest trace could hitherto be discerned, make their appearance upon the dorsal aspect of the thorax; two long tracheæ seem to be thus simultaneously produced, occupying the two sides of the body, and constituting the main trunks, from which large branches are given off to supply, in front, the head, the eyes, and the nascent limbs; while posteriorly they spread over the now conspicuous ovaries, and terminate by ramifying largely through the thin lamellæ that constitute the caudal appendages. In individuals subjected to microscopic examination within a very brief period after their assumption of the pupa state, the places originally filled by the air-sacs of the larva are found to be occupied by the lateral remnants of their external coats, clearly indicated by ragged membranes, covered with patches of black pigment, in the immediate vicinity of which numerous air-bubbles are met with, extravasating, as it were, into the cellular tissue.

The family Phryganeidce, the larvæ of which are aquatic, present almost as little resemblance to the imago as those of some metabolous insects. They are long, softish grubs, furnished with six feet, and with a horny head armed with jaws, generally fitted for biting vegetable matters, although some appear to be carnivorous. To protect their soft bodies, which constitute a very favourite food with fishes, the larva are always inclosed in cases formed of bits of straw and sticks, pebbles, and even small shells. The materials of these curious cases are united by means of fine silken threads, spun like those of the caterpillars of the Lepidoptera, from a spinnaret situated on the labium. In increasing the size of its case to suit its growth, the larva is sail to add to the anterior part only, cutting off a portion of the opposite extremity. When in motion, tho larva pushes its head and the three thoracic segments, 
which are of a harder consistence than the rest of the body, out of its case; and as the latter is but little, if at all, heavier than the water, the creature easily drags it along behind, thus keeping its abdomen always sheltered. It adheres stoutly to the inside of its dwelling by means of a pair of articulated caudal appendages, generally assisted hy three tubercles on the first abdominal segment. Before changing to the pupa state, the larva fixes his case to some object in the water, and then closes up the two extremities with a silken grating, through which the water necessary for the respiration of the pupa readily passes. The pupa is furnished with a large pair of hooked jaws, by means of which, when about to assume the perfect state, it bites through the grating of its prison, and thus sets itself free in the water. In this form the pupæ of some species swim freely through the water by means of their long hind legs, or creep about plants with the other four ; frequently rising to the surface of the water, they there undergo their final change, using their deserted skin as a sort of raft, from which to rise into the air ; others climb to the surface of aquatic plants for the same purpose.

The perfect insect (Phryganea grandis) has four wings, with branched nervures, the anterior pair of which, clothed with hairs, are more frequently used than the posterior. The organs of the mouth, except the palpi, are rudimentary, and apparently quite unfit for use. The head is furnished with a pair of large eyes, and with three ocelli; the antennæe are generally very long. Some species are so exactly like Moths, that they have often been supposed to belong to the Lepidopterous order ; in point of fact, these insects may be considered to form a connecting link between the Neuroptera and the Lepidoptera. The females have been seen to descend to the depth of a foot or more in the water, to deposit their eggs.

Eggs of Insects, Plate VI. No. 124, \&c.-In form, colour, and variety of design, the eggs of insects are nore surprisingly varied than those of the feathered tribes; but as from their smallness they escipe observation, our acquaintance with their structure and peculiarities is necessarily limited and imperfect. Although the eggs of the animal serics differ nuch in their external characteristics, 
they closely resemble each other while yet a part of the ovarian ova, and prior to their detachment from the ovary. At one period of their formation all eggs consist of three similar parts :-1st. The internal nucleated cell, or germinal vesicle, with its macula; $2 \mathrm{~d}$. The vitellus, or yolksubstance; and $3 \mathrm{~d}$. The vesicular envelope, or vitelline membrane. The germinal vesicle being the first produced may be regarded as the ovigerm; then comes the yolksubstance, which gradually envelopes it, or is deposited around it; and the vite!line membrane, the latest former, incloses the whole. The chemical constituents of the egrg contents are albumen, fatty matters, and a proportion of a substance precipitabie by water. The production of the chorion, or shell membrane, does not take place till the ovum has attained nearly the full size, and it then appear. to proceed, in part at least, from the consolidation over the whole surface of one or more layers of an albuminous fluiul serreted from the wall of the oviduct. The observations of H. Meyer have shown that a part of the outer membrane is derived from a conversion into it of the imner cellular or epithelial lining membrane of the oviduct, at the place where it is in closest contact with the surface of the ovum. Dr. Allen Thompson therefore thinks that " many of the varieties in the appearance and structure of the external covering of eggs may probably depend on the different modes of development of these ceils."

The embryo cell is so directly comnecter with the germinal vesicle that at a certain period it disappears altogether, and is absorbed into the germmal yolk, or rather hecomes the nucleus of the embryo, when a greater degree of compactness is observed in the yolk, and all that remains of the germinal vesicle is one or more highly refracting fat globules and albuminoid bodies. Towards the end of the period of incubation, the head of the young caterpillar, according to Meissner, lies towards the dot or opening in the lid, which he terms the micropyle, ${ }^{1}$ from its resemblance to a small gate, or opening through which the worm emerges forth. From a number of careful observa-

(1) The tern micropyle (a little gate) has heretofore only been usel in its re iation with the vegetable kinglom: it is used to denote the ovening or foramen towards which the radicle is always frointed. 
tions macle on the silkworm, we have not been able to satisfy ourselves of the correctness of these particulars: in every case, indeed, the young worm makes its way out from a point generally below this spot. Leuckart, how. ever, expresses his belief in this micropyle, and says, "It becomes at a later period converted into a funnel, which is connected directly with the mouth of the embryo, and serves to convey nourishment from without to it." We, on the contrary, look upon it as an involuted portion of membrane, indicating the spot where the formative process of the outer membrane terminated, or where at a still earlier stage, and while the ova was yet in the ova-sac, the sperinatozoa passed in to fecundate the yolk mass.

The germinal vesicle is very large and well marked, while the egg is yet in the ova-sac of the insect. By preparing sections, after Dr. Hallifax's method, ${ }^{1}$ we find that the germinal vesicle in the bee's egg is not situated immediately near or even below the so-called micropyle, but rather more to the side of the egg; just in the position which the head of the embryo is subsequently found to occupy when it arrives at maturity.

The egg membrane, or envelope, of all the Lepidoptera, is composed of three separate and distinct layers: an external slightly raised coat, tough and hard in its character, a middle one of united cells, and a fine transparent viteline lining membrane, perfectly smooth and homogenous in structure, imparting solidity, and giving a fine iridescent hue to the surface, such as most of us admire in old glass exhumed from the ruins of Pompeii. The germinal vesicle is of a proportionately large size for the egg, and its macula is at first single, then multiple. In the silk-worm's egg the outer membrane is comprised of an inner reticulated membrane of non-nucleated cells, and in the outer layer the cells are arranged in an irregular circular form, also non-nucleated, with ninute interstitial setæ or hairs projecting outward.

The outer surface of the egg-shell of Coccus Persica is

(1) Dr. Hallifax adopts the method of killing the insect with chloroform : he then immerses it in a bath of hot wax, in which it is allowed to remain until the wax becomes cold and hard: now with a sharp knife a section is easily made in the required direction without in the least disturbing any of the fragile parts, or interual organs. 
covered by minute rings, of which the ends somewhat overlap. These rings are thought to be identical in their character with the whitish substance which exudes through pores on the under-side of the body ; and it is more than probable that these layers of rings and their arrangement account for the beantiful prismatic hues which they present when viewed as opaqne objects under the microscope, and by the aid of the side-condenser. This white substance, it should be observed, becomes a part of the intimate structure of the egg-shell, and is in nowise affected by either strong spirit or dilute acids. Sir John Lubbock ${ }^{1}$ states that in the greenish eggs of Phryganea, "the colour is due to the yolk-globules themselves. In Coccus, however, this is not so ; the yolk-globules are slightly yellow, and the green hue of the egg is owing to the green granules, which are only minute oil globules. When, however, the egg arrives at maturity, and the upper chamber has been removed by absorption, these green granules will be found to be replaced by dark-green globules, regular in size, and about 1-8000th of an inch in diameter, and which appear to be in no way the same in the yolk of Phryganea eggs." Another curious fact has been noticed, which partially bears on the question of colour : the production of parasite bodies within the eggs of some insects. In the Cocens, for instance, parasitic cells of a green colour occur, "shaped like a string of sausages, in length about the 1-2000th of an inch by about the 1-7000th in breadth."

The eggs of Moths and Butterflies present many varying tints of colour; in speaking of this quality we do not restrict the term solely to' those prismatic changes to which allusion is so often made, and which are liable to constant mutations according to the accident of the rays of light thrown upon them; but we more particularly refer to the several natural transitions of colour, the prevailing tints of which are yellow, white, grey, and a light-brown. In some eggs the yellow, white, and grey are delicately blended, and when viewed with a magnifying power of about fifty diameters, and by the aid of a side-reflector (parabolicreflector), present many beautiful combinations. The sost delicate opalescent or rather iridescent tints appear

(1) Phil. Trans. 1859, p. 341. 
on others, of which the eggs of the feathered tribes furnish no example. The egg of the Mottled Umber Moth (Erannis defolaria), Plate VI. No. 137, is in every particular very beautiful. It is ovoid, with regular hexagonal retieulatious, and at each corner studded with a raised knob or button; the space within the hexagon is finely punctated, and the play of colours is exquisitely delicate. In this egg no micropyle can be seen. The egg of the Thorn Muth (Ennomos erosaria), Plate VI. No. 138, is of an elongated brick-looking form, one end of which is slightly tapered off, while the other in which the lid is placed is flattened and surrounded by a beautifully white-beaded border, having for its centre a slightly raised reliculated micropyle. The empty egg-shell gires a fine opalescent play of colours, while that containing the young worm appears of a brownish-yellow.

The egg of the Straw-belle Moth (Aspilates gidvaria), Plate VI. No. 139, is delicately tinted, somewhat long and narrow, with sides slightly flattened or rounded off; and irregularly serrated. The top is convex, and the base a little indented, in which are seen the lid and micropyle. The young worm, however, usually makes its way through the upper convex side : the indentation represented in the drawing shows the place of exit.

An example of those eggs possessing a good deal of natural colour is presented in that of the Common Puss Moth (C. Vinula), a large spheroidal-shaped egg, having, under the microseope, the appearance of a fine ripe orange; the micropyle exactly corresponds to the depression left in this fruit by the removal of the stalk; the surface is finely reticulated, and the natural colour a deep urange.

The egr of the Mottled Rustic Moth (Caradina Morpheus), No. 124, is subconical, and equally divided throughout by a series of ribs, which terminate in a wellmarked geometrically-formed lid. The Tortoise-shell Butterfly (Vanessa urtica), No. i25, presents us with a delicate ovoid egg, divided into segments, the ribs of which turn in towards the micropyle. 'The Common Footman (Lithosia campanula), No. 126, produces a perfectly globular egg covered with fine reticulations, and of 
a delicate buff colour. The egg of the Shark Moth ( $C u$ cullia umbratica), No. 127, is subconical in form, with ribs and cross-bars passing up from a flattened base to the summit, and turning over to form the lid. No. 136, the Egg of Blue Argus Butterfly (Poryummatus Argus). That of the Small Einerald Moth (JodisVernaria), No. 134, is an egg of singular form and beauty, - an oval, flattened on both sides, of silvery iridescence, and covered throughout with minute reticulations and dots. It is particularly translucent, so much so that the yellow-brown worm is readily seen curled up within. The lid or micropyle is not detected until the caterpillar eats its way out of the shell. It should be stated that the whole series of eggs in the plate are considerably over-coloured, and consequently lose much of their beautiful transparency. The eggs of Flies and Parasites also present much variety in form, colour, and coustruction. Many of their eggs are provided with a veritalle lid, which opens up with a hingelike articulation. The cover is shown in Plate VI. No. 144, Egg of Bot-fly, from which the larva is seen just escaping ; No. 146, Egg of Scatophaga ; No. 147, Egg of Parasite of Magpie. ${ }^{1}$

Moths and Butterflies supply the microscopist with some of the most beautiful objects for examination. What can be more wonderful in its adaptation than the antenna of the Moth, represented in fig. 271, No. 1, with a thin, finger-like extremity almost supplying the insect with a perfect and useful hand, moved throughout its extent by a muscular apparatus of the most exquisite construction! The tongue of Butterfly, No. 2, is evidently made for the purpose of dipping, into the interior of flowers and extracting the juices ; this is furnished with a spiral band of muscle: an enlarged view of a portion is given at No. 3. See also Plate V1. Nos. 132 and 133, Antennæ of Vapour Moth.

The inconceivably delicate structure of the maxillæ or tongues (for there are two) of the Butterfly, rolled up like the trunk of an elephant, and capable, like it, of every variety of movement, has been carefully examined and described by Mr. Newport. " Each maxilla is convex on

(1) A paper on the Eggs of Insects, giving many other forme wial be founil in thic Intellectual Observer, Oet. 1867. 


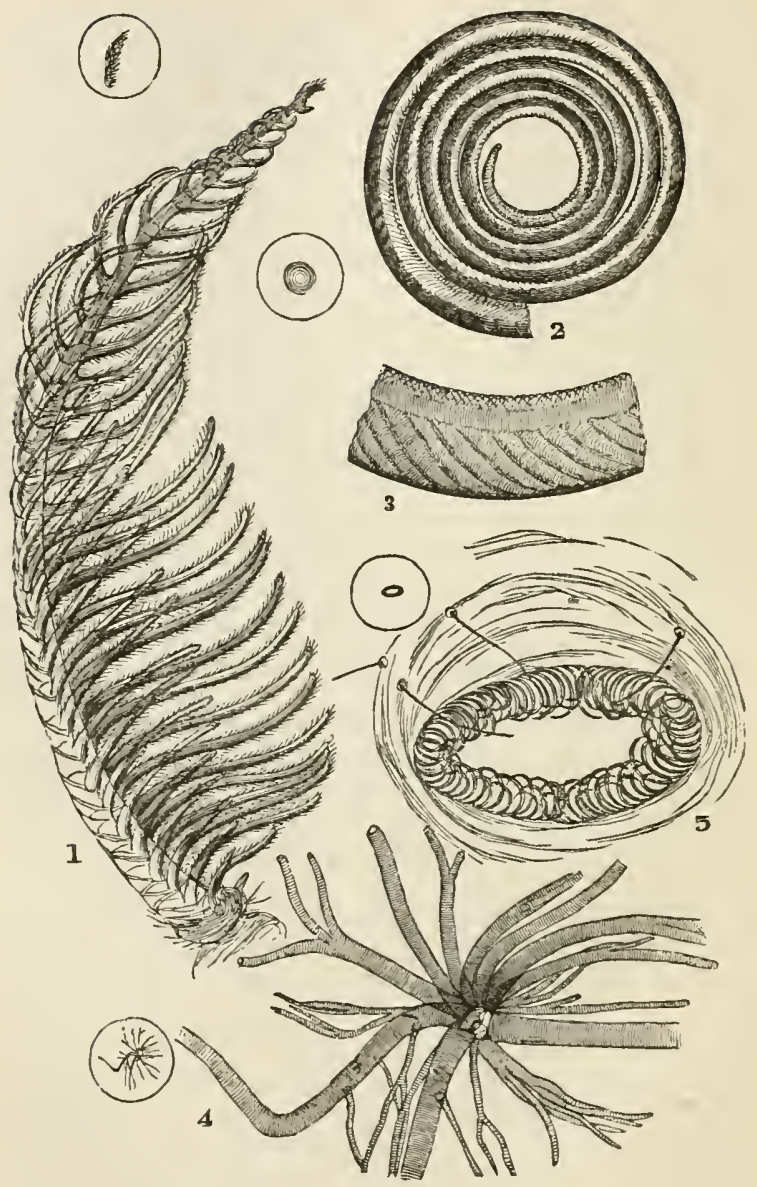

Fig. 271.

1. The Antenna of the Silkworm-moth. 2, Tongue of a Butterfly. 3, A purtion of the Tongue highly magnitied, showing its museular fibre. 4, The Trachea: of Silkworm. 5, The Foot of Silkworm. (The small circles enclose each somewhat near tho natural size). 
its onter surface, but concave on its inner; so that when the two are approximated, they form a tube by their union, through which fluids may be drawn into the mouth. The inner or concave surface, which forms the tube, is lined with a very smooth membrane, and extends throughout the whole length of the organ; but that each maxilla is hollow in its interior, forming a tube 'in itself,' as is generally described, is a mistake; which has doubtless arisen from the existence of large trachea, or breathingtubes, in the interior of each portion of the proboscis. In some species, the extremity of each maxilla is studded externally with a great number of minute papilla, or fringes-as in the Vanessa atalanta-in which they are little elongated barrel-shaped bodies, terminated by smaller papillæ at their extremities." Mr. Newport supposes that the way in which the insect is enabled to pump up the fluid nourishment into its mouth is this : "On alighting on a flower, the insect makes a powerful expiratory effort, by which the air is expelled from the interior air-tubes, and from those with which they are connected in the head and body; and at the moment of applying its proboscis to the food, it makes an inspiratory effort, by which the central canal in the proboscis is dilated, and the food ascends it at the same instant to supply the vacuum produced; and thus it passes into the mouth and stomach: the constant ascent of the fluid being assisted by the action of the muscles of the proboscis, which continues during the whole time that the insect is feeding. By this combined agency of the acts of respiration and the muscles of the proboscis, we are also enabled to understand the manner in which the Humming-bird sphynx extracts in an instant the honey from a flower while hovering over it, withont alighting; and which it certainly would be unable to do, were the ascent of the fluid entirely dependent upon the action of the muscles of the organ." 1

The wings of Moths and Butterflies are covered with scales or feathers, carefully overlapping each other, as tiles are made to cover the tops of houses. The iridescent variety of colouring on their wings arises from a peculiar wavy arrangement of the scales. Figs. 272 and 273 are magni.

(1) Cyclop. Anat. Physiol., article "Insecta." 
fied representations of $a$ few of them. No. 1 is a scale of the Mrorpto menelaus, taken from the side of the wing, of a
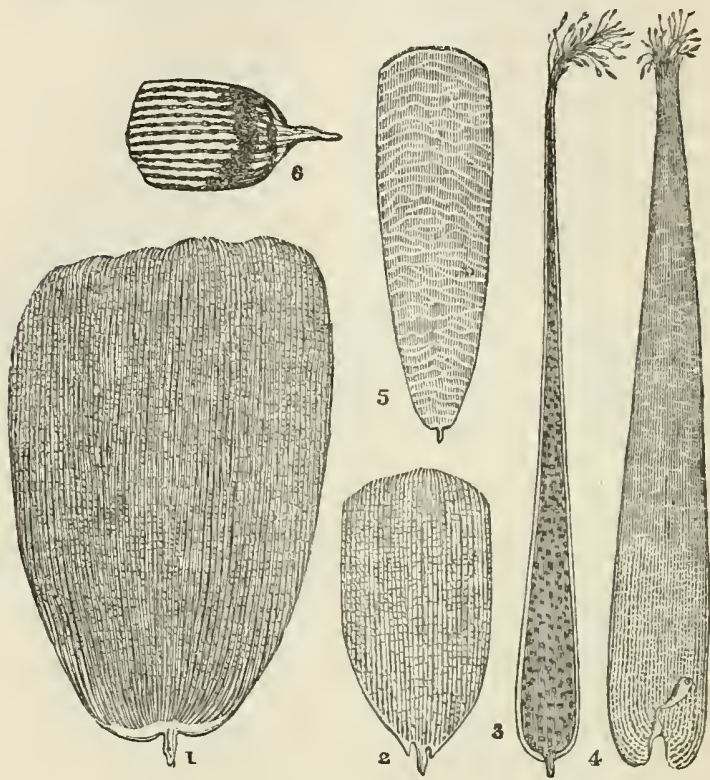

Fig. 372.-Scales from Bullerfies' and Moths' wings. (Magnified 200 dian eters.)

1, Scsie of Morpho Menelaus. 2, Large Scale of Polyommatus Argiolus, azure biue. 3, Hipparchia Janira Argiolus. 4, Pontia Brassica. 5, Podura Plumbea. 6, Small Scale of Azure Blue.

pale-blue colour: it measures about 1-120th of an inch in length, and exhibits a series of longitudinal stripes or lines, between which are disposed cross-lines or striæ, giving it the appearance of brick-work. The microscope should be enabled to make out these markings with the spaces between them clear and distiuct, as shown in fig. 273 , No. $1 a$.

Polyommatus argiolus, Azure-blue, Nos. 2 and 6, are large and small scales taken from the under-side of the wing of this beautiful blue butterfly; the small scale is covered with a series of spots, and exhibits both longitu- 
dinal and transverse stria, which should be clearly defined, and the spots separated : this is a good test of the defining power of a quarter-inch object-glass.

No. 3, Hipparchia janira, Common Meadow Brown Butterfly scale: on this we see a number of brown spots of irregular shape and longitudinal striæ.

No. 4, Pontia brassica, Cabbage-butterfly, affords an excellent criterion of the penetration and definition of a microscope: it is provided at its free extremity with a brush-like appendage. With a high power, the longitudinal markings appear like rows of little beads. Chevalier's test-object is the scale of the Pontia brassica, the granules of which must be rendered distinct. Mohl and Schacht use Hipparchia janira is a test for penetration, with a moderate angular aperture and oblique illumination. Amici's test-object is Navicula Rhomboides, the display of the lines forming the test; this is a very good test for angular aperture.
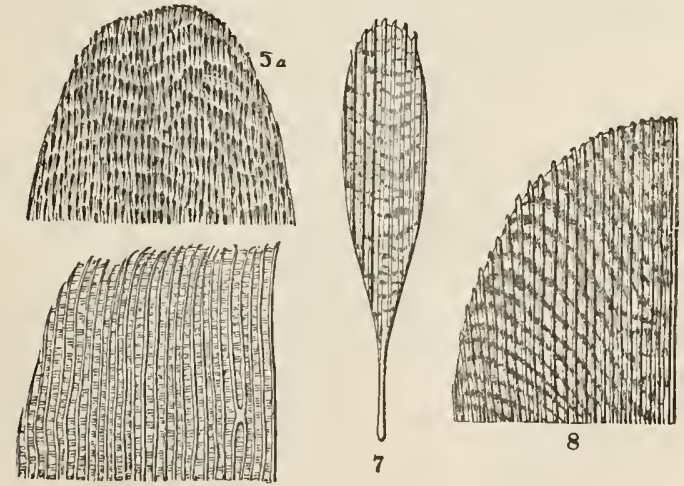

1a

Fir.273. - Portions of Scales, magnified 500 diameters.

la. Portion of Scale of Morpho Menelaus. 5a, Portion of Large Scale of Podura Plumbea. 7, Scale from the Wing of Gnat; its two layers are here represented. 8, Portion of a Large Scale of Lepisma Saccharina.

The Tinea vestianella, Clothes-moth, possesses very delicate and unique scales; two of these are imperfectly represented near the Acams taken from nne of these moths, 1. parn 64l. The feathers from the under-side of the R $\mathrm{R} 2$ 
wing are the best, requiring some management of illumination to bring out the lines sharp and clear.

The common Clothes-moth generally lays its eggs on the woollen or fur articles it is bent upon destroying; the larva begins to eat immediately it is hatched; then with the hairs or wool it first gnaws off, it forms a case or tube, under the protection of which it devours the substance of the article on which it fixes its abode. This tube is of parchment-like consistence, and quite white; is cylindrical in its shape, and furnished at both ends with a kind of flap, which the insect raises at pleasure, and crawls out; or it projects the front part of its body with its fore-feet through the opening, just enough to enable it to creep about without removing the rest of its body from the tube, which it draws after it. There are several kinds of Clothes-moths, the caterpillars of many bury them-

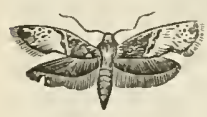

Fig. 274.-The Black Clothes-moth. selves in the article on which they feed, instead of making the tube before-mentioned. The moths also differ very much in appearance; the commonest is of a liglit buff colour ; one species, Tinea tapetzella, fig. 274, is nearly black, with the larger wings white tipped, or pale grey.

Mr. Topping, the well known preparer of microscopic objects, generally furnishes three kinds of test-oljects, which he covers with the thinnest glass, in order that objectglasses of the highest powers may be used in their examination. The following are arranged in their order of value as test-objects :

Amician test.

N. v. Rhomboides.

Grammatophora subtilisim\&.

Pleurosigma fasciola.

- cuspidata.

- angulatim.

- strigosum.

- prolongatum.

- Spencerii.

- Balticum.

- hippucampus.

- $\quad$ formosum.
SCA LES.

Meadow Brown.

Pontia Brassica.

Azure Blue.

From Gnat's Wing.

Tinea Vestianella.

Amathusia. Horsefieldii.

IIorplio Menelaus.

Podura plumbea.

Lepisma saccharina.

H AIRS,

Indian Bat.

, Mouse.

" Mole.

I.arva of Dermestes.

Mr. J. T. Norman, of City Road, preparer of specimens for the nuicroscope, furnished our Eggs of Insects. 
In a genus Coccina (Dorthesia), several species of which are found in this country, the female-although apterous and active in all stages-is completely covered with a snow-white secretion, which gives it more the appearance of a little plaster-cast than anything else.

In another tribe, the Phytophthiria, both sexes are either wingless or furnished with four distinctly veined wings. The rostrum springs apparently from the breast; the tarsi are two-jointed, and furnished with two claws. The most familiar species of this tribe are the Aphides, Plant-lice, which have ever been regarded with considerable interest by the naturalist. 'Their bodies are flaskshaped, and furnished with six feet, a pair of antennæ, and very generally with a pair of short tubes close to the extremity of the abdomen, from which a clear sweet secretion exudes. Both sexes are at one period winged, at another wingless; and individuals of the same species are often winged and apterous at different periods of the year. Perhaps the most remarkable phenomena in the history of generation was first observed in the Aphis, and to which great significance has been given by Professor ()wen, under the name Parthenogenesis or virgin-procreation. This mode of generation is not confined to insectlife, the same has been observed in the Salpce among mollusca, the Distomata among entozoa, each parent giving birth to an embryo different to itself; and this embryo living as an independent animal, gives birth, without intercourae, to another and to a successive series, until at length a progeny is formed bearing the likeness of the first parent. In the Aphis the ova are deposited by the parent insect, and in due time produce larval wingless Aphides. Each virgin Aphis of this brood will produce other similar broods without intercourse; this will go on to the eleventh generation, the last generation being winged, and male and femaie: these have intercourse, and then commence the same series of phenomena over again.

The Maple-aphis, better known as the Leaf Insect (Plate VI. No. 128), averages about the one-fiftieth of an inch in length, and, although long sold and exhibited under the name of the "Leaf Insect," nothing was known of its origin and history, with the exception of 
what the Rev. J. Thornton stated in 1852, to whom we owe its discovery on the leaves of the maple; he, believing it to be a species of Aphides, called it Phyllophorus testudinatus. Subsequently it attracterl the attention of the Dutch naturalist Van der Hoeven, who regarded it as the larval form of an undetermined species of Aphis, and named it Periphyllus. It has more recently engaged the attention of Dr. Palbiani and M. Siguoret, whose united investigations are given in the "Comptes Rendus" of June 17, 1867. They have positively ascertained that it is the larva of Aphis aceris; a brown species is also met with during a great part of the year upon the young shoots of the maple. At the same time a curious fact was made out, constituting a new and remarkable peculiarity in the development of this group of insects, already presenting so many curious phenomena in connexion with their reproduction. It rea!ly appears that the female produces two kinds of young-one normal, the other abnormal; the first are alone capable of continuing the course of their development, and capable of reproducing the species; whilst the latter retain their original form, which is never changed throughout their existence. They increase so little in size, that it appears almost doubtful whether they eat; the mouth is so very rudimentary that this surmise in some measure gains support from the circumstance; they undergo no change of skin, never acquire wings like the reproductive insect, and their antennæ always retain the five joints which are peculiar to all young Aphides before the first monlt. Neither are they all of the same colour, some being of a bright green, as represented in our Plate, while others are of a darker, or brownish-green, colour. The brown-green embryos differ from the adult female only in those characters analogous to all other species; and that chielly with regard to the hairs, which are long and simple. In the green embryos, in the place of hairs, the body is surrounded with scaly, transparent lamellæ, more or less oblong in shape, each of which is traversed by divergent ramifying nervures. These lamellæ not only occupy the body, but also the anterior part of the head, the first joint of the antennæ, and the outer edge of the tibire of the 
two anterior pair of legs. The dorsal surface in these insects is corered with a mosaic of hexagonal plates, very closely resembling the plates of the carapace of the tortoise. In this particular our artist has certainly fallen into an error. Another peculiarity is that the body is much flattened out, and looks so much like a scale on the surface of the leaf, that it requires considerable practice, as well as quickness of sight, to detect the young Mapleaphis. One of the lamellæ is seen highly magnified at $c$, and a tenent-hair at $b$. The antennæ taper off towards the apex, are serrate on both edges, and terminate in fine setæ, one of which is shown at $a$. Below the insertions of the antennæ, brilliant scarlet-coloured compound eyes are placed, which receive their nervous supply from the central ganglion.

Aphicles live upon plants, the juices of which they suck, and, when they occur in great numbers, cause considerable damage to vegetation; a fact well known to the gardener and farmer. Mary plants are liable to be attacked by swarins of these insects, which cause the leaves to curl up: they grow sickly, and their produce is greatly reduced. One striking instance is presented in the devastation caused by the Hop-fly (Aphis humuli).

The Aphrophora bifasciata, common Frog-hopper, has the antennæ placed between the eyes, and the scutellum visible-that is to say, not covered by a process of the prothorax. The eyes, never more than two in number, are sometimes wanting. These little creatures are always furnished with long hind legs, which enable them to perform most extraordinary leaping feats. The bestknown British species, because so very abundant in gardens, is the Cuckoo-spit, Froth-fiy, fig. 275. The names Cuckoo-spit and Froth-fly both allude to the peculiar habit of the insect, while in the larva state, of $a$, The frothy substance. $b$, The pupa. enveloping itself in a kind of frothy secretion, somewhat resembling saliva; and this, indeed, was at one time sup. 
posed to be the saliva of the curkoo, being found on the young shoots of plants just about the time the cuckoo is heard in the woods.

The IIymenoptera are distinguished from other insects with membranous wings by the presence of an ovipositor of peculiar construction at the extremity of the abdomen of the females, which serves for placing the eggs in the required position; and in the males of some (Bces, Wasps. rc.) constitutes a most formidable offensive weapon. As the structure of this organ, which is rarely absent, is essentially the same throughout the order, the form of its component parts being merely modified to suit the exigencies of the different insects, a short description of its structure will not be considered out of place. The ovipositor, borer, or sting, generally consists of five pieces : a pair of horny supports (6ig. 279) forming a sheath for the borer or ovipositor, being jointed at the point where they issue from the cavity of the last abdominal segment, and the last joint is usually as long as the borer itself. The latter consists of three bristles, of which the superior is grooved along its lower surface, for the reception of a pair of finer styles, and these are barbed at the point. The three pieces, when fitted together, form a narrow tube, through which the eggs pass to their destination, the poisonous fluid also, which renders the sting of the Bee so painful, is forced down the same into the wound. In the Saw-fly, a part of this organ remaius rudimentary, in other respects it does not very much differ.

The larvæ of most Hymenoptera are footless grubs, furnished with a soft head, and exhibiting but little, if any, advance upon those of Diptera (Plate VI. No. 141). In the Saw-fly, however, the larva, instead of being, as above described, a mere footless maggot, presents the closest resemblance to the caterpillar of the Lepidoptera; it is provided with a distinct head, with six thoracic legs, and in most cases, from twelve to sixteen pro-legs are appended to the abdominal segments.

The Saw-fly, fig. 276, most destructive to the gooseberry-bush, is remarkable for the way in which the female provides for the safety of her eggs. This fly has a flat yellow body, and four transparent wings, the outer two of 
which have brown edges. The female lays her eggs on the under-side of the leaf, along the projecting veins; these are firmly attached, and cannot be removed without

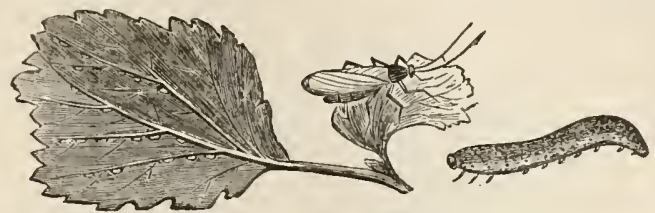

Kig. 276.-The Caterpillar and Saw-fly of Gooselierry-tree.

crushing. The instrument which the little insect uses for the purpose of cutting the leaf is the most remarkable piece of perfect mechanism imaginable; securely lodged,

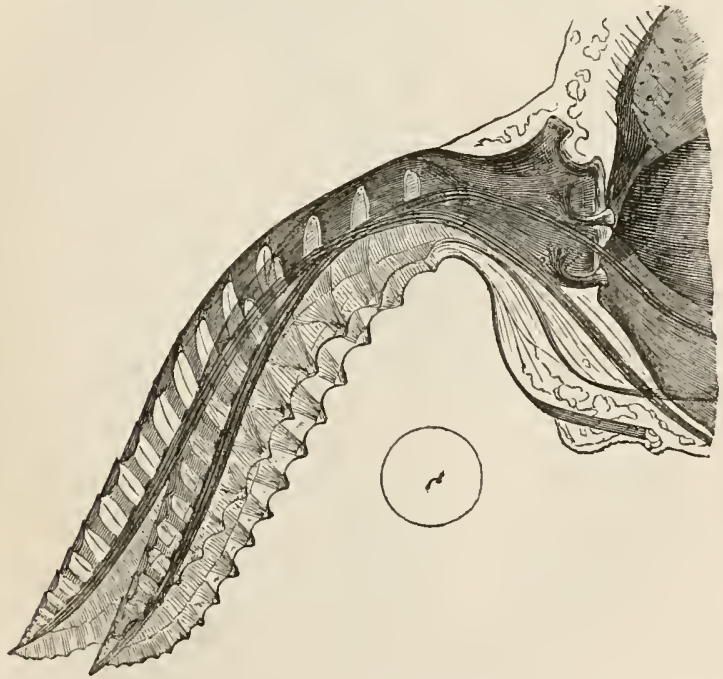

V'ir. 277.-Saws of Saw-fiy. (The small circle incloses the same nearly tho natural size.)

when not in use, in a long narrow slit beneath the abdomen, and protected by two horny plates, which at first appear io consist of a single piece ; but upon closer inspection 
four plates are seen to enter into their construction: namely, two saws, placerl side by side, as in fig. 277; and two supports, somewhat like the saws in shape. A deep groove runs along the thicker edge of the latter, which is so arranged that the saws glide backwards and forwards, without a possibility of running out of the groove. When the cut is made, the four are drawn together; and through a central canal, which is now formed by combining the whole, an egg is protruded into the fissure made by the saws in the leaf. The cutting edges of the saws are provided with about eighteen or twenty teeth : these have sharp points of extreme delicacy, and together make a serrated edge of the exact form given to the finest and best-made surgieal saws. In the summer-time the proceedings of this little insect can be watehed, and the method of using this curious instrument seen, by the aid of a hand magnifier; they are not easily alarmed when busy at their work.

Many other insects are provided with instruments for boring into the bark or solid wood itself. The Cynip bores

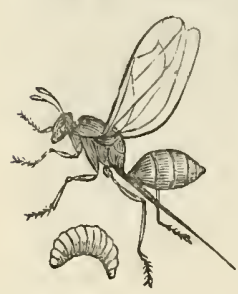

a hole into the side of the oak-apple, for the purpose of depositing her egg. The larva when hatched finds a comfortable lodging, and a good supply of food; when full grown, it eats its way out of the nut, and, dropping to the ground, it assumes the form of the per feet fly. The most important of this family is the Cynip galloe tinctorive. FIg. 27s.-Female Eglan- fig. 278, which is the cause of the gallti.ie tiall-fly and larru. nut, a nut most extensively employed in the manufacture of ink and for dyeing purposes.

Some of the Wasp tribe, so very peculiar in their habits, are artive agents in the economy of nature. The solitary Mason-wasps curiously construet nests in the form of cells, for the purpose of carefully rearing their young. The social-wasps, like bees, live in communities, and have nearly the same divisions of labour and regulations for the good government of the colony. The structure and mechanical contrivance of the wasp's sting can only be seen under the microscope. The sting consists of 
tw, barbed darts, which will penetrate the flesh deeply, and, from a peculiar arrangement of their serrated edges, their immediate withdrawal is prevented; by the great muscular effort required for this purpose, a small sac or

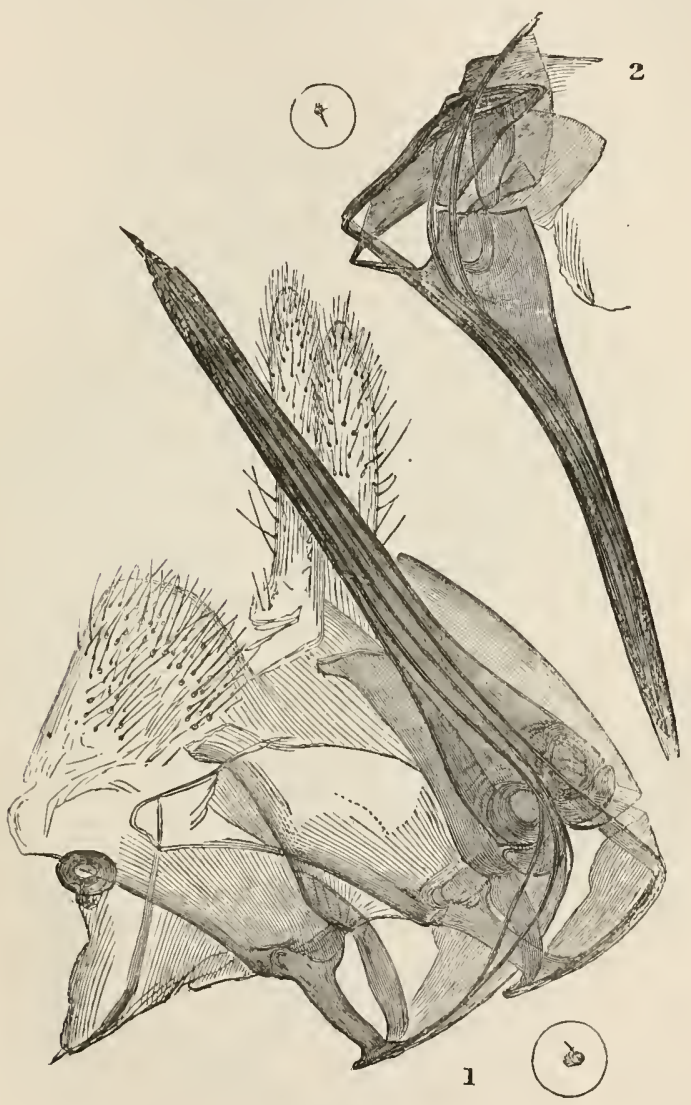

Fig. $2 \pi 9$.

1. Eting of Wasp. 2, Sting of Eee. (The small circles show each neariy the full size.) 
bag near the root is pressed upon, and its irritating con tents squeezed out into the wound. After the fluid is injected, the wasp has the power of contracting the barbed points, and then it withdraws the sting from its victim. In fig. 279 the sting of the wasp is shown, with its attachments and muscular arrangement; and it will be seen that the sting is most wonderfully adapted to become an instrument of a very effective and dangerous character. The palpi near it are placed there for the purpose of cleaning or wiping it; at all events, this appears to be one of the ises they are put to.

The proboscis or trunk of the Honey-bee next demands attention; this is used, with its curious accessories, to collect the honey while roving about from flower to flower. The proboscis itself (fig. 280) is very curiously diridel ; the divisions are elegant and regular, beset with triangular hairs, which, beirg numerous, appear at first sight as a number of different articulations. The two vutsicle lancets are spear-shaped, of a membranaceous or horny substance, set on one side with short hairs, and having their interior hollow; at the base of each is a hinge-articulation, which permits of considerable motion in several directions, and is evidently used by the busy insect for the purpose of opening the internal parts of flowers, and thus facilitating the introduction of its proboscis. The two shorter feelers are closely connected to the proboscis, and terminate in three-jointed articulations. Swammerdam thought these were used as fingers in assisting the remoral of obstructions; but it is more probable that they are mado use of by the insect for storing, and removing the bee-bread to and from the pocketreceptacles in the legs. The lower part of the proboscis is so formed that it can be considerably enlarged at its base, and thus made to contain a larger quantity of the collected juice of flowers; at the same time, it is in this cavity that the nectar is transformed into pure honey by some peculiar chemical process. The proboscis tapers off to a little nipple-like extremity, and at its base will be seen two shorter and stronger mandibles, which serve the iittle insects in the construction of their cells, and from between which is protruded a long and narrow tongue 
or lance; the whole is most ingeniously connected to the head by a horny sheath, and a series of muscles and ligaments. The proboscis, being cylinclrical, extracts the juice of the flower in a somewhat similar way to that of the butterfly; when loaded with honey, the insect's next

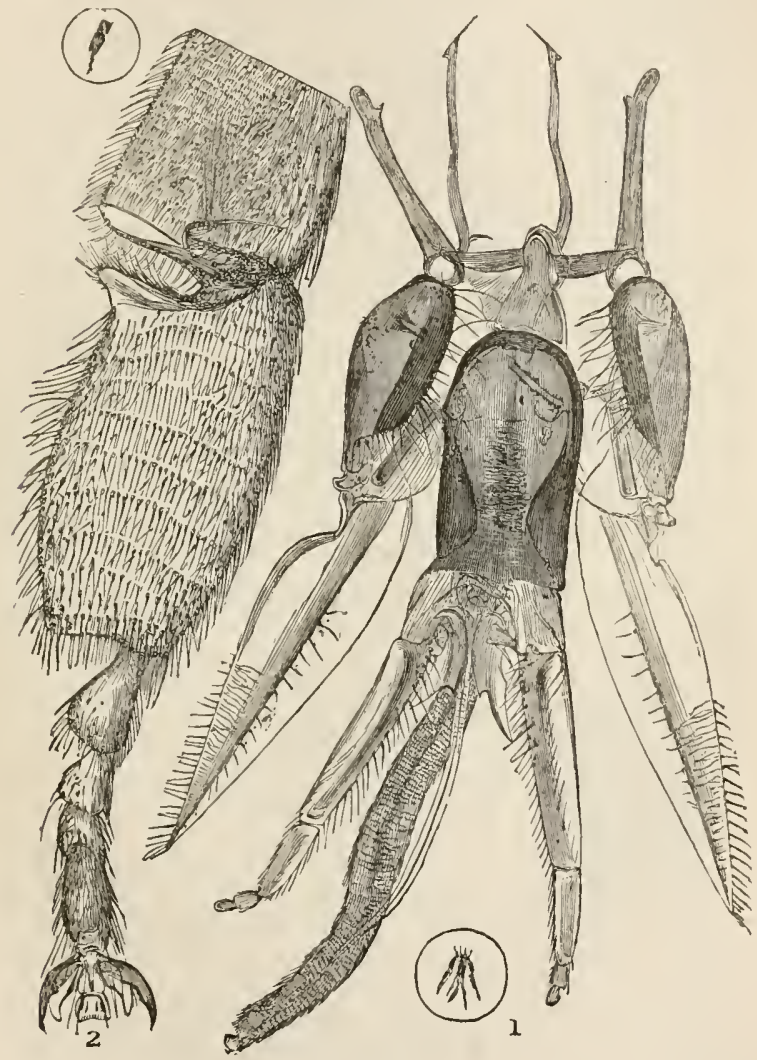

Fig. 2 So.

1. Honey-bee's tongue. 2, Leg, showing pocket for carrying the Bee-bread. (The small eircles show the objects about the natural size.) 
care is to fill the very ingenious pockets situated in its hind legs (one of which is shown at No. 2) with bee-bread; when these little pockets are filled with as much pollen as the bee can convenieatly carry, it flies back to the live with its valuable load, where it is speedily assisted to unload by its fellow-workers; the pollen is at once kneaded and packed closely in the cells provided for its prescrvation. The quantity of this collected in one day by a single hive during favourable weather is said to be at least a pound ; this chiefly constitutes the food of the working-bees in the hive. The wax is another secretion exuding through the skin of the insect; it is found in little pouches in the under-part of the body, but is not collected and bronght home ready for nse, as has been generally supposed. The waxen walls of the cells are, when completed, strengthened by a varnish, called propolis, collected from the buds of the poplar and other trees, and besmeared over by the aid of the wonderful apparatus represented in the engraving. If a bee is attentively observed as it settles down upon a flower, the activity and promptitude with which it uses the apparatus is truly surprising; it lengthens the tongue, applies it to the bottom of the petals, then shortens it, bending and turning it in all possible directions, for the purpose of exploring the interior, and removing the pollen. In the words of Brook:-

\footnotetext{
"The dainty suckle and the fragrant thyme,

By chemical reduction they sublime;

Their sweets with bland attempering suction strain,

And eurious through their neat alembies drain;

Imbib'd recluse, the pure secretions glide,

And vital warmth coneocts th' ambrosial tide."
}

The leading characteristic of the vast order Coleoptera, Beetles, consists in the leathery or horny texture of the anterior wings (elytra), which serve as sheaths for the posterior wings in repose, and generally meet in a straight line down the back.

The elytra present us with wing-eases of many Curculios, Diamond bectles, the most brilliant of opaque objects. Some are improved by being mounted in Canada balsam, whilst others are more or less injured by it: a trial of a small portion, by first touching it with turpentine, decides 
this point. Oblique or side illumination shows the play of colours on the scale to the greatest advantage.

To the genus Ptinus belongs a small beetle known as the Death-watch, fig. 281. This and the species Anobium are found in our houses, doing much injury whilst in the larval state. The eggs are often deposited near some crack in a piece of furniture, or on the binding of an old book. As the larvæ are hatched, they begin to eat their way into ally furniture on which they may lave been deposited; and, having attained a sufficient depth, they undergo transformation, and return,

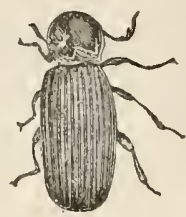

Fig. 281. by other passages, as perfect beetles. In Atropus, maguified. furniture attacked by them, small round holes, about the size of the head of a pin, may be seen, and to these holes the term worm-eaten has been applied; and the noise, made by the insect striking its head against the wood, has given rise to the name of Death-watch. The larva is called a Book-worm when it attacks books; old books and those seldom used, are often found bored through by it. Kirby and Spence mention, that in one ease twenty-seven folio volumes were eaten through, in a straight line, by this insect. The beetle is very small, and almost black. The head is particularly small; and from the prominence of the thorax, looks as if it were covered with a hood. The Anobium puniceum, fig. 282, attacks dried objects of natural history, and all kinds of bread and biscuits, particularly sailors' biscuits, in which maggots frequently abound. In collections of insects, it first consumes the interior; when the larva assails birds, it is generally the feet that it devours first; and in plants, the stem or woody part. The larva is a small white maggot, the body

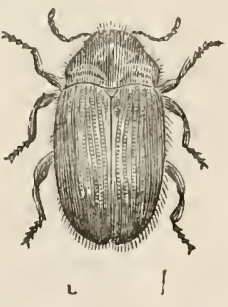

Fig. 282,-Anobium pus niccum, magnified. of which is wrinkled, and consists of several segments covered with fine hairs; its jaws are strong and horny, and of a dark brown. The body is white, and so transparent, that the internal organs of the insect can ko seen 
through it. The beetle itself is of a reddish-brown colour, covered with fine hairs.

The Bacon-beetle (Dermestes lardarius) is another of the destructive beetle family. The larva of this beetle is

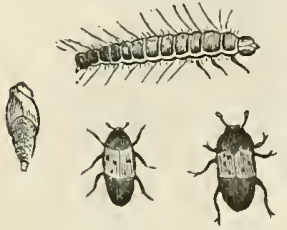

Fig. 283.-Dermestes lardarius. Larva, pupa, and imago. particularly partial to the skin of any animal that may fall in its way; consequently it destroys stuffed animals and birds in collections of natural history, whenever it can gain access to them. It attacks hams and bacon for the skin's sake; and, being a very glutton, extends its ravages to the flesh. This larva is long and slender, its body nearly round, and is divided into thirteen segments, of a blackish-brown in the middle, and white at the edge; the whole being fur. nished with bristle-shaped reddish-brown hairs.

The Dermestidce belong to the family of Necrophagous beetles, six genera of which have been found in Great Britain. The $D$. lardarius is black about the head and tail, with an ash-grey band across the back, having three black spots on each wing-case. Sometimes this band takes a yellowish tinge, and then the hairs, which are here disposed in tufts, are likewise of a yellowish-grey colour. The beetle is most destructive in spring. The larvæ, like those of the clothes moths, are but seldom seell, being careful to conceal themselves in the bodies they attack, and their presence can only be guessed at by finding occasionally their cast-off skins, which they change several times during their larvæ state. Specimens of hair put up by the mounters and labelled "Hair of Dermestes," do not belong to the species at all. These hairs, long favourite objects with microscopists, and placed by them among test-objects, may, it is believed, be those found in tufts at the extremity of the body of Anthrenus museorum. Westwood says that the larvæ of these beetles are furnished with tufts of hairs, which are "individually formed of a series of minute conical pieces placed in succession, the base being very slender, and the extremity a large oblong knot, placed on a slender footstalk." This 
description comes very near to that of the hair in question. It is also suggester that the larvæ of another genus, that of Tiresius serra, furnish these hairs; but, however that may be, it is quite clear that the hairs called "Dermestes" (fig. 307) are not obtained from the Bacon-beetle.

In the Gyrinus, Whirligig, we have a combination of contrivanees to facilitate the creature's movements in the element in which it lives. The hind legs are converted into a pair of oars of remarkable efficieney, the point of

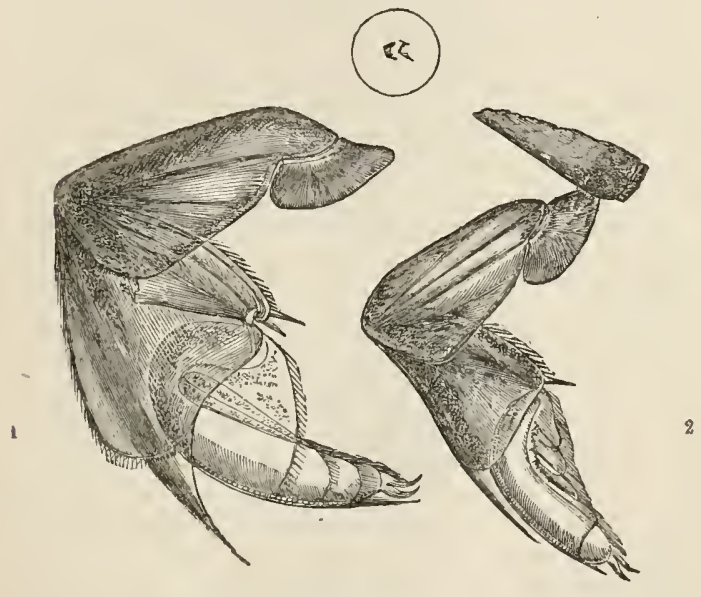

Fig. 284.

1, Leg of Gyrmus, Whirligig, with paddle expanded. 2, With paddle closed up.

their connexion with the body being adapted with great precision to ensure the most effectual application of the propelling power; as it strikes out behind in the act of swimming, a membranous expansion of a portion of the legs enables the insect to move about with great rapidity; upon the legs being drawn back again towards the body, the membrane closes up, and thus offers but a small resistance to the water (fig. 284). The eyes are not the least curious part of the merry little creature; while one 
portion of them, that fitted for seeing in the air, is fixed on the upper part of the head, the lower portion, for seeing under the water, is placed at the lowest part, a thin division separating the two.

Sir John Lubbock, writing of aquatic insects, says : ${ }^{1}$ "Though most of the great orders are represented, no aquatic Hymenoptera or Orthoptera had till now (1864) been discovered. Great, therefore, was my astonishment, when I saw a small Hymenopterous insect, evidently quite at its ease, and actually swimming by means of its wings. At first I could hardly believe my sight; but having found several specimens, and shown them to some of my friends, there can be no doubt about the fact. Moreover, the same insect was again observed, within a week, by another entomologist, Mr. Duchess, of Stepney. It is a curious coincidence that, after remaining so long unnoticed, this little insect should thus be found almost simultaneously by two independent observers. Mr. Walker at first considered the insect to be Polynema fuscipes, but though allied to that species, it is not identical with it. So completely aquatic is it in its habits that it can remain immersed for at least twelve hours; but it nevertheless requires to come to the surface at certain intervals to renew the air in its tracheæ. It is uncertain whether $P$. natans can also use its wings in flight. They are at any rate not easily incited to do so. It is a very minute species, and well fitted for microscopical observation, the female measuring 0.38 of an inch, and the male $0.42 . "$

Dytiscus marginalis, derived from dutes, a diver. Larva narrow, body composed of twelve segments, including head, which is large and strong, bearing antennæ, and armed with two powerful jaws. Several varieties of this beetle are met with in fresh and still waters. The larvæ feed upon other aquatic larvæ, such as the Gnat, Dragonfly, \&c. The suckers on the legs, the feet, \&c. are very interesting objects, and should be mounted for viewing both as transparent and opaque objects.

To the Orthoptera belong Locustina, Gryllina, and Achesina, all herbivorons insects. The first is represented by our well-known Grasshopper (Gryllus viridissimus), the secord,

(1) Journ. Micros. Soc. vol. iv. p 139. 18604. 
the Gryilina, appear to frequent trees and shrubs more than the other tribes, the members of which generally keep among herbage; and, in accordance with this habit, many of the exotic species have wing-cases which present the most perfect resemblance to leaves both in colour and venation. Of the Achetina, the common Cricket (Acheta domestica), fig. 285, the noisy little denizen of the kitchen. hearth, is the best example. These insects have the antennæe slender and tapering, and often considerably .onger than the body. 'They agree with the Gryllina in the structure of their singing apparatus; but the wings, instead of being arranged in the form of a high-pitched zoof, are laid flat upon the back. Some of them possess ocelli, whilst others are destitute of those organs. The wings are very long, and folded up in such a manner as to project beyond the wing-cases in the form of a pair of tapering tails; the abdomen is also furnished, in both sexes, with a pair of pilose, bristle-shaped, caudal appendages: in the female these form a long slender ovipositor, the two filaments being placed side by side, and somewhat thickened at the tip. The tarsi are three-jointed. The horny covering and muscular apparatus under the wingcases of the Cricket are very curious, and will repay microscopical examination. The Crickc'chas two wings,

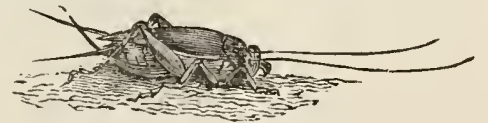

Fig. 286. - The Cricket. covered by elytra or wing-cases of a dry membranous consistency, near the base of which is a horny ridge having transverse furrows, exactly resembling a rasp or file; this it rubs against its body with a very brisk motion, and produces the well-known merry chirp; the intensity of which is increased by a hollow space, called the tympanum, acting as a sounding-board. The gastric teeth are numerous.

In Thysanura there is a remarkable diversity of structure; they undergo no metamorphosis, and have no wings. This order contains two families, Spring-tails, Poduridoe, and Lepismence. In the former, the caudal appendage has the form of a forked tail (Podura, fig. 286), which is bent under the body when not in use; by its sudden extersion the insect causes itself to spring to a very great 
distance, in comparison with its size. The body is covered with numerous minute scales, mostly of a beautiful silvery or pearly lustre, and curiously striated.

Podura plumbea, Lead-colour Springtails, are generally found in damp places, leaping about like fleas. They

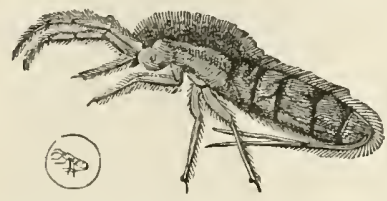

Fis. 2s6.-Todura plumbea. (In the small

circle the insect alpears life-size.) prefer a moist atmosphere, some take to the surface of the water in secluded places; their food seems to be vegetable matter of any kind in a stage of decay; the little active creatures are seen to leap about if a stone in a damp situation in the garden is turned up, or if a dark, damp corner of the cellar, about the beer-barrel, is searched; or if we peep among the roots of the ferns in the fern-case. Poduridæ, varying in form, colour, \&c. are produced from eggs, undergo no metamorphosis, are not parasitic, have from twelve to sixteen simple eyes; are furnished with strong mandibles, and a broad, curious-looking snout, and a rather long body, terminating in a bifid tail, which by alternately expanding and contracting, enables them to leap great distances. The antennæ are very long, and covered with scales and fine hairs. To obtain the scales from the body without damage-which is certain to occur if the Podura is touched by the fingers-take a small test-tube and quickly place it over the insect, when it instantly springs up and clings to the side of the tube; insert a thin glass. cover beneath, and close up the open end. One drop of chloroform carefully administered instantly kills the in-. sect; in a very short time this evaporates and leaves the tube quite dry. By gently shaking the tube a number of scales will drop off and adhere to the thin glass cover; remove this, and make it secure to the ordinary glass-slip.

Mr. R. Beck says, "that the best scales are obtained from insects found in comparatively dry places." Mr. S. J. MIcIntire, in an interesting paper on the Podura, ${ }^{\mathbf{1}}$ confirms this statement, but believes that the "test-scale" figured by Mr. Beck belongs to a distinct species. The

(1) Science Gossip, March, 1867. 
markings on the scale are better seen when an achromatic condenser is employed with a good objective. Under a power of 500 diameters, the surface appenr's to be covered with extremely delicate longitudinal and wavy lines. The smaller scales are much more difficult to resolve than the larger, and these form a good test of the defining power of a 1-8th or 1-12th object-glass. No. 5 a, fig. 274 , is a portion of a large scale. Fig. 273, No. 5, the longitudinal markings are shown under a lower power. "But the transverse strie on the scale of the speckled Podura are rendered more distinct when the central rays are stopped out. Any error in the correction of the lenses, whether iu the manufacture or in the adjustment of the thin covering glass, is immediately detected by the peculiar appearance which these markings present."

Lepisma saccharina has a spindle-shaped body covered with silvery scales, the sides of the abdomen being furnished with a series of appendages or false feet, with long-jointed bristle-like organs at their extremities. The head is concealed under a pro-thorax ; the eyes are usually compound, and generally occupy the greater part of the head. The antennæ are very long, and composed of numerous joints; the maxillary palpi, which are from five to seven jointed, are very conspicuous. These insects are also inhabitants of moist places. The Leprisma saccharina is commonly found about houses, in sash-frames, old sugarcasks, \&c.; from the latter circumstance it derives its name. The scales (fig. 273, No. 8) liave long been favourite objects, and much used for testing the power of penetration and definition of object-glasses. The scales should be mounted under thin glass covers; oblique light shows some portions of the scale to advantage; other parts are rendered more distinct when the central rays of the achromatic condenser are stopped out.

The metamorphosis is complete in the Suctoria, or Siphonaptera, a wingless family-the larva, pupa, and imago of which are very distinct in their appearancesthe well-known Flea is the best example of this small group. By many authors these insects have been arranged with the Diptera : this is most decidedly incorrect, since they differ in many particulars. The external covering of 
the Flea (fig. 287) is a horny case, divided into distinct segments; those upon the thorax being always disunited. Although apterous, the Flea has the rudiments of four

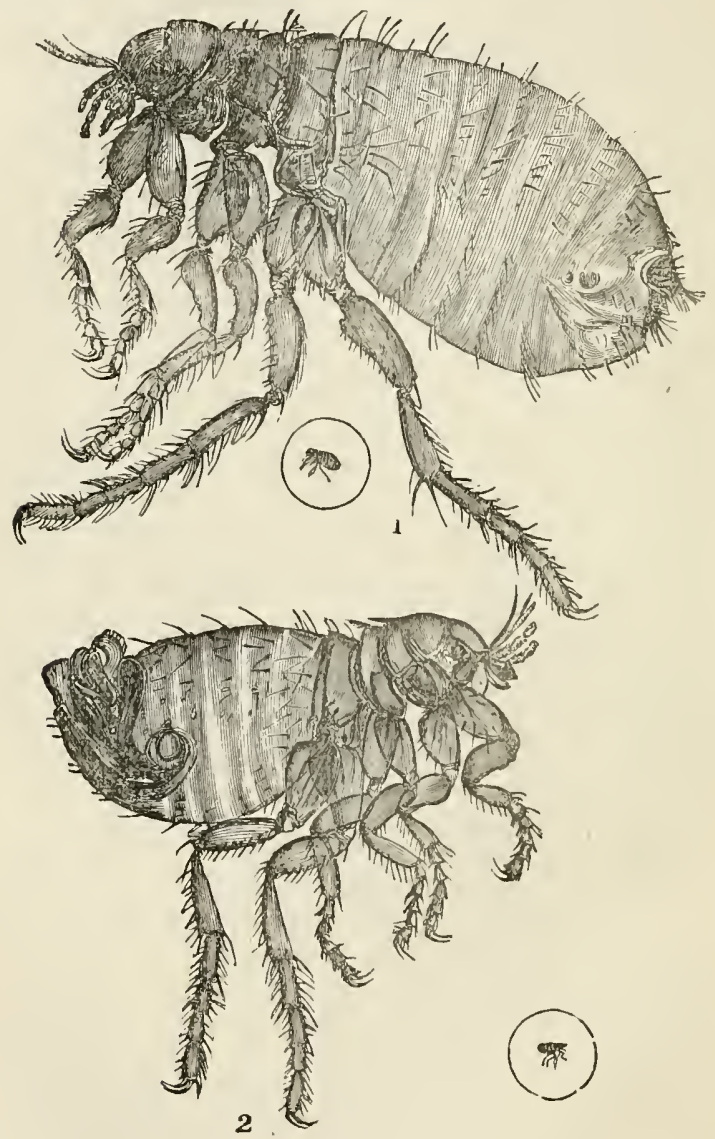

Fig. 287

1, Female Flea. 2 Male Flea. (The small circles enclose fleas the actual or life size.) 
wings, in the form of horny plates on both sides of the thoracic segments. Its mouth consists of a pair of swordshaped mandibles, finely-serrated; these, with a sharp, penetrating needle-like organ, constitute the formidable weapons with which it pierces through the skin.

The neck is long, the body covered over with scales, the edges of which are set with short spikes or hairs; from its head project a pair of antennæ, feelers or horns, a proboscis, which forms a sheath to the pair of lance-shaped weapons. On each side of the head a large compound eye . is placed. It has six many-jointed powerful legs, terminating in two-hooked claws; a pair of long hind legs are kept folded up when the insect is at rest, which in the act of jumping it suddenly straightens out, at the same time exhausting all its muscular force in the effort. The female Flea, fig. 287, lays a great number of eggs, sticking them together with a glutinous secretion; the Flea infesting the dog or cat glues its eggs fast to the roots of the hairs ; in four days' time the eggs are hatched, and a small white larva or grub is seen crawling abont, and feeding most actively. No. 4 (fig. 289) is a magnified view of one, covered with short hairs, doubtless for the purpose of preventing its dislodgment. After remaining in this state about nine or ten days, it assumes the pupa form; this it retains four days; and in nine days more it becomes a perfect Flea. The head of the Flea found in the cat (No. 3, fig. 289) somewhat differs in form from that of the species infesting the human being. Its jaws are furnished with more formidable-looking mandibles, and from between the first and second joints behind the head short strong spines project.

Arachida. - The animals forming the class Arachnida include spiders and their allies, most of which are looked upon with disgust and aversion by the generality of mankind. Arachnida are divided into two orders, Trachearia and Pulmonaria. The first includes the Acaridae or Mites, in which we find tracheæ, as in insects, but no distinct vascular apparatus : in the second, spiders and scorpions are included, and these have a pulmonary cavity, and a well-developed circulatory system. The above are distinguished from Podopthalmia or Arthropoda by their 
aerial respiration, their possession of four pairs of legs attached to an anterior division of the body, and the total absence of antenuæ. The body is also covered with a softish skin, which sometimes attains a horny consisteney, but nothing more. In the higher forms, the body is divided into two parts, the anterior of which, as in Crustacea, consists of a thoraeic segment, amalgamated with the head, and forming together a whole called the cephalothorax. In the highest classes the division of the thorax into separate segments becomes apparent; the anterior segment is, however, amalgamated with the head. The structure of the abdomen varies greatly. In some cases it forms a soft round mass, without any traces of segmentation; whilst in others, as seorpions, it is continued into a long flexible jointed tail.

Acarea, an order of animals not strictly belonging to insects, but rather to Arachnida, Spiders, Scorpions, \&c. The general description of the class is that the head is united with the thorax, forming a cephalo-thorax; no antennæ, simple eyes, body presenting transverse striæ or furrows between the second and third pair of legs, which are eight in number, terminated by an acetabulum and claws. These animals are commonly called mites, and the best known species is that found in cheese, the Acarr. domesticus. Most of the speeies are oviparous and viviparous, their eggs are very numerous. The spider envelopes its eggs in a beautiful silken cocoon. Seurpions produce their young alive, and it is deserving of notice that in this family the embryo is developed in the ovum while it still remains in the ovary. The existence of a mieropyle has not yet been made out in the ova of Arachnida. For the sake of convenience we have included Parasites in this part of our work, and in Plate VI. Nos. 144 to 147 are representations of their eggs, from some of which the larva are just emerging.

The Malophagus, or Sheep-tick, fig. 288, is apterous, and seems to be a connecting link between acarina and insects proper. The Sarcoptes scabiei produces the itch in the human being : it is also found to be the cause of mange in the dog. In one pustule on a dog suffering from this disease, as many as thirty parasites have been found. 
The Louse (fig. 290, No. 1).-Whenever wretehedness, disease, and hunger seize upon mankind, this horrid parasite seldom fails to appear in the train of such calamities, and to increase in proportion as neglect of

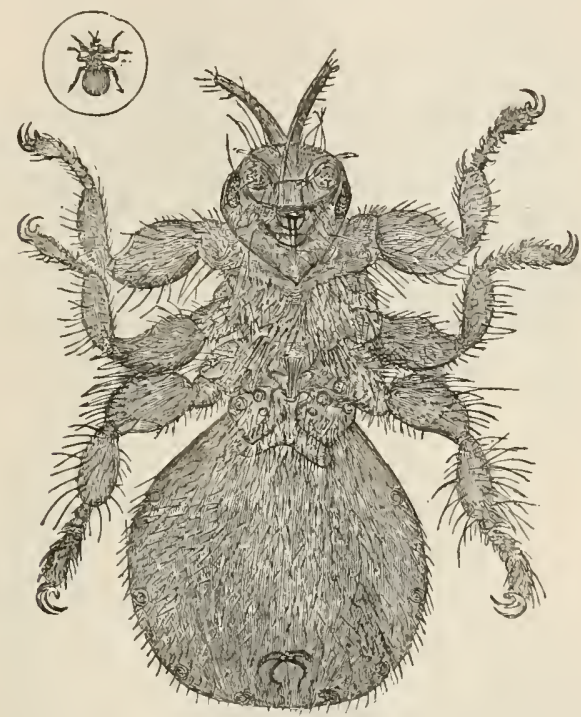

Fis. :8s.-Malophagus ovinus, Sheep-tick. (The small cirele encluses ore of life size.)

personal cleanliness engenders loathsome disease. When examined under the microscope, our disgust of it is in no way diminished. In the head may be distinguished two large eyes, and near to them are the two antennie; the front of the head is long, and somewhat tapering off to form a snout, which serves as a sheath to the proboscis and the instrument of torture with which it pierces the flesh and draws the blood. To the fore part of its body six legs arc aftixed, having each five joints, terminated by two unequal hooks; these, with other portions, are covered with short hairs. Around the outer margin of 
the body may be seen small circular dots, the breathing apertures, with which all the class are freely provided, rendering them very tenacious of life, and difficult to kill. There is another louse, rather differing in its characteristics

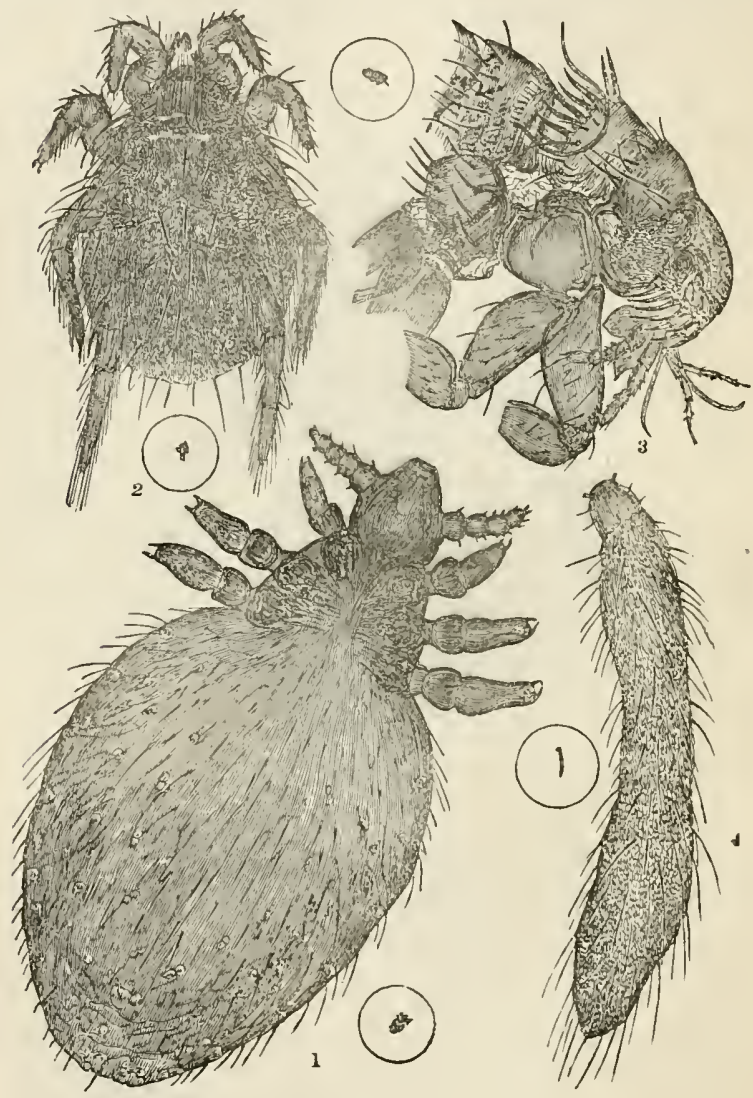

Fig. 289.

1, Dog's parasite. 2, Rat Acarus. 3, Head of Cat-Flea. 4, Larva, or grub of Flea. (The life size of each is given in the small circles.) 


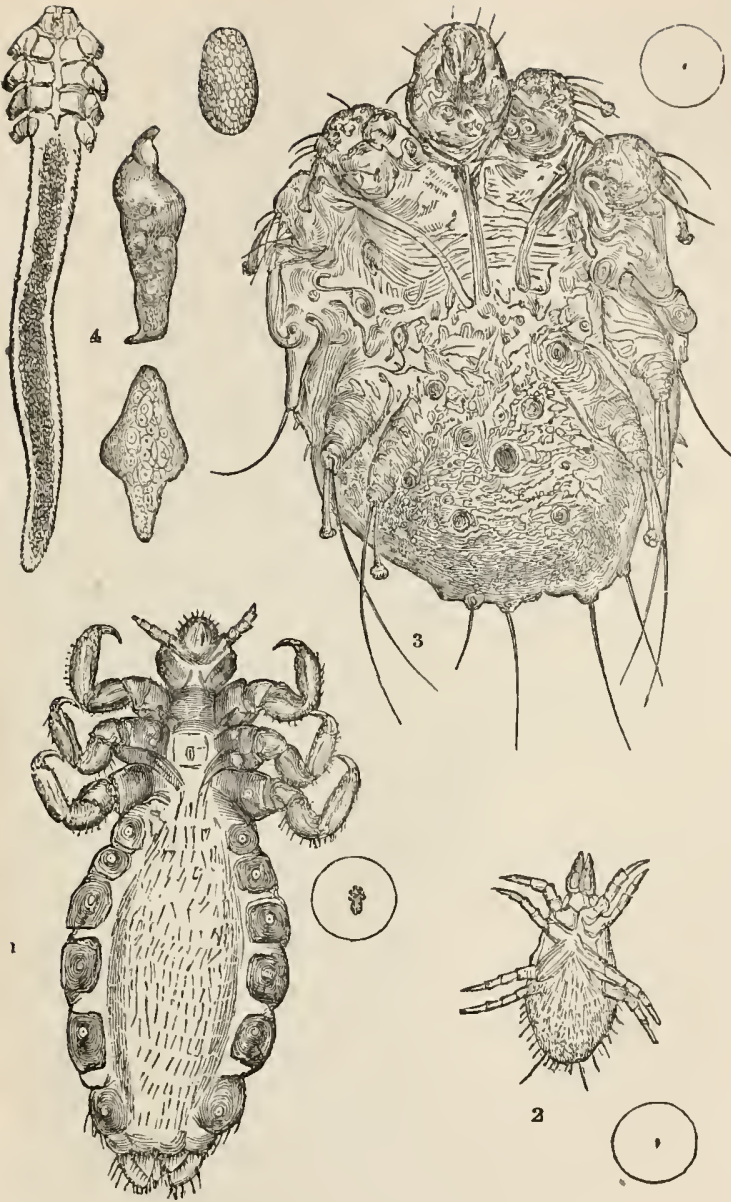

Fig. 990.-Furusites. Acarina.

1, inนsค, Hluman ; magnified 50 diameters. 2, Acarus domesticus, CheeseMite: under surface. 3, Acorus Scabiei, Iteh-Insect; magnified 350 diameters. 4, Entozonn folliculorum, Grub from the human skin in various stages of existence, from the egg nuwards: magnified 350 diameters. (Tho small circles near represent the uljects about the natural size.) 
from this, found upon the body of the very poor and dirty, known as the body or crab-louse. Leeuwenhoek carried his researehes on the habits of these insects further than most investigators, even allowing his zeal to overeome his disgust for such creatures as the louse. In describing its mode of taking food, \&c., he observes: "In my experiments, although I had at one time several on my hand drawing blood, yet I very rarely felt any pain from their punctures; which is not to be wondered at, when we consider the excessive slendemess of the piercer; for, upon comparing this with a hair taken from the luak of my hand, I judged, from the most accurate computation I could form by the microscope, that the hair was 700 times larger than this incredibly slender piercer, which consequently by its punctures must excite little or no pain, nuless it happens to touch a nerve. Hence I have been induced to think that the pain or uneasiness those persons suffer who are infested by these creatures, is not so much prodnced from the piercer as from a real sting, which the male louse carries in the hinder part of nis body, and uses as a weapon of defence." He found, from experiments made to ascertain the possible increase of these pests, that from two females he obtained in eight weeks the almost incredible number of 10,000 eggs.

The Itch-insect, Acarus scabiei (fig. 290, No. 3, magnified 350 diameters). Dr. Bononio was among the first to detect the parasitic character of the disease known as the itch. On turning out one of the pustules, or little bladders, from between the fingers, with the points of very fine needles, under the microscope, a minute animal was discovered, very nimble in its motion, covered with short hairs, having a short head, a pair of strong mandibles or cutting jaws, and eight legs, terminating in remarkable appendages, each provided with a sucker and setæ.

It has no eyes; but when disturbed it quickly draws in its head and feet, and then somewhat resembles the tortoise in appearance, its march is precisely that of the tortoise. It usually lays sixteen eggs, which are earefully deposited in furrows under the slin, and ranged in pairs; these hateli in about ten days.

To find the itch-insect, the operator must examme care- 
fully the parts surrounding each pustnie, he will then see a red line or spot communicating with it; this part, and not the pustule, must be probed with a fine-pointed instrument; the operator must not be disappointed by repeated failures. Dr. Bourgnignon bestowed much time in sturlying the habits of this troublesome parasite. To arrive at a knowledge of its haunts he arranged his microscope so as to enable him to observe it under the skin of the diseased person. The rays of light from a lamp or' candle must be carefully brought to a brilliant focus by means of the condensing or bull's-eye lens upon the chosen point of observation; a Leiberkuihn should also be attacherl to the object-glass.

No. 4, fig. 290, Demodex folliculorum, is another remarkable parasite found beneath the skin of man : it is sometimes obtained from a spot where the sebaceous follicles, or fat glands, are abundant; such as the forehead, the side of the nose, and the angles between the nose and lip ; if the part where a little black spot or a pustule is seen be squeezed rather hard, the oily matter there accnmulated will be forced out in a globular form; if this be laid on a glass slide, and a small quantity of oil added to it, to cause the separation of the harder portions, the parasite in all probability will float out; after the addition of more oil, it can then be taken away from the oily matter by means of a fine-pointed sable pencil-brush, and transferred to a clean slide; when dry it should be immersed in Canada balsam, covered over with thin glass, and mounted in the usual way.

The Cheese-mite, Acarus domesticus (fig. 290, No. 2), has a peculiar elongation of its snout, forming strong, cutting, dart-shaped mandibles, which it has the power of advancing separately or together. The powder of old and dry cheese almost entirely consists of mites and their eggs, which are hatched in about eight days; if deprived of food, they have been seen to kill and eat each other. scari infest almost the whole of sur driecl articles of food. Ac. passularum has two very long buccal bristles; it lives upon dried figs, and other saccharine fruits. Ac. destructor has long black hairs; it feeds upon the contents of entomolngizal cabinets, especially butterflies; Ac. pru- 
norum is found on dried plums, \&c.; Ac. favorum finds its food in old honeycombs ; Acarus sacchari (fig. 291) is commonly present in the more impure kinds of sugar. The discovery of the general prevalence of this acarus rests, we believe, with Dr. Hassall.

The Sugar acarus approaches somewhat, in its organisation and habits, A carus domesticus; it attains to a size so considerable, that it is plainly visible to the unaided sight.

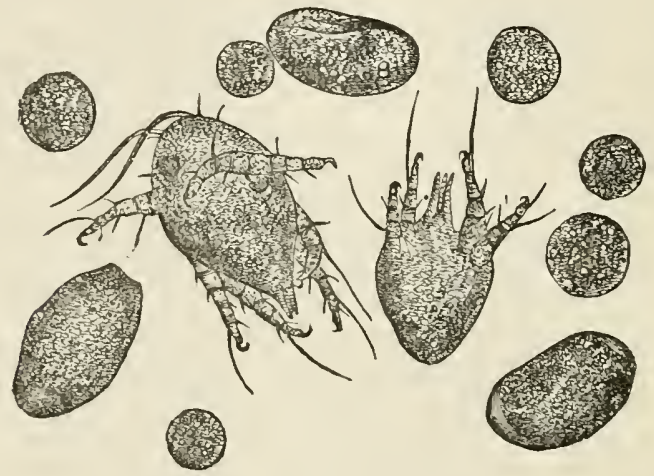

Fig. 291.

Ova and young of the Acamus sacchari, Sngar-Insect, after Hassail.

When present in sugar, it may always be detected by the following proceeding : two or three drachms or teaspoonfuls of sugar should be dissolved in a large wine-glass of tepid water, and the solution allowed to remain at rest for an hour or so; at the end of that time the acari will be found, some on the surface of the liquid, some adhering to the sides of the glass, and others at the bottom, mixed up with the copious and dark sediment, formed of fragments of cane, woody fibre, grit, dirt, and starch-granules, which usually subside on the solution of even a small quantity of sugar in hot water. The Acarus sacchari, when first hatched, is scarcely visible; as it grows it becomes elongatod and cylindrical, until it is about twice as long as 
broad; after a time the legs and proboscis begin to protrude. The body is partially covered by setre, and the feet terminate in hooks. These stages of the development of the acarus are exhibited in fig. 291.

The Acarts farino, Flour-mite. - This is of occasional occurrence in flour, but is never present unless it has become damaged. Any flour, therefore, containing the animal in question is in a state unfit, for consumption. We believe that it is found more frequently in the flour of the Leguminosa than that of the Graminea.

This acarus differs considerably in structure from the Sugar-mite, particularly so in its pennate setæ.

Dr. Burmett established to his satisfaction the following facts : "1. That though there are single species of parasites peculiar to particular animals, there are others which are found on different species of the same genus; as is the case in the parasites living on birds of the genus Larus (gulls), and the diurnal birds of

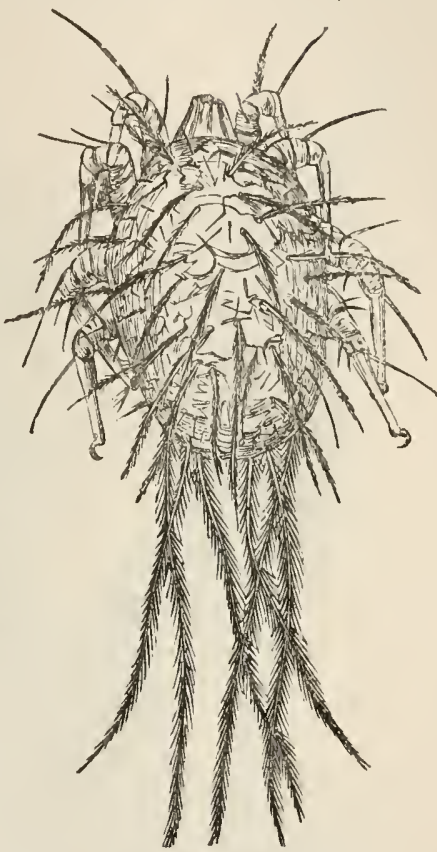

Fig. 292,-Acarus farina, Meal-Mite, Inagnified 2.50 diameters.

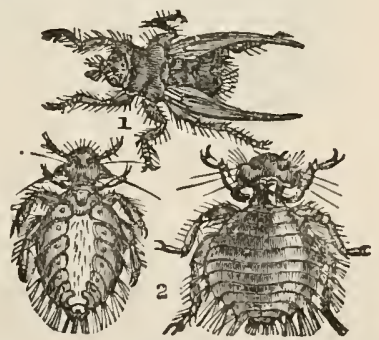

Fig. 293

1, Hippobosca Hirurdinis. 2, Nirmi, mals and female, parasites infesting Swallows. 
prey. 2. The parasites of the human body confine themsalves strictly to particular regions; when they are found

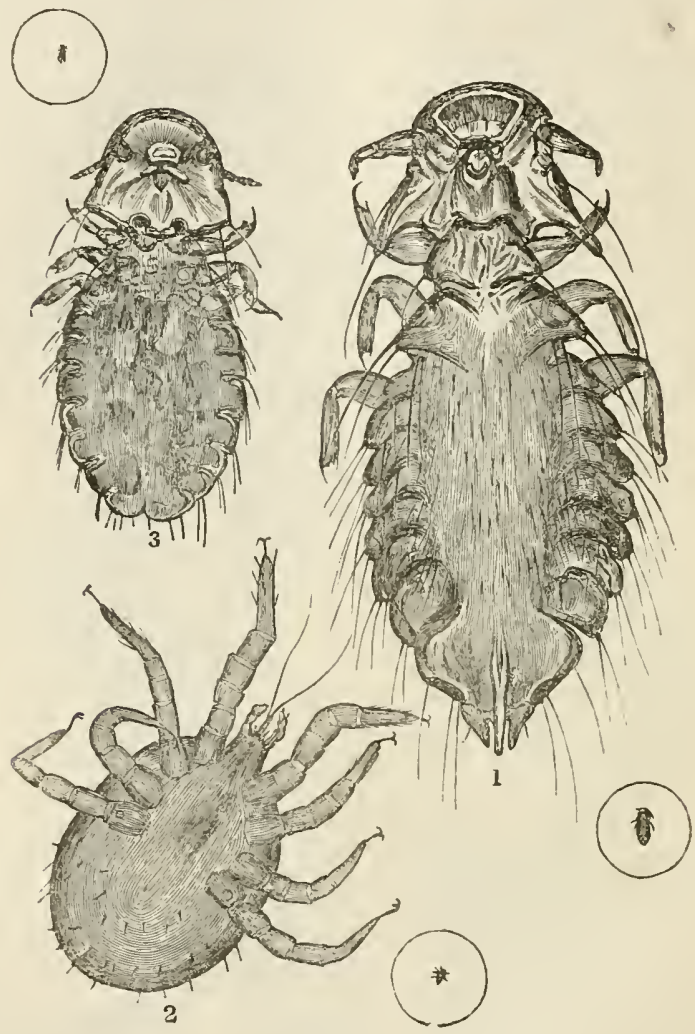

Fig. 294.

1, Parasite of Turkey. 2, Acarus of common Fowl, under surface. 3, Parasito of Pheasant. (The small circle encloses each about life size.)

elsewhere, it is the result of accident. Thus, the Pediculi capitis live in the head; $P$. vestimenti, upon the surface of the body; the $P$. tabescentium, on the bodies of those dying of marasmus; and the $P$. inguinalis, about the groins, arm- 
pits, mouth, and eyes." From an examination of the structure of these parasites, Dr. Burnett is of opinion that

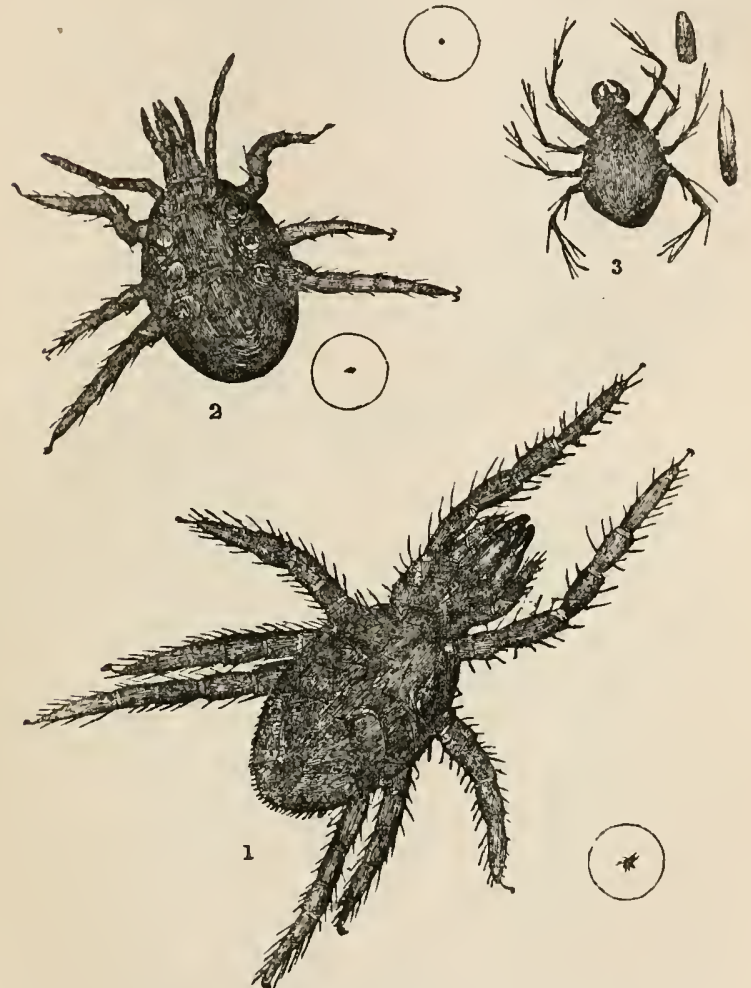

Fig. 295.

1, Acarus of Beetle. 2, Acarus of Fly. 3, Acarus of Clothes-Moth. (The circles enclose each about life size.)

they should be placed in an order by themselves, closely allied to Insecta; the mandibulate parasites occupying the highest, and the haustellate the lowest, position in the orde: : thus confirming to some extent the observations made by Mr. Denny.

There is a remarkable species of acarus described by 
Dr. Robins, found spinning a white silken web on the baso of the sparrow's thigh, or on the fore-part of its body; on raising this delicate web, you perceive that it is filled with minute erga, from which the young issue, being in due time inatched by the warmth of the body it is destined to

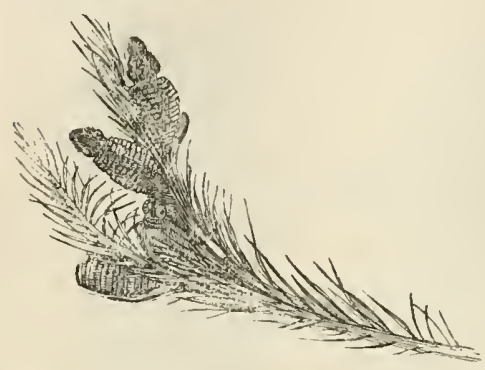

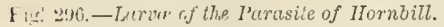
annoy. In fig. 296 are seen some eggs of a parasite infesting the hornbiil ; they are glued to the feathers near the head of the bird; the larvæ are ready to leave the egg in two days. Another, curiously enough, selects the pulmonic orifice of the snail: when the animal dilates this oivice, for the purpose of allowing the air to penetrate its respiratory cavity, the female acarus slips through the opening, and lays her eggs in the folds of the mucous membrane, where they are gradually developed. The young, upon issuing forth from the eggs, select some portion of the snail's body upon which to feed and perfect their growth.

Ixodidoe are furnished with a powerful rostrum, armed with recurvate spines, with which they pierce the skin of the unfortunate animal upon whose blood they live. So firmly do these anchor-like organs retain their holi, that if the parasite is pulled away it usually carries a portion of the skin of its victim with it. These creatures live upon a great variety of animals. The dog is very liable to their attacks, and many species fix themselves exchusively upon serpents and other reptiles. Glyciphagus cursor is found in the feathers of the owl, and in the cavities of the bones of skeletons. Gamaside are furnished with a sucking apparatus very similar to that of Ixodida, usually attaching themselves to the bodies of beetles; the common Dungbeetle (Geotroupes) is often found with its lower surface nearly covered with them.

There are other families leading a more active life, being furnished with eyes. One family, Hydrachnillx, 
Water-mites, inhabit the water, where they swim about with considerable rapidity by means of their fringed lega
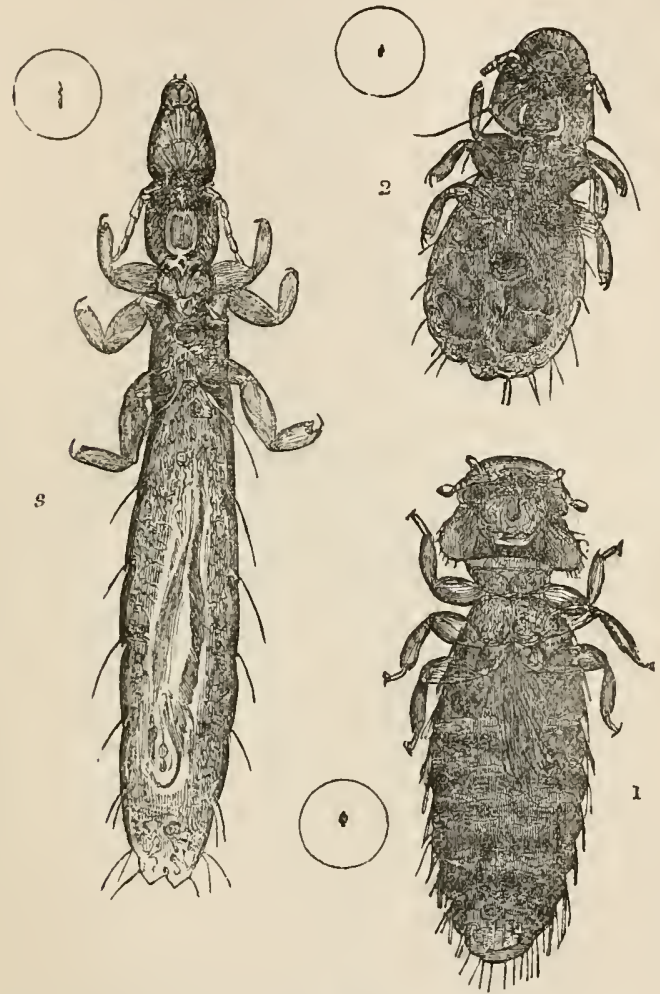

Fig. 297.

1, Farasite of Eagle. 2, Parasite of Vulture. 3, Parasite of Pigıon. (T'ne eircles znclose each about life sizc).

In their young state, they attach themselves parasitically to aquatic animals; they then possess only six legs, and pass through a quiescent or pupa state before acquiring the fourth pair. Orbitade, unlike other Acarea, live upon vegetable matter, principally damp leaves and moss; 
they have a mouth adapted for biting such food, and are covered with a hard and very brittle skin. The Bdellidae live among damp moss, have the body divided apparently into two parts by a constriction, and the rostrum and palpi very long; whilst Trombidiidae, of which the little scarlet mite so often seen in gardens is an example, have their palpi converted into little raptorial organs.

Another family of parasites are commonly met with in the bodies of fishes, attaching themselves to the branchiæ, to the soft skin under the fins, or to the eyes, much to the annoyance of the unfortunate victim. Some of these found on fresh-water fish are sufficiently transparent to show the circulation of their fluids-most interesting objects for the microscope.

The Water-snail, Limnous, is tormented by a larva of the family Amphistoma, which attaches itself by a series of hooklets and bristles to various parts of the body and mantle; under a low magnifying power, when congregated together, they appear somewhat like tufts of threads. ${ }^{1}$

Arachnida, Spiders. - Epeira diadema is the best known of the British species of Garden Spiders: it is readily recognised by the beantiful little gem-like marks on its body and legs. Spiders abound on every shrub; and if we consider that the Spider is destitute of a distinct head, without antennæ, one-half of its body attached to the other by a very slender connexion, and so soft as not to bear the least pressure,-its limbs so slightly attached to its body that they fall off at a very slight touch,-it appears ill-adapted either to escape the many dangers which threaten it on all sides or to supply itself with food; and the economy of such an animal is deserving of the microscopist's attention.

The several important organs peculiar to the Spider tribe are represented in fig. 299. Of these, No. 1 show the spinning apparatus; four only are the spinnarets, or organs by which their silky threads are emitted. Their structure is very remarkable ; the surface of each spinnaret is pierced by an infinite number of minute holes, shown

(1) The earliest known account of the parasite tribes is given in Redi's Treatise de Generatione.Insectorum; see also H. Denny's Monographia A noplurorum Britannios. 1842. 
in No. 2, from each of which there escapes as many little drops of a liquid as there are holes, which, drying the moment they come in contact with the air, immediately form so many delicate threads. Immediately after the filaments have passed through the pores, they unite first together, and then with those of the next, to form one common thread; so that the thread of the spider is com-

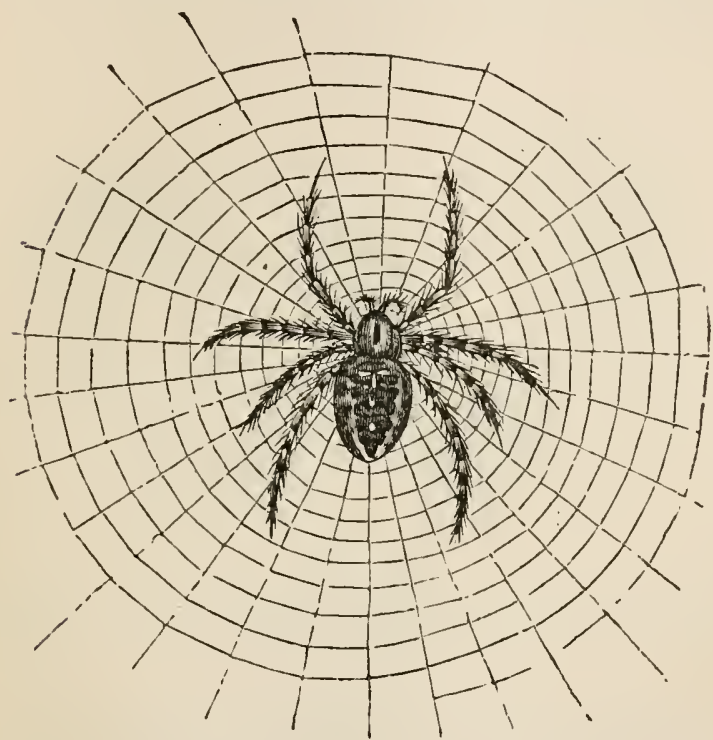

Fig. 298.-Epeira diadema, Diadem Spider.

posed of a large number of minute filaments, perhaps many thousands, of such extreme tenuity that the eye cannot detect them until they are twisted together into the working thread. In the two pairs of spinnarets a different anatomical structure can be detected; the pair above, which are a little the longest, show a multitude of small perforations, the edges of which do not projest, and which therefore resemble a sieve. The shorter pair have projecting tubes independent of the perforations which exist 
in those above. The tubes are hollow, and perforated at their extremities; and it is supposed that the agglutinating threads issue from these tubes, while those emitted from the perforations do not possess that property. It will be seen, by throwing a little dust on a circular Spider's web, that it adheres to the threads which are spirally disposed, but not to those that radiate from the centre to the cir cumference; the latter are also the stronger of the set.

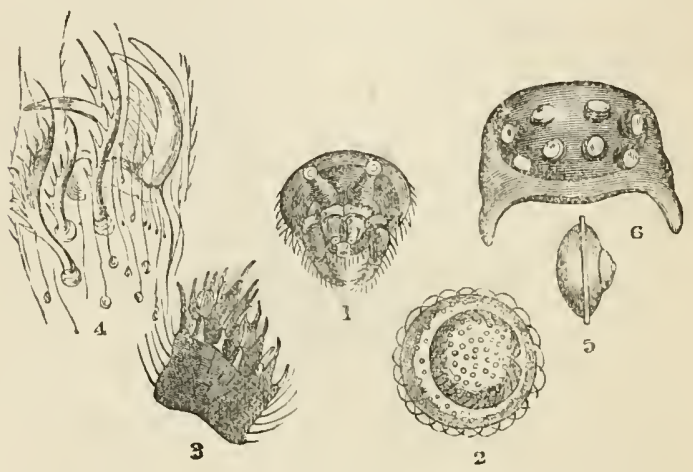

Fig. 299.

1, Spinnarets of Spider. 2, Extreme end of one of the upper pair of spimnrets. 3, End of under pair of spinnarets. 4, Foot of Spider. 5, Side view of eye. 6 , The arrangement of the four pairs of eyes.

The rapidity with which these webs are constructed is as astonishing as is the accuracy with which the webs are formed. There are many different kinds of Spiders ; but nearly all of them envelop their eggs in a covering of silk, forming a round ball, which the Spider takes care to hang up in some sheltered place till the spring. The mode in which the ball is formed is very curious: the mother Spider uses her own body as a guage to measure her work, in the same way as a bird uses its body to guage the size and form of its nest. The Spider first spreads a thin coating of silk as a foundation, taking care to have this circular by turning its body round during the process. In the same manner it spins a raised border 
round this till it takes the form of a cup; it is at this stage of the work that it begins to lay its eggs in the cup, and not content to fill it up to the brim, it also piles up a large round heap, as high as the cup is deep. Here, then, is a cup full of eggs, the under half covered and protected by the silken sides of the cup, but the upper still bare and exposed to the air and the cold. She now sets to work to cover thesc also: the process is similar to the preceding, that is, she weaves a thick web of silk all round them, and, instead of a cup-shaped nest, like some birds, the whole partakes of the form of a ball much larger than the boly of the Spider.

The feet of the Spider, one of which is represented at No. 4, are curiously constructed. Each foot, when magnified, is seen to be armed with strong horny claws, with serrations on the under-surface. By this arrangement the Spider is enabled to regulate the issue of its rope from the spinnarets. Some have, in addition, a remarkable comblike claw, for the purpose of separating certain threads which enter into the composition of their delicate webs.

One of the most remarkable members of the family, the A rgyroneta aquatica, Diving Spider, weaves itself a curious little bell-shaped globule, which it takes with it to the bottom of the water, whither it retires to devour its prey. Notwithstanding its aquatic habits, this, like the rest of its order, is fitted only for aerial respiration; it therefore fills its miniature balloon with air, which it carries down with it entangled amongst the hairs of its body. This closely resembles the earliest diving-bells.

Mr. Quekett recommended the following as a simple method of obtaining a perfect system of tracheal tubes from the larvæ of insects :- $A$ small opening having been made in the body, it is to be placed in strong acetic acid, which softens or decomposes all the viscera: the trachea must then be well washed with a syringe, and removed from the body, by cutting away the connexions of the main trunks with the spiracles, by means of fine-pointed scissors. For mounting, they should be floated on to the glass-slide, and laid out in the position best adapted for displaying them. If we wish to mount them in Canada balsam, they should be allowed to dry upon the slide, 
but their natural appearance is best preserved by mounting in weak spirit and water, or Goadby's solution, using a very shallow cell, to aroid pressure. The spiracles should be dissected out with a fine knife and scissors.

Mr. Hepworth's Mode of Preparing and Mounting Insects.-He destroys life with sulphuric ether, then washes the insects thoroughly in two or three waters in a widenecked bottle; he afterwards immerses them in caustic potash or Brandish's solution, and allows them to remain in it from one day to several weeks or months, according to the opacity of the insect; with a camel-hair pencil he then presses the contents of the abdomen and other soft parts dissolved by the potash out in a saucer of clean water, holding the head and thorax with one brush, and gently pressing the other with a rolling motion against the body from the head to the extremities. The potash must afterwards be completely washed out, or crystals may form. The insects must then be dried, the more delicato specimens being spread out or floated on to glass-slides, covered with thin glass and tied down with thread. When dry, they must be immersed in rectified spirits of turpentine, and placed under an air-pump. When sufficiently saturated they are ready for mounting in Canada balsam; but they may be retained for months in the turpentine without injury. Before mounting, as much turpentine as possible must be drained and cleaned off the slide; but the thin glass must not be removed, or air would be readmitted. Balsam thinned with chloroform is then to be dropped on the slide so as to touch the cover, and it will be drawn under by capillary attraction. After pressing down the cover, the slide may be left to dry and to be finished off. If quicker drying be required, the slide must be warmed over a spirit-lamp, but not made too hot, os boiling disarranges the object. Vapours of turpentine or chloroform may cause a few bubbles, but these disappear when condensed by cooling. ${ }^{1}$

TRANSFORMATION OF INSECTS.

The metamorphoses of the insect race offer some of the most curious and wonderful of nature's phenomena for

(1) Journ. Micros. Soc. vol. i. p. 73. 
contempiation. "We see," says an old author, "some of these creatures crawl for a time as helpless worms upon the earth, like ourselves ; they then retire into a covering, which answers the end of a coffin or a sepulchre, wherein they are invisibly transformed, and come forth in glorious array, with wings and painted plumes, more like the inliabitants of the heavens than such worms as they were in their former state. The transformation is so striking and pleasant an emblem of the present, intermediate, and glorified state of man, that people of the most remote antiquity, when they buried their dead, embalmed and enclosed them in an artificial covering, so figured and painted as to resemble the caterpillar in the intermediate state; and as Joseph was the first we read of that was embalmed in Egypt, where this custom prevailed, it was probably of Hebrew origin."

Faint and imperfect symbol though it be, yet it may, perchance, offer a glimpse of the metamorphosis awaiting our own frail bodies. Between the highest and lowest degree of corporeal and spiritual perfection, that there are many intermediate degrees, the result of which is one universal chain of being, no one can for a moment gainsay. Thus the angel Raphael is made to say, in Milton's Paradise Lost,-

"What surmounts the reach
Of human sense, I shall delineate so,
By likening spiritual to corporeal forms,
As may express them best: though what if earth
Be but the shadow of heaven, and things therein
Each to other like, more than on earth is thought !"

The great class of insects, which furnishes four-fifths of the existing species of the animal kingdom, has two chief divisions. In the one, the Ametabola, we have an imperfect, in the other, the Metabola, a perfect metamorphosis; that is, in the former there is no quiescent pupa state, and the metamorphosis is accompanied by no striking change of form ; in the latter, there is an inactive pupa that takes no nourishment, and so great a change of form, that only by watching the progress of the metamorphosis can we recognise the pupa and the imago as belonging to the same animal.

The degree of metamorphosis is, however, very different 
in different groups of insects. In its most complete form, as exemplified in the Butterflies, Moths, Beetles, and many other insects, the metamorphosis takes place in three very distinct stages. In the first, which is callerl the larma state, the insect has the form of a grub, sometimes furnished with feet, sometimes destitute of those organs. Different forms of insects in this state are popularly known as Caterpillars, Grubs, and Maggots. During this period of its existence, the whole business of the insect is eating, which it usually does most voraciously, changing its skin repeatedly, to allow for the rapid increase in its bulk; and after remaining in this form for a certain time, which varies greatly in different species, it passes to the second period of its existence, in which it is denominated a pupa. In this condition the insect is perfectly quiescent, neither eating nor moving. It is sometimes completely enclosed in a horny case, in which the position of the limbs of the future insect is indicated by ridges and prominences; sometimes covered with a case of a softer consistence, which fits elosely round the limbs, as well as the body, thus leaving the former a certain amount of freedom. Pupae of this description are sometimes enclosed within the dried larva skin, which thus forms a horny case for the protection of its tender and helpless inmate. After lying in this manner, with scarcely a sign of life, for a longer or shorter period, the insect, arrived at maturity, bursts from its prison in the full enjoyment of all its faculties. It is then said to be in the imago or perfect state. This metamorphosis is one of the most remarkable phenomena in the history of insects, and was long regarded as perhaps the most marvellous thing in nature; although recent researches have shown that the history of many of the lower animals presents us with circumstances equally if not more wonderful, nevertheless the metamorphosis of the higher insects is a phenomenon which cannot fail to arrest our attention. To see the same animal appearing first as a soft worm-like creature, crawling slowly along, and devouring everything that comes in its way, and then, after an intermediate period of death-like repose, emerging from its quiescent state, furnished with wings, adorned with brilliant colours, and confined in its choice cf food to the 
most delicate fluids of the vegetable kinglom, is a spectacle that must be regarded with the livghest interest; especially when we remember that these dissinilar creatures are all composed of the same elentents, and that the principal organs of the adult animal were in a manner shadowed out in all its previous stages.

Nor is the singularity of their natural history the only claim that these insects have upon our attention. Lowly as they seem in point of organization, there are few animals that exceed them in commercial importance. To give an instance or two; the finest red dyes known to our manufacturers are derived from insects. The Lecanium ilicis, an inhabitant of the Ilex, Evergreen-oak, growing in countries near the Mediterranean, was employed for this purpose by the ancient Greeks and Romans, as it is still by the Arabs; and, until the introduction of the Mexican cochineal, another species, the Coccus polonicus, living on the roots of the Scleranthus perennis in Central Europe, was much used for the same purpose. The Mexican cochireal, which has driven all other kinds out of the market, is one of the species Coccinia; this pretty insect was long regarded as a parasite upon the Cactus opuntia, Pricklypear-a plant common in Central America. The commercial importance of this insect is shown by a single fact: in 1850 , no less than $2,514,512$ lbs. of cochineal were imported into Great Britain alone (value about 7s. per lb.); and as abont 70,000 insects are required to weich a pound, we may form some idea of the almost countless numbers annually destroyed. For many years the cultivation, or rather feeding, of cochineal was entirely confined to Mexico; but the insect has lately been introduced into Spain, and the French possessions in Africa, with every prospect of success. A fourth species, of great importance, is the Lac insect, Coccus lacca, an inhabitant of the East Indies, where it feeds upon the Banian-tree, Ficus religiosa, and other trees. It is to this insect we are indebted, not only for the dye-stuffs known as lac-dye and lac-lake, of which npwards of $18,000 \mathrm{cwts}$. were imported in 1850, but also for the well-known subsubstance called shell-lac, so much used in the preparation of sealing-wax and varnishes. It is somewhat remark- 
able that only the female insects yield a good colouring matter.

Of all the secretions peculiar to insects, silk may well be regarded as the most valuable, since it has become as much an essential to the purposes of mankind as to the economy of its producers. The fluid, before it comes in contact with the air, is viscous and transparent in the young larva, but thick and opaque in the more mature. It is found, by chemical analysis, to be chiefly composed of Bombic acid, a gummy matter, a portion of a substance resembling wax, and a little colouring matter. Silk may be placed in boiling water without undergoing any change ; the strongest acids are required to dissolve it; and it has never yet been imitated artificially. More than 500,000 human beings derive their sole support from the culture and manufacture of silk; and the importance of the Silkworm to Great. Britain alone is represented by the large sum of $16,500,000 l$. annually. Then we have large sums of money changing hands from the labours of the useful little Bee; tons' weight of honey and wax are yearly consumed; England pays more than $50,000 l$. for foreign honey and wax, in addition to her own valuable produce. A great variety of scents, which from their agreeable odours are much used in perfumery, are manufactured from insects. The Spanish $\mathrm{Fly}$ is an indispensable article in the treatment of certain forms of disease; and that invaluable agent, Chloroform, was first made from formic acid; an acid discovered in the Formic ant, and from which it has derived its name. Then there are Gall-nuts, produced by a small fly, for which a substitute could not be found in dyeing and ink-making.

"Much more extensive and important than any of the foregoing, but, as less palpable, even more disregarded, are the general uses of insect existence. Disease, engendered of corruption in substances animal and regetable, would defy all the precautions of man, unless these were aided by scavenger-insects, those myriads of flies and carrion beetles, whose perpetual labours, even in our tempered climate-but infinitely more so in warmer regions-are essentially important to cleanliness and health.

"A use of this nature, and one performed perhaps to an extent we little think of is the purification of standing 
waters by the innumerable insects which usually inhabit them. We have witnessed ample proof of the efficacy in this respect of Gnat larva, when keeping them to observo their transformations. Water swarming with these 'lives of buoyancy' has been perfectly sweet at the end of ten days; while that from the same pond, containing only vegetable matter, has become speedily offensive.

"We have already pointed out the utility of insects in affording ever-new subjects of interesting inquiry. And let those who will look scorn upon our pursuit; but few are more adapted to improve the mind. In its minute details, it is well calculated to give habits of observation and of accurate perception; while, as a whole, the study of this department of nature, so intimately linked with others above and below it has no common tendency to lift our thoughts to the great Creative Source of Being, to Him who has not designed the minutest part of the minutest object without reference to some use connected with the whole."

"The shspely limb anl jubricated joint

Within the small dimensions of a joint,

Muscle and nerve miraculously spun,

Hrs mighty work, who speaks, and it is done :

The invisible in things scarce seen revealed,

To whom an atom is an ample field."

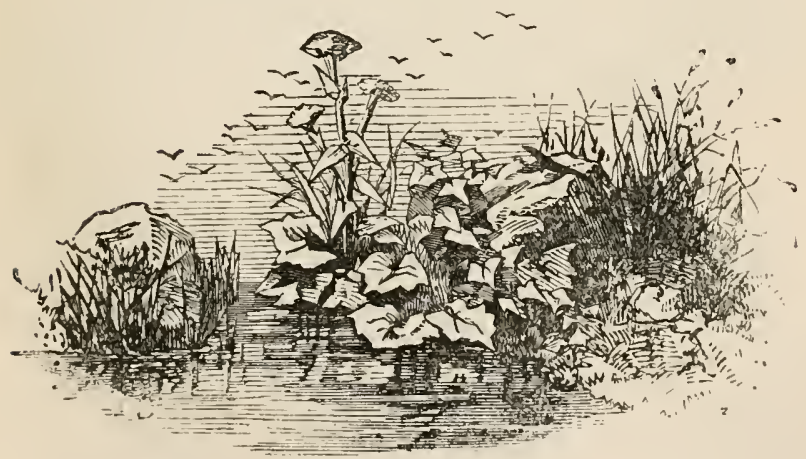




\title{
CHAPTER V.
}

\author{
VERTEBRATA.
}

\begin{abstract}
PEYSIOLOGY-I, ISTOLOGY-BOUNDARY BETWEEN THF TWO KINGDONB-CELL DEVELOPIEN L - GROWTH OF TISSUES - SKIN, CARTILAGE, TEETH, BONE, ETC.
\end{abstract}

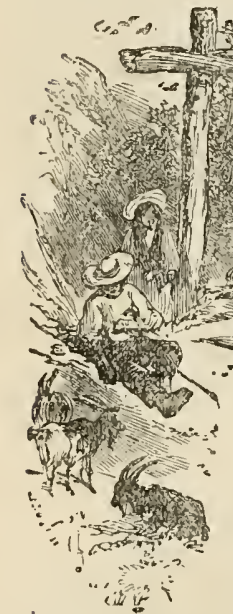

HE most complicated state in which matter exists, is where, under the influence of life, it forms bodies with a curious internal structure of tubes and cavities, in which fluids are moving and producing incessant internal changes. These are called organised bodies, beeanse of the $\because$ various organs which they contain, and they form two remarkable classes; those of the lowest class are for the most part fixed to the soil, and are recognised as vegetalles, - the structure of these we have already considered; those of the higher order are endowed with power of locomotion, and are called animals. Some of the peeuliarities and minute strusture of the invertebrate animals have aiready been made the subject of investigation, and we now propose to extend our observations to the vertebrate.

The study of the Science of Life, or the building up of the living structure, is termed Physiology, or Biology; ${ }^{1}$ and that part of it more particularly relating to the minute structure of the organs of animals has been termed II istulogy. ${ }^{2}$

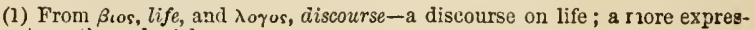
sive term than physiology.

(2) From ioros, a tissue, or web, and toros, a discourse. 

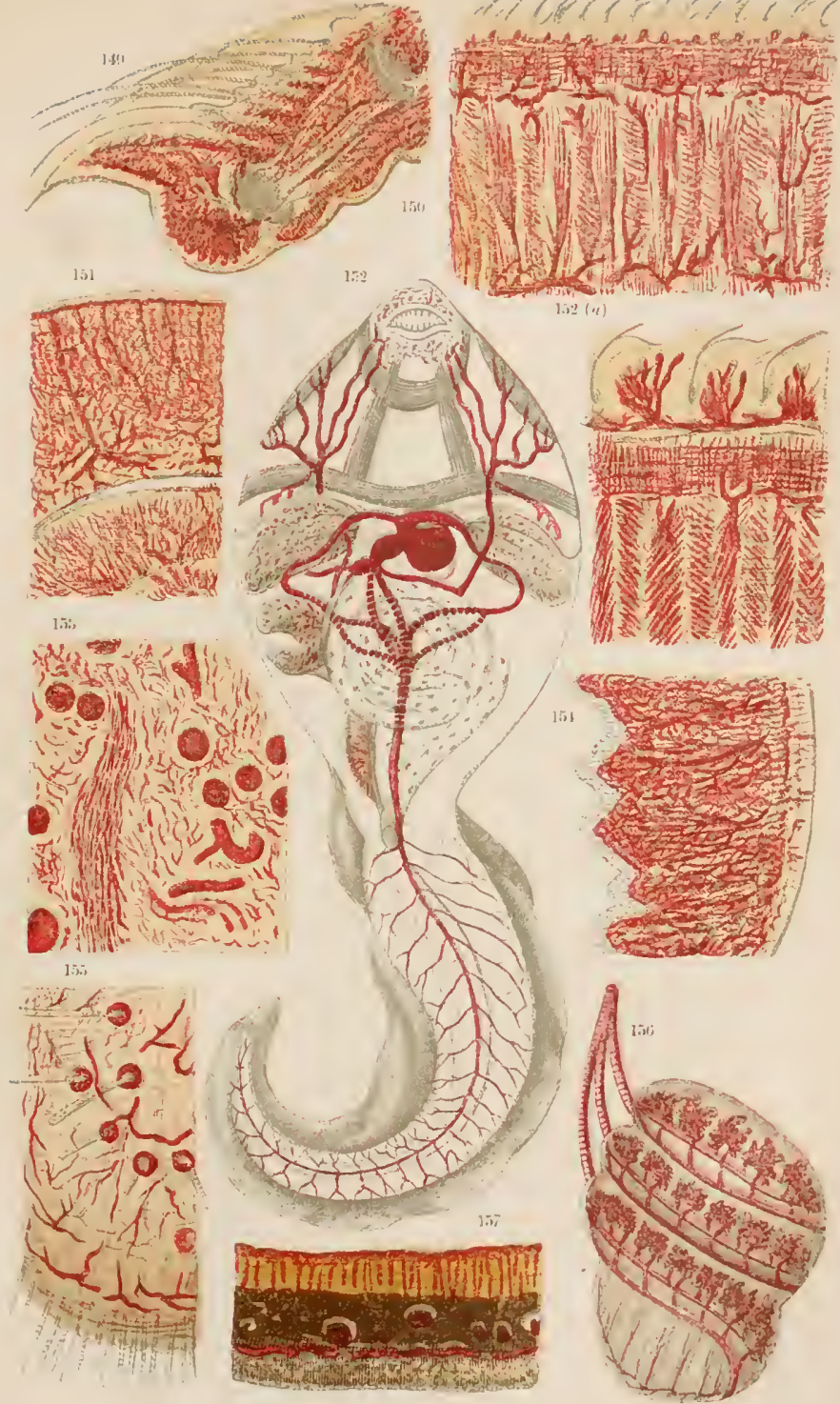

PAATE VIH. 

Physiology has for its object the scientific co-ordination of the phenomena and laws of life; yet, writes Mr. Lewes, "the attempts to define what we are to understand by Life, have hitherto proved almost if not quite valueless." In our previous investigations, we must have seen the value and advantage of "studying Life in its simpler forms, if Life is to be understood in its more complex; and no sooner do we comprehend the fact that the lower animals present to us the nore important phenomena of Life under simpler forms and conditions, than we at once recognise the study as indispensable."

It was Ehrenberg who first asserted that there was an absolute boundary between animals and plants; finding even, as he fancied he did, in the smallest of the former,the Infusoria,-which had previously been regarded as mere unorganised masses of mueus, the same systems of organs as those by which the most highly-developed animal is characterised, that is to say, distinct nutritive, motile, vascular, sexual, and sensitive systems. Siebold called the existence of these organs in question, regarding the organisation of the Infusoria as a homogeneous parenclyyma, in which he recognised only a nucleus, and in one division a mouth and œesophagus. Nevertheless he asserted that plants and animals were essentially distinct, and that there was no transition from one to the other, the nature of the plant being always immotile and rigid, whilst the animal possessed the faculty of contracting and expanding its body. This contractility is, in his opinion, alone to be taken as the characteristic feature. It is not, however, the animal organisation itself which is contractile, but only a single tissue in it; all the rest, skin, bones, and connectire tissue, are as rigid or passive as the vegetable membrane, or, at most, only elastic ; in the higher animals the muscles only are contractile, and in those of the lowest classes, viz. the Infusoria, the entire body.

Whence Ecker assumed the existence of a special contractile substance, which sometimes occurs in a formed state, as a contractile cell or as muscular substance, sometimes amorphous, as in the bodies of the Infusoria, Rhizopoda, and Hydrozoa. Kolliker confirmed this view, and carried it out, particularly in the case of the Infusoma, 
which he at one time declared to be unicellular animals with a contractile cell-membrane and contents. The contractile substance is characterised by the following attributes: it is homogeneous, or finely granular, transparent, of the consistence of albumen, gelatiniform, soft, more refractive than water, but less so than oil ; insoluble in water, but gradually decomposed; destroyed by caustic potash; coagulated and contracted by carbonate of potash, as well as by alcohol and nitric acid; having the power of furming aqueous cavities, which originate either by the separation of the water contained in it, or by its reception from without; owing to which the remainder becomes denser and more granular, and lastly, it represents the appearance, in water, of contractile drops, which move like an Amœba. All these properties had already been observed by Dujardin, in a substance of which the Infusoria and Rhizopoda are principally composed, and which he termed "sareode;" the aqueous spaces or hollows he named "vacuoles," regarding them as the most characteristic features of the substance; these spaces had been erroneously regarded by Ehrenberg as stomachs. All these properties, however, are possessed by a substance in the plant-cell, which must be regarded as the prime seat of almost all vital activity, but especially of all the motile phenomena in its interior-the protoplasm. Not only do its optical, chemical, and physical relations coincide with those of the "sarcode," or contractile substance, but it also possesses the faculty of forming "vacuoles" at all times, and even externally to the cell; a property, it is true, which has for the most part been hitherto overlooked or misinterpreted. These clear, aqueous spaces, the sotermed vesicular contents, are present in all young cells, and play a considerable part in cell-division, and the sap-currents; they are in all respects analogous to the vacuoles of the sarcode. ${ }^{1}$

(1) Mr. Huxley has satisfied himself that in all the animal tissues the socalled nucleus (endoplast) is the homologue of the primordial utricle, with nucleus and contents (endoplast) of the plant, the other histological elements being invariably modifications of the periplastic substance. Upon this view we find that all the discrepancies which had appeared to exist between the animal and vegetable structure disappear : and it becomes easy to trace the absolule identity of plan in the two, the differences between them being produced merely by the nature and form of the deposits in, or modifications of, the periplastic 
In organised beings, the way in which nature works out her most secret processes is by far too minute for observation by unassisted vision; even with the aid of the improved microscope, comparatively a very small portion has, up to this time, been revealed to us. To point out in detail the discoveries made through the employment of this instrument, as regards physiology, would be to give a history of modern biological science; for there is no department in this study which is not more or less grounded upon the facts and teachings of the microscope.

To the casual observer, the brain and nerves appear to be composed of fibres. The microscope, however, reveals to us, as was first pointed out by Ehrenberg, that these supposed fibres do not exist, or rather, that they all consist of numerous tubes, the walls of which are distinct, and contain a fluid which may be seen to flow from their broken extremities on pressure. In looking at a muscle, it appears to be made up of fine longitudinal fibres only. The microscope tells us that each of these supposed fine fibres is composed of numerous smaller ones, and that these are crossed by lines which have received the name of transverse striæ; that muscular contraction, the cause of motion in animals, is produced by the relaxation or approximation of these transverse striæ.

The microscope has shown us that a distinct network of vessels lies between the arteries and veins, partaking of the properties of neither, and possessed of others peculiar to themselves. These have been denominated intermediary vessels by Berres, and, serving to connect the arterial with the venous system, are commonly known as capillaries.

On regarding with the naked eye the different glands in which the secretions are formed, how complex they appear, how various in cunformation! The microscope teaches us that they are all formed on one type ; that the

substance. In both plants and animals there is but one histological elementthe endoplast-which does nothing but grow and vegetatively repeat itself: the other element-the periplastic substance-being the subject of all the cheuical and morphological metamorphoses in conseqnence of which specific tissues arise. The differences between the two kingdoms are mainly, first, That in the plant the endoplast grows, and the primordial utricle attains a large comparative size, while in the animal the endoplast remains small, the principal bulk of its tissues being formed by the preriplastic substance; and secondly, In the nature of the chemical changes which take place in the peri plastic substance in each case. 
ultimate element of every gland is a simple sacculated membrane, to which the blood-vessels have access; and that all glands are formed from a greater or less number, or different arrangement only of the primary structure.

Our notions respecting the skin were vague until the microscope discovered its real anatomy, and showed $n$ the existence and relations of the papille, of the sudorific organs and their ducts, the inhalent muscular apparatus, and so on. All our knowledge of epidermic structures, such as hair, horn, feather, \&c., the real structure of cartilage, bone, tooth, tendon, cellular tissue, and, in a word, of all the solid textures, has been revealed to us by the same agency; so that it may be truly said, that all our real knowledge of structural anatomy, and all onr acquaintance with the true composition of every organ in the body, have been arrived at by means of the microscope, and could never have been known without it.

In addition to this, and what is of greater importance, after having studied the healthy structure of the body, most beneficial aid is afforded in the investigation of changes produced by disease. We may cite one notable example. Dr. Andrew Clarke, after having carefully studied the appearances of sputa from patients under his care, says, "that the microscopical inspection of expectoration affords, at a very early period of consumption, definite information, not otherwise attainable, regarding the nature of the malady; and at all times must furnish valuable aid in forming a prognosis regarding the cause of the complaint." The expectoration generally shows pus, cells, lung tissue, blood corpuscles, and granular material, mixed with, at times, a small amount of fat corpuscles.

The space allotted to this division of our subject enables us to give only a short and imperfect sketch of a few of the fundamental tissues of the animal body. First, enumerating merely the elementary substances recognised by chemistry as entering into the formative processes, we shall proceed to inquire into that most interesting aud wonderful starting-point of life, the cell; almitted to be, and inleed demonstrable as, the common centre alike of animal and verretable organisms. 
THE HUMAN BJDY, ITS PHYSIOLOGICAL IOMPOSITION AND CHARACTER.

The elementary substances found in the human body are oxygen, hydrogen, carbon, nitrogen, phospkorus, sulphur, rhlorine, fluorine, iron, manganese, titanium, and calcium. silenium is found in the hair, and fluorine in combination
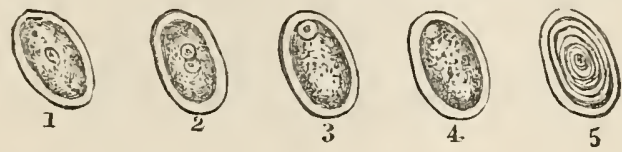

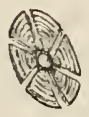

6

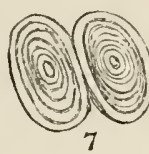

7
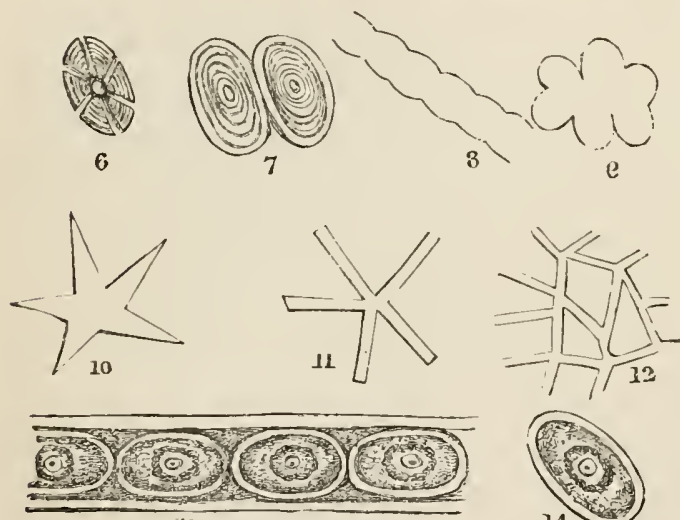

1.3

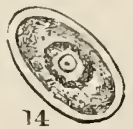

Fig. 300.-Diagram showing various forms of developmenl in Animal Cells.

1, Shows a newly formed cell. 2, Subdivision of the nucleus. 3, The nucleus changes its situation, and at 4, subdivides and disappears. 5, The walls of the cell increase in thickness. 6, the cell becomes branched, or slellate. 7 , Two cells are seen to coalesce. 8, They have-coaleseed and run into each other. 9, Again they take another form and become multiloeular. 10, 11, 12, Cells sprouting out to form membrane and vessels. 14, Development of complicated cells, which, at 13 , have coalesced to form tissue.

with lime forms the enamel of the teeth. Iron is ths chief colouring-matter of the blood, the black pigment of the choroid of the eye, and the skin of the negro.

\section{U U 2}


Cells. - All animal and vegetable structures, the microscope has revealed to us, are developed from cells or their nuclei ; and the materials for building up animal structures are furnished from the yolk and the blood.

The animal nucleated cell, fig. 300 , is more or less of a globular form; within the delicate cell-wall a granular matter is inclosed suspended in a fluid; the wall being somewhat darker than the rest. There are usually one or two spherical masses termed nuclei ; these enclose central dots, termed the nucleoli. The size of a cell may be 1-300th part of an inch in dialueter, some are larger, some smaller; the nucleus may be 1,3000 th of an inch in diameter; the nucleolus 1-10,000th of an inch in diameter, more or less.

Of the Cell.-Dr. Beale's views of the cell are so original, and his theory of its development so carefully studied and worked out, that we shall attempt, in as few words as possible, to place them before our readers. The cell has always been considered, and is still so, by many good authorities, to consist, as just stated, of certain definite parts, viz. cell-wall, cell-contents, and nucleus ; to each of which various different functions have been assigned. Many affirm that the vital force is resident in the nucleus alone, while others attribute to the cell-wall, or even to the inter-cellular substance, the power of producing chemical and other changes. Dr. Beale considers this view to be entirely erroneous, and states that every cell or "anatomical elementary part" consists of matter in two different states or stages of existence-matter which lives (germinal, matter), and matter which is formed (formed material), and which has ceased to manifest purely vital phenomena; all living entities, from the smallest living particle to the most complex cell, consist of matter in these two states, the relative proportions of which differ at different periods of the life of the cell, and vary with the different conditions under which it may be placed. If a tissue be examined before development has proceeded to any great extent, masses of germinal matter will be found almost cartinuous with each other, without any appearance of the cells from which all tissues are said to be originally formed. 
As growth proceeds, these masses become seprarated, and the small processes or tubes which connected them are drawn out, as it were, and become thimer and thinner; so that, for instance, in the formation of stellate tissue, so far from the rays having been shot out by unequal growth from special points of the original cell-wall, they have been continuous from the very first.

Of the Structure and Formation of the simple Cell Mncedines.-The mucedines, commonly called mildews, are among the simplest living things known, and are therefore well adapted for observation. If the membranous investment of a fully developed spore taken from one of these fungi be ruptured, innumerable minute particles, some not more than 1-100,000th of an inch in diameter, are set free: these constitute the living growing matter, in contradistinction to the envelope or onter part of the cells. "Germinal matter" may always be readily distinguished from formed material by its capability of becoming dyed by an alkaline solution of carmine, while the latter remains perfectly colourless. Directly such a minute living particle comes into contact with air or water, a thin layer upon its outer part becomes changed into a soft, passive, transparent homogeneous substance (cell-wall), exhibiting a membranous character, which protects the matter within. Nutrient matter passes through this into the interior, and there becomes changed into living matter; so that growth takes place, not by additions to the outside, but by the introaluction of new matter into the interior. If pabulum be abundant, and the external conditions (temperature, moisture, \&c.) favourable, it readily passes through the thin external membrane, and the living matter rapidly increases. But if the external conditions be unfavourable, less pabulum transudes, and the living matter within dies, layer after layer, until the envelope becomes very much thickened, with a proportionate decrease in the size of the living matter. If all this living matter die, and only formed material remain, no increase can take place; but if the smallest particle remain alive, any amount of living matter, and afterwards of tissue or formed material, may be produced. The position of the germinal matter in the cell varies with the direction in which the pabulum reaches 
it; thus in the columnar epithelial cells covering the villi of the intestines, in which the pabulum flows from the free surface towards the attached extremity, the germinal mass is found near the centre or near the free edge of the cells; but in the mucus-forming cells from the mouth and fauces, in which the pabulum flows in the opposite direction, the germinal mass is found to be placed quite at the attached end of the cells, where it has consequently easier access to the pabulum, upon which its growth and secretive jower depends.

Cell-wall and Cell-contents. - In the mucus cells above mentioned, and in many other cells, two kinds of formed material are produced from the original germinal matter; these are spoken of as the cell-wall, and the peculiar matter found inclosed in it, the cell-contents. For instance, in the starch-containing cell of the potato, the cell-wall is formed around and invests the germinal matter, while the starch is deposited as small insoluble particles in the very substance of the germinal matter. So that by the death of particles on the surface of the cell-wall the cellulose cell-wall is produced, while by the death of some of the particles further inwards, and therefore under different conditions, starch is formed. This outer part of the germinal matter, which eventually lies between the starch grains on the inside and the cell wall on the outside, is known as the "primordial utricle" of the regetable cell. Fat-cells or adipose vesicles are formed in precisely the same way; fat may, moreover, be deposited amongst the germinal matter of other cells, such as the cartilage or nerve cell.

Of the so-called Intercellular Substance.-In cartilage, tendon, and some other tissues there is no line of sepa. ration between the portions of formed material which belong to each respective mass of germinal matter; and hence it has been supposed that these tissues were developed in a different way to the epithelial structures. A "cell," or elementary part of adult cartilage or tendon, merely differs from the epithelial cells, spoken of above, in not having a distinct margin around its own particular formed material, and if a line were drawn midway between the various germinal unasses, it would roughly. mark out 
the point to which the former? material corresponding to each extended; and each vell would then be exactly analogous to the mucous-forming cells. In all of these cells, of mucous membrane, tendon, cartilage, muscle, \&c., there is no abrupt demarcation between the germinal matter and the formed material, but the one passes gradually into the other. All living cells consist of matter in these two different states; the one being an active condition, vital; the other merely passive, in which no vital actions are exhibited: upon matter in this first state, all growth, multiplication, conversion, all life depends; while in the second condition, matter may exhibit many very peculiar properties, but it does not grow or multiply, or convert or form; in short, it does not live, though it may increase by new matter being superadded to it.

Of the Nucleus and Nucleolus.-A mass of germinal matter, besiles increasing in quantity, may divide into several, and thus cell-multiplication may occur; and in all cases it is to be observed that this multiplication is not due to a "growing-in," or constriction of the cell-wall or formed material, but entirely to changes occurring in the germinal matter. In many cases a smaller spherical mass may be observed in the centre of the germinal mass, which often divides before the parent mass itself does; but it is by no means a necessary part of the process, for division as often takes place where no such bodies are to be seen; and it frequently happens that these small bodies may make their appearance only after the division of the original mass. And again, within these, other still smaller ones are sometimes produced. The former are termed nuclei, the latter nucleoli. These are to be regarded as but new living centres appearing in centres already preexisting, and may perhaps mark the commencement of a set of changes differing in some minor particulars from. the first that have occurred. But although both nuclei and nucleoli are germinal or living matter, they are not undergoing conversion into formed material. Nuclei do not always exhibit their vital powers, but under certain circumstances they may do so, and then they exhibit the characters of ordinary germinal matter; they absorb pabulum and-increase in size, and the original germinal matter 
becomes changed into formed material, while fresh nuclei and nucleoli are developed. And so far from nuclei being formed first, and the other elements of the cell depositerl around them, they always make their appearance in the substance of pre-existing matter, and have neither a different constitution to ordinary germinal matter, nor perform any special function.

Of the Increase of Cells. - Several distinct modes of cellmultiplication have been described, but in all cases the germinal matter divides, and is the only material actively concerned in the process; which may, however, take place in different ways.

1. The parent mass may simply divide into two equal parts, apparently in obedience to a tendency of the portions to move away from each other as soon as the original nrass has reached a certain size.

2. The parent mass may divide in three, four, or more equal portions.

3. From every part of the parent mass protrusions may occur, each of which, when detached, absorbs nutrient matter, and soon attains the same size as its parent. During these processes of increase and multiplication, the formed material is perfectly passive, and when a septum or partition exists, it does not result from a "growing-in" of this dead structure, but is produced by the conversion of part of the germinal matter into a thin layer of formed material.

Of the Changes of the Cell in Disease.-If the conditions under which cells ordinarily live be modified beyond a certain extent, a morbill change may result. For instance, if cells, which normally grow slowly, be supplied with an excess of nutrient pabulum, they grow-that is, convert certain of the constituents of the pabulum that come into contact with them into matter like themselves-at an increased rate. In this way the inflammatory produrt pus resuilts. "The abnormal pus-corpuscle may be produced from the germinal or living matter of a normal epithelial cell, the germinal matter of which has been supplied with pabulum much more freely than in the normal state." In cells in which the access of nutrient pabulum is more restricted than in the abnormal state, as in normal cells 
passing from the embryonic to the adult state, the outer part of the germinal natter undergoes conversion into formed material, which increases as the supply of pabulum becomes reduced.

Dr. Beale, in short, considers that "all formed matter results from changes in the germinal matter, and that the action of the cell really consists in a change from the living to the lifeless state of the matter of which it is composed; and that the products formed by the cell do rot depend upon any metabolic action exerted by the cell-wall or nucleus upon the pabulum, nor are they simply separated from, or deposited by, the blood." And he looks upon the "living cell" as a minute body, consisting partly of living matter influenced by vital force, and partly of lifeless matter resulting from the death of the first, in which chemical and physical changes occur, which may be modified by the influence of surrounding substances and external forces.

In pursuing the subject of cell development, we shall proceed by the aid of our old lights in this intricate path of physiological science. And it is only right that we should add, that the views we have endeavoured to place fairly before our readers have not been unhesitatingly accepted, but, on the contrary, those of the German school are greatly preferred by many physiologists.

Change of Cells into Tissues. - This may take place by a joining together or coalescence of cells in a rudimentary state. Cells may meet, and at the point of contact coalesce and run into each other, thus forming a tube; indeed, in this manner minute tubular structures are formed. Another mode is : cells aggregate into a mass, and at the point of contact run into each other, thus producing a multilocular cavity; No. 9, fig. 300. Glandular structures are formed in this way. Membrane is formed of a deposit from the cytoblastema; before the cell-membrane is formed, the substance from the cytoblastema coalesces with those particles close at hand, thus forming a delicate film-like membrane. This membrane Professor 'T. Wharton Jones calls endosmotic, retentive membrane. TVe liave the cells coalescing to form a filament or fibre. The nucleus may disappear, or form another structure; and where. 
regeneration of tissue is proceeding, there is found a larger number of granules.

According to some histologists, cells may be formed in cytoblastema independent of any pre-existing cells; this is cited as an instance of that mysterious agency designated spontaneous generation. There are cells which, so far as we know, after full development, undergo no further metamorphosis; such as those of the epithelium, the blood corpuscles, \&c.

As an instance where previously-existing cells exert an influence on those about to be formed, we may adduce a fractured bone, between the ends of which osseous matter is deposited. We infer from this, that the substance of the bone determines, as it were, the formation of other cells, first into cartilage, and then into bone. Generally, however, where a part has to be repaired, it does not seem to determine the generation of a texture similar to itselffor example, muscle and skin. We have an exception to the last observation in the case of nerves, which if cut across, a substance is formed between the ends which transmits the nervous influence, but the ends must not be separated to any great distance, or this will not occur: 'The same remark applies to bone. There may be a single layer of cells so arranged side by side, and presenting a columnar or basaltic form; this arrangement is seen in the cells of the intestinal tract, fig. $303 \alpha$. Another change of cell is this: they shoot out processes from certain parts of them, as seen in fig. 300, Nos. 10, 11, this kind of formation occurs on the imner surface of the sclerotic coat of the eye. The cylindrical form of cell is found with delicate processes shoting out from the broad end; these are called ciliated, fig. $30: 3 d$, and the cilia having a vibratile motion urge on the secretions of the part in a particular direction.

In some cases the walls of the cell increase in thickness (fig. 300, No. 5). Unier the microscope. some cells appear to be composed of concentric laminue. In plants this is the common mode of increase in the thicliness of the cell, but the deposit does not take place entirely around, but only here and there, so that vacint spaces are left which form canals, and mus become branched: 
these canals are named pore-canals (fig. 300, No. 6). They do not perforate the outer layers; consequently the blind ends seen through the outer membrane, and which were supposed to be apertures, are nothing of the kind. Henle believes he has found eanals in cells in animals similar to those in vegetables-in the cartilage of the epiglottis, for instance. In other instances, cells may be aggregated, like a bunch of raisins, and the parts in contact with each other disappear, so constituting a multilocular cavity: examples of this are seen in the racemose glands (fig. 300, No. 9). Schwann conjectures another mode of coalescence. From cells formed as usual, processes spront out; but this change takes place at the expense of the cell-membrane itself, and when it has gone on to some extent, we have the appearance of a net-work formed (fig. 300, No. 11, and 12). Capillary vessels are formed in this way. Cells, we thus perceive, coalesce to form tissues, when they have not attained their full growth as such; or when they have been fully formed they become flattened, and assume the solid form. Deposits of matter may take place from the cytoblastema with similar adjoining substance, constituting a delicate membrane, with here and there nuclei, as in the capsule of the lens, the membrane of the aqueous humour of the eye, or sheath of the prinitive fasciculus of musele; or the cells may coalesce in the linear series, to form fibre.

Development of Complicated Cells.-IIere the nucleated cell is surrounded by a deposit, and that again surrounded so as to constitute a membrane; so that the nucleated cell may be looked upon as the nucleus to the cell so formed (fig. 300, Nos. 13 and 14). Sometimes the nucleus undergoes important changes in the development of tissues, as well as the cell itself. In some cases, where the cells have joined in the linear series, the nneleus becomes oval, elongated, so that the nucleus of one cell tends to meet the nucleus of another cell; they subsequently coalesce, and thus fibre is said to be formed.

Action of Cells.-The subsequent changes of the cell depend in a great degree on endosmosis, or diffusion. The nature of the membrane is a necessary condition, for it 
determines the way in which the stream should pass; and we find in general that the current is from the rarer to the denser fluid. If we immerse a porous tube half filled with a strong solution of common salt in a jar of water, we notice that the level of the fluid inside the tube rapidly rises above the outside, while the water becomes slightly salt to the taste. It is not a constant circumstance that the stream is from the rarer to the denser fluid; with alcohol and water, for instance, the stream is from the latter to the former. Mineral substances, even pipeclay and chalk, permit of endosmosis in a low degree. In glands, the cells, being filled with their peculiar fluid, are conveyed to the wall of the intercellular passage, and through this the secretion arrives at the surface of the body.

The Epithclium. - If we cut very thin slices from the superficial portions of the skin, we can raise from it a delicate membrane; or, what is better, by using chemical or mechanical irritation, we obtain what is ordinarily called a blister ; to it we give the name of epidermis. The microscope has shown this to be a tissue of high and remarkable organisation ; being, in point of fact, an aggregation of cells, differing, in different

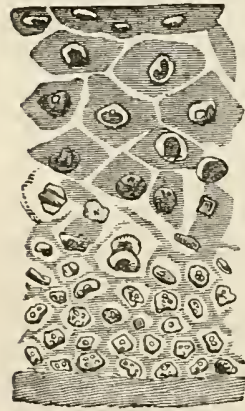

Hig. $301 .-4$ section of the Epidcrmis. situations, in regard to form, colour, and eomposition. These laminated elementary cells, found on the surfaces, have generally nuclei. The form of the nucleus is rounded or oval, and is the 1-3000th to 1-5000th of an inch in diameter. Each nucleus has two or three nucleoli, with outlines more or less irregular. The epithelium cells may be divided into three kinds: the 1st is termed the tesselated or pavement; $2 \mathrm{~d}$, the columnar or basaltic; $3 \mathrm{~d}$, the ciliated or vibratile epithelium. Some make a 4th, combining the tesselated and the columnar: this niay be considered as transition epithelium, and is found only in certain mucous passages. These various cells are represented in fig. $303, a, b, c$, and $d$. 
Tesselated epithelium is the simplest form, and, as its name implies, resembles flags of parement, overlapping each other at their edges. They assume, more or less, the polygowal form, and their size varies in the different membranes. The cells of the pericardium, or covering membrane of the heart, are much smaller 1 , than those of the covering membrane of the lungs, \&c. On some surfaces we have many layers-in the skin, for instance; if a vertical section of such be made, and viewed nuder the microscope, it will be seen to be conuposed of number-ess layers, shown in fig. 304. The skin taken from the sole of the foot, in consequence of the continued pressure there
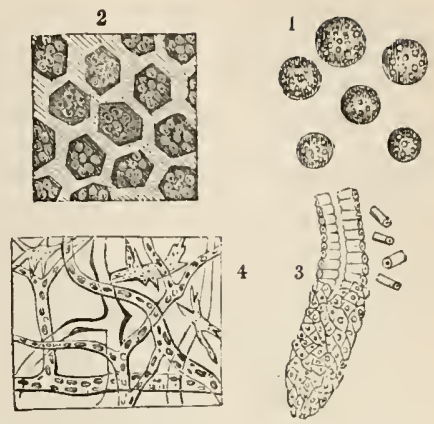

Fig. 302 .

, Simple isolatell cells containing reproductive granules. 2, Ineous membrane of stomach, showing nucleated cells. 3, One of the tubular follicles from a pig's stomach. 4, Seetion of a lyniphatie, mignified 50 diameters.
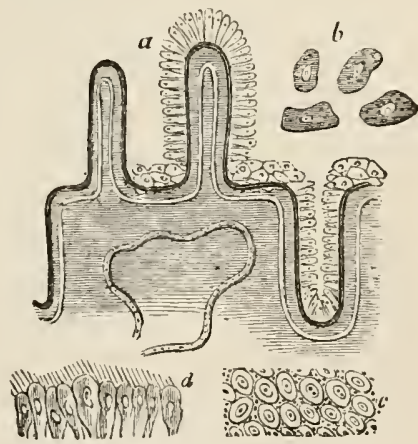

Fig. 303. (1)

(1) $a$ is a diagram of a portion of the involuted muenus membrane, showing the continuation of its elements in the follicles and villi, with a nerve entering its submueous tissue. The upper surface of one villus is seen covered with cylindrieal epithelium; the other is denuded, and with the dark line of basement membrane only running round it; $b$, pavement cpithelium sealos, separated and magnified 200 diameters ; in the centre of each is a nucleus, with a sualler spot in its interior, called the nuclenti. c, pavernent epithelium scales. from the unueous membrane of the bronchial or air tubes of the lung; $d$ represents another form of epithelium, termed the vibratile or ciliated: the nuclei are visible, with cilia at their upper or free surfaces, magnified 250 diameters. 
experiesced, presents a distinctly stratified appearance. These layers of cells are held together by intercellular substance, which exists in quantities; if the epithelium be taken from these membranes it is more easily seen, because the cells are not so closely aggregated together as in the skin; therefore a piece of epithelium from the mouth. is recommended for display under the microscope, and by the addition of a drop of the solution of iodine the cells are much better seen. The cells from serous and mucous membranes are acted upon by acetic acid, and dissolved if the acid be of considerable strength : but if the acid be weaker, the cells swell up. Cells are not affected by alcohol, ether, ammonia, or its salts; but they are dissolved by caustic potash, which also dissolves the intercellular substance.

Columnar or Cylindrical Epithelium, Fig. 303, a.-The nucleus is generally better seen than in the former kind of cells, although formed from them. If we examine a portion sideways, it resembles those at $d$, the upper part being broader, and the nuclens being midway between the two extremities. When the cells of the cylindrical epithelium are closely aggregater together, they become compressed into the prismatic form; when they are less so, the rounded shape prevails. Consequently, when we take a bird's-eye view of them, from above or below, they appear like the pavement epithelium, at $c$, and thus error might creep in ; but we must become fully satisfied by examining them sirleways, and with various reagents. Their chemical composition is the same, and the cells dissolve in strong acetic a:il. As examples of the situations in which this form of epithelium is found, we may instance the intestinal tract along the ducts of the glands, as the liver, \&c.

In no situations do we find these two kinds of epithelium terminating abuptly the one in the other; but there is a gradual change of the one kind into that of the adjoining; for example, where the tesselated epithelium is gradually supplanted by the cylindrical, as it passes from the cesophagus to line the interior of the stomach; it is then termed transition epithelium.

Caliated Epithelium, Fig. 303, d.- The cells do not differ materially from those of the cylindrical ; the great distinction between lie two is, that in the former there are no 
cilia attached to the broad end. Examples of the situations in which these are found are, investing membrane of the respiratory passages, upper part of the pharynx, larynx, bronchi, and the lateral ventricles of the brain, \&c.

Epithelium is found to grow from the surface of the cutis ontwards-in most places it is constantly growing outwards, and as continually being thrown off from the surface: it must at the same time be remembered, that though the epithelium is in close contact with the cutis, or true skin, it is not a deposit from it, but derives only its materials of formation and nourishment from it.

The epidermis is destitute of sensibility, yet it invests very sensitive parts : it is not vascular, but invests very vascular parts. Its exfoliation takes place regularly, as may be exampled in reptiles and the Batrachia, who throw off their skin : the moulting of birds is analogous. In the early periods of life in the human subject, exfoliation takes place from the surface of the skin; from the mouth the morsel of food is always mixed with detached cells. In the process of digestion the same thing occurs-in fact, it is only when the epithelium cells are thrown off that the gastric juice is secreted by the tubes of the stomach.

Cilia.-The most remarkable circumstance in connexion with cells is the movement of their cilia. There are three ways in which the cilia ordinarily move-the rotatory, the undulatory, and the wavy, like a field of wheat set in motion by a steady breeze. No satisfactory explanation has been given of the cause of this vibratile motion. The current produced by them is from within outwards, in most places; in the respiratory passages, on the contrary, it is from without inwards. In the Frog's mouth, it takes the same course. The ciliary motion may be seen in the kidney of the Frog or Newt; the cilia in the latter continue in active motion for some minutes after the animal is dead. Make a very thin section of the kidney with a sharp knife, and take care to disturb the structure as little as possible; then moisten it with a little of the serum of the animal, place it in a glass cell, ancl cover with thin glass and a inagnifying power of 250 diameters.

Pigment.-Pigment gramules are found in greater or less yuantities in the skin and bolies of white and dar's 
races. In the eye there is pigment, and it affords a good example of nucleated cells, in which are contained the pirment particles, fig. 304. These are placed there for an

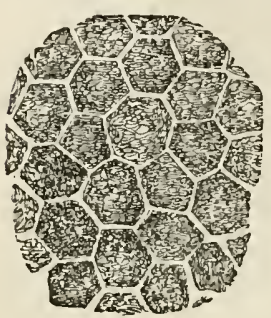

Fig. 304.-Pigment cells from the eye.

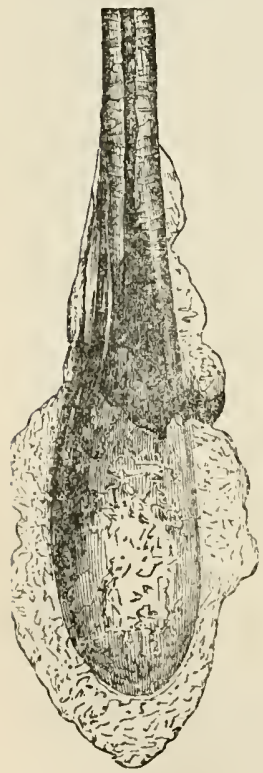

Fin. seen near its bulb. optical purpose, that of absorbing the rays of light. In the peculial colouration found in the eyes of some animals, called Tapetum lucidum, the colour is not owing to the pigment particles, but to the interference of the light: it is reflected from it, as in mother-of-pearl, coloured feathers, scales of fishes, \&c. The colour of the skin is owing to the granular contents of the pigment cells; these are like ordinary elementary granules, with the addition of colour; and this latter may be removed by the action of chlorine.

The Nails are appendages to the epidermis, and present a mould of the cutis beneath; from the cutis the materials are furnished for the formation and growth of the nail. Like the epidermis, the nail is stratified, the markings are parallel to the surface, and the appearance is produced by the coalescence of the cells and their lying over each other. See Plate VII., No.149, toe of mouse. The stratified arrangement when a section is examined by polarised light, presents the appearance seen in the processes of the cat's tongue, Plate VIII. No. 174.

Hairs.-The form and structure of hairs differ much; some are cylindrical, others flattened. A hair is divided into a body or shaft, and a root which is in the skin (fig. 305.) The shaft is again divided into two parts: the external is termed the cortical portion, and the internal the medul- 
lary portion; the latter does not usually exist in the whole laygth of the shaft. The cortical part consists of fibres,
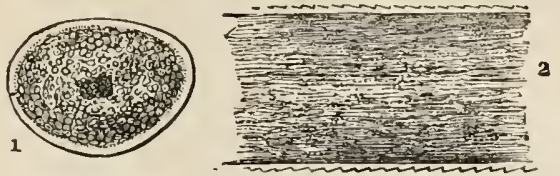

Fig. 306.

1, Transverse section of human hair, showing the cylinder of medullary aubstance. 2 Longitudinal section, showing the fibrous character of the samo pigment or colouring matter, and serratcd edges.

arringed parallel to each other: besides these there are, on the exterior, minute epithelial scales, which are arranged like the tiles on a house, producing the appearance of tronsverse markings. The fibres gradually expand out,

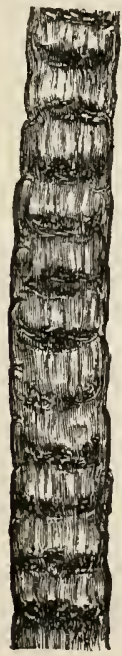

3

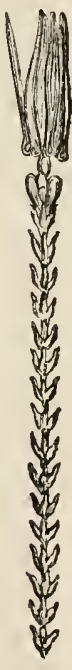

8

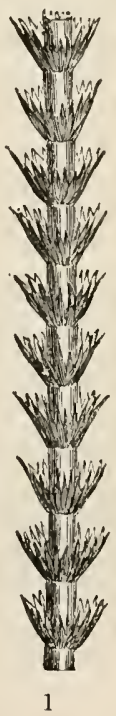

Fig. 307 .

Hair from the Indian Bat, magnified 500 diameters. 2, Hair supposed in belong to (Dermestes?) Anthrenus, magnitied 250 diameters. 3, Hair from the Mulase, sagnitied 250 diameters. 
forming a wall to the bulb enclosed in its capsule. The development of a hair commences at the bottom of the follicle, and by the aggregation of successive cytoblasts, or new cells, are gradually protruded from the follicle, both by the elongation of its constituent cells, and by the addition of new layers of these to its base; the apes and shaft of hair being formed before the bulb, just as the crown of a tooth is before its fang. The cytoblasts are round and loose at the base of the hair, but are more compressed and elongated in the shaft; and by this rectilinear arrangement the hair assumes a fibrous appearance. Of sixteen species of the Bat tribe, the hairs of which were examined by the late Professor Quekett, all were analogous in structure; and the diversity of surfaces which these hairs present are in reality owing to the development of scales on their exterior. By submitting hairs to a scraping process, these minute scale-like bodies, tolerably constant as regards their size and figure, can be procured; so that Bats' hair may be said to consist of a shaft invested with scales, which are developed to a greater or less degree, and varying in their mode of arrangement in the different species of the animal; that part of the hair nearest the bulb is nearly free from scales, but as we proceed toward the apex the scaly character becomes evident. Many of the scales are not unlike those procured from the wings of butterflies, but, being very much smaller, exhibit no trace of striæ on their surfaces; those taken from darkcoloured hairs have colouring-matter deposited on them in small patches. In some cases they appear to terminate in a pointed process; in others the free margin is serrated. By

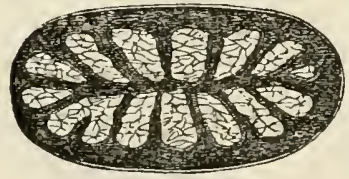

Fig. 308. Transvcrse section of Hair of Pecari, showing its fibrous and cellular structure. scraping, many of them will be detached separately ; but in some few cases, as many as four or fivewill be found joined together: in the larger hairs, the cellular structure of the interior, as well as the fibrous character of the shaft, are better seen after the scales have been removed.

The hair owes the greater part of its colour to pigment- 
cells: as these decay, and become gradually divested of their colouring-matter, they appear whitened, or "turn grey." These hexagonal cells also give colour to the skin of the negro, and are situated immediately beneath the transparent coat. A small portion is shown in fig. 309 , the vacant space denoting the situation of a lost hair.

Certain parts of the skin and mucous membranes are especially supplied with papillæ, which serve as organs of touch ; throughout the greater part of the skin there are papillce more or less sensitive, but only at the extrenities of the fingers, lips, and in a few other situations, are these highly dev loped, as in fig. 310. Papillæ are either filiform or tubiform,

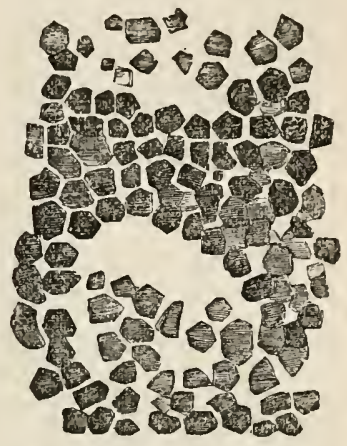

Fig. 309.-Pigment Cells from the skin. and have entering into them nerves and blood-vessels; the former supplying the sensibility of the skin, and terminating in loops, as shown in fig. 310. Papillæ injected are shown in Plate VII. No. 150, tongue of mouse ; villi from small intestine of rat, No. 154 .

The skin is the seat of two processes in particular; one of which is destined to free the blood from a large quantity of fluid, and the other to draw off a considerable amount of solid matter. To effect these processes we meet with two distinct classes of glandulæ in its substance: the sudoriferous, or sweat glands; and the sebaceous, or oil glands. They are both

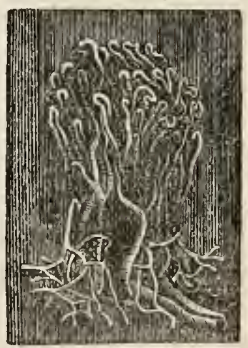

Fig. 310.-A section of shin from the finger, slowing the vascular network of papilla, at the surface of the cutis. formed, however, upon the same simple plan, and can frequently be distinguished only by the nature of their secreted product. The oil-glands of the skin ais $x \times 2$ 
similar in structure to the perspiratory ducts, being composed of three layers derived respectively from the searf-

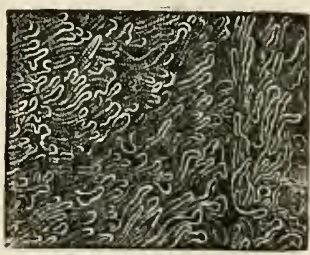
skin, which lines their interior; the sensitive skin, which is the medium of distribution for the vessels and nerves; and the corium, with its fibres, giving them strength and support. Like the sudoriferous ducts, they are in some situations spiral; Fig.311.-Capillary networkand dis- but this is not a constant featribution of popillce over the tongue. ture; more frequently they pass directly to their destination; they are also larger, as shown in fig. 312 , proceeding from the oil or fat vesicle

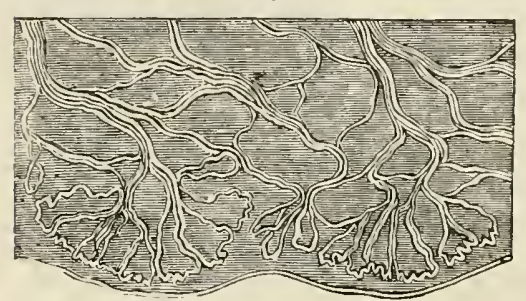

Fj: 312.-Distribution of the tactile nerves at the extremity of the fingers, as seen in a thin perpendicular section of the skin. situated at its lower extremity. Oil - glands are freely distributed to some parts, whilst in others they are entirely absent: in a few situations they are worthy of particular notice, as in the eyelids, where they possess great clegance of distribution and form, and open by minute pores along the edges of the lids; in the ear-passages, where they produce that amber-coloured substance known as the wax of the ears; and in the scalp, where they resemble small clusters of grapes, and open in pairs into the sleath of the hair, supplying it with a pomade of Nature's own preparing.

Internal parts of the body. - We shall now have under consideration cells of a much higher order than any before referred to ; the cell found floating in the animal fluids is known as the blood-cell, and requires a vascular system of its own for distribution over the whole animal body. line red blood cells, or corpuscles, have a circular form, somewhat llattened; their size is about 1-3,200th of an inch in diameter. It is well known that the blood-cor- 
puscles, when floating in their own serum, or after having been treated with acetic acid or water, appear to be furnished with perfectly plain coverings, composed of a simple homogeneous membrane, without distinction of parts. But, when the blood is treated with a solution of magenta (nitrate of rosanilin) or with a dilute solution of tannin, the corpuscles present changes which seem irreconcileable with such a proposition. Dr. W. Roberts, of Manchester, communicated an arcount of his observations on this suhject to

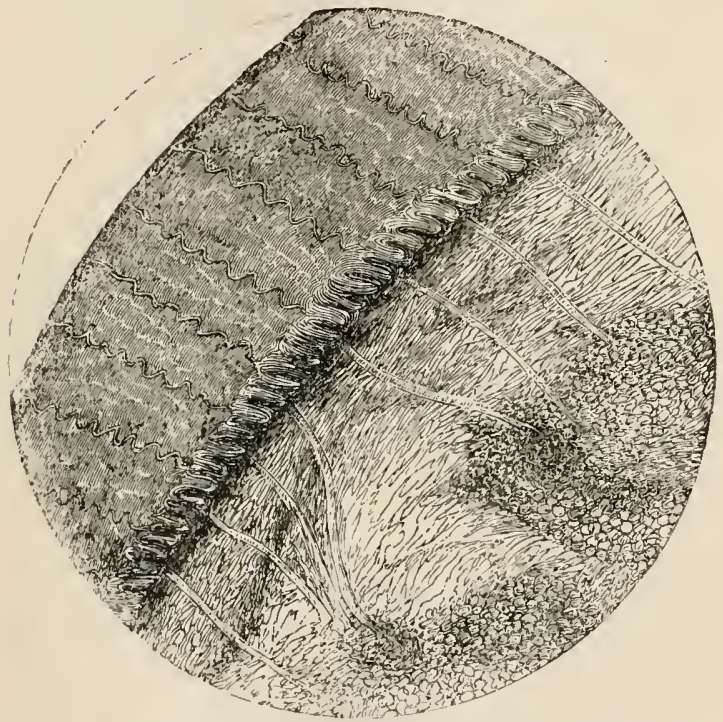

Fig. 313.-A vertical section of the Human Skin, showing the sweat-glands, surrouuded by fat-globules, the duets passing upwards through the epitielici: layer to the epidermis or external cuticle, magnified 250 diameters.

the Royal Society (Proc. Roy. Soc. vol. xii. p. 481), in which he shows that nearly every disc possessed a parietal macula, capable of being stained by a dye. It was commonly of a lenticular shape, but sometimes square, and as a rule very minute, not covering more than 1-20th or 1-30th of the circumference. Moreover, in the blood of many of 
the lower animals a similar tinted particle appeared wheu the corpuscles were treated with magenta. The conclusion to be drawn from this and other observations noted by Dr. Roberts, is that a duplication at one, or at most at two points only, is indicated by direct proof; but certain appearances occasionally observed fivour the notion of a complete duplication.

The wall of the cell is a transparent structureless memhrane, and is of greater thickness than we find the analogous membrane of cells to be generally. The red corpuscles of hirds, reptiles, \&c. possess a distinct nucleus; but, on examining those of the human subject and other Mam. malia, no distinct nucleus can be made out. By applying hilute acetic acid, the red corpuscle becomes bleached, and its walls distended, but no nucleus appears. If a red corpuscle from the Frog be treated in the same manner, we see a nucleus, and the red colouring matter is drawn out by exosmosis. Water canses the corpuscle to swell up, aud the colouring-matter disappears, but its real nature is masked; upon employing a drop of solution of iodine, the wall is coloured or tinged, and made distinct. The cells themselves have a tendency to undergo spontaneously certain changes, one of the most common is a wrinkling up of the walls, with a surface somewhat like that of a mulberry ; this may also be prodnced by mechanical pressure, the addition of oil, \&c.

There is anther set of corpuseles, slightly larger than the red set; these are termed zolourless corpuscles, which, when distended by the action of water, are seen as nucleated cells, whose diameter is about the 1-2,500th of an inch, and a double contour of the walls is observed; sometimes there is a slight tinge of colour to be seen in the nucleus. There is a third kind of corpuscles in the blood, more numerous than those above referred to, but of about the same diameter; when distended, they are seen to be cells filled with granular matter; sometimes a clear spot is noticed on one side; very dilute acetic acid being applied, the granules are dissolved out, and a clear central nucleus remains, if the acid be used stronger, an appearance is seen as if there were several nuclei aggregated together. This latter appearance was considered to be the natural 
state of the nucleus, the particles of which were either. tending to unite with one another, or there was a separation of the nucleus into several smaller portions. Wharton Jones, however, says there is no subdivision of. the nucleus.

If we examine a drop of blood under the microscope,

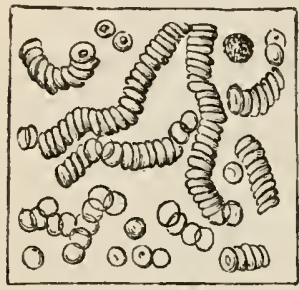

3
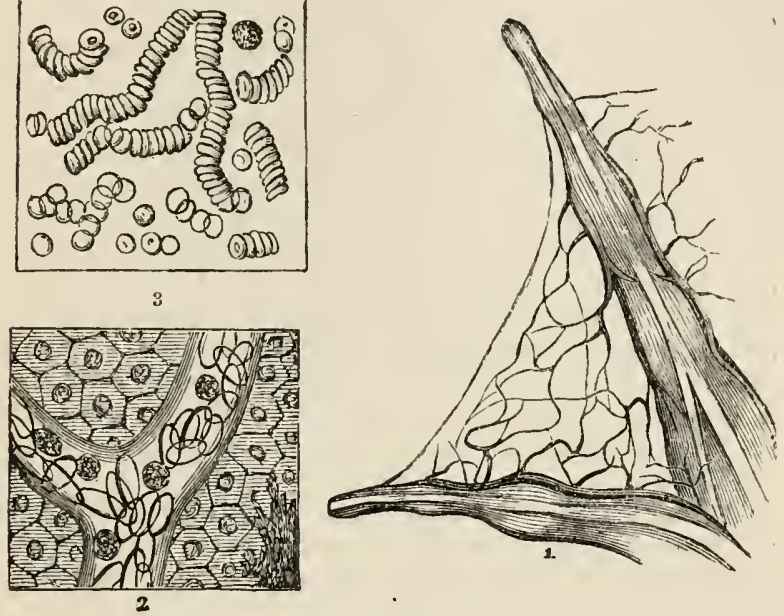

Fig. 314.

1, A portion of the web of a Frog's foot, spread out and slightly magnified to show the distrilution of the blood-vessels. 2, A portion magnified 250 diameters, showing the ovoid form of the blood-dises in the vessel, beuenth which a layer of hexagonal nucleated epithelium-cells anpear. 3, Human blood dises, as they appear when fresh drawn (magnified 250 diameters).

the corpuscles aggregate themselves together like rolls of coins, fig. 314 , No. 3 , presenting a kind of network so long as they remain suspended in their liquor sanguinis. After the lapse of a few minutes, the fibrin, from its elasticity, contracts more and more, and a yellow fluid, called serum, is pressed out,- - or, in other words, the components of the liquor sanguinis, with exception of the fibrin,-and only a shrunken, jelly-like mass remains.

The blood-corpuscles of the lower animals Mr. Gulliver has especially studied. In the blood-corpuscles of bird3, 
and animals below them, there are nuclei; but the cells, instead of being circular, as in the human subject, are elliptical, and larger. The corpuscles in Mammalia in general are like those of man in form and size, being a little larger or smaller. The most marked exception is in the blood of the Musk-deer, in which the corpuscles are of extreme smallness, about the 1-12,000th of an inch in diameter. The Elephant has the largest, which are about 1.2,000th of an inch in diameter. The Goat, of all common animals, has very small corpuscles; but they are, withal, twice as large as those of the Musk-deer. Another exception in regard to form is in the Camel tribe, where they are oval, and resemble those of the oviparous Vertebrata, as the Frog, shown in fig. 314, No. 2. In the Meno. branclius lateralis, they are of a much larger size than in any animal, being the 1-350th of an inch; in the Proteus, the 1-400th of an inch in the longest diameter; in the Salamander, or Water-newt, 1-600th; in the Frog, 1-900th; Lizards, 1-1,400th ; in Birds, 1-1,700th ; and in Man, the 1-3,200th of an inch. Of Fishes, the cartilaginous have the largest corpuscles; in the Gold-fish, they are about the $1-1,700$ th of an inch in their longest diameter.

The large size of the blood-discs in reptiles, especially'in the Batrachia, has been of great service to the physiologist, by enabling him to ascertain many particulirs regarding their structure which could not have been otherwise determined with certainty. The value of the spectroscope in the chemical examination of the blood has been already pointed out in our remarks on the application of this instrument to the microscope. See page 119 .

An interestingsubject to Physiologists has been noticedthe production from the blood, under certain conditions, of red albuminous crystals, - which, although formed from animal matter, and sometimes, in all probability, during life, have the same regular forms as inorganic crystals. Virchow was the first who paid particular attention to their actual nature, and proved them to differ from saline or earthy crystals. If we add water to a drop of blood spread out under the object-glass of the microscope, as the drop is beginning to dry up, the edges of the heaps of bloodcorpuscles are seen to undergo a sudden change: a few 
corpuscles disappear, others have dark thick edges, become angular and elongated, and are extended into small welldefined rodlets. In this manner an enormous quantity of crystals are formed, which are too small to enable us to determine their shape; they rapidly move lengthways, the entire field of vision being gradually covered by a dense network of acicular crystals, crossing one another in every direction, with other crystals presenting a rhomboid form.

Dr. Garrod discovered, that by a slow evaporation of portions of the serum of blood taken from patients labouring under gout, he could obtain strings of crystals of uric acid. His mode of procedure is to pour a little serum into a watch-glass, and add a few drops of acetic acid; in this mixture place a few very fine filaments of silk or tow, and stand it by for twenty-four hours under a glassshade. Upon removing the glass and submitting the filaments to microscopical examination, they are found studded with minute crystals.

A peculiar fatty matter was discovered by Virchow in the tissues of the human body and in certain nerves which he termed Myelin. In the liver it exists in large quantities, and much is found in the yolk of the egg. It is colourless, glistening, semi-fluid, prone to form drops, and capable of being drawn out into long threads, which curve and twist into very peculiar forms. The masses often exhibit double contours, or many lines are observed equi-distant from one another and varying in thickness. Cholesterin is a component of all forms of nyelin; Beneke has, in fact, shown that it is a mechanical mixture of cholesterin and cholate of lipyl. No. 1, fig. 314, the foot of the Frog, when stretched out, shows the

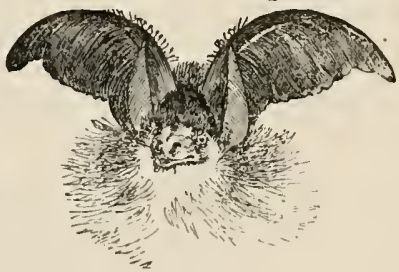

Fig. 315.-Head of Long-eared Bat. (Plecotus A uritus.) distribution of the blood-vessels in the web: the two sets of vessels - the arteries and veins-are very readily made out when kept steadily on the stage of the microscope; the rhythm and valvular action of the latter may be observed, although they are much better seen in the ear 
or wing of the Long-eared Bat, as Professor Wharton Jones pointed out.

To view the circulation of blood in the Frog's foot, the older microssopists, Baker, Adams, and otlıers, were in the habit of tying the frog to a frame of brass; at the present time the entire body of the animal, with the exception of the foot about to be examined, is secured in a black silk bag, and this is fastened to a plate, termed the frogplate, shown at a a $a$ in fig. 316 . The bag provided should

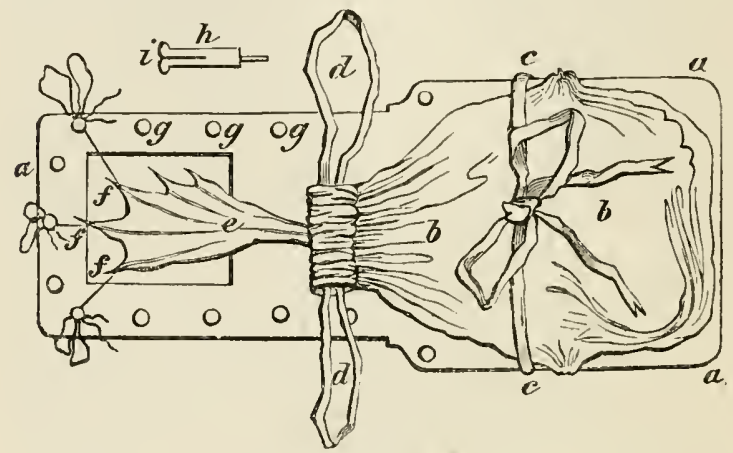

Fig. 316 .

he from three to four inches in length, and two and a half inches broad, shown at $b b$, having a piece of tape, $c c$, sewn to each side, about midway between the mouth and the bottom; and the mouth itself capable of being closed by a drawing-in string, $d d$. Into this bag the frog is placed, and only the leg which is about to be examined kept outside; the string $d d$ must then be drawn sufficiently tight around the small part of the leg to prevent the foot from being pulled into the bag, but not to stop the circulation; three short pieces of thread, $f f f$, are now passed around the three principal toes; and the bag with the frog must be fastened to the plate $a$ a by means of the tapes $c c$. When this is accomplished, the threads $f f f$ are passed either through some of the holes in the edge of the plate, three of which are shown at $g g g$, in order to keep the web open; or, what answers better, in a series 
of pegs of the shape represented by $h$, each having a slit $i$ extending more than half-way down it; the threads are wound round these two or three times, and then the erid is secured by putting it into the slit $i$. The plate is now ready to be adapted to the stage of the microscope : the square hole upon which the foot must be placed is brought over the aperture in the stage through which the light passes to the object-glass, so that the web may be strongly illuminated by the mirror.

The circulation of the Tadpole is best seen by placing the creature on its back, when we immediately observe the beating heart, a bulbous-looking cavity, formed of the most delicate, transparent tissues, through which are seen the globules of the blood, perpetially, but alternately, entering by one orifice and leaving it by another. The heart is enclosed within an envelope or pericardium; and this is, perhaps, the most delicate and certainly the most elegant thing in the creature's organism. Its extreme fineness makes it often elude the eye under the single microscope, but under the binocular its form is distinctly revealed. Passing along the course of the great blnodvessels to the right and left of the heart, the eye is arrested by a large oval body, of a more complicated structure. This is the inner gill, which, in the tadpole, is formed of delicate, transparent tissue, traversed by arteries, and presenting a crimson network of blood-vessels.

The Tadpole is hatched with respiratory and circulatory organs resembling those of the fish. It lives in, and breathes oxygen from the air contained in, the water, and during the early period of its existence respires ex. clusively by gills.

It will be remembered that in nearly all fish the heart has but two cavities, an auricle and ventricle; that the blood of the latter is returned by the veins to the auricle, passes into the ventricle, is then transmitted to the gills, where, being exposed to the air contained in the water, it becomes deprived of carhonic acid, aerated, and rendered fit for recirculation through the system. In the reptile we find a modification of plan. The heart has three cavities, two auricles and one ventricle; by this contrivance there is a perpetual mixture in the heart of the impure car- 
bonized blood which has already circulated through the body, and flows into the ventricle from the right auricle, with the pure aerated blood returned from the lungs, and which also flows at the same instant into the ventricle from the left auricle. Thus the habitual circulation of this "cold-blooded" mixture is the cause of the low tone of vitality that distinguishes reptiles.

For the purpose of observation the tadpole must, of course, be selected during the period in which the skin is perfectly transparent. The first examinations reveal plainly enough the appearances already described of the form and situation of the heart, and the three great arterial trunks proceeding (right and left) from it. Nany observations are required to arrive at the true anatomical arrangement of these vessels. First, they are closely connected with the corresponding gill. The upper one (the cephalic) runs along the upper edge of the gill, and gives off, in its course, a branch which ascends to the mouth, which, with its accompanying vein, is termed the labial artery and vein. The oephalic artery continues its course around the gill, until it suddenly curves upwards and backwards, and reaches the upper surface of the head, where it dips between the eye and the brain, towards which it is evidently travelling.

It would be a mistake to suppose that you can make this out distinctly, in the average of tadpoles taken, without some preparation. The great obstacle is the large coil of intestines, usually distended with dark-coloured food. This must be got rid of by making your tadpoles live on plain water for some days. Plate VII. No. 15:, exhibits the view of the vessels obtained under the influence of low diet, and we are now enabled to trace the course of the three large arteries. The third trunk, traversing the lung, is seen to emerge from the lower edge and descend into the abdomen to form the great abdominal aorta. A small black-looking starved little tadpole shows the heart beating and the blood circulating, but the latter is quite colourless, not a single red globule visible anywhere. The globules chase one another away like globules of water, the heart is a colourless globe, the gills two transparent ovals, and the bowels a colourless, 
transparent coil. 'Through the empty coil the artery is seen on each side descending from the gills, converging to the spine, meeting its fellow, and with it uniting to form the abdominal aorta, the large central vessel coloured red in the figure. After the aorta has supplied the abdominal viscera, a prolongation, or caudal artery is seen descending to the tail, the all-important organ of locomotion in the tadpole. This artery, entering the root of the tail, is imbedded deeply in the Hesh, whence it emerges, and then continues its course, closely accompanied by the vein, to within a short distance of the tail's extremity, where, being reduced to a state of extreme fineness, it terminates in a capillary loop, which is composed of the end of the artery and the beginning of the vein. The artery, in its conurse, gives off branches continually to supply the neighhouring tissue. We may often observe that the blood current in the tail, even in the main artery or vein, is sluggish or even still. This occurs independently of the heart, which may continue to beat as usual; it happens, because the circulation in the tail depends very much on the motion of the organ. When this is suspended (as in confining the tadpole under the microscope), the blood moves sluggishly, or stops, till the tail regains its freedom and motion, when the activity of the current is restored. This fact is thus alluded to by Dr. Grant:- "It is the restless activity of the worm and of the insect that makes every fibre of their body, as it were, a heart to propel their blood and circulate their fluids, while the slow-creeping snail that feeds upon the turf has a heart as complicated as that of the red-blooded, vertebrated fish, that bounds with such velocity through the deep. It is because the fish is muscular and active in every point that it requires no more heart than a snail to keep up the necessary movements of its blood."

Having traced the arterial system, which conveys the blood from the heart to the extremities, we will now note its return by the veins back again to the heart.

The caudal vein runs near to the artery during the greater part of its course, with its stream, of course, towards the heart. This stream is swollen by perpetual tributaries from vessels so numerous that their loops form a network which 
covers the entire surface of the tail. As the vein approaches the root of the tail it lies superficially to the artery, and diverges from it at the point of entering the abdomen. Here it approaches the lidneys, sends off branches to then, while the main trunk contines its course onward; and, passing upwards behind a coil of intestine, it approaches the liver, and runs in a curved course along the margin of that organ. This blood is seen to enter the vena cava by numerous fine channels, which converge towards the great vein as it passes in close proximity to the organ. Beyond the liver the vena cava continues its course upwards and inwards to its termination in the sinus venosus or rudimentary auricle of the heart. This termination is the junction of not less than six distinct venous trunks, incessantly pouring their blood into the heart. The circulation in the fringed lips forms a most complicated network of vessels, out of which proceeds a vein corresponding to the artery already traced. This descends in a direct course till it joins the principal vein of the head, which corresponds to our own jugular.

Thus we have traced the blood through its main channels, and completed the circle of its course.

The blood is driven by the heart into each inner gill through three large blood-vessels, which arise directly from the truncus arteriosus, and may be called the afferent vessels of the gill. (See enlarged view of gill, Plate VII. No. 156).

It will be seen that "each internal gill or entire branchial organ consists of cartilaginous arches (No. 156), with a piece of additional framework of a solidly triangular form, stretching beyond the arches, and composed of semi-transparent, gelatinous-looking material. 'These parts, forming the framework of the organ, support upon their unper surface the three rows of crests with their vascular network, and the main arterial and venous trunks which lie parallel with and between them. The three systemic arteries arising, right and left, from the truncus arteriosus, enter each gill on its carliac side, and then follow the course of the crests, lying in close proximity to them. The upper of these branchial arteries runs alono on the outside of the upper crest, and another branch leaving the trunk and passing into the network of the 
crest, whence a returning vessel may be traced carrying back the blood across the branchial artery, and to a vessel lying close to and taking the same course as the artery itself. Carrying the eye along the latter vessel wo find, at a short distance from the first of these crest branches, a second, which leaves the main trunk and enters the crest, when a corresponding returning vessel conveys the blood across the arterial trunk into the vessel lying beside it, as in the former instance. A succession of these branches (cach taking a similar course) may be traced from one end of the crest to the other. But it is now to be observed that the trunk from which these arterial branches spring diminishes in size as it proceeds in its conrse (like the gill artery in fishes), while the vessel running parallel to it and receiving the stream as it returns from the crest enlarges in the same degree. Thus, the artery or afferent vessel which brings the blood to the gill is large at its entrance, but gradually diminishes and dwindles to a point at the opposite end of the crest; while the venous or efferent vessel, beginning as a mere radical, gradually enlarges, and thus becomes the trunk that conveys the blood out of the gill to its ultimate destination. Calling this vessel the upper branchial vein as long as it remains in contact with the gill, we subsequently change its name when it leaves the gill and winds upwards for distribution to the head, and then designate it the cephalic artery. The middle branchial artery and vein proceed in like manner in connexion with the middle crest, and the lower artery and vein in connexion with the lower crest. The middle and lower venous trunks, having reached the extremity of the crests, curve downwards and inwards, and then leave the gill. The former trunk, converging towards the spine, meets its fellow, and with it forms the ventral aorta. The latter gives origin to the pulmonary artery, and supplies also the integuments of the neck. Curious and beautiful is the final stage of the metamorphosis, when the waning tadpole and incipient frog coexist, and are actually seen together in the same subject. The dwindling gills and the shrinking tail-the last remnants of the tadpole form-are yet seen, in company with the coloured, spotted skin, the newly-formed and slender legs, the flat head, the wide and toothless 
mouth, and the crouching attitude of the all but perfect reptile.

"By the process now described, the three systemio arteries become continuous with the corresponding efferent trunks that convey the blood for distribution through the body, while, simultaneously, the vital fluid is being abstracted from the special trunks belonging to the gill and its vascular crests. These, with the gill structure ronnected with and dependent upon them, being thus leprived of their blood, shrink, become absorbed, and disappear. Such appears to be the beautifully simple mechanism by which the transition in the type of the respiratory function from fish to reptile is accomplished. If we take a tadpole a few days old, when the outer gills are fully developed, and immerse it for another few days in a weak solution of chromic acid (Mr. Archer's method), we may, by placing the tadpole under a dissecting microscope, and with the aid of a needle and camel-hair brush, then remove the integuments, disclose the tufts of the inner gills, and by carefully getting rid of a prominent roll of intestine that occupies the upper part of the abdomen, succeed in revealing the incipient lungs. These are situated behind the gut and close to the spine, and appear as a vair of minute tubular sacs, united at their upper and open extremities. The chromic acid renders the tissues friable, so that they can be readily peeled away."

That we may see how the circulation is carried on during life in the gills, the outer covering must be carefully raised, or even stripped off. This can be accomplished by putting the Tadpole nnder the influence of chloroform-a drop of the fluid is sufficient for the purpose. ${ }^{1}$

The blood-vessels of mammals are divided with reference to their structure into arteries, capillaries, and veins ; yet these three divisions are by no means broadly separated from each other, inasmuch as tbe capillaries are continued into the veins just as impercertibly as they arise from the arteries. But with reference to their structure, while the capillaries possess only a single coat without any special structure, the larger vessels present, with but Sew excep-

(1) We refer the reader to Mr. W. U. Whitney's highly interesting and valuable paper in the Trans. Bicros. Soc. for 1861 and 1867. 
tions, three layers, which are designated as the inner coat, or tunica intima; the middle coat or circular fibrous coat, tunica media; and an outer coat, tunica externa or adventitia. The first is the thinnest coat, and consists of a cellular layer, the epithelium ; generally, also of an elastic coat, with the fibres disposed in a longitudinal direction. The second, middle coat, is a thicker layer, and the principal seat of the muscular fibres of the vessels; in the veins, however, it contains numerous longitudinal fibres, and in the largest vessels more or less elastic elements and connective tissue. The third coat has its fibres again arranger? for the most part longitudinally, and it is as thick as, or even thicker than, the middle coat, and consists of connective and elastic tissues. This coat, the turica adventitia of large arteries, contains muscular fibres in animals, but none in man. According to J. Lister (Quart. Joum. Micros. Scien. 1857, p. 8), the smallest arteries of the frog's web show contractile fibre-cells, which measure from the 1-100th to 1-200th of an inch, and run in a spiral direction, making one and a half up to two and a half turns round the inner coat of the vessel, and such fibre-cells in a single layer constitute the only muscular elements of the vessel.

The blood-vessels of the eye are extremely numerons, and present different conditions in the several parts. In the choroid coat, they are arranged in a most beautiful stellate manner, and the capillary network of the innermost layer of the choroid coat, when injected, forms an intractive object. A vertical section of the eye of a cat, showing its several vascular and nervous coats, is given in Plate VII. No. 157.

The circulation in the foot of the Frog and the tail of the Newt is, for the most part, the capillary circulation. The ramifications of the minute arteries form a continuous network, from which the small branches of the veins take their rise The point at which the arteries terminate and the minute veins commence, cannot be exactly defined; the transition is gradual ; but the intermediate network is so far peculiar, that the small vessels which compose it maintain nearly the same size throughout; they do not diminish in diameter in one direction, like arteries and veins; hence the term capillary, from 
capillus, a hair. (Fig. 317.) The size of the capillaries is proportioned in all animals to that of the blood-corpuscles; thus, amongst the Reptilia, where the blood-corpuscles are the largest, the capillaries are also the largest:

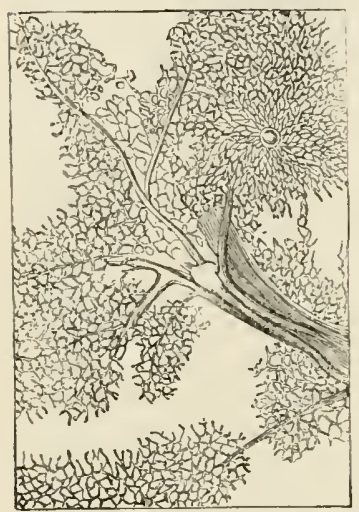

Fir. $317 .-A$ network of capillaries conveying blood to the lungs.

but it does not follow that they should be always of the same size in all the tissues of ono and the same animal; for if we examine and carefully measure in the human subject their sizes in different tissues, we shall find that they vary greatly even in individual tissues, and, at a rough estimate, examples may occur as larga as a thousandth, whilst others are as small as the fonr or fivethousandth of an inch. They should be measured, if possible, in their natural state; when injected, their size is slightly increased; but, when dried, they diminish so considerably that in some specimens vecsels imperfectly filled with injection have been known to shrink from the three to the twenty-thousandth of an inch.

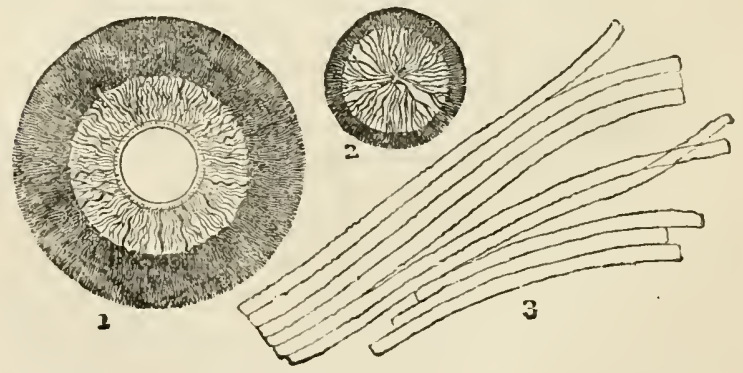

Fig. 31 .

1, Blood-vessels of the Ere; back view of the Iris and ciliary processes, 2, Vessel of the membrana pupitlaris, from the eye of a Kitten. 3, Fibres or tubes froin the lens of the Ox. (A sectional view of a Cat's eye is given in Plate VII. No. 157.) 
Capillaries are, with very few exceptions, always supported by an areolar network, which serves not only as an investment to them, but connects them intimately with the tissues they are destined to supply. A possibility arises, in first examinations, of mistaking or confounding capillaries with nerves, especially if the part under observation should have been left for some time in strong preserving or alkaline solutions.

A weak solution of caustic soda, and also another of acetic acid, are both of use ; the first is available for the purpose of tracing nerves; the latter in making out vessels, structure of papillæ, unstriped muscle, \&c., inasmuchasitrenders

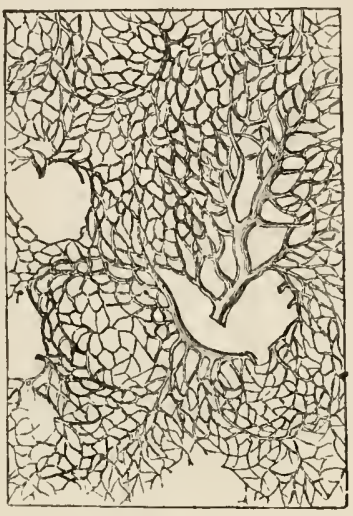
their nucleimore obvious, Fig. 319.-The bronchi, a fine network of while soda thickens and air-tubesfor supplying the lungs with air. makes them less so. It is very useful sometimes to use these re-agents alternately; the rule is, to apply them to the object while under the microscope, so as to watch their gradual operation.

It is not in the hlood alone that cells fioat in a fluid; the chyle and lymph are colourless corpuscles flowing along their especially - adapted tubes and ducts, and carrying the nutritive particles gathered from the food to the blood-vessels, for the

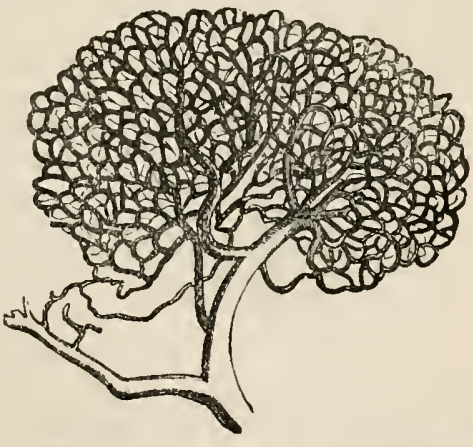

Fig. 320.-A capillary of blood-vessels distributed to the fat tissue. Better seen in some of tho is jected specimens, Plate VII. 
reparation of the framework, or growth that incessantly goes on in the animal body.

Classification of the Animal Tissues.-Professor Schwann classifies the fundamental tissues of the human body as follows:-and it will be seen that more than half are made up of cellular tissue or simple membrane.

1. Simple membrane : employed alone Fxamples: Walls of cells, capsule of in the formation of compound lens of the eye, sarcolemma of membranes . . . . . . muscle, \&c.

2. Fibrous tissues.

Examples: White and yellow fibrous

\&c.
Examples: Cartilage, fat, 1igment,

3. Cellular tissues. . . . . . - Examples: Cartilage, \&c. 4. Scierous, calcareous, or hard tissues\} Examples: Rudimentaly skeleton of

5. Compound membranes: composed of simple meinbrane and a layer of cells of various forms (ephitlielium or epidermis), or of areola or con-

nective tissue and epithelium . .

Examples: Mucous membrane, skin, true or secreting glands, serous and synovial membranes.

6. Compound tissues; $a$, those composed of tubes of homogenous membrane, containiug a peculiar substance........ . .

$b$, those composed of white fibrous Example: Fibro-cartilage. tissues and cartilage . . . . .

Examples : Muscle, nerve.

Cellular or Formative Membrune.-Areolar or connective tissue is generally distributed throughout the body, and

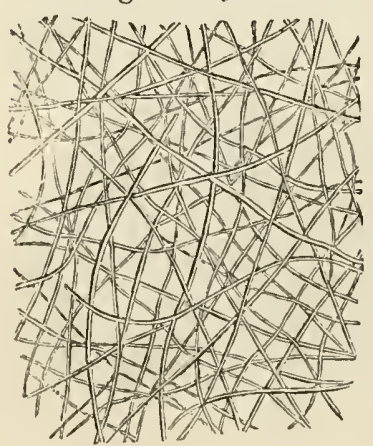

Fig. 321.-Fibrous tissue, lining the interior of the eggshell, the lime having heen previousiy removed by immersion in dilute hydrochloric acid. various forms of this tissue are found ; it is seen uniting together component parts, filling up interstices between them, and affording a support to the blood-vessels and nerves, before they are distributed to the various organs. This tissue is soft, clear, smooth, and extremely minute, being the 1-12,000th of an inch in diameter, sometimes less. The fibre is usually found united together in bundles; if these be acted upon by dilute acetic acid, they swell up, become transparent, and the appearance of fibrous structure is no longer seen, although 
some fibres that were not previously observed may become more distinct. The first kind does not refract the light strongly; the second kind does, showing some chemical difference in their composition.

Cellular tissue, if dried, becomes a yellowish, brittle, transparent mass; but regains its former state if placed in water. The fibres have a remarkable arrangement and disposition. They are often deposited in a spiral manner; at other times they are regularly undulating. In fibres taken from some parts of the body, we find a fasciculus wound round in a spiral form. As a consequence, when acetic acid is applied, we perceive projections of swollen cellular tissue, and the depressions, from not having been acted on, have a constricted appearance. The fibrous tissue lining the eggshell, fig. 321 , is the simplest form in which it is found.

Fat is generally found in the cellular tissue; it is not secreted from it, but is contained in its proper cells, and termed adipose tissue; the elementary cells of which are from the 1-300th to the 1-600th of an inch in diameter (fig. 322). The cell-wall is very delicate and transparent; sometimes there are one or two nuclei enclosed. AEther dissolves out the fat-cells from the tissues. Acetic acid acts upon the cell-wall, and causes the contents to pass from within outwards.

Fibrous tissue, elastic and non-elastic, is usually divided into white and yellow fibrous tissue. The yellow is elastic, and of great strength, consisting of bundles of fibres which are highly elastic. (Fig. 324, No. 2.) The white (fig. 324, No. 1), though non-elastic, is of great

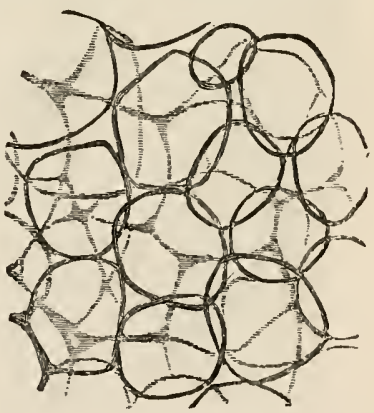
strength, and of a shining, Fig. 322.-Cells from adipose tissue, silvery appearance.

These two kinds of fibrous tissue differ from each other in many respects, but chiefly in their ultimate structure, their physical properties, and their colour: both are largely 
employed in those parts subservient to the organs of locomotion.

The white fibrous tissue is (when perfectly cleared of the arcolar) of a silvery lustre, and couposed of bundles of fibres rumning, for the most part, in a parallel direc-

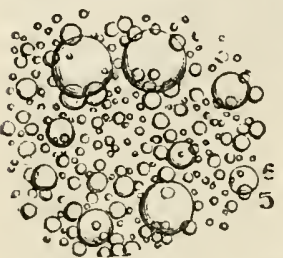

Fig. 323. The contents of a single fut-cell, separated and magnified 250 diameters. tion; but if there be more than one plane of fibres they cross or interlace with each other. In some specimens it is very difficult to make out the fibres distinctly, except with oblique light; from this circumstance it would appear that this tissue is composed of longitudinally striated membrane, which is often found split up into fibres. The white fibrous tissue is principally employed in the formation of ligaments and tendons-a purpose for which it is admirably fitted on account of its inelasticity: it also enters into the formation

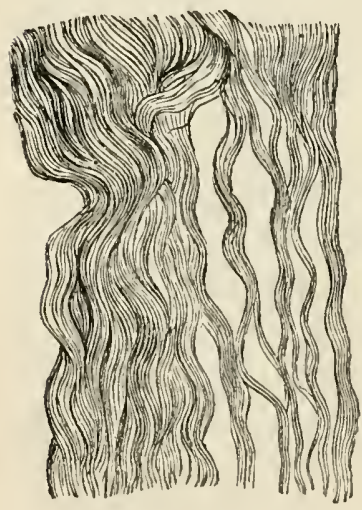

1

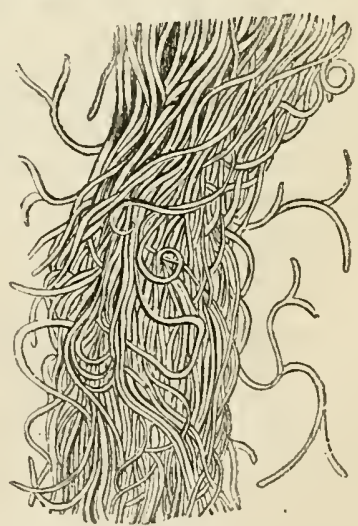

2

Fig. 328.

1, White fibrous or non-elastic tissue. 2, Yellow fibrous or elastic tissue taken near a ligament.

of fibrous membranes, viz. the pericardium, dura mater, periosteum, perichondrium, sclerotic coat of the eye, and 
all the fascixe. It is sparingly supplied with capillaries and nerves: the former always run in the areolar tissue, comnecting the bundles of fibres togrether"; in the generality of the fibrous tissues, the capillaries are not well seen, except in that of the dura mater and periosteum; in other parts it must be injected to show them.

The yellow fibrous tissue is highly elastic; it consists of bundles of fibres covered with, and connected together by, areolar tissue: the fibres are of a yellow colour, some round, others flattened; they are not always parallel, but frequently bifurcate and anastomose with neighbouring fibres. It is always difficult to separate the fibres from each other ; and when separated, the elasticity of each individual fibre is shown

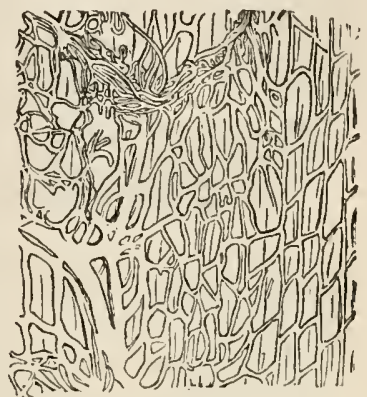

Fig. 325.-White fibrous tissue from the sclerotic coat of the eye.

by its tendency to curl up at the end. The fibres in the human subject vary in diameter from the 1-5,000th to 1-10,000th of an inch. Acetic acid of ordinary strength does not act on yellow fibrous tissue; nor for a very long time after maceration in water or spirit does its elasticity diminish. Very long boiling extracts from it a minute quantity of a substance nearly allied to gelatine; neither nuclei nor a trace of a cell can be seen in it after the addition of acetic acid: both are readily seen when white fibrous element is treated with this acid.

Muscular Fibre.-There are three different kinds of muscular fibre found in the animal body: 1st, in the muscle of the skeleton; $2 \mathrm{~d}$, in the muscle of the heart; and $3 \mathrm{~d}$, in the stomach, intestine, \&c. The functions of muscular fibre may be referred to two kinds-voluntary and involuntary. The muscles endowed with voluntary power are those of the skeleton; the involuntary are those of the heart, stomach, intestine, \&c.

Muscular fibre is held together by a very delicate tubular sheath, nearly rescmbling simple structureless membrane. 
This cannot always be discerned; but when the two ends are drawn asunder it will be perceived to rise up in wrinkles, or the fragments of the torn muscle will be seen to be connected by the untorn membrane, as at fig. 326. This membrane is termed Myolemma. It is best seen when a piece of muscle is subjected to the action of

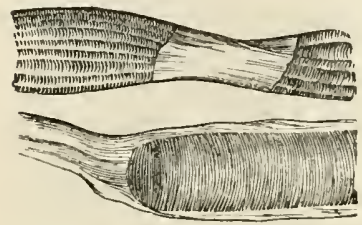

Fig 326.-Muscuhtfibuluchen weross, the frugments connected by the untorn structureless membrane, myolemima. (Magnified 100 diameters.) fluids, as diluted acetic or citric acid, or the fluid alkalies; which occasion it to swell and become easy of separation. It has no share in tlse contraction of the muscle itself, which is made up of a series of bundles of highly elastic fibres : portions of a separated bundle are shown at fig. 327 , and the ultimate structure of

a fibre, under a magnifying power of 600 diameters, at fig. 328, No. 1.

Dr. Hyde Salter pointed out, that in the tongue the muscles pass directly into the bundles of the submucous

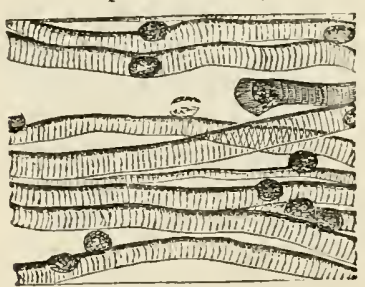

Fing 327.-Museular fibre, broken up into irregular and distinet bands: a few blood globules are distributed al,out. (Magnified 200 diameters). connective tissue, which serve as their tendons. Such a transition is shown in fig. 328, No. 2 ; the tendon, the lower part of which may be seen passing insensibly into the striped musele, the glandular sarcous elements of the latter appearing, as it were, to be deposited in the substance of the tenclon (just as calcareous particles are deposited in bone), at first leaving the tissue about the walls of the cavities of the endoplasts, and that in some other directions, unaltered. These portions, which woukl have represented the elastic element in ordiuary counective tissue, disappear in the eentre of the muscular bundle, and the endoplasts are immediately surrounded by muscle; just as, in many specimens of bone (see figs. of bone), the lacunæ have no distinguishable walls. On the other haud 
at the surface of the bundle the representative of the elastic element remains, and often becones as much developed as its sarcolemma. There is no question here of muscle resulting from the contents of fused cells. It is obviously and readily seen to be but a metamorphosis of the periplastic substance, in all respects comparable to that which occurs in ossification, or in the develop. ment of tendon. In this case, we might expect that, as there is an areolar form of connective tissue, so should
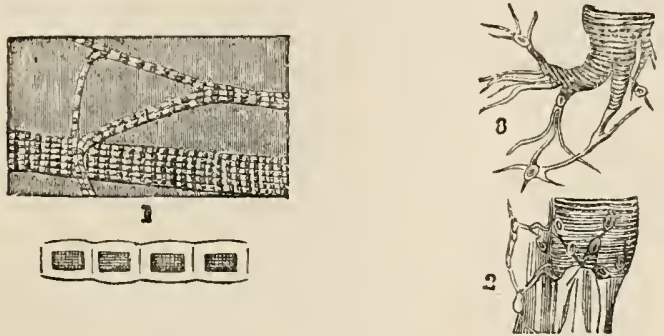

Fig. 328.

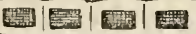

- IInspular filre, anrl a fascirulus of a muscle taken from a young l'ig. (Matg-ified 600 diameters.) 2, Muscular tibre from the tongue of Lant, showing zontinuity of the upper portion, with connective tissue of the lower portion. 3 , Branched muscle, ending in stellate connective cells, from the upper lip of the Rat.

we find some similar arrangement of muscle; such may, indeed, be seen very beautifully in the termination of the branched muscles, as they are called. In fig. 328, No. 3, the termination of a muscular fibre from the lip of a Rat, is shown: and the stellate "cells" of areolated connective tissue are seen passing into the divided extremities of the muscular bundle, becoming gradually striated as they (lo so. In the muscle it is obvious enough, that whatever homology there may be between the stellate "cells" and the muscular bundles with which they are continuous, there is no functional analogy, the stellate bodies having no contractile faculty. The nervous tubule is developed in essentially the same manner as a muscular fisciculus, the only difference being, that fatty matters take the place of syntonin. Now it commonly happens that the nerve-tubule terminates in stellate bodies (fig. $330)$ of a precisely sinilar nature; these are supposed to 
possess important nervous functions; and are now known as " ganglionic cells."

The muscular fibre, known as the non-striated, or involuntary, consists of a series of tubes presenting a flattened appearance, without the transverse striæ so characteristic of the former: elongated nuclei immediately appear upon the application of a little dilute acetic acid. Professor Wharton Jones first demonstrated this structure in his lectures at Charing Cross Hospital, about 1843 : he was led to infer, from appearances in very young fibre, that the striped muscular fibre is originally composed of similar elements to the unstriped, or plain muscular tissue, which, in the process of deve-

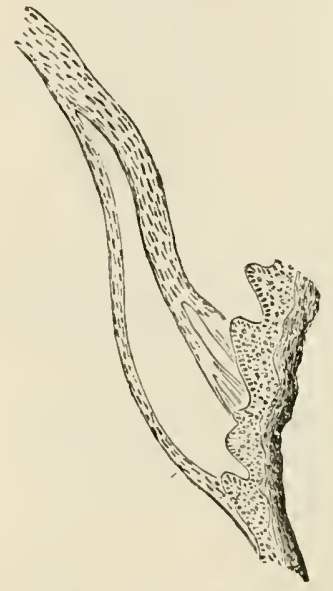

Fig. 329.-A portion of the involuntiry muscular fibre surrounding the hair. lopment, becomes enclosed in a sarcolemma (simple membrane) common to many of them; the fibres then split into smaller fibres (fibrillae). Thus accounting for the nuclei of striped muscular tibre; which, according to his views, are "the persistent nuclei of the primitive muscular-nibre cells."

The non-striated fibre is beautifully seen in connexion with the slsin surrounding the hairs of the head, a few fibres of which are separately shown in fig. 329. Professor Kölliker originally described these muscles of the skin, of which there appear to be one or two in connexion with each hair-follicle, arising from the more superficial parts of the onter skin, then passing down to the root of the hair, close behind the fat-gland, and there embracing it. It is incleed most remarkable that skin, when covered with hair, should alone be provided with these muscular fibres; the effect of the contraction of which must be to thrust up the hair-follicles and depress the intermediate portions of skin, and thus produce that peculiar 
and before unaccountable state of the surface known as goose-skin.

Nerves. - The nervous system consists of brain, spinal marrow, and nerves. There are two sets of nerves in the body; in the one set the nerves are white, firm, shining, more or less rounded, with transverse markings; in the other, they are softer, not so consistent, of a reddish grey colour, and generally flat.

Under the microscope, nerves are seen to be composed of minute fibres or tubules, full of nervous matter, arranged in bundles, and connected by an intervening fibro-cellular tissue, through which capillaries ramify. A layer of the same, or of a more delicate, transparent, structureless tissue surrounds the whole nerve, forming a sheath. The slight pressure of the thin glass, when placed on the nerve-fibre, causes nearly the whole of the contents to flow out in the form of a granular material ; it therefore becomes necessary to exercise considerable care in breaking up structures to view these tubules, which should be immersed in a very weak solution of spirit and water.

On Microscopical Examination of Nerves-It would scarcely be necessary to insist on the great importance of removing and examining the nervous centres as soon as possible after death, were it not that the practice is too often neglected. Great caution also should be exercised to avoid injuring the parts, so that when hardened, perfect or entire sections of them may be obtained for examination

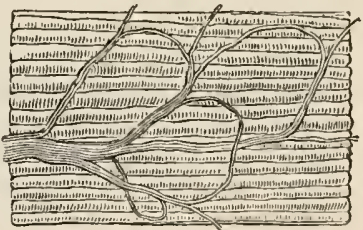

Fig. 331.-Termination of nerve-loops in muscles.

under the microscope. After they have been carefully examined externally, by the assistance of a lens, if necessary, incisions should be made, not at random, but in a 
regular manner through them; and wherever there is reason to suspuct the existence of disease, small portions should be removed, and examined while perfectly fresh under high magnifying powers. The nature of the lesion having been thus ascertained, the morbid parts, with some of the surrounding healthy tissne, should be removed, and after being divided, if necessary, into smaller portions, should be macerated in a weak solution of chromic acil. It is very important to cut and subdivide the parts, when necessary, in such a manner, that their relation to the rest may be recognised after they have become hardened for the purpose of making sections. Thus,unless the locality of the lesion require a different course, -one incision may be made through the crura cerebri, immediately in front of the small or motor roof of the trifacial nerves, and a third through the base of the anterior pyramids, immediately below the attachment of the sixth pair of cerebral nerves. With regard to the spinal cord, if it be cut up into small portions and hardener in chromic acid, it will be found better to divide it in three places: 1st, through the middle of the cervical enlargements; 2d, through the middle of the dorsal region; and $3 \mathrm{rd}$, through the middle of the lumbar enlargement.

If, however, the lesion be suspected to exist at another point, the cord must be divided there; as it is highly important that the nature of any morbid portion that may be found should be examined under the microscope in a perfectly fresh state; for the nerve-fibres undergo a considerable and very deceptive alteration by the action of chromic acid. But it is no less important, and indeed is absolutely necessary for an exact and complete investigation, that entire portions of the cord, in the locality of the lesion, bo hardened in chromic acid, so that thin, but perfect sections may be made for examination under the microscope.

"The strength of the chromic acid solution should differ for different parts of the cerebro-sjinal centres. For the convolutions of the cerebral hemispheres and cerebellum, the proportions should be one of the crystallised acid to about four hundred of water, while for the pons varolii, 
medulla oblongata, and spinal cord, the strength of the solution may be in the proportion of one of the acil to about three or even two hundred of water. It is best, however, to begin with the weaker solution and increase its strength at the end of some hours. If the cerebral and cerebellar hemispheres be hardened in a solution of greater strength, they become friable and unfit for making perfect sections." 1

Method of Preparing Sections of the Spinal Cord.-By a peculiar method of preparation, Mr. Lockhart Clarku obtains beautiful sections showing the arrangement of the nerve-tibres and vesicles in the spinal cord and other parts of the nervous system. The results are recorded in the Philosophical Transactions for 1851, Part 2. The cord it appears is first hardened in acetic acid and alcohol, when excessively thin sections may be readily obtained with a sharp knife. These are then soaked in pure spirit, which permeates the texture in every part, and drives out the acetic acid, and afterwards transferred to turpentine which expels the spirit, and lastly the sections are mounted in Canada balsam. By this plan the tissues of the embryos of manımalian animals can also be rendered so transparent that the smallest ossific points can be seen in the temporary cartilages. To render the specimen more transparent immerse it in alcohol to which a few drops of a solution of soda have been added, and allow it to remain quietly for a few days. When sufficiently acted on, remove it, and preserve permanently in weak spirit. The principle of the action of the fluid may be explainerl thus: alcohol alone tends to coagulate albuminous textures and render them opaque, at the same time that it hardens them. The alkali, on the other hand, renders the tissues soft and transparent, and if time were allowed, would cause their complete solution. These two fluids in conjunction harden the texture and at the time make it clear and transparent. Many soft tissues may thus be hardened sufficiently to enable us to cut very thin sections.

Nerves may be examined in thin sections of the skin after the addition of acetic acid and a solution of soda Gerber boils the slin until it becomes quite transparent,

(1) Lockhart Clarke on the Microscopical Examination of Nerves. 
then allows it to remain a few hours in turpentine, or until the nerves are seen to be white and shining, when they are ready for cutting into perpendicular sections with the double (Valentine's) knife.

It is proposed to employ chloride of gold to stain the tissues of the body. Tissues soaked in a weak sclution of from one to two per cent. of this salt in distilled water, and afterwards exposed to light, are found to exhibit certain parts, as nerve-fibres, connective tissue, corpuscles, and cells generally, stained of violet-red colour, while other parts, as intercellular substance, \&c. are left untouched. The soaking must be continued until the tissue assumes a straw-yellow colour, then taken out of the solution and placed in dilute acetic acid of one to two per cent. The colour will be scen to gradually develop itself, and Kölliker and Colnnheim, who have tried it, say that nerve-fibre, \&c. are exceedingly well shown in this way.

Consolidated Tissues.- Such tissues are formed by a chemical combination with the albumen and gelatine of the fibre; this in cartilaginous

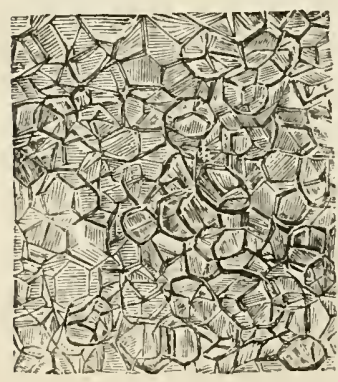
formations is termed chondrine, the cells of which become consolidated by calcareous deposits, and a gradual transition results therefrom. Cartilage is the firmest structure next to bone met with; it is very elastic, and, as an intercellular substance, is generally divided into two kinds. Between the ribs we find this substance presenting a uniform bluish appearance, Fig. 332-Cartilage from ear of and slightly granular; this is Mouse, resembling a section of
vegetable tissue, with superins- true, or white cartilage. The posed layers. other form of intercellular substance is developed in fibrous substances; and it is in this peculiarly-formed felt-work that cells with nuclei are found. This is known as yellow, fibrous, or spongy cartilage, the yellow colour depending on the mode of fibrous arrangement of the intercellular substance : it is found in the ears, and other parts. 
Cartilage forms the entire skeieton in some kinds of fishes, the Skate, Lamprey, \&c. ; and it is nourished with-

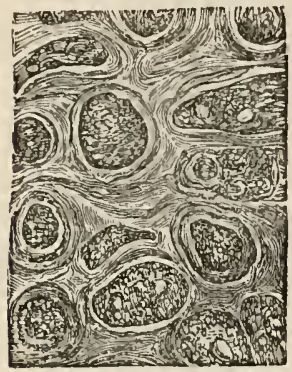

1

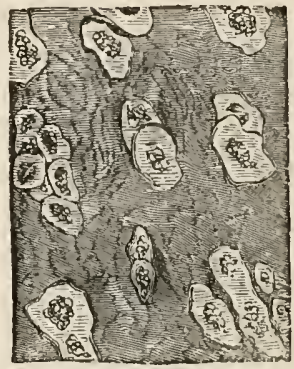

2

Fig. 333.

1, Cartilage from Rabbit's ear, showing larte cells embedded in a fibrous matrix. 2, Cartilage from Human ribs, with cells in groups, each having a granular nucleus. (Dlagnified 200 diameters.)

out coming into direct contact with the blood-vessels, therefore it is said to be non-vascular; nourishment is derived by imbition from the surrounding blood-vessels. When examined microscopically, the simplest form of cartilage is seen to resemble in a striking manner the cellular tissue of vegetables; it consists of an aggregation of cells of a spherical or oval form, capable in some cases of heing separated from each other, and every cell having a nucleus, with a nucleolus in its interior. In figs. 332, 333, and 334 , we have varieties of this structure. In the more highly organized scale of animals, a strong fibrous capsule, or sheath, surrounds the cartilagecells; some of the fibres dip in amongst the cells, and bind them firmly

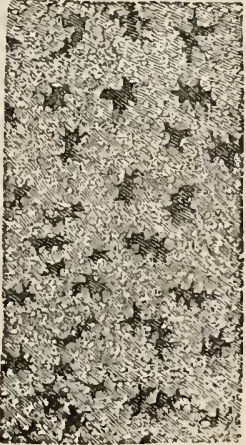

Fig. 334.-Cartilage froin the Cuttle-fish, showing stellate form of cells. together. In those inhabitants of the water, the Ray and Shark, the entire skeleton being cartilaginous, the cell is imbedded in a matrix, which mav 
be strictly termed iutercellular. Cells are frequently or entirely isolated, as in the section from the ear of a Mouse (fig. 332), they then rarely become converted into bone. In the highest animals it is generally invested by a fine

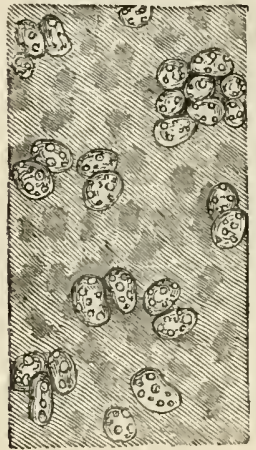

I
Fig. 335.

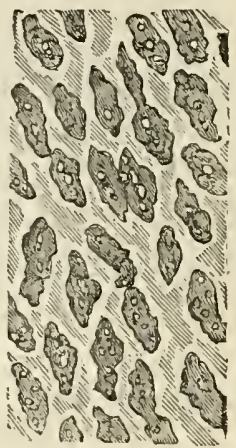

2

1, Cartilage from the lieal of Skate, with elusters of nucleated cells and nucleoli inclosed. 2, Cartilagu from the Frog, with cells having nucleoli, magnified 200 diameters.

and delicate membrane, termed perichondrium, which brings the blood-vessels in close contact with the cartilage; and, when in actual contact with the extremities of bones, is covered by a vascular membrane having a large number of vessels terminating in it, for the purpose of supplying a lubricating fluid to the end of the bones: this, the synovial membrane, is a very beautiful structure when injected and viewed under a 1-inch power.

In the earliest stages of existence, the entire framework-or a very large proportion-is composed of cartilage, which, by a gradual addition of earthy matter, becomes consolidated into bone. The mode of development, and the change from one to the other, is represented in the section (fig. 336); it will there be seen that the calcareous matter is deposited in nearly straight lines, which stretch from the ossified surface into the substance of the matrix of the cartilage, the amount of calcareous matter in which gradually diminishes as we recede from the ossified part. If the deposit has taken place to any great extent, the 
calcareons matter becomes crowded and consolidated; as the process advances, the bone thickens, and a series of grooves, of a stellate form as in the annexed cut (fig. $3 \dot{3} 7$, No. 2), are found upon its surface, which become gradually converted into canals for the passage of blood-vessels.

In certain forms of disease, many of the soft parts of the human body are converted into eartilaginous and bony masses, which have received the name of Enchondroma (figs. 338 and 339). The microscopical characteristics of this change have been described by the author in the Transactions of the Pathological Society of London, vol.iv.

Teeth.-It is desirable to be. come acquainted with the structure of teeth under the microscope ; they are highly interesting to the physiologist, and important guides to the naturalist in the classification of animals. Professor Owen has said, "If the microscope is essential to the full and true interpretation of the vegetable remains of a former world, it is not less indispensable to the investigator of the fossilised parts of animals. It has sometimes happened that a few scattered teeth have been the only indications of animal

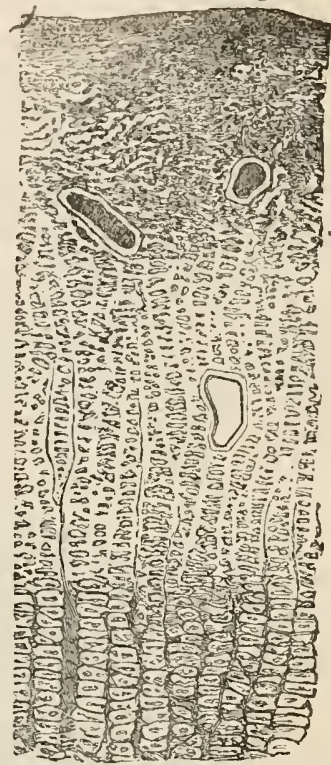

Fig. 336.-A vertical section o; cartilage, with clusters of cells arranged in columns previous to conversion into bone, which is scen consolidated at the upper surface. The greater opacity of this portion is owiug to the increase of osseous fibres, the epacity of the cell contents, and the multiplieation of oilglobules; the dark intereellular spaces become occupied by vessels.

life throughout an extensive stratum; and when these teeth happened not to be characterised by any well-marked peculiarity of external form, there remained no other test by which their nature could be ascertained than that of the microscopic examination of their intimate tissue. By the microscope alone could the existence of Keuper. 
reptiles in the Lower sandstones of the New red system,
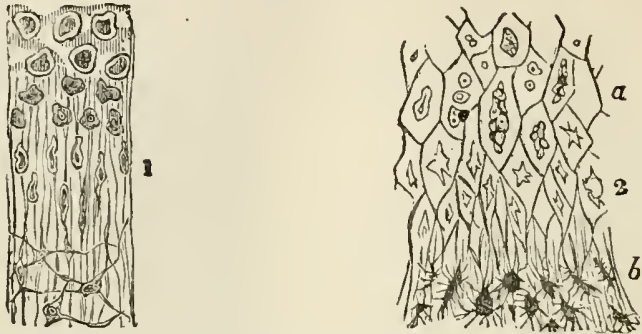

Fig. 337 .

1, Section of the tendo-Achillis as it joins the oscalcis; showing the stellate cells of tendon gradually coalescing to form the round or oval cells of the cartilage. 2, A small transverse section of enchondroma, showing the gradual change of the cartilage-cells at $a$ into the true bone-cells, termed lacunce, at $b$, with their characteristic canaliculi.

in Warwickshire, have been placed beyond a doubt. By

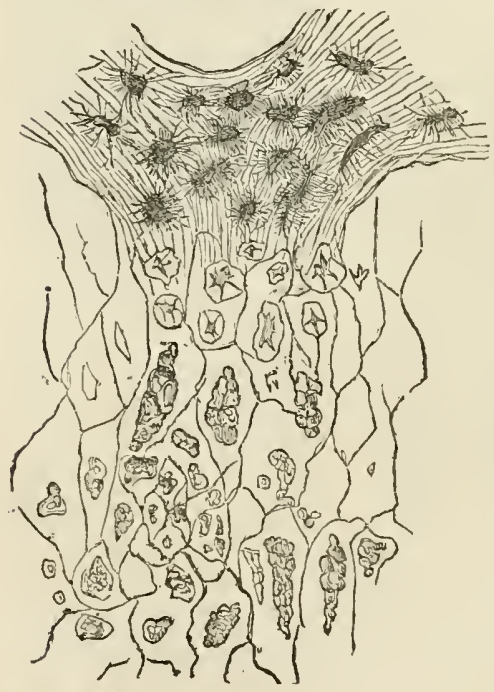

Fig. 3E, Microscopical character of Enchondroma, fr: $m$ a finger. The cartilage undergoes a graan change, and is 3 seen converted into bone at the wper portion. the microscope, the supposed monarch of theSaurian tribes -the so-called $\mathrm{Ba}$ silosaurus - has been deposed, and removed from the bead of the Reptilian to the bottom of the Mammalian division. The microscope has degraded the Saurocephalus from the class of reptiles to that of fishes. It has scttled the doubts entertained by some of the highest authorities in palæontology as to the true affinities of the gigantic $\mathrm{Me}$ gatherium ; and by 
demonstrating the identity of its dental structure with that of the Sloth, has yielded us an unerring indication of the true nature of its food."

The teeth of Man and of most of the higher animals are composed of three different substances, Dentine (known as ivory in the tusk of the elephant), Enamel, and Cementum, or crusta petrosa. These are variously disposed, according to the purposes which the tooth is to serve: in Man the whole crown of the tooth is corcred with enamel, shown in the darker marginal part of fig. 340 ; its root or fang is covered with cementum,

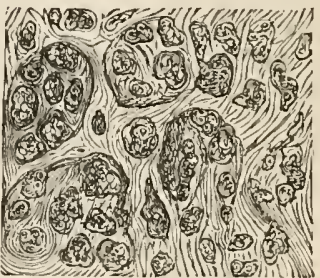

Fig. 339.-Portion of an enchondromatous mass, nucleatcd cells, and fibrous tissue.

whilst the substance or body of the tooth is composed of dentine.

In the human subject, two sets of teeth are developed, the milk and permanent : the first are formed from one

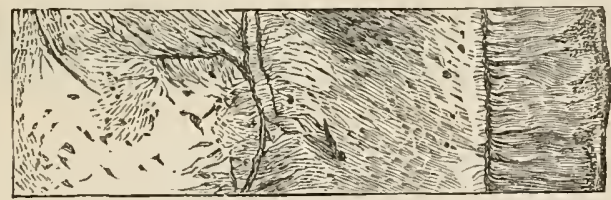

1

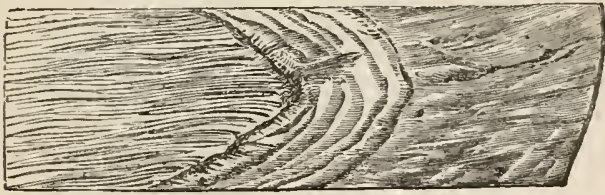

2

Fig. 340.-Sections of Fuman Molar Tooth (magnified 50 diameters) 1, Vertical Section. 2, Horizontal section.

set of bulbs, which in time shrink, and let the teetli fall out; the permanent set is then produced from now bulba, 
situated by the side of the old ones. Blandin was the first to point out that the teeth are developed in the mucous membrane, in a similar way to hair and nails. Other observers have been led to the same conclusion; and, more lately, Professor Goodsir demonstrated that the teeth are first formed in grooves of the mucous membrane, and subsequently converted into closed sacs by a process of involution, and that their final adhesion to the jaw is a comparatively late part of the process. It is now generally conceded that teeth belong to the muco-dermoid, and not
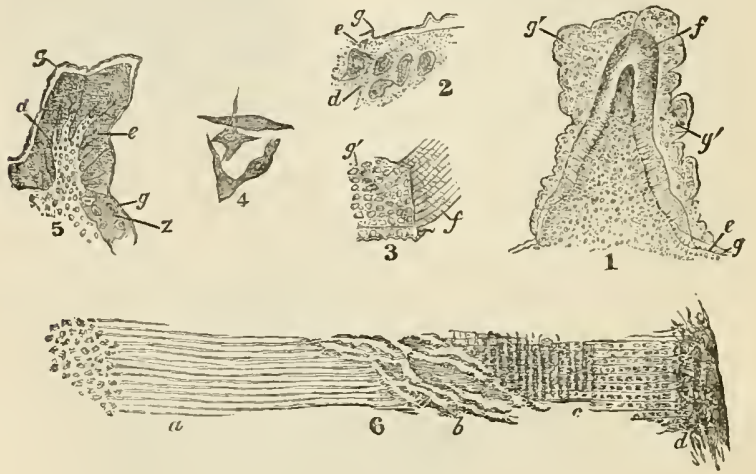

Fig. 341.

1. A section of a cusp of the pasterior molar, upper jaw of a Child. The inner outline represents it before the addition of acetic acid - the outer afterwards, when Nasmyth's membrane $g$ is seen raised up in folds; $f$, the enamel organ; $e$, the dentine. The central portion is filled up with pulp. 2. Edge of the puly of a molar cusp, showing the first rudiment of the.dentine, commencing in a perfectly transparent layer between the nuclei of the pulp and the membrana preformativa. 3, Nasmyth's membrane detached from the subjacent enamel by acetic acid. 3 , The stellate-cells of the enamel-organ. 5. Tooth of the Frog, acted on by dilute hydrochloric acid, so as to dissolve out the enamel and free Nasmyth's membrane. The structure of the dentine $e$ is rendered indistinct. At the base, Nasnyth's membrane is coutinued over the bony substance at $z$, in which the nuclei of the lacuna are visible. (After Huxley.) 6. Decalcified tooth-structure; $a$, the dentine; $b$, enamel organ; $c$, enamel ; $d$, Nasmyth s membrane.

to the periosteal, series of tissues; that, instead of standing in close relation to the endo-skeleton, they are part of the dermal or exo-skeleton; their true anologues being the hair, and some other epidermic appendages. Professor Huxley has proved that, although teeth are developed in 
two ways, these are mere varieties of the same mode in the animal kingdom. In the first, which may be typified by the Mackerel and the Frog, the pulp is never free, but from the first is inclosed within the capsule, seeming to sink down as fast as it grows. In the other, the pulp projects freely at one period above the surface of the mucots membrane, becoming subsequently included within a capsule formed by the involution of the latter; this occurs in the human subject. The Skate offers a sort of intermediate stage.

The enamel forms a continuous layer, and invests the crown of the tooth; it is thickest upou the masticating surface, and decreases towards the neck, where it usually terminates. The external surface of the enamel apperis
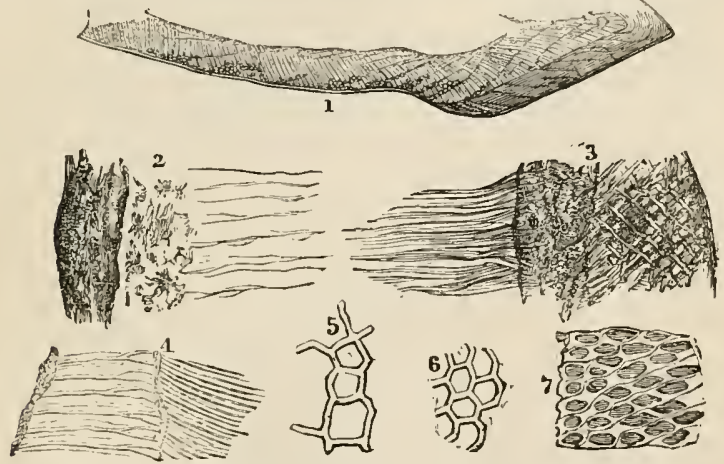

Fig. 342.-Tooth Structure.

1. Longitudinal section of superior canine tooth, exhibiting feneral arrangement, and contour markings, slightly magnificd. 2 aud 3 , l'urtions from silue, bighly magnified, showing the relative position of bonc-cells, cementum at 2, dentine fibres, and commencement of enamel at 3. 4, Dentiue tibres decalcified. 5, Nasmyth's membrane separated and the calcareous matter dissolved out with dilute acid. 6, Cells of the pulp lying between it and the ivory. 7 , A transverse section of enamel, showing the sheaths of fibres, contents removed, and magnificd 300 diameters.

smooth, but is always marked by delicate elevations and transverse ridges, and covered by a fine membrane ( $\mathrm{Na}$ smyth's membrane), containing calcareous matter: this membrane is separable after the action of hydrochloric acid; it then appears like a network of areolar tissue, shown in fig. 342 , No. 6 ; which is Huxley's "calcified memorana 
mreformativa," of the whole pulp. Nasmyth says: "In all cases where this covering has been removed by means of asid, it has, of course, the appearance of a simple membrane, in consequence of the earthly deposit having been dissolved out, and the animal tissue only remaining. The structure and appearance of the covering detached in this manner from the enamel is the same in every respect as that observed in the capsule of the unextruded tooth, and consisting, like it, of two layers, fibrous externally, and having on its external surface the peculiar reticulated appearance common to both."

"On examining carefully fine sections of several teeth under the microscope, I perceived here also," observes Nasmyth, "that the structure in question was continuous with the crusta petrosa of the fang of the tooth."

The enamel has a fibrous bluish aspect, is very brittle, and much harder than the other dentinal structures; it is, indeed, so hard, that it strikes fire with steel; if an attempt be made to cut it without the application of water to keep it cooled down, it burns with an ammoniacal odour, such as we perceive when horse-hoof is burnt. It is composed of prisms, about 1-5,000th of an inch in breadth, more or less wavy, and transversely striped. Two kinds of bands or stripes are seen traversing enamel, the direction of one of which nearly coincides with that of the dentine fibres; the other set of stripes indicate the laminated structure of enamel. Under polarised light, a third set become visible, arising from the variable inclination of the axes of the fibres to the plane of polarisation. The enamel is often traversed by cracks or fissures, mostly running parallel with one set of the fibres: these are sometimes described as canals; but as they resemble splits, and are seldom seen in young teeth, it is more likely that they are cansed by the nature of the food and drink, which is taken into the mouth at temperatures varying many degrees; we also trace the commencement of disease from a fissure in the enamel. When a section of the enamel is cut obliquely, it has somewhat of a hexagonal or sixsided appearance. The dentine consists of a transparent basement membrane, with alternating layers of calcareous matter, traversed by very fine branching tubuli, 
which commence at the pulp cavity, and pass up to the enamel.

Czermak discovered that the curious appearances of globular conglomerate formations in the substance of dentine depend on its mode of calcification and the presence of earthy material ; and he attributes the contour lines to the same cause. Contour markings vary in intensity and number; they are most abundant in the root, and most marked in the crown. Vertical sections exhibit them the best; as fig. 342, No. 1 . In preparing a specimen, first make the section accurately, then decalcify it by submersion in dilute muriatic acid; then dry it and mount in Canada balsam with continned heat, so as to allow the specimen to soak in the fluid resin for some time before it cools. It is the white opacity at the extremity of the contour markings which gives the appearance of rings on the tooth-fang.

"The tooth-substance appeara," says Czermark, "on its inner sur-

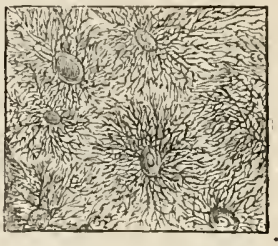

Fig. 343.-Transverse section of Tooth of Pristis, showing orifices of medullary canals, with systems of radiating fibres (tubuli) analogous to the Haversian canals in true bone.

face, not as a symmetrical whole, but consisting of balls of various diameter, which are fused together into a mass with one another in different degrees, and on which the dentinal tubes in contact with the germ cavity are terminated. By reflected light, back-ground illumination, one perceives this stalactite-like condition of the inner surface of the tooth-substance very distinctly, by means of the varied illumination of the globular elevations, and by the shadows which they cast. Here one has evidently to do with a stage of development of the tooth-substance; for the older the tooth is, the less striking in general are these conditions, and the more even is the surface of the wall of the germ-cavity. In very old toeth considerable unevenness again makes its appearance; these, however, are not globular, but have a cicatrised, distorted appearance. It is best to make the preparation from a tooth of which the root is not perfectly completed. With such preparations, one is readily convinced that the ground. 
substance of the last-formed layer of the tooth-substance appears, at least partly, in the form of balls, which are fused one among another, and with the balls of the penultimate layers; and one also pereeives that in general their diameter becomes less and less, somewhat in the form of a point, towards the periphery of the tooth-substance. To obtain specimens, procure a tooth of which the fang is half-grown; then introduce the point of a penknife into its open extremity, and scraping the inner surface, detach suall portions, which exhibit the globules admirably." 1

The cementum is the

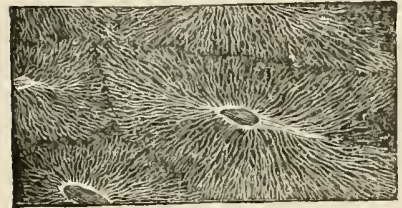

Fig. 344.-Transverse section of Tooth of Myliobates, Eagle-ray, viewed as an opaque object to show its radiating fibrous structure. cortical layer of osseous tissue, forming an outer coating to the fangs, which it sometimes cements together. It commences as a very thin layer at the part where the enamel ceases, and increases in thickness towards the ends of the fangs. Its internal surface is intimately united with the dentine, and in many teeth it would appear as if the earliest determined arrangement of the fibres of the dentine started from the canaliculi, as they radiate from the lacunæ in the cement. The interlacunar layer is often striated, and exhibits a laminated structure: sometimes it appears as if Haversian canals were running in a perpendicular direction to the pulp cavity. The canaliculi frequently run out into numerous branches, connecting one with another, and anastomising with the ends of the dentine fibres. The thick layers of cement which occur in old teeth show immense quantities of aggregated lacunæ of an irregular and elongated form. Professor Owen believes that by age the pulp ceases to produce or nourish the dentine, which then becomes converted into osteo-dentine, and thereby the layer of crusta is so much increased as often to fill up the pulp cavity of tho tooth. Professor Simonds assures us that this is not the case in the Herbivora. For instance, in the horse, the oblitera-

(1) Czermak: translated by James A. Salter, M.B., Quarterly Journal of Mi icroseopical Science, July, 1S:3. 
tion of the cavity is gradually effected by an increased formation of dentine; and this is not supplanted by ar abnormal or diseased growth, as would be the case wero the pulp to become ossified, but as the pulp diminishes, so is the supply of nutriment to the tooth lessened, and at length entirely cut off from the interior. "To provide for the vitality of the tooth under these circumstances, the crusta increases in quantity on the fang, at the expense of the perfectly-formed dentine, which is lying in immediate contact with its iuner surface. Through the medium of the canals in the crusta, which open on its borders, the tooth now draws its nourishment from the bloodvessels of the socket; and thus it continues, long after the obliteration of its pulp cavity, to serve all the purposes as a part of the living organism." I

Bone.-The elements of bone are lamellæ and small corpuscles; the latter are possibly merely spaces between the former, in which is deposited the earthy substance. The lamellæ have for their basis a cartilaginous substance combined with earthy matter, or salts. These salts are chemically combined with the organic basis. Acids dissolve only the earthy salts, and leave the organic basis of the same form as the bone itself. The lamella are homogeneous throughout, like the intercellular substance of cartilage, but chemically different, being resolved by boiling in water into colla, whereas cartilage is resolved into chondrine.

(1) Professor Simonds, on the "Structure and Development of Teeth of Arimals." 
Professor Quekett's paper in an early number of the

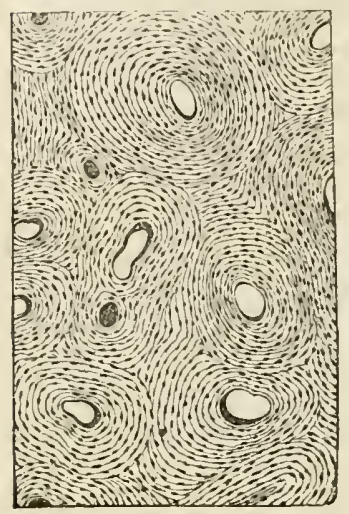

Fis. 316-The same, viewed under a lower power, appear to be a series of small black dots.

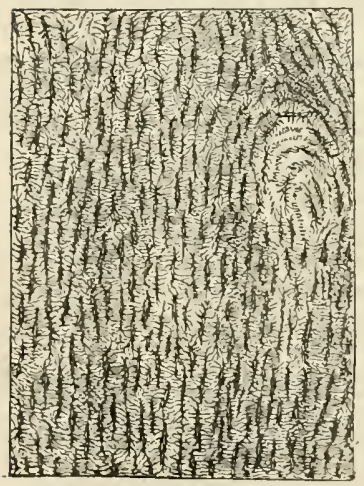

Fig. 347.-A transverse section of the Humerus, or fore-arm bone, of a Turtle (Chelonia mydas). It exhibits traces of Haversian canals, with a slight tendency to a concentric arrangement of bone-cells around them. The bone-cells are large and very numerous, but occur for the most part in parallel I0WB.
Micros. Soc. Trans. gave an excellent account of the "Intimate structure of Bone." To this paper we are indebted for the following microscopical investigation of bone :-

Bone consists of a hard and soft part; the hard is composed of carbonate, phosphate, and fluate of lime, and of carbonate and phosphate of magnesia, deposited in a cartilaginous or other matrix; whilst the soft consists of that matrix, and of the periosteum which invests the outer surface of the bone, and of the medullary membrane which lines its interior or medullary cavity, and is continued into the minutest pores. If we take for examination a long bone of one of the extremities of the human subject, or of any mammalian animal, we shall find that the bony substance, or shaft, is slightly porous, or rather occupied, both on its external and internal surfaces, by a series of very minute canals, which, from their having been first described by our countryman Clopton Havers, are termed to this day the Haversian canals, and serve for the transmission of blood-vessels into the interior of the bone. If now a thin transverse section of the same bone be made, and be examined by the micro- 
scope with a power of 200 linear, we shall sce the Haversian canals very plainly, ancl around them a series of concentric bony lamine, from three to ten or twelve in number. If the section should consist of the entire circle of the shaft, we shall notice, besides the concentric laminx round the Haversian canals, two other series of laminæ, the one around the outer margin of the section, the other round the inner or medullary cavity. Between the laminæe is situated a concentric arrangement of spider-like looking bodies, which have, by different author's, received the name of osseous corpuscles, lacunæ, or bone-cells, according as to whether they were ascertained to be solid or hollow: these bone-cells have little tubes or canals radiating from them, which are termed canaliculi. The average length of the lacunæ, or bone-cells, in the human subject is the $1-2,000$ th of an inch; they are of an oval figure, and somewhat flattened on their opposite surfaces, and are usually about one-third greater in thickness

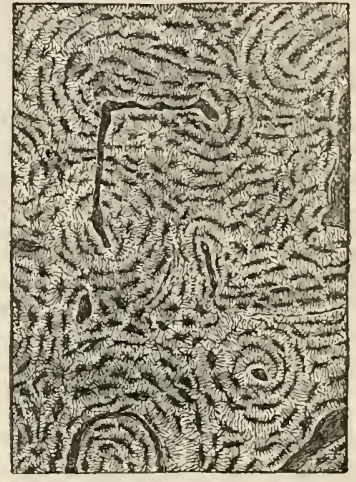

Fig. 348.-A transverse section lof the Femur, or leg-bone of an Ostrich (magnified 95 diameters). When contrasted with the preccding figure, it will be noticed that the Haversian canals are much smaller and more numerous, aud many o. them run in a transverse direction. than they are in breadth; hence, as will be presently shown, it becomes necessary to know in what direction a specimen is cut, in order to judge of their comparative size. The older anatomists supposed them, from their opacity, to be little solid masses of bone; but if the section be treated with spirits of turpentine coloured with alkanet-rcot, or if it has been soaked in very liquid Canada balsam for any great length of time, it can then be unequivocally demonstrated that both these substances will gain entrance into the bone-cells through the canaliculi. The bone cells, when viewed by transmitted light, for the most part appear perfectly opaque; and they will appear the more opaque the nearer the section of 
them approaches to a transverse one: for when the cells are cut through their shorter diameter, they are often of such a depth that the rays of light interfere with each other in their passage through them, and darkness results; whereas if the section be made in the long diameter of the cells, they will appear transparent. When viewed as an opaque object, with a dark ground at the back and condensed light, the bone-cells and canaliculi will appear quite white, and the intercellular substance, which was trans. parent when viewed by transmitted light, is now perfectly

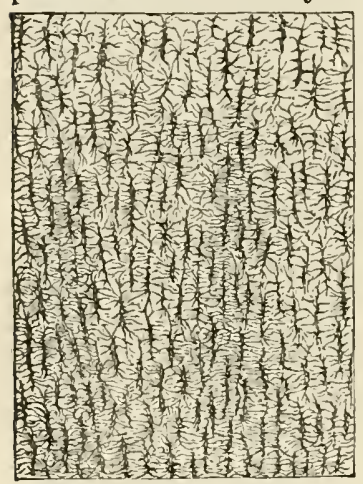

Fig. 349.-A horizontal section of the lower Jaw-bone of a Conger eel, which exhibits a single plane of bone-cells arranged in parallel lines. There are no Haversian canals present: and when this specimen is contrasted with that of fig. 347 , it will be noticed that the canaliculi given off from each of the bone-cells of this fish are very few in number in comparison with that of the reptile. dark. The soft part consists of the periosteum, which invest the outer, and of the medullary membrane, which invests the inner surface, lines the Haversian canals, and is continued from them, through the canaliculi, into the interior of the bone-cells; and of the cartilaginous or other matrix, which forms the investment of the minute ossific granules. The earthy matter of the bone may be readily shown by macerating the section for a short time in a dilute solution of caustic potash. The animal matter may be procured by using dilute hydrochloric acid instead of caustic potash ;when all the earthy matter is removed the section will exhibit nearly the same form as when the earthy constituent was present; and if then viewed microscopically, it will be noticed that all the parts characterising the section previous to its maceration in the acid will be still visible, but not so distinct as when both constitutents were in combination. When, however, the animal matter is removed, the bone will not exhibit the cells and the canaliculi, but is opaque and very brittle, and nothing but the Haversian canals and a 
granular structure can be seen. The parts which a transverse or a longitudinal section of a long bone of a mammalian animal exhibits, will be the Haversian canals, the concentric bony laminæ, the bonecells and their canaliculi; even these, except the bony laminie, may be seen in all mammalian bones (fig. 345). Whether long or otherwise, they are, nevertheless, so differently arranged in the flat bones, such as those of the skull, and in the irregular bones, as the vertebræ, as to require notice. Those of the head are composed of two thin layers of compact texture; enclosed between which is another layer of variable thickness, of a cellular or cancellated structure. The two outer layers are called tables-the one being the outer, the other the inner table; and the middle or cancellated layer is termed the diploe: in this last the principal blood-vessels ramify. The outer table of the skull is less dense than the inner; the latter, from its brittleness, is termed by anatomists the vitreous table. When a vertical section of a bone of the skull is made so as to include the three layers above mentioned, bone-cells may be seen in all ; but each

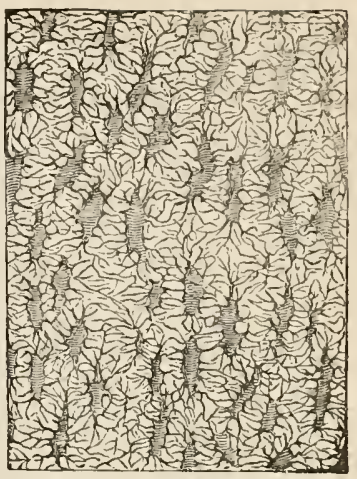

Fig. 350.-A portion of the Cranium of a Siren (Siren lacertina), remarkable for the large size of the bone-cells and of the canuliculi, which are larger in this animal than in any other yet examined. As in the proceding specimen, no Haver sian canals are present.

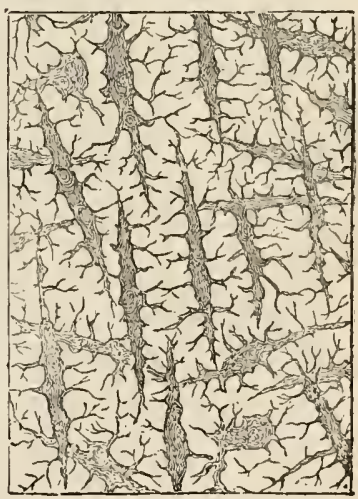

Fig. 351. $-A$ small portion of bone taken from the exterior of the shaft of the Humerus of a Pterodactyle; this exhibits the elongated bone. cells characteristic of the order Reptilia. of the three layers differ in structure: the middle or can- 
cellated structure will be found to resemble the cancellated structure in the long bones-viz. thin plates of bone, with one layer of bone-cells without Haversian canals; the outer layer will exhibit Haversian canals of large size, with bonecells of large size, and a slightly laminated arrangement; but the inner or vitreons layer resembles the densest bone, as the outer part of the shaft of a long bone, for instance, and will exhibit both smaller Haversian canals and more numerous bone-cells of ordinary shape around them.

A transverse section of the long bone of a bird, when contrasted with that of a mammal, exhibits the following

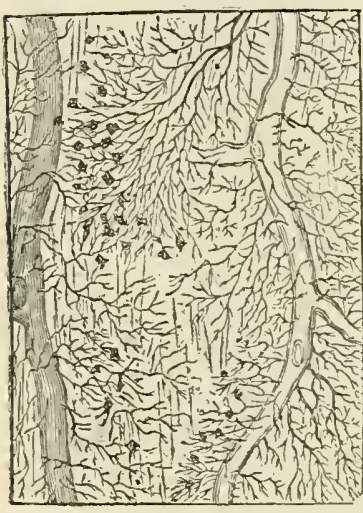

Fig. 352.-A horizontal section of a scale, or flattened spine, from the exhibits large Haversian canals,

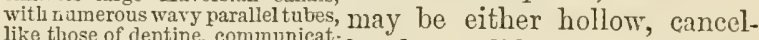
like those of dentine, eommunicat. lated, or solid; but the specific
ing with them. This specimen late shows, besides these wavy tubes, gravity is not so great as that
numcrous bone-cells, whose cana-grat liculi communicate with the tubes, of birds or mammals. The as in many specimens of dentine. short bones of most of the Chelonian reptiles are solid, but the long bones of the extrenities are either hollow or cancellated; the ribs of the Serpent tribe are hollow, the medullary cavity performing the office of an Haversian canal; the bone-cells are accordingly arranged in concentric circles around the canal. The vertebre of these animals are solid; and the 
bone, like that of some of the birds, is remarkable for it density and its whiteness. When a transverse section is laken from one of the long bones, and contrasted with that of a mammal or bird, we shall notice at once the difference which the reptile presents: there are very few, if any, Haversian canals, and these of large size; and at one view, in the section, fig. 347 , we shall find the canals and the bone-cells arranged both vertically and longitudinally: the bone-cells are most remarkable for the great size to which they attain; in the Turtle they are 1-375th of an inch in length, the canaliculi are extremely numerous, and are of a size proportionate to that of the bone-cell.

In fishes we have a greater variation in the minute structure of the skeleton than in either of the three classes already noticed. Of all the varieties of structure in the bones of fishes, by far the greater number exhibit nothing more than a series of ramifying tubes, like those of teeth; others exhibit Haversian canals, with numerous fine tubes or canaliculi, like ivory tubes, connected with them; a few consist of Haversian canals, with fine tubes and bonecells, fig. 349; and a rare form, found only as yet in the sword of the Swordfish (Istiophorus), exhibits Haversian canals and a concentric laminated arrangement of the bone, but no bone-cells. The Haversian canals, when they are present, are of large size, and very numerous, and then the bone-cells are, generally speaking, either absent or but few in number; their place being occupied by tubes or canaliculi, which are often of a very large size. The bone-cells are remarkable for their graduate figure, and the canaliculi which are derived from them are few in number; they are seen to anastomose freely with the canaliculi given off from neighbouring cells; and if the specimen under examination is a thin layer of bone, such as the scale of an osseous fish, from the cells lying nearly all in one plane, the anastomoses of the canaliculi are seen beautifully distinct. In the hard scales of many of the osscous fishes, such as the Lepidosteus and Calicthys, and in the spines of the Siluride, the bone-cells are beautifully seen; in the true bony scales comprising the exo-skeleton of the cartilaginous fishes, the bone-cells are 
to he seen in great numbers. In the spines of some of the Ray family may be noticed a peculiar structure : the Haversian canals are large and very numerous, and communicating with each canal are an infinite number of wavy tubes, which are connected with the canals in the same manner as the dentinal tubes of the teeth are connected with the pulp-cavity; and if such a specimen were placed by the side of a section of the tooth of some of the Shark tribe, the discrimination of one from the other would be no easy matter. In the spine of a Ray, fig. 352, the analogy between bone and the ivory of the teeth is made more evident; for in this fish we have tubes, like those of ivory, anastomosing with the canaliculi of bone-cells.

Now, if we proceed at once to the application of the facts which have been laid down, and make a fragment of bone of an extinct animal the subject of investigation; we first find that the bone-cells in Mammalia are tolerably uniform in size ; and if we take 1-2,000th of an inch as a standard, the bone-cells of birds will fall below that standard; but the bone-cells of reptiles are very much larger than either of the two preceding; and those of fishes are so entirely different from all three, both in size and shape, that they are not for a moment to be mistaken for one or the other; so that the determination of a minute yet characteristic fragment of fishes' bone is a task easily performed. If the portion of bone should not exhibit bone-cells, but present either one or other of the characters mentioned in a preceding paragraph, the task of discrimination will be as easy as when the bone-cells exist. IVe have now the mammal, the bird, and the reptile to deal with; in consequence of the very great size of the cells and their canaliculi in the reptile, a portion of bone of one of these animals can readily be distinguished from that of a bird or a mammal; the only difficulty lies between these two last: but, notwithstanding that on a cursory glance the bone of a bird appears very like that of a mammal, there are certain points in their minute structure in whicb they differ; and one of these points is in the difference in size of their bone-cells. To determine accurately, therefore, between the two, we must, if the section be a transverse one, also note the comparative sizes of the Haversian 
canals, and the tortuosity of their course; for the diameter of the canal bears a certain proportion to the size of the bone-cells, and after some little practice the eye will readily detect the difference.

A curious modification of horn is presented in the appendage borne by the Rhinoceros upon its snout, which in many points resembles a bundle of hairs. When a transverse section is made and viewed by polarised light, each cylinder is seen to have a cross diverging from a central spot; the lights and shadows of this cross are replaced by bands of contrasted complementary colours, if the selenite plate is interposed (fig. 353). See Plate VIII. No. 178. Whale-Fig. 353-Transvcrse section of Itwm bone is almost identical in light. structure, and is similarly affected by polarised light.

A knowledge of the form and structure of scales of fishos (fig. 354), like that of teeth, has been "shown by M. Agassiz to afford an unerring indication of the particular class to which the fish may belong: in the examination of fossil remains, the application of this knowledge has been attended with extraordinary results. As a class of objects for the microscope, the scales of fishes are exceedingly curious and beautiful, especially when mounted in fluid or Canada balsam, and viewed by polarised light. Many are seen best as opaque objects, and are then mounted dry between

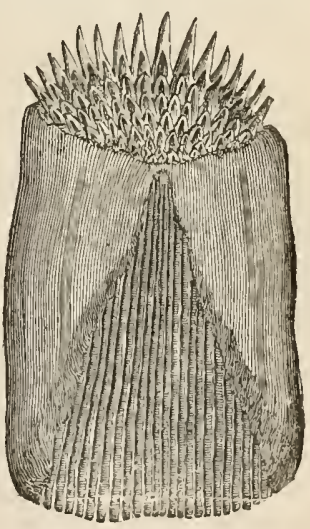

Fig. 354.-Scale of Sole. glasses. МГ. Agassiz divides the scales into four order's, which he names Placoid, Ganoid, Ctenoid, and Cycloid; in the first two the scales are more or less coated with 
enamel, in the others they are of a lorny nature. 'To the Placoid order belong the Skates, Dog-fish, Ray, and Sharks; cartilaginous fishes, having skins covered with small prickly or flattened spines. To the Ganoid belong the Sturgeon, Lepidosteus, Hassar-fish, and Polypterus; fishes of this order are more generally found in a fossil state, and the scales are of a bony character. To the Ctenoid belong the Pike, Perch, Pope, Basse, IVeaver-fish, \&c. ; their scales are notched like the teeth of a comb. To the Cycloid belong the Salmon, Herring, Eel, Carp, Blemny, and the majority of our edible fishes; their scales are circular and laminated. The scales of the Eel tribe are of an oral figure, and are among the most remarkable that can be selected for microscopic examination. To procure them, a sharp knife must be passed beneath the epidermal layer, and a portion of it raised, in a similar manner as directed for tearing off the cuticle from plants: after $a$ few trials some will be detached. They are of an oval figure, rather softer than the scales of other fishes, and in some parts of the skin do not form a continuous layer. When the skin has been stripped off, previous to the fish being cooked. the scales can be obtained from the under surface, with a lnife or pair of forceps. The scales of the viviparous Blenny are of a circular figure, situated under the epidermal layer; they were described by Mr. Yarrell as mucous glands, from their figure and small number. The surface of the skin of this fish, when fresh, appears to be covered with follicles ; if, however, a portion be scraped off, it will be found to be a mass of delicate circular scales. A piece of the skin, when dried, exhibits the scales to great advantage, and, like those of the Eel, are beautiful objects for polarised light. The prismatic colours exhibited by fish are said to be due to the presence of fatty matter in the skin; but the beautiful metallic tints displayed by so many of them are rather due to the numerous microscopic plates, or scales, distributed over its surface.

Having thus brought our brief examination of a few of the more important structures of the animal economy to a close, it only remains for us to express a hope that it will be found to smooth the way, or in some degree assist the investigations of the student to a better and more 
general survey of the whole fabric. Such a survey will not be unattended with its difficulties and disappointments, but it will bring its own reward for any amour:t of labour bestowed. To the medical student, desirous of obtaining further information in his especial department of microscopy, we recommend Dr. Beale's book on "The Microscope, and its Application to Clinical Medicine." 1

The importance of becoming thoroughly familiar with the structural and microscopical characters of any particular organ in a healthy condition, cannot be too strongly urged upon the attention of the student; as to a want of this knowledge must be attributed many erroneous descriptions of morbid appearances. All who wish to use the microscope successfully, with reference to the examination of organs in a diseased state, will do well to acquaint themseives with minute anatomy generally, not ouly of the human subject, but of the lower animals; without such knowledge it will be found impossible to study pathology, or prosecute pathological inquiries with any degree of success.

A large amount of wrong observation has been recorded on cells and cellular structures : since Schwann announced his "cell theory," almost everything round has been regarded as a cell; any single body within this, or where there are several, the largest, has been regarded as a nucleus, and any spot within the nucleus has been viewed as a nucleolus. Whereas many of the so-called cells are homogeneous spheres; many of the nuclei are vacuoles, and so forth.

Such errors are natural, at first inevitable; they can be corrected only by practice, by testing observations in other ways, especially by chemical re-agents, and by comparison with the observations of others. "The marvel is not that the microscope should suggest false views-do not our eyes play us that trick?-but that it should reveal so many astounding facts as it really does; and the one con-

(1) The Cyclopoedia of Anatomy and Physiology will be found a most valuable book of reference for the student in all matters relating to physiology and minute anatomy. Numerous valuable papers are distributed throughout the Trans. of the Royal Micros. Soc. : Huxley's Lectures on Comparative Anatomy: Owen's Lectures on Comparative Anatomy; Carpenter's Physiology, edited by $\mathbf{H}$. Power, and Kölliker's Manual of Human Microscopic Anatomy. 
solatory reflection which accompanies the difficult task of microscopic investigation is the unanimity which now reigns among observers on so vast a body of observations. If we read in physiological works of the yolk cells and coloured oil globules of the yolk, and the beautiful function of assimilation which has been attributed to them, they exist but in the imagination of the authors who have regarded the one as cells simply because they are round, and the other as consisting of fat because they are highly refractive, - such errors of interpretation do not discredit it any more than the mis-interpretations, which have helped to make Ehrenberg's name at once famous and suspicious, alter the facts which he saw, and could not rightly interpret. In truth, the eye is only a preliminary instrument in science. What we see has to be interpreted; and, as it is very difficult to confine ourselves to pure observation unmixed by hypothetical interpretation, we need many collateral confirmations."

The principal physical characters to be regarded in microscopic examinations may be summed up as follows :-

1. Shape.-Accurate observation of the shape of bodies is very necessary, as many are distinguished by this physical property. Thus the human blood-globules present a round biconcave dise, and are in this respect different from the oval corpuscles of birds, reptiles, and fishes. The distinction between round and globular is very requisite. Human blood corpuscles are round and flat; but they become globular on the addition of water. Minute structures seen under the microscope may also be likened to the shape of well-known objects, such as that of a pear, balloon, kidney, heart, \&c.

2. Colour.-The colour of structures varies greatly, and often differs under the microscope from what was previously conceived regarding them. Thus the coloured corpuscles of the blood, though commonly called red, are, in fact, yellow. Many objects present different colours, according to the mode of illumination; that is, as the light is reflected from or transmitted through their substance, as in the case of certain scales of insects, feathers of birds, \&c. Colour is often produced, modified, or lost, by re-agents ; as when iodine comes in contact with starch-granules, when 
nitric acid is added to chlorophyle, or chlorine-water to the pigment-cells of the choroid, and so on.

3. Edge or Border.-This may present peculiarities worthy of notice. Thus, it may be dark and abrupt on the field of the microscope; so fine as to be scarcely visible; or it may be smooth, irregular, serrated, beaded, \&c.

4. Size.-The size of the minute bodies, fibres, or tubes, which are foumd in the various textures of animals, can only be determined with exactitude by actual measurement. It will be observed, for the most part, that these minute structures vary in diameter; so that when their medium size cannot be determined, the variations in size from the smaller to the larger should be stated. Human blood-globules in a state of health have a pretty general medium size, and these may consequently be taken as a standard with advantage, and bodies described as being two, three, or more times larger than this structure; or all may be measured with a micrometer, as explained at page 51 .

5. Transparency.-This physical property varies greatly in the ultimate elements of numerous textures. Some corpuscles are quite diaphanous; others are more or less opaque. The opacity may depend upon corrugation or irregularities on the external surface, or upon contents of different kinds. Some bodies are so opaque as to prevent the transmission of the rays of light; in this case they look black when seen by transmitted light, though white if viewed by reflected light; others, such as fatty particles and oil-globules, refract the rays of light strongly, and present a peculiarly luminous appearance.

6. Surface.-Many textures, especially laminated ones, present a different structure on the surface from that which exists below. If, then, in the demonstration, these have not been separated, the focal point must be changed by means of the fine adjustment. In this way the capillaries in the web of the Frog's foot may be seen to be covered with an epidermic layer, and the cuticle of certain minute Fungi or Infusoria to possess peculiar markings. Not unfrequentiy, the fracture of such structures enables us, on examining the broken edge, to distinguish the difference in structure between the surface and the deeper layers of the tissue under examination. 
7. Contents.-The contents of those structures which consist of envelopes, as cells, or of various kinds of tubes, are very important. These may consist of included cells or nuclei, granules of different kinds, pigment matter, or crystals : a fair illustration of the changes effected by disease is given in fig. 256, from a cyst in a diseased liver: occasionally their contents present definite moving currents, as in the cells of some vegetables; or trembling rotatory molecular movements, as in the ordinary globules of saliva in the mouth.

8. Effects of Re-agents. -These are most important in determining the structure and chemical composition of numerous tissues. Thus water generally causes cell-formations to swell out from endosmosis; while syrup, gumwater, and concentrated saline solutions, cause them to collapse from exosmosis. Acetic acid possesses the valuable property of dissolving coagulated albumen, and in consequence renders the whole class of albuminous tissues more transparent. Thus it operates on cell-walls, causing them either to dissolve, or become so thin as to display their contents more clearly. Ether, on the other hand, and the alkalies, operate on fatty compounds, causing their solution and disappearance. The mineral acids dissolve most of the mineral constituents that are met with; so that in this way we are enabled to tell with tolerable certainty, at all events, the group of chemical compounds to which any particular structure may be referred.

Many, if not all, animal structures require examination in several ways before an accurate idea of their general structure can be obtained. It is of much importance to examine a body by reflected light, transmitted light, and by polarised light; when immersed in water, or in a highly refracting fluid, such as glycerine, oil, turpentine, and Canada-balsam; with a cover and without a cover, with the application of distilled water and of chemical re-agents. The most scrupulous cleanliness must always be observed in microscopical examinations; many crrors have arisen in consequence of a want of sufficient care not having been taken to prevent the admixture of various accidental substances. The better way of avoiding errors from this canse is to become familiar with the characters 
of common substances which are likely to be mixed up with preparations, as the following:-oil globules, air bubbles, portions of worsted, cotton, and linen, silk and wool fibres, hairs from plants, vegetable tissues, human hair, portions of feathers, and the starches-wheat and potato starch from bread crumbs. In taking fluids from bottles and vessels, the possibility of mixing small portions of their contents must be avoided; the pipette should be washed immediately after it has been used. Another fallacy arises from the great transparency of some structures ; a membrane may appear perfectly elear and transparent when in reality it is covered with a delicate layer: of epithelium, which only becomes visible after the application of some chemical re-agent; on the other hand, by the action of re-agents, a fibrous appearance is produced. Acetic acid, when added to many preparations, frequently produces a swelling of the tissue, which looks like basement membrane, but which in reality has been formed by the action of the acid. The mechanical pressure of the thin glass, if pressed down tightly, alters structures very much; the appearance of the blood dises are, at times, much distorted in this way, and lead to false conclusions and erroneous descriptions.

Should it be desirable to make an examination of the vital fluid, blood, the smallest drop, caused by the prick of a fine needle, may be placed on a strip of glass, and waved backwards and forwards, that the blood may dry as quickly as possible; in this way the corpuscles or blood-discs retain their form ; and if the preservation of the specimen is wished, a thin glass cover carefully placed over it, and cemented down, in the way directed at page 217.

To the advanced observer, the examination of the mucous membrane will afford some instruetion. Should the specimen be small, it will be better to pin it to a piece of cork; then well wash it by means of a small syringe. If the investing epithelium is required for examination, a portion can be detached from the surface by a knife; when placed on a glass slide, add a drop of iodine solution, then view it with a $\frac{1}{4}$-inch power. Villi and papillæ are best made out in injected specimens, as will be seen on reference to Plate VII. 


\section{CHAPTER VI.}

THE MINERAL KINGDOM.

IXORGANIC MATTER-FORMATION OF CRYSTALS-POLARIBATION, ETC.

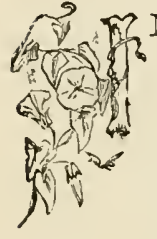

$\mathrm{N}$ our meagre search through organic nature we met with an endless variety of beautiful and instructive materials for the employment of the microscope. If we now turn our attention to the inorganic or mineral kingdom we shall find a vast storehouse filled with objects of unsurpassed interest to the microscopist. In the examination of the many beatiful forms presented to us in the phenomena of crystallisation, and the study of the varied chemical combinations, the student will discover a never-ending source of useful occupation.

We are as yet in great ignorance of the manner in which the majority of crystals belonging to the mineral kingdom are formed: we know, however, that very few can be reproduced by the chemist. But, although ignorant of the means whereby the great majority of crystals have been formed in the vast laboratory of nature, we can crystallise an immense number of substances, watch their numerous intricate modes of formation; and that in the smallest appreciable quantities, when aided by the microscope. Among natural crystals those employed in the formation of rocks open up a wide field to our view. The varieties of granites present us with the earliest crystallised condition of the earth's crust as it cooled down, the structure of which is beautifully exhibited under polarised light. Plate VIII. No. 160 , is a section of a graviłe 
Indurated Sundstone, Howth.

Iudurated Sandstone, Bromsgrove. Gibraltar rock.

Granite, various localities. No. 160.

Hornblend Schist.

Labrador Spar.

Norway Rock.

Quartz Rock, yarious. No. 159.

Quartzite, Mont Blanc.

Sandstone, Plate VIII. No. 158.

Satin Spar.

Selenites, various colours.

Tin Ore, with Tourmalin.

\section{VEGETABLE SUBSTANCES.}

Çuticle of Leaf of Correa Cardinaliz.

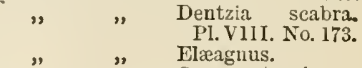

Equisetum. "No. 174.

Fibro cells from orchid. No. 169.

" Oncidium bicallosum.

Scalariform vessels from Fern.

Seyllium. No. 177.

Silicious Cuticles.-Various.

Starch.-Various. No. 167.

Very interesting results will be obtained by combining two or more ehemical salts. Mr. Davies ${ }^{1}$ succeeded in forming numerous beautiful double salts in the following manner. To a nearly saturated solution of the sulphate of copper and sulphate of magnesia add a drop, on the glass-slicle, and dry quickly. To effect this, heat the slide so as to fuse the salts in its water of crystallisation, and there remains an amorphous film on the hot glass. Put the slide aside and allow it to cool slowly; it will gradually absorb a certain amount of moisture from the air, and begin to throw out crystals. If now placed under the microscope, numerous points will be seen to start out here and there. The starting points may be produced at pleasure by touching the film with a fine needle point, so as to admit of a slight amount of moisture being absorbed by the mass of salt. Development is at once suspended by applying gentle heat; cover the specimen with balsam and thin glass. The balsam should completely cover the edges of the thin glass circle, otherwise moisture will probably insinuate itself, and destroy the form of the crystals.

Mr. Thomas succeeded in crystallising "the salts of the magnetic metals" at very high temperatures, which gave interesting results, and produced eurious forms of crystals. Plate VIII. No. 163 are representations of crystals of sulphate of iron and cobalt, No. 165, of nicke? and potash, obtained in the following manner :-To form the sulphate of iron crystal, add to a concentrated solution of iron a small quantity of sugar, to prevent oxidation. Put a drop of the solution on a glass slide, and drive out the 
water of crystallisation as quickly as possible, by the aicl of a spirit lamp; then with a Buusen's burner bring the plate to a high temperature. Immediately a remarkable change is seen to take place in the form of the crystal, and if properly managed the "fulration" represented in the plate will be fairly exhibited. The slide inust not be allowed to cool down too rapidly or the crystals will probably absorb moisture from the atmosphere, and in so doing the crystals alter their forms. Immerse them in balsam, and cover in the usual way before quite cold.

Sublimation of Alkaloids.-Dr. Guy a fow years ago directed the attention of microscopists to the fact that the crystalline shape of bodies belonging to the inorganic world might lead to their detection. Subsequently, Dr. A. Helwig, of Mayence, took the matter up, and showed that the plan was applicable not only to inorganic but also to organic substances, and especially to the poisonous alkaloids. By improving on Dr. A. Helwig's process, and substituting a bit of porcelain, Dr. Guy has been able to watch the process more minutely, and to regulate it more exactly. He has by this means been able to obtain characteristic crusts composed of crystals of strychnine weighing not more than 1-3,000th or 1-5,000th of a grain. Morphia gives equally characteristic results. For the examination of these, Dr. Guy recommends the use of a binocular microscope with an inch object-glass. But it is not to crystalline forms alone that one need trust; the whole behaviour of a substance as it melts and is converted into vapour is eminently characteristic, and when once deposited on the microscopical slide, under the objectglass, the application of re-agents may give still more satisfactory results. The re-agents, however, which are here to be applied are not of the kind ordinarily employed. Colourtests under the microscope are, comparatively speaking, useless: those that give rise to peculiar crystallime forms are rather to be sought after. For instance, the crystals produced by the action of carbazotic acid on morphia are by themselves almost perfectly characteristic. These experiments should not, however, be undertaken for medicolegal purposes by one unskilled in their conduct, for the effects of the re-agents themselves might be mistaken by 
the uninitiated for the result of their action on the substances under examination.

Dr. Guy's ${ }^{1}$ method of procedure is as follows :- "Pror vide small crucibles, covers, slabs, or fragments of white porcelain ; a few microscopic cell-glasses, with a thickness of about one-eighth of an inch and a diameter of circle of about two-thirds of an inch : and discs of window-glass about the size of a shilling. Place the porcelain slab on the ring of a retort-holiler or other convenient support; then the glass cell ; and upon the porcelain in the centre of the cell a minute portion of the alkaloid or other white powder, or crystal reduced to powder; then pass the clean glass dish through the flame of the spirit-lamp till the moisture is driven off, and adjust it with the forceps over the glass ring; now apply the flame of the spirit-lamp to the porcelain, underneath the powder or crystal, and continue the heat till the powder undergoes its characteristic change and gives off vapour. Watch the deposit of this vapour on the glass dish, and remove the spirit-lamp, either directly or after a short interval, as experience may determine.

"The white surface of porcelain being visible through the glass dise, as through a window, the behaviour of the substance under examination is easy to observe. It may be driven off without undergoing change or leaving residue, and the disc may be covered with crystals, as happens with arsenious acid, or with an amorphous sublimate, as happens with calomel; it may coalesce, throw out long silky crystals, to be gradually transferred as crystals to the glass disc, as is the case with corrosive sublimate; and it may melt, with or without previous change of colour, retain or shift its place, deposit carbon more or less abundantly, and yield a sublimate of detached crystals (veratrine), twigs (solanine), tufts (meconine), branching patterns (strychnine, morphine, cryptopia, \&c.), watered patterns with or without crystalloids (several alkaloids and glucosides), the melting and deposition of carbon being a common property of the alkaloids and of some analogous active principles.

(1) Dr. W. A. Guy, F.R.S. \&c. on the "Sublimation of the Alkaloids."Pharmaceutical Journal, June and August, 1867. Micros. Jour. Dec. 1867 
"The principal precaution to be observed in the application of heat is, that it should be moderate and gradual. It is best to act on the assumption that the substance under examination may be one of a considerable group of bodies, some of which siblime at very moderate temperatures. The spirit-lamp should, therefore, be placed at first three or four inches below the slab of porcelain, so that the point of its flame may not touch it; and if, under this low temperature, the disc of glass is not dimmed, the lamp should be raised by degrees till the mist makes its appearance. Then, as a general rule, the lamp should be withdrawn, the disc removed, and a new one put in its place. It may be well to state that, as the disc has been passed through the flame to drive off moisture, and has in this way been heated, the flame of the spirit lamp should not be allowed to play on the porcelain slab after the mist has appeared on the disc, at least not for any length of time; for, if this precaution be neglected, it may happen with the alkaloids as with arsenious acid or corrosive sublimate, that the mist does not form at all, or that it is driven off as soon as it is deposited. Perhaps, too, it may not be quite unnecessary to recommend that each dise of glass, as it is removed, should be placed with the sublimate upwards against a glass slide or fragment of porcelain; and that in this position (sublimate upwards) it should be retained. If this very simple precaution be overlooked, it is quite possible that we may mistake one surface for the other, and find ourselves applying our re-agents to the wrong one. The chief precaution relating to the examination and disposal of the sublimates consists in measures for preserving their identity during the examination to which we may have to subject them. This is best done by writing the names and that of the reagents on dises of paper, and placing paper and disk together in sunken grooves or circular spaces.

"As a precaution omitted by an experimenter so practised as Dr. Helwig is very likely to be overlooked by others, it is well to insist upon and to prescribe as the first step to be taken with a crystalline solution which wo are about to use as a test, the determination of its proper crystalline form or forms as evaporated on a flat surface of 
glass. Another mistake, arising out of a similar want of caution, may consist in confounding the effect of some saline re-agent with that of the water which holds it in solution.

"Now these remarks have a direct practical bearing on the selection of tests. A preference ought to be given to re-agents which leave no residne of their own : to distilled water, to alcohol, ether, chloroform, benzole, and fusel oil; and to acetic acid and the dilute mineral acids. Then those salts should be preferred of which the solutions yield dry residues of one or two definite forms, not such as put on many different shapes, are deliquescent themselves, and are likely to leave moist and unstable compounds. Nor is the strength of the solution a matter of little or no importance; for it should be borne in mind that the sublimates to which we apply them contain very minute fractions of a grain; and that a very strong solution, after acting on this minute quantity, would leave a coarse deposit of its own, both over the general surface and at the margin of the spot, which, blending with the reaction, would obscure and confuse it. As a general rule, therefore, solutions of a moderate strength are to be preferred, such as 1 grain of carbazotic acid to 250 of water, and 1 grain of bichromate of potash to 100 , the same of the red prussiate of potash, and of the nitro-prusside of sodium."

Other than the alkaloids and volatile metallic poisous were found to yield sublimates when heated, as urea, uric acid, hippuric acid, alloxan, uramile, \&c.; but these results scarcely prepared one to expect a sublimate from a blood-stain. Yet, on separating the fibres of a small spot of a cotton texture stained with blood about twenty-five years since, and submitting a section of the fibre an eighth of an inch long to heat, a figured pattern of the colour of blood was obtained, such as might be caused by a solution of blood in some thin oily liquid: this figured pattern was surrounded by a colourless border, having bright figured patterns such as those which mark the less characteristic portions of crystalline sublimates. Dr. Guy, on repeating his experiments, found the results constant; and on conducting them with care, and under the guidance of microscopic examinations, two sublimates were uniformly ob- 
tained, the first colourless and apparently crystalline, the second, under a high temperature, of the colour of the blood-stain from which it was procured, and of the figured pattern mentioned.

Mr. Sorby's attention has been directed to obtain a definite method of qualitative analysis of "animal and vegetable colouring matters," and of animal substances generally, by means of the spectrum microscope. He has also so combined the spectroscope with the binocular microscope as to make it available for the purpose of distinguishing minute portions of coloured minerals in thin sections of rocks and meteorites. $\mathrm{He}$ employs an ordinary large binocular microscope, with an object-glass of about threeinches focal length, corrected for looking through glass an inch thick; the lenses being at the top, and as far as possible from the slit. This objective is placed at the focus, and between it and the lenses, at a distance of about half an inch from them, is a compound prism, composed of a rectangular prism of flint-glass and two of crown-glass, of about $61^{\circ}$, one at each end. This arrangement gives direct vision and a spectrum of a suitable size for these inquiries, since a wide dispersion often produces indistinctness of the absorption bands. That we may have the opportunity of comparing two spectra side by side, a small rectangular prism is fixed over half the slit, and with the acute angle parallel to it and passing beyond it. ${ }^{1}$

"This gives an admirable result, the only defect being that, when the spectra are in focus, their line of junction is some distance within it ; and therefore to correct this employ a cylindrical lens of about two feet focal length, with its axis in the line of the slit, which can easily be fixed at such a distance between the slit and the prisms as to bring the spectra and their line of contact to the same focus. In front of the slit, close to the small rectangular prism, is a stop with a circular opening, to shut out lateral light, and a small achromatic lens of about half an inch focal length, which gives a better field, and counteracts the effect of the concave surface of the liquid in the tubes used in the experiments, if they are not

(1) Mr. Sorby's prisms and their arrangement have been entrusted to Mr. Browning's skilful hands, who is prepared to adapt them to any microscope. 
quite full. These are cut from barometer-tubes, having an internal diameter of about one-seventh of in inch, and an external diameter of about three-sevenths of an inch. They are made half an inch long, ground flat at each end, and fixed with Canada balsam on slips of glass two inches long and about six-tenths of an inch wide, so that the centre of the tube is about one-fourth of an inch from one edge. By this arrangement the liquid may be examined through the length of the tube by laying the slip of glass flat on the stage of the microscope, or through the side of the tube, by placing the slip vertical and the tube horizontal. Cells of this size can be turned upside down and deposits removed without any liquid being lost; and the upper surface of the liquid is sufficiently flat, even when inclined at a considerable angle. If requisite, small bits of thin glass can be laid on the top, which are held on by capillary attraction, or fastened on with gold-size, if it be desirable to keep the solution for a longer time. When the depth of colour is too great in the line of the length of the cell, we can at onee see what would be the effect of about one-fourth of the colour by turning it sideways; and thus we can save much time, and quickly ascertain what strength of solution would give the best result. Very frequently an excellent spectrum is obtained in one direction with one reagent, and in the other with another, without further trouble.

"The seale of measurement consists of two small Nicol's prisms, and an intermediate plate of quartz. If white light, passing through two such prisms, without the plate of fuartz, be examined with the spectrum-microscope, it of ourse gives an ordinary continuous spectrum; but if we place between the prisms a thick plate of quartz or sek nite, with its axis at $45^{\circ}$ to the plane of polarisation, thr r.gh no difference can be seen in the light with the naksd eye, the spectrum is entirely changed. The light is still white, but it is made up of alternate black and coloured bands, evenly distributed over the whole spectrum. The number of these depends on the thickness of the depolarising plate, so that we may have, if we please, almost innumerable fine black lines, or fewer broader bands, black in the centre and shaded off at each side. 
These facts are of course easily explained by the interference of waves. It would be impossible to have a more convenient or suitable scale for measuring the speetra of coloured solids and liquids. If we use a mierometer in the eyepiece, an alteration in the width of the slit modifies the readings, and the least movement of the apparatus may lead to error, whereas this scale is not open to either objection. Besides this, the unequal dispersion of the speetrum makes the blue end too broad, so that a given width, as measured with a micrometer in the eyepicee, is not of the same optical value as the same width in the red. The divisions in the interference-spectrum bear, on the contrary, the same relation to the length of the waves of light in all parts of the spectruu, and 110 want of adjustment in the instrument alters their position. As will be seen from the diagram (fig. 355), the unequal dispersion makes the distance between the bands in the blue about twice as great as in the red. The perfection of a spectrum would be one in which they were all at equal intervals; but possibly no such uniform dispersion could be produced. By having a direct-vision prism, composed of one of flintglass of $60^{\circ}$, and two of crown-glass of suitable angle, we can place it over the eyepiece, and can diminish the dispersion at the blue end, or increase that at the red end, by turning it in one position or the other, and thus see either end to the greatest advantage.

"Since the number of divisions depends on the thickness of the interference-plate, it became necessary to decide what number should be adopted. Ten it was thought would be most suitable ; but, on trying, it appeared to be too few for practical work. Twenty is too many, since it then becomes extremely difficult to count them. Twelve is as many as can be easily counted ; it is a number easily remembered, gives sufficient aceuracy, and has a variety of other advantages. With twelve divisions the sodium-line D comes very accurately at $3 \frac{1}{2}$; and thus, by adjusting the plate su that a bright sodium-light is hid in the centre of the band, when the Nicol's prisms are crossed, it is accurately at $3 \frac{1}{2}$, when they are arranged parallel, so as to give is wider field. The general eharacter of the seale will 
be best understood from the following figure, in which the bands are numbered, and given below the principal Fraunhofer lines. The centre of the bands is black, and

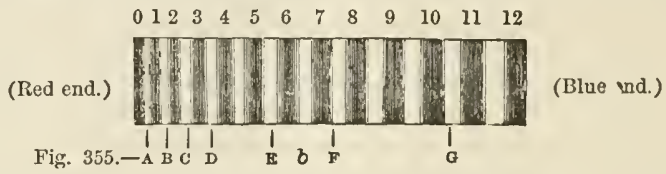

they are shaded off gradually at each side, so that the shaded part is about equal to the intermediate bright spaces. Taking, then, the centres of the black bards as $1,2,3, \&$ c., the centres of the spaces are $1 \frac{1}{2}, 2 \frac{1}{2}, 3 \frac{1}{2}$, \&c., the lower edges of each $\frac{3}{4}, 1 \frac{3}{4}, \& c$., and the upper $1 \frac{1}{4}, 2 \frac{1}{4}$, $\&$ c., we can easily divide these quarters into eighths by the eye; and this is as near as is required in the subject before us, and corresponds as nearly as possible to 1-100th part of the whole spectrum, visible under ordinary circumstances by gaslight and daylight. Absorption-bands at the red end are best seen by lamplight, and those at the blue end by daylight.

On this scale the position of some of the principal lines of the solar spectrum is about as follows :-
A....... $\frac{3}{4}$
B...... $1 \frac{1}{2}$
$b \ldots . . .6 \frac{3}{16}$
F....... $2 \frac{3}{8}$
D...... $3 \frac{1}{2}$
G..... $10 \frac{5}{8}$

At first plates of selenite, which are easily prepared, were used, because they can be split to nearly the requisite thickness with parallel faces; but its depolarising power varies so much with the temperature, that even the ordinary atmospheric changes alter the position of the bands. However, quartz cut parallel to the principal axis of the crystal is so slightly affected in this manner as not to be open to this objection, but is prepared with far greater difficulty. The sides should be perfectly parallel, the thickness about $\cdot 043$ inch, and gradually polished down with rouge until the sodium-line is seen in its proper place. This must be done earefully, since a difference of 1-10,000th inch in thickness would make it decidedly incorrect.

The two Nicol's prisms and the intervening plate are mounted in a tube, and attached to a piece of brass in such 
" manner that the centre of the aperture exactly corre. sponds to the centre of any of the cells used in the experiments, which are all made to correspond in such a manner that any of them, or this apparatus, may be placed on the stage and be in the proper place without further adjustment, which, of course, saves much time and trouble. ${ }^{1}$

In the preparation of vegetable colouring matters for the spectroscope, care must be taken to employ a small quantity of spirits of wine; filter the solution, and evaporate it at once to dryness at a very gentle heat, otherwise if we attempt to keep the colouring matters in a fluid state they quickly decompose. It is necessary to employ various reagents in developing characteristic spectra. The most valuable reagent is sulphite of soda, which admits of the division of colours into groups. Of the mode of applying reagents, ample directions are given.

Mr. Sorby's qualitative analysis is just the kind of thing to employ in detecting adulterations in many substances met with in commerce, as well as in inquiries where very small quantities of inaterial are ai command. By this method we might be able in a few minutes to form a very satisfactory opinion, or at least one that might meet all practical requirements, and narrow the inquiry to a surprising extent; if this can be said even now, surely further research cannot fail to make it most useful in cases where ordinary chemical analysis would be of little or no use; for in this way we may be able to detect the presence of chlorophyll in some of the lower animal forms; as the amœba, hydra, \&c., or, on the other hand, the red colouring matter of the blood, cruorine, in worms, moiluscs, and insects. A number of colouring matters can be obtained, by using ether, from sponges, polyzoa, and the crustaceans; these, if examined in this way, may give unexpected results.

For further information on this interesting subject we must refer the reader to Mr. Sorby's paper "On a Definite Method of Qualitative Analysis of Vegetable and Animal Colouring Matter by means of the Spectrum Microscope," published in the Proc. Roy. Soc. No. 92, 1867.

(1) See a paper by Dr. Gladstone, on the Spectra of Solutions of Salts. Quurt. Journ. Chem. Soc. vol. xi. p. 36 . 
It would be a vain attempt were we to try to convey to our readers any idea of the great discoveries which have been made by the microscope, or of the important purposes to which it has been applied. Second only to the telescope, though in many respects superior to it, the microscope transcends all other instruments in the scientific value as well as in the social interest of its results. While the human eye, the telescope and microscope combined, enables us to enjoy and examine the scenery around us, to study the forms of life with which we are more immediately connected, it fails to transport us into the depth of space, to throw into relief the planets and the stars, and to indicate the forms and arrangements in the worlds of life and motion, which distance diminishes and conceals. To these mysterious abodes, so long unrevealed, the telescope has at last conveyed us. It has shown us those worlds and systems, of which our own earth and our own system are the types; but it fails to enlighten us respecting the nature and constitution of the celestial bodies, and the forms of life for which they are created.

In its downward scrutiny, as well as in its upward aspirations, the human eye has equally failed. In the general view which it commands of animal, vegetable, and mineral structures, it cannot reach those delicate organizations on which life depends, or those structures of inorganic matter from which its origin and composition can be derived. Into these mysterious regions, where the philosopher has been groping his way, the microscope now conducts him. The dark abodes of unseen life are lighted up for his contemplation,-organizations of transcendent beauty appeal to his wonder-new aspects of life, new forms of being, new laws of reproduction, new functions in exercise, reward the genius of the theoretical and practical optician, and the skill and toil of the naturalist. With wonders like these all nature is pregnant : the earth, the ocean, and the air-times past and times present, now surrender their secrets to the microscope.

What we know at present, even of things the most near and familiar to us, is so little in comparison of what we know not, that there remains an illimitable scope for our inquiries and discoveries; and every step we take serves to 
enlarge our capacities, and give us still more noble and just ideas of the power, wisdom, and goodness of God. This marvellous universe is so full of wonders, so teems with objects of latent beauty, that perhaps eternity alone will open up and develop sufficient opportunities to enabls: us to survey and admire and appreciate them all.

\footnotetext{
"And lives the man, whose universal eye

Has swept at once th' unbounded scheme of things, Mark'd their dependence so, and firm accord, As with unfaltering accent to conclude That this availeth nought? Has any seen The mighty chain of beings, lessening down From infinite perfection to the brink of dreary nothing, desolate abyss ! From which astonish'd thougbt, recoiling, turns? Till then, alone let zealous praise ascend, And hymns of holy wouder, to that Power, Whose wisdom shines as lovely on our minds As on our smiling eyes his servant sun."-THomson
}

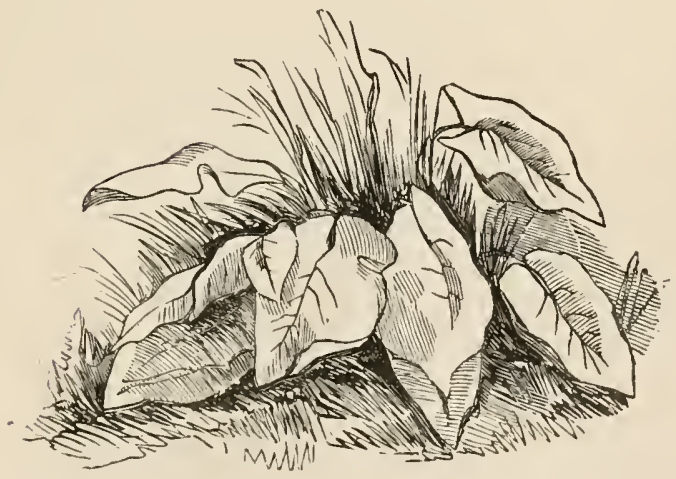





\section{APPENDIX.}

As increasing demand for good, useful, and moderately priced instruments fit for students and others, naturally enough has had the effect of inducing makers to vie with each other in their endeavours to give a better class microscope for a small sum. Among those manufacturers who have effected much for the microscope, not only in the way of improvements, but in its production at a moderate cost, we should have mentioned Messrs. R. and I. Béck. Their Popular Microscope will be readily understood on reference to fig. 357.

The body, a (in the illustration shown as binocular), is carried by a strong arm, B; and this is attached to a square bar, c, which may be moved up or down by a rack-work and pinion in the lower part of the stand, where the stage, D, and the mirror, E, are attached.

The base, $F$, is triangular; and it is connected with the parts of the instrument already descrihed by a broad stay, G, which moves on centres at the top and bottom, so as to allow the end of the tube, $\mathrm{H}$, to fit by its projecting pin into various holes along the medial line of the base. With this arrangement, if the body of the microscope be required in a more or less inclined position as in the figure, four holes are provided near the extremity of the base for the pin of the tube to fit into. A hole near the stout pin, $\mathrm{L}$, is used when a vertical position is wanted; while to obtain the horizontal position, the pin of the tube is placed in a hole in the stud, $\mathrm{K}$, the inner surface of the stay, $\mathrm{G}$, resting at the same time on the top of the stout pin, $\mathrm{L}$. This form of construction is entirely new, and has the following advantages : it is strong, firm, and yet light; the instrument cannot alter from any particular inclination it is put into, 
which is not unfrequently the case when the ordinary

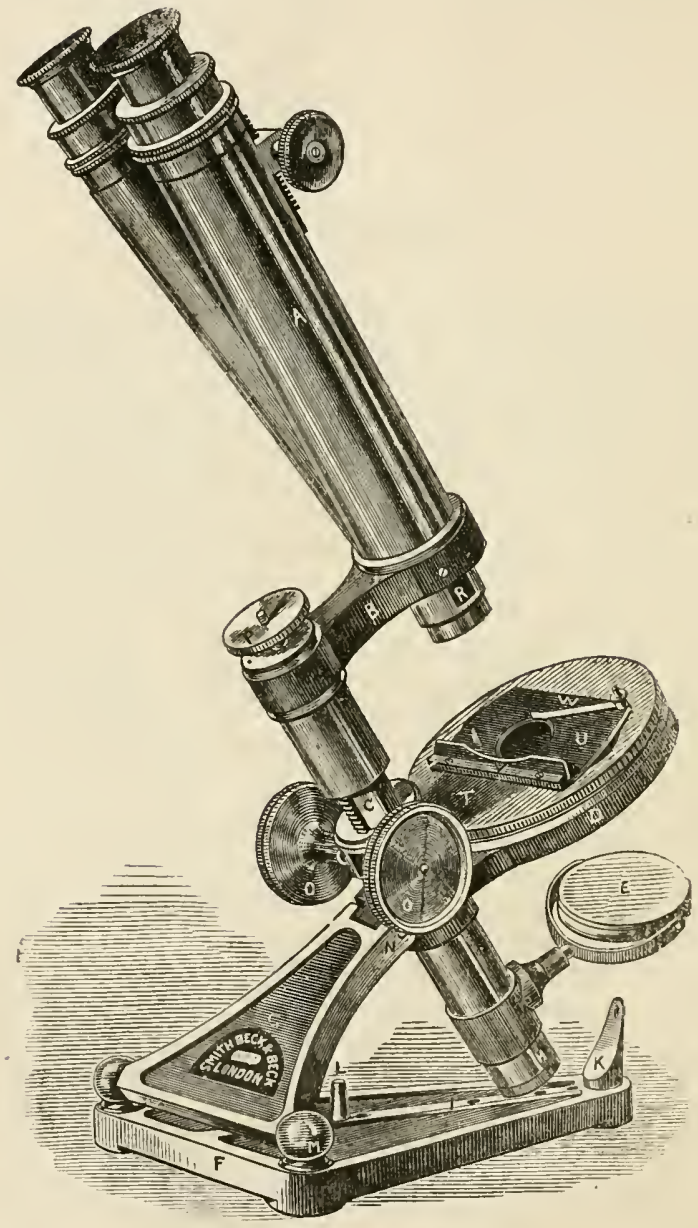

Fig. 357.-Beck's Popular Microscope.

joint works loose; and in every position the heavier part 
of the stand is brought over the centre of the base, to ensure an equality of balance.

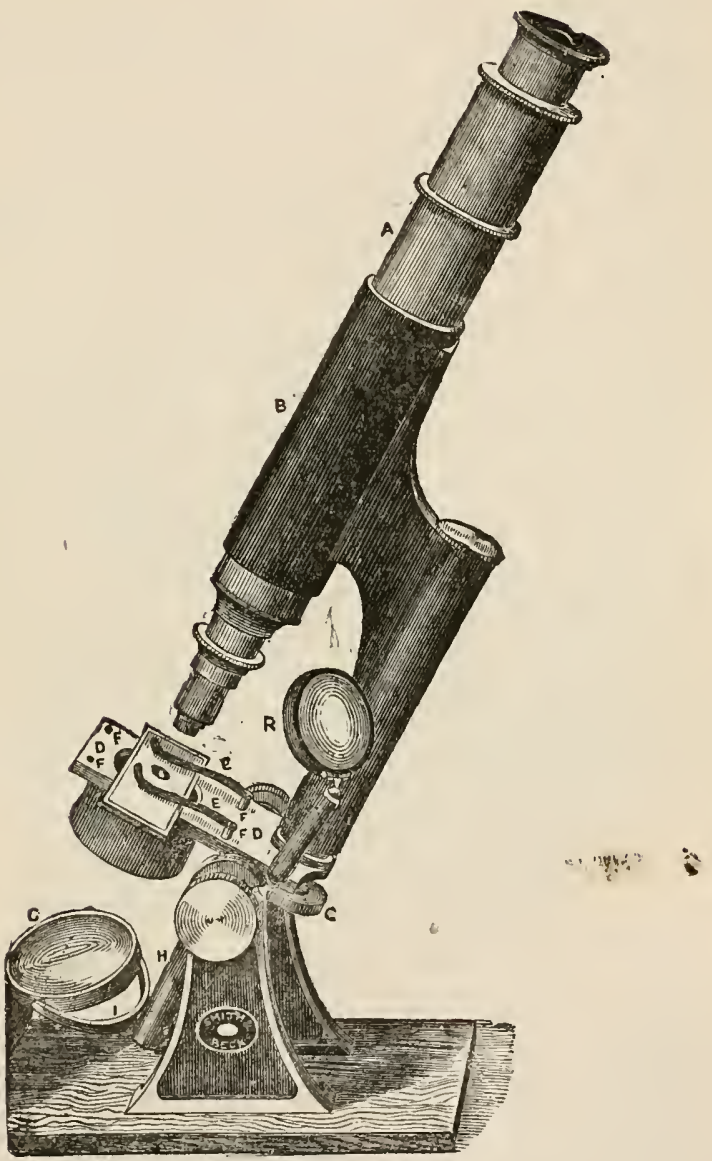

Fg 358. Beck's S'tudent's M icroscope. 
To adjust the focus of the object-glass, turn the milled heads o for a quick movement, or the milled head $\mathrm{P}$ for a slow one.

The stage, D, is circular, and upon it fits a plate, $\mathbf{T}$; this again carries the object-holder, $\mathrm{v}$, which is provided with a ledge, $v$, and a light spring, $w$; it is held on the plate $T$ by a spring underneath, so that it can be noved about easily and smoothly by one or by both hands. The small spring, $w$, is fastened to the object-holder by a milled head, which will unscrew; so that the position of the spring may be altered to give more or less pressure upon the edge of the object, or it may be removed altogether, if necessary.

When a stage with only a flat surface is required, the object-holder, U, may be removed by unscrewing, from the under side of the plate $\mathrm{T}$, two small milled heads which connect a circular spring with the object-holder; or, by removing the plain stage altogether, an extra simple flat plate may be substituted.

Beneath the stage there is a cylindrical fitting for the reception of a diaphragm, or for any additional apparatus that may be required in that position.

The mirror, E, besides swinging in a rotating semicircle, will slide up or down the tube, H, or it will turn on either side for oblique illumination.

Their Educational and Student's Microscopes, and larger instruments are well known, and therefore need no particular mention.

\section{CROUCH'S CHEAP BINOCULAR MICROSCOPE.}

The instrument (fig. 359) manufactured by Mrr. H. Crouch, London Wall, possesses many important advantages as a cheap microscope. First it combines perfect optical performance with good workmanship in the construction of the stand. It is well balanced, and stands firmly in any position. The polariscope can be used (the analyser being adapted to binocular) with the 2-inch. The stage is of a new construction, giving universal movements of $1 \frac{1}{2}$ inch in extent. The condenser is on a separate 
stand, and the accessory apparatus can be adapted at any time, without returning the instrument to the maker's hands.

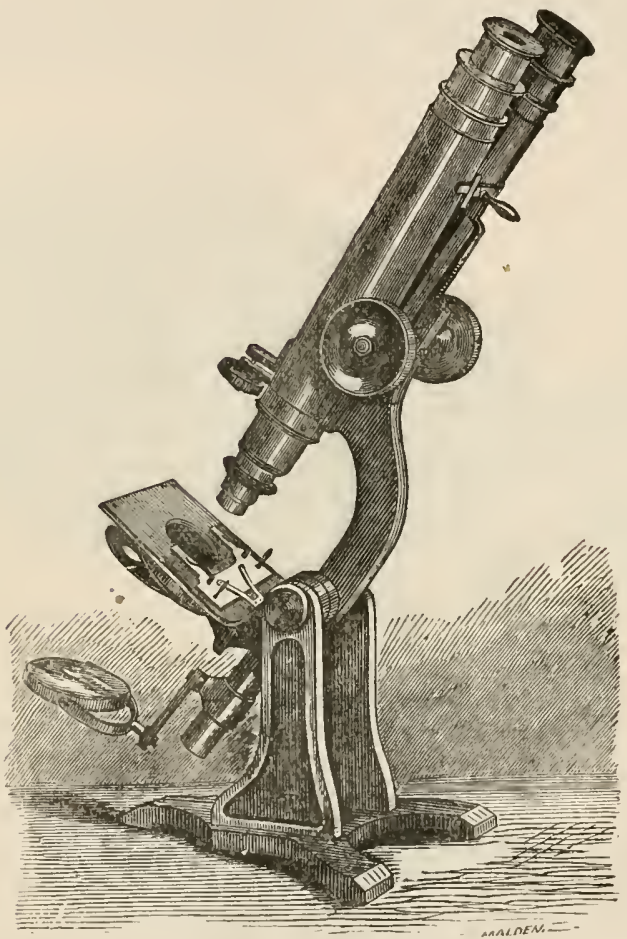

Fig. 359.-Crouch's Microscope. Scale $\frac{1}{5}$.

The latest improvement effected in this cheap instrument is the adaptation of a concentric rotating stage, a most convenient and useful addition. This stage (fig. 360) is an adaptation of Nachet's principle, and consists of : $\Delta$, a lower plate or stage attached to stand in the ordinary manner, with fitting beneath $(F)$ to receive the accessory illuminating apparatus. $\quad B$ is an upper plate rotating con- 
centrically with optic axis of microscope. c a plate of polished glass let in and secured to upper surface of 1 . D D, a v spring attached to onter edge of the plate $\mathrm{B}$ and rotating with it, having at the outer extremities projecting "ivory" points $d d$. $\mathbf{E}$ the object-carrier, consisting of another plate of polished glass, mounted in brass frame,

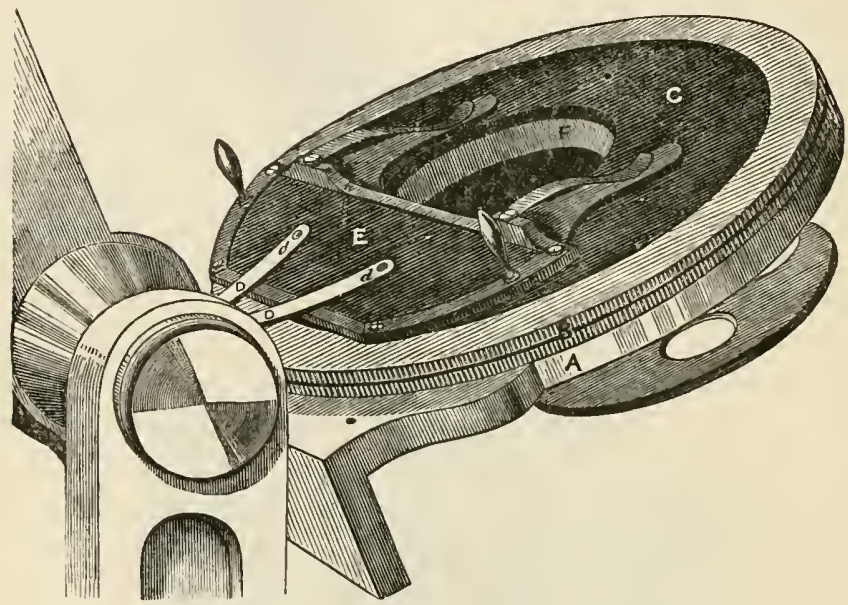

Fig. 360.-Crouch's Concentric Stage. Scalez.

having an attachment for holding the object. The plate E projecting slightly on the lower surface from the brass frame is kept in contact with the circular plate c (also slightly projecting above $\mathrm{B}$ ), the two glass surfaces being in contact, and kept so by the pressure of the "ivory points" $d d$; thus securing a beautifully smooth and delicate-thongh firm-adjustment, which admits of an object being found with extreme facility under the highest powers. It is particularly adapted for the examination of objects in fluid of any kind, the adjustments not being subject to oxidation; any minute object may very readily be found, and the slightest change in the field is easily effected; there is no slipping back or recoil. 
The whole stage may also be concentrically rotated; this is most essential in the examination of the markings on certain test objects ; and, what is very important, there is no danger of the apparatus getting out of order. For histological work, and for the teacher who wishes to demonstrate any peculiar feature of an object, it is

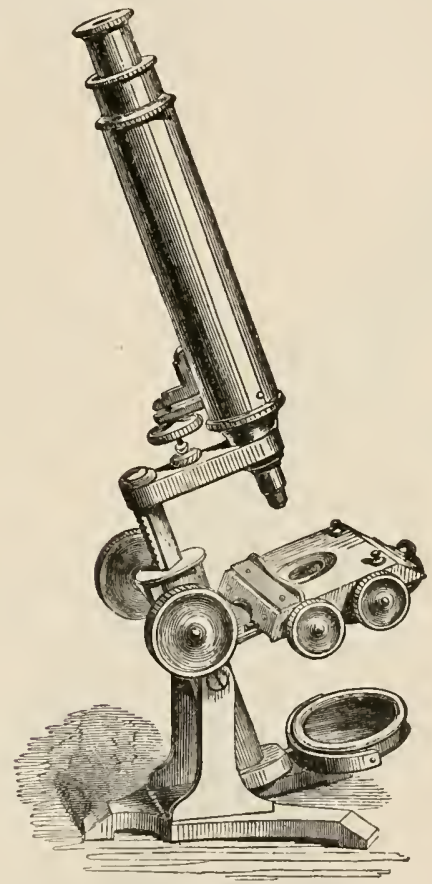

Eig. 361,-Browning's Student's Microscope.

certain to prove exceedingly useful. The stage car. be added to any instrument.

BROWNING'S COMPLETE MICROSCOPE.

Mr. Browning's Student's Complete Microscope differs from the educational instruments of other makers, possessing 
mechanical motions to the stage. It has a handsome brass stand, large body, with coarse and fine adjustments, and drawer tube to eye-piece, and separate 1 -inch and $\frac{1}{4}$-inch objectives of very superior quality, of moderate angular aperture, cut to the Microscopic Society's gauge.

The stage is arranged to receive the polariscope, and the whole instrument is so made that additional apparatus may at any time be added to it, at pleasure. The performance of the objectives is unusually good ; the instrument is well

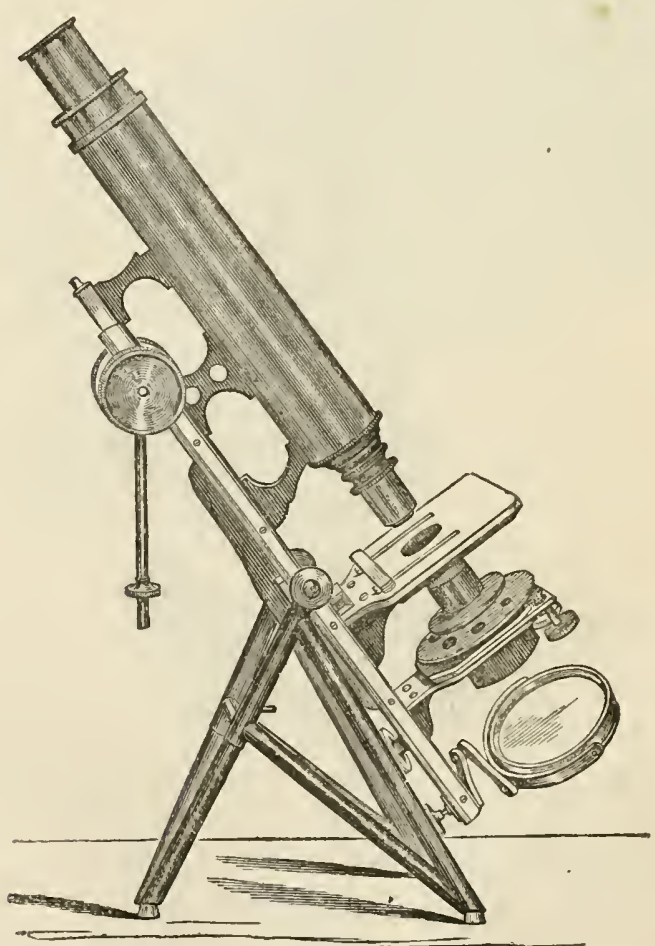

Fir. 3i2. -Ladd's Microseope. 
made, and remarkably cheap at the price, $£ 66 s$. ; we very cordially recommend it for general use.

Mr. Ladd has lately effected improvements in the manufacture of his instruments in mechanical details, and in obtaining a perfect centre of gravity. In his best instrument, such as that represented in fig. 362 , he has taken great care to obtain a perfect balance in any position, even when placed on its horizontal axis; no instrument can be better adapted than this to all the wants of the microscopist, and which is one combining many of the advantages of the more expensive forms.

Nachet and Hartnack of Paris, and Merz of Munich, hold an almost equal rank as makers of first-class microscopes, and in point of excellence of workmanship fairly rival those of English makers. Hartnack's latest improvement consists in an exquisitely-fitted concentric stage, which works with marvellous neatness and precision. Nachet has also effected considerable improvements in his circular stage, as well as in the arrangement of his binocular form of body; for fuller particulars of which we must refer the reader to the Montlly Microscopical Journal.

\section{IMMERSION OBJECTIVES.}

Messrs. Hartnack and Merz and others have likewiseadded greatly to their well-earned reputations by the construction of immersion objectives of a very superior order. We have had especial opportunities of comparing the respective merits of Hartnack's $\frac{1}{10}$ and $\frac{1}{18}$, Merz's $\frac{1}{18}$, and Noberts's $\frac{1}{20}$, of which we can speak in the highest terms of praise. All work through ordinary thick glass covers with great facility, and are therefore adapted either for the examination of pathological specimens or for the most delicately-lined test-objects. The low price at which they are sold is no unimportant consideration to the student in microscopical science; the $\frac{1}{18}$ of either Hartnack or Merz can be purchased for about $£ 66$ s., while their other powers are equally cheap. Amici nearly fifty years ago demonstrated the value of the immersion system : and Scmmering, writing of one of Amici's microscopes, observes: "Its magnifying power, and the admirable precision and clearness with which the object is seen, seems astonishing." 
It is not difficult to see that Amici's method of connecting the objective with the cover-glass by a film of water must necessarily very much diminish the reflection which usually takes place in the incidence of oblique light when the ordinary or dry objective is employed. The limiting angle of refraction of water being about 48 degrees, it follows that whatever is the degree of obliquity in the incident light falling on the object, the immersion lens never has to deal with rays of greater obliquity than 48 degrees. To this, as well as the greater number of parallel rays brought to a focus, and the increased angle of aperture, is due the greater clearness and precision of the image obtained.

"The advantages," writes Mr. J. MIayall," "mainly claimed for the immersion objectives are: greater working distance between the object and the objective, increase of light, superior definition and clearness in the optical image, which image is obtained by much simpler illuminating apparatus, and with less manipulative skill than that considered indispensable in using high-power dry objectives."

We are pleased to add that Messrs. Powell and Lealand are turning their attention to the construction of objectives on the immersion system. This cannot however be effected by making the front lens moveable, so that the objective may be used either wet or dry.

(1) The Bonthly Microscopical Jourmal, 1869, p 82. 


\section{N D E X.}

Aberration, chromatic, 26. of lenses, 21. spherical, 26.

Abraham's reflecting prism, 172 .

Absorption bands, 1:S.

Acalephæ, 491.

Acarina. 632.

Acarus beetle, 455 . cloth moth, 611 .

domesticus, 637 .

of $\operatorname{dog}, 634$.

farinæ, 639 .

of $\mathrm{fly}, 64 \mathrm{l}$.

of fowl, 640 .

of rat, 634 .

sacchari, 638.

scabiei, 635 .

of swallow, 639 .

Achetina, 627.

Achnanthes Longipes, 420.

Achorion, 296.

Achromatic condenser, 166. corrections, 40.

Illuminator, 167.

object-glasses, 42.

Acineta-form infusoria, $40 \mathrm{~s}$. vorticella, 447

Acineta tuberosa, 411, 449.

Actinia actinozoa, 465 .

— rubra, 483.

- bellis, 484 .

Actiniforum zoophytes, 485 .

Actinophrys sol, 374.

Actinotrocha, 578 .

Actinozoa, 485 .

Adams' microscope, 10.

Adipose tissue, 693 .

Adulteration of food, 345 .

Existes, 451.

Ecidium, 293.

Agates, 399.

Alcyonella stagnorum, 526.

Alcyonidæ, 489, 524.

Alcyonium digitatuin, 489. polypidnins, preparation of, 509 .

Alder, section of, 334 .

Algæ, devclopment of, 269.

Alkaloids, sublimation of, 731 .

Amici's microscopes, 12. prisms, 82.

Amphistome conicum, 565.

Amphitetras, 416.
- Amœba, 373.

Amceboid state of volvox, $27 \%$.

Anacharis alsinastrum, 321 .

Analysis, spectrum, 735 .

Angle of aperture, 44

- of incidence, 16.

— of refraction, 17.

- mode of measuring, 45 .

Anguillulæ, $5 \pi$. .

Anguinaria spatulata, 520 .

Animalcule, sun, 374 .

Animalcules, 402.

— eage, Varley's, 195.

- collecting, 193.

- history of, $4 \mathrm{C3}$. infusorial, 414.

Animal cell, 659 .

— action of cilia in, 671 .

bodies, injecting, 231 .

- cells, change into tissues, 665 .

cellular inembrane, 692 .

- connective tissue, 662 .

- elementary substance, 659 .

epithelium, 668 .

fibrous tissue, 694.

- kingdom, division of, 866 .

- life, 655 .

- structure, 661.

- structure, mode of investigating. 724.

— tissues classified, 692.

- tissue consolidated, 702.

tissues, staining, 225 .

Annelida, 575 .

Annulosa, 559 .

Anobium, 633 .

Antennæ of insects, 607 .

Amyot,'s finder, 188 .

Aphides, 613.

Aphrophora bifasciata, 615.

Aplanatic doublet iens; 26.

Aplysia, 529.

Aquatic box, 194.

Arachnida, 631, 6\$4.

Arachnoidiscus, 432 .

Archer on amoboid bodien, 277.

Arcella acuminata, 372 .

Arenicola, 576.

Aristoplanes, microscope known t.?, 2.

Artemiæ, 557.

Articulata, 579.

Asci of lichens, 305. 
Aspergillus, 301.

Astasia, 412.

Asteroidea, 495.

Atropus, 623 .

Atlantic soundings, 382 .

BAKER on the microscope, 10 .

Baker's microscopes, 86. brachionus, 456.

Baccillaria paradoxa, 269.

Bacterium, 412.

Baird, Dr., on entomostraca, 557.

Balanidæ, 555.

Balbiani, Dr., on paramæcium, 410.

Barnacle, 554 .

De Bary on fungi, 291.

Bat, liead of, 681 .

Beale, Dr. on cell development, 659. - on a new object glass, 73 .

- on magnifying power, 77 .

- on glycerine for preserving objects, 224. on method of staining tissues, 225. on injecting, 237.

Beck, Mr. R., on errors of interpretation, 64.

Bee's tongue, leg, \&c., 612.

$\longrightarrow$ eye, 586 .

Beetles, 623.

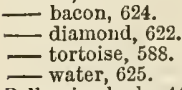

Bell-animalcule, 445.

Berg-mehl, 432.

Bergh, Dr., on urticating organs, 545. on podura scales, 628

Berkeley on two new British fungi, 304. on fungi, 290.

Beroë, 492.

Bichromate of potash injection, 236.

Biddulphia, 437.

Bilharzia hæmatobra, 567.

Binocular microscope, 117.

Bird's-head coralline, 518.

Blood-corpuscles, 678.

- erv'stallization of, 680 , disc, size of, 680 . spectrum, 128

- vessels, structure of, 688 .

Blow-fly, 590.

Bockett's lamp, 185.

Bone, 714.

- cutting sections of, 208.

- eel, 716 .

- fishes, 719 .

- human, 713 .

- ostrich, 715 .

— reptiles, 714 .

- sting ray, 718 .

Bonnani's microscope, 8.

Butrytis, 301.

Bot-fly, egg of, 607 .

Buurgingnon, Dr., on acarus scabiei, $6: 35$.
Bowerbank on sponges, 386 .

Bowerbankia, 512 .

Brachionus, 455.

Brachiopoda, 535 .

Brewster, Sir David, on viewing objects, 160 .

- Nineveh lens, 3 .

on double refractory crystals, 147.

Bridgeman's reflector, 181 . finder, 190.

Brightwell ou navicula, 419 ,

Brooke, Mr., on opaque objects, 183.

- on test objects, 57 .

Browning's spectro-microscope, 120. oblique illuminator, 171.

Bryozoa Bowerbankia, 512.

Buccinuen undatum, palate, 538.

Burnett, Dr., on parasites, 639.

Busk, Mr., on anguinaria spatulata, 520 .

- on starch granules, 342.

- on echinococci, 570 . volvox, 276.

Butterflies, eggs of, 605 .

— tongues of, 607 .

Calepteryx viroo, 597.

Caleification of animal tissues, 552.

Callithamuion, 273.

Camera-lucida, 129.

Carapanularia volubilis, 481.

- gelatinosa, 481 .

Campilodiscus clypeus, 439.

Cancer-cell, 296.

Cane, scction of, 341 .

Capillaries, 689.

in fat, 691 .

Carbonate of lime in shell, 528.

Carpenter, Dr., on volvocineæ, 275

- on diatomacem shell, 553.

- on tomopteris, 576 .

- on eozoon, 379 .

Cartilage, 702.

- from ear of mouse, 702.

-.. rabbit's ear, 703 .

Carter, Mr., on chara development, 318 on spongilla, 391.

Cassida viridis, 588 .

Cedar, stem of, 357.

Cell formation, 659 .

- action of, 667 .

- animal, 660 .

- changes, 258, 661 .

changes in disease, 664.

- contents, 662.

- development, 239, 659 .

- nueleus, 663 .

pigment, 671 .

Cells, complicated, 667 .

- for desmids, 230.

for mountiug, 213.

- growing, 198.

- motile, 261. Suffolk's, 221. vegetable, 257 
Cellularia, 518. avicularia, 518 .

Cellular tissue of plants, 323. in animals, 662 .

Cements, 219.

Cephalopod, tongue of, 540 .

Cephalopoda, 550.

Cephalosiphon, 451.

Charace:e, 315. - antheridia of, 317 . development, 318.

Cheesc-mite, 637 .

Chemical re-agents, 245.

China-grass, 353.

Chitonidxe, 537.

Chlnride of gold, 701.

Chloroform and balsam mixture for mounting, 227.

Cilia, 404, 671 .

- movement of, mode of exhibiting, 406.

Ciliated epithelium, 670.

Cimex lecticularis, egg of, 113.

Circulation of blood in frog, to view, 682.

Cirrhopoda, 554.

Clarke, I.ockhart, on the preparation of spinal cord, 761 .

Clematis, sectiou of, 333,357 .

Clepsinidæ, 574 .

Clionæ, 395.

Clip for mounting, 214.

Closterium lunula, 285.

Clothes moth, 612 .

Clypeastrodea, 501.

Coal, structure of, 363 .

Cobbold, Dr., on helminths, 567 .

Cocconena, 437.

Coccus persicæ, 604 . egg of, 605 .

Cochineal insect, its value, 651 .

Cocoa, adulteration of, 346 .

Colenterata, 462.

Coddington's lens, 34.

Coffee, structure of, 347 .

Cohn on stephanospliæra, 265.

Coleoptera, 622.

Collecting objects, 248.

— animalcules, 248.

bottle, 192.

net, 193.

stick, Mr. Williamson's, 193.

Collins' mounting case, 254. microssope, 95.

Collodion for multiplying objects, 585 . Comatula, 496.

Compressorium, 195.

Condensers, Gillett's, 163.

Powell and Lealand's, 169.

Collins's, 169.

Ross's, 167.

- J. B. Readc's, 174 .

bulı's-eye, 182.

Eonfervoidex, 267.

Conjugation in desmids, 279.
Conochilus, 448.

Consolidated tissues, 702.

Coral reefs, 491.

Corals, 490 .

Cordylophora, 463.

Corcthra plumicornis larva, 600 .

Corn-blights, 293.

Corymorpha nutans, 476.

Coryne-stauridia, 475 .

Coscinodiscus, 440.

Cosmarium, 282.

Crabshell, 528.

Craytish, 553.

- shell of, 553.

Cricket, 627 .

Crinoidea, 505.

Crisiadæ, 519.

Cristatella mucedo, 524.

Crustacea, 554.

Crystals, formation of, 729 . of snow, 153 .

- quinine, 151.

—_ urinary salts, 149.

- mode of showing optical axis, 145 .

Cuckoo-spit, 615.

Cules, head of, 599.

Cutleria dichotoma, 273.

Cutting sections, 203.

_- glass cells, 213.

Cuttlefish-bone, 546 .

Cyclops, 556.

Cymba olla, tongue of, 542.

Cymbella Ehrenbergii, 418.

Cynips gallæ, 618 .

Cypridæ, 556 .

Cystic disease of liver, 570 .

Cysticercus fasciolaris, 564.

- pisiformis, 564

Czermak on tooth substance, 711.

DAlyell, Sir J. G., on tubularidæ, 474.

on actiniæ, 467.

Dana, Prof., on corals, 490.

Daphnia pulex, 557.

Darker's selenite stage, 142 .

Darwin on infusoria, 433.

Dasy Kuitzingiana, 272.

Davis on a new rotifer, 451 .

Death-watch beetle, 623 .

Deep-sea soundings, 381 .

Delabarre's microscope, 10.

Delessaria, 274.

Demodex folliculorum, 637.

Dentine, 707.

Deparia prolifera, 313.

Dermestes lardarius, 624.

Dermestidæ, 624 .

Designing from microscopic forms, 440

Desmidiaccx, 278. finding and preserving, 288.

Deutzia scabia, 339.

Dinmond beetle, scales of, 622 .

Diaphragm, description of, 163.

Diatomaceæ, 416.

_ Kützing on, 417 . 
Diatumaceir, on collecting, 428.

Diatoms, movements of, 423.

Difflugir, 372 .

Diffraction of light, 68.

Dlphydæ, 464.

Dipping tubes, 191.

Directions for keeping instrument, 159.

- for mounting and preparing objects, 215.

Disc, circular, 212.

Dissecting knives and needles, 198.

— microscopes, 98.

— under water, 200.

- scissors, 202.

Distoma, 567 .

Divini's mieroscope, 7 .

Doris tuberculata, palate of, 538 .

Dragon-1ly, 597.

Drone-fly, 591.

Dytiscus marginalis, 626.

—-sucker from leg of, 588 .

ECHINTDE, 498.

Echinococci, 569.

Echinodermata, 494.

Echinorlyynchus, 571.

Ecker, on protozoa, 373.

Eel, scales of, 722 .

Eggs of insects, 602 .

- of gasteropoda, 545 .

Egyptian cloth, 354 .

Ehrenberg's brachionus, 455.

- on boundary betweer plants and animals, 655 .

Eider-root, section of, 363 .

Elm, section of, 350 .

Enamel, 709.

Enchondroma, 704.

Encrinus, 506.

Entomostraca, 555.

Entozoa, 565. :

Eozoon canadense, 378.

Epeira cíadem, 645.

Epithelium, tesselated, 668.

- columuar, 670 .

Equisetaceæ, 311.

Equisetum, section of, 332 .

Errors of interpretation, 65, 723.

Escharidæ, 521.

foliacea, 522 .

Euastrum, 280.

Eucratiadæ, 519.

Euglena, 412.

Eunotia, 437.

Euphorbia neriifolia, 329.

Euplectella, 402.

Eye-glass, 31.

Eye, human, vessels in, 689. pigment, 672.

section of cat's, 689 .

Eye-piece, the, 47.

- Huyghenian, 47.

Ramsden, 50.

micrometer, Jackson's, 52 .

Kelner, 78 .
Eyes of insects, 584 .

Fasciola hepatica, $56 t$

Fat cells, 694 .

Favus fungus, 296.

Feather-star, 496.

Feet of insects, 587.

Ferns, 311.

- section of root, 335 .

Fibre, muscular, 695.

in the tongue, 697 .

involuntary, 698.

Fibrous tissue, 692 . white and yellow 694

Finders, 187.

Anyot's, 188.

Maltwood's, 189.

Fishes' scales, 721 .

Flatness of field, 72

Flax, 353.

Flea, 630 .

— cat's, 634 .

- larva of, 634.

Flora of coal measure, 364 .

Florideæ, 271.

Flower buds, 351 .

Flowers, colouring matter of, $35 q$

Flukes, 565.

Floscularia ornata, 458.

Floseulariæ, 453.

Flustre, 521.

- avicularis, 523.

- chartacea, 523 .

- foliacea, 522 .

Fly, foot and leg of, 587, 59]

tongue, 595 .

fungoid disease of, 596 .

Tsetse, of Africa, 596.

Follicles of pig's stomach, 669

Foraminifera, 375 .

akeletons of, 381 ,

fangasina, section of, 380 .

fossil, 377 .

Forceps for holding objects, 190.

Fossil infusoria, 431. inode of preparing, 434 .

Fossil plants, 362 .

Fraunhöfer's glasses, 10. lines, 738.

Frog-plate, 632.

- bit, 321 .

- circulation, 679 .

hopper, 615 .

Fungi, 290.

-Berkeley on, 290.

- De Bary on, 291.

floating, 295 .

Fungix, 484.

Fungoid growths, 296.

Furze's spotted lens, $15 \mathrm{~s}$

Fragillaria pectinalis, 437 .

Funaria, capsule of, 309.

GALL-FLY, 618.

Gallionclla sulcata, 438. 
Garrod's, Dr., crystals in blood, 681 .

Gas-lamp, 185.

Gasteropoda, 537.

- lingual bands of, 538 .

Gemmules of sponges, 400 .

Geodia Barretti, 390.

Gibbous's section-cutter, 205.

Gillett's illuminator, 163 .

- measuring angle of aperture, mode of, 45 .

Gill of tadpole, circulation in, 686 .

Glass-rope sponge, 401.

Glauconia, 414.

Globigerina, 384.

Giossina morsitans, 596.

Glycerine for mounting, 224.

- Rimmington's, 223.

Gnat, description of, 598 .

Goadby's flutid, 227.

- process of injecting, 235.

Gomphonema, 419.

Goniometer, Schmidt's, 56 .

Gordiacea, $56^{\prime} 2$.

Gorgonidæ, 4 S7.

spiculæ from, 487.

Gorhain, T, on eyes of insects, 584 .

Goring, Dr., achromatic object-glasses, 13.

Gosse on notommata, 456 .

- on cellularia, 518 .

- on coryne stauridia, 475 .

-.. on melicerta ringens, 459.

Gourd seed, section of, 335 .

Graminaceæ, 340 .

Grant, Dr., on flustra, 521 .

— on alcyonidæ, 489 .

- on plumularia, 478 .

- on sponges, 386 .

Grass, cuticle of, 339.

Gray, Dr., on mollusca, 539.

Gregarinida, 367 .

Gregarina of earth-worm, 270.

Growing cell, 198.

Guinea-worm, 563.

Gulliver on raphides, 337.

- on blood dises, 679 .

Guy, Dr., on subimation, 731.

Gyrinus, paddle of, 625.

HAIR, HUMAN, 672.

bat, Indian, 673 .

- gregarires in, 369.

-- moss, 311.

- mouse, 673 .

pecari, 674 .

Haliotus splendens, 536 .

tuberculatus, tongue of, 543 .

Hallifax, Dr., on preparing insccts, 604.

Hand magnifiers, 33.

Hard tissues, preparation of, 208.

Hartce elegans, 524.

Hassall, Dr., on the adulteration of food, 349 .

Henfrey, Prof., on ferns, 313.

Hemp, 353 .
Hepaticæ, 308.

Hepworth, Mr., on mounting insects 648

Herapath's, Dr., polarising crystal, 142. iodo-quinine, 149.

on polarisation as a test, 148 .

Hermia glandulosa, 475 .

Herschel's a planatic doublet lens, 26.

$\longrightarrow$ A., spectroscope, 122 .

Hey's mounting fluid, 227.

Hicks, Dr. B., on amoboid bodies, 227

- ou lichens, gonida of, 306.

Highley's gas-lamp, 185.

microscopes, 99 .

photographic camera, 157.

Hill's microscone, 10.

Hipparchia janira, 611 .

Hirudinid $2,573$.

Holland's eye-piece, 33.

Holothuridæ, 50 .

drawing of, life-size, 507.

Honey-bee, 620 .

Hooke's microscope, 7 .

How's microscope, 101.

Horn, rlinoceros, 721 .

Human body, composition of, 659 .

Huxley, T. H. on animal tissues, 656

- on echinidæ, 494.

- on tongue of mollusca, 538.

on actinozoa, 468.

on rotifera, 560 .

- on tooth development, 708.

Huyghenian eye-piece, 47.

Hyalonema, 401.

Hydatids, 570.

Hydra, 446, 466, 470 .

Hyulrachnidæ, 642 .

Hydractinia, 473.

Hydroplilus, trachea of, 587 .

Hyınenoptera, 616.

- aquatic, 626 .

ICELAND SPAR, 135.

Improvements in microscope, 10.

India-rubber tree, 329. section of leaf, 334 .

Indicators, 187.

Infusoria, 402.

- cilia, 405 .

fossil, 431 .

listory of, 403.

Infusorial animalcules, 402.

- Pasteur's researches on, 414.

Injecting, mode of, 231.

- lower animals, 244.

- mercurial, 239.

- steps of the process, 241.

the ducts and glands, 243.

Injected preparations, to mount, 244.

Injections, transparent, 226. coloured, 232.

Insects, 579 .

- commercial importance of, 651 . dissection of, by Swammerdam, 679. 
Insects' eggs, 602 .

- eyes, 584 . feet, 587 .

- hairs, tenent, 589. heads, 582 .

itch, 635 .

- injecting, 240.

- ovipositors, 616 .

- preparing and mounting, 590, 648. proboscis, 599.

— spiracles, 586 .

— stings, 619 .

- tongues, 582,607 .

- transformations of, $64 \mathrm{~S}$.

- wings of, 597 .

Interpretation, errors of, 67 .

Intercellular substance, 662 .

Iodo-quinine, 151.

Iris-leaf, 336.

Isthmia enervis, 432.

Ivory nut, section of, 350 .

Ixodidæ, 642 .

JACKson's micrometer, 51.

Jelly-fish, 491.

Johnstone, Dr., on sponges, 385.

- on hydra, 471.

on campanularia, 481.

Jones, Wharton, on non-striated muscular fibre, 698 .

- Rymer, on the coretha plumicornis, 600 .

Jungermannia bidentata, 310 .

KeTtledrum condenser, 174.

Kelner eye-piece, 78.

Knives for dissecting, 119.

Kölliker on the muscles of the skin, 698.

Kützing on vegetables and animals, 256.

- on diatomaceæ, 417 .

LadDs's microscopes, 84 .

Lamps, 184.

Iamp-shells, 535 .

Lankester, E. R., on gregarina of earthworm, 370 .

Lathe for polishing, 209.

Leaf-insect, 613 .

Leech, medicinal, 5 73.

Leeuwenhoek's microscope, 6.

Lenses, achromatic, 28.

- chromatic, aberration of, 26.

Coddington, 34.

concavo-convex, 19.

condensing, 183 .

correction of, 28.

curvature of, 22.

double convex, 18.

forms of, 18 .

maguifying nower of, 29.

meniseus, 25.

tracing rays through, 17 .
Lenses, periscopic, 33.

- plano-conrex, 24.

- refraction of light through, 16.

- spherical aberration of, 26 .

Stanhope, 35.

Lepidoptera, eggs of, 605 .

tongue of, 608.

wing-scales of, 609 .

Lepisma, 529 . seale of, as a test, 611 .

Lepralia nitida, 517.

Leuchart on echinorlynchus, 571.

Lewes on actinix, 467.

- on life, 655 .

- thread cells, 467.

Libellulidæ, wings of, 597 .

Lichens, 304.

Lieberkủhm, Dr. N., on gregarina, 369

- microscope, 5 .

- on spongilla, 389.

Life, animal, 655 .

Light, diffraction of, 68 ,

- polarisation of, 132 .

Limax rufus, 529.

Limnæa stagnalis, 545.

Limnias ceratophylli, 458.

Lister's lenses, 13.

- achromatic corrections, 40.

List of ohjects, Wheeler's, 253.

Live-box, 194.

Liver, diseased, 567.

Liverworts, 308 .

Lobb, Mr., on polarising erystals, 145 61. on the illumination of test objecte, micrasterias, 279

Lob-worm, 576 .

Loligo, palate of, 541.

Lophopus erystallum, 525 .

Loven on mollusca, 538 .

Louse, 633.

Lubbock, Sir J., on the eggs of insests, 605.

on aquatic insects, 626 .

Lucernaridæ, 493.

Luidia fragilissima, 496 .

Lung, capillaries of, 690 .

Lymphatic, vessels of, 669 .

MADREPORIDE, 486.

Maddox, Dr., wire clip, 214.

Magnifiers, hand, 33 .

Magnifying power of single lenses. 19

— of object-glasses, F.oss's, 55 .

Powell's, 56.

Maltwood's finder, 189.

Maple-aphis, 613.

Marchantia polymorplia, 308 .

Mechanical arrangements, 70 .

Medusæ, 492.

Meissner on the micropyle, 603.

Melicerta ringens, 459 .

Melolantha, eye of, 585 .

Melting vessel, 233.

Mercurial injections, 239. 
Mesoglia vermicularis, 268.

Micrometers, 51.

Microscope, Baker on the, 10. Baker's binocular, 89 .

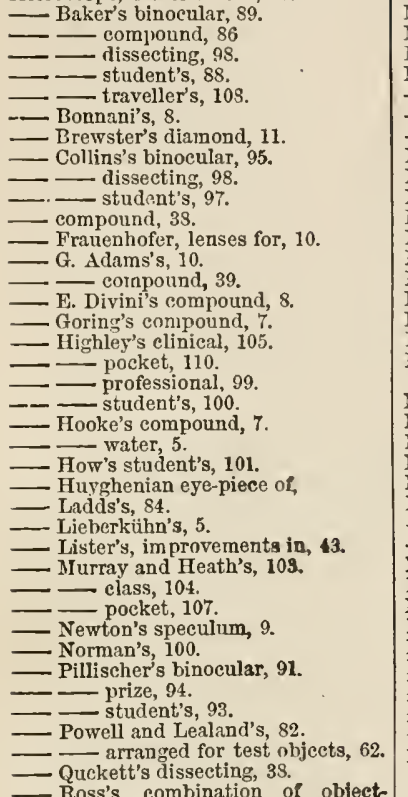

- Ross's, combination of objectglasses, 43 .

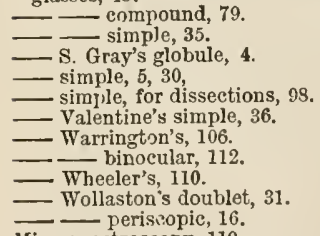

Microspectroscopy, 119.

Micrasterias, 280.

Miller, Hugh, on fossil plarts, 364 .

Mineral kingdom, 728.

Mite, cheese, e37.

- flour, 639.

Molccular movements, 67.

Mollusca, 511.

-_ injecting, 240.

_ tongues of, 539 .

Monads, 411.
Morpho Menelans, scale of, 611.

Moss-agates, 399.

Mosses, 309.

Moths and butterflies, 607.

Motile organs in cells, 262.

Mottled umber moth, 606 .

Mounting and preparing, 215. spring elip for, 214. injections, 244.

- case, Collin's, 254.

Movements of diatoms, 423 .

Mucidines, development of, 661 .

Mucor mucedo, 292.

Mncous membrane, 669.

Murray and IIeath's microscopes, 103.

MIuscular fibre, 695 .

Musliroom spawn, 343 .

Mussel, 531.

Myelin, 681 .

Myolemma, 696 .

Mytilus, 535.

NA ILS, the, 672 .

Naviculæ, 419.

Neckera antipyretica, 310.

Nernatoidea, 563.

Nemertidæ, 562 .

Nerves, 699. perfection of sections, 701 .

stellate corpusele, 699.

Newport on moths' tongues, 607 .

Nicol's prism, 136.

Nitella, 320 .

Nobert's ruled micrometer lines, 54.

Noctiluca miliaris, 409.

Norman on collecting diatoms, $42 \mathrm{~S}$.

Norman's microseope, 100.

Notommata aurita, 456 .

Nudibranchiata, 530 . tongues of, 541 .

Nummnlites, 377.

OBJECT-GLA SSES, forms of, 72 . Ross's, 55.

Objects, Shadbolt on collecting, $24 \mathrm{~S}$.

- directions for viewing, 160 .

mounting and preserving, 215.

Oblique illumination, 171.

-J. N. Tomkins's double prism, it4

Ojdium, 293.

Opuntia, 356.

Onion, section of, 338 .

Ophiocoma, 495.

Ophiura, 495.

Orchideæ, 350.

Orthoptera, 626.

Osborne, Lord S. G., on fungi, 295.

- on closteriuin lurula, 284 .

Oscillatoriæe, 266.

Ostracoda, 556 .

Ova inollusca, 545 .

Owen, Major, on foraminifera, 383 . Professor, on animaleule Jife, 444 on microscopic investigation, 705

Oxytricha, 414. 
Oyster, 533.

- fry, 554.

- pearl, 533 .

Palates of mollusca, 539.

Panax Lessonii, 329.

Papillæx of skin, 675.

Parabolic reflectors, 179.

Paramæcium, 409.

Parasites, 612.

- of dog, $634 . '$

— of eagle, 643

- eggs of, 607.

- of fowl, 640 .

- of hornbill, 642 .

- human, 633.

- of pheasant, 640 .

— of pigeon, 643 .

- of sheep, 633 .

— of swallow, 639 .

— of turkey, 640 .

- of vulture, 643 .

Parasitic fungi, 293.

Parmelia, 305.

stellata, 306 .

Patella, tongue of, 541.

Pear cells, 338 .

Pearl oyster, 533.

Pearls, artificial, 533.

Pedicellariæ of starfish, 496.

Pediculi, 636.

Penetrating power of object-glasses, 75 .

Penium, 283.

Pennatulidx, 488. phospliorea, 488.

Peziza, 30 \%.

Peronosjoræ, 290.

Pholas dactylus, 531.

Photography applied to microscope, 155.

Pliryganea, eggs of, 605.

Phryganeidæ, 601.

Phyllactinia, 294.

Pigment cells from skin, 675 .

Pillisclier's microscope, 91.

Pinna, 530.

Planariæ, 562.

Plants and animals, 257.

- annular vessels, 358 .

- cellular tissue of, 323 .

- circulation in, 320 .

- crystals in, 337.

— fossil, 362 .

- hairs of, 324 .

- lactiferous tissue of, 334 .

- lice, 613.

- pollen graius, 361 .

- pollen and seeds from, 361.

- preparation of tissues, 359 .

- raphides in, 337.

- cutting sections of, 205

— silicia in, 340 .

- silicions cuticle of, 339 .

- starch in, 341.

— starch of potato, 345 .
Plants and animals, starch, wheat flour, 344 .

- stellate tissue, 333.

- structure of, 323 .

- unicellular, 260.

- - vascular system of, 327.

- vascular tissue of, 324.

— vital characteristies, 257 . woody tissue, 353 .

Phosphorescence, inarine, 408.

Pleurolranelus, mandible of, $5+2$.

Pleurosigma, 421.

- as a test object, 59 .

Plumatella repens, 527.

Plumularia, 478.

- piunata, 480.

Podura plumbia, 628.

- scale, as a test, 610 .

Polarisation of light, 132, 729.

Polarised light, objects for, 729 .

Polariser, 137

Polyzoa, 512.

- mounting, 227.

- fresh-water, 524.

Polycystina, 384 .

Polygastrica of Ehrenberg, 416.

Polyommatus argiolus, 610 .

Polypifera, 462.

Polythalarnia, 376.

Pontia brassica, 611 .

Potato mould, 290. starches, 343 .

Powell and Lcaland's microscopes, 82

Powell's condenser, 168.

Preparation of insects, 648.

Preparing sections of bone, 209.

- tongues of mollusca, 544.

vegetable tissues, 359 .

Prism for binocular, 117.

— for micro-spectroscope, 121. polarising, method of using, 138. Sollitt's, 175.

Propita gigantea, 493.

Proteus, the, 373 .

Protococcus pluvialis, 259.

Protozoa, 363.

Prussian blue for injections, 238.

Pteropoda, 532.

Puccinia, 292.

buxi, 294.

Pumpkin fungi, 301.

QUEKETT on the vascular tissue of plants, 358 .

- on polarised light, 154. on fossil infusoria, 438 .

- on the structure of bone, 714

— dissecting microscópe, 33. on resolving test objects, 61 .

Quinine, its detection, 149.

Quinidine, test for, 151.

- erystals of, 151 .

RalNEY, on artificial shell, 551.

Ralt's, Mr., on desmidiaceæ, 288. 
Ralfs, MIr., on preservation of algæ, 228. $\mid$ Skin, section of, 677.

Raphides in plants, 337

Reade's, Rcv. J. B., hemispherical condenser, 174.

Re-agents, effects of, 726 .

Redfern on separating naviculæ, 435.

Red seaweeds, 271 .

Reflector, parabolic, 179, 1 S2. Shadbolt's, Mr., 179. side, 18 .

Rhinoceros liorn, 721.

Rhizopoda, 372 .

Rhubarb cells, with raphides and starch, 388.

- spiral vesscls, 355 .

Ringworm, 296.

Roberts, Dr., on blood cell, 677 .

Robertson, $\mathbf{J}$, , on pholas, 531 .

Ross's compressorum, 195. microscopes, 78 object-glasses, 74 .

Rotiferæ, 452.

Rush, stem of, 333

SAlter, Dr. Hyde, on muscle, 496.

Salts, urinary, 149.

Samuelson, J., on infusoria, 415.

Sandstone, 729.

Sarcina ventriculi, 295.

Sarcode, 656.

Saw-fly, 616.

Scales of fish, 721.

- of lepidoptera, 609.

- of podura, 628 .

Scapander, tongue of, 542 .

Schultze, Dr., on rhizopoda, 375.

- on the diatom valve, 422 .

Schwann's classification of tissues, 692 .

Scissors, dissecting, 202.

Sea-cucumbers, 508 .

—- lilies, 497, 505.

—_ jelly-fish, 491 .

— slug, 529 .

— soundings, 383.

urchins, 498.

Sections of tissues, $24 i$.

Section-cutting machines, 208.

Selenite, 14I.

Sepia, palate of, 541.

Serpula, 575.

Sertulariadæ, 476.

‥ pumila, 477 .

- setacea, 477 .

Shadbolt, Mr., on collecting, 248.

Sheep-tick, 633.

Shell, structure of, 546 .

artificial formation of, 551

Sheppard,J.B., on colouredinonads, 413. Silk, 354.

Silkworm moth, antenna of, 608.

Silkworm's brcathing aperture, 586 .

Single microscope, 4 .

Skin, capillaries of, $6: 5$.

- fungoid diseases of. 296.

- nerves of, 670.

Slack, H. J., on a new rotifer, 451.

Smith and Beck's troughs, 197. oblique illuminator, 170 .

Smith's, Prof., condenser, 170. growing slide, 198.

- mechanical finger, 436.

- Mr. J., section-cutter, 203.

Snow, crystals of, 152.

Sollitt on diatoms as test objects, 427 .

Sollitt's prism illumination, 175.

Sorby's standard interfercrice spec-

trum, 738.

spectroscope, 123.

Sori of ferns, 315 .

Sphacelaria cirrhosa, 269.

Spectro-microscopy, 119.

Spectrum analysis, 735 .

Speculum, Lieberkiihn's, 181.

Spencer, Herbert, on the formation of fibre in plants, 325 .

Sphagnum, 309.

Sphæriacei, 294.

Sphærosira volvox, 276 .

Spiders, 644.

- diving, 647 .

Spiderwort, 350.

Sponges, 385.

- motile phenomena in, skeletons of, 396.

spicnla from, $387,390$.

Spongia coalita, 388. geodia, 390 .

Spongilla cinera, 391.

- alba, 392.

development of, 389

gemmules of, 400 .

Spring clips, 214.

Starches, 343 .

- in plants, 342 .

- potato, polarised, 154

- tests for, 283.

Star-fishes, 495.

Staulastrum, 282.

Stellate tissue from a rush, 333.

Stentors, 450 .

Stephanoceros, 458.

Stephanosphæra pluvialis, 265.

Stichostegidr, 276.

Stinging-nettle hairs, 324.

Stokes's, Prof., absorption bands, 128.

Stomach, mucous membrane of, 669 ,

Structure of shell, 529 .

Suctoria, 629.

Sugar insect, 638.

- cane, section of, 336 .

Surirclla constricta, 420 .

Swammerdam's dissection of insecta 579.

Synapta chirorlota, 503.

Synaptidx, 508.

TADPOLE, circulation of, 683 .

gill of, 686 .

Tæniadie, 563. 
Tapeworms, 563.

Tea, adulterated, 349 .

Teeth, preparing sections of, 209.

- of mollusca, how to prepare, 544 . sections, mounting of, 211.

Terebratula, 536 .

Teredo, 531 .

Test objects, $57,61,65$.

Testacella, tongue of, 542 .

Thomas, Mr., on crystallization, 730 .

Thompson on mollusca tongues, 543 . Prof. W., on sea-lilies, 497 .

Thread cells, 544

— in actiniæ, 467.

- in mollusea, 544 .

Thuiarea, 478.

Thysanura, 627.

Tinea vestianella, 611 .

Tissue, woody, 353. animal, 6 .

Tissues, consolidated animal, 702. elastic and non-elastic, 694.

Tomato, diseased, 300 .

Tomkins, Mr. J. N. on aleyonella, 526. double prism illumination, 174 .

Tomopteris onisciformis, 576 .

Tooth, section of, 707 .

- structure, 709 .

— of prestis, 711 . of eagle-ray, 712 .

Topping's test objects, 612. section-cutter, 206.

Torala cererisia, 300 .

- diabutica, 295.

Tourmaline, 138. artificial, 143.

Tradescantia, circulation in, 350 .

Transformation, insect, 648 .

Transparent injections, 226.

Trematoda, 563.

Trembley on hydra, 471.

Triceratium, 419 .

Trichobasis, 294.

[richocera hyemalis, 598.

Frichoda, 411.

Triclina spiralis, 568 .

Trochell, Dr., on mollinsca, 539.

Troughs for exhibiting circulation, 197

Truffles, 302.

Tsetse-fly of Africa, 596 .

Tnbercibarium, 302.

Tubicola, 5 i4.

Tubiporidæ, 4 S9.

Tubularidæ, 473.

Tnbularia Dumortierii, 497.

Tunicata, 532 .

Turbellaria, 561.

Turbo marmoratns, tongue of, 543 .

ULVE, development, 271.

Uredo, 291.

Urinary salts, 149.

Uromyces, 291.
Urticating organs in actinæ, 467

— in mollusea, 544.

VALENTINE'S knife, 201.

Vallisneria, 321.

Varley's animaleulæ cage, 195.

- on chara, 315.

Vauclieria, 274.

Vegetable structure, 323.

- cell, 255 .

- cellular tissue, 360 .

kingdom, 255.

256.

— preparation of, 359 .

- preparation of tissues, 359

- starch, 341.

- vascular tissue, 324.

Velutina, palate of, 540 .

Vertebrata, 654.

Vibrio spirilla, 412 .

Viewing objects, directrons for, 160

Virchow on trichina, 569 .

Volvox globator, 275 .

Vorticellidæ, 445.

WALKER's trough for fish, 197.

Warington's microscope, 106.

Wasp, 618.

tongue of, 582 .

Water-beetle, 625 .

Water-microscope, 5 .

Water-mites, 643.

Wenham on photography applied to microseopic drawing, 156.

- on the binocular microscope, 113.

- on object-glasses, 64 .

- on a parabolic reflector, 179 .

West, Tuffen, on feet of flies, 58 .

Wheat, portion of hrsk of, 340 .

- flonr, adulterations of, 344 .

Wheeler's microscopes and eabinets, 110.

list of objects, 253

Whelk, palate of, 537 .

Whirligig, 625 .

Whitney, W. U., on the tadpole, 683 .

Williamson's collecting stick, 193.

Wing-shell, 530 .

Wollaston's lenses, 36 .

Wood, cutting sections of, 206.

Woodward, Mr., on polarised light, 139.

Wool, 354 .

Wright, Dr., on alcyonida, 524.

XANTHIDIN, 2S2, 441.

YEAST-PLANT, 299.

Yew, section of, 356 .

ZOOFHYTES, canal-bearing, 355 .

- a fresl-water group, 463.

- preservation of polypidoms of, 509. skeletons of, 490

Zygoceros rhombus, 432 .

Woodfall and Kinder Printers, Milford Lane, Strand, London, W. 

t 

QH

205

Hogg, Jabez

$\mathrm{H} 72$

The microscope

1867

BioMed

PLEASE DO NOT REMOVE CARDS OR SLIPS FROM THIS POCKET

UNIVERSITY OF TORONTO LIBRARY 
